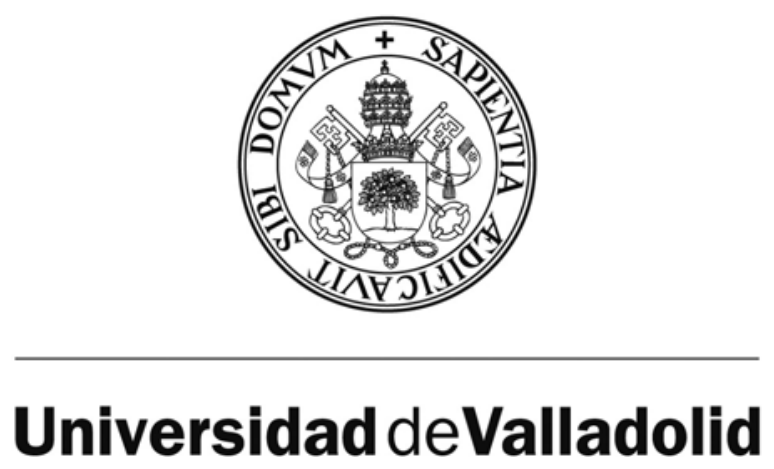

FACULTAD DE CIENCIAS

DEPARTAMENTO DE FÍSICA DE LA MATERIA CONDENSADA MINERALOGÍA Y CRISTALOGRAFÍA

TESIS DOCTORAL:

\title{
IMPLEMENTACIÓN DE UN SISTEMA DE EVALUACIÓN SENSORIAL ELECTRÓNICO PARA EL CONTROL DE CALIDAD DE VINOS.
}

Presentada por Natalia Prieto Castañeda para optar al grado de doctora por la Universidad de Valladolid

\author{
Dirigida por: \\ María Luz Rodríguez Méndez
}





\title{
Tabla de Contenídos
}

\author{
Implementación de un Sistema de \\ Evaluación Sensorial electrónico para \\ el Control de Calidad de Vínos
}

Página

Capítulo

Introducción

1.1. INTRODUCCIÓN

$\begin{array}{ll}\text { 1.2. OBJETIVOS } & 7\end{array}$

1.3. EL VINO 10

1.3.1. Gestión del oxígeno y capacidad antioxidante de los vinos 12

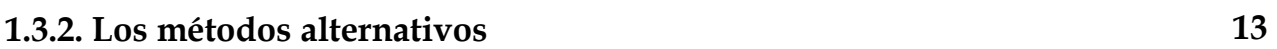

1.4. EL PANEL DE CATA 17

1.4.1. La nariz electrónica 17

$\begin{array}{ll}\text { 1.4.1.1. Los sensores } & 19\end{array}$

1.4.1.2. Los sistemas de inyección $\quad 25$

1.4.1.3. Tratamiento de los datos $\quad 27$

1.4.1.4. Narices electrónicas comerciales $\quad 36$

1.4.1.5. Combinación de sistemas 39

1.4.1.6. Aplicaciones de las Narices electrónicas 39

1.4.2. La lengua electrónica $\quad 42$

1.4.2.1. Tratamiento de datos $\quad 45$

1.4.2.2. Aplicaciones de la lengua electrónica 48

1.4.3. El ojo electrónico $\quad 49$

1.4.3.1. Percepción del color $\quad 50$

1.4.3.2. Medición del color $\quad 53$

$\begin{array}{lc}\text { 1.5. REFERENCIAS } & 57\end{array}$ 
Capítuloll

Desarrollo experimental

2.1. ANÁLISIS FÍSICOS-QUÍMICOS DEL VINO 76

2.1.1. Parámetros estándar $\quad 76$

$\begin{array}{ll}\text { 2.1.2. Cromatografía de Gases } & 78\end{array}$

2.1.2.1. Condiciones experimentales para la GC-MS 83

2.1.2.2. Condiciones experimentales para la GC-O 86

2.2. PANEL DE CATA ELECTRÓNICO 91

2.2.1. Nariz electrónica $\quad 91$

2.2.2. Lengua electrónica $\quad 102$

2.2.3. Ojo electrónico 106

2.3. ANÁLISIS DE LOS DATOS 106

$\begin{array}{ll}\text { 2.3.1. Pretratamiento señales nariz electrónica } & 108\end{array}$

2.3.2. Pretratamiento señales lengua electrónica 111

2.3.2.1. Selección de variables para la lengua electrónica 113

2.3.3. Pretratamiento datos espectroscopía, GC-MS, GC-O 116

2.3.4. Análisis exploratorio $\quad 116$

2.3.5. Validación cruzada $\quad 119$

2.3.6. Análisis multi-way: Tucker3 122

2.3.7. Anova-PCA 125

2.3.8. Implementación 132

2.4. REFERENCIAS 132

Capítulo Ill

Evaluación de la influencia del oxígeno en las características organolépticas de un vino tinto mediante un panel de cata electrónico

3.1. GENERALIDADES 139

3.1.1. Revisión teórica $\quad 141$

3.1.1.1. Efectos del oxígeno en el color del vino 141

3.1.1.2. Efectos del oxígeno en el gusto del vino 143

3.1.1.3. Efectos del oxígeno en el aroma del vino 143

3.1.1.4. Aplicación del tratamiento de microoxigenación 144

3.1.1.5. Efecto del oxígeno durante el envejecimiento en botella 145

3.1.1.6. Incorporación del oxigeno a través de los tapones 146 sintéticos porosos

3.2. DESARROLLO EXPERIMENTAL 149

3.2.1. Características de las muestras de vino $\quad 149$

3.2.2. Características de los tapones sintéticos porosos 150

3.2.3. Medidas del oxígeno durante el envejecimiento en botella 154

3.2.4. Medidas de los compuestos polifenólicos 155 
3.3. RESULTADOS

3.3.1. Análisis Exploratorio

3.3.1.1. Coordenadas CIELab.

3.3.1.2. Nariz electrónica. $\quad 159$

$\begin{array}{ll}\text { 3.3.1.3. Lengua electrónica. } & 161\end{array}$

3.3.1.4. Panel de Cata. $\quad 162$

3.3.2. Análisis Supervisado 164

3.3.2.1. Análisis Cualitativo: Clasificación (PLS-DA) 164

3.3.2.2. Análisis Cuantitativo: PLS2 168

3.4. CONCLUSIONES 178

3.5. REFERENCIAS 179

Capitulo IV

Evaluación, caracterización y análisis comparativo de vinos tintos tratados con sistemas alternativos a la barrica de roble

4.1. GENERALIDADES 183

4.1.1. Revisión teórica 184

4.1.1.1. Técnicas alternativas para el envejecimiento del vino 185

4.1.1.2. Efectos de la madera sobre el vino 186

4.1.1.3. Compuestos cedidos por la madera al vino 189

4.1.1.4. Tendencias actuales de consumo y el posicionamiento de 192 métodos alternativos

4.2. DESARROLLO EXPERIMENTAL 195

4.2.1. Preparación de las muestras de vino 195

4.3. RESULTADOS 197

4.3.1. Parámetros Químicos 197

4.3.2. Coordenadas CIELab 200

4.3.3. Cromatografía de Gases Masas 204

4.3.4. Cromatografía de Gases-Olfatometría 207

4.3.5. Nariz electrónica 213

4.3.5.1. Análisis exploratorio. PCA 214

4.3.5.2. Análisis supervisado. PLS-DA 216

4.3.6. Correlaciones entre sistemas 221

4.3.6.1. Regresión Lineal por mínimos cuadrados PLS2 221

4.3.6.2. Modelo Tucker3 227

4.3.6.3. ANOVA-PLS 236

4.3.7. Tratamientos de datos complementarios 240

4.4. CONCLUSIONES 249

4.5. REFERENCIAS 251 


\section{Capítulo V}

Estudio de los efectos de la fermentación en barrica en la composición volátil de vinos blancos.

5.1. GENERALIDADES

5.1.1. Revisión teórica

5.2. DESARROLLO EXPERIMENTAL 261

$\begin{array}{ll}\text { 5.2.1. Preparación de las muestras de vino } & 261\end{array}$

5.3. RESULTADOS 262

5.3.1. Parámetros Químicos 262

5.3.2. Coordenadas CIELab 265

5.3.3. Cromatografía de Gases Masas 268

5.3.4. Olfatometría 270

5.3.5. Nariz electrónica $\quad 272$

5.3.5.1. Análisis supervisado. PLS-DA 274

5.3.5.2. Regresión Lineal por mínimos cuadrados PLS2 275

5.4. CONCLUSIONES 275

5.5. REFERENCIAS 276

Capitulo VI

Conclusiones

6.1. CONCLUSIONES 

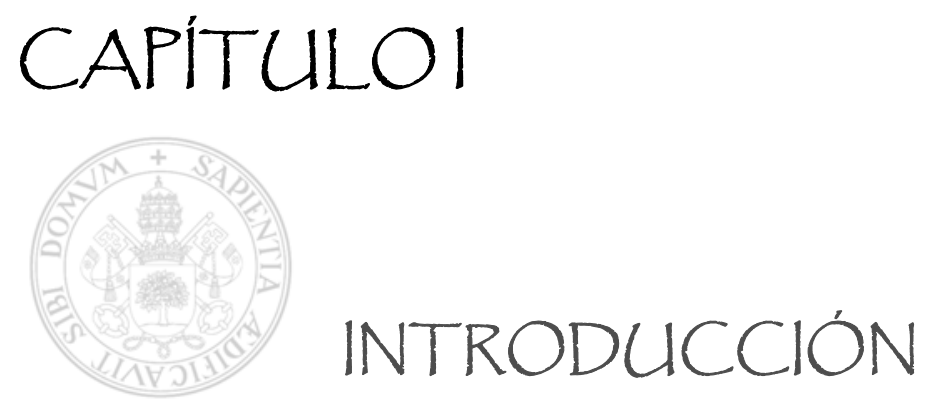

Página

1.1. INTRODUCCIÓN

1.2. OBJETIVOS

1.3. EL VINO

1.3.1. Gestión del oxígeno y capacidad antioxidante de los vinos

12

1.3.2. Los métodos alternativos

1.4. EL PANEL DE CATA

1.4.1. La nariz electrónica

1.4.1.1. Los sensores

1.4.1.2. Los sistemas de inyección

1.4.1.3. Tratamiento de los datos

1.4.1.4. Narices electrónicas comerciales

1.4.1.5. Combinación de sistemas

39

1.4.1.6. Aplicaciones de las Narices electrónicas

1.4.2. La lengua electrónica

$\begin{array}{ll}\text { 1.4.2.1. Tratamiento de datos } & 45\end{array}$

$\begin{array}{ll}\text { 1.4.2.2. Aplicaciones de la lengua electrónica } & 45 \\ \end{array}$

1.4.3. El ojo electrónico $\quad 49$

1.4.3.1. Percepción del color $\quad 50$

1.4.3.2. Medición del color 



\section{Implementación de un sistema de evaluación sensorial electrónico para el control de calidad de vinos}

\subsection{INTRODUCCIÓN}

Esta memoria se encuadra en los trabajos que realiza el Grupo de investigación en sensores de la Universidad de Valladolid, cuya principal línea de investigación es el desarrollo de métodos sensoriales electrónicos (sistemas olfativos, y gustativos electrónicos) para la valoración organoléptica y caracterización de alimentos, y en particular, vinos.

El análisis de vinos mediante sistemas olfativos electrónicos, tiene un alto grado de complicación debido a la alta complejidad de la composición de los vinos, en la que participan cientos de compuestos. En relación con esta evaluación es importante considerar que las diferencias sutiles (pero importantes) existentes en las propiedades organolépticas entre distintos vinos, según su calidad, denominación de origen D.O., cosecha, tipo de uva, etc. son consecuencia de variaciones en la composición de una serie de compuestos que se encuentran en 
una proporción minoritaria. Por otra parte el vino debe de ser considerado como un producto "vivo", ya que su composición química y por tanto sus características organolépticas varían con el tiempo.

Tradicionalmente se utilizan dos métodos para conocer la evolución y características del vino: el análisis sensorial (panel de expertos) y el análisis físicoquímico convencional. Estos procedimientos, aunque de un valor evidente, tienen ciertas limitaciones. Por ejemplo, no es obvio que una caracterización química, por rigurosa que sea, facilite información que pueda ser correlacionada con la apreciación sensorial humana; tampoco es fácil disponer de un comité de cata que continuamente pueda valorar el proceso de elaboración de un vino. Estos problemas, junto con la necesidad de la industria de disponer de métodos de control de calidad rápidos, baratos, objetivos, precisos y de fácil interpretación, ha animado el desarrollo de sistemas de análisis sensorial electrónico.

El grupo de Sensores de la Universidad de Valladolid es pionero en este campo, y en el momento de mi incorporación al grupo, había desarrollado un primer instrumento específico para el análisis de aromas de vinos. En el caso concreto de la "nariz electrónica" se había diseñado un sistema basado en una red de sensores resistivos, sensibles a la composición aromática del vino y capaz de eliminar las interferencias causadas por el agua y el etanol. Respecto a la "lengua electrónica" se estaba diseñando una red de sensores modificados con diversos materiales electroactivos, que permite discriminar vinos y obtener datos relacionados con la composición fenólica. Con la nariz electrónica en aquel momento había sido posible diferenciar vinos monovarietales elaborados con uvas provenientes de diferentes Denominaciones de Origen (D.O.) españolas y envejecidos bajo idénticas condiciones (en barrica de roble americano con tostado medio); también había sido posible discriminar vinos elaborados en diferentes tipos de barricas, así como seguir la elaboración y evolución de los vinos.

Es este estado de cosas, se me encomendaron dos tareas fundamentales. Por una parte, hacerme cargo de la línea de investigación en el campo de la nariz electrónica y colaborar en las investigaciones del grupo en el campo del análisis organoléptico de vinos. En esta línea, se me atribuyó la tarea avanzar en los métodos quimiométricos de tratamiento de datos que permitieran mejorar la discriminación y clasificación de muestras así como tratar datos provenientes de redes híbridas de nariz electrónica, lengua electrónica y ojo electrónico.

Es obligado decir que las investigaciones llevadas a cabo en esta Tesis Doctoral han sido financiadas a través de dos proyectos del Plan Nacional del Ministerio de Educación y Ciencia relacionados con la evaluación de sistemas alternativos a la barrica de roble (AGL2006-05501/ALI) y el análisis de la capacidad antioxidante de los vinos (AGL2009-12600/ALI). Además, hemos contado con la colaboración de las Estaciones Enológicas de la Rioja (Haro) y de Castilla y León 
(Rueda) que han realizando los análisis químicos a las muestras de vino y han participando en la asesoría enológica. Citar también las facilidades y muestras aportadas por la industria Nomacorc y el laboratorio investigación enológica de la E.T.S. Ingenierías Agrarias de Palencia y por la cooperativa Agrícola la Castella (Rueda).

Es conveniente especificar que el número de muestras que dispondremos para este estudio no es el mejor desde el punto de vista estadístico. Contar con un amplio número de vinos elaborados especialmente para una investigación es muy complicado y más aún si tenemos en cuenta que el vino es un material en constante evolución. Para compensar esta dificultad decir que, las muestras que manejamos son representativas de cantidades relativamente importantes de vino y además dentro de cada estudio se aplicarán diferentes métodos para establecer comparaciones entre modelos resultantes. En todo momento se ha intentado tener un error experimental mínimo, realizar un amplio número de repeticiones y ser cauteloso con las conclusiones que se puedan alcanzar con este estudio.

\subsection{OBJETIVOS}

El objetivo principal de esta Tesis doctoral es profundizar en el conocimiento de los sistemas olfativos electrónicos y su aplicación al análisis de vinos. Con este fin general, se han llevado a cabo investigaciones con dos enfoques complementarios. El primero de ellos se basa en establecer la capacidad de nuestra nariz electrónica (de forma aislada o de forma conjunta con nuestro panel de cata electrónico) para discriminar y clasificar vinos de interés industrial. Por otra parte, como hemos dicho anteriormente otro de nuestros objetivos ha sido desarrollar tratamientos quimiométricos que nos permitieran validar nuestras medidas electrónicas y aumentar la capacidad de discriminación y clasificación de los sistemas electrónicos desarrollados en nuestro grupo.

Este objetivo general, se alcanza a través de los siguientes objetivos específicos:

Evaluación de la capacidad de discriminación de los sistemas electrónicos frente a tres conjuntos de muestras de interés industrial.

En primer lugar, se analizará la capacidad de una nariz electrónica y de un panel de cata electrónico para evaluar el estado de oxidación y contenido fenólico de vinos tintos, preparados mediante diferentes métodos que incluyen variaciones en la maceración, en la microoxigenación durante su elaboración, y en la nanooxigenación 
controlada mediante el uso de tapones con diferentes capacidades de transmisión del oxígeno durante su envejecimiento en botella.

En esta fase, los datos serán tratados mediante técnicas convencionales: la evaluación de la capacidad de diferenciación entre un conjunto muestras del panel de cata electrónico se realizarán mediante el método de análisis exploratorio PCA (análisis de componentes principales). Un análisis supervisado PLS-DA (análisis discriminante basado en la técnica de regresión por mínimos cuadrados) valorará la capacidad del sistema para clasificar los vinos de acuerdo con las diferentes problemáticas planteadas en esta investigación. Finalmente la aplicación del método PLS2 establecerá correlaciones con los métodos analíticos tradicionales y datos químicos relacionadas con el nivel de polifenoles y los niveles de oxigeno.

$\checkmark$ En segundo lugar, se evaluará la capacidad de una nariz electrónica para discriminar vinos envejecidos mediante el método tradicional en barricas de roble de vinos y por métodos alternativos (en depósitos de acero a los que se añaden trozos de madera a la vez que se microoxigenan para simular la difusión de oxígenos a través de las poros). Además de la aplicación del panel de cata electrónico, para esta valoración se utilizarán técnicas de cromatografía de gases y olfatometría para cuantificar los compuestos volátiles significativos en los vinos y su evolución durante su elaboración.

$\checkmark$ Estudiar el efecto de la fermentación de vinos blancos en barricas de roble y realizar una comparación de los resultados obtenidos con la nariz electrónica con aquellos provenientes de los métodos analíticos tradicionales. Finalmente, mediante los diferentes algoritmos de reconocimiento de patrones, evaluar la capacidad de discriminación de nuestro sistema de análisis sensorial. Incidiendo en la valoración asociada a las variaciones del color ("ojo electrónico") tan interesantes en los vinos blancos.

\section{Implementación de nuevas herramientas para el tratamiento de datos}

Establecer correlaciones entre los datos proporcionados por los sistemas olfativos electrónicos y los resultados que proporcionan las técnicas convencionales de análisis como la cromatografía de gases y la olfatometría. Para ello se aplicarán nuevos métodos como el multiway (Tucker3) y ANOVA-PLS cuya función será evaluar los efectos de determinados parámetros experimentales y al mismo tiempo la capacidad del sistema electrónico para seguir las variables significativas en el marco de cada experimento. 
$\checkmark$ Aplicar el método de los algoritmos genéticos (PLS-GA) para reducir el alto número de variables derivadas que hacen parte de las señales electroquímicas provenientes de medidas efectuadas con una lengua electrónica, para así facilitar el modelamiento de los datos que arroja el sistema y mejorar su capacidad de clasificación.

Establecidos concisamente los puntos importantes del trabajo, los diferentes capítulos del mismo tratan de dar respuestas a todos los objetivos expuestos. Se ha creído conveniente estructurar la exposición del trabajo en cinco capítulos de acuerdo con el siguiente esquema:

Capítulo I. Introducción. Esta primera parte comienza con una presentación de los problemas enológicos que plantea la caracterización organoléptica de vinos y en particular, nos centraremos en los retos que supone la utilización de nuevas tecnologías durante su elaboración y envejecimiento. A continuación se describirán las características generales de un panel de cata electrónico, presentando el estado actual del arte, incidiendo en los problemas particulares que presenta el análisis de vinos. Además se incluye un resumen general de los diferentes modelos y trabajos que se han publicado en el área de la quimiometría y reconocimiento de patrones, especialmente aquellos adaptables a los tres sistemas nariz, lengua y ojo electrónico.

Capítulo II. Desarrollo experimental. En este segundo Capítulo se describen los materiales y métodos utilizados en esta Tesis. Se dedica un espacio a resumir la metodología de nariz, lengua y ojo electrónicos, y las herramientas estadísticas, utilizadas para el análisis de vinos. La descripción particular de las muestras de vino analizadas en nuestra investigación se realizará en los capítulos siguientes.

Capítulo III. Evaluación de la influencia del oxígeno en las características organolépticas de un vino tinto mediante un panel de cata electrónico. Este capítulo contempla la aplicación de un panel de cata electrónico (nariz, lengua y ojos) en el proceso de microoxigenación del vino, en muestras elaborados con la misma variedad de uva pero vinificados con distintas técnicas de extracción (distinto contenido en polifenoles), diferentes grados de microoxigenación, y envejecidos en botella con corchos sintéticos de diferente porosidad.

Capítulo IV. Evaluación, caracterización y análisis comparativo de vinos tintos tratados con sistemas alternativos a la barrica de roble. En esta parte de la memoria se refieren las investigaciones que venimos realizando comparando las 
características organolépticas de vinos tratados con virutas de roble con las de otros vinos equivalentes envejecidos en barrica convencional, en muestras extraídas durante etapas significativas en ambos tipos de procesos. En la valoración organoléptica el elemento principal de análisis ha sido la nariz electrónica. Así mismo, se han utilizado medidas físico-químicas estándar y valoraciones de la fase volátil mediante olfatometría que han permitido profundizar en los mecanismos de elaboración y percepción sensorial.

Capítulo V. Estudio de los efectos de la fermentación en barrica en la composición volátil de vinos blancos. Constituye una investigación adicional que tiene por objeto confirmar el potencial de nuestro sistema de análisis sensorial electrónico para la caracterización de vinos en general y en particular para evaluar las características y evolución de vinos blancos fermentados en barricas nuevas y utilizadas. En el caso de las barricas usadas también se valorará el efecto de la adición de chips de roble, las cuales han sido incorporadas con la idea de paliar el agotamiento de la barrica por su uso precedente. La evaluación de estos procedimientos se realizó a través de caracterizaciones organolépticas (aroma y color) en muestras extraídas durante las primeras etapas de la fermentación. En esta estimación el elemento principal de análisis han sido medidas de la evaluación de la composición volátil utilizando una nariz electrónica, cromatografía de gases-masas, olfatometría, así como medida colorimétricas complementarias. Así mismo, se han utilizado medidas físicoquímicas estándar y la espectroscopia UV-Visible, técnicas que nos han permitido profundizar en los mecanismos de elaboración y en la percepción sensorial final de esta variedad de vinos.

Capítulo VI. Conclusiones. En este capítulo se recogen las conclusiones más relevantes alcanzadas en esta investigación.

\subsection{EL VINO}

Al ser objetivo de esta investigación desarrollar y validar nuevas herramientas para el análisis de vinos, consideramos importante dedicar un apartado a describir algunos aspectos interesantes de esta muestra, extraordinariamente compleja con notorias dificultades de manejo, y de gran interés en nuestro país, y específicamente en la región de Castilla y León. Por otra parte, esta descripción nos va a permitir matizar conceptos vinculados al tipo de muestras en nuestra investigación. 
Según el Ministerio de Industria, Turismo y Comercio, en la ley 24/2003 de 10 de julio, de la Viña y del Vino, el vino puede definirse como el producto obtenido exclusivamente por fermentación alcohólica parcial o total de uva fresca, estrujada o no, o de mostos de uva. En otras palabras, esta definición presenta al vino como el producto alcohólico derivado del zumo de uva tras sufrir una transformación enzimática. La fermentación es un proceso bioquímico natural y aparentemente sencillo en el que están implicados diferentes microorganismos, levaduras, bacterias y enzimas. A lo largo de su desarrollo, este proceso sigue los principios y leyes de la Termodinámica y Cinética Químicas, y, como suele suceder en la gran mayoría de los procesos de la Naturaleza, su aparente sencillez intrínseca va acompañada de un elevado grado de complejidad.

Es a partir de aquí cuando, ese mosto fermentado, junto con su posterior y formidable protocolo de maduración y envejecimiento, pasa a ser un casi inagotable reservorio de conceptos científicos, técnicos, sociales y comerciales, que da lugar a numerosas vías de aproximación, todas ellas suficientemente autónomas. Así, un buen "observador" con una copa de vino en la mano, tratará de hallar una respuesta fría, calculada y contrastada acerca de la naturaleza, composición, utilidad, estado sanitario o conveniencia del consumo del caldo. En cambio, un "degustador" añadirá aspectos relacionados al placer de su consumo, asumiéndolo a un cierto entorno social. Por otra parte, un "catador" experimentado pretenderá dar respuestas según diversos descriptores organolépticos bien definidos, emitiendo una opinión en la que destacará las características favorables y negativas que exaltan las virtudes y defectos del vino, respectivamente. Por último, un "investigador" desarrollará su capacidad de observación y medida, aplicando conocimientos químicos, físicos y biológicos con rigor, que apoyen y justifiquen sus apreciaciones acerca de las propiedades del vino.

Las técnicas vitivinícolas y enológicas persiguen mejorar las características organolépticas de los vinos, que están estrechamente ligadas a su composición y calidad. Algunas de estas técnicas son ampliamente aceptadas y han sido reguladas por organismos internacionales, mientras que otras son objeto de constante controversia.

Describir estas técnicas y estrategias está fuera del ámbito de esta Tesis Doctoral. En esta memoria, nos hemos centrado en dos aspectos.

1. El uso de técnicas de control del oxígeno (microoxigenación o utilización de tapones sintéticos) para mejorar la capacidad antioxidante de vinos y valorar su estado de oxidación durante su elaboración. 
2. La aplicación de técnicas de envejecimiento alternativas a la tradicional barrica de roble, utilizando tanques de acero a los que se añaden trozos de madera con un adecuado grado de tostado.

\subsubsection{Gestión del oxígeno y capacidad antioxidante de los vinos}

Durante los últimos años se ha estudiado en profundidad la acción de los radicales libres en los organismos vivos. Su acción destructora puede ser contrarrestada por sustancias antioxidantes, un amplio grupo de sustancias biológicamente activas que se encuentran ampliamente distribuidas en la naturaleza [1]. El consumo habitual y moderado de vino, especialmente de vino tinto, puede producir efectos beneficiosos para la salud debido a su contenido en polifenoles, particularmente en quercitina y resveratrol. Estudios epidemiológicos relacionan la ingestión de polifenoles y la disminución del riesgo de cáncer y cardiopatía coronaria en humanos. Los polifenoles también modifican la calidad y características organolépticas de los vinos tintos afectan también a su capacidad antioxidante. La contribución de cada componente depende de su poder antioxidante, concentración e interacción con su microambiente [2].

Con el fin de conocer dicha capacidad antioxidante de los vinos se han empleado distintos métodos [3], sin embargo, no existe un método único y los índices obtenidos para una muestra dependen del procedimiento utilizado para evaluarla. Es necesario indicar que la capacidad antioxidante de una mezcla, no está dada solo por la suma de las capacidades antioxidantes de cada uno de sus componentes; también depende del microambiente en que se encuentra el compuesto, ya que los compuestos interactúan entre sí pudiendo producirse efectos sinérgicos o inhibitorios. Los métodos de determinación de la actividad antioxidante se basan en comprobar cómo un agente oxidante induce daño oxidativo a un sustrato oxidable, daño que es inhibido o reducido en presencia de un antioxidante. Las caracteristicas esenciales de cualquier prueba de evaluación de la capacidad antioxidante son un sustrato adecuado para monitorear la inhibición de la oxidacion, un iniciador de la oxidacion (radical libre) y una adecuada medida del punto final de la oxidacion, que puede llevarse a cabo por métodos químicos, instrumentales o sensoriales. A la hora de abordar el estudio de la actividad antioxidante del vino es aconsejable emplear varios métodos, ya que cada uno ofrece información diferente. Hay compuestos antioxidantes que no reaccionan con determinadas especies oxidantes y sí con otras, pudiéndose obtener así valores dispares entre los distintos métodos. La 
actividad antioxidante puede ser evaluada mediante la medida de la capacidad de absorbancia del radical oxigeno ORAC (oxygen radical absorbance capacity) y la capacidad antioxidante equivalentes trolox TEAC (trolox equivalent antioxidant capacity) [3]. Por otro lado, la medida de fenoles mediante el metodo de FolinCiocalteu, basada en una reaccion de oxido-reduccion, también es considerada una forma de medir actividad antioxidante en función del contenido de fenoles [4-7]. Los primeros (ORAC y TEAC) son adecuados para medir capacidad antioxidante en alimentos y fluidos biológicos, mientras que Folin es un indicador de la capacidad antioxidante en función del contenido de polifenoles totales en el alimento, por ello teniendo en cuenta que la actividad antioxidante de los vinos viene dada fundamentalemnte por la composición fenólica, algunos autores emplean esta evaluación como indicadora de la actividad antioxidante de los vinos. Sin embargo, en trabajos recientes se estima que el perfil antioxidante completo de los vinos tintos podría ser establecido por evaluación del ABTS y por algún tipo de método con bioindicador del estrés oxidativo, preferentemente la inhibición de la peroxidación de lípidos. La evaluación del contenido en polifenoles totales (PT) es también recomendable, porque este método evalúa el poder reductor de los vinos [8]. El conocimiento del perfil fenólico de los vinos (fenoles sencillos, flavonoides, proantocianidinas, catequinas, taninos, antocianos, etc. y su relación con la capacidad antioxidante es de gran interés para evaluar las modificaciones que sufrirá en el vino a lo largo de su vida.

Por otra parte, la evolución del vino es extremadamente dependiente de la cantidad de oxígeno que se le aporte durante su proceso de elaboración [9]. Dentro del proceso de asimilación del oxígeno existen dos etapas claramente diferenciadas. La primera de ellas es cuando se producen las operaciones de transferencia de oxigeno durante la fermentación y envejecimiento del vino; la segunda etapa se produce cuando el vino es almacenado en botellas, el vino se encuentra en un medio reductor y la cantidad de oxigeno que recibe y necesita, depende de la eficacia de sellado que pueden llegar a propiciar los tapones.

\subsubsection{Los métodos alternativos}

La crianza de los vinos en barrica persigue varios objetivos que permiten potenciar sus características organolépticas favoreciendo un posterior envejecimiento en botella $[10,11]$. Este proceso redunda en beneficio de una complejidad en su composición química que da lugar a vinos más armoniosos y equilibrados [12-14]. En efecto, durante el envejecimiento de los vinos en barrica, el paso de oxígeno a través de las duelas produce reacciones químicas que lentamente confieren a los vinos estabilidad y una mejora en sus características sensoriales. La duración de esta etapa, el origen de la madera de roble y el volumen de la barrica de envejecimiento, así como su grado de secado y tostado, 
son factores que deberán adaptarse a la personalidad y características intrínsecas de cada vino. Esto implica que la crianza de los vinos debe regularse adecuadamente y por esto es necesario entender en profundidad los numerosos factores que intervienen, desde un punto de vista tecnológico, para el conocimiento de la composición del vino destinado al envejecimiento, las características de acuerdo con las barricas de roble (tipo de madera, espesor, edad y tamaño, quemado, etc.), las condiciones ambientales de la bodega, periodicidad de los trasiegos, etc., y también desde un punto de vista académico la valoración de los componentes químicos fundamentales y el seguimiento de su evolución durante el proceso de envejecimiento $[15,16]$.

Como ya hemos indicado, en la crianza de los vinos en barrica se producen una serie de fenómenos o transformaciones de carácter físico, químico o incluso biológico. Todos estos sucesos se producen de una manera simultánea, por lo que pueden influir unos sobre otros; en términos generales estos procesos deben ser modificados y controlados continuamente por el enólogo de la bodega, para así mejorar las prestaciones buscadas con la crianza.

Los fenómenos que suceden durante la crianza en barrica tradicional pueden resumirse en los siguientes:

- Entrada de aire u oxígeno a través de la madera (duelas) o durante los trasiegos.

- Pérdida de vino a través de la madera o de los trasiegos.

- Precipitación de diversas sustancias del vino en la barrica.

- Cesión de sustancias contenidas en la madera.

- Formación de esteres y transformación de los polifenoles.

Es evidente que toda la etapa de envejecimiento en barrica da lugar al aumento del coste del producto, y en consecuencia un incremento en su precio final en el mercado. Además es un proceso complicado desde el punto de vista de la organización de la bodega y siempre conlleva la inmovilización de un capital. Por estas razones, solamente un porcentaje de su producción suele ser destinado a este tipo de elaboración. De hecho en Francia, país de gran tradición en la crianza de vino, se considera que tan sólo entre el 20 y $25 \%$ de los VCPRD es criado en barricas de roble. También se tiene referencia de otros porcentajes más elevados como en la D.O. Ribera del Duero de esta comunidad en el año 2009, fueron alrededor de un $50 \%$ de la producción total de vino [17]. Un porcentaje mayor de vino reservado para el envejecimiento en barrica es cuestionable y tiene que condicionarse a balances económicos en cada bodega. 
Por esta razón, los productores siempre han buscado alternativas a la crianza del vino en barricas de roble, que fuesen más sencillas y sobre todo más baratas. Las iniciativas en este sentido han sido múltiples y han partido tanto de los países tradicionalmente elaboradores como de aquellos que recientemente han entrado en el mercado vitivinícola con productos de buena calidad. No obstante, la tradición existente en Europa ha supuesto un cierto freno a su implementación, mientras que en los nuevos países productores el empleo de estos métodos alternativos ha sido más ágil. Aún así, se puede afirmar que en la actualidad, la utilización de estas técnicas es amplia en todo orbe, si bien en muchas ocasiones, y como salvaguarda de la imagen tradicional, su práctica permanece oculta al consumidor.

Básicamente las alternativas a la crianza del vino en barricas tienen como condición la necesidad de cubrir dos aspectos. En primer lugar es preciso lograr la estabilización de la materia colorante y la suavización de la astringencia, y en segundo lugar es necesario aportar al vino aromas y los taninos elágicos que complementen sensorialmente al vino. Por esta razón las técnicas alternativas a la crianza en barrica tienen que contemplar dos aspectos fundamentales y complementarios: la microoxigenación y la aromatización con fragmentos de madera de roble [18]. Otras opciones están relacionadas con la fermentación de vinos en barricas viejas a las que se añaden pequeños trozos de madera de roble con un adecuado grado de tostado. Estos procesos un algunos casos implican también una especifica dosis de microoxigenación.

La práctica del envejecimiento con técnicas alternativas hoy día en España se considera fraudulenta, principalmente para los vinos acogidos a D.O., tal y como se recogen en sus reglamentos, por lo que es de gran interés para los Consejos Reguladores poder diferenciar a los vinos tratados con virutas o travesaños de los envejecidos bajo el sistema tradicional.

La posibilidad de que estos métodos alternativos sean aprobados hace necesario investigar en este tipo de elaboraciones [19, 20]. Es de gran interés abordar el estudio de la influencia de parámetros como son las características de los chips (origen, tamaño, tostado, origen), tiempos de contacto, estabilidad de los vinos obtenidos, etc. Es también necesario destacar nuevamente que en la crianza en barricas, otro factor importante es la entrada de oxígeno por las duelas, paredes de madera, o a través del tapón de la barrica [21]. Por esta razón, se debe incluir como variable adicional la utilización de una microoxigenación controlada durante el uso de chips en depósitos de acero inoxidable.

En este capítulo se realizará una valoración de las características del vino durante su permanencia en contacto con la madera de las barricas, así como en el caso de utilizar trozos de madera de roble de diferente tamaño (chips, staves). Igualmente 
significativo, es el estudio posterior de su estabilidad y el seguimiento de la evolución en botella.

El análisis sensorial y el análisis físico-químico convencional, son esenciales para conocer la evolución del vino. Estos métodos, aunque de un valor evidente, tienen ciertas limitaciones. Por ejemplo, no es obvio que una caracterización química, por rigurosa que sea, facilite información que pueda ser correlacionada con la apreciación sensorial humana [22]; tampoco es fácil disponer de un comité de cata que continuamente valore la bondad de la elaboración de un vino. Los problemas de los métodos tradicionales, junto con la necesidad creciente por parte de la industria de disponer de métodos rápidos y objetivos para el control de calidad exigen pensar en la posibilidad de diseñar un nuevo tipo de instrumento que sea capaz de evaluar con máximo rigor las principales características organolépticas de los vinos.

Dados los buenos resultados obtenidos hasta el momento con nuestro sistema de análisis sensorial electrónico, parecía interesante evaluar su potencial aplicación en el análisis de otras características de los vinos asociadas a su envejecimiento. Además, existe un gran interés por correlacionar los resultados obtenidos con este instrumento, con los alcanzados mediante el análisis químico y técnicas como la olfatometría.

Resumiendo，

i) Estamos ante un movimiento de cambio en la elaboración de vinos en diversos países, importante sobre todo por el volumen de sus producciones y por sus precios competitivos.

ii) La defensa a ultranza de unos métodos de producción tradicionales, puede generar falta de competitividad, especialmente en las gamas medias, frente a los que nuestras bodegas deben de estar prevenidas. En los próximos años cada bodega y para cada gama de vinos tendrá que adoptar una estrategia de producción frente a esta competencia (combatiéndola o adecuándose al nuevo sistema).

iii) El conocimiento profundo de los mecanismos que rigen estas elaboraciones alternativas así como la puesta a punto de nuevos sistemas, rápidos y objetivos, que permitan diferenciar los tratamientos que han seguido estos vinos han de ser importantes herramientas en la valoración de estos caldos. 


\subsection{EL PANEL DE CATA ELECTRÓNICO}

Constituye un equipo desarrollado en los últimos 5-10 años por nuestro grupo de investigación que ha venido siendo aplicado a la caracterización organoléptica de varias bebidas y alimentos [23-25] Está formado por conjunción de tres partes complementarias (nariz, lengua y ojo) cuyas valoraciones independientes son mezcladas sinérgicamente a través de los tratamientos de datos quimiométricos. Finalmente, citar también diferentes trabajos realizados por otros grupos de investigación. Estos esfuerzos están encaminados a combinar la nariz y lengua electrónica entorno al análisis de agua, zumos y leche [26, 27], control de calidad y producción alimentos [28, 29].

\subsubsection{La nariz electrónica}

El sentido del olfato es quizás el menos apreciado de los cinco sentidos, y sin embargo, es de gran utilidad en numerosas áreas de la industria, entre ellas en perfumería, automoción, minería, diagnóstico clínico, o en la monitorización medioambiental. El análisis de olores es de particular importancia en la industria de la alimentación, donde junto al sabor y color son los atributos organolépticos que definen la calidad de un producto.

Tradicionalmente, el análisis de las características de un alimento se realiza bien mediante un panel de cata formado por expertos (análisis sensorial) o bien a través del análisis químico estándar. La práctica tradicional que se lleva a cabo para realizar una evaluación sensorial consiste en recurrir a un grupo de personas entrenadas capaces de percibir y distinguir olores (incluso a bajas concentraciones), de caracterizar su intensidad y además, de describir y cuantificar con ambas sensaciones. Disponer de un panel de expertos requiere reunir una serie de 6-15 personas cualificadas y bien entrenadas, lo que resulta caro y además, a pesar de su profesionalidad, puede que en algunos casos se de lugar a un cierto nivel de subjetividad debido al estado físico del experto, o a sus gustos personales [22].

Por otra parte, el análisis químico también tiene un alto grado de dificultad, ya que la composición química de los alimentos es compleja y además, no existe una relación inmediata entre la composición química y las percepciones humanas. Mientras que existen compuestos que no tienen olor o sabor y tienen altas 
concentraciones dentro de una determinada matriz, existen otros que dan lugar a sensaciones intensas aunque estén en bajas concentraciones.

Además, el sentido del olfato es extremadamente sensible, y así, el umbral de detección para muchas sustancias está por debajo de cantidades en unidades de $\mu \mathrm{g} / \mathrm{L}$. Además, para mayor complejidad, la percepción humana depende de interacciones mutuas entre estos compuestos (sinérgicas, aditivas, antagónicas o compensatorias), lo que hace muy complejo correlacionar los resultados de un análisis químico con las percepciones humanas [30].

Como se mencionó en la introducción de esta memoria, los problemas de los métodos tradicionales, junto con la necesidad creciente por parte de la industria de disponer de métodos para el control de calidad rápidos, baratos y objetivos, llevaron a pensar en la posibilidad de diseñar un nuevo tipo de instrumento que fuese capaz de emular al sistema olfativo humano. Para lograrlo, estos sistemas deben cumplir dos requisitos: en primer lugar el aroma debe analizarse de forma conjunta sin tener que ser separado en sus componentes (como ocurre en la cromatografía), y además, el sistema tiene que ser capaz de detectar, discriminar y cuantificar olores, de forma rápida y objetiva.

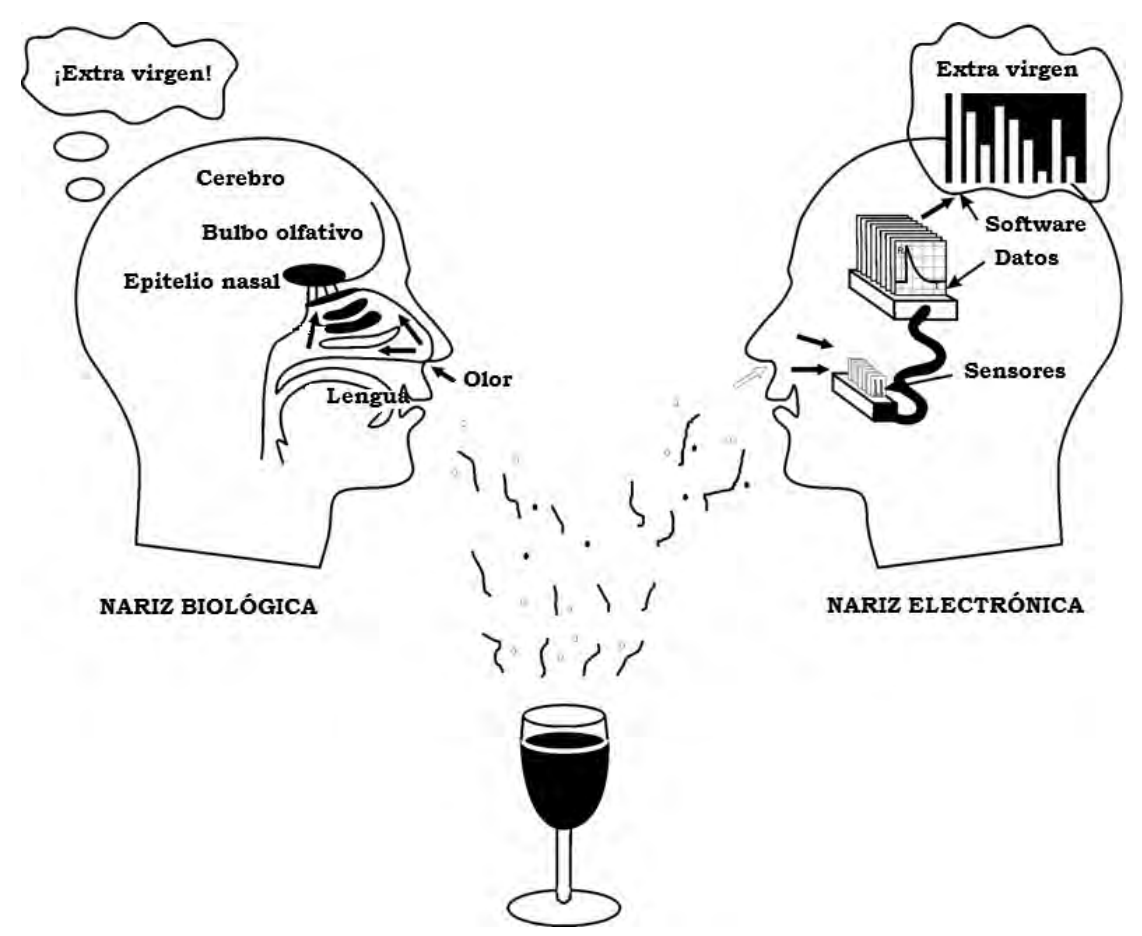

Figura 1.1. Esquema comparativo de una nariz electrónica

El primer sistema capaz de analizar aromas de forma artificial se diseñó en la Universidad de Warwick en 1982 y recibió el nombre de nariz electrónica [31]. Desde entonces numerosos grupos de investigación industriales y académicos trabajan en este campo. Se han desarrollado cientos de narices electrónicas 
diferentes que se han aplicado a campos muy diversos. Estos trabajos se han recogido en diferentes libros y revisiones que se han publicado recientemente [32-40].

Una nariz electrónica consta de una red de sensores, con especificidad parcial (realizan una función similar a los receptores olfativos inespecíficos de la pituitaria), que reaccionan con los olores y producen una señal que pueda ser amplificada y medida; las señales producidas por los sensores son procesadas por un software de reconocimiento de patrones, capaz de reconocer y clasificar los olores (de forma análoga a como lo hace el cerebro). Además, una nariz electrónica necesita de un hardware que permita exponer los sensores a los aromas de forma controlada y reproducible, así como registrar las señales producidas por los sensores [41, 42]. En la Figura 1.1. se muestra un esquema general de una nariz electrónica.

En este sentido, podemos afirmar que el uso que comúnmente se hace para el rotulo "nariz electrónica" se hace con el fin de explicar más fácilmente la mayoría de las propiedades que tienes este dispositivo. Este apelativo no debería ser entendido como una reproducción exacta de la nariz humana; significa más bien que existe una analogía en el funcionamiento de ambos sistemas.

Muchos autores sugieren utilizar el término "nariz electrónica" cuando se quiera hacer una referencia a la primera denominación histórica que le fue asignada a este dispositivo. Sin embargo, estos autores argumentan que actualmente es más conveniente hablar de una red de sensores de gases o de aroma, que además es un título que describe, desde un enfoque más científico, las propiedades de este sistema. Por simplificación textual nosotros abundaremos en la denominación histórica aún entendiendo la mayor precisión del término "red de sensores" [43].

Hoy día, el diseño clásico de una nariz electrónica con una red de sensores químicos, se ha extendido también a nuevas tecnologías como la espectrometría de masas (MS) y la espectrometría de movilidad de iones (IMS).

\subsubsection{Los sensores}

Los sensores son dispositivos fabricados con un material activo que se deposita en forma de película sobre un sustrato. Cuando el material sensible entra en contacto con los volátiles responsables de los olores, se produce una interacción física/química que da lugar a un cambio en alguna de sus propiedades. Así, pueden registrase cambios en las propiedades ópticas (sensores ópticos), en las 
propiedades electroquímicas (sensores electroquímicos), en la masa (sensores másicos), etc. [40-46].

Uno de los tipos de sensores más utilizados en narices electrónicas son aquellos en los que la interacción gas-sensor produce un cambio en la resistividad del material sensible [47-51]. También se han usado extensamente sensores másicos, basados en resonadores de cuarzo o cantilevers micromecanizados [52-55] o sensores ópticos en diferentes narices electrónicas [34, 56, 57].

Otra subdivisión dentro de los sistemas de detección, consiste en los dispositivos basados en biosensores. En este caso, se incorpora un elemento biológico sensible posicionado cerca del transductor para aumentar la sensibilidad y especificidad del sistema hacia un analito objetivo $[58,59]$.

En el campo de los sensores basados en resonadores de cristal de cuarzo podemos nombrar aquellos del tipo SAW (onda acústica de superficie), y BAW (onda acústica de volumen) dentro de la cual se encuentra la QCM (Microbalanza de cristal de cuarzo). Este tipo de sensores han sido utilizados especialmente para detectar explosivos, hidrocarburos y alcoholes. Con este tipo de sensores se han conseguido resoluciones en el cambio de la señal característica en pico gramos, lo que ha motivado a extender cada vez más su aplicación a la evaluación de diversas características de alimentos [60-63].

Respecto a los sensores ópticos sensores, se ha desarrollado hasta el momento dispositivos colorimétricos con aplicaciones en la identificación de gases nocivos, y verificación de pureza de un amplio tipo de gases en industrias químicas. Hasta el momento este tipo de sensores han demostrado buena selectividad en la detección de monóxido de carbono, sulfuro de hidrogeno, amoniaco y gases similares, sin embargo son sensores que dependen bastante de las condiciones del entorno como la temperatura y la humedad $[64,65]$. Dentro de esta categoría se hace cada vez más referencia a los sensores infrarrojos y basados en fibras ópticas. Habitualmente este tipo de tecnología estaba relacionada con un instrumento como tal, sin embargo la miniaturización y el diseño de dispositivos micromecanizados consigue que sean considerados también como sensores [66].

Como hemos comentado, los sensores más utilizados en narices electrónicas, están basados en sensores resistivos y dentro de esta categoría los más utilizados son los sensores basados en Óxidos Metálicos Semiconductores (MOX) [67-71], aunque existen numerosos trabajos que utilizan otros materiales como nanotubos de carbono [72-78], polímeros conductores [79-82] o ftalocianinas [83, 84].

Centrándonos en los sensores basados en MOX, decir que estos materiales se usan desde 1960 como sensores de gases en las alarmas de incendios [85]. Consisten en un sustrato cerámico cubierto de una película del óxido 
semiconductor. Este óxido se puede depositar mediante diversas técnicas como la evaporación, la técnica CVD (Chemical Vapor Deposition), las técnicas de spray, etc. El óxido metálico puede ser de tipo n (óxido de zinc, de estaño, dióxido de titanio $\mathrm{u}$ óxido de hierro) y responde a compuestos oxidantes, o de tipo $\mathrm{p}$ (óxido de níquel o de cobalto) y a gases reductores. También se han utilizado otros óxidos como el óxido de wolframio y perovsquitas. Además, los sensores están provistos de un sistema de calentamiento, ya que su temperatura óptima de funcionamiento está entre 200 y $650^{\circ} \mathrm{C}[47,86]$.

Para conseguir sensores con diferente selectividad hacia distintos compuestos químicos, la película de óxido metálico se dopa con metales nobles catalíticos como platino o paladio. También es posible variar su selectividad modificando el tamaño de grano del semiconductor policristalino.

La Figura 1.2. ilustra la estructura básica de un sensor MOX de la serie TGS2600. Como se aprecian en esta figura la estructura del sensor consiste en la base sobre la cual se encuentra el elemento de detección que contiene material de sensible, con una resistencia calefactora que regula automáticamente su temperatura óptima de funcionamiento; todo el dispositivo está protegido por una cápsula que permite el paso de los gases al interior de sensor y previene su contaminación.

\section{Principio de operación de los sensores MOS}

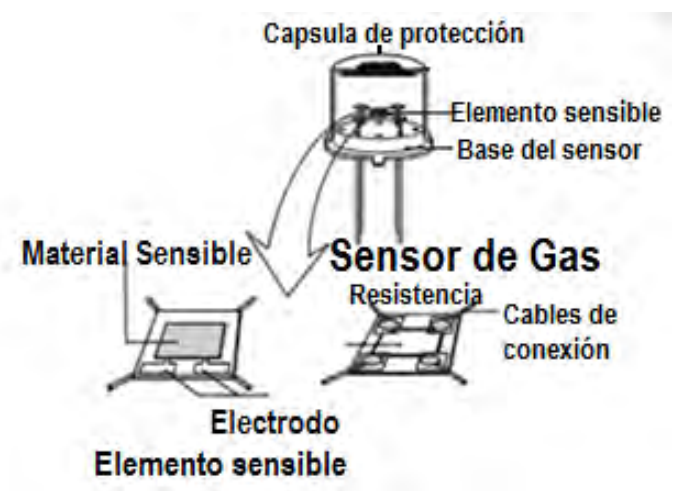

Figura 1.2. Estructura del sensor

Los detalles en profundidad sobre los sensores químicos y su principio de funcionamiento pueden ser consultados en las referencias que se citan a continuación [34, 87-89]. A manera de resumen, el principio de detección de gases de estos sensores se basa en una reacción mediada por el óxido de la superficie y el oxígeno ionizado adsorbido con las moléculas oxidantes o reductoras de la muestra que está siendo detectada. La respuesta de los sensores es la medida del cambio en la resistencia entre dos electrodos como un resultado de las reacciones químicas en la superficie del semiconductor de óxido metálico. 
Este mecanismo de detección se representa en la Figura 1.3.

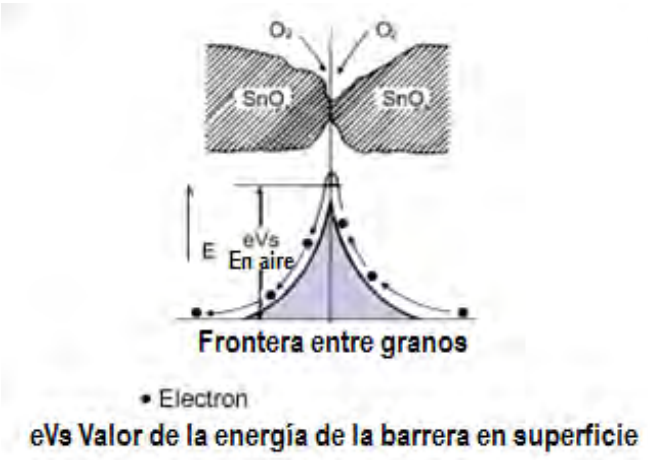

Modelo del sensor en ausencia de gases

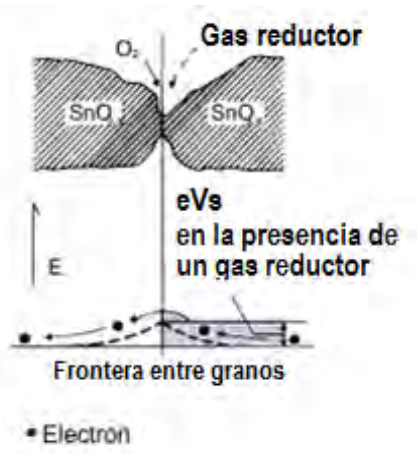

Modelo del sensor en un gas reductor

Figura 1.3. Interacción del sensor con la atmósfera. Tomada de [85].

En una atmósfera oxidante los átomos de oxígeno (resultantes de la descomposición de las moléculas de oxígeno en el ambiente) u otros aceptores de electrones se adsorben en la región superficial y atrapan los electrones libres de la banda de conducción del material semiconductor; por lo tanto, la resistencia del dispositivo se incrementa. En el caso contrario, en una atmósfera reductora, los átomos de oxígeno adsorbidos reaccionan con las moléculas reductoras presentes en el medio, liberando los electrones atrapados en el material y de esta manera la resistencia disminuye. La señal de salida se obtiene por un cambio en la conductividad del óxido causado por la reacción entre el oxígeno adsorbido y las moléculas entrantes en la capa sensible.

La relación entre la resistencia del sensor y la concentración del gas reductor puede ser descrita para ciertos niveles de concentraciones en gases, a través de la siguiente ecuación:

$$
R_{s}=A[C]^{-\alpha}
$$

Donde $\quad R_{\mathrm{s}}$, resistencia eléctrica del sensor

A, constante

[C] concentración del gas

a constante experimental obtenida en función del gas evaluado

Hemos de notar que el valor de $a$ depende de la naturaleza del gas de entrada para cada material sensorial. Cuando se utilizan semiconductores tipo $n$ como $\mathrm{SnO}_{2}$ o $\mathrm{WO}_{3}$, el factor a determinado empíricamente para el $\mathrm{O}_{2}$ es $1 / 2,1$ para el $\mathrm{NO}_{2}$ al igual que para el $\mathrm{O}_{3}$ y alrededor de $-1 / 2$ para gases reductores como el $\mathrm{H}_{2}$ y CO [87-91]. 


$$
\begin{gathered}
1 / 2 \mathrm{O}_{2}+\left(\mathrm{SnO}_{2-x}\right)^{-} \rightarrow \mathrm{O}^{-} \mathrm{ad}\left(\mathrm{SnO}_{2-x}\right) \\
\mathrm{CO}+\mathrm{O}^{-} \operatorname{ad}\left(\mathrm{SnO}_{2-x}\right) \rightarrow \mathrm{CO}_{2}+\left(\mathrm{SnO}_{2-x}\right)^{-} \\
\text {-electrón libre }
\end{gathered}
$$

Figura 1.4. Esquema de la reacción entre el CO y el oxigeno adsorbido en la superficie del material sensible

Los sensores MOX dominan el mercado de las aplicaciones por su bajo coste y su uso en un amplio rango de posibilidades. Desde hace ya varias décadas existen un número importante de compañías que se encargan de la comercialización de este tipo de sensores; entre ellas podemos nombrar: Figaro Inc. (Japón), Capteur and Analyser (UK), Sinostone Inc (China), Fis Inc (Japón), Nemoto (Japón), Microsens (Suiza), Micromechemical SA (Suiza), etc.

Si bien estos sensores están muy desarrollados (existen muchos tipos diferentes con diferentes sensibilidades), presentan el inconveniente de que, al trabajar a altas temperaturas, requieren un gasto adicional de energía. Por otra parte, estos sensores son altamente sensibles a compuestos como el etanol, lo que supone un problema por ejemplo en el caso del análisis de aromas de vinos donde el etanol enmascara la respuesta de los sensores [92].

Las investigaciones actuales entorno a los sensores MOX van dirigidas a la configuración de su estructura en nanogranos, nanocintas, o nanohilos. Este tipo de nanoestructuras tienen como ventaja principal poseer una relación superficie/volumen que permite aumentar el número de sitios activos de la superficie sensible. En relación a los sensores análogos microestructurados, la sensibilidad de este tipo de sensores puede aumentar incluso en varios órdenes de magnitud. Este tipo de estructuras además se pueden obtener mediante diferentes técnicas, entre ellas por ejemplo electrospinning o sol-gel [94, 95, 96, 97].

Como resultado de los nuevos avances alcanzados, estas estructuras se consideran una plataforma para la próxima generación de los sistemas de evaluación sensorial electrónica: las nano-narices. Uno de los esfuerzos con mejores resultados hasta el momento es la microrred de sensores KAMINA (KArlsruhe Micro NAse) basada en nanohilos distribuidos al azar con contacto entre sí y que forman múltiples caminos para la detección del analito [93]. Un esquema de las propiedades estructurales y funcionales de esta nueva red es reportado en la Figura 1.5. Sin embargo, a pesar de lo motivante que resultan estas mejoras en las estructuras de óxido de metal nanométricas, todavía existe una brecha tecnológica entre la elaboración en un laboratorio de estas piezas, y su fabricación a gran escala, al igual que su capacidad de operar en un determinado entorno. 


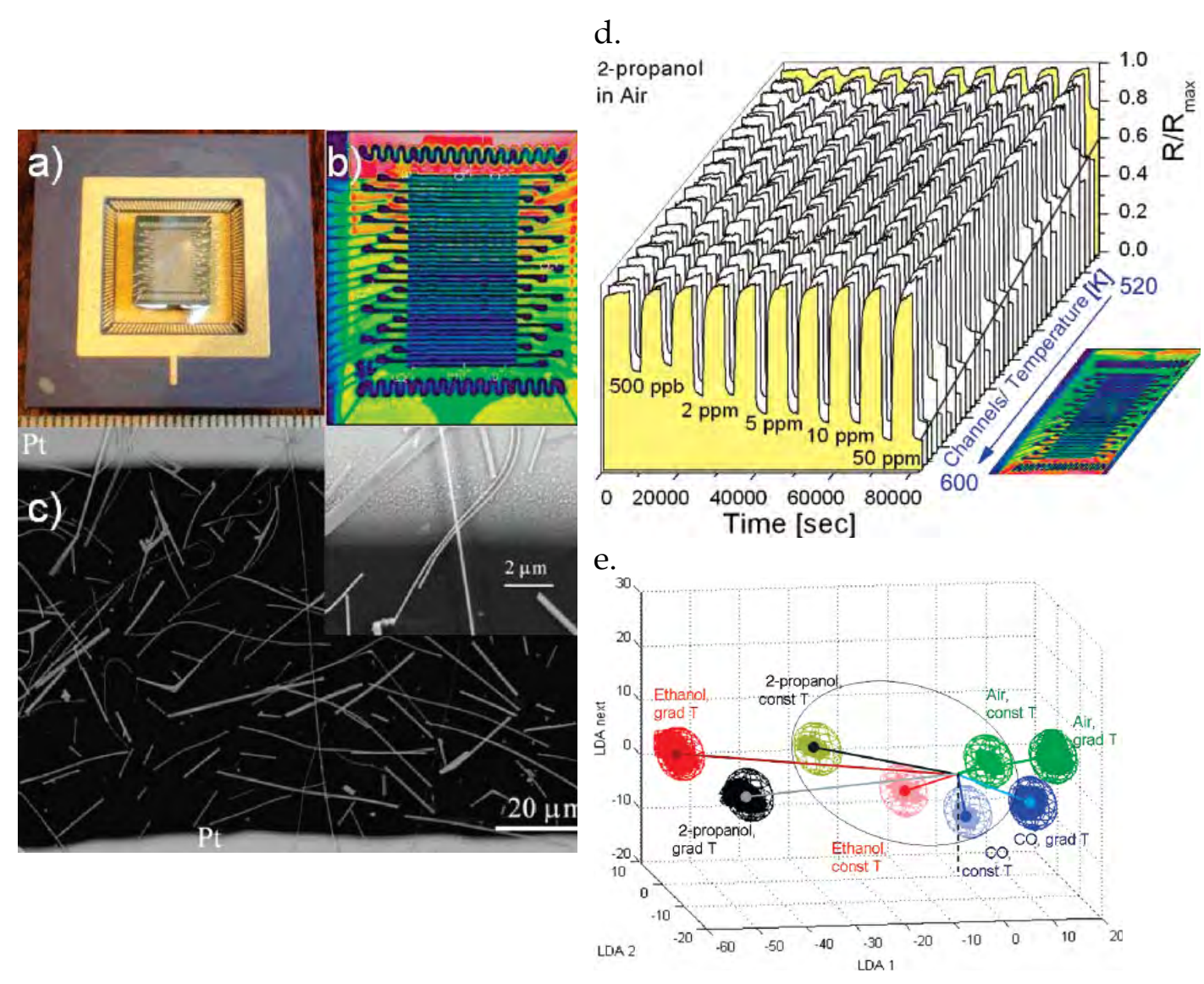

Figura 1.5. a. Microred KAMINA con nanhilos de $\mathrm{SnO}_{2}$. b. Imagen IR a una temperatura de 520 K. c. Imagen SEM de los nanohilos de $\mathrm{SnO}_{2}$. d. Señal respuesta de 38 sensores (nanohilos) a la inyección de 2-propanol. e. Análisis LDA para los patrones obtenidos con la red de 38 nanohilos de $\mathrm{SnO}_{2}$. Tomada de [93].

Ahora bien, otros instrumentos de análisis sensorial electrónico como los basados en la detección de iones en función de su relación masa/carga (MS) mediante aplicación de un campo magnético también han demostrado buenos resultados. Sin embargo las redes de sensores de gases clásicas siguen siendo el método más común en la literatura, posiblemente debido a las diferencias en costo y manejo de ambos sistemas. Las narices electrónicas basados en MS presentan como principales ventajas: (i) la capacidad de adaptación, mediante la selección de un conjunto óptimo de iones, (ii) la sensibilidad, al rechazar los iones de los componentes potencialmente interferentes, y (iii) la versatilidad, ya que se puede aplicar a una amplia gama de muestras de alimentos. De igual forma, los sensores de gas tienen varias ventajas demostradas: (i) son más fácil de utilizar en comparación con los complejos dispositivos de MS, (ii) tienen un precio más bajo tanto en coste como en mantenimiento, y (iii) la posibilidad de su utilización en línea de producción, debido a su portabilidad [39]. 


\subsubsection{Los sistemas de inyección}

La exposición de los sensores a los aromas es un punto clave en el diseño de una nariz electrónica, ya que la sensibilidad de los sensores es muy alta y su respuesta es muy rápida, de modo que el correcto funcionamiento del instrumento requiere disponer de un sistema de inyección de gases reproducible y de gran precisión. Además, es necesario utilizar sistemas que permitan recoger muestras representativas de los volátiles que producen los aromas.

Los sensores se colocan en una cámara de reacción de pequeño volumen, donde se inyectan los aromas mediante sistemas que son análogos a los utilizados en cromatografía de gases.

A continuación describiremos las principales características los métodos de muestreo de los compuestos volátiles.

1. Espacio de cabeza estático (SHS), esta técnica consiste en colocar la muestra en un vial herméticamente cerrado y, a continuación, una vez se ha establecido el equilibrio entre la matriz y la fase gaseosa, se toma la muestra del espacio en cabeza. Mediante este método es posible inyectar pequeños volúmenes de muestra con gran precisión y posibilitan la concentración de los aromas de manera previa a la inyección en la cámara de reacción. En este caso la temperatura de la muestra, tiempo de equilibrio, el tamaño del vial y la cantidad de la muestra son los principales parámetros que han de ser optimizados. Debido a la baja repetibilidad de la inyección manual del espacio en cabeza, se recomienda utilizar un muestreador automático HS [63, 98, 99].

2. Purga y trampa (P \& T) y técnicas de espacio de cabeza dinámicas (DHS), este tipo de técnicas se han diseñado para aumentar la sensibilidad, ya que proporcionan una preconcentración de compuestos volátiles. En estos sistemas, los componentes volátiles se purgan con una corriente de gas inerte y se atrapan con un materia adsorbente. En el caso de P \& T, el flujo de gas se inyecta a través de la muestra, mientras que, en el caso de DHS, sólo el espacio en cabeza se purga con el gas. Por ejemplo, cuando el material a evaluar es el vino estas trampas retienen los VOCs, pero no el etanol y el agua. La etapa posterior de desorción térmica, permite inyectar en la cámara de reacción los volátiles aromáticos prácticamente libres de agua y etanol, evitándose así el enmascaramiento de la señal de los sensores [100, 101].

Aparte de la elección de trampa, los principales parámetros para optimizar son la temperatura de la muestra, el tiempo de equilibrio, la velocidad de flujo del gas de extractor y el tiempo de purga del espacio en cabeza. 
3. Microextracción en fase sólida (SPME) es una de las técnicas más recientes y con mayores perspectivas para la extracción y concentración de los analitos que están contenidos en la matriz de una determinada muestra, como puede ser el vino. Su funcionamiento se basa en el empleo de una fibra de sílice fundida que está recubierta de un adsorbente, en la mayoría de los casos es un polímero, que designaremos como la fase estacionaria de este dispositivo [102]. Este proceso está seguido por la desorción de los compuestos volátiles mediante un incremento en la temperatura o mediante extracción en un disolvente orgánico $[103,104]$. Aparte de la naturaleza del adsorbente depositado sobre la fibra, los principales parámetros para optimizar el tiempo de equilibrio son, la temperatura de la muestra y la duración de la extracción.

La selección del tipo de fibra adsorbente depende de las características de los analitos de interés, y ello es debido a que su funcionamiento se basa en el principio de la similitud química; es decir, en la semejanza existente entre los compuestos medidos y el material adsorbente con respecto a cualquiera de las características que puedan favorecer la interacción entre ellos. Es por ello que las fibras apolares retienen preferiblemente compuestos apolares; mientras que los compuestos polares son retenidos selectivamente por fibras polares. En el caso de las fibras bipolares (mezcla de las anteriores) retienen compuestos no polares y también extraen eficientemente compuestos polares. La selectividad, también se puede modificar cambiando el espesor del recubrimiento; esto significa que los compuestos con mayor volatilidad requieren una capa en la fase estacionaria mucho más gruesa que los compuestos semi-volátiles [105].

Actualmente, se dispone de fibras con diferentes recubrimientos de manera que la SPME pueda usarse para determinar un amplio grupo de compuestos. Es común que los recubrimientos polares como el poliacrilato (PA) o carbowax (CW) sean usados para retener analitos polares como fenoles y los recubrimientos apolares como PDMS para analitos apolares como PAHs o BTEX (benceno, tolueno, xileno y etilbenceno) [106].

4. Extracción sobre barras magnéticas (SBSE) esta técnica ha sido desarrollada recientemente, y consiste en una barra magnética recubierta con polímeros, la cual retiene los compuestos volátiles y utiliza también la técnica del espacio en cabeza para concentrar estos compuestos. Su capacidad de adsorción es mucho mayor que la de SPME [107, 108]. Sin embargo, técnicas como el espacio de cabeza dinámico (DHS) y la barra magnética de agitación para adsorción (SBSE), han sido pocas veces reportadas para aplicaciones en el ámbito de las narices electrónicas, en parte debido al incremento en el costo y tiempo necesarios para realizar una determinada medida. 
5. Preconcentración dinámica (INDEX). En este caso se utilizan unas agujas que contienen una fase de polímero que absorbe los compuestos, los cuales son forzados a pasar través de la aguja por movimientos repetidos de aspiración y expulsión debido al movimiento del émbolo de la jeringa. La ventaja potencial de este sistema es su robustez mecánica y la posibilidad de aumentar la cantidad de polímero absorbente, así como el área superficial disponible para adsorber los compuestos volátiles [109].

La elección del método para la concentración de volátiles para narices electrónicas, depende en buena medida del tipo de aplicación al cual será destinado este sistema. En el caso del análisis de bebidas alcohólicas, técnicas tales como el DHS y la SPME han demostrado que son capaces de concentrar compuestos volátiles de una manera eficiente y de eliminar parte del contenido de agua y etanol; además ofrecen la posibilidad de un control automático para la inyección de los compuestos volátiles.

\subsubsection{Tratamiento de los datos}

En principio, el análisis de los patrones de las respuestas de una red de sensores, en primera aproximación, debe realizarse visualmente, comparando las gráficas obtenidas en cada caso. Comparar dos gráficas es relativamente sencillo, a pesar de que hay que tener en cuenta numerosos parámetros (intensidad de la respuesta, forma de la curva, cinética de desorción reversibilidad, etc.). Sin embargo, en una red de 16-32 sensores, el número de variables a estudiar es demasiado alto, por lo que es necesario recurrir finalmente a métodos de análisis de datos quimiométricos, que contemplen las diferencias entre series de datos como un todo, en lugar de comparar las respuestas individuales de cada sensor $[110,111]$.

Por otra parte, la evolución de los sistemas electrónicos para análisis sensorial ha estado sujeta al avance alcanzado por las distintas técnicas de tratamiento de datos multivariantes, ya que es necesario aprovechar al máximo la información que pueda llegar aportar cualquier tipo de sensor. En palabras de Haswell y Walmsley [112], la quimiometría puede ser considerada clave en la perfeccionamiento de las redes de sensores.

La quimiometría, de acuerdo D.L. Massart [113], uno de los fundadores de esta disciplina, es el área de la química que utiliza la matemática, la estadística y la lógica formal y ello implica: a. diseñar o seleccionar procedimientos experimentales óptimos, b. proporcionar la máxima información química relevante a partir del análisis de datos químicos, c. y obtener conocimientos de sistemas químicos. 
Parte de la quimiometría se dedica al desarrollo y estudio de técnicas multivariantes, las cuales son capaces de detectar las relaciones ocultas existentes en los datos y eliminar la información redundante. Por lo tanto, disminuyen la dimensionalidad de la matriz de datos y facilitan la interpretación de los resultados $[114,115]$.

Podemos definir tres etapas importantes en el tratamiento de datos, y aplicación de las técnicas multivariantes como se ilustra en el diagrama de bloques de la Figura 1.6. El bloque inicial indica que una vez las señales son adquiridas y guardadas es necesario realizar un análisis estadístico tradicional calculando la desviación estándar para el conjunto de medidas, para que sea posible una primera aproximación que nos permita valorar la calidad de las muestras medidas y además detectar cualquier tipo de error sistemático a nivel experimental. A continuación se efectua el preprocesamiento de la señal, que tiene varios propósitos, los cuales incluyen las compensaciones de la deriva en las líneas bases, la selección de parámetros descriptivos y la preparación de la matriz de datos para la reducción de dimensionalidad. La matriz resultante es utilizada para representar análisis tipo clustering, discriminación, clasificación, regresión o predicción en función de la problemática seguida[116].

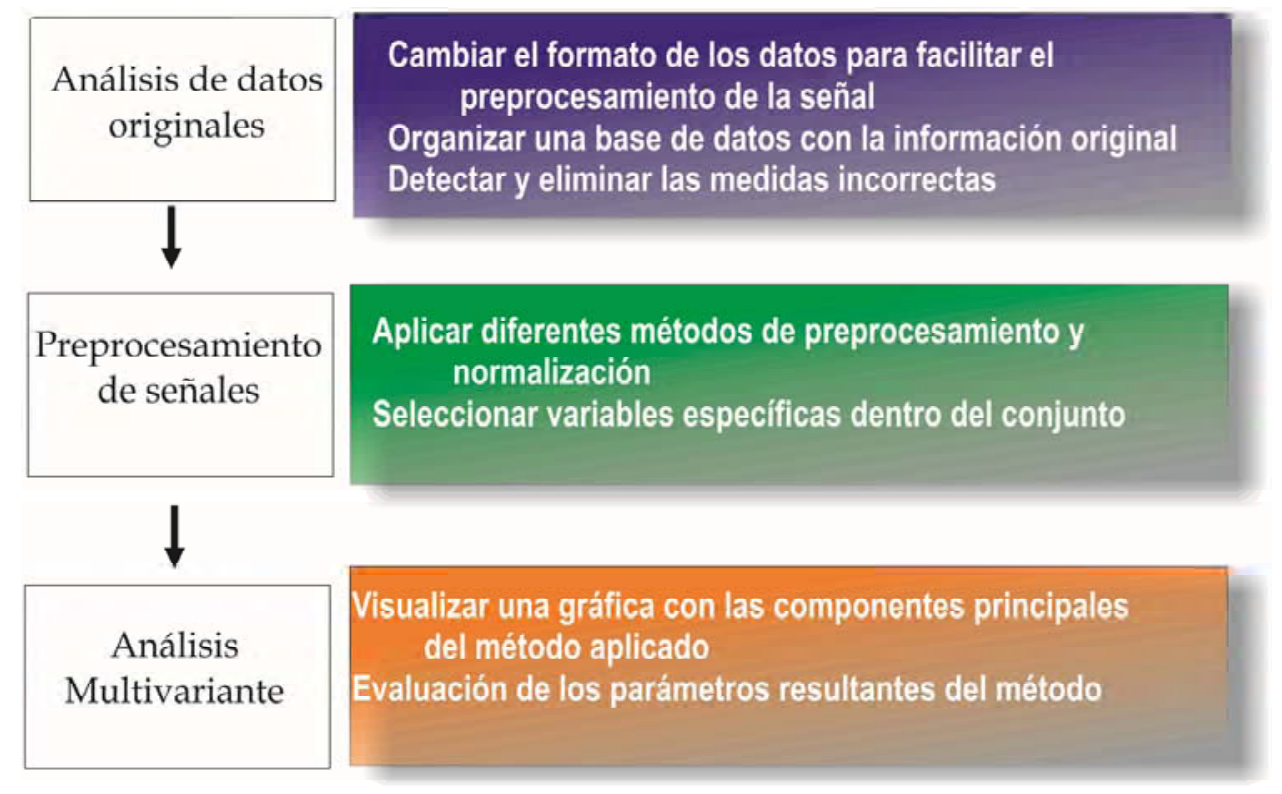

Figura 1.6. Procedimiento para el análisis de datos

\section{Técnicas de preprocesamiento de datos}

El propósito principal de esta etapa es la selección de un número de parámetros que describan todas las características de la señales bajo estudio. Dentro del 
preprocesado de la señal, algunos autores diferencian tres fases [117], cada una de las cuales repercute en los resultados que posteriormente serán obtenidos tras la aplicación de los oportunos algoritmos quimiométricos. Tales fases son:

I. Acondicionamiento de los resultados conseguidos en la fase previa: procesos relacionados con el centrado y normalización de datos, corrección de línea base, etc.

II. Disminución y extracción de determinados parámetros de las señales originales registradas.

III. Cuando sea necesario, establecer criterios de selección de variables en función de la información o ruido que aportan.

La importancia de esta fase es primordial para el éxito de una aplicación particular. En esencia, este paso permite garantizar la calidad de los datos y hacerlos más representativos. Como una consecuencia de la dependencia que tiene esta etapa en función del tipo de datos a tratar, los detalles de cada una de las técnicas de preprocesamiento serán discutidos en las secciones específicamente dedicadas al tratamiento de datos para narices y lenguas electrónicas.

\section{Pretratamiento de las señales de la nariz electrónica}

Como se ha indicado anteriormente, el propósito principal de la etapa de preprocesamiento de la señal es manipular la línea base y seleccionar cuidadosamente un número de parámetros que sean representativos de la respuesta de la red de sensores.

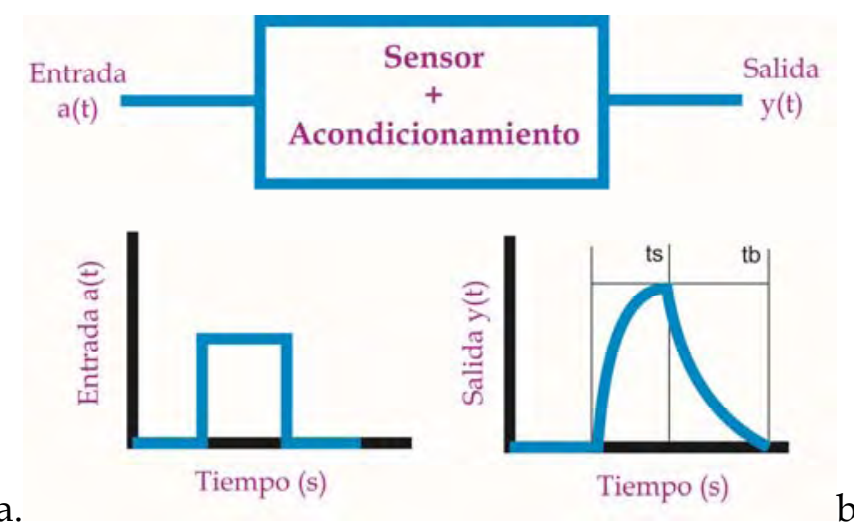

Figura 1.7. Respuesta genérica, en un sistema dinámico, de un sensor de gas a un cambio en la concentración del aroma: (a) concentración de aroma sobre el sensor, (b) respuesta resistiva. 
En el caso de los sensores MOS, cuando el aroma interacciona con la red de sensores, la resistividad eléctrica del sensor se modifica y esta variación puede ser medida. A su vez, esta señal es implementada en la electrónica asociada al sensor para convertirla en una señal eléctrica amplificada [118] (Figura 1.7).

Posteriormente, mediante una etapa de post-procesado, la señal analógica, se convierte en una digital para adecuarla al microprocesador y así tener una elevada velocidad de cálculo.

En estas condiciones, cuando todos los sensores tienen valores de resistencia parecidos, se podría hacer comparaciones entre ellos sin más que superponer las diferentes gráficas; pero en la mayoria, sus valores difieren en varios órdenes de magnitud lo que dificulta la interpretación de los resultados. Por este motivo, después de que las señales de los sensores han sido registradas y almacenadas en el ordenador, normalmente se realiza una corrección de la línea base. Mediante esta operación se transforma la respuesta de los sensores utilizando como referencia su línea base (respuesta de los sensores al gas portador). Los algoritmos utilizados para la manipulación de la línea base pueden ser diversos: resistencia relativa [119], conductancia relativa [68, 120, 121], porcentaje de resistencia relativa [122], el cociente entre la resistencia al aroma y en condiciones normales $[123,124]$, etc.

\section{Análisis multivariante}

Actualmente se cuenta con un importante número de herramientas estadísticas, que confieran la posibilidad de alcanzar diferentes resultados cuando se realiza el análisis de un conjunto de datos. Podemos decir que estás técnicas tienen las siguientes características principales [115]:

$\checkmark$ Identificación de estructuras (clusters, correlaciones) entre los objetos y/o variables estudiadas.

$\checkmark$ Modelamiento de respuestas cualitativas.

$\checkmark$ Procesamiento de datos para la predicción de respuestas cuantitativas.

En lo concerniente al tratamiento de datos para sistemas de análisis sensorial electrónico, las técnicas disponibles se pueden dividir en dos grupos básicos:

$\checkmark$ Análisis multivariante: Análisis de componentes principales (PCA), análisis de cluster (CA), análisis discriminante (CDA), modelado suave independiente por analogía de clases (SIMCA), regresiones lineales por mínimos cuadrados (PLS), análisis muti-way. 
$\checkmark$ Herramientas basadas en algoritmos de inteligencia artificial: Redes neuronales (ANN), algoritmos genéticos (GA), funciones de base radial (RBF), algoritmos basados en lógica difusa, perceptrones multicapas (MLP), mapas de autoorganizados (SOM) y máquinas de soporte vectorial (SVM).

Estos tipos de técnicas multivariantes existentes, se pueden clasificar también en técnicas supervisadas y no supervisadas [115]. Las técnicas no supervisadas, también denominadas técnicas de análisis exploratorio de los datos (AED), identifican distintas clases o categorías sin indicar a priori información de la naturaleza de las muestras [42]. En el caso de las técnicas supervisadas es necesario adicionar a la matriz de datos una variable categórica. Esta variable categórica contiene tantas categorías como sean necesarias para describir los objetos utilizados en la clasificación [125]. Además, las técnicas supervisadas a diferencia de las técnicas no supervisadas, requieren un aprendizaje previo del sistema (una base de datos) con conjuntos de muestras de calibración que definirán cada clase o grupo de muestras. Por tanto, una muestra desconocida puede compararse con el modelo y ser clasificada en una de las clases o grupo de muestra del entrenamiento [126]. El objetivo es, por tanto, la clasificación de muestras desconocidas, en otras bien conocidas que han sido caracterizadas anteriormente durante un proceso de entrenamiento. Dentro de este tipo de técnicas están los mínimos cuadrados parciales (PLS) y el análisis discriminantePLS (PLS-DA).

A continuación describiremos las principales características de algunos de los métodos de análisis de datos multivariantes citados hasta el momento.

Análisis de componente principales (PCA). El uso de esta técnica permite la visualización de la estructura latente de datos mediante graficas, cuyas interpretaciones permiten un entendimiento más profundo del que es posible obtener cuando se evalúan las variables en solitario, ya que permite analizar varias variables simultáneamente. El PCA como análisis exploratorio es el punto de partida conveniente dentro de los métodos de datos multivariantes [127].

Simultáneamente al proceso de reducción de variables, el PCA permite encontrar tendencias de agrupamiento entre los objetos (muestras problema) bajo análisis, ya que las nuevas variables reducidas hacen referencia a las direcciones de máxima varianza entre objetos. Es decir, el PCA explica la varianza existente entre los datos originales, y es por ello que suele emplearse en la obtención de patrones de clasificación entre muestras.

Algoritmos de clustering (CA). El proceso de clustering consiste en la división de los datos en grupos de objetos similares. Para medir la similitud entre objetos se 
suelen utilizar diferentes formas de distancia: distancia euclídea, de Manhatan, de Mahalanobis, etc. La representación de los datos por una serie de clusters, conlleva la pérdida de detalles, pero consigue la simplificación de los mismos [128].

Análisis discriminante (CDA). Hay dos tipos de funciones, las lineales y cuadráticas, para ambos casos se supone que la distribución de los datos es normal. El Análisis Discriminante normalmente es usado para: i. Determinar si existen diferencias significativas entre los "perfiles" de un conjunto de variables de dos o más grupos definidos a priori. ii. Determinar cual de las variables independientes cuantifica mejor las diferencias entre un grupo $\mathrm{u}$ otro. iii. Establecer un procedimiento para clasificar una muestra con base a los valores de un conjunto de variables independientes $[129,130]$.

Modelado suave independiente por analogía de clases (SIMCA). El nombre se debe a la adaptación al castellano de las siglas SIMCA ("Soft Independent Modelling of Class Analogy"). Se trata de un método de clasificación o discriminación basado en la construcción de modelos independientes para cada una de las clases existentes mediante PCA. Es un método supervisado porque se conocen a priori las clases adjuntas en la matriz de datos experimental. Ya que el PCA busca las variables latentes o PCs de las clases, si éstas son realmente diferentes, los modelos matemáticos que definen cada una de ellas serán necesariamente distintos. Los nuevos objetos (muestras) se asignarán a las clases cuyos modelos se ajusten mejor a sus características [131, 132].

Regresión por mínimos cuadrados (PLS). Esta es otra de las técnicas supervisadas, la cual utiliza un método de proyección basado en la reducción de variables por medio del PCA. Este método está fundamentado en algoritmos multivariantes lineales que, a partir de conjuntos de variables independientes $X$, construyen modelos de predicción de otras variables $\mathrm{Y}$, que son conocidas. En este caso, no se utilizan las componentes principales para crear un modelo de regresión sino que se emplean una serie de variables latentes que capturan la máxima varianza posible relacionando las variables predictivas y las variables respuesta (o variables a predecir). Es decir, la información de los datos originales se proyecta sobre un pequeño y manejable número de variables latentes (VLs) [116]. Los puntos proyectados sobre las nuevas latentes se conocen como gráfico de puntuaciones o "scores", equivalente al PCA. Esta representación permite establecer asociaciones o agrupamientos entre muestras, en este caso, en función de los datos originales y los conocidos a estimar.

Otra herramienta visual de gran utilidad para evaluar los resultados del PLS es el gráfico de valor real en función del valor predicho, representación que permite establecer y comparar con qué variables y métodos se obtienen los mejores ajustes o estimaciones. 
Esta técnica no está restringida a un número de variables determinado a modelar con respecto al número de objetos o muestras, pudiendo además establecerse predicciones o correlaciones entre una o varias variables dependientes simultáneamente. En esta investigación se ha utilizado esta técnica para el establecimiento de correlaciones entre la respuesta de los sensores y diversos parámetros fisicoquímicos y organolépticos de las muestras de vino.

Análisis Multi-way. Un análisis multi-way es una extensión natural del análisis multivariado, sólo que en este caso los datos están dispuestos en matrices de más de dos dimensiones. Los fundamentos para el desarrollo y uso de los métodos multi-way se debe principalmente a: i. El desarrollo de nuevos técnicas instrumentales que obtienen información aún más descriptiva del fenómeno a evaluar pero al mismo tiempo aún más información multivariada y compleja, ii. La necesidad de un método que facilite modelos parsimoniosos (principio según el cual cuando dos teorías en igualdad de condiciones tienen las mismas consecuencias, la teoría más simple tiene más probabilidades de ser correcta que la compleja), robustos e interpretables, y que por lo tanto entreguen mejores predicciones y mejores posibilidades para explorar los datos [133-136].

Redes neuronales (ANN). Es un tipo de algoritmos inspirado en las redes neuronales biológicas, ajustan sus modelos de forma experimental, sin necesitar un conocimiento previo de las características de los datos que debe procesar ni de los mecanismos que lo producen. Este método ofrece una valiosa capacidad de mapeo no-lineal, muchos tipos de redes neuronales se han empleado hasta el momento para realizar análisis de clasificación con los datos de la nariz electrónica, incluidas las redes de Kohonen, el vector de aprendizaje (LVQ) y sus variaciones de backpropagation (BP) y el algoritmo ART [131-142].

Una red neuronal puede considerarse como un sistema de computación compuesto por una serie de elementos más simples (neuronas) que se interconectan entre sí. La señal de una neurona sirve de señal de entrada a todas las neuronas de la columna inmediatamente a su derecha, existiendo unos pesos que ponderan esa señal [143].

Algoritmos genéticos (GA). Esta tipo de algoritmo opera sobre una población de posibles soluciones que aplican el principio de la supervivencia del más apto, y de esta manera producir sucesivas aproximaciones a una solución mejor. Esta técnica puede proporcionar y asegurar la selección de número óptimo de características que permitan una mejor discriminación entre muestras evaluadas $[138,144-146]$.

Mapas de autoorganizados (SOM). Es un algoritmo de tipo de agrupamiento y se utiliza para el análisis exploratorio de datos. Con esta técnica se elige un 
amplio número de clusters y se colocan en forma de una red bidimensional. La idea es que los representantes de cada grupo (o pesos, según la notación de Kohonen) estén correlacionados espacialmente, de modo que los puntos más próximos ven la rejilla sean más parecidos entre sí que los que estén muy separados

Máquinas de soporte vectorial (SVM). Conforman una familia de algoritmos de clasificación y regresión desarrollados por Vapnik y colaboradores. Los SVM se comenzaron a usar como método para el tratamiento de datos de redes de sensores desde el año 2003 [147, 148]. Entre las características interesantes de estos métodos para su aplicación en este campos figuran que es un algoritmo no paramétrico, permite una separación no lineal, aunque en principio son métodos para resolver problemas de clasificación también pueden ser aplicados como técnicas de regresión.

La decisión sobre el tipo de método que debe ser usado depende de si se desea hacer un análisis descriptivo o cuantitativo de las muestras evaluadas [149]. A modo general el tratamiento de datos dentro del entorno de los sensores tiene la misma función: extraer información y buscar correlaciones con otros sistemas, existe una diferencia importante entorno a su aplicación, y esta radica precisamente en el pretratamiento de la señal. No es lo mismo tratar una señal propia de una lengua electrónica basada en sensores electroquímicos, como lo es un voltamograma, que tratar una señal característica de una nariz electrónica basada en sensores MOS, la cual es algo más sencilla pues se trata del registro del cambio de la resistencia en función del tiempo.

Tabla 1.1 Análisis de datos para las señales provenientes de una nariz electrónica.

\begin{tabular}{|c|c|c|}
\hline $\begin{array}{c}\text { Método de } \\
\text { análisis de datos }\end{array}$ & Autores & Año \\
\hline
\end{tabular}

\begin{tabular}{|l|l|l|l|}
\hline PCA para el análisis de mezclas de gases & U. Weimar et al. & 1990 & {$[150]$} \\
\hline Redes neuronales & J. Gardner et al. & 1991 & {$[151]$} \\
\hline PCA y análisis de Clusters & J. Gardner et al. & 1991 & {$[128]$} \\
\hline Mapas de auto-organización (SOM) & Di Natale et al. & 1997 & {$[152]$} \\
\hline SOM+ árbol de expansión mínima & A. Ortega et al. & 2000 & {$[153]$} \\
\hline ANOVA-PLS & M. G. Sullivan et al. & 2003 & {$[154]$} \\
\hline Máquinas de vectores de soporte (SVM) & M. Pardo et al. & 2005 & {$[155]$} \\
\hline Modelos para el procesamiento de datos & Skov et al. & 2005 & {$[156]$} \\
\hline PLS & J. Lozano et al. & 2008 & {$[157]$} \\
\hline Reducción y selección de variables & D. Ballabio et al. & 2008 & {$[158]$} \\
\hline PCA, bi-plot & R. Jónsdóttir et al. & 2008 & {$[159]$} \\
\hline Preprocesamiento de datos & M. Padilla et al. & 2009 & {$[160]$} \\
\hline Análisis three-way & C. Burian & 2010 & {$[161]$} \\
\hline
\end{tabular}


Respecto a la tercera etapa del tratamiento de datos (Figura 1.6.). La Tabla 1.1. presenta un resumen sobre como se han ido incorporando diferentes métodos del análisis multivariante al área de los sensores de gases. Una de las mayores tendencias en el tratamiento de datos para estos sistemas de análisis sensorial electrónico radica en el desarrollo de métodos capaces de realizar reconocimientos de olores, es decir que el reto no está únicamente en distinguir y clasificar entre un determinado grupo de muestras sino además en la descripción de sus principales características organolépticas.

Ryman [162] reportó las mejoras en la identificación de un olor a través de una nariz electrónica, utilizando redes neuronales para el procesamiento de las señales. Wang [163] combinó una red neuronal de base radial con el algoritmo "fuzzy c-means" para mejorar la clasificación de patrones de olor con el empleo de narices electrónicas. La utilización combinada de redes neuronales y la lógica fuzzy ha demostrado ser más eficiente en el proceso de reconocimiento del olor que las técnicas convencionales, usando narices electrónicas [164]. Una red neuronal híbrida para la clasificación de olores que aprovecha los beneficios de los mapas auto organizativo de variables fue desarrollada por Di Natale con resultados satisfactorios [152].

La utilización de las técnicas de reconocimiento de patrones se ha puesto también de manifiesto en la selección o comprobación del funcionamiento del complejo de sensores. Polikar comparó la eficiencia de un algoritmo basado en árboles de decisión versus el algoritmo conocido como escalamiento de montaña para la selección de los componentes de una red de sensores destinada a la identificación de componentes volátiles orgánicos [165].

La figura 1.8 explica como se ha venido generando algunas de las nociones del tratamiento de datos, los cuales en los últimos años han sido un aporte significativo en el avance del desarrollo de narices y lenguas electrónicas. Recientemente Skov, [156] ha conseguido aplicar este concepto del análisis multiway en el manejo de datos provenientes de narices electrónicas. Generalmente los datos generados por las narices electrónicas tienen la posibilidad de ser arreglados en más de 2 dimensiones (muestra $x$ tiempo $x$ sensores), pero sin embargo en su procesamiento y específicamente en el caso de la extracción de variables, generalmente se simplifica el ordenamiento de los datos en una matriz de 2 dimensiones, y ello es debido a las limitaciones que presentan muchos software comerciales integrados en los equipos narices electrónicas. El aporte de Skov en su trabajo consistió en investigar la posibilidad de la utilización de los modelos de procesamiento de datos multiway como los del modelo PARAFAC-2 que permite modelar los datos de narices electrónicas en una manera más adecuada que el convencional tratamiento con un arreglo de dos dimensiones. 


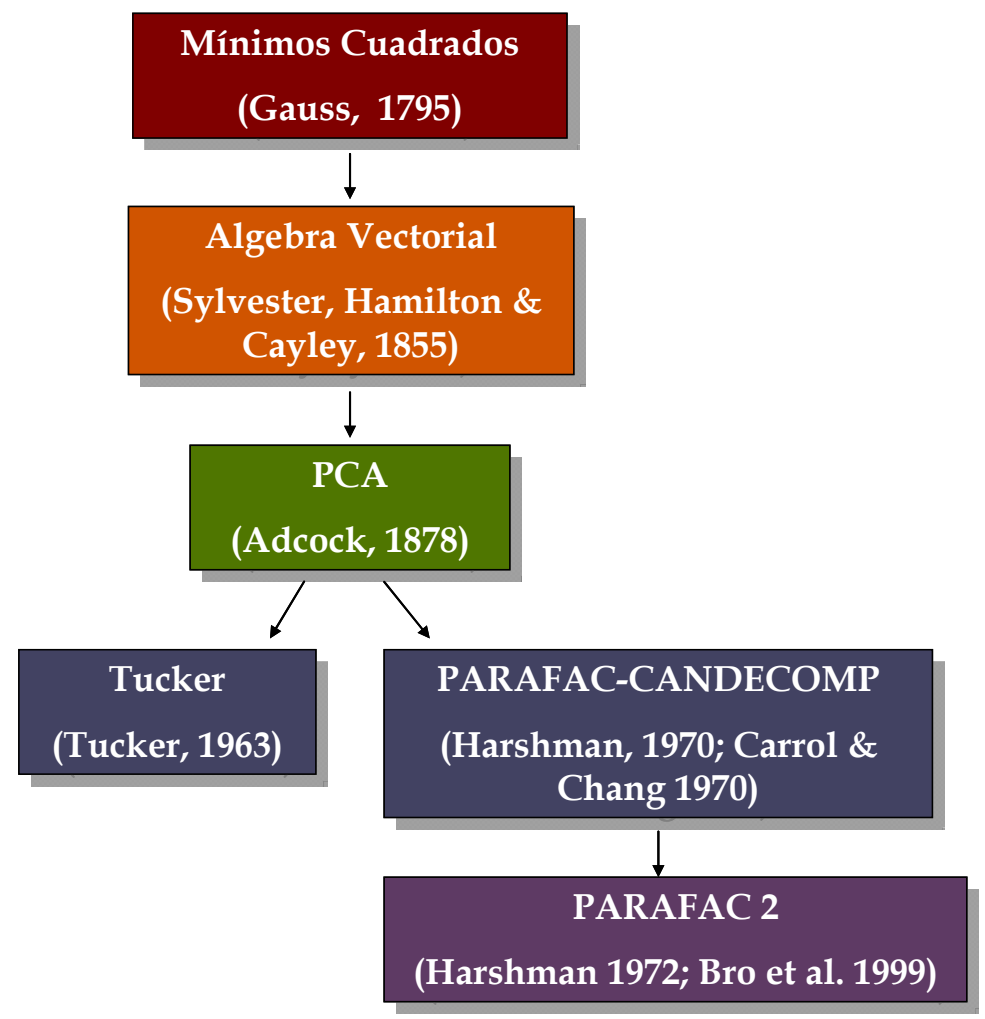

Figura 1.8. Desarrollo histórico de las herramientas matemáticas para el tratamiento de datos aplicados a los sistemas de análisis sensorial electrónico.

La aplicación de este tipo de modelo demostró ser capaz de brindar una nueva e interesante parametrización de datos provenientes de narices electrónicas, lográndose rescatar una mayor información de significación para el proceso de clasificación que se perdía con la utilización de los arreglos bidimensionales.

\subsubsection{Narices electrónicas comerciales}

Actualmente existen varias narices electrónicas comerciales cuyo funcionamiento general se basa en las características que acabamos de describir, y que se están utilizando en una gran variedad de aplicaciones (alimentación, seguridad, aplicaciones militares, medioambiente, industria farmaceutica, medicina, diagnóstico, etc.).

En la Tabla 1.2. se recoge una descripción de las narices electrónicas más destacadas hasta el momento incluyendo, el fabricante, tipo de sensores (resistivos, opticos, másicos, etc) y composición básica. El uso de las narices electrónicas ha aumentado conforme se han ido comprobando sus posibilidades en diferentes campos. El número de unidades vendidas de este sistema por diferentes fabricantes ha dependido en gran medida del tipo de tecnología 
aplicada a cada instrumento, el costo por unidad, y su aplicación de acuerdo a necesidades específicas $[40,166]$.

Tabla 1.2. Diferentes modelos Comerciales de narices electrónicas. Tomada de [35].

Tipo de

Fabricante

Modelos producidos

Composición

instrumento

\begin{tabular}{|c|c|c|c|}
\hline & Airsense Analytics & i-Pen, PEN2, PEN3 & Sensores MOX \\
\hline & Alpha MOS & FOX 2000, 3000, 4000 & Sensores MOX \\
\hline & Applied sensor & Air quality module & Sensores MOX \\
\hline & Bloodhound Sensors & BH114 & Polímeros conductores \\
\hline & Chemosensing & ChemSensing & Sensores colorimétricos \\
\hline & CogniScent Inc & ScanTrak & Polímeros conductores \\
\hline & CSIRO & Cybernose & Biosensores \\
\hline & Cyrano Sciences & Cyranose 320 & Polímeros conductores \\
\hline & $\begin{array}{l}\text { Daimler Chrysler } \\
\text { Aerospace }\end{array}$ & Sistema SAM & QCM, SAW, MOX \\
\hline & Dr. Födisch AG & OMD 98, 1.10 & Sensores MOX \\
\hline & $\begin{array}{l}\text { Electronic Sensor } \\
\text { Technology }\end{array}$ & zNose & SAW \\
\hline & Element & Frésense & Sensores MOX \\
\hline Nariz & $\begin{array}{l}\text { Forschungszentrum } \\
\text { Karlsruche }\end{array}$ & SAGAS & SAW \\
\hline electrónica & Gerstel GMBH Co. & QSC & Sensores MOX \\
\hline & $\begin{array}{l}\text { GSG Mess-und } \\
\text { Analysengeräte }\end{array}$ & MOSES II & $\begin{array}{l}\text { QCM, MOS, sensores } \\
\text { electroquímicos }\end{array}$ \\
\hline & Illumina Inc. & ONose & $\begin{array}{l}\text { Sensores ópticos } \\
\text { basados fluorescencia }\end{array}$ \\
\hline & $\begin{array}{l}\text { Marconi Applied } \\
\text { Technologies }\end{array}$ & e-Nose 5000 & $\mathrm{CP}, \mathrm{MOS}, \mathrm{QCM}$ \\
\hline & Microsensor & Hazmatcad & SAW \\
\hline & Osmetech Plc & Aromascan A32S & Polímeros conductores \\
\hline & Quartz Technology & QTS-1 & QCM \\
\hline & Sacmi & EOS 835 & Sensores de gases \\
\hline & Scensive Technol. & Bloodhound ST214 & Polímeros conductores \\
\hline & Smiths Group plc & Cyranose 320 & Polímeros conductores \\
\hline & Sysca AG & Artinose & Sensores MOX \\
\hline & Systems Inc. & MiniCAD mk II & SAW \\
\hline & Technobiochip & LibraNose 2.1 & Sensores QMB \\
\hline & Airsense Analytics & GDA 2 & MOX, EC, IMS, PID \\
\hline & Alpha MOS & $\begin{array}{l}\text { RQ BOX, } \\
\text { Prometheus }\end{array}$ & MOX, EC, PID, MS \\
\hline Tecnología & HKR Sensorsysteme & QMB6 & QCM, MS \\
\hline $\begin{array}{l}\text { combinada } \\
\text { (nariz }\end{array}$ & $\begin{array}{l}\text { Electronic Sensor } \\
\text { technology }\end{array}$ & $\begin{array}{l}\text { Znose 4200, 4300, } \\
7100\end{array}$ & SAW, GC \\
\hline electrónica + & Environics Industry & MGD-1 & IMCELL \\
\hline $\begin{array}{c}\text { otros } \\
\text { sistemas) }\end{array}$ & Microsensor System & $\begin{array}{l}\text { Hazmatcad Plus CW } \\
\text { Sentry 3G }\end{array}$ & SAW, EC \\
\hline & Rse Systems & RAE monitor & $\mathrm{CB}, \mathrm{O}_{2}, \mathrm{EC}, \mathrm{PID}$ \\
\hline & RST Rostock & FF2, GFD1 & MOS, QMB, SAW \\
\hline & SMart Nose & Smartnose 300 & MS \\
\hline
\end{tabular}


En los últimos años, la compañía Applied Sensor ha vendido la mayor cantidad de unidades, una cantidad mayor a 100.000 unidades de su dispositivo "Air Quality Module electronic nose". Desde finales de la década de los 90, la investigación relacionada con estos sistemas se ha centrado en evaluar y comparar las capacidades de detección y su potencial dentro de las distintas aplicaciones posibles. Las características de los sistemas diseñados en los últimos años se discuten a continuación.

La nariz electrónica Fox de Alpha-MOS (Toulouse, Francia) fue diseñada en colaboración con las Universidades de Warwich y Southampton. El modelo Fox 2000 emplea seis sensores de óxido metálico, 12 en el caso del Fox 3000, y 18 para el Fox 4000. Todos estos dispositivos tienen la posibilidad de ser utilizados con un sistema de inyección flujo conectado a un gas portador externo, o a un controlador de flujo másico.

El modelo Aromascan A32S (Osmetech Plc, Reino Unido) se basa en un detector con una red de 32 sensores desarrollados con polímeros conductores, y tiene como punto de partida un diseño realizado en el Instituto de Ciencia y Tecnología de la Universidad de Manchester. Los polímeros conductores utilizados para la fabricación de los sensores que constituyen la matriz fueron producidos por electropolimerización de polipirrol, polianilina o derivados politiofeno.

Este instrumento ya no está comercialmente disponible debido a que la compañía encargada de su comercialización Osmetech Plc interrumpió la producción y reorientó su negocio hacia el desarrollo y producción de instrumentos para aplicaciones en el sector biomédico.

El nuevo énfasis de este sistema está orientado a la detección de diferentes metabolitos que se producen durante una infección urinaria, o la detección temprana de la neumonía, por ejemplo. El nuevo sistema ha sido llamado Osmetech Microbial Analyser (OMA), y su sistema de detección contiene 48 sensores situados en un substrato circular con un controlador de la temperatura.

GSG Mess- und Analysengeräte. Sistema diseñado en una compañía fundada por un par de investigadores del proyecto MOSES II de la Universidad de Tübingen. Entre los diferentes dispositivos desarrollados se incluye un sistema portable basado en una matriz de sensores Tagushi y sensores másicos.

Cyrano Science, Inc. Este sistema lleva el nombre de la empresa Cyrano Science, la cual fue fundada en 1997 utilizando el diseño elaborado por Nathan Lewis et al. en el Instituto de Tecnología de California. El dispositivo Cyranose 320 comprende una matriz de 32 sensores fabricados con polímeros conductores. 


\subsubsection{Combinación de sistemas}

La tabla 1.2. incluye un amplio número de ejemplos de dispositivos formados por redes de sensores con un determinado tipo de tecnología y sistemas combinados con instrumentos de analíticos tradicionales u otro tipo de sistemas de evaluación sensorial electrónica que trabajan de manera paralela a una nariz electrónica.

La necesidad adicional de identificar compuestos volátiles de manera individual o componentes dentro de una mezcla, y al mismo tiempo evaluar las características globales de una determinada muestra, recientemente ha causado una fusión de las tecnologías de la nariz electrónica con instrumentos analíticos.

Este tipo tecnológicas combinadas han dado lugar a un nuevo tipo de instrumentación para las cuales no es fácil determinar si hacen parte de la clasificación de las narices electrónicas y/o de los instrumentos analíticos convencionales.

En otro sentido, la mayoría de las narices electrónicas tienen un número limitado de sensores, mientras que el sistema biológico utiliza un gran número de sensores con diversos tipos receptores sensibles. Con esta idea se ha trabajado en un sistema artificial que consigue imitar el sistema biológico como lo hace el sistema portátil e-mucosa (PeM). Este sistema se basa en tres redes con un importante número de sensores, cada una con 200 sensores resistivos combinados con dos columnas recubiertas con diferentes materiales adsorbentes encargados de retener compuestos polares y no polares. De esta manera ha sido posible mejorar el poder de discriminación en comparación con las narices electrónicas tradicionales [167].

Otra innovación en este campo es la denominada "Deferential e-nose (Dennose)" con dos redes de sensores para discriminar olores [168].

La diferencia entre las señales entregadas por dos conjuntos de sensores independientes e idénticos se somete a una deconvolución en dos dimensiones. Este método mejora la sensibilidad, y la capacidad de reconocer diferencias entre olores similares. Optimización del sensor también es importante y se incorporan a veces en el software de e-nariz, el análisis de datos

\subsubsection{Aplicaciones de las narices electrónicas}

Las narices electrónicas son utilizadas actualmente en una gran variedad de aplicaciones de determinación de olores y compuestos volátiles. Las más 
comunes están operativas en los sectores industria alimenticia y control de calidad, diagnóstico médico, monitoreo del medio ambiente, control de procesos industriales, desarrollo de fragancias y cosméticos, seguridad y toxicología, detección de narcóticos, sistemas de acondicionamiento de aire, etc [32, 35, 36, 39, 169].

Las narices electrónicas son particularmente útiles en el control de calidad de los productos alimenticios. Su inclusión en este sector de aplicaciones está siendo impulsada por su confiabilidad, objetividad, costo y respuesta casi instantánea (real time). En principio, podrían ser utilizadas para analizar los aromas de productos muy diferentes, desde vinos a quesos, pasando por aceites, perfumes, etc. Sin embargo, los nuevos avances en este campo han demostrado que para obtener buenos resultados, tanto los instrumentos como los sensores, deben ser diseñados específicamente para una aplicación concreta. No es lo mismo detectar los aromas de un aceite de oliva que el aroma de un vino, donde los volátiles responsables del olor están acompañados de agua y etanol en proporciones muy altas $[40,71]$.

Entre las aplicaciones especificas dentro de la industria de la alimentación está la determinación de la calidad materias primas [170-175], evolución durante la producción, control de procesos de cocción [46], monitoreo de procesos de fermentación [176], control del calidad de pescados [177-181], valoración de ranciedad [182], deterioro por envejecimiento [183], verificación de ingredientes y evaluación de zumos [184, 185] monitoreo de bebidas [186, 187], graduación alcohólica de licores, inspección de olores en contenedores y "packaging" de alimentos y determinación del tiempo en que se produce la pérdida de cierto tipo de aroma en productos conservados [70, 188-190]. Un área emergente es en el desarrollo de productos incorporando nuevos aromas, buscando un buen ajuste entre los aromas sintéticos y los aromas naturales, o bien desarrollando nuevos aromas estudiando la relación con los existentes [191]. Reiteramos que los mayores éxitos se han logrado utilizando sistemas específicamente diseñados para una aplicación concreta por ejemplo en el caso de la valoración del agua mineral, la cerveza y el café, en algunos casos incluso existen productores que ya utilizan las narices electrónicas como sistema para el control de la calidad de sus bebidas [31, 141, 176, 192-195]. También se utilizan para separación de aromas de aceites de oliva extra virgen, normal y de baja calidad y la identificación de su denominación de origen [60, 82, 139, 196-200].

En el sector enológico, la principal aplicación de una nariz electrónica está orientada hacia el control de calidad de los caldos a diferentes niveles. La tabla 1.3. resume las algunas de estas aplicaciones.

Todas estas aplicaciones tienen un gran interés para poder controlar y garantizar la autenticidad de los vinos. En estos casos la fiabilidad de los resultados 
obtenidos depende fundamentalmente del desarrollo de un diseño específico y de la representatividad de los vinos seleccionados para el estudio, por lo que es necesario disponer de un gran número de muestras. Los estudios efectuados con un número reducido de vinos tienen especialmente un interés científicoacadémico.

Tabla 1.3. Principales aplicaciones de las narices electrónicas en la evaluación de vinos.

\begin{tabular}{|c|c|c|c|c|c|}
\hline Muestras & Tipo de estudio & $\begin{array}{c}\text { Pre- } \\
\text { concentración }\end{array}$ & $\begin{array}{l}\text { Sistema de } \\
\text { detección }\end{array}$ & $\begin{array}{l}\text { Tratamien } \\
\text { to de } \\
\text { datos }\end{array}$ & Ref. \\
\hline Vinos italianos & $\begin{array}{l}\text { Reconocimiento de } \\
\text { adulteración }\end{array}$ & SHS & $\begin{array}{l}4 \text { películas } \\
\text { delgadas } \\
\text { MOS }\end{array}$ & $\begin{array}{l}\text { PCA, BP- } \\
\text { ANN }\end{array}$ & [137] \\
\hline Vinos españoles & $\begin{array}{l}\text { Clasificación de } \\
\text { variedades de } \\
\text { vinos }\end{array}$ & $\begin{array}{l}\text { SHS, P\&T, } \\
\text { SPME }\end{array}$ & $\begin{array}{l}16 \text { sensores } \\
\text { MOS }\end{array}$ & $\begin{array}{l}\text { PCA, } \\
\text { PNN }\end{array}$ & [201] \\
\hline Vinos españoles & $\begin{array}{l}\text { Clasificación de } \\
\text { variedades de } \\
\text { vinos }\end{array}$ & SHS & $\begin{array}{l}8 \text { sensores } \\
\text { MOS }\end{array}$ & $\begin{array}{l}\text { PCA, } \\
\text { PNN }\end{array}$ & [202] \\
\hline Vinos españoles & $\begin{array}{l}\text { Clasificación de } \\
\text { variedades de } \\
\text { vinos }\end{array}$ & SHS & $\begin{array}{l}\text { sensores } \\
\text { SAW ZnO }\end{array}$ & $\begin{array}{l}\text { PCA, } \\
\text { PNN }\end{array}$ & [62] \\
\hline Vinos españoles & $\begin{array}{l}\text { Clasificación de } \\
\text { variedades de } \\
\text { vinos }\end{array}$ & SHS & MS & $\begin{array}{l}\text { PCA, } \\
\text { SIMCA }\end{array}$ & [203] \\
\hline $\begin{array}{l}\text { Vinos tintos } \\
\text { italianos }\end{array}$ & $\begin{array}{l}\text { Predicción de } \\
\text { descriptores } \\
\text { sensoriales }\end{array}$ & DHS & $\begin{array}{l}\text { PEN 2: } 10 \\
\text { sensores } \\
\text { MOS }\end{array}$ & GA & [204] \\
\hline Vinos & $\begin{array}{l}\text { Evaluación del } \\
\text { envejecimiento de } \\
\text { un vino tinto }\end{array}$ & SHS & $\begin{array}{l}16 \text { películas } \\
\text { delgadas de } \\
\text { óxido de } \\
\text { estaño }\end{array}$ & PLS & [157] \\
\hline Vinos italianos & $\begin{array}{l}\text { Clasificación de } \\
\text { variedades de } \\
\text { vinos }\end{array}$ & HS & $\begin{array}{l}\text { Sensores } \\
\text { QMB }\end{array}$ & ANN & [24] \\
\hline Vinos australianos & $\begin{array}{l}\text { Clasificación de } \\
\text { sauvignon blanc } \\
\text { por regiones }\end{array}$ & SPME & $\begin{array}{l}\text { Sensores } \\
\text { MOS- MS }\end{array}$ & $\overline{\mathrm{LDA}}$ & [205] \\
\hline Vinos españoles & $\begin{array}{l}\text { Evaluación del } \\
\text { envejecimiento de } \\
\text { un vino tinto }\end{array}$ & SPME & $\begin{array}{l}16 \text { Sensores } \\
\text { MOS }\end{array}$ & PCA, PLS & [206] \\
\hline
\end{tabular}

Los últimos retos trazados en este sentido están entorno a la detección de defectos aromáticos. La nariz electrónica ha mostrado su potencial en la detección del TCA y en la detección del defectos aromático causado por las Brettanomyces [207]. 


\subsubsection{La lengua electrónica}

Las lenguas electrónicas trabajan bajo los mismos principios selectivos que las narices electrónicas, usando una red de sensores no específicos, que esta vez se sumergen en la disolución a analizar, acoplada a un software de reconocimiento de patrones, que permite reconocer disoluciones simples o complejas. Recientemente, se han publicado numerosas revisiones sobre el tema [208214].Los sensores más utilizados en las lenguas electrónicas son los electroquímicos (potenciométricos, amperométricos o impedimétricos) [215-217].

También se han desarrollado lenguas electrónicas que utilizan otros sistemas de transducción, incluyendo redes basadas en ISFETS [218-220] o transducción óptica [66]. Aunque como ya hemos comentado, los sistemas más comunes trabajan con sensores electroquímicos. La mayor parte de estos instrumentos utilizan sensores potenciométricos, en los que se mide el potencial creado por la difusión de iones a través de una membrana sensible. Estas membranas pueden prepararse con diferentes materiales, y ello permite obtener sensores con distinta selectividad. Por esta existe una investigación muy activa en la búsqueda de nuevos ionóforos capaces de detectar iones de manera específica [213, 221].

Otro sistema bastante utilizado, es el basado en medidas de impedancia electroquímica, con la que se puede detectar la adsorción de sustancias a la superficie del electrodo a diferentes potenciales. Las lenguas electrónicas basadas en espectroscopía de impedancias (en las que el grupo de los profesores. O.N. Oliveira, A. Riul y C.J.L. Constantino son líderes mundiales) constan de una red de electrodos recubiertos de diferentes materiales sensibles (polímeros conductores, nanotubos de carbono o ftalocianinas entre otros). Este grupo ha prestado una especial atención al análisis de vinos [222-225]

Un tercer tipo de sensores electroquímicos son los amperométricos. En este grupo de sensores se valora la oxidación/reducción de especies electroactivas presentes en la disolución, cuando se aplica un cierto potencial; la corriente registrada $(\mu \mathrm{A})$ es una medida directa de la concentración del analito presente en la disolución. La modificación de los electrodos permite obtener redes de sensores con diferente selectividad [215, 226]

Finalmente, en los sensores voltamétricos, se realiza un barrido de voltaje y mientras se mide la intensidad de corriente en cada punto. Cuando se alcanza el valor de voltaje adecuado, las especies redox presentes en la disolución se oxidan/reducen, dando lugar a una variación de la corriente. Así, los voltamogramas muestran picos asociados a los procesos redox de las especies electroactivas disueltas en la muestra. La intensidad y la posición de los picos 
dependen de las condiciones experimentales $(\mathrm{pH}$, fuerza iónica) y de la naturaleza del electrodo de trabajo, de modo que electrodos de distinta naturaleza han de proporcionar respuestas diferentes. Además, es posible aplicar el voltaje siguiendo diferentes funciones (por ejemplo, voltametría cíclica, voltametría de onda cuadrada o voltametría de pulsos). Según la técnica utilizada, la información obtenida es diferente $[209,227]$. Teniendo en cuenta estas ideas generales, se han diseñado y construido lenguas electrónicas voltamétricas utilizando redes de electrodos fabricados con una importante cantidad de materiales (platino, oro, acero inoxidable, grafito materiales carbonosos, etc.), utilizando diferentes técnicas voltamétricos (voltametría cíclica, onda cuadrada o de pulsos) o combinando la voltametría con la potenciometría [228] o adaptando sistemas de inyección de flujo (FIA) [229, 230].

Los sensores voltamétricos son inespecíficos en muchos casos, lo que limita bastante la capacidad de discriminación de estas lenguas electrónicas.En esta filosofía, nuestro grupo ha desarrollado en estos últimos años un novedoso tipo de sensores voltamétricos, que permiten detectar especies iónicas (como hacen los sensores potenciométricos e impedimétricos), a la vez que detectan especies electroactivas (tal y como hacen los sensores amperométricos y voltamétricos comunes). Estos sensores consisten en electrodos químicamente modificados con diversos materiales electroactivos, fundamentalmente ftalocianinas, y están proporcionando excelentes resultados, en particular en el análisis de vinos ya que como se comprenderá tras la lectura de los siguientes párrafos, son capaces de detectar compuestos que juegan un papel fundamental en las características organolépticas de vinos [231, 232]. El principio de funcionamiento de estos sensores se resume a continuación.

Como hemos indicado, nuestra red de sensores está formada por electrodos recubiertos de materiales electroactivos, es decir, materiales que pueden oxidarse y reducirse. Cuando los electrodos se sumergen en la disolución y se realiza un barrido de voltaje, el voltamograma presenta dos tipos de picos, aquellos asociados a la oxidación/reducción del electrodo y los relacionados con la oxidación/reducción de especies redox presentes en la disolución.

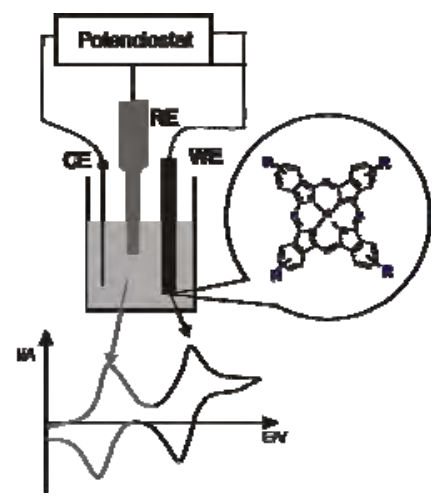

Figura 1.9. Esquema del funcionamiento de una lengua electrónica. 
Pero el aspecto más importante, es que el electrodo puede interaccionar con la disolución, y esto hace que el mismo electrodo produzca respuestas diferentes cuando se sumerge en disoluciones diferentes. Estas interacciones incluyen entre otras:

- El material del electrodo (en nuestro caso las ftalocianinas) poseen un efecto electrocatalítico que facilita la oxidación de ciertas especies presentes en la disolución.

- La coordinación de iones al metal central de la ftalocianina puede modificar el potencial de oxidación del electrodo.

- Cuando el electrodo se oxida (o reduce), se produce una difusión de iones hacia dentro (fuera) del electrodo, que es necesario para mantener la electroneutralidad del electrodo. Por esta razón la respuesta depende de la naturaleza de los iones presentes en la disolución [233].

- Si en la disolución hay disueltas especies oxidantes (o reductoras), su presencia, facilitará (o dificultará) la oxidación del material del electrodo. De forma similar, el $\mathrm{pH}$ puede afectar al potencial de oxidación del electrodo [234].

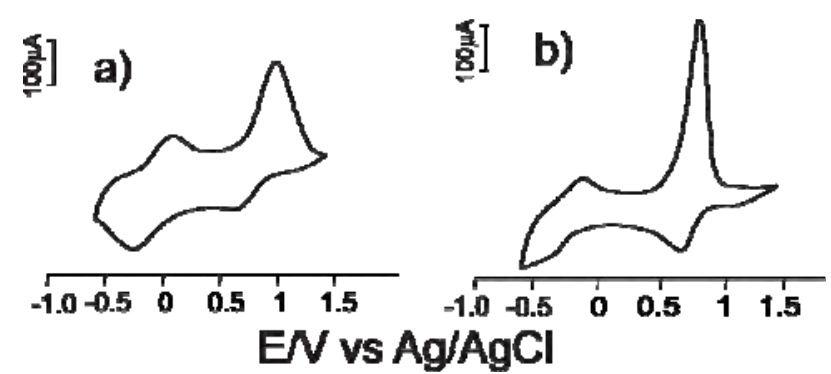

Figura 1.10. Voltamogramas de un sensor basado en LuPc2 expuesto a 0.1 mol.L-1 (a) $\mathrm{KCl}$. (b) $\mathrm{MgCl}$.
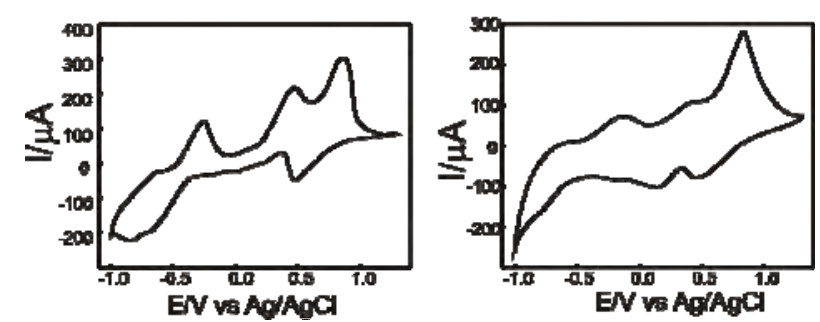

Figura 1.11. Voltametría cíclica de un electrodo LB basando en GdPc2 inmerso en una solución de $10^{-2} \mathrm{~mol} . \mathrm{L}^{-1} \mathrm{KCl}$ y $10^{-3} \mathrm{~mol} . \mathrm{L}^{-1}$ de: a. ácido ascórbico, b. Catequina. Scan rate 0.1 V.s.

Según lo anteriormente expuesto, las redes de sensores voltamétricos modificados químicamente, dan respuestas complejas con un alto grado de 
selectividad. Además, nuestra red es sensible a especies importantes en el vino, tanto iónicas $(\mathrm{pH}, \mathrm{Na}+$, etc.) como especies con actividad antioxidante (polifenoles, sulfuroso, etc.)

\subsubsection{Tratamiento de los datos}

Fila

Figura 1.12. Efecto de algunos preprocesamiento y normalización de datos de filas en un voltamograma ejemplo cuando se presenta un desplazamiento, deriva o un efecto en la intensidad de la curva. Tomada de referencia [115]. 
Los sensores voltamperométricos proporcionan voltamogramas muy complejos (presentan variedad de picos en diferentes posiciones y con diferentes intensidades). La complejidad intrínseca, la riqueza y la selectividad cruzada de las señales generadas por el conjunto de electrodos voltamétricos pueden constituir una ventaja, ya que el conjunto de datos contiene una gran cantidad de información sobre una muestra evaluada, si se tratan adecuadamente. Pero al mismo tiempo el hecho de que el conjunto de datos contenga excesiva información puede dificultar el tratamiento de datos. En consecuencia a este hecho, se requiere una etapa de pretratamiento para reducir el número de variables, sin que esto implique una pérdida de información.

Adicionalmente, como se mencionó en la primera parte relacionada con la nariz electrónica, la etapa inicial del procedimiento del análisis de datos consiste en la valoración de la señal original. A titulo de ejemplo, la Figura 1.12. presenta los efectos que puede tener en una señal voltamétrica cualquiera la aplicación de un determinado preprocesamiento y normalización, cuando se presenten tres problemas típicos en señales electroquímicas (efecto aditivo, multiplicativo o en la intensidad global) [115].

En un artículo publicado por Brown y Bear Jr. se hizo una revisión del uso de métodos quimiométricos en electroquímica y se declaró, entre otras conclusiones, que la cantidad de artículos publicados en química electroanalítica que reportan alguna aplicación de herramientas quimiométricas es mucho menor que la observada para cualquier área de la química analítica. En este trabajo también se explica que se han dado muchos progresos en la aplicación de quimiometría para la resolución de problemas electroquímicos, demostrando además que es un área con un enorme potencial de exploraxión para contribuir en el avance de la electroquímica.

La dificultad de la aplicación de las herramientas quimiométricas no viene determinada por los métodos como tal, por el contrario y como se mencionó anteriormente, se debe al tipo de señales que corresponde analizar y la manera como se deberían pretratar. Comenzando por decir que en una medida electroquímica las fuentes de ruido son aún mayores que en las medidas que se realizan con otro tipo de sensores. El origen del ruido en estas medidas se debe a la instrumentación utilizada y a las corrientes parásitas. Así por ejemplo, si la corriente a medir es de naturaleza farádica, que es el caso más habitual en aplicaciones electroanalíticas, la corriente capacitiva que se presenta debido a la carga y descarga en el espacio existente en la interfaz electrodo-solución puede ser considerada como ruido. La reducción de estas corrientes capacitivas ha sido una de las razones principales para el desarrollo de muchas técnicas electroanalíticas relevantes. Entre las alternativas que se han propuesto existe una que ha resultado especialmente beneficiosa, la aplicación de técnicas pretratamiento de las señales para la eliminación del ruido y de outliers. A esta 
práctica se han ido adicionando métodos como la aplicación de métodos para la sustracción de la línea base, suavizado, transformación y la reducción o selección de los datos.

En esta misma dirección, se han implementado diversos métodos conocidos en el tratamiento digital de señales para obtener un conjunto de datos análogo a la señal electroquímica, y de esta manera hacer más sencillo el posterior análisis de los datos. Para realizar estas operaciones se cuentan con métodos clásicos, como los filtros Savitzky-Golay o Kalman (KF) [235], la transformada rápida de Fourier (FFT) [236].

Entre los métodos recientemente desarrollados podemos nombrar la transformación Wavelet (WT), técnica que permite la eliminación de los efectos de línea de base, disminuyendo el ruido y reduciendo el número total de variables. Con este método es posible además analizar también el ancho de los picos, el grado de suavidad de la curva, si existe alguna discontinuidad y otros aspectos referentes a la forma del voltamograma [209].

Finalmente citar un procedimiento que hemos adecuado a nuestro tratamiento de datos que consiste en el preprocesamiento de los voltamogramas por la adaptación de una técnica de reducción basada en una respuesta predefinida bellshaped-windowing de curvas definidas como "kernels" [231].

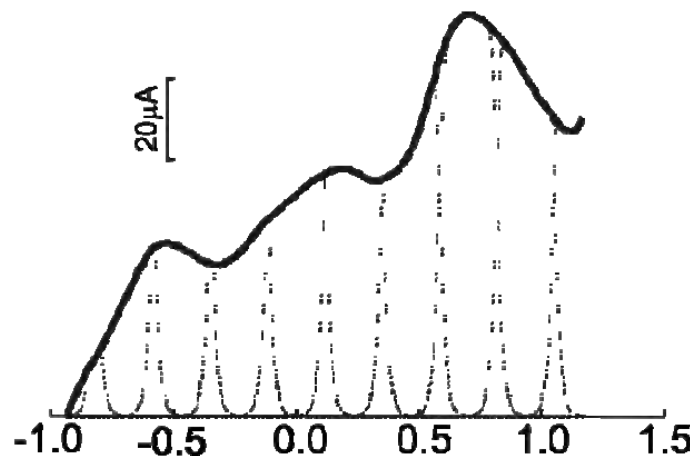

Figura 1.13. Voltametría de onda cuadrada y los "kernels" para obtener 10 parámetros por curva.

Aplicando este método, hemos recurrido a la voltamperometría de onda cuadrada (SWV) en lugar de la voltametría cíclica, ya que los voltamogramas cíclicos son curvas bievaluadas muy difíciles de procesar. La curva de SWV (i vs E) se multiplica por un número de 10 funciones bellshaped-windowing, y se integran con respecto al potencial. La idea detrás de esta técnica de preprocesamiento es capturar la información sobre la respuesta global y obtener 10 parámetros estraidos de toda curva (Figura 1.13). 
Respecto a la selección de variables de una señal voltamométricas, decir que este es un tema relativamente menos estudiado en comparación con otras áreas del análisis físico-químico como es la espectroscopia, de ahí el gran potencial que tiene la aplicación y la experiencia existente, en el campo de la electroquímica de métodos como el fuzzy artmap, redes neuronales, forward selection, backward elimination y stepwise selection y los algoritmos genéticos.

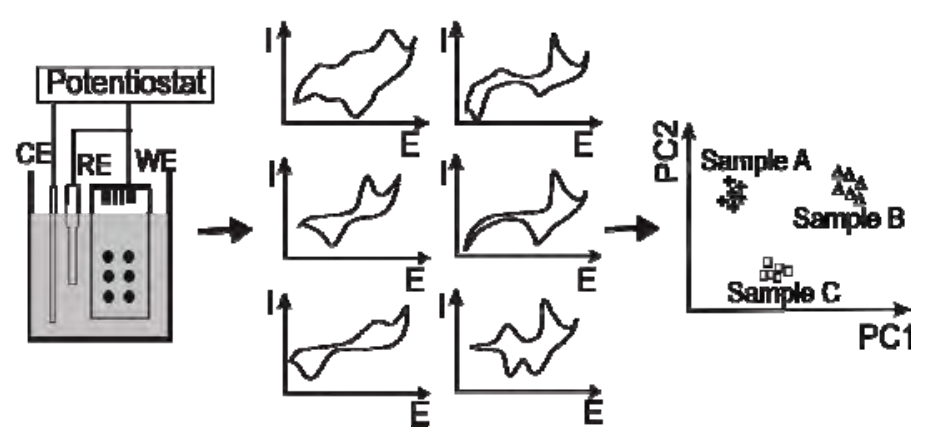

Figura 1.14. Ilustración de una lengua electrónica basada en ftalocianinas y su tratamiento de datos.

Una vez que los voltamogramas han sido preprocesados y el número de variables reducidas, estas se utilizan como entrada para el análisis estadístico como la PCA, PLS-DA o cualquier otro tipo de herramientas análisis multivariante. Es posible decir que sin importar el tipo de técnica electroquímica o el analito evaluado, los métodos electroquímicos analizados básicamente son los mismos durante los últimos años. En el caso específico de las lenguas electrónicas alrededor del $40 \%$ de las investigaciones publicadas utilizan como técnica de reconocimiento de patrones una PCA [237]. Esto significa que aún hay un tema abierto a todo tipo de posibilidades, y a una exploración que permita estudiar las ventajas y desventajas de implementar nuevos métodos diseñados para otras áreas e incluso, por que no, al diseño de nuevos métodos específicos.

\subsubsection{Aplicaciones de la lengua electrónica}

El desarrollo de las lenguas electrónicas es muy actual, es posterior al de las narices electrónicas; es por ello que sus aplicaciones son mínimas. En este apartado se citan exclusivamente nuestras investigaciones en este campo.

Nuestra lengua electrónica ha permitido discriminar vinos tintos con diferentes características organolépticas [238] y de discriminar vinos tintos con diferente capacidad de oxidación [239].

En manera intensiva se ha estudiado, desde hace años el papel de diversas ftalocianinas como material sensible. También se han utilizado otros materiales como perilenos o polímeros conductores y hemos construido redes híbridas 
combinando electrodos de diversos materiales. Con estos sistemas ha sido posible discriminar vinos elaborados con diferentes variedades de uvas [231], detector adulteraciones [240] o realizar un seguimiento del envejecimiento de vinos tintos [241].

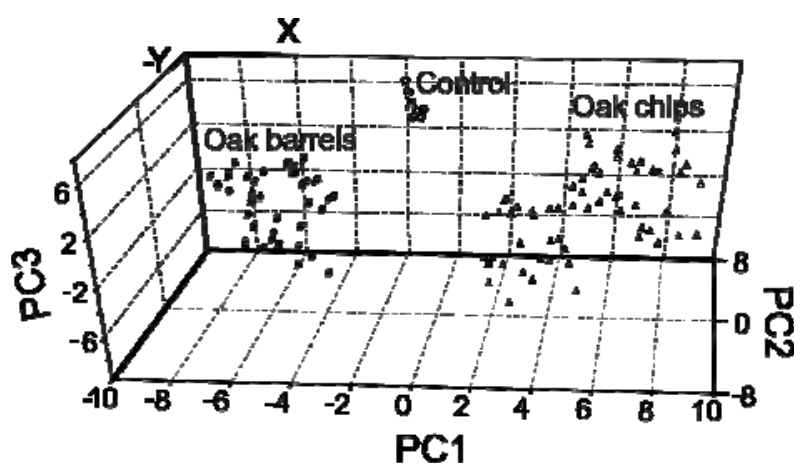

Figura 1.15. PCA de un conjunto de señales derivado de la aplicación de una lengua electrónica para el análisis de vinos tintos.

También en el sector alimentación las redes de sensores basados en ftalocianinas se han sido utilizadas con éxito para discriminar entre diferentes tipos de aceites de oliva [242], control de calidad en la producción de cervezas [243] o en evaluación de la frescura del pescado a través de los niveles de aminas biógenas [244].

\subsubsection{El ojo electrónico}

En este apartado se ilustra a un breve desarrollo conceptual de las diferentes expresiones asociadas al concepto de color en general y en particular al color de los vinos.

En el campo de la física se vincula el color de un material a las variaciones en las distribuciones espectrales que medimos al observarlo, tanto si son emitidas directamente por fuentes como si lo son indirectamente reflejadas o transmitidas. En un lenguaje más químico el color se asocia a las diferencias espectrales debidas a variaciones en la composición molecular y configuraciones electrónicas de los compuestos químicos constituyentes de la muestra [245]. En sociología el color es un aspecto de la respuesta de un observador humano, una percepción cultural que tiene lugar en el cerebro del observador como resultado de la estimulación visual [246]. No es trivial compatibilizar estas definiciones.

En nuestro estudio nos centraremos en dos nociones de estudio diferentes, por una parte explicaremos la percepción del color, que estará centrada en los 
elementos que son necesarios para la definición de un color, y que nos llevará al segundo conceptos presentado: la medida del color. Es decir, que llegados a este punto seamos capaces de relacionar una condición sicológica (perceptiva) con un fenómeno físico-químico, a través de una serie de funciones matemáticas denominados valores triestímulos [247].

\subsubsection{Percepción del color}

En la definición del color de un determinado objeto, después de fijar un observador, es necesario precisar la combinación espectral de la fuente de luz con la cual se ilumina; es decir que este factor actúa como el punto de referencia de nuestro problema.

La fuente de luz en este caso se refiere a una fuente física de luz visible, es decir que sólo cubre la parte del espectro electromagnético que comprende las longitudes onda entre 300 y $900 \mathrm{~nm}$. La Figura 1a. muestra la energía relativa de la luz solar para cada longitud de onda; es una forma representativa que permite cuantificar las características espectrales de una determinada fuente de la luz, en este caso es el sol.

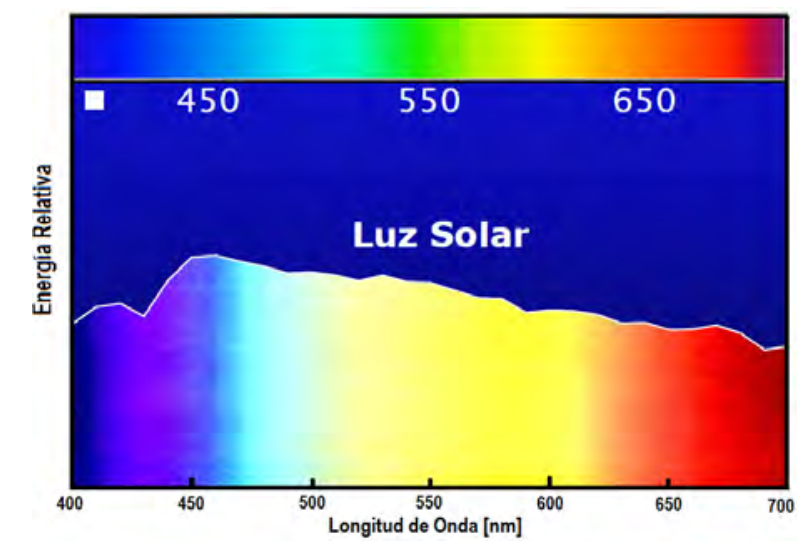

a. Distribución espectral de la energía de luz solar

Iluminante para tres fuentes diferentes

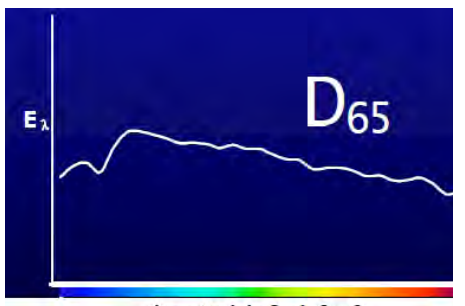

Lonaitud de Onda [nm]

Fuente luz solar

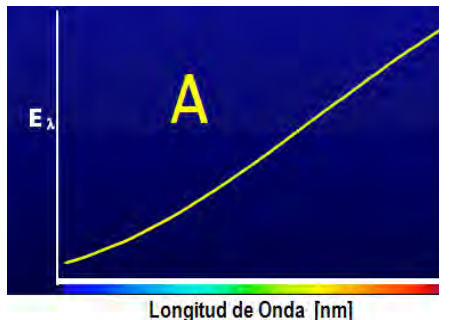

Fuente de tungsteno

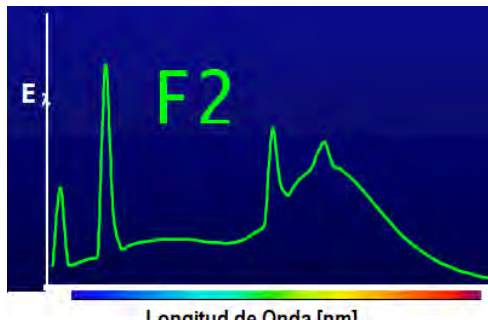

Longitud de Onda [nm] Fuente fluorescente

Figura 1.16. Fuentes de Luz frente a Iluminante 
En el laboratorio, para efectos de la cuantificación y estandarización de la observación visual se han establecido ciertas fuentes de luz como iluminantes, esto significa que se han establecido por acuerdo internacional unos iluminantes patrón denominados A, B, C, D65, y E. En la (Figura 1.16) hemos recogido algunos de estos factores.

Iluminante A. Es la luz de una lámpara de incandescencia de filamento de wolframio, alimentada a una tensión tal que adquiere una temperatura de color $2856 \mathrm{~K}$.

Iluminante B. Representa en promedio la luz del sol directo al medio día con un cielo claro. Puede obtenerse esta luz haciendo pasar el iluminante A utilizando dos disoluciones estándar. Su temperatura de color es de $4874 \mathrm{~K}$.

Iluminante $C$. Representa la luz de cielo norte sin sol directo en un día claro. Su temperatura de color es de $6774 \mathrm{~K}$

Iluminante $D_{65}$. Iluminación con una composición espectral tipo luz de día, correspondiente a una temperatura de color $6504 \mathrm{~K}$. Pretende emular un promedio de luz diurna.

Iluminante E. Representa un espectro equienergético teórico y puede obtenerse aproximadamente filtrando la luz A por disoluciones estándar. Temperatura de color 5500K.

Respecto a otra de las partes que constituye este proceso, como lo es el objeto, se sabe que este absorbe y refleja selectivamente ciertas longitudes de onda de la luz incidente y transmite sus complementarias, como un efecto de su composición atómica y propiedades estructurales. Un objeto entonces quedará perfectamente definido por su espectro de reflectancia -en el caso de sólidos-, y de transmitancia -en el caso de líquidos- a lo largo de todo el espectro visible. Utilizando un espectrómetro UV-Visible con lectura entre 380 y $780 \mathrm{~nm}$ con una iluminación estándar se puede definir el espectro visible del objeto, y de esta manera seremos capaces de cuantificar el segundo elemento de la observación visual.

Por último, aunque no menos significativa está la intervención del observador, la cual se cuantifica valorando la distinta sensibilidad relativa del ojo humano a cada longitud de onda de la luz. Esta sensación es también referenciada como una medida de la sensibilidad del ojo a la luminosidad. Los valores de la sensibilidad del ojo para todas las longitudes de onda fueron determinadas a nivel experimental por la Comisión Internationale de l'Éclairage (CIE). Los 
resultados de estos modelos patrón establecido pueden observarse en la Figura 1.17 [247].

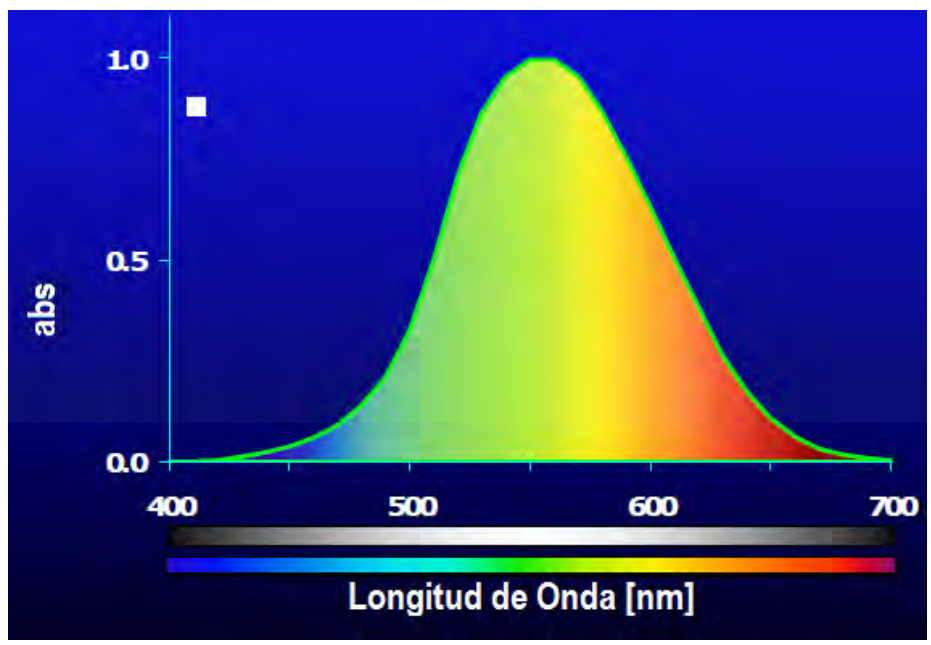

Figura 1.17. Sensibilidad de Ojo Humano a los colores espectrales

Ahora bien en 1931, apoyándonos en los conocimientos fisiológicos sobre la sensibilidad de los conos del ojo a los colores rojo, verde y azul del observador humano promedio, fueron desarrolladas experimentalmente las funciones $X, Y, Z$ por la CIE para cuantificar la habilidad del ojo humano de percibir el color rojo, verde y azul, bajo una iluminación $\mathrm{D}_{65}$; estos colores fueron definidos como primarios. Cuando se llevaron a cabo los experimentos en 1931 del observador patrón bajo un ángulo de $2^{\circ}$, se pensó que los conos se concentraban solamente en una pequeña región del ojo, sin embargo más tarde cuando se demostró que esta región era más extensa se volvieron a repetir los experimentos resultando de estos el observador patrón CIE $10^{\circ}$, típico en la mayoría de las aplicaciones en investigación y también a nivel industrial (Figura 1.18.).

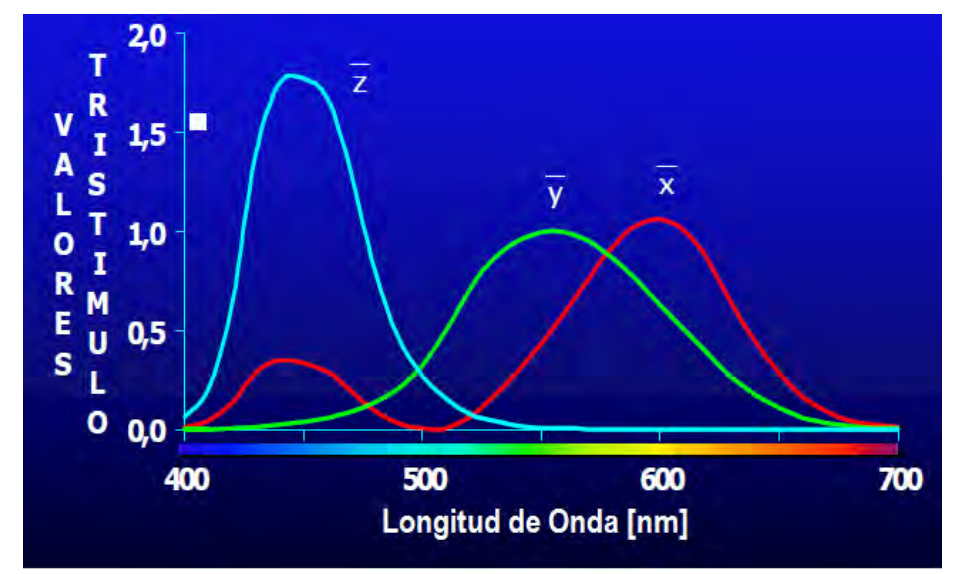

Figura 1.18. Observador colorimétrico patrón CIE a $10^{\circ}$ 


\subsubsection{Medición del color}

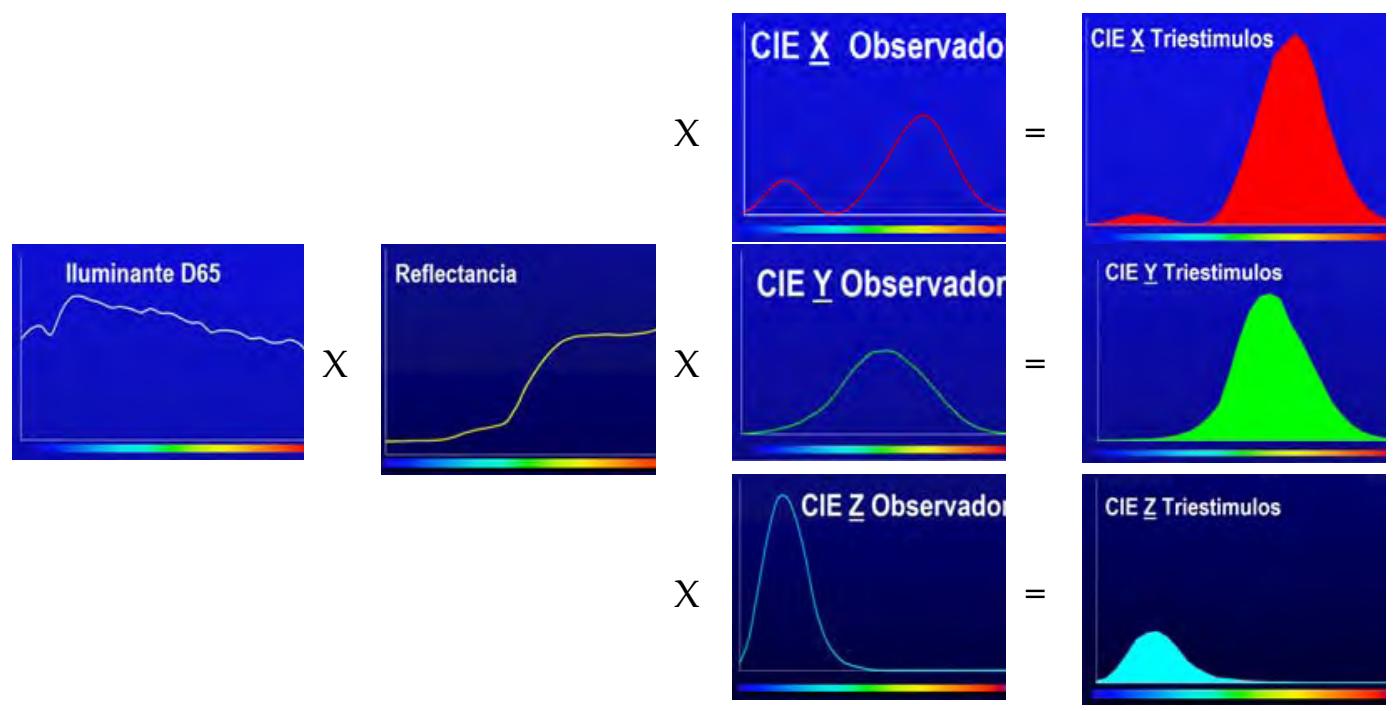

Resumiendo los valores triestímulos pueden describirse considerando los siguientes parámetros:

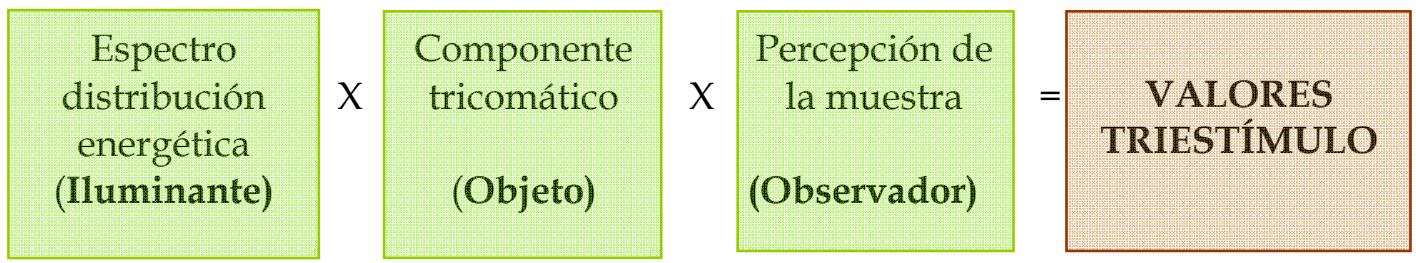

Figura 1.18. Parámetros X, Y, Z CIE triestímulo de cualquier color

Para diseñar una técnica capaz de modelar la percepción humana del color, cada elemento constituyente del proceso de la observación visual debería representarse como una relación matemática. Hasta el momento se han descrito los espectros característicos asociados a cada una de las partes; a manera de resumen podemos destacar lo siguiente: la fuente que se cuantifica de acuerdo con el espectro generado iluminante seleccionado por el usuario, el objeto se cuantifica midiendo su reflectancia o transmisión y el observador se cuantifica según la sensibilidad del observador patrón CIE para cada uno de los colores básicos [248].

Así pues, el producto de los valores para el iluminante, la reflectancia o transmisión del objeto y las funciones del observador patrón integrados al espectro visible permiten describir los valores triestímulo del color, definidos como valores X, Y, Z CIE. Para conseguir especificar con un único valor cada color básico, se suma el producto de los tres espectros para cada longitud de onda en el rango visible. La Figura 4 describe las características generales de los valores triestímulos X, Y, Z CIE [247, 249]. 
En términos matemáticos los valores triestímulos están definidos por las siguientes relaciones:

$$
\begin{gathered}
X=k \sum_{380}^{780}[\mathrm{~S}(\lambda) \cdot \overline{\bar{x}}(\lambda) \cdot \mathrm{T}(\lambda)] Y=k \sum_{380}^{780}[\mathrm{~S}(\lambda) \cdot \overline{\bar{y}}(\lambda) \cdot \mathrm{T}(\lambda)] \quad Z=k \sum_{380}^{780}[\mathrm{~S}(\lambda) \cdot \overline{\mathrm{Z}}(\lambda) \cdot \mathrm{T}(\lambda)] \\
\text { Siendo } k=\frac{100}{\sum_{380}^{780}[S(\lambda) \cdot \bar{y}(\lambda)]}
\end{gathered}
$$

En estas expresiones, $S(\lambda)$, es la distribución energética espectral del iluminante; $\bar{x}(\lambda), \bar{y}(\lambda), \bar{z}(\lambda)$, son los valores obtenidos de la sensibilidad que tiene un observador a los colores primarios de acuerdo a la valoración realizada por la CIE; $\mathrm{T}(\lambda)$, está definida por los valores experimentales medidos en transmitancia de un determinado objeto; y finalmente $k$, es el factor de normalización suponiendo que $Y=100$, es decir para el caso de un difusor ideal [246].

No obstante, este sistema conceptualmente importante no es cómodo en la práctica ya que no consigue reflejar totalmente la sensación visual percibida por el observador, característica que en nuestro caso es importante porque necesitamos definir cualidades usuales de los vinos. Es por ello que se recurre a una nueva representación de estos valores en el denominado sistema CIELab, propuesto como una alternativa más completa a la descripción de una observación visual [250].

\section{Método CIELab}

La revisión de los conceptos realizada hasta el momento, es un punto de partida para la descripción de uno de los métodos más utilizados para evaluar el color de los vinos, simplemente porque es el mejor modelo capaz de expresar la sensación psicológica percibida. Los parámetros denominados CIELab [251], basan su ejercicio en la teoría de la percepción de los colores opuestos; es decir por ejemplo que un color no puede ser verde y rojo al mismo tiempo, ni tampoco azul y amarillo a la vez. Todo el proceso necesario para obtener una descripción del color mediante parámetros CIELab se esquematiza en la Figura 1.19.

Las nuevas coordenadas $a^{*}, b^{*}, L^{*}, C, H$ y $S$ se obtienen a partir de las coordenadas $\mathrm{X}, \mathrm{Y}, \mathrm{Z}$ y relaciones mostradas en la primera parte de la Tabla 1.4. 


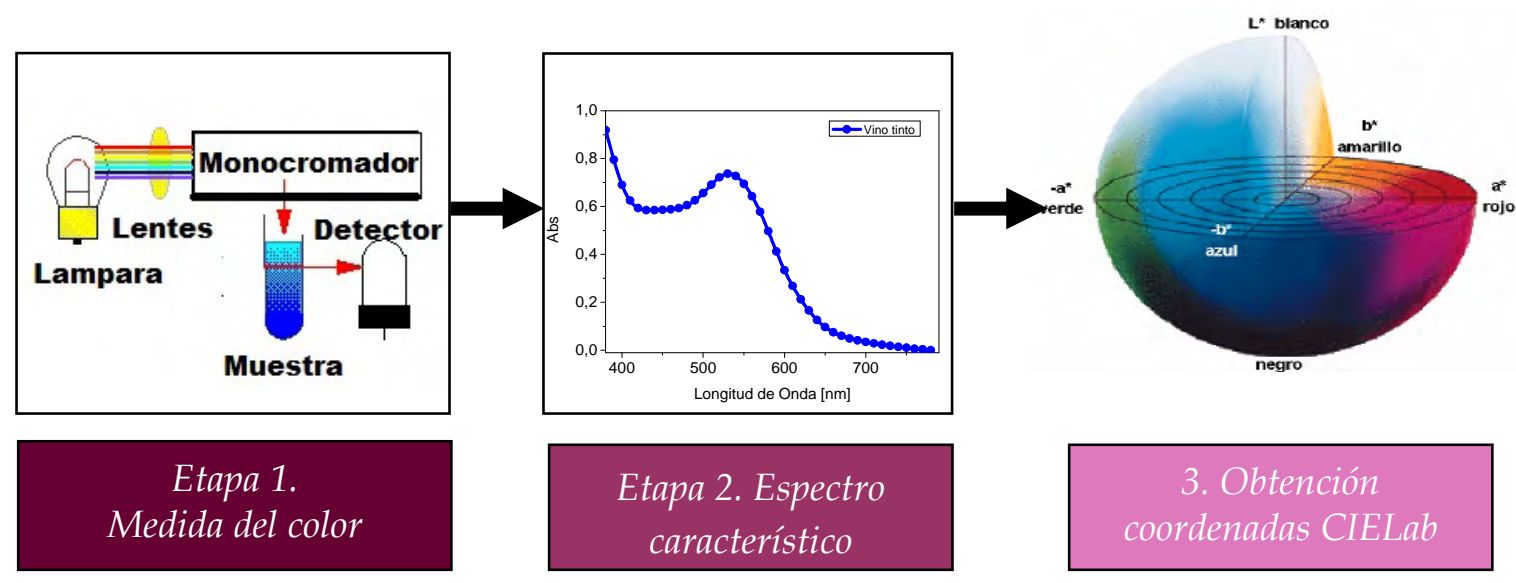

Figura 1.19. Procedimiento experimental para el análisis del color

Tabla 1.4. Ecuaciones matemáticas para la obtención de la coordenada CIELab

\begin{tabular}{|ll|}
$a^{*}=500\left(\sqrt[3]{\frac{X}{X_{n}}}-\sqrt[3]{\frac{Y}{Y_{n}}}\right)$ & $C=\sqrt{a^{*^{2}+b^{* 2}}}$ \\
$b^{*}=200\left(\sqrt[3]{\left.\frac{Y}{Y_{n}}-\sqrt[3]{\frac{Z}{Z_{n}}}\right)}\right.$ & $H=\operatorname{arctg}\left(\frac{b^{*}}{a^{*}}\right)$ \\
$L^{*}=116\left(\sqrt[3]{\frac{Y}{Y_{n}}}-0.1379\right)$ & $S=\frac{C^{*}}{L}$ \\
\hline
\end{tabular}

En este nuevo espacio el eje vertical $L^{*}$ es una medida de la luminosidad, y tiene una variación desde la opacidad total (valor 0) hasta la transparencia total (valor 100). Para los círculos de tonalidad, $\mathrm{a}^{*}$ es una medida de la intensidad de color rojo ( $\mathrm{y}-\mathrm{a}^{*}$ de color verde), y el $\mathrm{b}^{*}$ de la intensidad de color amarillo ( $\mathrm{y}-\mathrm{b}^{*}$ de color azul). La tonalidad $(\mathrm{H})$ y la saturación o croma $(\mathrm{C})$ se obtienen a partir de las coordenadas $\mathrm{L}^{*}, \mathrm{a}^{*} \mathrm{y} \mathrm{b}^{*}$, por sustitución en las ecuaciones matemáticas indicadas en la tabla anterior [251-252].

El parámetro $\mathrm{H}$ está asociado especialmente a pigmentos amarillos que absorben la luz a longitudes de onda cercanas $420 \mathrm{~nm}$ 253. La saturación o croma (C), indica la contribución de $\mathrm{a}^{*}$ (color rojo) y b* (color amarillo) al color total del vino.

Todos los resultados referentes al color de los vinos publicados en esta tesis serán descritos en términos de estas coordenadas CIELab. La Figura 1.20 resume la función de cada uno de los parámetros que conforman este método. 

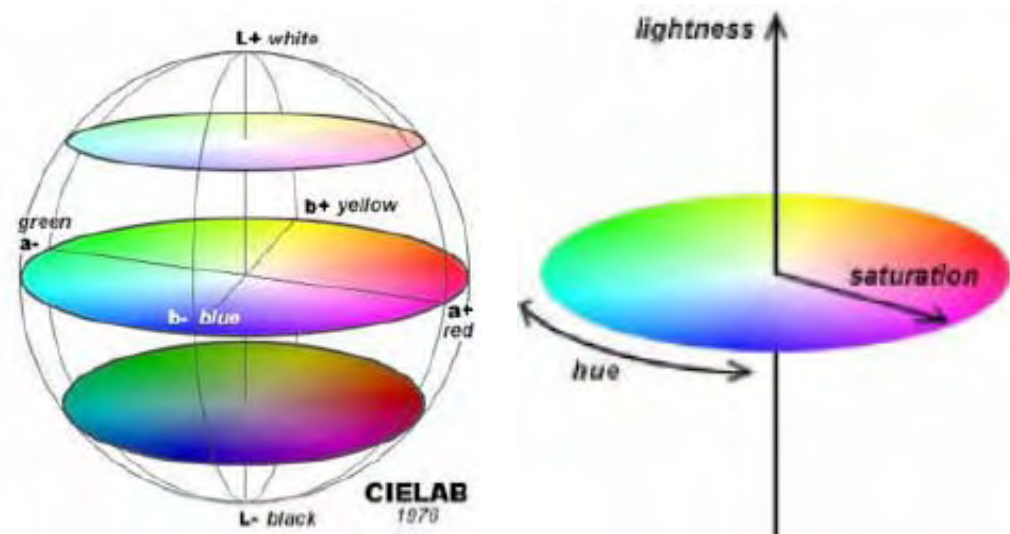

Figura 1.20. Coordenadas CIELab

\section{Índices colorimétricos del vino}

A lo largo de esta memoria también serán expuestos otros métodos que no tienen relación con los métodos oficiales de determinación de color de la CIE, pero que los enólogos utilizan por su rapidez y sencillez de cálculo al verificar la evolución de la materia colorante durante la crianza o envejecimiento del vino.

Estos métodos simplificados se fundamentan en índices que evalúan la medida de la absorbancia de las muestras a longitudes de onda específicas de $520 \mathrm{~nm}$ y $420 \mathrm{~nm}$. Sudraud sugiere que se calcule la densidad de color (definida como la suma de las absorbancias a $520 \mathrm{~nm}$ y $420 \mathrm{~nm}$ ) como índice "intensidad de color", y el índice "tonalidad" (definida como la razón entre las absorbancias a $420 \mathrm{~nm}$ y $520 \mathrm{~nm}$ ) que expresa el matiz de color [254].

Por otra parte Glories propone una nueva "intensidad colorante" correspondiente a la suma de las absorbancias a $420 \mathrm{~nm}, 520 \mathrm{~nm}$ y $620 \mathrm{~nm}$, es decir:

$$
I C=A_{420}+A_{520}+A_{620}
$$

Otro índice que se corresponde aproximadamente con el color rojo, lo define Glories, a partir de

$$
d A(\%)=\left(A_{520}-\frac{A_{420}+A_{620}}{2}\right) * \frac{1}{A_{520}} * 100 \%
$$

Si el vino presenta un color rojo vivo, $d \mathrm{~A}(\%)$ es elevado, entre 60 y $80 \%$, mientras que si el vino presenta un color rojo teja $\mathrm{dA}(\%)$ es inferior a $40 \%$.

Dentro de los índices utilizados para evaluar la evolución de los vinos también se evalúa el porcentaje de amarillos, el porcentaje de rojos y el porcentaje de azules, como lo expresan las siguientes ecuaciones [255]. 
Índices del color:

\%amarillos $=\frac{A_{420}}{A_{420}+A_{520}+A}$ \%rojos $=\frac{A_{520}}{A_{420}+A_{520}+A_{6}}$ \%azules $=\frac{A_{620}}{A_{420}+A_{520}+A_{6}}$

Los porcentajes elevados de rojos reflejan juventud en el vino; mientras que porcentajes elevados de amarillos implican pérdida de juventud.

\subsection{REFERENCIAS}

[1] B. N. Ames, M. K. Shigenaga \& T. M. Hagen, Oxidants, antioxidants, and the degenerative diseases of aging, Proceedings of the National Academy of Sciences of the United States of America, 1993, 90, 7915-7922.

[2] F. Leighton, I. Urquiaga \& M. S. Diez, Presentación a la $77^{a}$ Asamblea General de la OIV y al XXII Congreso Mundial de la Vid y del Vino, Buenos Aires, 1997.

[3] K. J. Stutts \& R. M. Wightman, Electrocatalysis of ascorbate oxidation with electrosynthesized, surface bound mediators, Analytical Chemistry, 1983, 55, 1576-1582.

[4] A. M. Alonso, D. A. Guillén, C. G. Barroso, B. Puertas \& A. García, Determination of of antioxidant activity of wine by products and its correlation with with polyphenolic content, Journal of Agricultural and Food Chemistry, 2002, 50, 5832-5838.

[5] M. Antolovich, P. D. Prenzler, E. Patsalides, S. McDonald \& K. Robards, Methods for testing antioxidant activity, Analyst, 2002, 127, 183-198.

[6] J. Burns, P. T. Gardner, D. Matthews, G. G. Duthie, M. E. J. Lean \& A. Grozier, Extraction of phenolics and changes in antioxidant activity of red wines during vinification, Journal of Agricultural and Food Chemistry, 2001, 49 5797-5808.

[7] M. S. Fernández-Pachón, D. Villaño, A. M. Troncoso \& M. C. García-Parrilla, Revisión de los métodos de evaluación de la actividad antioxidante in vitro del vino y valoración de sus efectos in vivo, Archivos Latino Americanos de nutrición, 2006, 56, 2.

[8] M. D. Rivero-Pérez, P. Muñiz \& M. L. González-San José, Antioxidant profile of red wines evaluated by total antioxidant capacity, scavenger activity, and biomarkers of oxidative stress methodologies, Journal of Agricultural and Food Chemistry, 2007, 55 5476-5483.

[9] J. Ribereau-Gayon, Dissolution d'oxygene dans les vins. In contribution a l'etude des oxidations et reductions dans les vins. Application a l'etude de vieillissement et des casses, Delmas, Bordeaux-France, 1933.

[10] P. Pontallier, M. H. Salagoity-Auguste \& P. Ribereau-Gayon, Intervention Du Bois De Chêne Dans L'Evolution Des Vins Rouges Elevés En Barriques, Connaissance de la Vigne et du Vin, 1982, 16, 45-61. 
[11] P. Pontallier, Practiques Actuelles D’Elevage En Barriques Des Grands Vins Rouges, Journal International Des Sciences De La Vigne Et Du Vin, 1991, 16, 115-122.

[12] N. Vivas, Y. Glories \& J. Francois, Mise Au Point Sur L’Elevage Des Vins Rouges En Fûts De Chêne", Revue Francaise d'oenologie. , 1991, 62, 17-21.

[13] J. Artajona, Caracterización del Roble según su origen y grado de tostado mediante la utilización de G.C. Y H.P.L.C., Viticultura/Enologia Profesional, 1991, 14, 61-72.

[14] P. Chatonnet, J. N. Boidron \& M. Pons, Maturation of red wines in oak barrels - evolution of some volatile compounds and their aromatic impact, Sciences Des Aliments, 1990, 10, 565587.

[15] J. Cacho, El roble, la barrica, y la crianza del vino tinto, Institución "Fernando el Católico", Zaragoza, 2009.

[16] M. A. Sefton, P. J. Spillman, K. J. Pocok, Francis Il \& P. J. Williams, The influence of oak origin, seasoning, and other industry practices on the sensory characteristics and composition of oak extracts and barrel-aged white wines, Australian Grapegrower \& Winemaker, 1993, 335.

[17] C. Marquet, Las nuevas tendencias del mercado del vino en España ACE Revista de Enología, 2007.

[18] M. Del Álamo, I. Nevares, L. Gallego, B. Fernández de Simón \& E. Cadahía, Microoxygenation strategy depends on origin and size of oak chips or staves during accelerated red wine aging, Analytica Chimica Acta, 2010, 660, 92-101.

[19] T. Dubordieu \& V. Moine, Role des manoproteines et levures sur la stabilite tartrique des vins, Revue Francaise d'oenologie., 1997, 85, 17-23.

[20] I. Apetrei, C. Apetrei, I. Nevares, M. Del Alamo, V. Parra, M. L. Rodríguez-Méndez \& J. A. De Saja, OIV Conference. ( Logroño) 2006.

[21] M. J. Cejudo-Bastante, I. Hermosin-Gutierrez \& M. S. Perez-Coello, Micro-oxygenation and oak chip treatments of red wines: Effects on colour-related phenolics, volatile composition and sensory characteristics. Part II: Merlot wines, Food Chemistry, 2011, 124, 738-748.

[22] E. Peynaud, El gusto del vino, Ediciones Mundiprensa, 1987.

[23] M. S. Cosio, D. Ballabio, S. Benedetti \& C. Gigliotti, Evaluation of different storage conditions of extra virgin olive oils with an innovative recognition tool built by means of electronic nose and electronic tongue, Food Chemistry, 2007, 101, 485-491.

[24] C. Di Natale, R. Paolesse, A. Macagnano, A. Mantini, A. D'Amico, M. Ubigli, A. Legin, L. Lvova, A. Rudnitskaya \& Y. Vlasov, Application of a combined artificial olfaction and taste system to the quantification of relevant compounds in red wine, Sensors and Actuators B-Chemical, 2000, 69, 342-347.

[25] M. L. Rodriguez-Mendez, A. A. Arrieta, V. Parra, A. Bernal, A. Vegas, S. Villanueva, R. Gutierrez-Osuna \& J. A. de Saja, Fusion of three sensory modalities for the multimodal characterization of red wines, Ieee Sensors Journal, 2004, 4, 348-354. 
[26] M. Cole, J. A. Covington \& J. W. Gardner, Combined electronic nose and tongue for a flavour sensing system, Sensors and Actuators B-Chemical, 2011, 156, 832-839.

[27] C. Di Natale, R. Paolesse, A. Macagnano, A. Mantini, A. D'Amico, A. Legin, L. Lvova, A. Rudnitskaya \& Y. Vlasov, Electronic nose and electronic tongue integration for improved classification of clinical and food samples, Sensors and Actuators B-Chemical, 2000, 64, 15-21.

[28] T. Sundic, S. Marco, J. Samitier \& P. Wide, Electronic tongue and electronic nose data fusion in classification with neural networks and fuzzy logic based models, Instrumentation and Measurement Technology Conference, 2000. IMTC 2000. Proceedings of the 17th IEEE, 2000, 3, 1474 - 1479.

[29] P. Wide, F. Winquist, P. Bergsten \& E. M. Petriu, The human-based multi-sensor fusion method for artificial nose and tongue sensor data, Instrumentation and Measurement Technology Conference, 1998. IMTC/98. Conference Proceedings. IEEE, 1998, 1, 531 - 536.

[30] P. N. Bartlett, J. M. Elliott \& J. W. Gardner, Electronic noses and their application in the food industry, Food Technology, 1997, 51, 44-48.

[31] T. C. Pearce, J. W. Gardner, S. Friel, P. N. Bartlett \& N. Blair, Electronic nose for monitoring the flavor of beers, Analyst, 1993, 118, 371-377.

[32] J. W. Gardner \& P. N. Bartlett, Electronic noses: principles and applications, Oxford University Press Oxford, 1999.

[33] K. C. Persaud \& J. P. Travers, Arrays of broad specificity films for sensing volatile chemicals, (Ed. Kress-Rogers), CRC Press, Frankfurt, 1997, pp. 563-592.

[34] T. C. Pearce, S. Schiffman, H. Troy Nagle \& J. W. Gardner, Handbook of Machine Olfaction: Electronic Nose Technology, John Wiley \& Sons 2003.

[35] A. D. Wilson \& M. Baietto, Applications and advances in electronic-nose technologies, Sensors, 2009, 9, 5099-5148.

[36] E. A. Baldwin, J. Bai, A. Plotto \& S. Dea, Electronic Noses and Tongues: Applications for the Food and Pharmaceutical Industries, Sensors, 2011, 11, 4744-4766.

[37] M. L. Rodríguez-Méndez, C. Apetrei \& J. A. de Saja, Electronic tongues purposely designed for the organoleptic characterization of olive oils., Eds.: V. R. Preedy and R. R. Watson), Academic Press, 2009, pp. 525-532.

[38] N. Barsan, D. Koziej \& U. Weimar, Metal oxide-based gas sensor research: How to?, Sensors and Actuators B-Chemical, 2007, 121, 18-35.

[39] M. Peris \& L. Escuder-Gilabert, A 21st century technique for food control: Electronic noses, Analytica Chimica Acta, 2009, 638, 1-16.

[40] F. Roeck, N. Barsan \& U. Weimar, Electronic nose: Current status and future trends Chemical Reviews 2008, 108, 705-725.

[41] J. W. Gardner \& P. N. Bartlett, A brief-history of electronic noses, Sensors and Actuators B-Chemical, 1994, 18, 211-220. 
[42] C. Di Natale, A. Macagnano, R. Paolesse, A. Mantini, E. Tarizzo, A. D'Amico, F. Sinesio, F. M. Bucarelli, E. Moneta \& G. B. Quaglia, Electronic nose and sensorial analysis: comparison of performances in selected cases, Sensors and Actuators B-Chemical, 1998, 50, 246-252.

[43] M. Vinaixa, Improving MS-Sensor technoloogies for food quality assessment, Doctoral Thesis, Rovira i Virgili, Tarragona, 2008.

[44] M. E. H. Amrani, R. M. Dowdeswell, P. A. Payne \& K. C. Persaud, An intelligent gas sensing system, Sensors and Actuators B-Chemical, 1997, 44, 512-516.

[45] R. W. Cattrall, Chemical Sensors, Oxford University Press, Oxford, 1997.

[46] S. Mannino, S. Benedetti, S. Buratti \& M. S. Cosio, Gas and liquid sensors for quality control in food processing, 2005.

[47] P. T. Moseley, A. M. Stoneham \& D. E. Williams, Oxide semiconductors: patterns of response behaviour according to materials type, Eds.: P. T. Moseley, J. Morris and D. Williams), Adam Hilger, Bristol, 1991, p. 108.

[48] D. James, S. M. Scott, Z. Ali \& W. T. O'Hare, Chemical sensors for electronic nose systems Microchimica Acta, 2005, 149, 1-17.

[49] A. Mandelis \& C. C., Physics, Chemistry and Technology of Solid State Sensor Devices, John Wiley \& Aons, Inc., New York, 1993.

[50] J. W. Gardner, Microsensor Array Devices, Wiley, Chichester, 1994, pp. 279-302.

[51] E. Garcia-Berrios, T. Gao, D. Walker, B. S. Brunschwig \& N. S. Lewis, Composites of carboxylate-capped $\mathrm{TiO}(2)$ nanoparticles and carbon black as chemiresistive vapor sensors, Sensors and Actuators B-Chemical, 2011, 158, 17-22.

[52] R. Paolesse, C. Di Natale, A. Macagnano, F. Davide, T. Boschi \& A. D'Amico, Selfassembled monolayers of mercaptoporphyrins as sensing material for quartz crystal microbalance chemical sensors, Sensors and Actuators B-Chemical, 1998, 47, 70-76.

[53] A. D'Amico, C. Di Natale, R. Paolesse, A. Macagnano \& A. Mantini, Metalloporphyrins as basic material for volatile sensitive sensors, Sensors and Actuators B-Chemical, 2000, 65, 209-215.

[54] T. Alizadeha \& S. Zeynali, Electronic nose based on the polymer coated SAW sensors array for the warfare agent simulants classification, Sensors and Actuators B-Chemical, 2008, 129, 412-423.

[55] D. Matatagui, J. Marti, M. J. Fernandez, J. L. Fontecha, J. Gutierrez, I. Gracia, C. Cane \& M. C. Horrillo, Chemical warfare agents simulants detection with an optimized SAW sensor array, Sensors and Actuators B-Chemical, 2011, 154, 199-205.

[56] S. Kladsomboon, M. Lutz, T. Pongfa \& T. Kerdcharoen, An Optical Artificial Nose System for Odor Classifications Based on LED Arrays, Electrical Engineering/Electronics, Computer, Telecommunications and Information Technology (ECTI-CON), 2011 8th International Conference on 2011, 145-148. 
[57] B. A. Suslick, L. Feng \& K. S. Suslick, Discrimination of complex mixtures by a colorimetric sensor array: Coffee aromas, Analytical Chemistry, 2010, 82, 2067-2073.

[58] E. A. H. Hall, Biosensors, Open University Press, Buckingham, 1990.

[59] C. Wu, L. Du, D. Wang, L. Zhao \& P. Wang, A biomimetic olfactory-based biosensor with high efficiency immobilization of molecular detectors, Biosensors \& Bioelectronics, 2012, 31, 4448 .

[60] M. E. Escuderos, S. Sanchez \& A. Jimenez, Quartz Crystal Microbalance (QCM) sensor arrays selection for olive oil sensory evaluation, Food Chemistry, 2011, 124, 857-862.

[61] M. Garcia, M. J. Fernandez, J. L. Fontecha, J. Lozano, J. P. Santos, A. Aleixandre, I. Sayago, J. Gutierrez \& M. C. Horrillo, Differentiation of red wines using an electronic nose based on surface acoustic wave devices, Talanta, 2006, 68, 1162-1165.

[62] J. Lozano, M. J. Fernandez, J. L. Fontecha, M. Aleixandre, J. P. Santos, I. Sayago, T. Arroyo, J. M. Cabellos, F. J. Gutierrez \& M. C. Horrillo, Wine classification with a zinc oxide SAW sensor array, Sensors and Actuators B-Chemical, 2006, 120, 166-171.

[63] N. Barié, M. Bücking \& R. Rappa, A novel electronicnose based on miniaturized SAW sensor arrays coupled with SPME enhanced headspace-analysis and its use for rapid determination of volatile organic compounds in food quality monitoring, Sensors and Actuators B-Chemical, 2006, 114, 482-488.

[64] X. Huang, J. Xin \& J. Zhao, A novel technique for rapid evaluation of fish freshness using colorimetric sensor array, Journal of Food Engineering, 2011, 105, 632-637.

[65] Komyo Kitagawa http://www.itraders.biz/komyo/index.html.

[66] M. Gutierrez, A. Llobera, J. Vila-Planas, F. Capdevila, S. Demming, S. Buttgenbach, S. Minguez \& C. Jimenez-Jorquera, Hybrid electronic tongue based on optical and electrochemical microsensors for quality control of wine, Analyst, 2010, 135, 1718-1725.

[67] M. C. Cerrato Oliveros, J. L. Pérez Pavón, C. García Pinto, M. E. Fernández Laespada, B. Moreno Cordero \& M. Forina, Electronic nose based on metal oxide semiconductor sensors as a fast alternative for the detection of adulteration of virgin olive oils, Analytica Chimica Acta, $2002,459,219-228$.

[68] V. V. Malyshev \& A. V. Pislyakov, Dynamic properties and sensitivity of semiconductor metal-oxide thick-film sensors to various gases in air gaseous medium, Sensors and Actuators BChemical, 2003, 96, 413-434.

[69] M. Graf, A. Gurlo, N. Barsan, U. Weimar \& A. Hierlemann, Microfabricated gas sensor systems with sensitive nanocrystalline metal-oxide films, Journal of Nanoparticle Research, $2006,8,823-839$.

[70] N. El Barbri, E. Llobet, N. El Bari, X. Correig \& B. Bouchikhi, Electronic nose based on metal oxide semiconductor sensors as an alternative technique for the spoilage classification of red meat, Sensors, 2008, 8, 142-156.

[71] S. Villanueva, A. Guadarrama, M. L. Rodriguez-Mendez \& J. A. de Saja, Use of an array of metal oxide sensors coupled with solid phase microextraction for characterisation of wines Study of the role of the carrier gas, Sensors and Actuators B-Chemical, 2008, 132, 125-133. 
[72] C. Po-Chiang, N. I. Fumiaki, C. Hsiao-Kang, R. Koungmin \& Z. Chongwu, A nanoelectronic nose: a hybrid nanowire/carbon nanotube sensor array with integrated micromachined hotplates for sensitive gas discrimination, Nanotechnology, 2009, 20, 125503125511

[73] I. Sayago, E. Terrado, E. Lafuente, M. C. Horrillo, W. K. Maser, A. M. Benito, R. Navarro, E. P. Urriolabeitia, M. T. Martinez \& J. Gutierrez, Hydrogen sensors based on carbon nanotubes thin films, Synthetic Metals, 2005, 148, 15-19.

[74] R. Leghrib, R. Pavelko, A. Felten, A. Vasiliev, C. Cane, I. Gracia, J. J. Pireaux \& E. Llobet, Gas sensors based on multiwall carbon nanotubes decorated with tin oxide nanoclusters, Sensors and Actuators B-Chemical, 2010, 145, 411-416.

[75] M. Castro, B. Kumar, J. F. Feller, Z. Haddi, A. Amari \& B. Bouchikhi, Novel e-nose for the discrimination of volatile organic biomarkers with an array of carbon nanotubes (CNT) conductive polymer nanocomposites (CPC) sensors, Sensors and Actuators B-Chemical, 2011, $159,213-219$.

[76] B. Kumar, Y. T. Park, M. Castro, J. C. Grunlan \& J. F. Feller, Fine control of carbon nanotubes-polyelectrolyte sensors sensitivity by electrostatic layer by layer assembly (eLbL) for the detection of volatile organic compounds (VOC), Talanta, 2012, 88, 396-402.

[77] M. D. Shirsat, T. Sarkar, J. Kakoullis, Jr., N. V. Myung, B. Konnanath, A. Spanias \& A. Mulchandani, Porphyrin-Functionalized Single-Walled Carbon Nanotube Chemiresistive Sensor Arrays for VOCs, Journal of Physical Chemistry C, 2012, 116, 3845-3850.

[78] P. Lorwongtragool, A. Wisitsoraat \& T. Kerdcharoen, An Electronic Nose for Amine Detection Based on Polymer/SWNT-COOH Nanocomposite, Journal of Nanoscience and Nanotechnology, 2011, 11, 10454-10459.

[79] K. C. Persaud, S. M. Khaffaf, P. J. Hobbs \& R. W. Sneath, Assessment of conducting polymer odour sensors for agricultural malodour measurements, Chemical Senses, 1996, 21, 495-505.

[80] M. Bernabei \& K. Persaud, Development and test of a large array of conductive polymers sensors resembling the redundancy of the biological olfactory system, Chemical Senses, 2011, 36, E18-E18.

[81] Bo Li, Suresh Santhanam, Lawrence Schultz, Malika Jeffries-EL, Mihaela C. Iovu, Genevieve Sauvé, Jessica Cooper, Rui Zhang, Joseph C. Revelli, Aaron G. Kusne, Jay L. Zinder, Tomasz Kowalewski, Lee E. Weiss, Richard D. McCullough, Gary K. Fedder \& D. N. Pages, Lambeth Inkjet printed chemical sensorarray based on polythiophene conductive polymers, Sensors and Actuators B-Chemical, 2007, 123, 651-660.

[82] A. Guadarrama, M. L. Rodríguez-Méndez, C. Sanz, J. L. Ríos \& J. A. de Saja, Electronic nose based on conducting polymers for the quality control of the aroma of olive oil. Discrimination of quality, variety of olive and geographic origin, Analytica Chimica Acta, 2001, 432, 287- 296.

[83] N. Prieto, M. L. Rodriguez-Mendez, R. Leardi, P. Oliveri, D. Hernando-Esquisabel, M. Iniguez-Crespo \& J. A. de Saja, Application of multi-way analysis to UV-visible spectroscopy, gas chromatography and electronic nose data for wine ageing evaluation, Analytica Chimica Acta, 2012, 719, 43-51. 
[84] N. Gutierrez, M. L. Rodríguez-Méndez \& J. A. de Saja, Array of sensors based on lanthanide bisphthalocyanine Langmuir-Blodgett films for the detection of olive oil aroma, Sensors and Actuators B-Chemical, 2001, 77, 437- 442.

[85] F. E. INC., Operating principle of semiconductor type gas sensors, 2008, http://www.figaro.co.jp/en/item2.html.

[86] D. Kohl, Fundamentals and Recent Developments of Homogeneous Semiconducting Sensors, (Ed. W. a. B. Gardner, P. N. ), Kluwer Academic Publishers, Dordrecht, 1992, pp. 53-60.

[87] R. Díaz Delgado, Sensores de gases basados en óxidos de estaño: una aproximación electroquimica, Tesis doctoral, Universidad de Barcelona, Barcelona, 2002.

[88] A. Heilig, N. Barsan, U. Weimar, M. Schweizer-Berberich, J. W. Gardner \& W. Gopel, Gas identification by modulating temperatures of SnO2-based thick film sensors, Sensors and Actuators B-Chemical, 1997, 43, 45-51.

[89] T. Sahm, A. Gurlo, N. Barsan \& U. Weimar, Basics of oxygen and SnO2 interaction; work function change and conductivity measurements, Sensors and Actuators B-Chemical, 2006, $118,78-83$.

[90] W. Schmid, N. Barsan \& U. Weimar, Sensing of hydrocarbons with tin oxide sensors: possible reaction path as revealed by consumption measurements, Sensors and Actuators BChemical, 2003, 89, 232-236.

[91] N. Barsan \& U. Weimar, Conduction model of metal oxide gas sensors, Journal of Electroceramics, 2001, 7, 143-167.

[92] S. Villanueva, A. Vegas, J. A. Fernandez-Escudero, M. Iniguez, M. L. RodriguezMendez \& J. A. de Saja, SPME coupled to an array of MOS sensors - Reduction of the interferences caused by water and ethanol during the analysis of red wines, Sensors and Actuators B-Chemical, 2006, 120, 278-287.

[93] V. V. Sysoev, J. Goschnick, T. Schneider, E. Strelcov \& A. Kolmakov, A Gradient Microarray Electronic Nose Based on Percolating SnO2 Nanowire Sensing Elements, Nano Letters, 2007, 7, 3182-3188.

[94] H. Xing-Jiu \& C. Yang-Kyu, Chemical sensors based on nanostructured materials, Sensors and Actuators B-Chemical, 2007, 122, 659-671.

[95] X. Shi, L. Wang, N. Kariuki, J. Luo, C.-J. Zhong \& S. Lu, A multi-module artificial neural network approach to pattern recognition with optimized nanostructured sensor array, Sensors and Actuators B-Chemical, 2006, 117, 65-73.

[96] G. Sberveglieri, I. Concina, E. Comini, M. Falasconi, M. Ferroni \& V. Sberveglieri, Synthesis and integration of tin oxide nanowires into an electronic nose, Vacuum, 2012, 86, 532535.

[97] V. V. Sysoev, E. Strelcov, S. Kar \& A. Kolmakov, The electrical characterization of a multi-electrode odor detection sensor array based on the single $\mathrm{SnO}(2)$ nanowire, Thin Solid Films, 2011, 520, 898-903. 
[98] J. M. Cook, R. L. Karelitz \& D. E. Dalsis, Measurement of oxygen, nitrogen, and carbon dioxide in beverage headspace, Journal of Chromatographic Science, 1985, 23.

[99] K. Kolb \& L. Ettre, Static Headspace-Gas Chromatography: Theory and Practice, Wiley, 2006.

[100] A. Guadarrama, M. L. Rodríguez-Méndez, J. A. Fernández, J. Souto \& J. A. de Saja, Array of conducting polymer sensors for the characterisation of wines, Analytica chimica acta, 2000, 411, 193-200.

[101] E. Campo, V. Ferreira, A. Escudero \& J. Cacho, Prediction of the wine sensory properties related to grape variety from dynamic-headspace gas chromatography-olfactometry data, Journal of Agricultural and Food Chemistry, 2005, 53, 5682-5690.

[102] M. F. Alpendurada, Solid-phase microextraction: a promising technique for sample preparation in environmental analysis - Review, Journal of Chromatography A 2000, 889, 314.

[103] J. Pawliszyn, Solid phase microextraction, theory and practice, Wiley, New York, 1997.

[104] J. Pawliszyn, Applications of Solid Phase Microextraction, The Royal Society of Chemistry, Cambridge, 1999.

[105] M. Decker, Solid Phase Microextraction: A Practical Guide, SueAnn Scheppers Wercinski, 2000.

[106] L. Maggi, A. Zalacain, V. Mazzoleni, G. L. Alonso \& M. R. Salinas, Comparison of stir bar sorptive extraction and solid-phase microextraction to determine halophenols and haloanisoles by gas chromatography-ion trap tandem mass spectrometry, Talanta, 2008, 75, 753-759.

[107] M. A. Pedroza, A. Zalacain, J. F. Lara \& M. R. Salinas, Global grape aroma potential and its individual analysis by SBSE-GC-MS, Food Research International, 2010, 43, 1003-1008.

[108] K. MacNamara, R. Leardi \& F. McGuigan, Comprehensive investigation and optimisation of the main experimental variables in stir-bar sorptive extraction (SBSE)-thermal desorption-capillary gas chromatography (TD-CGC), Analytica Chimica Acta, 2009, 636, 190197.

[109] S. Ampuero, S. Bogdanov \& J. O. Bosset, Classification of unifloral honeys with an MSbased electronic nose using different sampling modes: SHS, SPME and INDEX European Food Research and Technology, 2004, 218, 198-207.

[110] B. K. Lavine \& C. E. Davidson, Classification and Pattern Recognition, (Ed. P. Gemperline), CRC PRESS, New York, 2006.

[111] S. M. Scott, D. James \& Z. Ali, Data analysis for electronic nose systems, Microchimica Acta, 2007, 156, 183-207.

[112] S. J. Haswell \& A. D. Walmsley, Analytical division diary, Analytical Proceedings, $1991,28,115$.

[113] D. L. Massart, B. G. M. Vandeginste, L. M. C. Buydens, S. De Jong, P. J. Lewi \& J. Smeyers-Verbeke, Handbook of Chemometrics and Qualimetrics, Elsevier Science, Amsterdam, 1997. 
[114] K. L. Goodner, J. G. Dreher \& R. L. Rouseff, The dangers of creating false classifications due to noise in electronic nose and similar multivariate analyses sensors and Actuators BChemical, 2001, 80, 261-266.

[115] P. Oliveri, C. Casolino \& M. Forina, Chemometric Brains for Artificial Tongues, (Ed. A. Press), 2010, pp. 57-117.

[116] K. R. Beebe, P. R. \& M. B. Seasholtz, Chemometrics: A practical Guide, Wiley New York, 1998.

[117] B. Raman, P. A. Sun, A. Gutierrez-Galvez \& R. Gutierrez-Osuna, Processing of chemical sensor arrays with a biologically inspired model of olfactory coding, Ieee Transactions on Neural Networks, 2006, 17, 1015-1024.

[118] C. Distante, M. Leo, P. Siciliano \& K. B. Persaud, On the study of feature extraction methods for an electronic nose, Sensors and Actuators B-Chemical, 2002, 87, 274-288.

[119] M. L. Olvera, A. Maldonado \& R. Asomoza, Películas delgadas de SnO2:Ga utilizadas como sensores de oxígeno, Superficies y Vacío, 1999, 8, 33-36.

[120] S. H. Hahn, N. Bârsan \& U. Weimar, Investigation of CO/CH4 mixture measured with differently doped $\mathrm{SnO} 2$ sensors, 2001, 78, 68.

[121] N. G. Patel, P. D. Patel \& V. S. Vaishnav, Indium tin oxide (ITO) thin film gas sensor for detection of methanol at room temperature, Sensors and Actuators B-Chemical, 2003, 96, 180189.

[122] K. Chatterjee, S. Chatterjee, A. Banerjee, M. Raut, N. Pal \& H. S. Maiti, The effect of palladium incorporation on methane sensitivity of antimony doped Tin, Materials Chemistry and Physics, 2003, 81, 33-38.

[123] J. V. Burlachenko, B. A. Snopok, S. Capone \& P. Siciliano, Performance of Machine Olfaction: Effect of Uniqueness of the Initial Data and Information Coding on the Discrimination Ability of Multisensor Arrays, Ieee Sensors Journal, 2011, 11, 649-656.

[124] P. Ivanov, E. Llobet, X. Vilanova, J. Hubalek, I. Gracia, C. Cane, X. Correig \& Ieee, Gas sensors micro-array for air quality monitoring based on pure and doped $\mathrm{SnO} 2$ thick sensing films, 2005.

[125] M. Forina, P. Oliveri, M. Casale \& S. Lanteri, Multivariate range modeling, a new technique for multivariate class modeling - The uncertainty of the estimates of sensitivity and specificity, Analytica Chimica Acta, 2008, 622, 85-93.

[126] M. Forina, P. Oliveri, H. Jager, U. Romisch \& J. Smeyers-Verbeke, Class modeling techniques in the control of the geographical origin of wines, Chemometrics and Intelligent Laboratory Systems, 2009, 99, 127-137.

[127] K. H. Esbensen \& P. Geladi, Principal Component Analysis: Concept, Geometrical Interpretation, Mathematical Background, Algorithms, History, Practice, (Ed. Elsevier), 2009

[128] J. W. Gardner, Detection of vapors and odors from a multisensor array using patternrecognition .1. Principal component and cluster-analysis, Sensors and Actuators B-Chemical, 1991, 4, 109-115. 
[129] R. A. Fisher, The statistical utilization of multiple measurements, Annals of Eugenics, $1938,8,376-386$.

[130] E. Anderson, The irises of the Gaspe Peninsula, Bulletin of the American Iris Society, $1935,59,2-5$.

[131] M. J. Benito, M. C. Ortiz, M. S. Sanchez, L. A. Sarabia \& M. Iniguez, Typification of vinegars from Jerez and Rioja using classical chemometric techniques and neural network methods, Analyst, 1999, 124, 547-552.

[132] M. E. Melendez, M. C. Ortiz, M. S. Sanchez, L. A. Sarabia \& M. Iniguez, Chemometric characterization of the claretes and rose wines of the certified denomination of origin Rioja using CieLab parameters, Quimica Analitica, 1999, 18, 119-126.

[133] R. Bro, PARAFAC. Tutorial and applications, Chemometrics and Intelligent Laboratory Systems, 1997, 38, 149-171.

[134] C. A. Andersson \& R. Bro, Improving the speed of multi-way algorithms: Part I. Tucker3, Chemometrics and Intelligent Laboratory Systems, 1998, 42, 93-103.

[135] R. Bro \& C. A. Andersson, Improving the speed of multiway algorithms part II. Compression, Chemometrics and Intelligent Laboratory Systems, 1998, 42, 105-113.

[136] R. Bro, Multi-way Analysis in the Food Industry. Models, Algorithms, and Applications, Ph.D. thesis, University of Amsterdam, 1998.

[137] M. Penza \& G. Cassano, Recognition of adulteration of Italian wines by thin-film multisensor array and artificial neural networks, Analytica Chimica Acta, 2004, 509, 159-177.

[138] M. Aleixandre, I. Sayago, M. C. Horrillo, M. J. Fernandez, L. Ares, M. Garcia, J. P. Santos \& J. Gutierrez, Analysis of neural networks and analysis of feature selection with genetic algorithm to discriminate among pollutant gas, Sensors and Actuators B-Chemical, 2004, 103, $122-128$.

[139] M. S. Cosio, D. Ballabio, S. Benedetti \& C. Gigliotti, Geographical origin and authentication of extra virgin olive oils by an electronic nose in combination with artificial neural networks, Analytica Chimica Acta, 2006, 567, 202-210.

[140] A. Amari, N. El Barbri, E. Llobet, N. El Bari, X. Correig \& B. Bouchikhi, Monitoring the freshness of Moroccan sardines with a neural-network based electronic nose, Sensors, 2006, 6, 1209-1223.

[141] T. Michishita, M. Akiyama, Y. Hirano, M. Ikeda, Y. Sagara \& T. Araki, Gas Chromatography/Olfactometry and Electronic Nose Analyses of Retronasal Aroma of Espresso and Correlation with Sensory Evaluation by an Artificial Neural Network, Journal of Food Science, 2010, 75, S477-S489.

[142] H. Men, H. Liu, Y. Pan, L. Wang \& H. Zhang, Electronic Nose Based on an Optimized Competition Neural Network, Sensors, 2011, 11, 5005-5019.

[143] J. Brezmes, Diseño de una nariz electrónica para la determinación no destructiva del grado de maduración de la fruta, Tesis Doctoral, Universitat Politécnica de Catalunya, 2002. 
[144] D. B. Hibbert, Genetic algorithms in chemistry Chemometrics and Intelligent Laboratory Systems, 1993, 19, 277-293

[145] J. W. Gardner, P. Boilot \& E. L. Hines, Enhancing electronic nose performance by sensor selectionusing a new integer-based genetic algorithm approach Sensors and Actuators BChemical, 2005, 106, 114-121.

[146] R. Leardi, Genetic algorithms in chemistry, Journal of Chromatography A, 2007, 1158, 226-233.

[147] S. A. Khalifa, S. Maldonado-Bascón \& J. W. Gardner, Identification of CO and NO2 using a thermally resistive microsensor and support vector machine, IEE Proceedings Science, Measurement \& Technology, 2003, 150, 11-14.

[148] C. Distante, N. Ancona \& P. Siciliano, Support vector machines for olfactory signals recognition, Sensors and Actuators B-Chemical, 2003, 88.

[149] E. Schaller, J. O. Bosset \& F. Escher, Electronic noses an their application to food, Lebensm-Wiss u Technol, 1998, 31, 305-316.

[150] U. Weimar, K. D. Schierbaum, W. Gopel \& R. Kowalkowski, Pattern-Recognition methods for gas-mixture analysis - application to sensor arrays based upon $\mathrm{SnO}$, Sensors and Actuators B-Chemical, 1990, 1, 93-96.

[151] J. W. Gardner, E. L. Hines \& M. Wilkinson, Application of artificial neural networks to an electronic olfactory system, Measurement Science \& Technology, 1990, 1, 446-451.

[152] C. Di Natale, A. Macagnano, A. D'Amico \& F. Davide, Electronic-nose modelling and data analysis using a self-organizing map Measurement Science and Technology 1997, 8, 1236.

[153] A. Ortega, S. Marco \& J. Samitier, New pattern recognition systems designed for electronic noses Sensors and Actuators B-Chemical 2000, 69, 302-307.

[154] M. G. O'Sullivan, D. V. Byrne, M. T. Jensen, H. J. Andersen \& J. Vestergaard, A comparison of warmed-over flavour in pork by sensory

analysis, GC/MS and the electronic nose, Meat science, 2003, 65, 1125-1138.

[155] M. Pardo \& G. Sberveglieri, Classification of electronic nose data with support vector machines, Sensors and Actuators B-Chemical, 2005, 107, 730-737.

[156] T. Skov \& R. Bro, A new approach for modelling sensor based data, Sensors and Actuators B-Chemical, 2005, 106, 719-729.

[157] J. Lozano, T. Arroyo, J. P. Santos, J. M. Cabellos \& M. C. Horrillo, Electronic nose for wine ageing detection, Sensors and Actuators B-Chemical, 2008, 133, 180-186.

[158] D. Ballabio, T. Skov, R. Leardi \& R. Bro, Classification of GC-MS measurements of wines by combining data dimension reduction and variable selection techniques, Journal of Chemometrics, 2008, 22, 457-463.

[159] R. Jónsdóttir, G. Ólafsdóttir, E. Chanie \& H. J.E, Volatile compounds suitable for rapid detection as quality indicators of cold smoked salmon (Salmo salar). Food Chemistry, 2008, 109, 184-195. 
[160] M. Padilla, A. Pereral, I. Montoliu, A. Chaudry, K. Persaud \& S. Marco, Improving Drift Correction by Double Projection Preprocessing in Gas Sensor Arrays, Eds.: M. Pardo and G. Sberveglieri), 2009, pp. 101-104.

[161] C. Burian, J. Brezmes, M. Vinaixa, N. Canellas, E. Llobet, X. Vilanova \& X. Correig, MS-electronic nose performance improvement using the retention time dimension and two-way and three-way data processing methods, Sensors and Actuators B-Chemical, 2010, 143, 759768 .

[162] N. Ryman-Tubb, Computers learn to smell and taste, Expert Systems, 1995, 12, 157-161.

[163] P. Wang \& X. Jun, A novel recognition method for electronic nose using artificial neural network and fuzzy recognition, Sensors and Actuators B-Chemical, 1996, 37, 169-174.

[164] D. Vlachos \& J. Avaritsiotis, Fuzzy neural networks for gas sensing Sensors and Actuators B-Chemical, 1996, 33, 77-82.

[165] R. Polikar, R. Shinar, L. Udpa \& M. D. Porter, Artificial intelligence methods for selection of an optimized sensor array for identification of volatile organic compounds, Sensors and Actuators B-Chemical, 2001, 80, 243-254.

[166] F. A. M. Davide, C. Di Natale, A. D'Amico \& Biosens. Bioelectron. , 203-218., Selforganizing sensory maps in odour classification mimicking, Biosensors \& Bioelectronics, 1995, 10, 203-218.

[167] F. K. Che Harun, J. A. Covington \& J. W. Gardner, Portable e-Mucosa System: Mimicking the biological olfactory, Procedia Chemistry, 2009, 1, 991-994.

[168] K. Brudzewski, S. Osowski \& J. Ulaczyk, Differential electronic nose of two chemo sensor arrays for odor discrimination, Sensors and Actuators B-Chemical, 2010, 145, 246-249.

[169] M. A. Craven, J. W. Gardner \& P. N. Bartlett, Electronic noses - Development and future prospects, Trac-Trends in Analytical Chemistry, 1996, 15, 486-493.

[170] A. Amari, N. El Barbri, N. El Bari, E. Llobet, X. Correig \& B. Bouchikhi, Potential application of the electronic nose for shelf-life determination of raw milk and red meat, Eds.: M. Pardo and G. Sberveglieri), 2009, pp. 457-460.

[171] H. H. Jelen, A. Ziolkowska \& A. Kaczmarek, Identification of the Botanical Origin of Raw Spirits Produced from Rye, Potato, and Corn Based on Volatile Compounds Analysis Using a SPME-MS Method, Journal of Agricultural and Food Chemistry, 2010, 58, 12585-12591.

[172] L. Abbey, D. C. Joyce, J. Aked, B. Smith \& C. Marshall, Electronic nose evaluation of onion headspace volatiles and bulb quality as affected by nitrogen, sulphur and soil type. , Annals of Applied Biology 2004, 145, 41-50.

[173] F. Sinesio, C. Di Natale, G. B. Quaglia, F. M. Bucarelli, E. Moneta, A. Macagnano, R. Paolesse \& A. D'Amico, Use of electronic nose and trained sensory panel in the evaluation of tomato quality, Journal of the Science of Food and Agriculture, 2000, 80, 63-71.

[174] C. Di Natale, A. Macagnano, E. Martinelli, E. Proietti, R. Paolesse, L. Castellari, S. Campani \& A. D'Amico, Electronic nose based investigation of the sensorial properties of peaches and nectarines, Sensors and Actuators B-Chemical, 2001, 77, 561-566. 
[175] C. Di Natale, A. Macagnano, E. Martinelli, R. Paolesse, E. Proietti \& A. D'Amico, The evaluation of quality of post-harvest oranges and apples by means of an electronic nose, Sensors and Actuators B-Chemical, 2001, 78, 26-31.

[176] L. Vera, L. Acena, J. Guasch, R. Boque, M. Mestres \& O. Busto, Characterization and classification of the aroma of beer samples by means of an MS e-nose and chemometric tools, Analytical and Bioanalytical Chemistry, 2011, 399, 2073-2081.

[177] C. Di Natale, G. Olafsdottir, S. Einarsson, E. Martinelli, R. Paolesse \& A. D'Amico, Comparison and integration of different electronic noses for freshness evaluation of cod-fish fillets, Sensors and Actuators B-Chemical, 2001, 77, 572-578.

[178] G. Olafsdottir, P. Nesvadba, C. Di Natale, M. Careche, J. Oehlenschager, S. V. Tryggvadottir, R. Schubring, M. Kroeger, K. Heia, M. Esaiassen, A. Macagnano \& B. A. Jorgensen, Multisensor for fish quality determination, Trends in Food Science \& Technology, 2004, 15, 86-93.

[179] N. El Barbri, E. Llobet, N. El Bari, X. Correig \& B. Bouchikhi, Application of a portable electronic nose system to assess the freshness of Moroccan sardines, Materials Science \& Engineering C-Biomimetic and Supramolecular Systems, 2008, 28, 666-670.

[180] T. Miyasaki, M. Hamaguchi \& S. Yokoyama, Change of Volatile Compounds in Fresh Fish Meat during Ice Storage, Journal of Food Science, 2011, 76, C1319-C1325.

[181] X.-Y. Tian, Q. Cai \& Y.-M. Zhang, Rapid Classification of Hairtail Fish and Pork Freshness Using an Electronic Nose Based on the PCA Method, Sensors, 2012, 12, 260-277.

[182] M. Vinaixa, E. Llobet, J. Brezmes, X. Vilanova \& X. Correig, A fuzzy ARTMAP- and PLS-based MS e-nose for the qualitative and quantitative assessment of rancidity in crisps, Sensors and Actuators B-Chemical, 2005, 106, 677-686.

[183] L. Gil-Sanchez, J. Soto, R. Martinez-Manez, E. Garcia-Breijo, J. Ibanez \& E. Llobet, A novel humid electronic nose combined with an electronic tongue for assessing deterioration of wine, Sensors and Actuators a-Physical, 2011, 171, 152-158.

[184] I. Sayago, M. C. Horrillo, J. Getino, J. Gutierrez, L. Ares, J. I. Robla, M. J. Fernandez \& J. Rodrigo, Discrimination of grape juice and fermented wine using a tin oxide multisensor, Sensors and Actuators B-Chemical, 1999, 57, 249-254.

[185] H. Reinhard, F. Sager \& O. Zoller, Citrus juice classification by SPME-GC-MS and electronic nose measurements, Lwt-Food Science and Technology, 2008, 41, 1906-1912.

[186] Y. X. Li, X. J. Liang, Y. Y. Ni \& J. M. Li, Progress in the application research of electronic nose for drinks aroma evaluation, 2005.

[187] J. A. Ragazzo-Sanchez, R. Chalier, D. Chevalier, M. Calderon-Santoyo \& C. Ghommidh, Identification of different alcoholic beverages by electronic nose coupled to GC, Sensors and Actuators B-Chemical, 2008, 134, 43-48.

[188] A. K. Deisingh, D. C. Stone \& M. Thompson, Applications of electronic noses and tongues in food analysis., International Journal of Food Science \& Technology, 2004, 39, 587-604. 
[189] S. Marin, M. Vinaixa, J. Brezmes, E. Llobet, X. Vilanova, X. Correig, A. J. Ramos \& V. Sanchis, Use of a MS-electronic nose for prediction of early fungal spoilage of bakery products, International Journal of Food Microbiology, 2007, 114, 10-16.

[190] C. Cevoli, L. Cerretani, A. Gori, M. F. Caboni, T. G. Toschi \& A. Fabbri, Classification of Pecorino cheeses using electronic nose combined with artificial neural network and comparison with GC-MS analysis of volatile compounds, Food Chemistry, 2011, 129, 1315-1319.

[191] T. M. Dymerski, T. M. Chmiel \& W. Wardencki, Invited Review Article: An odorsensing system-powerful technique for foodstuff studies, Review of Scientific Instruments, 2011, 82.

[192] S. Singh, E. L. Hines \& J. W. Gardner, Fuzzy neural computing of coffee and taintedwater data from an electronic nose, Sensors and Actuators B-Chemical, 1996, 30, 185-190.

[193] L. D. Bonifacio, G. A. Ozin \& A. C. Arsenault, Photonic Nose-Sensor Platform for Water and Food Quality Control, Small, 2011, 7, 3153-3157.

[194] M. Ghasemi-Varnamkhasti, S. S. Mohtasebi, M. L. Rodriguez-Mendez, J. Lozano, S. H. Razavi \& H. Ahmadi, Potential application of electronic nose technology in brewery, Trends in Food Science \& Technology, 2011, 22, 165-174.

[195] J. W. Gardner, H. V. Shurmer \& T. T. Tan, Application of an electronic nose to the discrimination of coffees, Sensors and Actuators B-Chemical, 1992, 6, 71-75.

[196] A. Cimato, D. Dello Monaco, C. Distante, M. Epifani, P. Siciliano, A. M. Taurino, M. Zuppa \& G. Sani, Analysis of single-cultivar extra virgin olive oils by means of an Electronic Nose and HS-SPME/GUMS methods, Sensors and Actuators B-Chemical, 2006, 114, 674-680.

[197] P. Kadiroglu, F. Korel \& F. Tokatli, Classification of Turkish Extra Virgin Olive Oils by a SAW Detector Electronic Nose, Journal of the American Oil Chemists Society, 2011, 88, 639-645.

[198] E. J. Hong, S. J. Park, J. Y. Choi \& B. S. Noh, Discrimination of palm olein oil and palm stearin oil mixtures using a mass spectrometry based electronic nose, Food Science and Biotechnology, 2011, 20, 809-816.

[199] M. Cano, J. Roales, P. Castillero, P. Mendoza, A. M. Calero, C. Jimenez-Ot \& J. M. Pedrosa, Improving the training and data processing of an electronic olfactory system for the classification of virgin olive oil into quality categories, Sensors and Actuators B-Chemical, 2011, 160, 916-922.

[200] V. Messina, A. Biolatto, A. Sancho, A. Descalzo, G. Grigioni \& N. W. de Reca, Odor Profile of Different Varieties of Extra-Virgin Olive Oil During Deep Frying Using an Electronic Nose and SPME-GC-FID, (Ed. P. Gouma), 2011, pp. 228-229.

[201] J. Lozano, J. P. Santos, M. Aleixandre, I. Sayago, J. Gutierrez \& M. C. Horrillo, Identification of typical wine aromas by means of an electronic nose, Ieee Sensors Journal, 2006, 6, 173-178.

[202] M. Aleixandre, J. Lozano, J. Gutierrez, I. Sayago, M. J. Fernandez \& M. C. Horrillo, Portable e-nose to classify different kinds of wine, Sensors and Actuators B-Chemical, 2008, 131, 71-76. 
[203] M. P. Martí, O. Busto \& J. Guasch, Application of a headspace mass spectrometry system to the differentiation and classification of wines according to their origin, variety and ageing, journal of Chromatography A, 2004, 1057, 211-218.

[204] S. Buratti, D. Ballabio, S. Benedetti \& M. S. Cosio, Prediction of Italian red wine sensorial descriptors from electronic nose, electronic tongue and spectrophotometric measurements by means of Genetic Algorithm regression models, Food Chemistry, 2007, 100, 211-218.

[205] A. Z. Berna, S. Trowell, D. Clifford, W. Cynkar \& D. Cozzolino, Geographical origin of Sauvignon Blanc wines predicted by mass spectrometry and metal oxide based electronic nose, Analytica Chimica Acta, 2009, 648, 146-152.

[206] M. L. Rodriguez-Mendez, C. Apetrei, I. Apetrei, S. Villanueva, I. J. A. de Saja, I. Nevares \& M. del Alamo, Combination of an electronic nose, an electronic tongue and an electronic eye for the analysis of red wines aged with alternative methods, 2007.

[207] T. Garcia-Martinez, A. Bellincontro, M. de las Nieves Lopez de Lerma, R. Andres Peinado, J. Carlos Mauricio, F. Mencarelli \& J. Jose Moreno, Discrimination of sweet wines partially fermented by two osmo-ethanol-tolerant yeasts by gas chromatographic analysis and electronic nose, Food Chemistry, 2011, 127, 1391-1396.

[208] Y. Vlasov, A. Legin, C. Rudnitskaya, C. Di Natale \& D'Amico, Non-specific sensor arrays («electronic tongue») for chemical analysis of liquids, Pure and Applied Chemistry, 2005, 77, 1965-1983

[209] F. Winquist, Voltammetric electronic tongues-basic principles and applications, Microchimica Acta, 2008, 163, 3-10.

[210] K. Hayashi, M. Yamanaka, K. Toko \& K. Yamafuji, Multichannel taste sensor using lipid-membranes, Sensors and Actuators B-Chemical, 1990, 2, 205-213.

[211] L. Escuder-Gilabert \& M. Peris, Review: Highlights in recent applications of electronic tongues in food analysis, Analytica Chimica Acta, 2010, 665, 15-25.

[212] A. Riul Jr, C. A. R. Dantas, C. M. Miyazaki \& O. N. Oliveira Jr, Recent advances in electronic tongues, Analyst, 2010, 135, 2481-2495.

[213] P. Ciosek \& W. Wroblewski, Potentiometric electronic tongues for foodstuff and biosample recognition-An overview, Sensors, 2011, 11, 4688-4701.

[214] M. del Valle, Bioinspired Sensor Systems, Sensors, 2011, 11, 10180-10186.

[215] C. Krantz-Rulcker, M. Stenberg, F. Winquist \& I. Lundstrom, Electronic tongues for environmental monitoring based on sensors arrays and pattern recognition: A review, Analytica Chimica Acta, 2001, 426.

[216] P. Ciosek \& W. Wróblewski, Sensor arrays for liquid sensing - electronic tongue systems, Analyst, 2007, 132, 963-978.

[217] A. Gutes, F. Cespedes \& M. del Valle, Electronic tongues in flow analysis, Analytica Chimica Acta, 2007, 600, 90-96.

[218] M. Gutierrez, A. Llobera, A. Ipatov, J. Vila-Planas, S. Minguez, S. Demming, S. Buttgenbach, F. Capdevila, C. Domingo \& C. Jimenez-Jorquera, Application of an E-Tongue to the Analysis of Monovarietal and Blends of White Wines, Sensors 2011, 11, 4840-4857. 
[219] R. Paolesse, C. Di Natale, M. Burgio, E. Martinelli, E. Mazzone, G. Palleschi \& A. D'Amico, Porphyrin-based array of cross-selective electrodes for analysis of liquid samples, Sensors and Actuators B-Chemical, 2003, 95, 400-405.

[220] L. Lvova, R. Paolesse, C. Di Natale, M. Burgio, E. Martinelli, G. Pennazza \& A. D'Amico, Detection of alcohols in beverages: An application of porphyrin based electronic tongue, Sensors and Actuators B-Chemical, 2006, 118, 439-447.

[221] A. Bratov, N. Abramova \& A. Ipatov, Recent trends in potentiometric sensor arrays-A review, Analytica Chimica Acta, 2010, 678, 149-159.

[222] M. Ferreira, A. Riul, K. Wohnrath, F. J. Fonseca, O. N. Oliveira \& L. H. Mattoso, High-performance taste sensor made from Langmuir-Blodegtt films of conducting polymers and a ruthenium complex, Analytical Chemistry, 2003, 75, 953-958.

[223] M. J. Ju, K. Hayama, K. Hayashi \& K. Toko, Discrimination of pungent-tasting substances using surface-polarity controlled sensor with indirect in situ modification, Sensors and Actuators B-Chemical, 2003, 89, 150-157.

[224] M. Cortina-Puig, X. Muñoz-Berbel, M. del Valle, F. J. Muñoz \& M. A. AlonsoLomillo, Characterization of an ion-selective polypyrrole coating and application to the joint determination of potassium, sodium and ammonium by electrochemical impedance spectroscopy and partial least squares method, Analytica Chimica Acta, 2007, 597, 231-237.

[225] G. Pioggia, F. Di Francesco, A. Marchetti, M. Ferro \& A. Ahluwalia, A composite sensor array impedentiometric electronic tongue: Part I. Characterization, Biosensors \& Bioelectronics, 2007, 22, 2618-2623.

[226] T. A. Nguyen, S. Kobot, D. M. Ongarato \& G. G. Wallace, The use of chronoamperometry and chemometrics for optimization of conducting polymer sensor arrays, Electroanalysis, 1998, 11, 1327-1332.

[227] P. Ivarsson, S. Holmin, N. E. Höjer, C. Krantz-Rülcker \& F. Winquist, Discrimination of tea by means of a voltammetric electronic tongue and different applied waveforms, Sensors and Actuators B-Chemical, 2001, 76, 449-454.

[228] S. Holmin, F. Bjorefors, M. Eriksson, C. Krantz-Rulcker \& F. Winquist, Investigation of electrode materials as sensors in a voltammetric electronic tongue, Electroanalysis, 2002, 14, 839-847.

[229] F. Winquist, E. Rydberg, S. Holmin, C. Krantz-Rülcker \& I. Lundström, Flow injection analysis applied to a voltammetric electronic tongue, Analytica Chimica Acta, 2002, $471,159-172$.

[230] A. Gutes, A. B. Ibañez, M. del Valle \& F. Cespedes, Automated SIA e-tongue employing a voltammetric biosensor array for the simultaneous determination of glucose and asorbic acid, Electroanalysis, 2006, 18, 82-88.

[231] V. Parra, A. Arrieta, J. A. Fernández-Escudero, H. García, C. Apetrei, M. L. Rodríguez-Méndez \& J. A. de Saja, E-tongue based on a hybrid array of voltammetric sensors based on phthalocyanines, perylene derivatives and conducting polymers: Discrimination capability towards red wines elaborated with different variety of grapes, Sensors and Actuators B-Chemical, 2006, 115, 54-61. 
[232] V. Parra, A. Arrieta, J. A. Fernández-Escudero, M. Iñiguez, M. L. Rodríguez-Méndez \& J. A. de Saja, Monitoring of the ageing of red wines in oak barrels by means of an hybrid electronic tongue, Analytica Chimica Acta, 2006, 563, 229-237.

[233] A. Arrieta, M. L. Rodriguez-Mendez \& J. A. de Saja, Langmuir-Blodgett film and carbon paste electrodes based on phthalocyanines as sensing units for taste, Sensors and Actuators B-Chemical, 2003, 95, 357-365.

[234] C. Apetrei, S. Casilli, M. De Luca, L. Valli, J. Jiang, M. L. Rodríguez-Méndez \& J. A. de Saja, Spectroelectrochemical characterisation of Langmuir-Shaeffer films of heteroleptic phthalocyanine complexes. Potential applications, Colloids and Surfaces A: Physicochemical and Engineering Aspects, 2006, 284, 574-582.

[235] Y. N. Ni, P. Qiu \& S. Kokot, Simultaneous voltammetric determination of four carbamate pesticides with the use of chemometrics, Analytica Chimica Acta, 2004, 516, 7-17.

[236] D. Koziej, N. Barsan \& U. Weimar, CO Sensing with Tin Dioxide Sensors; Effect of Fabrication Technology and Operation Conditions as Revealed by Diffuse Reflectance Infrared Fourier Transformed Spectroscopy and Conductance Measurements, Sensor Letters, 2008, 6, 817-820.

[237] V. Parra, Diseño y aplicación de sensores electroquímicos basados en compuestos orgánicos electroactivos para una lengua electrónica: discriminación y clasificación de vinos., Tesis doctoral, Universidad de Valladolid, Valladolid, 2005.

[238] V. Parra, T. Hernando, M. L. Rodriguez-Mendez \& J. A. de Saja, Electrochemical sensor array made from bisphthalocyanine modified carbon paste electrodes for discrimination of red wines, Electrochimica Acta, 2004, 49, 5177-5185.

[239] N. Prieto, M. Gay, S. Vidal, O. Aagaard, J. A. De Saja \& M. L. Rodríguez-Méndez, Analysis of the influence of the type of closure in the organoleptic characteristics of a red winenext term by using an electronic panel Food Chemistry, 2011, 129, 589-594.

[240] V. Parra, A. A. Arrieta, J. A. Fernandez-Escudero, M. L. Rodriguez-Mendez \& J. A. De Saja, Electronic tongue based on chemically modified electrodes and voltammetry for the detection of adulterations in wines, Sensors and Actuators B-Chemical, 2006, 118, 448-453.

[241] C. Apetrei, I. M. Apetrei, I. Nevares, M. del Alamo, V. Parra, M. L. RodriguezMendez \& J. A. De Saja, Using an e-tongue based on voltammetric electrodes to discriminate among red wines aged in oak barrels or aged using alternative methods - Correlation between electrochemical signals and analytical parameters, Electrochimica Acta, 2007, 52, 2588-2594.

[242] C. Apetrei, M. L. Rodríguez-Méndez \& J. A. de Saja, Evaluation of the polyphenolic content of extra virgin olive using an array of voltammetric sensors, Electrochimica Acta, 2008, $53,5867-5872$.

[243] M. Ghasemi-Varnamkhasti, S. S. Mohtasebi, M. L. Rodriguez-Mendez, M. Siadat, H. Ahmadi \& S. H. Razavi, Electronic and bioelectronic tongues, two promising analytical tools for the quality evaluation of non alcoholic beer, Trends in Food Science \& Technology, 2011, 22, 245-248. 
[244] M. L. Rodríguez-Méndez, C. Apetrei, M. Gay \& J. A. de Saja, Biogenic amines and fish freshness assessment using a multisensor system based on voltammetric electrodes. Comparison between CPE and screen printed electrodes, Electrochimica Acta, 2009, 54, 7033-7041.

[245] L. Usseglio-Tomasset, Quimica Enologica, Mundi-Prensa., 1998.

[246] P. Capilla, J. M. Artigas \& J. Pujol, Fundamentos de colorimetria, University of Valencia, Valencia, 2002.

[247] C.I.E., Colorimetrie, C.I.E. Central Bureau of the Comisión Internationale de L'Eclairage., Viena, 1986.

[248] F. Heredia \& M. Guzmán-Chozas, Reliability of the Spanish official method for colour of red wines in comparison with the CIE $1931(x, y)$ method. , Food Chemistry, 1991, 39.

[249] HunterLAB, Color Theory, 2008, http://www.hunterlab.com/.

[250] O.I.V, Recueil des Méthodes Internationales d'Analyse des vins et des moûts. , Office International de la Vigne et du Vin., Paris, 1990.

[251] R. Gil-Muñoz, E. Gómez-Plaza, A. Martínez \& J. López-Roca, Evolution of the CIELAB and other spectrophotometric parameters during wine fermentation., Food Research International, 1997, 30, 699-705.

[252] B. W. Zoecklein, K. C. Fugelsang, B. H. Gump \& F. S. Nury, Análisis y produccion de vino, Acribia 2001.

[253] R. Gawel, Red wine astringency: A review, Australian Journal of grape and wine Research, 1998, 4, 74-95.

[254] P. Ribéreau-Gayon, Y. Glories \& D. Maujean A, The chemestry of wine, stabilization and treatments, Wiley, 1999.

[255] A. Negueruela, J. Echávarri, F. Ayala \& M. Pérez, Propuestas de determinación de las características cromáticas del vino OIV, Valencia, 2001. 


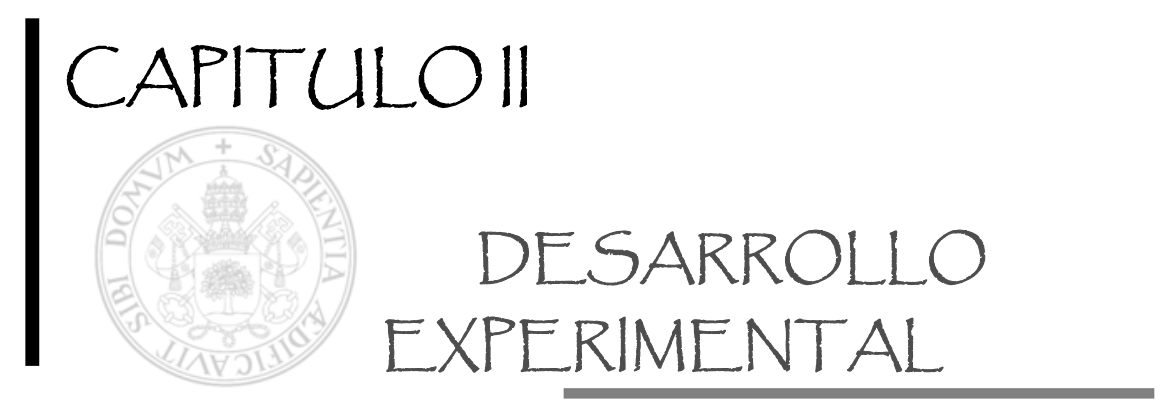

Página

2.1. ANÁLISIS FÍSICOS-QUÍMICOS DEL VINO 76

2.1.1. Parámetros estándar $\quad 76$

2.1.2. Cromatografía de Gases $\quad 78$

2.1.2.1. Condiciones experimentales para la GC-MS 83

2.1.2.2. Condiciones experimentales para la GC-O 86

2.2. PANEL DE CATA ELECTRÓNICO 91

2.2.1. Nariz electrónica $\quad 91$

2.2.2. Lengua electrónica 102

2.2.3. Ojo electrónico 106

2.3. ANÁLISIS DE LOS DATOS 106

2.3.1. Pretratamiento señales nariz electrónica 108

2.3.2. Pretratamiento señales lengua electrónica 111

2.3.2.1. Selección de variables para la lengua electrónica 113

2.3.3. Pretratamiento datos espectroscopía, GC-MS, GC-O 116

2.3.4. Análisis exploratorio 116

$\begin{array}{lr}\text { 2.3.5. Validación cruzada } & 119\end{array}$

2.3.6. Análisis multi-way: Tucker3 122

2.3.7. Anova-PCA 125

2.3.8. Implementación 132

2.4. REFERENCIAS 132 

En este capitulo se describen los materiales, instrumentación, programas, métodos y sistemas electrónicos que pretenden dar soluciones a las diferentes problemáticas mencionadas en el capitulo anterior entorno al control de calidad en vinos. El contenido que se va a desarrollar a continuación explicará todas las condiciones experimentales y los procedimientos de optimización llevados a cabo con cada una de las técnicas utilizadas en nuestra investigación.

Cada procedimiento experimental puede ser objeto de una discusión entorno a sus características, pero en este capitulo nos hemos limitado a una descripción general que estará conectada con citas bibliográficas que conducen al conocimiento en profundidad de cada tema.

Para comenzar, repasaremos las técnicas analíticas convencionales utilizadas en esta investigación y continuaremos con la descripción de las técnicas electrónicas específicas de análisis sensorial desarrolladas por el Grupo de Investigación de Sensores de la Universidad de Valladolid.

Una vez mencionadas las características de estos sistemas, pasaremos a hacer una revisión de los métodos quimiométricos utilizados para el análisis de datos. Todos los procesos analíticos y estadísticos del desarrollo experimental están totalmente conectados entre si, ya que un apropiado aprovechamiento de la instrumentación experimental depende de un certero análisis de datos; de igual manera, esta pauta funciona en el sentido contrario, de nada nos serviría tener las adecuadas herramientas computacionales sin tener una herramienta experimental perfectamente validada.

En este sentido, serán explicadas las técnicas quimiométricas que han sido aprovechadas para obtener mayor la información de los resultados provenientes de la nariz y lengua electrónica, además de la búsqueda de correlaciones con las demás sistemas empleados en esta tesis. Técnicas como el modelo Tucker3, el Anova-PLS y los GA-PLS, desarrolladas recientemente están ya consolidadas en varios tipos de aplicaciones concernientes a métodos analíticos y son objetos de constante discusión dentro del campo de la quimiometría. 


\subsection{ANÁLISIS FÍSICOS-QUÍMICOS DEL VINO}

Las técnicas y procedimientos convencionales para el análisis químico del vino tienen un carácter rutinario, pero no por ello menos significativo. Estas medidas son suficientes en bodega para identificar y cuantificar aspectos generales de la composición química del vino, a la vez que permiten verificar el cumplimiento de las exigencias comerciales marcadas por la legislación y el mercado.

En esta línea, todas las muestras de vinos preparadas para esta investigación fueron previamente evaluadas mediante análisis químico tradicional en las estaciones enológicas de Castilla y León y/o de la Rioja, con la colaboración de la E.T.S. de Ingenierías Agrarias de Palencia. Las valoraciones realizadas incluyen acidez total, acidez volátil, extracto seco, anhídrido sulfuroso total, grado alcohólico, glicerol, ácido tartárico, ácido málico, antocianos totales, polifenoles totales, $\mathrm{pH}$, polifenoles poco polimerizados, polifenoles muy polimerizados, catequinas, taninos, etc. Cada uno de estos parámetros se determinaron aplicando métodos estándares descritos por la O.I.V [1]. Se citan a continuación algunas de las características de estos ensayos y sus autores de referencia.

\subsubsection{Parámetros Estándar}

La densidad es un valor que nos proporciona la riqueza en azúcares del grano de uva expresada en grados Brix (o grado probable de alcohol, mediante la oportuna conversión). Se determina a partir de una medida de la densidad obtenida de la lectura en un aerómetro que flota de manera que es diferenciado al ser introducido en el vino [2].

La acidez total es el conjunto de todos los ácidos contenidos en el mosto, se mide con una valoración ácido-base. El sulfuroso libre y total se evalúa utilizando un método potenciométrico, mediante una hidrólisis básica seguida de una iodometría [3]. Tanto la acidez total, como el sulfuroso libre, sulfuroso total y $\mathrm{pH}$ se determinaron directamente en el sistema automático Metler Toledo Tritator DL77.

La acidez volátil y los azúcares reductores se evalúan por métodos enzimáticos, midiendo el cambio en la absorbancia de la muestra a una longitud de onda de $365 \mathrm{~nm}$ cuando se propicia un reacción enzimática NADH. El equipo utilizado para la determinación de estos parámetros es un espectrómetro UV-Vis [4]. 
El ácido hidroxicinámico, monómeros flavonoides y pigmentos totales se analizaron mediante un sistema de HPLC Waters Alliance 2690 equipado con un sistema de inyección automática, un detector con una red de fotodiodos Waters 996 y el software Millenium 32 (Waters, Milford, MA). La separación se consigue en una columna Atlántida DC18 $(250 \times 2,1 \mathrm{~mm}, 5 \mu \mathrm{m})$ protegida con una columna de seguridad del mismo material $(20 \times 2,1 \mathrm{~mm}, 3 \mu \mathrm{m})$ (Waters, Milford, MA). Las condiciones de elución fueron las siguientes: flujo de $0,250 \mathrm{ml} / \mathrm{min}$; temperatura del horno $30^{\circ} \mathrm{C}$; disolvente $\mathrm{A}$, agua/ácido fórmico (95:5 v/v); disolvente $\mathrm{B}$, acetonitrilo/agua/ácido fórmico (80:15:5 v/v/v) [5]. Otros compuestos evaluados mediante las cromatografía de líquidos fueron el porcentaje de ácido gálico, porcentaje de epigalocatequina y el grado de polimerización promedio.

Se realizaron diferentes rectas de calibrado con compuestos estandar comerciales de grado analítico con su respectiva lectura de la absorción a $520 \mathrm{~nm}$ (Extrasynthèse, Francia). Los compuestos utilizados fueron específicamente la epicatequina-etil-malvidina-3-glucósido, carboxi-piruvato-malvidina-3-glucósido (vitisin A) y Malvidin-3-O-glucósido-vinil aducto (vitisina B), para la valoración de los parámetros catequina, epicatequina, malvidina-3-O-glucósido unidos por puentes etilo y los piranoantocianos [5].

Taninos (g/l) Se realizó por el método de Lowenthal, modificado por Carpene [6], el cual se basa en la precipitación del tanino, disolución del precipitado en un medio ácido y valoración con permanganato potásico y sulfo-indigotina como indicadores.

Índice de polifenoles totales Para su determinación se ha utilizado el método basado en la medida del máximo de absorción a 275-280 nm que presentan los polifenoles del vino, y que resulta directamente proporcional a la cantidad de estos compuestos existente en el medio (Índice de Masquelier) [7].

Antocianos (mg/l) Se puede admitir que la variación de color provocada por la adición de un exceso de bisulfito, es proporcional al contenido en pigmentos. Esta variación de intensidad colorante se mide por espectrofotometría evaluando la absorción de la muestra a $520 \mathrm{~nm}$ [8].

Índice de polimerización de polifenoles Esta valoración está basada en la absorción de fracciones de polifenoles de cierta polimerización a través de polivinilpolipirrolidona, que actúa sobre los polifenoles como una molécula proteica [7]. 
Catequinas $(\mathrm{g} / \mathrm{l})$ La determinación de catequinas se basa en la reacción del anillo floroglucínico con la vainillina, que produce un color rojo relativamente estable, medible mediante la utilización de un espectrofotómetro de UV-Vis a $500 \mathrm{~nm}$ [4].

Edad química de vino se evaluó mediante el cálculo de la relación entre pigmentos poliméricos y el contenido de antocianos vino utilizando el método de Somers y Evans [9].

El porcentaje de los pigmentos de color por la sulfitos (PDSO2\%) se determinó según el método propuesto por Boulton [10]. La calculó también la relación entre taninos y antocianos para evaluar la estabilidad del color.

Todos los parámetros físico-químicos fueron estudiados periódicamente para garantizar un adecuado transcurso de todos los procesos que tienen lugar durante la vinificación. Estos datos además de ser una herramienta de control y validación de las muestres han sido utilizados para establecer correlaciones entre los sistemas de evaluación sensorial electrónicos con las medidas del color y los fenómenos que ocurren a nivel químico.

\subsubsection{Cromatografía de gases}

Como se ha comentado, el aroma del vino es el resultado de interacciones complejas entre muchos componentes químicos volátiles. Sólo en algunos casos particulares y sencillos es posible encontrar genuinos compuestos impacto capaces de transmitir al producto sus características aromáticas más específicas. En los productos más complejos y de mayor calidad, las notas sensoriales aparecen como consecuencia de la acción concertada de muchas moléculas y, de manera sorprendente, en muchos casos estas moléculas se encuentran en concentraciones muy cercanas a su valor umbral.

Desde el primer momento la técnica de cromatografía gaseosa capilar (1956) evidenció su potencial analítico para tratar de dilucidar la naturaleza química de los aromas. En esa década aparecen un gran número de trabajos producidos en California, Francia, Italia, Alemania, Australia y Sudáfrica, que hablan de la composición aromática de diferentes vinos habiéndose detectado unas 600 moléculas; posteriormente en el año 1989 el número ascendía a 800, aunque se sabía muy poco sobre el papel sensorial de las moléculas identificadas. Se conocían por ejemplo, el linalol y su papel en el aroma de los moscateles, o el papel de los ésteres etílicos en la fermentación en las notas frutales, así como algunos componentes relacionados con defectos o características de algunas 
variedades que las hacían poco habituales o poco válidas para el consumo de vino normal, como el furanol o los mercaptanos.

De estas moléculas, unas 20 compuestos se encuentran en todos los vinos, desde los más simples a los más complejos aromáticamente hablando, y sus valores de aroma son relativamente altos ${ }^{1}$. Estos 20 compuestos constituyen la base del aroma y se originan en la fermentación. Aparentemente, únicamente un compuesto, la ß-damascenona, proviene directamente de las uvas, ya que el resto se origina como consecuencia del metabolismo de las levaduras. Estas sustancias son alcoholes superiores (butírico, isoamílico, hexílico, feniletílico), ácidos (acético, butírico, hexanoico, octanoico, isovaleriánico), ésteres etílicos de los ácidos grasos, acetatos y sustancias como diacetilo, acetoína y acetaldehído. El olor conjunto es el que se denomina "vinoso", y es difícil describirlo, puesto que están perfectamente integradas las notas de sus componentes: notas frutales, a plátano, piña, manzana, alcohólicas, a polen, a ácidos o a frutas rojas.

Por otra parte también está el etanol, componente fundamental con dos funciones en la mezcla aromática. A nivel fisicoquímico aumenta la solubilidad de los odorantes en agua y ayuda a evaporar otros compuestos volátiles odoríferos. Y en el aspecto sensorial ayuda a la percepción de compuestos como el eugenol y el decanal (con olores a clavo o cítrico, respectivamente) aunque también puede enmascarar, e incluso llegar a anular, las notas afrutadas de los ésteres, con olor a manzanas.

Otros compuestos, alrededor de 16 se encuentran en casi todos los vinos con valores de aroma más bajos. Proceden tanto del proceso fermentativo como de las uvas y son los responsables de las notas sutiles en los vinos.

Actualmente se dispone de una amplia gama de posibilidades bien definidas conducentes a la clasificación química de los diferentes compuestos vinculados al aroma del vino [11-13]. El amplio desarrollo del que ha sido objeto esta técnica ha supuesto que esta parte de nuestra investigación estuviera exclusivamente dedicada a la definición de los parámetros óptimos de medición, cuyo fin fue mejorar la sensibilidad, selectividad, reproducibilidad, rapidez y automatización de cada uno de los análisis llevados a cabo. Bajo esta premisa, procederemos a dar una breve descripción conceptual de cada proceso, puntualizando las diferentes metodologías de trabajo y optimizaciones alcanzadas para dar solución a la problemática de la que es objeto esta tesis.

La evaluación cromatográfica de los volátiles de un vino consiste en desarrollar tres etapas sucesivas, como explica el esquema que mostramos a continuación:

\footnotetext{
79

${ }^{1}$ El valor de aroma, OVA, es la relación entre la concentración analítica de una sustancia y su umbral de detección.
} 


\section{Etapa 1.}

Extracción y

Concentración de Volátiles

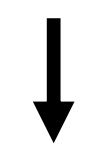

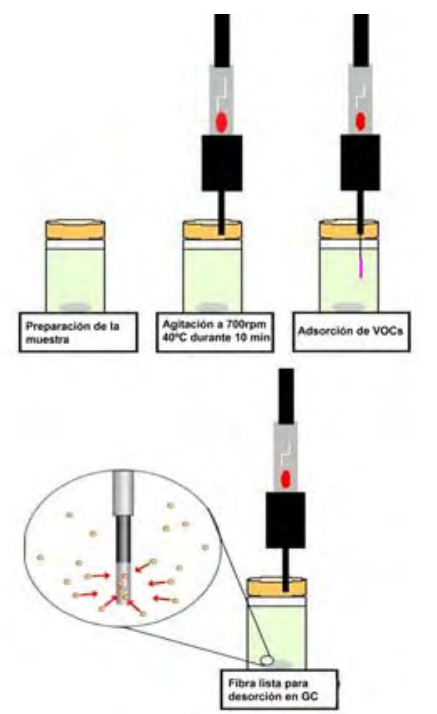

Microextracción en fase sólida (SPME)

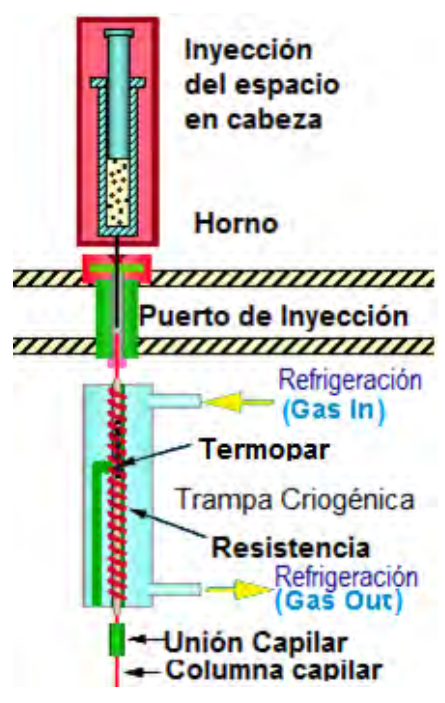

Espacio en cabeza
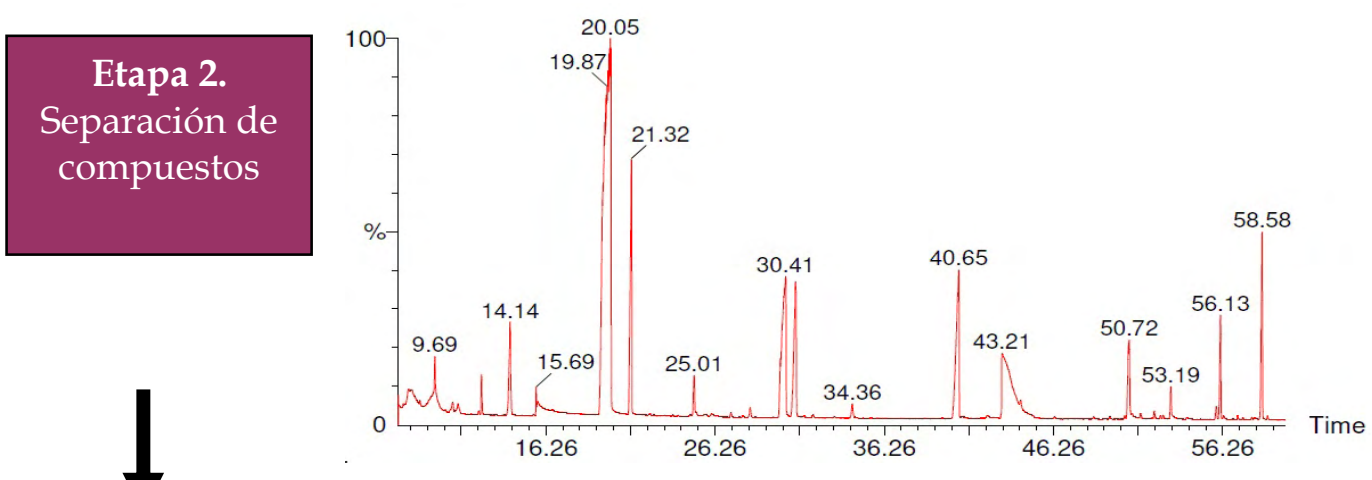

Cromatograma característico de un vino tinto

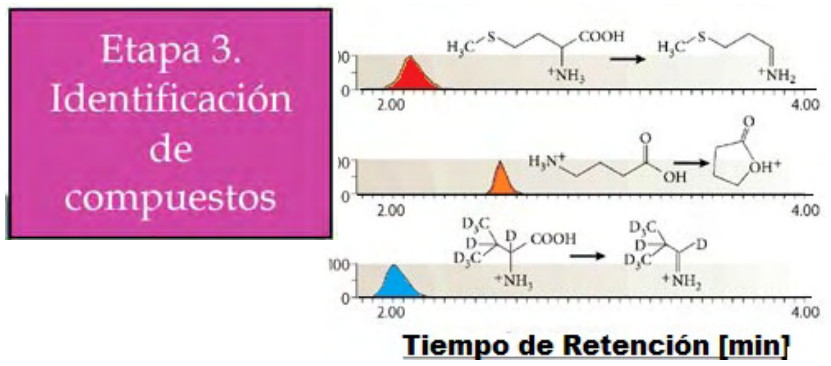

GC-MS. Identificación de compuestos por espectrometría de masas

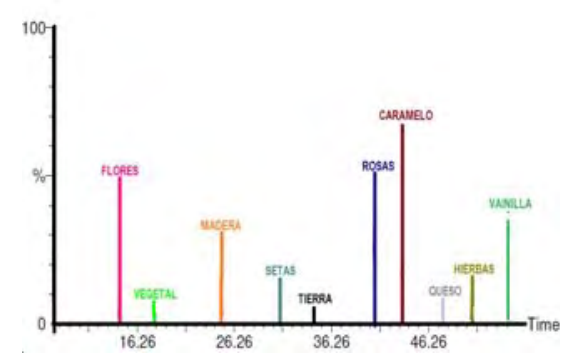

GC-O. Identificación de compuestos por su aroma característico

Figura 2.1. Principales condiciones experimentales de la cromatografia de gases (GC) 


\section{Etapa 1. Extracción y concentración de los compuestos volátiles}

En la Figura 2.1. es posible observar la primera etapa relacionada con la extracción de los compuestos volátiles. Para dar inicio a este proceso, las muestras de vinos han sido colocadas en viales de $10 \mathrm{ml}$ (Supelco) y cerradas herméticamente con un septum Termogreen LB-2 (Supelco) previamente perforado y cápsulas de aluminio magnéticas de $20 \mathrm{~mm}$ de espesor.

La selección del método de extracción está determinada por las características específicas de las aplicaciones requeridas. Es decir, que es necesario en nuestro caso, un sistema que elimine parte sustancial del agua y etanol y que además sea capaz de capturar y concentrar aquellos compuestos volátiles minoritarios. Entre las diferentes opciones posibles se optó por la microextracción en fase sólida (SPME) y el espacio en cabeza (HS), técnicas bien conocidas en el mundo de la cromatografía de gases.

La técnica SPME fue optimizada especialmente para la red de sensores MOS. Para alcanzar este objetivo fue indispensable todo un proceso de selección de parámetros adecuados que permitieron mejorar el análisis de compuestos volátiles con esta instrumentación. Este estudio se realizó comparando los resultados de la nariz electrónica y la cromatografía de gases, estudiando el efecto que tenia cada parámetro que es requerido dentro de la aplicación de la SPME en la capacidad extracción de volátiles. Este trabajo fue parte de algunos de los resultados de una anterior tesis doctoral realizada en nuestro grupo de investigación [14].

De los anteriores planteamientos y nuestra consecuente validación experimental se encontró que la mejor opción y pensando principalmente en las medidas a realizar con la nariz electrónica, se optimizó la extracción de los compuestos volátiles analizados en esta investigación mediante una fibra de Poliacrilato (PA) $[14,15]$. Todas las variables que intervienen en esta técnica fueron programadas en un automuestreador CTC Combi PAL (CTC Analytics AG) en modo SPME. El método definido comienza con un proceso de agitación constante de 700 r.p.m. a una temperatura de $40^{\circ} \mathrm{C}$ durante $10 \mathrm{~min}$; son las condiciones óptimas para favorecer un equilibrio entre la fase gaseosa de la muestra y la fase estacionaria de la fibra. De esta manera se propicia la transferencia de compuestos volátiles durante los siguientes 5 minutos en los que la fibra permanece sostenida en el espacio en cabeza de la muestra en el vial [16].

A continuación esta fibra debe ser introducida directamente en el inyector del cromatógrafo, donde los analitos son desorbidos a una temperatura de $250^{\circ} \mathrm{C}$ y arrastrados a la columna del cromatógrafo. La secuencia necesaria para llevar a cabo la extracción de volátiles con la GC-MS y GC-O se especifica en la parte superior de la Figura 2.1. 
Además, la mayor parte de las medidas seguidas con la GC-MS también fueron también realizadas con la técnica de extracción HS, en una estancia realizada en la estación enológica de Haro. Este instrumento está dedicado exclusivamente al análisis de vinos y su calibración se ha realizado según la norma UNE EN ISO/IEC 17025.

La preconcentración de compuestos volátiles con la técnica del espacio en cabeza, se ha llevado a cabo utilizando un equipo Perkin Elmer Turbomatrix 110 trap, las temperaturas utilizadas fueron las siguientes: aguja $110^{\circ} \mathrm{C}$, linea de transferencia $150^{\circ} \mathrm{C}$, horno $85^{\circ} \mathrm{C}$; trampa, $45^{\circ} \mathrm{C}$ (baja), $280^{\circ} \mathrm{C}$ (alta). Las presiones fueron: vial, 30 psi; en columna, 30 psi; desorción, 35 psi. Los tiempos estipulados para los distintos procesos que tienen lugar en el espacio en cabeza fueron: purga seca, 10,0 min; trampa a la máxima temperatura, 12,0 min; desorción, 10,0 min; termostatización, $45.0 \mathrm{~min}$.

\section{Etapa 2. Separación de compuestos}

Uno de los mayores inconvenientes que surgen a la hora de separar los compuestos de los vinos usando la CG, reside en fijar las condiciones necesarias para obtener separaciones bien definidas y por consiguiente una correcta identificación de los compuestos volátiles.

Existe una dificultad adicional al momento de evaluar una amplia variedad de compuestos: los factores de retención. Si estos factores son parecidos entre sí, se producen solapamientos y consecuentemente picos cromatográficos mal definidos. En consecuencia, ha sido necesario separar el desarrollo de la medida en tres etapas diferentes en función de los tiempos de retención conocidos para el conjunto de compuestos aromáticos de interés en este estudio.

Los estudios iniciales de esta parte de la investigación se apoyaron en la obtención de cromatogramas individuales por cada uno de los compuestos fundamentales en la conformación del aroma. Aunque se disponía de bases de datos y referencias bibliográficas que reportan los tiempos de retención de algunos de estos compuestos y sus respectivos espectros de masas característicos, dada la complejidad de una mezcla como el vino, era importante confirmar la correcta identificación de cada compuesto [17].

La Figura 2.2. especifica el programa de temperatura establecido para llevar a cabo la separación cromatográfica. El procedimiento han sido realizado en un equipo GC Perkin Elmer Clarus 500 de la estación Enológica de la Rioja, con una columna capilar de cristal de silicio supelcowax de $30 \mathrm{~m} \times 0.25 \times 0.25 \mu \mathrm{m}$. El programa de temperatura estándar para todas las valoraciones es el siguiente: 


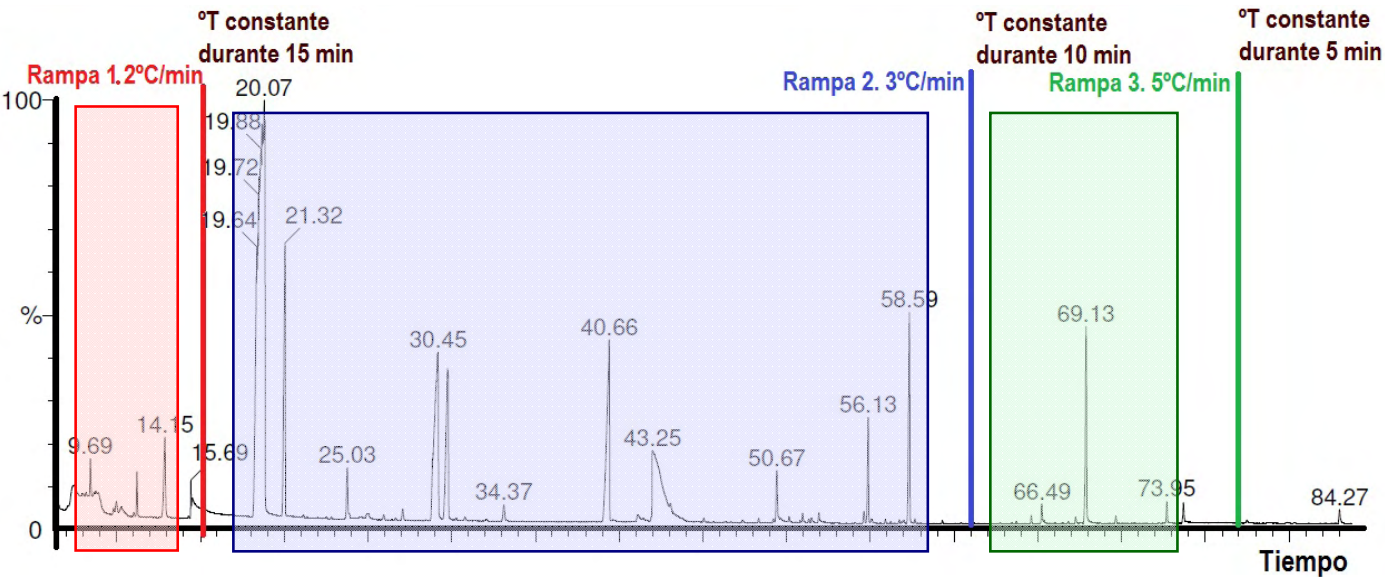

Figura 2.2. Programa de temperatura múltiple para la elución de los compuestos volátiles característicos del proceso de vinificación

Etapa 1, comienza con una temperatura inicial de $40^{\circ} \mathrm{C}$ que debe mantenerse constante durante $3 \mathrm{~min}$, luego incrementarse a una velocidad de $2^{\circ} \mathrm{C} / \mathrm{min}$ hasta alcanzar a los $80^{\circ} \mathrm{C}$, temperatura que se mantiene durante fija los siguientes 15 min, para así conseguir la elución de una parte importante de los compuestos primarios del vino. Etapa 2, en esta parte se aplica un calentamiento a una velocidad $3^{\circ} \mathrm{C} / \mathrm{min}$ hasta llegar a $\operatorname{los} 180^{\circ} \mathrm{C}$, temperatura que se mantiene durante $10 \mathrm{~min}$ y finalmente, la etapa 3 , en la cual se efectúa un calentamiento a una velocidad de $5^{\circ} \mathrm{C} / \mathrm{min}$ hasta alcanzar una temperatura de $210^{\circ} \mathrm{C}$, temperatura que se mantiene durante $5 \mathrm{~min}$ para asegurar la salida de todos los compuestos inyectados al dispositivo detector. El gas portador para estos análisis es helio.

\subsubsection{Condiciones experimentales para la GC-MS}

La detección por acoplamiento de GC y espectrometría de masas (MS) se basa en la introducción de la fase gaseosa eludida en la cámara de ionización de un espectrómetro de masas. En este dispositivo, las moléculas son convertidas en iones gaseosos mediante un bombardeo de electrones [18]. Seguidamente, los iones son acelerados y deflectados en función de su relación m/Z (masa/carga); de esta manera la detección consecutiva de los iones formados a partir de las moléculas de la muestra, suponiendo que se trate de una sustancia pura, produce el espectro de masas del compuesto, diferente para cada molécula, que constituye una identificación prácticamente inequívoca de los compuesto volátiles de la muestra.

Los espectrómetros de masas pueden trabajar según dos métodos: el método scan, en el que se registra la intensidad de todos los iones, y el método del ión selectivo 
(SIM), que es útil cuando se desea explícitamente localizar la presencia de uno o varios compuestos determinados, de espectro conocido, con la mayor rapidez o con la máxima sensibilidad posible [19]. Para nuestro caso se ha escogido el modo de operación SIM para la cuantificación de los compuestos que aparecen señalados en la Tabla 2.1 con sus respectivos iones característicos.

La cromatografía de gases acoplada a la espectrometría de masas ha sido aplicada en esta investigación utilizando un equipo Clarus 500 (PerkinElmer). Los espectros de masas se obtuvieron mediante ionización por impacto electrónico a $70 \mathrm{eV}$. La detección se realiza con un intervalo de muestreo de $0.5 \mathrm{~s}$ a un potencial de $1.4 \mathrm{KV}$. La temperatura de la línea de transferencia se establece en $250^{\circ} \mathrm{C}$.

Para identificar los VOCs del vino es suficiente trabajar en un rango de masa comprendido entre 55 hasta $200 \mathrm{~m} / \mathrm{Z}$. Se ha seleccionado este rango inferior a 200 $\mathrm{m} / \mathrm{Z}$ ya que por encima de dicho valor no hay fragmentaciones con una abundancia significativa. En cuanto al valor mínimo de fragmentaciones se han seleccionado la relación $\mathrm{m} / \mathrm{Z}$ igual a 55 . Por debajo de este valor aparecen las fragmentaciones del etanol, que pueden interferir en el análisis quimiométrico, debido a su elevada abundancia dentro de la constitución del vino y la escasa información que aportan el modelamiento de patrones relacionados con la maduración del vino.

\section{Calibración}

Las curvas de calibración de los compuestos de interés fueron elaboradas, en parte, para verificar si las condiciones seleccionadas para el proceso de cromatografía eran apropiadas. Además, este método proporciona las distintas funciones para calcular la concentración de los VOCs a partir de la medición del área bajo la curva a determinado tiempo de retención para las moléculas identificadas en el cromatograma. El método de calibración se realizó para cada uno de estos periodos como una alternativa para definir las posibles variaciones experimentales.

Para realizar las diferentes calibraciones se prepararon diferentes disoluciones en etanol al $12 \%$ (valor promedio del grado alcohólico de un vino) con 37 compuestos químicos aromáticos mayoritarios y minoritarios de diferente polaridad y volatilidad, adicionados en tres diferentes concentraciones [38]. A la hora de seleccionar estos compuestos aromáticos fue crucial el apoyo de las Estaciones Enológicas de Haro y de Castilla-León.

La Tabla 2.1 presenta las constantes para la ecuación en un ajuste lineal y los coeficientes de correlación de los compuestos de interés para este estudio. Como 
se puede observar en la Tabla 2.1, la relación entre la concentración de cada uno de los VOCs y el área reportada por el cromatógrafo es lineal, con coeficientes de correlación cercanos a 1 . Puede asegurarse entonces que el sistema cromatográfico y el procedimiento utilizado son idóneos para el análisis de los VOCs. De acuerdo a lo parámetros registrados en la Tabla 2.1. se puede concluir que el sistema cromatográfico utilizado es de alta precisión, además la desviación media para cada uno de los VOCs evaluados no supera el $4 \%$.

Tabla 2.1. Compuestos utilizados para calibración lineal.

\begin{tabular}{|l|l|l|l|c|}
\hline Compuesto & $\begin{array}{l}\text { Tiempo de } \\
\text { Retención }\end{array}$ & Pendiente & Intercepción & $\mathbf{R}^{2}$ \\
\hline
\end{tabular}

\begin{tabular}{|c|c|c|c|c|}
\hline Isovaleriato de etilo & 11.057 & $8.5342^{*} 10^{7}$ & 210480 & 0.99294 \\
\hline Acetato de isopentilo & 14.055 & $1.7132^{*} 10^{8}$ & $1.0300 * 10^{7}$ & 0.99265 \\
\hline Limoneno & 18.245 & $4.2186^{*} 10^{6}$ & 110235 & 0.98930 \\
\hline Hexanoato de etilo & 21.344 & $5.3897 * 10^{8}$ & $1.160^{*} 10^{6}$ & 0.99670 \\
\hline 1-Pentanol & 22.417 & $8.8746^{*} 10^{7}$ & 735824 & 0.99948 \\
\hline Acetato de hexilo & 23.810 & $4.2458^{*} 10^{8}$ & $1.520^{*} 10^{6}$ & 0.99437 \\
\hline Piruvato de etilo & 24.012 & $1.7567 * 10^{7}$ & $-3.2430^{*} 10^{6}$ & 0.98500 \\
\hline Heptanoato de etilo & 28.807 & $3.4082^{*} 10^{8}$ & $1.0079 * 10^{6}$ & 0.99120 \\
\hline Lactato de etilo & 30.494 & $8.2332^{*} 10^{6}$ & $6.5918^{*} 10^{7}$ & 0.96230 \\
\hline 1-Hexanol & 31.035 & $2.3503^{*} 10^{8}$ & $1.0625^{*} 10^{7}$ & 0.99859 \\
\hline E-3-hexen-1-ol & 32.053 & $5.2805^{*} 10^{7}$ & 24067 & 0.99987 \\
\hline Octannoato de etilo & 40.864 & $3.9593^{*} 10^{8}$ & $-8.8550 * 10^{6}$ & 0.99803 \\
\hline 1-heptanol & 43.064 & $1.3350^{*} 10^{8}$ & -156575 & 0.99960 \\
\hline Bezaldehido & 45.512 & $2.0026^{*} 10^{8}$ & 904862 & 0.99942 \\
\hline Pelargonato de etilo & 49.537 & $3.0685^{\star} 10^{6}$ & -570387 & 0.99946 \\
\hline Linalol & 50.894 & $3.3885^{\star} 10^{6}$ & $-276912 * 10^{6}$ & 0.99869 \\
\hline 1-Octanol & 51.362 & $3.5154^{*} 10^{8}$ & $-2.1614^{*} 10^{6}$ & 0.99901 \\
\hline Lactato de isoamilo & 52.169 & $4.44263^{*} 10^{7}$ & $-2.8018^{*} 10^{6}$ & 0.99132 \\
\hline Acetato de fenilo & 55.378 & $1.2377^{*} 10^{8}$ & -63429 & 0.99973 \\
\hline Decanoato de etilo & 56.038 & $1.5023^{*} 10^{8}$ & $-1.6870 * 10^{6}$ & 0.99876 \\
\hline Acido Butírico & 56.331 & $3.8909^{*} 10^{6}$ & $-1.0399^{*} 10^{6}$ & 0.98496 \\
\hline Succinato de dietilo & 58.477 & $2.5787^{*} 10^{7}$ & $6.12289 * 10^{6}$ & 0.99692 \\
\hline Citronelol & 62.676 & $8.7749^{*} 10^{7}$ & $1.35713^{*} 10^{6}$ & 0.99977 \\
\hline Nerol & 64.326 & $5.9710^{*} 10^{7}$ & 435786 & 0.99973 \\
\hline Acetato de 2-feniletilo & 64.931 & $1.0679^{*} 10^{8}$ & $8.75295^{*} 10^{6}$ & 0.99228 \\
\hline Decanoato de etilo & 65.821 & $1.3329^{*} 10^{8}$ & $-4.76418^{*} 10^{6}$ & 0.99230 \\
\hline Geraniol & 66.362 & $4.7340^{*} 10^{7}$ & -514855 & 0.99982 \\
\hline Guaiacol & 67.068 & $2.4164^{*} 10^{7}$ & $-1.2139 * 10^{6}$ & 0.99929 \\
\hline Alcohol Bencílico & 67.710 & $7.0360^{*} 10^{6}$ & 81476.5 & 0.99617 \\
\hline Cis-whiskylactona & 68.040 & $9.4578^{*} 10^{6}$ & 203518 & 0.99950 \\
\hline 2-Feniletanol & 69.057 & $1.1808^{*} 10^{7}$ & $1.3871 * 10^{7}$ & 0.995067 \\
\hline Ácido heptanoico & 70.671 & $8.5163^{*} 10^{6}$ & $-1.2397^{*} 10^{6}$ & 0.986357 \\
\hline Trans-whiskylactona & 70.864 & $5.7159^{*} 10^{6}$ & 110987 & 0.999450 \\
\hline 4-Etilguaiacol & 73.724 & $2.7036^{*} 10^{7}$ & $-2.67255^{*} 10^{6}$ & 0.998687 \\
\hline Ácido octanoico & 75.090 & $1.8738^{*} 10^{7}$ & $2.38555^{\star} 10^{7}$ & 0.992035 \\
\hline 2-Etilfenol & 75.595 & $4.8992^{*} 10^{7}$ & 347050 & 0.999771 \\
\hline Eugenol & 77.493 & $2.4316^{*} 10^{6}$ & -210597 & 0.995374 \\
\hline
\end{tabular}


Los resultados provenientes de esta técnica serán utilizados dentro del desarrollo de esta tesis con varios objetivos, el primero de ellos como medida de control y seguimiento del proceso de vinificación. Los siguientes enfoques están relacionados con las soluciones que puede generar esta técnica para identificar las diferencias entre los compuestos aromáticos de los vinos; partiendo de este punto se pueden buscar correlaciones entre la nariz electrónica y olfatometría.

\subsubsection{Condiciones Experimentales para la GC-O}

La detección olfatométrica o snifing GC-O está diseñado para introducir la opinión técnica de un evaluador que determine el perfil aromático de un muestra a partir de una previa separación cromatográfica de la mezcla volátil [20, 21].

La cromatografía de gases es una técnica válida para la clasificación y cuantificación de una de una mezcla de volátiles, pero no es útil para describir una apreciación sensorial de la muestra evaluada. Es por ellos que diversos autores han recurrido a análisis paralelos de GC y olfatometría [22]. Sin embargo debemos añadir que este tipo de valoraciones sensoriales presentan dificultades al momento de juzgar la relevancia de los compuestos volátiles con una repetibilidad óptima. Una posible solución a esta problemática se ha conseguido aplicando las técnicas de dilución, método que explicaremos al final de este apartado [23].

La olfatometría es un sistema dual, esto significa que conserva la características de la detección instrumental de la cromatografía convencional realizada bien sea por FID o por MS, y además adiciona un segundo puerto detector para la valoración aromática específica de cada componente previamente separada por la columna cromatográfica. En este sentido, se ha dispuesto en nuestra investigación que las condiciones aplicadas con la cromatografía de gases sean las utilizadas para realizar la olfatometría.

Las medidas de olfatometria las hemos realizado en nuestro laboratorio en un equipo provisto de una detección FID y un acoplamiento para evaluar sensorialmente los aromas separados por la correspondiente columna. Es evidente que la solución ideal habría sido acoplar el sistema olfatométrico en un equipo GC-MS pero la adaptación nos pareció viable y sobre todo nos permitió hacer estas experiencias olfatométricas en Valladolid, bien entendiendo que el estudio de cuantificación de los compuestos se realizó en la Estación Enológica de Haro. Añadir que el sistema FID, aún mas imperfecto que la separación magnética, es una suficiente referencia complementaria para la identificación de los volátiles, que además se podrán confirmar con nuestra experiencia sensorial desarrollada previamente cuando preparamos los compuestos independientemente para obtener las curvas de calibración de cada uno de ellos. 
Un óptimo desarrollo experimental para la posterior búsqueda de correlaciones entre sistemas requiere mantener el mayor número de similitudes posibles. En concreto, para la olfatometría se ha instalado una columna con las mismas características que la utilizada para la GC-MS (capilar de cristal de silicio supelcowax de $30 \mathrm{~m} \times 0.25 \times 0.25 \mu \mathrm{m}$ ). Además se ha usado el mismo programa de temperatura especificado en el apartado anterior.

Procederemos hacer referencia a aquellas características para las cuales ambos sistemas son diferentes. En este caso, se empleó un equipo de cromatografía Hewlett Packard HP 4890D, la columna cromatográfica fue divida por medio de un separador de cuarzo para obtener una respuesta simultánea en el detector fotométrico de llama (FID) y el puerto de detección olfatométrica ODO II diseñado por SGE (Ringwood, Australia).

En los primeros estudios en los que se empleó la GC-O, se evaluaba el efluente que salía directamente del cromatógrafo. Pero éste era un gas seco y muy caliente, lo que provocaba la deshidratación nasal de quienes llevaban a cabo los análisis. Además de ser un procedimiento sumamente incómodo, disminuía la capacidad olfativa de los analistas, lo que se traducía en problemas de reproducibilidad. Por ese motivo, en 1971 se diseñó el primer cromatógrafo de gases con detector olfatométrico, en el que se mezclaba el efluente caliente que salía de la columna con aire humidificado. Desde entonces se han introducido distintas mejoras y actualizaciones en la instrumentación para hacer más cómodo y ergonómico el análisis. En esencia, el sistema aquí descrito es el que se utiliza actualmente en casi todos los detectores olfatométricos.

El detector olfatométrico consiste en un tubo de aluminio $(8 \mathrm{~cm} \times 2,5 \mathrm{~cm}$ d.i.) que se mantuvo a $250^{\circ} \mathrm{C}$ con un sistema de humidificación constante. A la salida de la columna que conduce los VOCs al detector se ubica un panelista entrenado en la evaluación de notas arómaticas de los compuestos que eluyen de la columna. Una vez percibido un aroma, este se indica en el cromatograma y se describe la percepción olfativa. Ambos detectores se mantuvieron a una temperatura de 250 ${ }^{\circ} \mathrm{C}$. El FID actúo con una mezcla de hidrógeno $(20 \mathrm{ml} / \mathrm{min})$ y aire $(250 \mathrm{ml} / \mathrm{min})$ y como gas auxiliar el nitrógeno a un flujo de $40 \mathrm{ml} / \mathrm{min}$. La Figura 2.3. esquematiza algunas de las características del diseño experimental del sistema utilizado para el análisis olfatométrico.

Una vez identificados y valorados los compuestos de acuerdo con su actividad aromática, se procede a estimar la importancia de cada componente en cuanto al aporte que hace a la percepción global utilizando la técnica de dilución. Esta técnica permitió evaluar la percepción de una aroma desde diferentes grados de concentración de la fracción volátil, además de estimar su contribución a nivel global y la reproducibilidad respecto a los tiempos de retención. Con relación a la medición del error experimental, se realizaron un conjunto de tres repeticiones 
por cada medida. Por otra parte, el tiempo de evaluación del panelista por cada muestra, se decidió que no podía superar más de 20 minutos para prevenir la fatiga del evaluador.

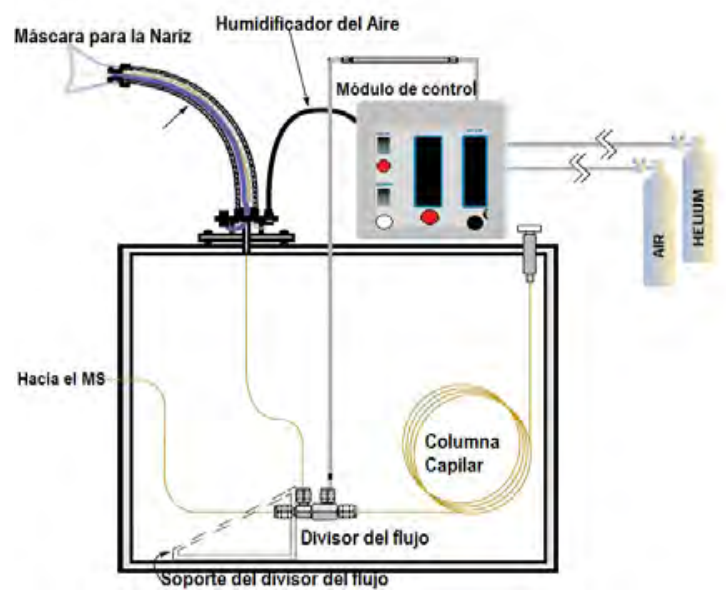

Figura 2.3. Sistema de análisis cromatográfico GC-FID-O

Los compuestos, una vez separados y eludidos de la columna, fueron cuantificados de acuerdo a sus propiedades aromáticas. Para distinguir entre aromas activos e inactivos se definió el parámetro "valor de actividad aromática" $(\mathrm{OAV})$, que mide la actividad aromática de un compuesto en una matriz determinada [24].

El uso de la olfatometría reiteramos supone tener una herramienta capaz de relacionar el análisis cualitativo y cuantitativo que aporta la cromatografía de gases con la percepción aromática humana. Hasta el momento se han desarrollado distintas técnicas olfatométricas que recopilan y procesan los datos obtenidos a través de la GCO, y que se clasifican en cuatro grupos en función del tipo de medida que lleven a cabo: las técnicas de dilución, las técnicas de frecuencia de detección, las técnicas de tiempo-intensidad y las técnicas de intensidad posterior [25]. A continuación daremos explicación a las dos primeras técnicas antes mencionadas, las seleccionadas es esta tesis, debido a que sus características son las que mejor se acoplan a los objetivos marcados en este trabajo.

\section{Técnicas de evaluación mediante dilución.}

Este tipo de análisis se realizó empleando uno de las métodos más conocidos hasta el momento, (AEDA) diseñado por Grosch y su grupo [26, 27]. El AEDA permitió la evaluación de la actividad aromática de compuestos oliendo una serie de disoluciones del extracto original hasta encontrar el límite de detección que se tiene para cada compuesto. 
La aplicación del método AEDA requiere la preparación de un grupo de muestras cada una de ellas con diferentes concentraciones. Seguidamente, se mezcla todo el conjunto de muestras para prevenir una sobrevaloración de las mismas y se siguen todas las condiciones especificadas en la separación cromatográfica.

La segunda parte del experimento es la referente a la adquisición de datos. Actualmente se dispone de programas que permiten simplificar la adquisición de datos. Estos programas se basan en sistemas de reconocimiento de voz para registrar la información que determina el evaluador. Sin embargo, en nuestro caso la adquisición se realizó manualmente; cuando el evaluador detectaba una aroma, describía sus respectivas características sensoriales y registraba el tiempo inicial y final de elución. Cada cualidad era puntuada en una escala categórica entre $0-3$; siendo 0.5 la calificación para un volátil débilmente detectable, 1 poco, 2 moderado, y 3 fuerte.

Algunos de los resultado publicados en la bibliografía dividen el análisis olfatométrico de acuerdo a la similitud de los compuestos (alcoholes, fenoles, ésteres, ácidos) para mejor la capacidad de análisis, en cuanto a la cantidad e intensidad de los compuestos percibidos [28]. En vista de que el propósito era buscar correlaciones para la valoración global del vino, se ha realizado un análisis conjunto aunque ello implicara una posible reducción del número de compuestos apreciados.

El entrenamiento del evaluador se realizo con una serie de mezclas con los compuestos citados en la Tabla 2, y siguiendo la técnica de AEDA. Para este desarrollo experimenta se utilizaron soluciones hidroalcohólicas mezclados con compuestos volátiles cada vez más diluidas, 1:4, 1:16, 1:64, 1:256 y 1:1024, hasta llegar a una dilución para la que ningún odorante es percibido. Para asegurar la repetibilidad de los resultados cada análisis se realizó con tres observadores diferentes y las valoraciones de los mismos fueron promediadas.

Finalmente, se ha calculado una magnitud que denominaremos factor de percepción, este valor depende del compuesto que haya alcanzado la mayor nota ente el intervalo de compuestos medidos. El factor se calculó dividiendo la evaluación de cada compuesto por la mayor nota registrada y de esta manera se obtiene el olfatograma con los valores característicos de cada vino.

Dentro de esta investigación, la olfatometría fue utilizada para identificar y cuantificar solamente los compuestos que son realmente activos desde el punto de vista de su percepción aromática en la matriz volátil del vino [23, 29, 30]. A partir de esta información cuantitativa ha sido posible la construcción de un perfil de aroma general de los vinos analizados y establecer algunas nociones de las relaciones existentes entre odorantes. 
Técnicas de evaluación mediante frecuencia de detección.

En estas técnicas un panel de analistas analiza un mismo extracto aromático mediante GCO. Para cada individuo se registran los tiempos de retención en los que se detectan olores y la descripción y duración de los mismos. Tras los análisis, se determina el número de analistas que perciben cada olor, de manera que los olores detectados con mayor frecuencia son los presentan una mayor importancia relativa. Esto se relaciona a su vez con una mayor intensidad aromática. Así se puede dibujar un "aromagrama", un perfil aromático del producto analizado en el que se relaciona la frecuencia de detección con los tiempos de retención.

Los resultados de los diferentes análisis realizados con esta técnica serán utilizados para la búsqueda de correlaciones con las señales resultantes de la evolución realizada con la nariz electrónica al mismo grupo de vinos.

\section{Limitaciones de la GCO}

Todas las técnicas olfatométricas desarrolladas al día de hoy presentan ventajas e inconvenientes, por otra parte cada una de ellas basa su funcionamiento en principios diferentes. De este manera, será más adecuado emplear una técnica u otra de acuerdo con los requerimientos que se impongan para seleccionar y clasificar los odorantes de acuerdo con su potencia y/o su intensidad aromática [31].

De forma general, las técnicas más rápidas son las de frecuencia de detección, mientras que las de dilución requieren tiempos de análisis considerablemente más largos.

En las técnicas de dilución, un analista puede no percibir un odorante en una dilución, pero sí detectarlo en diluciones superiores [32], motivo por el cual, en opinión de diversos autores, las técnicas de frecuencia de detección son más adecuadas a la hora de comparar muestras diferentes o realizar análisis cuantitativos [33-35].

Además de las limitaciones propias que presenta cada una de las distintas técnicas olfatométricas, es necesario considerar aquéllas que aparecen por el hecho de emplear la nariz humana como detector cromatográfico y que principalmente influyen sobre la reproducibilidad del análisis [36, 37], como son el cansancio y la saturación sensorial que puede sufrir el analista y que afectan negativamente a la calidad de los datos obtenidos. 


\subsection{PANEL DE CATA ELECTRÓNICO}

\subsubsection{Nariz electrónica}
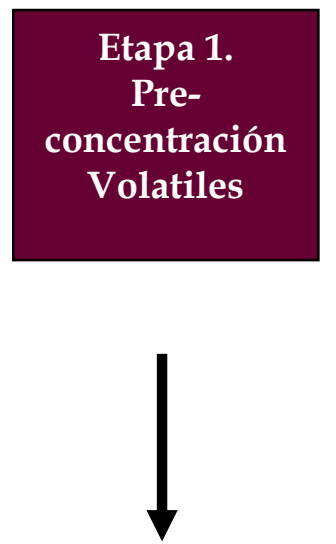

Etapa 2.

Desorción de

VOCs en el

sistema de

medida

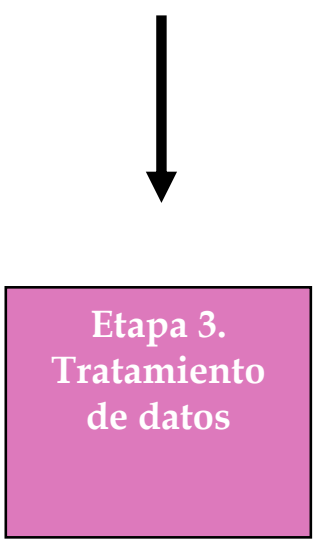

La selección del sistema

de concentración de

VOCs es muy importante en el caso de los vinos, ya que los sensores son muy sensibles al agua y el etanol presentes en el vino dificultando la capacidad de discriminación del sistema.

Procedimiento para el método SPME

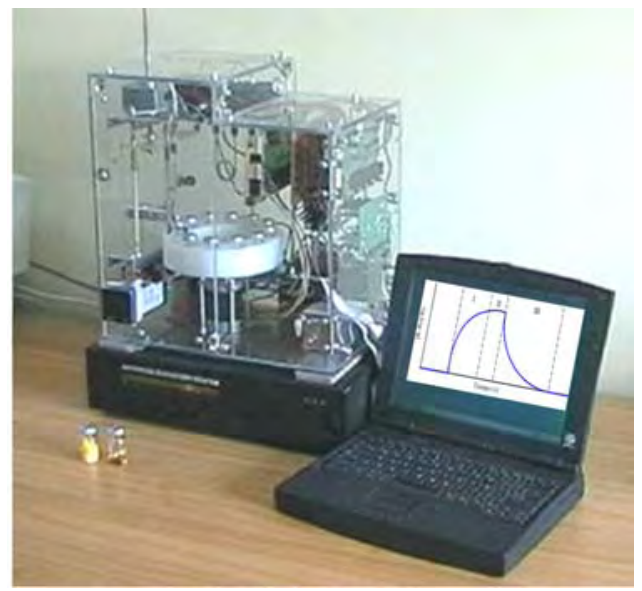

Prototipo de la Nariz electrónica desarrollado por nuestro grupo de investigación. Sistema formado por una red de sensores MOS.

\section{Sistema de medida}
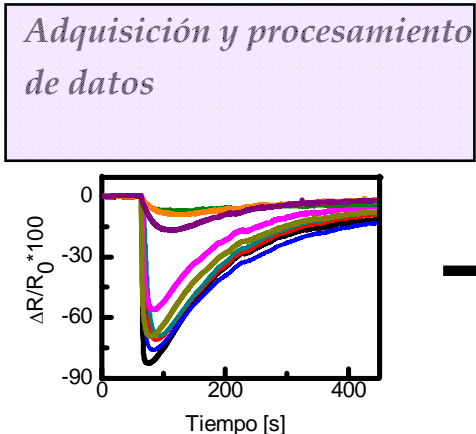

Respuesta típica de los sensores MOS
Identificación de las principales características de las muestras medidas

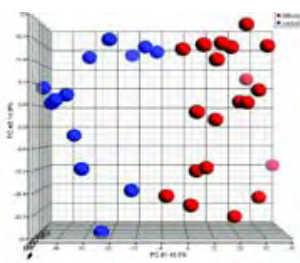

Análisis multivariante para la clasificación de muestras

Figura 2.4. Proceso de medida con una nariz electrónica 
La Figura 2.4 resume los tres bloques principales que constituyen una nariz electrónica: Sistema de inyección, sistema de detección, y sistema de reconocimiento de patrones. Podemos decir de esta manera que es un equipo que sirve para la caracterización de VOCs en un conjunto de muestras determinado; las valoraciones finales pueden obtenerse a través de técnicas de reconocimiento de patrones.

Ya hemos hecho mención a una conclusión importante después de años de investigación en el área de las narices electrónicas, la necesidad de diseños específicos, o al menos de protocolos de medidas que se acomoden a los requerimientos del tipo de productos que van a ser analizados.

En esta sección daremos explicación a las características principales y metodología de trabajo llevada a cabo con una nariz electrónica diseñada por este grupo de investigación para la valoración de vinos.

\section{Etapa 1. Sistemas de inyección}

Para transferir los compuestos volátiles de la muestra evaluada arrastradas por una corriente de $\mathrm{N}_{2}$ y aire hacia el sistema de detección, se requiere la práctica de dos procedimientos simultáneamente: el control del flujo de gases y la preconcentración de VOCs. La primera de las demandas implica el diseño de una línea de gases que era capaz de mantener un flujo constante (gases de arrastre más volátiles) y la prevención de humedad; en nuestro caso hemos seguido las características señaladas en la Figura 2.5. Con esta línea de gases se ha garantizado la estabilidad de las señales provenientes de la red de sensores, además una favorecer el arrastre de todos los volátiles durante la desorción térmica hasta la cámara de sensores; también en estas condiciones podemos asegurar que no existe contaminación alguna entre una medida y la siguiente (minimizar el efecto memoria) [39].

\section{Linea de gases}

La Figura 2.5. como se mencionó anteriormente, especifica el diseño de la línea de gases. Esta instalación está compuesta de tres bombonas que transfiere los gases por medio de una red de tubería de acero inoxidable y cobre (según sea requerido) de $1 / 8$ de pulgada hasta llegar a un puerto de inyección. De esta manera se conducen los VOCs a la cámara de sensores que tiene en su salida un rotámetro para la lectura del flujo del gas presente en el sistema.

Concretamente el transporte de los VOCs -desde el sistema de muestro a la cámara de sensores- tiene lugar gracias a un flujo dinámico de un gas portador 
de elevada pureza (Aire, $\mathrm{N}_{2}$ de la compañía Air Liquide). Salvo que se especifiquen otros requerimientos, en las condiciones experimentales de este estudio se han utilizado una mezcla de $100 \mathrm{~mL} \cdot \mathrm{min}^{-1}$ de gas inerte (nitrógeno) y $50 \mathrm{mLmin}^{-1}$ de aire. Se mantiene el flujo constante de gases durante toda la jornada de trabajo en la que se están realizando las diferentes medidas.
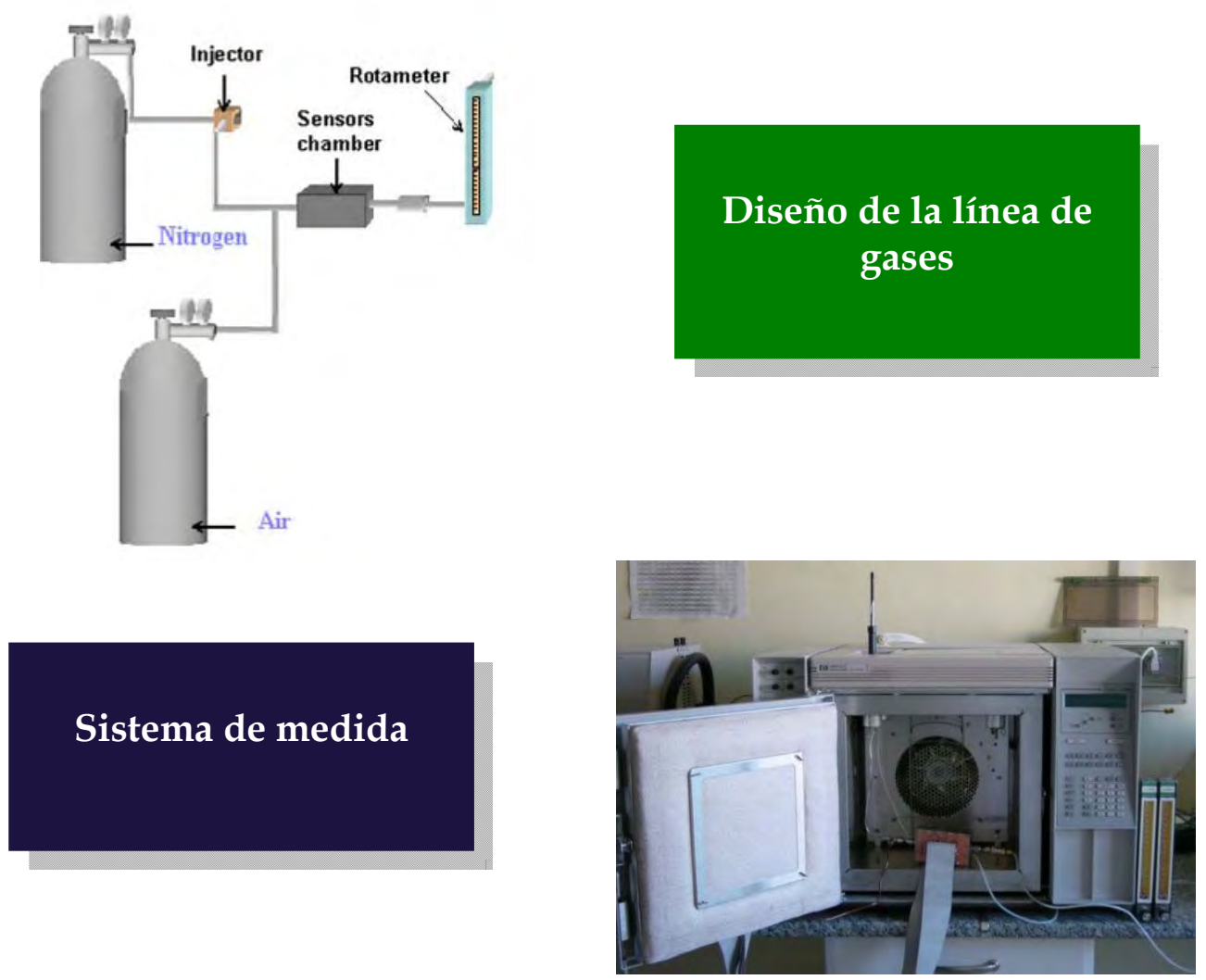

Figura 2.5. Linea de gases y sistema de medida

A continuación se da comienzo con el proceso de transporte de los compuestos volátiles desorbidos. Una de las particularidades a destacar de nuestro sistema es el uso el puerto de inyección de un GC (HP4890D) que permite asegurar el control de temperatura y la repetibilidad del experimento; con este fin la columna cromatográfica propia del GC situada en el interior del equipo, ha sido remplazada por la red de sensores, como se ilustra en la Figura 2.5. La conexión se realiza utilizando un adaptador de $1 / 16$ a $1 / 8$ de pulgada de diámetro y una tubería de acero inoxidable de $5 \mathrm{~cm}$ de longitud (1/16 pulgadas) que une el puerto de inyección del GC con la cámara donde se encuentra la red de sensores.

Cabe incluir en esta explicación que cuando se utiliza la técnica SPME para la desorción de VOCs se necesita la aplicación de temperaturas altas bajo una atmosfera inerte, para así prevenir la combustión de la materia orgánica, mientras que el proceso de adsorción característico de los sensores MOS requiere para su funcionamiento la presencia de aire. Atendiendo a este requerimiento, el sistema se diseñó de manera que el flujo de aire (gas auxiliar) se incorporara en el 
sistema después de la desorción de los volátiles a elevada temperatura. En este caso, la conexión consiste simplemente en un tubo de acero inoxidable de $1 \mathrm{~cm}$ de longitud (1/16 pulgadas $\varnothing)$ con tres salidas $[40,41]$.

\section{Preconcentración de VOCs}

\section{Microextracción en fase sólida (SPME)}

Una vez establecidas todas las condiciones concernientes a la línea de gases podemos continuar con la descripción de la técnica usada para la extracción, concentración y desorción de los volátiles que serán valorados con la nariz electrónica. En este punto hemos de recordar el contenido relacionado con el método SPME descrito en apartado 2.1.2, donde se especificaron los parámetros programados en un automuestrador para la inyección de la fase gaseosa de una muestra de vino con un equipo de cromatografía. A diferencia de la GC, tenemos que este dispositivo sensorial requiere una preconcentración manual, y por tanto, es muy importante el adecuado control de todas las condiciones de trabajo para alcanzar una reproducibilidad aceptable.

Aún así, el procedimiento experimental es prácticamente el mismo para ambos casos. Es necesario entonces comenzar con acondicionamiento de la fibra, seguido por la extracción de los VOCs de interés y finalizar con la desorción e inyección de los compuestos químicos en la cámara de sensores de la nariz electrónica.

El acondicionamiento de la fibra se realiza para prevenir contaminación en el análisis, esta limpieza consiste en dejar la fase estacionaria del sistema a una temperatura de $280^{\circ} \mathrm{C}$ durante una 1 hora.

A continuación, para la extracción de volátiles se utiliza un vial para una capacidad de $10 \mathrm{ml}$ colocando una cantidad de $3 \mathrm{ml}$ de vino en un baño termostatizado a $40^{\circ} \mathrm{C}$ durante 10 minutos. Durante este intervalo de tiempo, la disolución permanece en agitación homogénea a 700 r.p.m, para facilitar la transferencia de los analitos desde la matriz hasta la fase estacionaria de la fibra [42]. El control de temperatura y agitación se efectúa con un agitador magnético (RCT Basic IKA WERKE) y una barra imantada. Los periodos de tiempo y temperatura determinados para propiciar la adsorción de volátiles son iguales a aquellos especificados en la cromatografía de gases.

Una vez han sido extraídos los VOCs, se procede a su desorción térmica en el puerto de inyección del CG. Esta etapa consta de dos fases, la primera de ellas, denominada en esta investigación como fase de secado, en la cual los restos de agua y etanol débilmente adsorbidos en este tipo de fibra se eliminan colocando 
la fibra en el puerto de desorción secundario de GC, durante un minuto con un flujo de $33 \mathrm{mLmin}^{-1}$ a $50^{\circ} \mathrm{C}$ (los compuestos desorbidos en esta fase se descartan). A continuación, en la segunda fase de desorción, los volátiles se desorben a una temperatura de $300{ }^{\circ} \mathrm{C}$ (si no se especifica otra temperatura) durante 10 minutos. El septum utilizado en el portal de inyección está previamente perforado para evitar la ruptura de la fibra (Termogreen LB-2, Supelco). Seguidamente, los VOCS son arrastrados, mediante un flujo controlado de $100 \mathrm{ml} . \mathrm{min}^{-1}$ de nitrógeno, hasta la cámara de sensores; en una fase intermedia, a la corriente de compuestos químicos y nitrógeno, se inyecta un flujo de $50 \mathrm{mLmin}^{-1}$ de aire. Finalmente, esta mezcla de gases es conducida hasta la cámara de sensores, lugar donde se produce la interacción entre los sensores y la fase volátil del vino. Este mecanismo será comentado a continuación.

\section{Etapa 2. Sistema de detección}

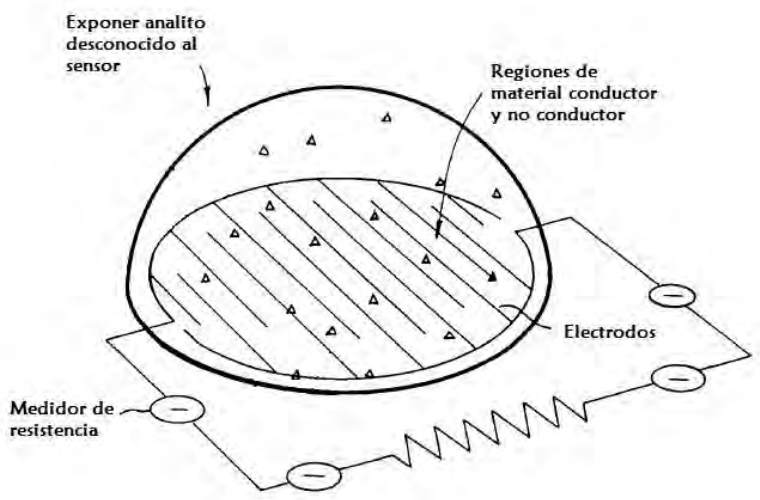

Figura 2.6. Diseño del sensor.

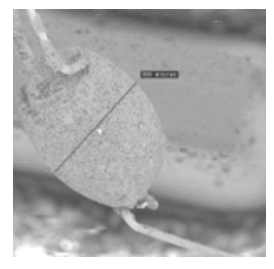

Sensores de la serie SB fis

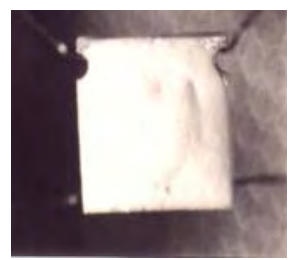

Sensores de la serie SP fis

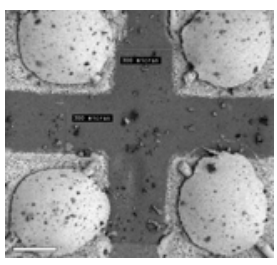

Sensores de la serie TGS-2600

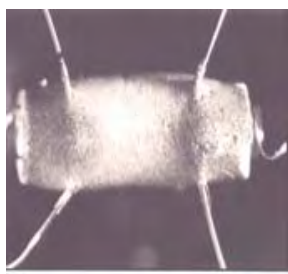

Sensores de la serie TGS-800

Figura 2.7. Vista desde arriba del elemento activo captado con un SEM Jeol JSM820 una vez eliminado el sistema de protección del sensor.

El segundo bloque del sistema es el relacionado con la red de sensores químicos inespecíficos, lo cuales son capaces de suministrar señales características para cada aroma. El término químico se usa para dar a entender que el dispositivo es capaz de cambiar algunas de sus propiedades químicas y consecuente modificación de su resistividad eléctrica cuando se expone a la interacción con 
compuestos volátiles. La Figura 2.6. y 2.7. esbozan el diseño habitual para un sensor MOS y las características del material sensible por cada tipo de sensor.

Descripción de la red de sensores.

Tabla 2.2. Relación de sensores utilizados para la caracterización de vinos.

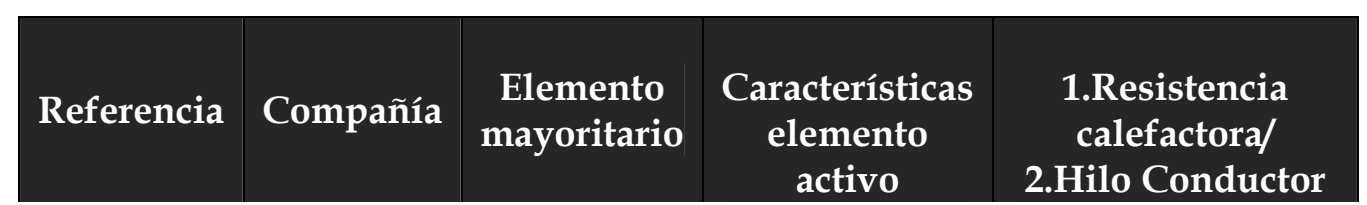

\begin{tabular}{|c|c|c|c|c|}
\hline$S P-31$ & \multirow{12}{*}{ fis } & \multirow{12}{*}{$\mathrm{SnO}_{2}$} & \multirow{7}{*}{$\begin{array}{c}\text { Sustrato } \\
\text { delgado de } \\
\text { dimensiones } 2 \\
\mathrm{~mm} \times 2 \mathrm{~mm} \\
\text { de } \mathrm{Al} 2 \mathrm{O} 3\end{array}$} & \multirow{7}{*}{$\begin{array}{l}\text { 1. película gruesa } \\
\text { de óxido de } \\
\text { rutenio o platino. }\end{array}$} \\
\hline$S P-15 A$ & & & & \\
\hline$S P-M W O$ & & & & \\
\hline$S P-53$ & & & & \\
\hline SP-19 & & & & \\
\hline$S P-12 A$ & & & & \\
\hline$S P-M W 1$ & & & & \\
\hline$S B-A Q 4 A$ & & & \multirow{5}{*}{$\begin{array}{l}0.5 \mathrm{~mm} \times 0.3 \\
\mathrm{~mm} \text { en forma } \\
\text { de ovillo }\end{array}$} & \multirow{5}{*}{ Platino } \\
\hline SB-95 & & & & \\
\hline$S B-11 A$ & & & & \\
\hline SB-50 & & & & \\
\hline$S B-A Q 1 A$ & & & & \\
\hline TGS-2600 & \multirow{7}{*}{ Fígaro } & \multirow{4}{*}{$\begin{array}{c}\text { Mezclas de } \\
\text { óxidos }\end{array}$} & \multirow{4}{*}{\multicolumn{2}{|c|}{$\begin{array}{l}\text { Sustrato de } \mathrm{Al} 2 \mathrm{O} 3 \text { que tiene } \\
\text { embebido en su interior la } \\
\text { resistencia calefactora. Tiene una } \\
\text { geometría en forma de cruz de } 300 \\
\mu \mathrm{m} \text { para cada brazo. }\end{array}$}} \\
\hline TGS-2610 & & & & \\
\hline TGS-2611 & & & & \\
\hline TGS-2620 & & & & \\
\hline TGS-880 & & - & \multirow{3}{*}{\multicolumn{2}{|c|}{$\begin{array}{l}\text { Un sustrato de } \mathrm{Al} 2 \mathrm{O} 3 \text { de geometría } \\
\text { cilíndrica } 10 \mathrm{~mm} \text {. resistencia } \\
\text { calefactora en el interior }\end{array}$}} \\
\hline TGS-822 & & $\mathrm{SnO}_{2}$ & & \\
\hline TGS-826 & & $\mathrm{WO}_{3}$ & & \\
\hline
\end{tabular}

En resumen, esta tabla 2.2. especifica los cuatro diferentes tipos de sensores clasificados de acuerdo a su diseño de fabricación: Figaro y Fis. Dentro del grupo de los sensores Figaro se tienen dos categorías, la serie 8 y 2000. Para el grupo de los sensores Fis se dispone de las series SP y SB. Las principales diferencias entre los sensores se deben el espesor del material sensible, el posicionamiento y la forma de la resistencia calefactora. 


\section{Cámara de sensores}

Respecto a otras condiciones técnicas, en esta investigación y para alojar los sensores se ha diseñado una cámara termostatizada de acero inoxidable con una capacidad de $75 \mathrm{cc}$. Como se puede observar en la Figura 2.8. esta cámara consta de sendas tapas donde se colocan los sensores. El cierre hermético de ambas partes se consigue gracias a una junta tórica de $3 \mathrm{~mm} \varnothing$. Además, la tapa posee conectores eléctricos y un circuito impreso (donde se posicionan los sensores) que permite la transmisión de datos desde la cámara hasta el ordenador.

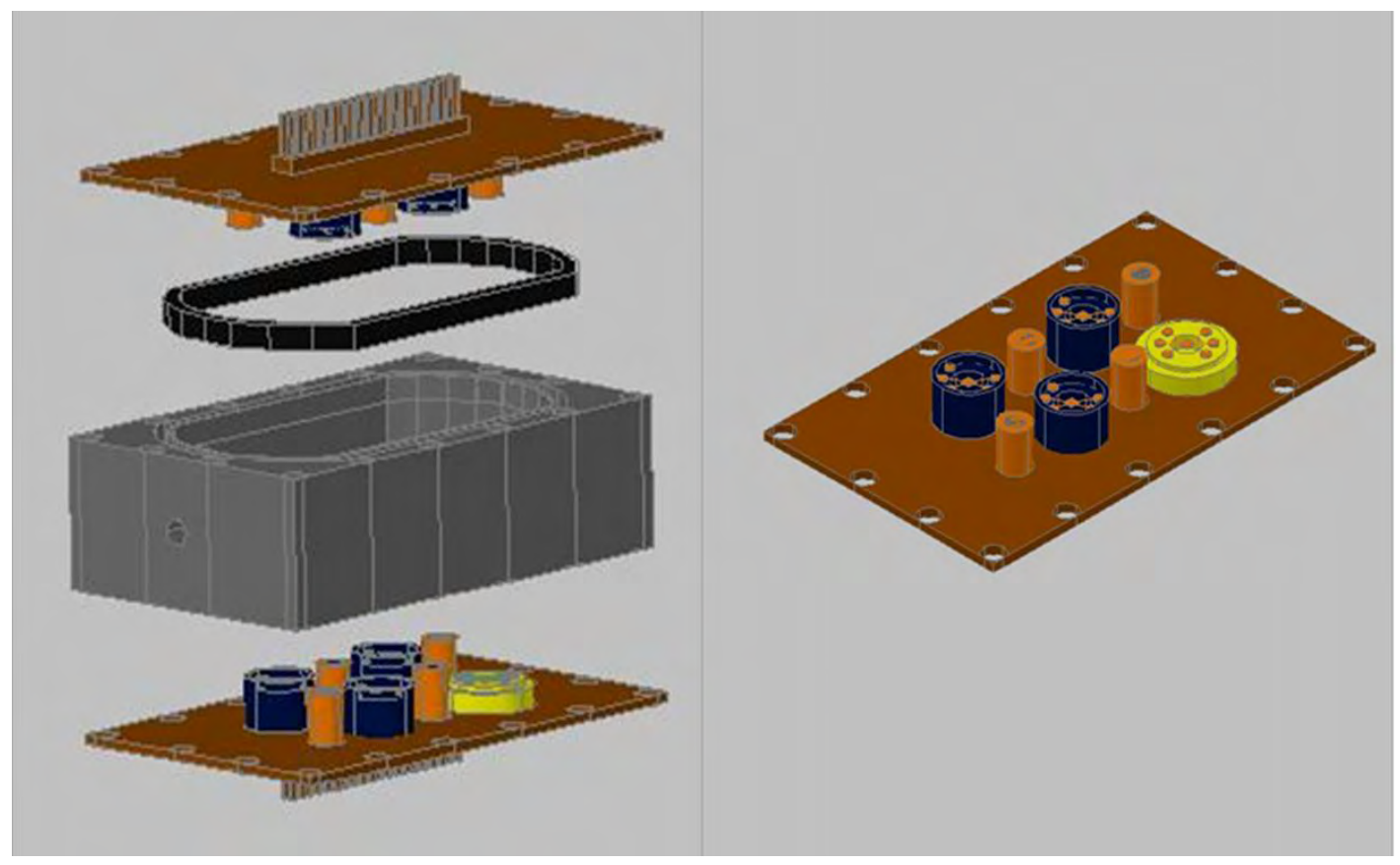

Figura 2.8. Diseño de la cámara de sensores: Tapa superior con el circuito impreso en el parte exterior; Junta tórica; Caja de acero inoxidable; Sensores; Tapa inferior.

La cámara de sensores se ha colocado en el interior del cromatógrafo para evitar las oscilaciones de su resistencia eléctrica con la temperatura ambiental. La cámara se ha mantenido durante todas las experiencias a $50^{\circ} \mathrm{C}$, temperatura aconsejada por los fabricantes de este tipo de sensores

\section{Etapa 3. Adquisición de datos.}

Como se ha indicado en los párrafos anteriores, ha sido diseñado un circuito eléctrico para el registro de los datos y para garantizar la temperatura de trabajo de los sensores. En esta investigación, ha sido necesario diseñar dos circuitos 
eléctricos diferentes en función de las especificaciones de los fabricantes de los sensores, Figaro y Fis.

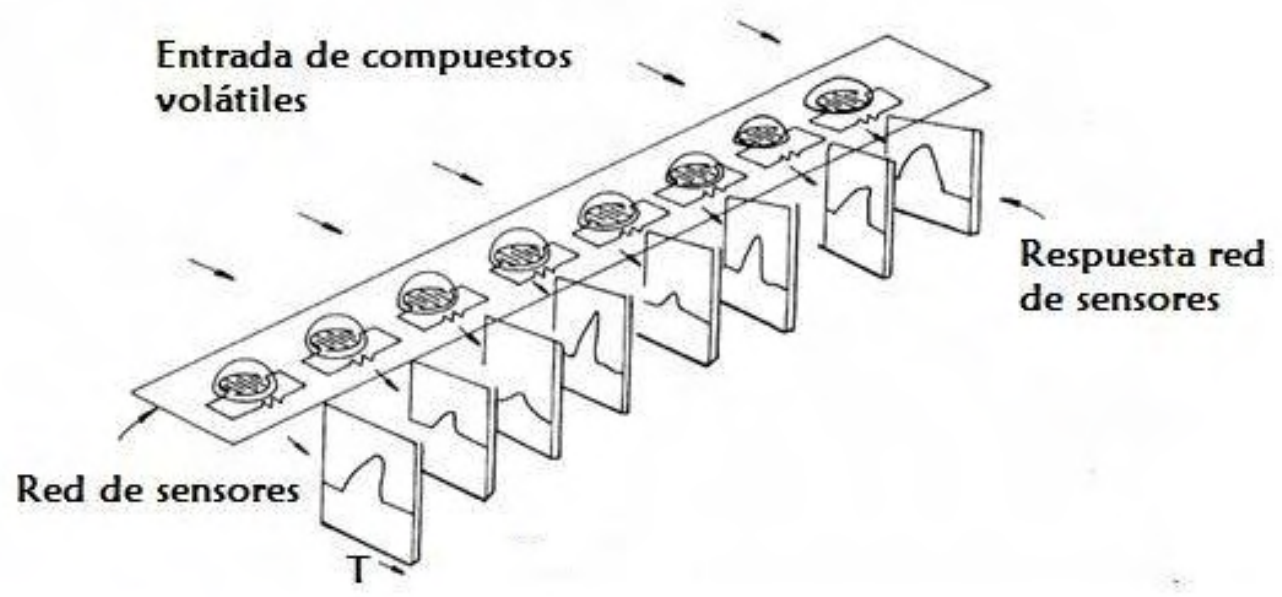

Figura 2.9. Adquisición de datos. Registro del cambio en la resistencia en función del tiempo

El circuito electrónico necesario para la medida entre los extremos de los sensores Fis se muestra en la Figura 2.10. Como se observa en esta figura, la diferencia de potencial en la resistencia del sensor es directamente proporcional a la variación relativa de la diferencia de potencial de la resistencia auxiliar. En efecto:

$\Delta V s=\frac{V s-V s O}{V s O} \times 100==_{1} \frac{\left(5-V_{A}\right)-\left(5-V_{A O}\right)}{5-V_{A O}} \times 100={ }_{2} \frac{-\left(V_{A}-V_{A O}\right)}{V_{A O}} \times 100 \times \frac{V_{A O}}{5-V_{A O}}=-\Delta V_{A} \frac{V_{A O}}{5-V_{A O}}$

(1) $\mathrm{V}_{\mathrm{T}}=\mathrm{V}_{\mathrm{A}}+\mathrm{V}_{\mathrm{S}}=5 \mathrm{~V} \Rightarrow \mathrm{V}_{\mathrm{S}}=5-\mathrm{V}_{\mathrm{A}}$

(2) Multiplicando y dividiendo por $\mathrm{V}_{\mathrm{A} 0}$

siendo, $\mathrm{V}_{\mathrm{T}}$, la diferencia de potencial total del sistema (5V); $\mathrm{V}_{\mathrm{SO}}$, la d.d.p. del sensor en el instante inicial; $V_{\mathrm{S}}$, la d.d.p. del sensor en el instante $t ; V_{\mathrm{AO}}$, la d.d.p de la resistencia auxiliar inicial; $\mathrm{V}_{\mathrm{A}}$, la d.d.p de la resistencia auxiliar en $\mathrm{t}$ y $\Delta \mathrm{V}_{\mathrm{S}}$, la variación relativa del potencial registrado por el sensor; $\Delta \mathrm{V}_{\mathrm{A}}$, la variación relativa del potencial de la resistencia auxiliar.

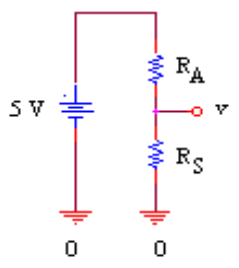

$\mathrm{R}_{\mathrm{S}}$ : resistencia del sensor

$\mathrm{R}_{\mathrm{A}}$ : resistencia auxiliar

$\mathrm{V}$ : Tensión total

v: Tensión de salida

Figura 2.10. Circuito eléctrico de los sensores de la compañía FIS.

En el caso de los sensores Fígaro, y también de acuerdo con las especificaciones del fabricante, requieren un sistema electrónico no muy diferente al de los 
sensores de la compañía FIS Inc. (Figura 2.11). Repitiendo el mismo procedimiento que en el caso de los sensores FIS, se tiene que la diferencia de potencial existente en la resistencia del sensor es proporcional a la diferencia de potencial de la resistencia auxiliar $\mathrm{R}_{\mathrm{A}}$. Aunque, como se puede observar, el término de proporcionalidad ha de modificarse debido a las diferencias en el circuito electrónico, resultando:

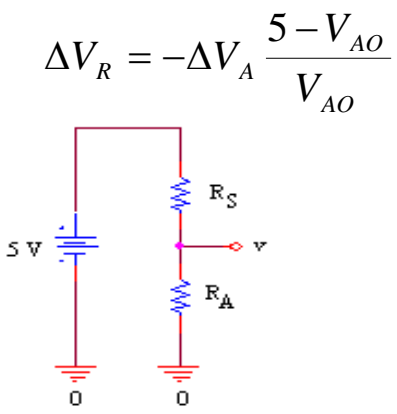

Figura 2.11. Circuito eléctrico de los sensores de la compañía Fígaro.

En todos los casos, con ayuda de una fuente de alimentación estabilizada en una disposición como la indicada en las figuras 2.10 y 2.11, se aplica una diferencia de potencial de $5 \mathrm{~V}$ entre los extremos del circuito. Además, esta fuente alimenta el sistema de calefacción del sensor que hace que se mantenga a su temperatura óptima de funcionamiento. Reiteramos que la diferencia de potencial en los extremos de los sensores se mide indirectamente en ambos casos a través de una resistencia auxiliar o resistencia de polarización $\left(R_{A}\right)$ en serie con la resistencia del sensor ( $\left.R_{S}\right)$.

En este trabajo se ha procurado en todo momento que el valor de cada una de las resistencias auxiliares fueran lo más parecidas a la resistencia inicial que presenta el sensor, de esta forma, la diferencia de potencial inicial de la resistencia auxiliar es en torno a $2.5 \mathrm{v}$ y las curvas registradas estarán tan sólo invertidas respecto de la forma habitual que tienen -debido al coeficiente de corrección que multiplica a $\Delta \mathrm{V}_{\mathrm{A}}$ para obtener $\Delta \mathrm{V}_{\mathrm{S}^{-}}$. Además, se ha comprobado que con resistencias por debajo de este valor se obtienen señales algo más intensas, pero con un peor grado de reproducibilidad; por el contrario, resistencias más elevadas conducen a señales mucho más pequeñas con menor grado de reproductividad [14].

El procedimiento experimental, desde que los sensores se conectan a la fuente de alimentación hasta la toma de los datos, consta de las siguientes etapas:

I. Estabilización del sistema de medida durante 48 horas que se alcanzan con una mezcla de nitrógeno (100 mL.min-1), aire (50 mL.min-1) y la fuente de alimentación conectada. 

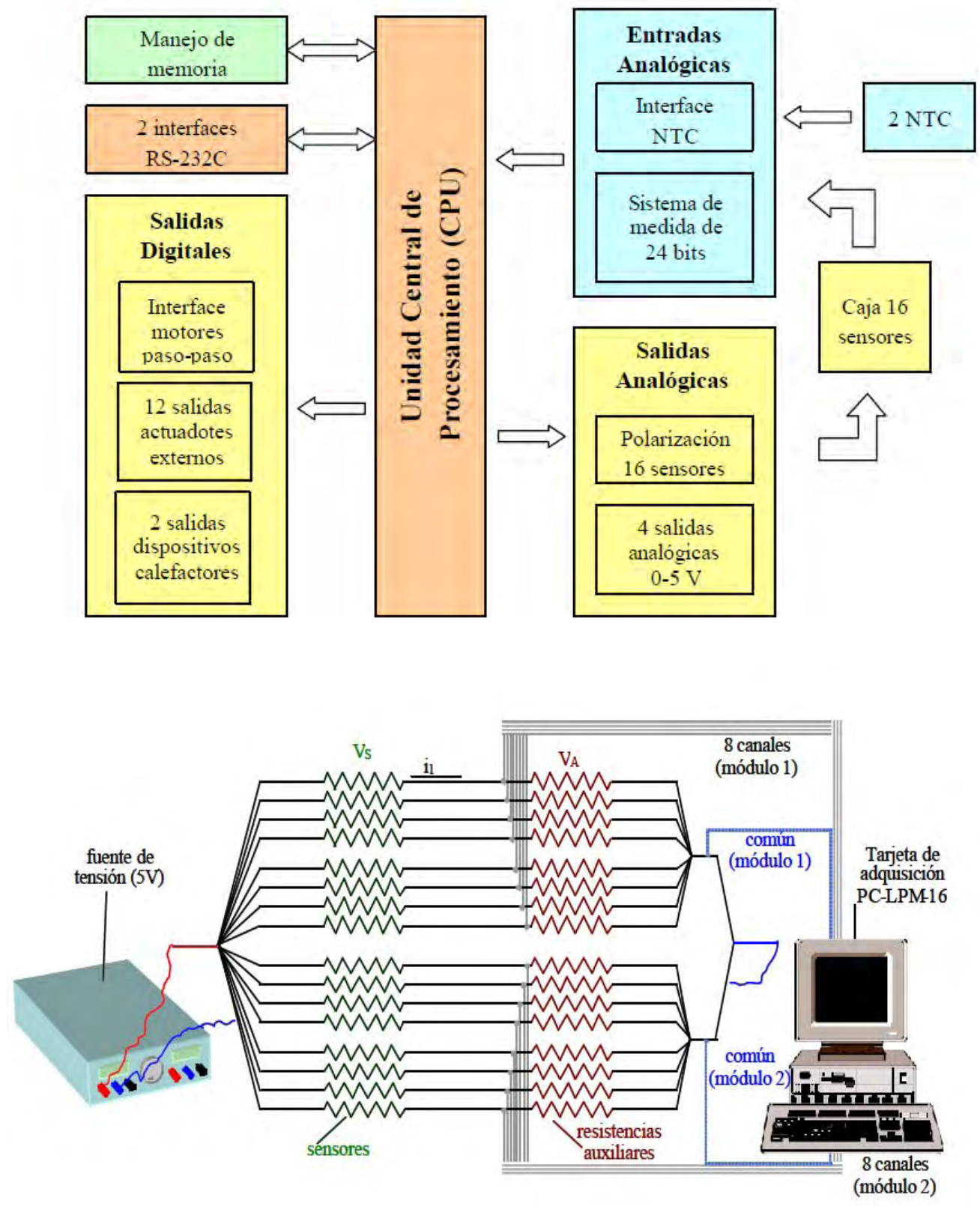

Figura 2.12. Diseño adquisición de datos

II. Adquisición de los datos un minuto antes de la inyección de los volátiles. En esta etapa, la medida de la diferencia de potencial en los extremos de los sensores se realiza a través de la tarjeta de adquisición de datos de Nacional Instrument (PCI-6013) insertada en la placa base de un ordenador, de acuerdo con el diseño que aparece en la Figura 2.12.

III. Mediante el software "NARIZ", programado por nuestro grupo en Visual $\mathrm{C}++, \mathrm{y}$ gracias a las funciones de biblioteca de "National Instrument", se registra y visualiza en tiempo real la diferencia de potencial de los 
distintos sensores (Figura 2.13). El intervalo de muestreo utilizado es de $0.5 \mathrm{~s}$ durante 15 minutos, salvo que se especifiquen otros intervalos.

Este software a su vez transforma los valores de la diferencia de potencial de los sensores en porcentaje de resistencia relativa -en formato compatible de EXCEL- [43]. Las ecuaciones que se aplican para dicha coversión de los datos son las siguientes:

$$
\begin{aligned}
& \Delta R_{S}=\frac{5\left(\Delta V_{A}\right)}{V_{A O}}\left(\frac{1}{\frac{5}{V_{A O}-\left(\Delta V_{A}\right)-1}}\right) \quad \Delta R_{s}=\frac{5\left(\Delta V_{A}\right)}{5-V_{A O}}\left(\frac{-1}{1+\left(\Delta V_{A}\right)}\right) \\
& \text { SENSORES FIS } \\
& \text { SENSORES FÍGARO } \\
& \Delta R_{s}=\frac{R-R o}{R o}
\end{aligned}
$$

Donde $\Delta R$ está definido como la resistencia relativa de los sensores, expresada en términos de la resistencia basal $\mathrm{R}_{\mathrm{o}}$ (en ausencia de aromas); $\mathrm{V}_{\mathrm{AO}}$, la d.d.p. de la resistencia auxiliar inicial y $\Delta \mathrm{V}_{\mathrm{A}}$, la variación relativa del potencial medido en la resistencia auxiliar.

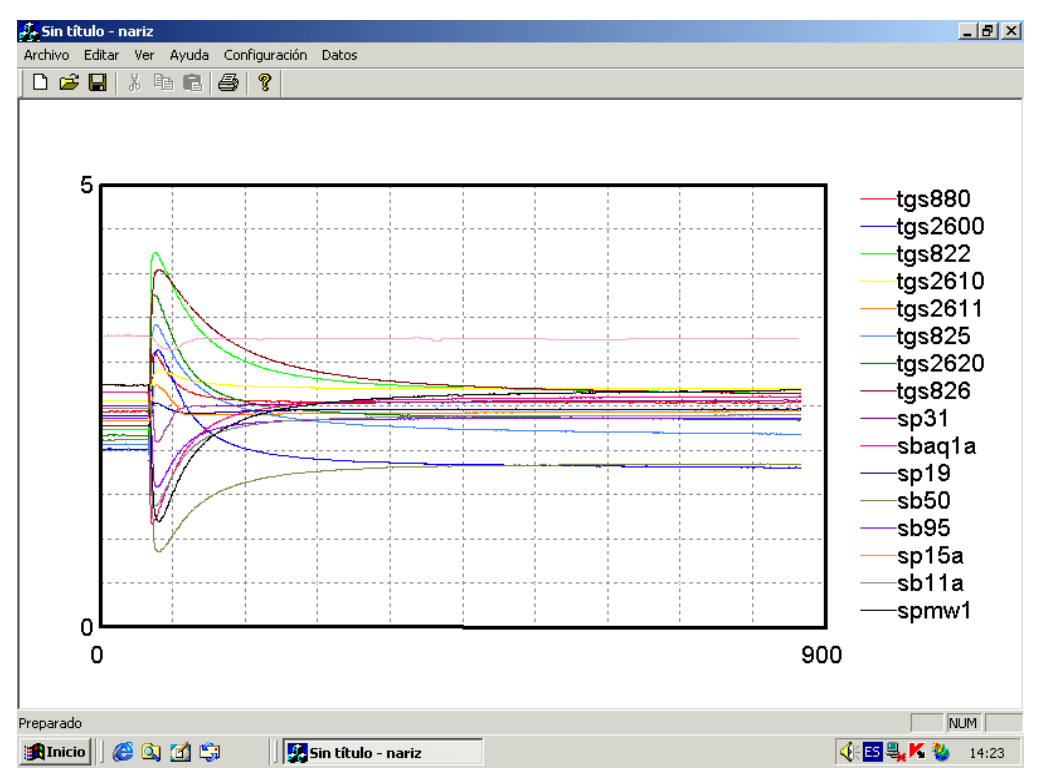

Figura 2.13. Representación típica de la adquisición de datos. Cambio de la resistencia de la red de sensores en función del tiempo.

IV. A partir de los valores de la resistencia relativa en función del tiempo, mediante una hoja Excel, calculamos la resistencia máxima (altura de pico) y los parámetros dinámicos de cada sensor [44]. Una vez realizados las medidas y cálculos pertinentes, un segundo programa extrae las 
características importantes y aplica algoritmos de reconocimientos de patrones, métodos que serán detallados en el apartado 2.3.

\subsubsection{Lengua electrónica}

\section{Sistema de detección}

La lengua electrónica empleada para el desarrollo de esta investigación tiene como electrodos de trabajo, sensores basados en una nueva familia de elementos, conocidos como electrodos modificados químicamente (CME) [45]. Específicamente la red ha sido formada con sensores con base de pasta de carbono, que denominaremos CPEs, elaborados de manera manual y con las características que se expondrán a continuación.

\section{Electrodos de Pasta de Carbono (CPE)}

Respecto a la fabricación de los sensores utilizados durante esta investigación, se ha trabajado esencialmente con un formato de sensor basado en CPEs macroscópicos o "de jeringa" (Figura 2.14). El material sensible esta formado por un sólido que generalmente es grafito, un líquido orgánico más o menos viscoso de carácter no electrolítico y el correspondientes modificador o dopante [45-47].

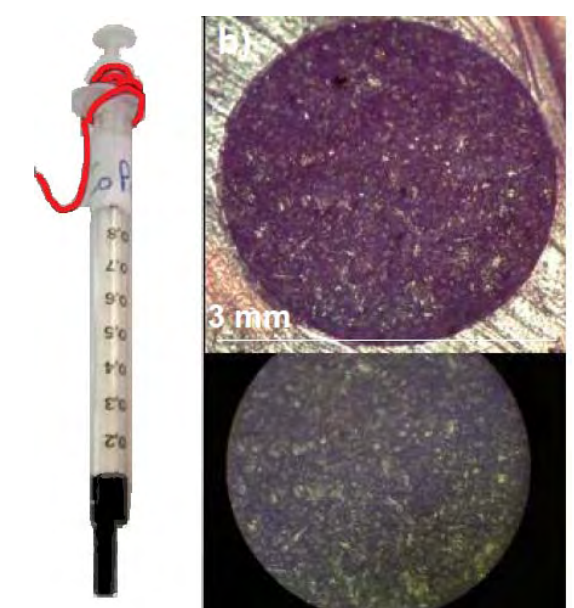

Figura 2.14. Micrografías de un CPE sin modificar de jeringa; aspecto macroscópico; micrografías ópticas de la superficie del electrodo.

El grafito es el material conductor por excelencia en la fabricación de pastas de carbono, comercialmente se expide en forma de polvo con un tamaño de partícula micrométrico (entre 5 y $20 \mu \mathrm{m}$ ), uniformemente distribuido. Numerosas empresas de productos químicos disponen de diversas variedades de grafito en 
polvo, por lo que la posibilidad de adquisición es sencilla. Las propiedades ofrecidas por las mezclas de grafito de calidad espectroscópica y aceites parafínicos (Nujol@) han resultado ser suficientemente satisfactorias en infinidad de aplicaciones. La presencia del agente conglomerante como el nujol, ralentiza el proceso de transferencia electrónica en comparación con el uso de electrodos de constitución homogénea [48,49]. Con respecto a las partes constituyentes, la Tabla 4 relaciona los elementos utilizados en la elaboración de los CPEs.

En cuanto a los compuestos orgánicos electroactivos, han sido incluidos una variedad de moléculas sensitivas, incluyendo ftalocianina, ferrocenos y perilenos debido a sus propiedades electroactivas complementarias. Concretamente, se han empleado bisftalocianina de lutecio $\left(\mathrm{LuPc}_{2}\right)$ un compuesto analogo sustitución de la anterior con 8 grupos tercbutilo $\left(\mathrm{LuPc}_{2 t}\right)$ y una monoftalocianina, la ftalocianina de cobalto (CoPc). En el caso de los perilenos, se han seleccionado el $\mathrm{N}, \mathrm{N}^{\prime}$-bis (piperidinometil)-3,4,9,10-perilenobis (dicarboximido)(BpPTCD), el Noctil-3,4,9,10-perylenobis (dicarboximida)(OcPTCMI) y el N-butil-3,4,9,10perylenobis(dicarboximida)(BuPTCMI). Se han incluído además en la red, dos sensores sin modificar; CPE y un electrodo de Platino [50].

Tabla 4. Productos utilizados como componentes de las pastas de carbono utilizadas

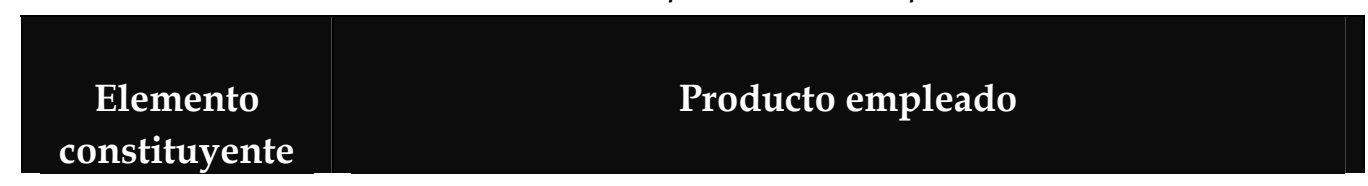

\begin{tabular}{|c|l|}
\hline $\begin{array}{c}\text { Matriz } \\
\text { conductora del } \\
\text { electrodo }\end{array}$ & $\begin{array}{l}\text { Grafito (Ultra Carbon Corp. USA); grado espectroscópico) } \\
\text { Nanotubos de carbono (Nanoledge [12]; SWNT 40\%: } \\
\text { MWNT 95\%) } \\
\text { Tinta de carbono C2000802D12 (Gwent Electronic } \\
\text { Materials) [13] }\end{array}$ \\
\hline $\begin{array}{c}\text { Aceite } \\
\text { conglomerante }\end{array}$ & $\begin{array}{l}\text { Nujol@ (Aldrich; densidad=0.862) Aceite de silicona: DC } \\
\text { 200 polidimetilsiloxano (Fluka; densidad=0.965) } \\
\text { Disoluciones EPDM2/ciclohexano y EPDM/o xileno }\end{array}$ \\
\hline Modificador & $\begin{array}{l}\text { - Sólidos en polvo de los compuestos orgánicos } \\
\text { electroactivos seleccionados. } \\
\\
\text { - Modelos redox clásicos: ferroceno (Fluka), hidroquinona } \\
\text { (Aldrich), ferrocianuro potásico (Aldrich), etc. } \\
\text { - Muestras de vino en sí }\end{array}$ \\
\hline
\end{tabular}

La mezcla de los materiales fue llevada a cabo mediante molienda de los sólidos en un mortero de ágata, ya que los materiales suministrados o bien sintetizados en nuestro laboratorio eran polvos o cristales de tamaño reducido. De esta manera se obtuvo un tamaño de partícula lo más reducido posible y una pasta convenientemente homogénea. El proceso de modificación electroactiva de la pasta comienza con la mezcla del compuesto orgánico en la menor cantidad de 
disolvente necesario, adición controlada, y agitación constante (manual o en baño de ultrasonidos), sobre la cantidad apropiada de pasta de carbono.

\section{Instrumentación electroquímica.}

Para llevar a termino las medidas con el conjunto de sensores preparados de acuerdo a las anteriores condiciones, se ha empleado una celda electroquímica voltamétrica, configurada por un receptáculo que contiene la disolución o muestra bajo estudio y tres electrodos (referencia, auxiliar o contraelectrodo y trabajo). Todos los experimentos de caracterización, puesta a punto y medidas con los sensores se han llevado a cabo empleando un potenciostato/galvanostato de la empresa EG\&G Princeton Applied Research [51], modelos 263A, controlado por el software M270, que opera dentro del entorno MS-DOS.

\section{Electrodos de referencia.}

Está constituido por un hilo de $\mathrm{Ag}$ recubierto por una fina capa de $\mathrm{AgCl}$ depositada electroquímicamente o bien por inmersión del hilo en la sal de plata fundida. Este es el electrodo más empleado para experimentos electroquímicos en medio acuoso, entre ellos lenguas electrónicas [52,53] debido a su simpleza, relativo bajo coste, estabilidad, robustez y nula toxicidad.

\section{Electrodos auxiliares.}

Se han utilizado distintos tipos de electrodos de Pt como contraelectrodos, en función del tipo de electrodo de trabajo a emplear. Se utiliza este material debido a su buena estabilidad e inercia química y electroquímica. Cuando se emplearon los sensores CPE, al igual que en el caso de las celdas, el contraelectrodo acompañante dependía del área del formato de CPE empleado, bien un hilo de $\mathrm{Pt}$ de $1 \mathrm{~mm}$ de diámetro y $6 \mathrm{~cm}$ de longitud, recubierto por una disolución $0.1 \mathrm{M}$ de $\mathrm{KCl}$ y separado de la muestra por una placa de vidrio poroso, o bien una placa de $\mathrm{Pt}$ de dimensiones relativamente elevadas (70x50 mm2).

La influencia ejercida por el electrodo de trabajo en la elección de la dimensión del electrodo auxiliar se debe a la naturaleza del experimento voltamétrico, en el cual se generan flujos de corriente que han de pasar por ambos electrodos, de manera que la densidad de dicha corriente ha de ser la misma en ambos casos, para así evitar problemas experimentales asociados a procesos electroquímicos no controlados (burbujeo de gases generados por electrólisis del disolvente, etc). Esto se consigue empleando un contraelectrodo de dimensiones comparables o 
superiores al de trabajo, descartando así contribuciones a la corriente única propia del circuito eléctrico en serie de la celda.

\section{Electrodos de trabajo}

Los electrodos de trabajo, son los responsables de evaluar las características electroquímicas del medio bajo estudio. Como se ha venido citando a lo largo de este apartado son sensores construidos utilizando como soporte una jeringuilla de PVC de $1 \mathrm{~mL}$ de capacidad donde se ha depositado la pasta de carbono, lo que le confiere unas buenas propiedades como electrodo de trabajo, ya que los procesos electroquímicos tienen lugar en la interfase creada entre superficie electródica y disolución. En este sentido, esta superficie favorece la acumulación de analitos para su detección, aunque consecuentemente se necesita una renovación de dicha superficie activa.

\section{Potenciostato/galvanostato.}

En un experimento voltamétrico, un potenciostato es un dispositivo electrónico que controla la diferencia de potencial existente entre el electrodo de trabajo y el electrodo de referencia, siendo la variable controlada el potencial de la celda y la variable medida, la corriente generada tras el paso de dicho potencial como consecuencia de los procesos redox que sean o no producidos. El sistema se encarga de efectuar este control por inyección de corriente eléctrica en la celda electroquímica a través del electrodo auxiliar.

Para mantener el electrodo de trabajo a un potencial constante, debe evitarse que circule corriente por el electrodo de referencia, ya que cambiaría su potencial, por lo que se incluye el electrodo auxiliar como tercera unidad, que hará las funciones de generador o sumidero de electrones en función del potencial oxidante o reductor que se esté aplicando al electrodo de trabajo, el cual es programado por el potenciostato en forma de barrido respecto del tiempo. Éste puede ser implementado de diversas formas (barrido lineal cíclico, pulsado, en onda cuadrada, etc.).

Potenciostato/galvanostato EG\&G PAR 263A. Se trata de uno de los equipos más empleados en los últimos años, tanto en electroanálisis como en otros muchos estudios afines (corrosión, etc.), permitiendo trabajar además en modos potenciostato o galvanostato. Entre sus especificaciones experimentales más importantes, podemos resaltar las siguientes:

- Potencial de salida: $20 \mathrm{~V}$.

- Corriente de salida: $20 \mathrm{~mA}$.

- Rango de barrido de potencial: $16 \mathrm{~V}$. 
- Rápida y notable capacidad de adquisición de datos: $30 \mu \mathrm{s}$.

Tales potenciales y corrientes de salida otorgan al EG\&G 263A gran capacidad de aplicación. Este equipo permite trabajar en el rango de $100 \mathrm{nA}$ (en escala total), lo que indica sensibilidad al nivel de nanoamperios. Además, bajo control vía software, es posible acceder a rangos de intensidad de 1 y $10 n \mathrm{~A}$, con resoluciones de hasta $2 p$ A.

\subsubsection{Ojo electrónico}

\section{Espectrometría UV-Visible. Condiciones experimentales}

En nuestra investigación, el color de un vinos se midió mediante técnicas de espectroscopia ultravioleta-visible (UV-VIS), a partir de los evaluaciones recogidas en la zona espectral de 380nm a $780 \mathrm{~nm}$ [54]. La información derivada de los espectros UV-Vis fue posteriormente procesada a través de las ecuaciones CIE hasta obtener las correspondientes coordenadas colorimétricas. Las medidas se realizaron en un equipo Shimadzu UV-Vis 2101. Para evitar problemas de saturación de la señal, se utilizaron cubetas de cuarzo de 1mm de espesor.

\section{Parámetros CIELab. Software de Análisis}

Una vez registrados los espectros, se aplicó un software de transformación de las señales espectrales en las coordenadas CIELab $\left(\mathrm{L}, \mathrm{a}^{*}, \mathrm{~b}^{*}, \mathrm{C}, \mathrm{H}, \mathrm{S}\right)$ utilizando un programa diseñado por nuestro grupo de investigación en un software Matlab versión 7.2.0.232 [14]. Todos estos parámetros fueron importantes para analizar cada etapa de los procesos de vinificación y dar una explicación a los diferentes fenómenos vinculados a las modificaciones colorimétricas que se presentan durante su elaboración. Además, han servido para obtener una consideración global de las muestras estudiadas usando correlaciones hechas con análisis mutivariantes.

\subsection{ANÁLISIS DE LOS DATOS}

Durante el desarrollo de este capítulo han sido explicadas las técnicas experimentales diseñadas por nuestro Grupo de investigación en Sensores y utilizadas para conseguir la caracterización organoléptica de los diferentes vinos. 
Llegados a este punto hemos de explicar las posibilidades y limitaciones de los métodos quimiométricos necesarios para aprovechar toda la información proveniente de cada uno de los sistemas. Además pretendemos contribuir a demostrar que estos métodos de trabajo podrían ser un soporte para el conocimiento y comprensión de los diferentes procesos en la elaboración de vinos.

Por lo tanto, comenzaremos por definir cada uno de los pasos del análisis exploratorio, para así tener la oportunidad de especificar como se realizó la selección de parámetros y el ajuste de los métodos para las aplicaciones particulares que son requeridas. El avance más importante que hemos propuesto en este punto de la investigación ha consistido en aplicación de métodos estadísticos que permitan retener mayor cantidad de información y conseguir que nuestros sistemas de caracterización sensorial electrónicos asocien información cualitativa y cuantitativa acerca del carácter gustativo de nuestras muestras.

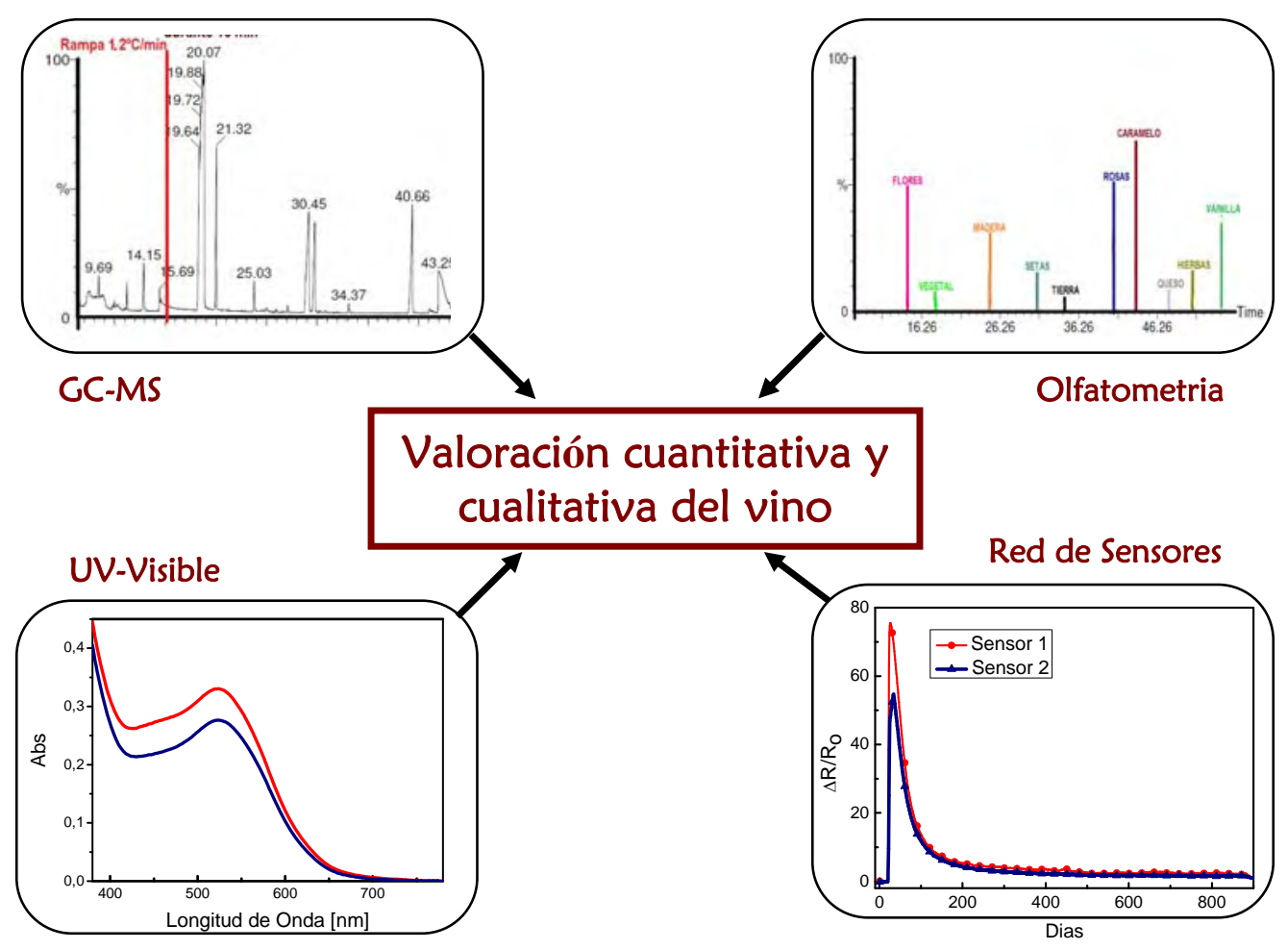

Figura 2.15. Esquema general de los resultados obtenidos para la caracterización de vinos

La Figura 2.15 ejemplariza el tipo de señales generales que hemos obtenido en nuestra investigación al realizar una valoración mediante espectroscopia, cromatografía, olfatometría o una red de sensores. Observando estos conjuntos de variables es posible hacernos a una idea de los retos marcados, desde los relacionados con el pretratamiento y selección de variables hasta la parte concerniente con el análisis clasificatorio, para el cual en primer lugar es 
necesario asegurar que no existe ninguna sobrevaloración de parámetros obtenidos en cada evaluación. Especialmente cuando intentamos realizar un análisis global de las muestras de vino, donde debemos precisar que cualquiera de las técnicas tenga el adecuado peso e influencia en la diferenciación de las muestras respecto a la problemática evaluada.

Es necesario resaltar una vez más la complejidad que tiene el tipo de muestra que se está evaluando en esta investigación, como es el vino. Una matriz de esta naturaleza, en la cual sólo alrededor del $2 \%$ de su composición es la encargada de marcar las diferencias entre variedades [35], necesita de métodos capaces de valorar la influencia que causa el cambio de un parámetro cualquiera durante su proceso de producción en las sensaciones perceptibles, en que consisten dichos cambios y el impacto que podrían tener en la percepción del consumidor sobre la calidad del vino.

\subsubsection{Pretratamiento de las señales de la nariz electrónica}

En nuestra investigación se ha seleccionado como algoritmo la variación relativa a la resistencia de cada sensor. Mediante esta medida relativa de la señal se consigue que todas las señales provenientes de los sensores presenten el mismo origen, y de esta manera se puede realizar fácilmente un estudio comparativo entre los diferentes sensores ante una muestra de aroma. Además, es un parámetro fácil de implementar y es adimensional. Una vez que se conoce la respuesta de los sensores se procede a extraer los parámetros más representativos de la misma. La elección de estos parámetros es clave, ya que si no son elegidos adecuadamente, el tratamiento de datos nos proporcionaría una información no apropiada, o lo que es lo mismo, tendríamos serios problemas en la etapa de clasificación de las muestras.

Como ya se sabe, la sensibilidad o resistencia relativa de un sensor está íntimamente relacionada con los procesos cinéticos que tienen lugar en su superficie, es decir, con la velocidad de adsorción y desorción y de las consecuentes reacciones entre las especies activas de la superficie del sensor y un determinado aroma (propiedad específica de los sensores). En esta investigación se han seleccionado como variables más representativas de la respuesta de la red de sensores la altura de pico y los parámetros dinámicos: tiempo de adsorción $\left(\tau_{0.9}\right)$ y de desorción $\left(\tau_{0.1}\right)[44]$.

Existen otros parámetros que se pueden extraer de la respuesta del sensor; sin embargo, la práctica común en nuestro grupo es la utilización de un solo parámetro de esta curva para dar una información suficiente sobre el comportamiento de los sensores frente a un determinado aroma [55]. 
A partir de los datos de resistencia relativa, mediante una hoja de EXCEL, se calcula el valor del máximo del pico y los parámetros dinámicos $\left(\tau_{0.9}\right.$ y $\left.\tau_{0.1}\right)$. De todos estos parámetros, únicamente el valor máximo de la altura de pico (por sencillez) es la que se ha utilizado en el tratamiento multivariante.

Casi todos los sensores de aromas requieren un periodo de estabilización inicial antes de ser utilizados para realizar medidas. En este periodo, el sensor equilibra sus condiciones de funcionamiento (p.e. temperatura, humedad y gas portador). Pero a pesar de esta etapa de acondicionamiento, los sensores de gases presentan variación de la línea base en el tiempo (drifts) [56, 57]. Estos cambios en el valor de la resistencia de la línea base a lo largo del tiempo están frecuentemente asociados con la contaminación o envejecimiento de los sensores.

La variación de la línea base en el tiempo debida a la contaminación, puede ser evitada en gran parte mediante una selección cuidadosa de los materiales sensores, controlando la temperatura [58], recurriendo a métodos multivariantes [59-61] o mediante la no utilización en la línea de arrastre de los gases de contaminantes conocidos para los óxidos semiconductores (siliconas y compuestos sulfurosos)[44].

Previamente al tratamiento de datos, y con el objetivo de tener estudios comparativos en el tiempo, se ha considerado importante calibrar el valor máximo del cambio de resistencia de los sensores cuando se inyecta un vino sintético, el cual puede ser preparado y valorado todos los días durante el trascurso de una serie de experimentos. Se emplean dos métodos; el primero de ellos se denomina método multiplicativo [62]. Este método está basado en el empleo de una muestra de referencia (en nuestro caso el vino artificial) que se utiliza para recalibrar la respuesta de los sensores MOS frente a muestras desconocidas utilizando la ecuación:

$$
\text { altura }=\frac{\Delta R \max _{21}}{\Delta R \max _{11}} \times \Delta R m_{21}(4.4)
$$

siendo, $\Delta R \max _{21}$, el valor de la resistencia relativa del sensor 1 al patrón el día 2; $\Delta R \max _{11}$, el valor de la resistencia relativa del sensor 1 al patrón el día $1 ; \Delta R m_{21}$, el valor de la resistencia relativa del sensor 1 a una muestra problema medida el día 2. Cuando el cambio en resistencia relativa de los sensores superaba el 10\%, el sensor era reemplazado por uno nuevo.

En segundo lugar, se utiliza un método multivariante [63], que asume que la deriva es la misma para todas las medidas. Luego, conociendo la dirección de la deriva en unas muestras de calibración (vino artificial) es posible minimizar la deriva de las muestras problema aplicando la ecuación: 


$$
X_{\text {compensación.deriva }}=x-\left(x \bullet v_{\text {cal }}\right) v_{\text {cal }}^{T}
$$

Aún existe otro factor que modifica la respuesta de los sensores a un mismo aroma en un periodo corto de tiempo: el agotamiento del material adsorbente empleado en el sistema de preconcentración (ya sea la fibra en SPME o la trampa en el sistema PT). Ello obliga a calibrar el sistema siempre que se quieran efectuar estudios comparativos en tiempos largos (como es el caso que nos ocupa). En nuestro caso, existe un protocolo de limpieza de la fibra, anteriormente mencionado, el que también especifica que la fibra no puede ser utilizada para un número mayor a 100 extracciones. Por otra parte cuando todos los sensores presentan un cambio significativo a la inyección del vino artificial, la fibra es reemplazada por una nueva.

Una vez seleccionadas las variables, minimizado la deriva de la línea base y calibrada la señal de los sensores se construye la matriz de datos correspondiente. Cada columna de la matriz de datos está constituida por las variables (V) seleccionadas. Y, en las filas se representa los objetos o muestras utilizadas (M). Como consecuencia, cada fila de la matriz de datos está constituida por las respuestas de las distintas variables a las diferentes muestras. En nuestro caso, el número de variables y objetos empleados en el tratamiento estadístico es elevado, lo que implica un tratamiento estadístico con matrices $n$-dimensionales $\left(V_{\text {variables }} \mathrm{x}\right.$ $\mathrm{M}_{\text {muestras})}$.

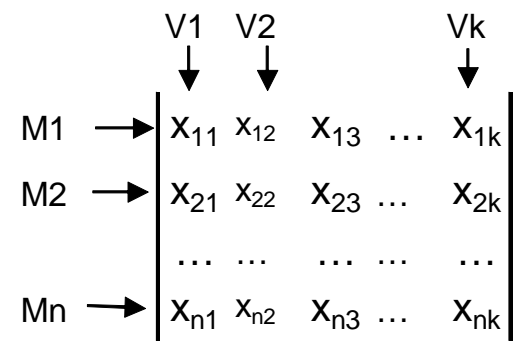

Cada $x_{n k}$ representa el valor que toma la variable k-ésima para la muestra nésima.

Previamente a la aplicación de técnicas estadísticas multivariantes se recomienda la normalización de los datos por diferentes razones, tales como la simplificación de los resultados y la corrección matemática de los problemas inherentes a las señales. El pretratamiento dirigido hacia la última de estas ideas se puede dividir en preprocesamiento de fila y de columna.

El pretratamiento de fila opera exclusivamente en cada línea de señales (las filas de la matriz de datos), independientemente de las demás. El preprocesamiento de columnas, en cambio, actúa individualmente en cada una de las variables de la medida (columnas de la matriz de datos). Dos procedimientos globales han sido aplicados a los datos de la nariz electrónica entre los distintos métodos de normalización existentes (sustracción de señal de fondo, centrado de datos, autoescalado, promediado de la señal, etc.). En esta investigación se ha 
seleccionado como pretratamiento de fila la transformación por medio de una variable aleatoria normal estándar (SNV) y como pretatamiento de columna el método de autoescalado.

\section{Pretratamiento de fila: Variable aleatoria normal estándar (SNV)}

Una discusión más profunda de cada tipo de pretratamiento tendrá lugar cuando comencemos a estudiar las señales resultantes de la lengua electrónica (apartado 2.3.2), para las cuales el efecto de las diferentes combinaciones entre pretratamiento de fila y columna es más significativo [64]. Sin embargo, entrando en consideraciones generales este preprocesamiento corrige las variaciones sistemáticas y efectos globales en la intensidad de una señal.

La transformación por medio del método de la variable aleatoria normal estándar también es conocida como autoscaling de filas, la aplicación de este método es bastante común en espectroscopía [65]. Con esta técnica cada señal $y_{i}$ es centrada dividiendo sus valores por la desviación estándar $\left(s_{i}\right)$. Los valores de la transformación SNV son obtenidos por medio de la siguiente ecuación:

$$
z_{i j}=\frac{y_{i j}-\overline{y_{i}}}{s_{i}}
$$

Donde $z_{i j}$, es la variable normalizada; $\overline{y_{i}}$, es la medida de la variable $y$.

\section{Pretratamiento de columna: Autoscaling}

En este caso el preprocesamiento de los datos de una variable se realiza restando la media de la variable y dividiendo por su desviación típica, de acuerdo a la expresión [66]:

$$
z_{i j}=\frac{X_{i j}-\overline{x_{i}}}{s_{i}}
$$

donde $z_{i j}$, es la variable normalizada; $x_{i j}$, es la variable sin normalizar; $\overline{x_{i}}$, es la media de la variable $x, s_{i}$ es la desviación típica de la variable.

\subsubsection{Pretratamiento de las señales de la lengua electrónica}

En este punto pretendemos explicar métodos quimiométricos que permiten mejorar las aplicaciones de la lengua electrónica en la industria enológica, esto 
nos obliga un poco a profundizar en las descripciones tanto a nivel electroquímico como desde el punto de vista quimiométricos.

A diferencia de lo que ha ocurrido con la nariz electrónica, las señales electroquímicas son más complejas desde el punto de vista de tratamiento de datos, es por ello que nos extenderemos un poco más en la discusión relacionada con el preprocesamiento de la curva, y una posterior selección de variables capaces de describir las características generales del voltamograma [67].

\section{Pretratamiento de filas.}

Nos volvemos a referir en primer término al tratamiento de fila, que para este caso además del método SNV, se han estudiado otras posibilidades como los son el centrado de datos, suavizado de datos usando la primera y segunda derivada y diferenciación entre el barrido anódico y catódico.

\section{Pretratamiento de columna}

En lo que respecta al pretratamiento de columnas, en el caso de la lengua electrónica estos métodos son principalmente utilizados para corregir las diferencias entre las intensidades registradas por cada sensor. Se realizado un estudio para conocer cual es combinación optima entre pretratamiento de fila y columna, ya que desafortunadamente no se existen reglas que determinen qué operación de transformación deber emplearse según el tipo de problema a resolver. En este acaso además de la técnica de autoscaling aplicada para los sensores de la nariz electrónica se ha utilizado la técnica del centrado de datos.

En el método de centrado de datos a cada variable se le sustrae el promedio de cada columna, los valores del centrado de columnas se obtienen mediante la siguiente ecuación:

$$
Z_{i j}=x_{i j}-\overline{x_{j}}
$$

donde $z_{i j}$, es la variable normalizada; $x_{i j}$, es la variable sin normalizar; $\overline{x_{i}}$, es la media de la variable $x$.

El paso previo al ejercicio de las técnicas de reconocimiento de patrones, tras la aplicación del escalado es la selección de variables. Los voltamogramas seleccionados están constituidos por un total de 920 puntos cada uno, correspondientes a pares de valores intensidad-potencial. Como es de suponer, este elevado número de variables registrado, incluido en una única medida, dará lugar a un inconmensurable conjunto de variables, más aún si se colectan señales de $n$ muestras diferentes con una red de $p$ sensores. Este hecho implica necesariamente la reducción de la información contenida en las señales para facilitar y/o incluso posibilitar la ejecución del tratamiento de datos. 


\subsubsection{Selección de variables para la lengua electrónica}

En esta investigación se ha escogido como método de reducción de variables los algoritmos genéticos, herramienta que permite hacer una revisión de la señal de manera global por medio de una evaluación que combina algunas de las variables al mismo tiempo y no por pasos separados, como sucede en algunos de los métodos clásicos anteriormente mencionados [68, 69].

Cualquier procedimiento para la selección de las variables basa su funcionamiento en dos aspectos fundamentales: un criterio de selección y un procedimiento de búsqueda. Para los algoritmos genéticos, ambos criterios están basados en los principios de la selección y la evolución natural, razón por la cual las posibles soluciones a un problema son codificadas en forma de cadenas binarias y la búsqueda se inicia con una población de posibles soluciones generada aleatoriamente; dentro de estas posibles soluciones se toman aquellas que son calificadas como las mejores de acuerdo con un criterio previo establecido [70-73].

En vista de que los GA-PLS son un método supervisado, también podemos decir que abren una posibilidad interesante en la búsqueda de correlaciones entre las señales electroquímicas con otras medidas analíticas, porque de cierta manera las variables seleccionadas pueden ser utilizadas en análisis posteriores y cumplir con tendencias paramétricas. Sin embargo el que sea un método supervisado también podría suponer una sobrestimación de la habilidad de predicción del sistema, por lo cual es necesario aplicar un cuidadoso proceso de validación, para estimar correctamente su capacidad de clasificación.

En esta investigación se han tenido en cuenta dos puntos importantes dentro de la aplicación de los algoritmos genéticos para encontrar un nuevo subconjunto de datos: no sobreestimar la capacidad de clasificación de la lengua electrónica, y que además el subconjunto encontrado sea interpretable desde el punto de vista electroquímico.

\section{Algoritmos genéticos}

En el problema de la reducción de variables utilizando algoritmos genéticos, cada posible combinación puede ser codificada con una cadena binaria tan larga como parámetros se consideraron para encontrar la combinación óptima de variables. En dicha cadena, cada variable tiene asignada una posición o bit, de manera que una posible solución venía descrita por una sucesión de unos y ceros indicando 
la presencia (con un 1) o la ausencia (con un cero) de cada una de las variables en esa combinación particular.

En este tipo de algoritmos, cada miembro de la población, que representa una posible solución, es evaluado con algún criterio objetivo, de manera que cada uno de los miembros de la población se valora en función de su "fitness" (valor del criterio). Este valor puede ser, como en nuestro caso, el error de predicción en cross-validation [74].

A las soluciones mejor valoradas se les permite permanecer y pasar a la siguiente iteración ("generación"), mientras que las soluciones con el peor fitness desaparecen para las sucesivas generaciones. El algoritmo genético continúa hasta que se cumpla un número predefinido de generaciones (Figura 2.16).

Para llegar a la solución óptima es necesario diseñar una estrategia de búsqueda. Las técnicas clásicas se caracterizan por tener estrategias con alta explotación y baja exploración; esto significa que desde un punto de partida, son capaces de encontrar el máximo local más cercano, pero una vez que lo han encontrado es probable que se queden anclados en ese punto. Por el contrario, técnicas como los algoritmos genéticos se basan sobre un compromiso entre exploración y explotación: esto significa que son capaces de detectar varios "máximos" locales, pero no es muy fácil que alcancen la cumbre de cada "máximo". Por tanto, es más bien intuitivo admitir que el acoplamiento con una técnica clásica puede producir una nueva estrategia que mejore tanto la exploración como la explotación [75].

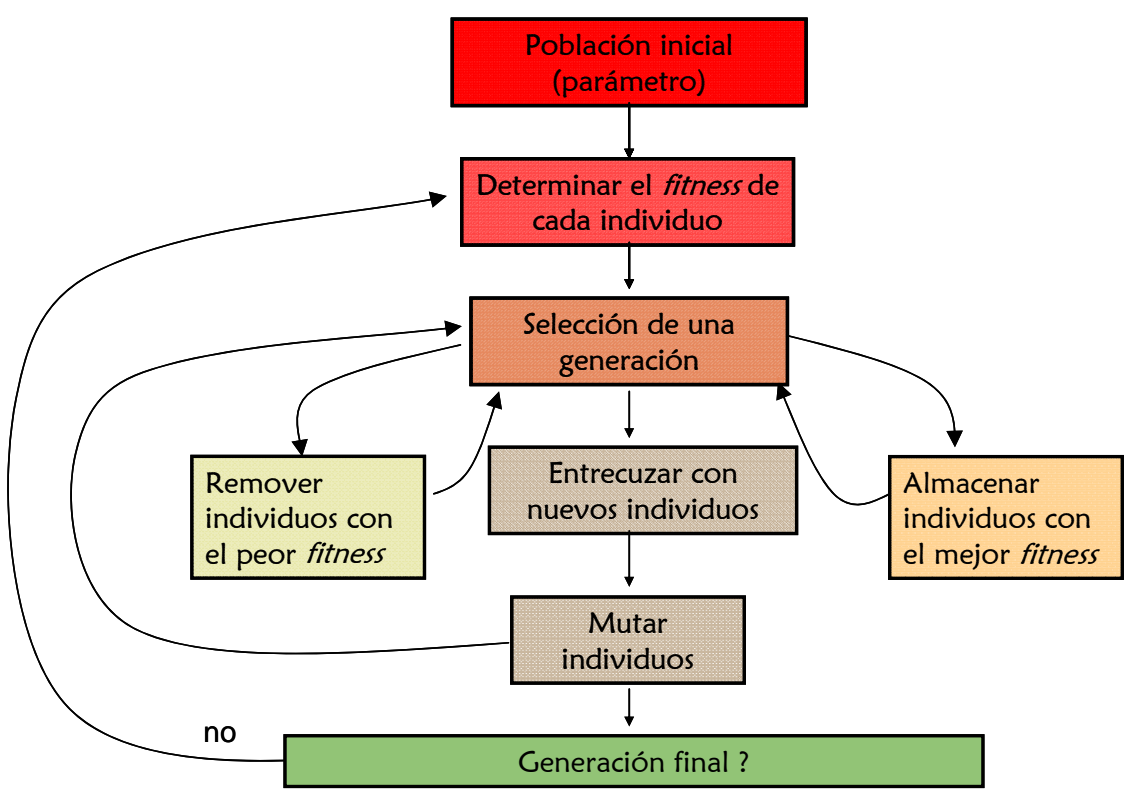

Figura 2.16. Esquema de la iteración de un algoritmo genético. 
La mayoría de las técnicas clásicas usan la selección progresiva, bien sea adicionando o eliminando variables, una a la vez. Este procedimiento continua iterando hasta encontrar que un paso donde los resultados de $n+1$ no sean mejores que los resultados del paso $n$ Ambas formas de estos métodos de búsqueda pueden ser muy útiles cuando se combinan con un GA.

Comenzando con algunos de los cromosomas de la población, el procedimiento de reducción permite limpiar el modelo eliminando aquellas variables que no dan información relevante pero que habían sido seleccionadas por azar. Por otro lado, el procedimiento de adición permite agregar aquellas variables que entregan información relevante pero que no habían sido seleccionadas. Un GA híbrido alterna generaciones de GA con ciclos de selección paso a paso, bien sea en su forma de reducción o adición de variables.

Una vez más debemos recordar que el principal riesgo respecto a la aplicación de este método es la sobreestimación de parámetros; la selección paso a paso contribuye demasiado al mejor ajuste del modelo, pero también puede permitir perdida de certeza al realizar una predicción. Para limitar este riesgo, sólo se aceptan los modelos para los cuales se produce una reducción importante del error de predicción (con respecto al error que genera el modelo parental). Otra manera de prevenir la sobreestimación del modelo es usar un conjunto de datos para el cual el número de muestras sea significativamente grande en comparación con el número de variables. Además es importante asegurar un bajo nivel de ruido en cada una de las medidas.

Puesto que las variables presentes en un cromosoma son generalmente un pequeño conjunto del total de variables, es evidente que el procedimiento de reducción se ejecuta con mayor frecuencia que el proceso de adición. Para describir el algoritmo definimos b como la frecuencia de reducción de variables paso a paso y f como la frecuencia de adición de variables [76].

La estructura general del algoritmo es la siguiente:

\section{Comenzar con el GA}

2. Después de la evaluación de $b$ cromosomas se realiza la eliminación progresiva de variables.

3. Si $b=f$ se realiza una selección de variables adicionando una variable por paso.

4. Se ejecuta el paso 1. (procedimiento del GA). 
Otra parte importante en el desarrollo de este GA híbrido es decidir cuando debe parar la adición de variables. El algoritmo general que se ha utilizado para un subconjunto de $s$ variables, es el siguiente:

1. Evaluar el modelo con $s$ variables

2. Repetir $t$ veces ( donde $t=s$ cuando se realiza el proceso de eliminación, $t=v$-s cuando se realiza la adición). Evaluar los modelos con $p$ variables $(p=s-1$ cuando se eliminan variables, $p=s+1$ cuando se adicionan variables).

3. Si el modelo evaluado en el paso 2 es mejor que el evaluado en el paso 1 (produciendo una reducción en el error de predicción más grande que el valor predefinido), volvemos al paso 1, con el correspondiente subconjunto de variables para recomenzar con el modelo.

\subsubsection{Pretratamiento de datos para la espectroscopía UV- Visible, cromatografía, y olfatometría.}

En el caso de las señales resultantes de estas tres técnicas se escogieron como variables las coordenadas CIELab (UV-Vis), los valores de los compuestos cuantificados (GC-MS), y los valores de intensidad de aroma (GC-O). Al igual que el preprocesamiento escogido para la nariz electrónica, con estas matrices de datos se ha realizado un pretratamiento de autoscaling para las filas, y el método SNV para las variables de cada columna perteneciente a las diferentes matrices que serían objeto del análisis multivariante.

\subsubsection{Análisis Exploratorio}

Una vez que los vectores respuesta de todas las variables están normalizados, dada la cantidad y complejidad de los datos obtenidos mediante redes de sensores, se requieren comúnmente métodos de análisis multivariantes para extraer con éxito la máxima información contenida en las señales resultantes de los diferentes análisis [77].

\section{Análisis de componente principales PCA. Algoritmo}

Para aplicar el PCA, es necesario una matriz de datos donde cada fila corresponde a la descripción completa de una medida, y cada columna a una 
variable indicadora concreta de cada experimento. En términos algebraicos, las filas hacen referencia a vectores pertenecientes a un espacio vectorial de dimensión p, en base canónica, en la que cada una de sus coordenadas se asocia a la respectiva variable descriptiva. El algoritmo PCA supone por lo tanto realizar un endomorfismo o cambio de base, desde la original canónica a otra generada por los vectores propios resultantes de la matriz de covarianzas de los datos. La expresión matemática se puede definir de manera esquemática como:

$E_{p}(X) \rightarrow E_{p}(Y)$

$X=t_{1} p_{1}+t_{2} p_{2}+\ldots+t_{k} p_{k}$

$Y=\left[t_{1} t_{2} \ldots t_{k}\right]$

Donde $\mathrm{X}$ es la matriz experimental (dimensión $n x p$ ).

- $t_{k}$ es el vector de coordenadas de las medidas sobre la componente principal késima (denominado "score" o puntuación).

- $p_{\mathrm{k}}$ es el vector de la componente principal k (denominado "loading" o peso), que es el vector propio de la matriz de covarianzas de $X$ y ortogonal al resto de vectores.

- Y es la matriz de coordenadas de las p medidas en función de la nueva base de variables, constituida por las componentes principales [78].

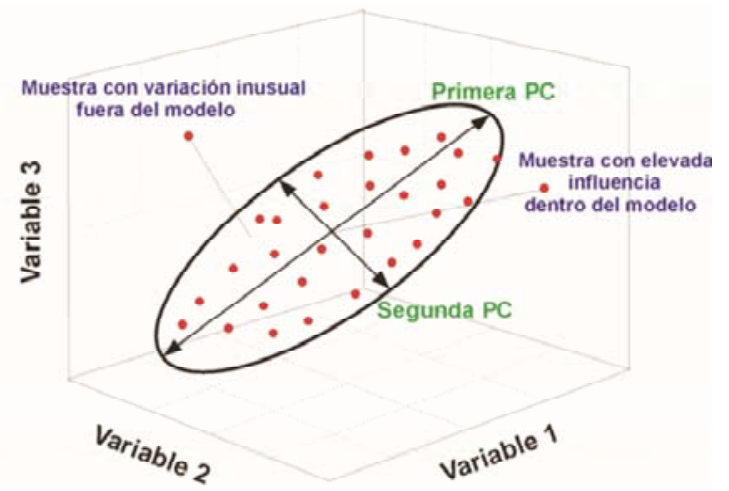

Figura 2.17. Ejemplo grafico de un PCA. Se observa cómo se proyectan las medidas sobre un eje.

De forma resumida, el PCA realiza proyecciones de los vastos datos originales sobre un nuevo espacio de reducidas dimensiones que recoge la máxima varianza entre los objetos (Figura 2.17). Los diferentes porcentajes de varianza descritos dependen de los valores propios que acompañen a sus respectivos vectores, estando definidos en orden descendente de peso, por lo que la mayor cantidad de varianza explicada es ofrecida por el primer vector o eje, que se denomina primera componente principal (PC); la segunda PC describe la mayor cantidad de varianza en una dirección ortogonal a la primera, y así 
sucesivamente hasta que el algoritmo encuentre el número necesario de PCs para explicar la matriz de datos [79].

\section{Regresión por mínimos cuadrados (PLS). Algoritmo}

Este es un método que intenta maximizar la covarianza. Para alcanzar este objetivo, se vale de las VLs que extraen la máxima varianza, escogiendo aquéllas cuya covarianza con las variables a estimar o predecir sea máxima. La Figura 34 representa gráficamente el procedimiento seguido por PLS.

PLS es un método bi-lineal, al igual que PCA, en el cual la información de los datos originales $\mathrm{X}$ se proyecta sobre un pequeño y manejable número de VLs, en ocasiones denominadas componentes PLS. La información de las variables dependientes $\mathrm{Y}$ se tiene constantemente en cuenta para estimar el número y la calidad de dichas VLs, y así asegurar que las primeras componentes sean las más relevantes para la predicción de las variables $\mathrm{Y}$ (basándose en la máxima covarianza).

En la Figura 2.18., la interrelación de las puntuaciones o "scores" de las VLs t y u resumen la relación existente entre $\mathrm{X}$ e $\mathrm{Y}$ a lo largo de una componente específica del modelo, dando lugar al conocido gráfico de puntuaciones, equivalente al de PCA. Esta representación permite establecer asociaciones o agrupamientos entre muestras, en este caso, en función de los datos originales y los conocidos a estimar. Además, como en PCA, se pueden obtener representaciones respecto de las primeras VLs (o PCs) de las variables predictivas ("loadings") y su relación con las variables a predecir, estableciéndose posibles correlaciones visuales entre ellas [80].

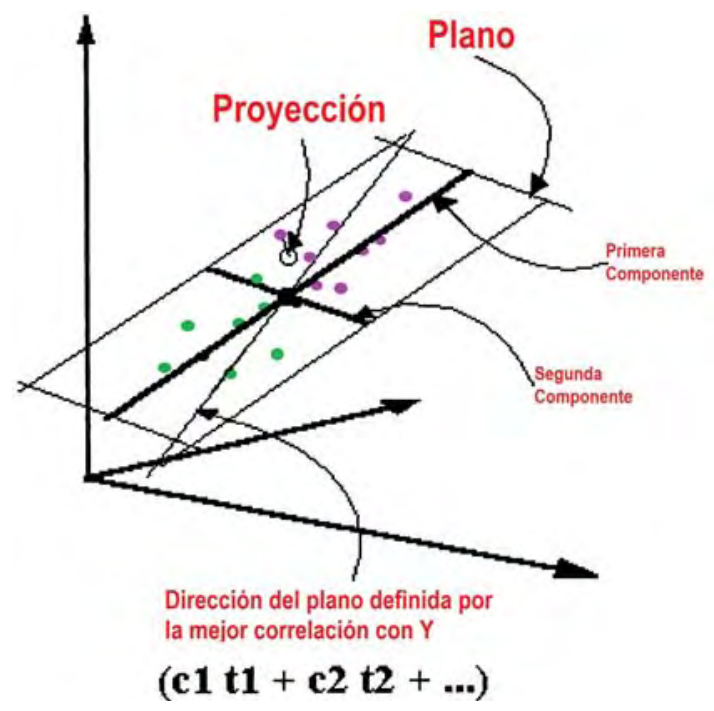

Figura 2.18. Ejemplo genérico de visualización de la regresión por MLR, PCR o PLS. 
Otra herramienta visual de gran utilidad para evaluar los resultados, empleada por los métodos de regresión comentados es el gráfico valor real vs. valor predicho, representación que permite esclarecer y comparar con qué variables y métodos se obtienen los mejores ajustes o estimaciones.

El eje horizontal de un tipo de análisis PLS representa el valor real (en este caso, en unidades arbitrarias) de la variable a estimar, mientras que el eje vertical, el valor predicho. La diagonal representa la línea de correlación lineal perfecta (pendiente 1 , ordenada en el origen 0 y coeficiente de correlación 1).

La determinación de la validez del proceso de modelización (o calibración) y de predicción, se realiza mediante el análisis de los errores en la regresión, como será expuesto posteriormente

Dada la complejidad esencial de los problemas llevados a cabo en esta investigación, se recurrirán a unas u otras herramientas entre las citadas con el fin de evaluar de la forma más ajustada las características y la capacidad de resolución de nuestros sensores en la valoración de las muestras seleccionadas.

\subsubsection{Validación cruzada}

Un último paso en el tratamiento de las señales es la validación de los métodos quimiométricos aplicados, etapa esencial cuando se pretende establecer modelos de clasificación o de predicción con los datos analizados. La validación es la vía de demostración de la capacidad de un modelo para ser generalizado en futuras aplicaciones con nuevas muestras "desconocidas". Para validar un método de reconocimiento de patrones se precisan normalmente dos tipos de información, unos datos de calibración ( $X$; entrenamiento o modelización) y otros de predicción o validación (Y; "test data") del modelo. Con el fin de obtener modelos fiables y útiles, es necesario que ambos conjuntos sean representativos del problema a analizar [81].

Entre los principales métodos de validación, mencionar especialmente dos tipos:

i) Validación por un conjunto de prueba separado: es el mejor procedimiento de validación de un modelo. Se emplean medidas separadas que no han sido utilizadas para construir el modelo y que comparten características muy similares al conjunto de datos de entrenamiento. Posee un inconveniente, en ocasiones insalvable: precisa un elevado número de datos de validación, por lo que puede ser inviable si se está tratando con muestras de elevado coste o de difícil adquisición. 
ii) Validación cruzada: para solventar el problema de las muestras, pueden utilizarse todas las medidas empleadas en la calibración. El fundamento de este proceso de validación es ir construyendo sub-modelos con diferentes secciones de los datos no utilizadas en cada uno de ellos. En la validación cruzada total, o validación "dejando uno fuera", frecuentemente aplicada, cada sub-modelo está definido por la totalidad de las medidas excepto una, hasta que todas queden en algún momento fuera. Así pues, se construyen tantos sub-modelos como medidas existen. Este procedimiento aproximado es extensamente aceptado y efectivo principalmente en aplicaciones en las que el número de medidas no es excesivamente elevado (Figura 2.19.).

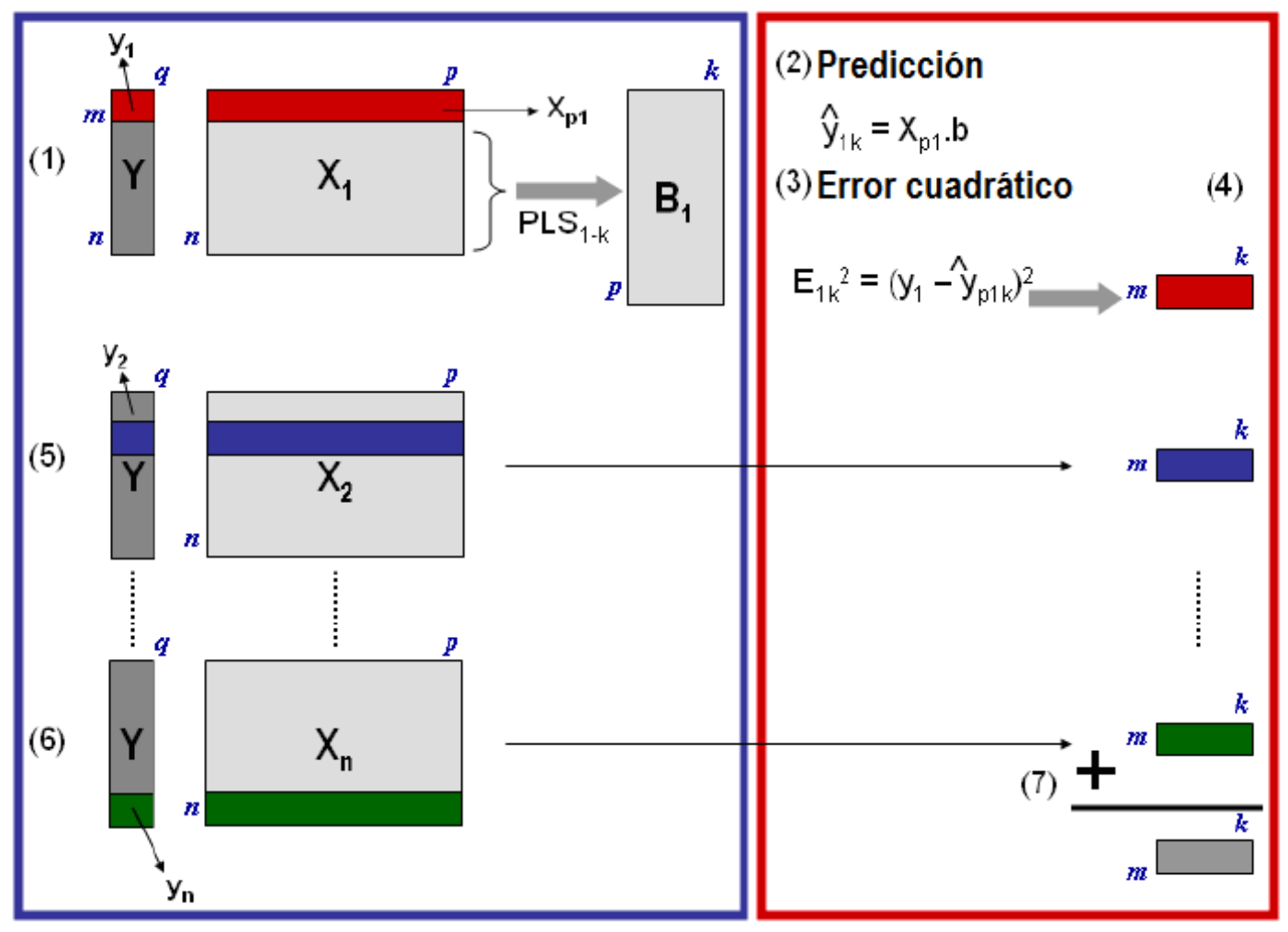

Figura 2.19. Esquema general de la validación cruzada. A la izquierda, modelos PLS con entre 1 y $k$ variables latentes y sus correspondientes coeficientes. A la derecha, la predicción de la muestra que no hacia parte de la calibración, el cálculo de los residuos cuadrados.

\section{Errores de validación y/o predicción.}

La medida de la capacidad de predicción de un modelo suele venir dada por el denominado RMSEP [81], cuyas siglas traducidas son la raíz cuadrada de la media de los cuadrados del error de predicción, estando éste definido por la diferencia en valor absoluto del valor predicho para la variable $Y$, respecto del conocido donde $\hat{y}_{i}$ es el valor predicho para la medida $i$ del conjunto de datos de prueba del modelo, es decir, la pésima fila de la matriz $X$ correspondiente a los datos de 
validación. $y_{i}$ es el respectivo valor verdadero, y $n$ es el número de medidas. El RMSEP es un valor medio, por lo que si el número de medidas es pequeño, puede obtenerse un resultado algo engañoso, con una varianza sensible. En estos casos, es de utilidad revisar el valor absoluto del error de predicción (PE), que es calculado para el conjunto de datos de prueba, y proporciona un valor individual para cada medida.

Previa estimación del error de predicción, debe tenerse en cuenta el error originado durante el proceso de modelización o calibración. Así, de manera similar, se define el RMSEC, como la raíz cuadrada de la media de los cuadrados del error de calibración [81]. Esta medida informa sobre cómo de bien modelada es la variable a estimar por el conjunto de datos de calibración. El RMSEC, sin embargo, no aporta información acerca del funcionamiento del modelo para datos futuros desconocidos. Esta característica es determinada por el RMSEP. El cálculo del RMSEC es análogo al correspondiente a la ecuación anterior con la diferencia de que las variables y pertenecen a los datos de calibración en lugar del conjunto de prueba. Al igual que para la predicción, es posible calcular el error absoluto de calibración $(C E)$, utilizando la misma expresión para $P E$, pero en esta caso con los datos de modelización.

\section{Número de variables latentes óptimas}

La calibración multivariante es una herramienta matemática para determinar las muestras que generan problemática cuando se pretende entrenar un sistema de medida para predecir alguna de las características evaluadas. En este caso cuando hablamos de problemática, nos referimos aquellos casos donde los valores de selección están por debajo de los calculados con un modelo de predicción ideal. Ahora bien, la cuestión es como desarrollar un criterio que permita evaluar el grado de selectividad de un modelo. La práctica más común dentro del análisis multivariante es utilizar la información derivada de una gráfica que relaciona RMSEC en función del número del variables latentes VLs. El RMSEC y el CE siempre decrecen conforme lo hace la complejidad del modelo.

Cuando el número de VLs es excesivamente elevado, el modelo se sobreajusta a los datos de calibración, lo que significa que el modelo funciona adecuadamente para el conjunto de datos sobre el cual ha sido construido, pero carecerá de validez y aplicación cuando se introduzcan nuevos datos. El sobreajuste del modelo se debe a que se incorporan incorrectamente los residuales existentes en el conjunto de datos de modelización, ocasionando una pérdida en la capacidad futura de predicción de dicho modelo, el cual se basa en información no correspondiente a la estructura real de los objetos bajo estudio. Por otro lado, si la complejidad del modelo no es lo suficientemente alta, el modelo se infraajusta a 
los datos de modelización, con la consecuente pérdida de capacidad de predicción [78].

Por ello, para decidir el número óptimo (y correcto) de VLs o PCs que se ajusten al modelo en cuestión, suelen emplearse los valores de RMSEP, aunque el uso de los PE es equivalente. El mínimo de la curva RMSEP vs. complejidad del modelo, brinda su número de componentes correctos. Es decir, si se pretende construir un modelo PLS para predecir el valor de una cierta variable y, deben emplearse el número de VLs que correspondan al menor RMSEP (al primer mínimo local de la curva, en el supuesto que existan más posteriormente).

\subsubsection{Análisis de datos a través del modelo tucker3}

\section{Estructura del modelo}

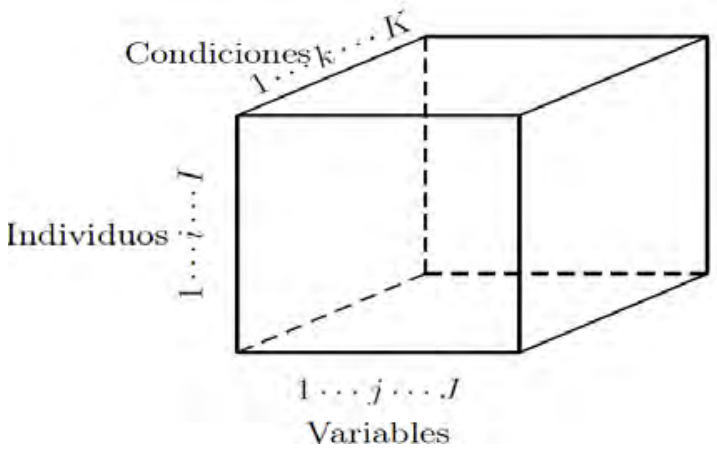

Figura 2.20. Representación de una matriz de datos en tres vías

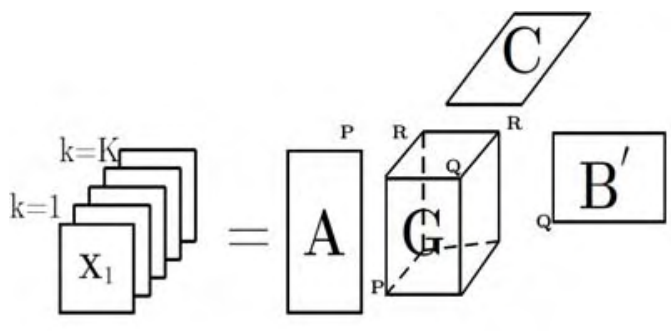

Figura 2.21. Representación del modelo Tucker 3.

Con el fin de realizar un estudio exploratorio, se efectúo un análisis de datos tipo multi-way que indicaron los patrones interpretables fundamentales en el fenómeno de la maduración del vino, en función del tiempo y del tipo de envejecimiento, que además especificó que variables son significativas y que impacto tienen a lo largo del proceso. En términos generales, el objetivo de aplicar este tipo de herramientas desarrolladas recientemente, era conseguir el modelamiento de la información proveniente de tres técnicas analíticas complementarias para encontrar las diferencias de matices existentes entre los vinos.

Tucker3 es uno de los métodos mas conocidos del análisis multi-way, y puede ser considerado una extensión del análisis bilineal de componentes principales PCA. Hasta el momento, el análisis multi-way ha sido exitosamente aplicado en diferentes técnicas instrumentales, principalmente las espectroscópicas [82, 83], pero son escasas las aplicaciones que han sido reportadas en cromatografía y aún 
menos los trabajos que se pueden referenciar en la línea de las narices electrónicas [84]. Estos trabajos han implementado correlaciones entre la composición química y las propiedades sensoriales de productos alimenticios [85], la influencia y evolución de compuestos volátiles para la discriminación entre distintos tipos de vinagre [86], la correspondencia entre atributos y jueces para la valoración de diferentes muestras de pan blanco [87] y la clasificación de aceites de oliva utilizando una nariz electrónica basada en espectroscopía de masas [88].

En nuestro caso el modelo Tucker3 permitió el estudio de información expresada con tres índices diferentes: uno que identificó las muestras i $(i=1, \ldots, I)$, otro las variables $\mathrm{j}(\mathrm{j}=1, \ldots, \mathrm{J})$ y un tercero que correspondía a la condiciones $\mathrm{k}(\mathrm{k}=1, \ldots, \mathrm{K})$; de esta manera se consiguió un arreglo tridimensional: individuos, variables y condiciones (Figura 2.20). Para la solución específica de nuestro problema la matriz está arreglada en términos de las muestras de vinos tratadas con diferentes métodos, $v$ variables (nariz electrónica, cromatografía y coordenadas CIELab) y los periodos de muestreo.

El método Tucker3 es una generalización del modelo de componentes dos vías al modelo de componentes tres vías. La generalización se puede representar con el siguiente modelo:

$$
x_{i j k}=\widehat{x}_{i j k}+e_{i j k} ; \quad i=1, \cdots, I ; \quad j=1, \cdots, J ; \quad k=1, \cdots, K
$$

En este modelo, se realiza una descomposición completa del conjunto tridimensional $\mathrm{X}$ en tres matrices ortonormales: una matriz $\mathrm{A}$ de dimensión $\mathrm{I} \times \mathrm{P}$ que implica a los coeficientes para los componentes del modo individuos, una matriz B de tamaño $\mathrm{J} \times \mathrm{Q}$ que contiene los coeficientes para los componentes del modo variables, una matriz $C$ de tamaño $K \times R$ que agrupa los coeficientes para los componentes del modo condiciones, y un arreglo central $\mathrm{G}$ de dimensión $\mathrm{P} \times$ $\mathrm{Q} \times \mathrm{R}$ (Figura 2.21).

$$
x_{i j k}=\sum_{p=1}^{P} \sum_{a=1}^{Q} \sum_{r=1}^{R} g_{p q r}\left(a_{i p} b_{j q} c_{k r}\right)+e_{i j k} ; \quad i=1, \cdots, I ; \quad j=1, \cdots, J ; \quad k=1, \cdots, K
$$

[Ecuación 1]

Donde $a_{i p}, b_{j q}, c_{k r}$ denotan los elementos de A, B y $\boldsymbol{C}$ respectivamente y $g_{p q r}$ corresponde a los elementos de la matriz central G.

Analizando el modelo entorno a A la ecuación toma la forma:

$$
x_{i j k}=\sum_{p=1}^{P} a_{i p} \sum_{q=1}^{Q} \sum_{r=1}^{R} g_{q r}\left(b_{j q} c_{k r}\right)+e_{i j k}
$$

[Ecuación 2] 
Donde $\boldsymbol{X}_{a}$ es una matriz de dimensión $I \times J K$ obtenida por el modelo entorno a $\mathbf{A}$ del arreglo $\mathbf{X}, \mathbf{G}_{a}$ es una matriz de dimensión $P \times Q R$ obtenida por matriciación por el modo $A$ del arreglo central y $\boldsymbol{E}_{a}$ es la matriz de residuales de dimensión $I$ x JK (Tucker 1966)

Análogamente, utilizando la matriciación del modo $\boldsymbol{B}$, el modelo tiene la forma:

$$
x_{i j k}=\sum_{q=1}^{Q} b_{j q} \sum_{p=1}^{P} \sum_{r=1}^{R} g_{p r}\left(a_{i p} c_{k r}\right)+e_{i j k}
$$

[Ecuación 3]

Donde $\boldsymbol{X}_{b}$ es una matriz de dimensión $I \times J K$ obtenida por el modelo entorno a $\boldsymbol{B}$ del arreglo $X, \mathrm{G}_{\mathrm{b}}$ es una matriz de dimensión $Q \times P R$ obtenida por matriciación por el modo $\boldsymbol{B}$ del arreglo central y $\boldsymbol{E}_{b}$ es la matriz de residuales de dimensión $J \mathbf{x}$ $I K$

Utilizando la matriciación del modo C, el modelo es:

$$
x_{i j k}=\sum_{r=1}^{R} c_{k r} \sum_{p=1}^{P} \sum_{q=1}^{Q} g_{p q}\left(a_{i p} b_{j q}\right)+e_{i j k}
$$

[Ecuación 4]

Donde $\boldsymbol{X}_{c}$ es una matriz de dimensión $K \times I J$ obtenida por el modelo entorno a $C$ del arreglo $\boldsymbol{X}, \mathbf{G}_{\mathrm{c}}$ es una matriz de dimensión $R \times P Q$ obtenida por matriciación por el modo $C$ del arreglo central y $\boldsymbol{E}_{c}$ es la matriz de residuales de dimensión $K \mathrm{x}$ IJ.

Utilizando los cálculos anteriores se obtiene la ponderación para las combinaciones de las componentes.

$P, Q$, y $R$ son el número de factores que se extraen por cada modo; el arreglo central G está compuesto por valores singulares y representa los valores por el cual la componente individual es pesada. El cuadrado de los elementos de este arreglo central es proporcional a la varianza explicada por la combinación de los factores correspondientes a cada índice.

\section{Preprocesamiento de los datos para el Tucker3}

Para este procedimiento se consideraron los conceptos previamente definidos en el apartado 2.5.2., especialmente los relacionados con el centrado y escalado de datos. La principal diferencia que se presenta entre los métodos bidimensionales y lo métodos multi-way, es que estos últimos requieren una etapa previa de matriciación, que corresponda con el modo para el cual se desea realizar el centrado. En la mayoría de los casos corresponde al modo $A$, generando la matriz 
$X_{a}$ de dimensión $I \times J K$. El Tucker3 puede realizar centrado de cualquiera de las matrices $X_{a}, X_{b}$ o $X_{c}$. Sobre la matriz centrada el método realiza la matriciación por el modo variables (matriz de dimensión $J \times I K$ ) y escala dividiendo cada fila por la desviación estándar correspondiente. Si por ejemplo se comienza con la matriz $X_{a}$, el método de centrado se aplica a las muestras y el escalado para las variables.

\subsubsection{ANOVA-PLS}

El análisis de la varianza ANOVA permite evaluar la existencia de diferencias significativas entre grupos de muestras evaluados en función de los distintos niveles que se puedan presentar para uno o más factores. En el análisis univariado, lo que significa que se realiza el análisis de una variable a la vez, utilizando una hipótesis (hipótesis nula) para verificar que las diferencias significativas entre los diferentes grupos de muestras no se presentan únicamente por cuestión de azar. Para decidir sobre la aceptación de la hipótesis nula se realiza una prueba de Fisher. En el caso de que el valor de F calculado sea menor que el valor crítico, el resultado no es significativo, y por lo tanto se concluye que los grupos no son diferentes.

En el caso multivariado, otros métodos como el ANOVA-PCA o el ANOVA-PLS, se propusieron para comparar las variaciones de los factores en relación con la varianza residual. El procedimiento se divide en dos pasos:

- Separación de la matriz de datos en un conjunto de matrices que representan los factores, como parte del diseño de experimentos, y una matriz de residuos.

- Un análisis de componentes principales en cada una de las matrices de los factores añadidos de la matriz de residuos.

\section{Método}

Las observaciones, de un experimento factorial de dos factores, pueden describirse con el modelo estadístico lineal.

$$
Y_{i j k}=\mu+_{i}+\beta_{j}+(\tau \beta)_{i j}+\varepsilon_{i j r}\left\{\begin{array}{l}
i=1,2, \ldots, a \\
j=1,2, \ldots, b \\
r=1,2, \ldots, n
\end{array}\right.
$$


donde $\mu$ es el efecto promedio global, $\tau_{i}$ es el efecto del $i$-ésimo nivel del factor A, $\beta_{j}$ es el efecto del $j$-ésimo nivel del factor $\mathrm{B},(\tau \beta)_{\mathrm{ij}}$ es el efecto de la interacción entre A y $B$ y $\varepsilon_{\mathrm{ijr}}$ es un componente de error aleatorio que tiene una distribución normal con media cero y varianza $\sigma^{2}$. Además, cada factor A y B tiene a y b niveles, respectivamente.

Otro posible diseño de experimentos se obtiene al tener en cuenta tres factores y se puede describir el modelo subyacente como sigue

$$
Y_{i j k}=\mu+\tau_{i}+\beta_{j}+\gamma_{k}+(\tau \beta)_{i j}+(\tau \gamma)_{i k}+(\beta \gamma)_{j k}+(\tau \beta \gamma)_{i j k}+\varepsilon_{i j r}\left\{\begin{array}{l}
i=1,2, \ldots, a \\
j=1,2, \ldots, b \\
k=1,2, \ldots, c \\
r=1,2, \ldots, n
\end{array}\right.
$$

[Ecuación 2] donde los efectos $\mu_{i}, \beta_{j}, \mathrm{y} \gamma_{k}$ corresponden a los efectos principales y $(\tau \beta)_{i j},(\tau \gamma)_{i k}$, $(\beta \gamma)_{i k} \mathrm{y}(\tau \beta \gamma)_{i j k}$ corresponden a la interacción entre los diferentes efectos.

La prueba de hipótesis consiste en probar que no existen efectos del factor A, del factor $\mathrm{B}$ y del factor $\mathrm{C}$ sobre la respuesta y además de que no existe interacción entre los factores A, B y C, por parejas o en conjunto. Esta hipótesis es probada haciendo uso del análisis de varianza (ANOVA) de dos factores y tres factores. Antes de iniciar el ANOVA para estos diseños de experimentos es importante determinar si los factores son de tipo fijo o aleatorio.

Un factor es fijo cuando los niveles de cierto factor, son seleccionados de manera específica por el experimentador, de tal forma que las inferencias están limitadas únicamente a estos niveles. En lo que se refiere a factores aleatorios, éstos corresponden a situaciones donde existen una gran cantidad de niveles posibles para el factor de interés, y el experimentador debe escoger al azar de toda la población un subconjunto de estos niveles, tal que, a partir de este subconjunto, se puedan obtener conclusiones para todos los posibles niveles del factor [89].

El propósito de ANOVA es probar significancia estadística de las diferencias entre las medias de los grupos o niveles de un factor. Esto se logra analizando la varianza, es decir, diviendiendo la varianza total en la componente que se debe a un error aleatorio de la medida y la componente que se debe a las diferencias entre las medias de los niveles del factor.

Finalmente, estas varianzas son comparadas para probar la hipótesis nula de que no hay diferencia entre las medias, y en caso de que resulten significativamente diferentes, se rechaza dicha hipótesis. Si hablamos por ejemplo, de los datos medidos para un diseño de experimentos de dos factores se definen $y_{i}$ como la suma total de las observaciones tomadas en el $i$-ésimo nivel del factor $\mathrm{A}$; $y_{j}$, la 
suma total de observaciones tomadas en el $j$-ésimo nivel del factor $\mathrm{B}$; $y_{i j}$, la suma total de las observaciones en la ij-ésima observación, y como el total de todas las observaciones.

Para probar la diferencia estadística entre los niveles de un factor se pueden tener en cuenta tres casos, se prueba el efecto de los factores individualmente, se prueba el efecto de la interacción entre los pares de factores posibles y se prueba el efecto de la interacción entre los tres factores presentes en el estudio

Para el primer caso, cada factor individual debe ser evaluado para probar su efecto sobre la medición $Y_{i j k r}$ (ver ecuación 2). En particular, para el factor $\tau$ la hipótesis nula, denotada como $\mathrm{H}_{0}$, consiste en que todos los a niveles del factor son iguales a 0 . Es decir, que no hay diferencias significativas entre dichos niveles. Mientras que si al menos para un nivel i no se cumple esta igualdad, se rechaza la hipótesis nula y se acepta la hipótesis alternativa $H_{1}$. Una descripción similar puede hacerse para los demás factores tenidos en cuenta. A continuación se presenta la notación habitual de las hipótesis a probar para cada uno los factores principales $\tau, \beta, \gamma$ :

1. $H_{0}: \tau_{1}=\tau_{2}=\cdots=\tau_{a}=0$

$H_{1}$ : al menos para un nivel $i \tau_{i} \neq 0$

2. $H_{0}: \beta_{1}=\beta_{2}=\cdots=\beta_{b}=0$

$H_{1}$ : al menos para un nivel $j \beta_{j} \neq 0$

3. $H_{0}: \gamma_{1}=\gamma_{2}=\cdots=\gamma_{c}=0$

$H_{1}$ : al menos para un nivel $k \gamma_{k} \neq 0$

Para el segundo caso, se prueba el efecto de la interacción entre cada par de factores sobre la medición $Y_{i j k r}$. Los posibles pares entre los factores $\tau, \beta$ y $\gamma$ corresponden a $\tau \beta$ con $i j$ niveles, $\tau \gamma$ con $i k$ niveles y $\beta \gamma$ con $j k$ niveles. En particular, para el factor de interacción $\tau \beta$, la hipótesis nula $H_{0}$ expresa que no hay diferencias entre los $a b$ niveles de interacción entre los factores $\tau$ y $\beta$. Si alguno de los niveles no cumple esta hipótesis se rechaza la hipótesis nula y se acepta la hipótesis alternativa $H_{1}$. Las hipótesis de los demás factores de interacción se describen de forma similar. A continuación se presentan las pruebas hipótesis de interacción que se tienen en cuenta en un diseño de experimentos con tres factores.
4. $H_{0}:(\tau \beta)_{11}=(\tau \beta)_{12}=\cdots=(\tau \beta)_{a b}=0$
$H_{1}$ : al menos para un par de niveles $i j(\tau \beta)_{i j} \neq 0$
5. $H_{0}:(\tau \gamma)_{11}=(\tau \gamma)_{12}=\cdots=(\tau \gamma)_{a c}=0$
$H_{1}$ : al menos para un par de niveles $i k(\tau \gamma)_{i k} \neq 0$
6. $H_{0}:(\beta \gamma)_{11}=(\beta \gamma)_{12}=\cdots=(\beta \gamma)_{b c}=0$
$H_{1}$ : al menos para un par de niveles $j k(\beta \gamma)_{j k} \neq 0$ 
El tercer caso consiste en probar la interacción entre los tres factores. La hipótesis nula $H_{0}$ consiste en probar que no hay diferencias entre los abc niveles de interacción. Si no se cumple dicha hipótesis se acepta la hipótesis alternativa $H_{1}$ de que el efecto para al menos una combinación de niveles ijk no es igual a los demás efectos.

$$
\begin{aligned}
& \text { 7. } H_{0}:(\tau \beta \gamma)_{111}=(\tau \beta \gamma)_{112}=\cdots=(\tau \beta \gamma)_{a b c}=0 \\
& H_{1}: \text { al menos para una combinación de niveles } i j k(\tau \beta \gamma)_{i j k} \neq 0
\end{aligned}
$$

La variabilidad total se mide con la suma total de cuadrados de las observaciones así

$$
S S_{T}=\sum_{i=1}^{a} \sum_{j=1}^{b} \sum_{r=1}^{n}\left(y_{i j r}-\bar{y} \ldots\right)^{2}
$$

mientras que la descomposición de la variabilidad está definida de la siguiente manera para un análisis de varianza de dos factores

$$
\begin{aligned}
\sum_{i=1}^{a} \sum_{j=1}^{b} \sum_{r=1}^{n}\left(y_{i j r}-\bar{y}_{\ldots}\right)^{2}= & b n \sum_{i=1}^{a}\left(\bar{y}_{i . .}-\bar{y}_{\ldots}\right)^{2} \\
& +a n \sum_{j=1}^{b}\left(\bar{y}_{\cdot j .}-\bar{y}_{\ldots}\right)^{2} \\
& +n \sum_{i=1}^{a} \sum_{j=1}^{b}\left(\bar{y}_{i j .}-\bar{y}_{i . .}-\bar{y}_{. j .}+\bar{y}_{\ldots}\right)^{2} \\
& +\sum_{i=1}^{a} \sum_{j=1}^{b} \sum_{r=1}^{n}\left(y_{i j r}-\bar{y}_{i j} .\right)^{2}
\end{aligned}
$$

[Ecuación 4]

Esta suma de cuadrados puede ser fácilmente extendida al caso de tres factores. Es así como la suma total de cuadrados queda dividida en una suma de cuadrados debida al factor A $\left(S S_{A}\right)$, una suma de cuadrados debida al factor $\mathrm{B}$ $\left(S S_{B}\right)$, una suma de cuadrados debida a la interacción entre A y B $\left(S S_{A B}\right)$ y una suma de cuadrados debida al error $\left(S S_{E}\right)$.

Un análisis tipo regresión lineal es generalmente usado para determinar las relaciones entre los tipos de datos obtenidos de las muestras objeto del estudio. En estos trabajos, el objetivo principal de la regresión no es predecir el valor de algún parámetro, en realidad este método se utiliza para obtener relaciones confiables y validadas que pueden ser estudiados más a la interpretación de las diferencias entre muestras respectos a las variables evaluadas. 
La identidad de la suma de cuadrados de la ecuación 4 puede escribirse de manera simbólica como

$S S_{T}=S S_{A}+S S_{B}+S S_{A B}+S S_{E}$

[Ecuación 5]

Existen en total $a b n-1$ grados de libertad. Los efectos principales A y B tienen $a$ -1 y $b-1$ grados de libertad, mientras que el efecto de interacción AB tiene ( $a-$ $1)(b-1)$ grados de libertad. Para cada una de los valores $a b$ celdas, existen $n-1$ grados de libertad entre las $n$ réplicas, y las observaciones en la misma celda sólo pueden diferir debido al error aleatorio. Por consiguiente, existen ab(n - 1) grados de libertad para el error. En consecuencia, los grados de libertad están divididos de acuerdo con

$a b n-1=(a-1)+(b-1)+(a-1)(b-1)+a b(n-1)$

[Ecuación 6]

Si se divide cada una de las sumas de los cuadrados del lado derecho de la ecuación 5 entre el número de grados de libertad correspondiente, se obtienen las medias de los cuadrados de A, B, la interacción y el error:

$$
\begin{aligned}
M S_{A}=\frac{S S_{A}}{a-1} & M S_{B}=\frac{S S_{B}}{b-1} \\
M S_{A B}=\frac{S S_{A B}}{(a-1)(b-1)} & M S_{E}=\frac{S S_{E}}{a b(n-1)}
\end{aligned}
$$

[Ecuación 7]

La variabilidad (SSE) dentro del grupo se conoce como la varianza del error. La variabilidad $S S A, S S B, S S A B$ se deben a las diferencias en las medias entre los grupos. Para probar que los efectos de los niveles para cierto factor son todos iguales a cero $(\mathrm{HO}: \mathrm{\tau i}=0)$, se emplea el cociente

$F_{0}=\frac{M S_{A}}{M S_{E}}$

[Ecuación 8]

el cual tiene una distribución F con $a-1$ y $a b(n-1)$ grados de libertad si

$H 0:$ ti $=0$ es verdadera.

Esta hipótesis nula se rechaza con un nivel de significancia a si fo $>\mathrm{f}_{\alpha,}, a-1, a b(n-1)$. De manera similar se define el cociente, la prueba de hipótesis y los grados de libertad para los demás efectos e interacciones.

Las pruebas de hipótesis se basan en la comparación de las varianzas debido a la variabilidad dentro de los grupos (MSA) con respecto a la variabilidad entre grupos (MSE). Bajo la hipótesis nula, de que no hay diferencias en la media entre grupos para cierta población, aún se esperaría alguna fluctuación aleatoria en las 
medias para los dos grupos cuando se toma una muestra pequeña. Por lo tanto, bajo la hipótesis nula, la varianza estimada con base en la variabilidad dentro de los grupos debe ser la misma que la varianza debido a la variabilidad entre grupos. Se puede comparar esos dos estimadores de varianza usando la prueba F, que prueba en qué casos esa relación de las dos estimaciones de varianza es significativamente más grande que 1 .

\section{Análisis de componentes simultáneo (ASCA): PCA-ANOVA y PLS-ANOVA}

Se trata de un método supervisado, donde los objetos son representados de diferentes niveles de cada uno de los factores que se definen en un diseño experimental. En una primera fase, se utiliza la varianza para evaluar las contribuciones de los factores y sus interacciones se evalúan en una segunda fase, estas contribuciones son comparadas con la varianza residual del PCA.

El modelo propuesto por [90] consiste en usar un PCA sobre una matriz de datos $Y$ de dimensiones $\mathrm{a}{ }^{*} \mathrm{~b}{ }^{*} \mathrm{n} \times \mathrm{p}$ (catadores* productos*réplicas $\times$ atributos), es decir el número de columnas corresponden al número de atributos y el número de filas corresponden a todas las combinaciones de catadores, productos y réplicas. Estos datos se pueden expresar usando un modelo MANOVA (análisis multivariante de varianza) de la siguiente forma:

$Y=1 \mu^{t}+\tau+\beta+\tau \beta+\varepsilon$

[Ecuación 9]

Lo anterior implica que PCA produce unos componentes que son combinaciones de todos los efectos del modelo anova multivariado, ver (9). Cuando se calculan los factores de PCA, pueden ser interpretados con el uso del gráfico de los scores y además relacionarlos con la matriz del diseño de los efectos usando ANOVA de dos factores.

El modelo ANOVA de la ecuación (9) puede ser reescrito reemplazando $Y$ en la ecuación de una PCA del tipo: $T=Y P$, donde $T$ es la matriz de componentes principales, $\mathrm{Y}$ es la matriz de datos estandarizada y $\mathrm{P}$ es la matriz de transformación formada por los vectores propios correspondientes a cada valor propio $\lambda j$ de la matriz de covarianza $\sum Y$.

$T=Y P=\left(1 \mu^{t}+\tau+\beta+\tau \beta+\varepsilon\right) P$

Se puede trabajar sobre esta matriz empleando ANOVA regularmente, es decir, se obtienen todos los cálculos de los efectos y pruebas de hipótesis sobre la matriz $T$. Debido a que los factores $T$ de PCA son ortogonales, se justifica el empleo de ANOVA separado para cada factor [91]. 


\section{Procedimiento A-PCA.}

APCA calcula sucesivamente una serie de matrices correspondientes al promedio de las variables para cada nivel de cada factor $y$, a continuación se resta este promedio a cada variable de la matriz original para obtener el error residual de muestra.

En la fase de "análisis de varianza", se calculan los promedios para cada nivel del factor y se crea una matriz donde cada objeto contiene la media correspondiente a su nivel. Restando esta matriz (Factor 1) en la matriz anterior (en este caso, $\mathrm{T}$ ) se obtienen los residuos. El procedimiento es el mismo para todos los demás factores e interacciones, hasta obtener la matriz representante de la varianza residual $\varepsilon$ [92]. Cada matriz de los residuos se añade a cada matriz de factores e interacciones para dar una matriz "factor + residuos."

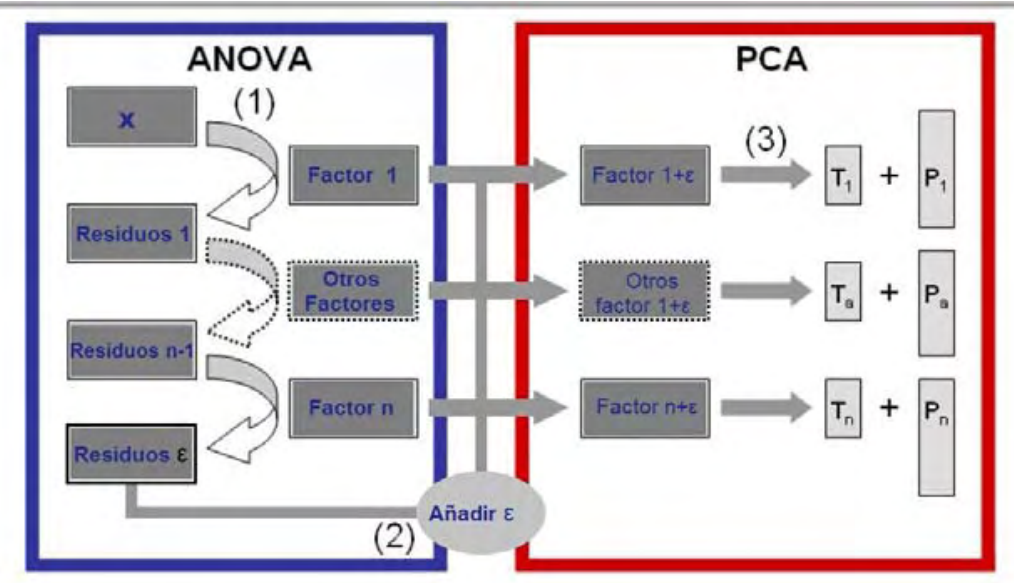

Figura 2.22. fase azul: ANOVA, se obtiene una matriz de residuos. Después de agregar la matriz de los residuos en cada factor se calcula la matriz en una segunda fase (fase roja PCA), un PCA para cada matriz individual resultante..

La PCA se aplica a cada matriz de "factor + residuos." Después de la inspección de proyecciones gráficas de coordenadas factoriales 1 y 2 , es posible llegar a una conclusión sobre la significancia de cada factor con respecto al ruido (residuos). Las contribuciones factoriales correspondientes son útiles para determinar qué variables son responsables del comportamiento de los objetos del factor en cuestión. La representación de este procedimiento puede ser observada en la Figura 2.22.

Similar al procedimiento ANOVA-PCA, el método ANOVA-PLS funciona como una combinación de la descomposición de la varianza para extraer diferentes efectos y un posterior análisis estadístico. 


\subsubsection{Implementación}

Todos los algoritmos aplicados en esta tesis para la interpretación de los datos resultantes de cada uno de los sistemas utilizados para el control de calidad de vinos ha sido implementado en el entorno de programación que ofrece el software Matlab 7.9. Las subrutinas básicas del análisis de datos se llevaron a cabo utilizando un paquete multivariante denominado PLS-Toolbox 4.2 desarrollado por Eigenvector Research Inc. Sin embargo, en vista de los objetivos de esta tesis fue necesario programar una serie de funciones de adicionales para facilitar la integración de los diferentes sistemas y para obtener la valoración final desde el punto de vista sensorial.

Con respecto a la aplicación de los algoritmos genéticos y el modelo tucker3 fueron muy importantes las rutinas facilitadas por el profesor R. Leardi del Departamento de Química y Tecnología Farmaceutica y Alimentaria de la Universidad de Genova. Finalmente, en el caso de los estudios realizados con el método ANOVA-PLS se ha utilizado el software Unscrambler® $X$.

\section{Análisis de datos}

Como conclusión de este Capítulo, creemos conveniente constatar que en estos párrafos se han descrito de forma general las pautas necesarias que deben adoptarse cuando nos enfrentemos a matrices de datos de compleja naturaleza, como son claro ejemplo las puestas en juego en esta investigación. La selección de una u otra técnica de pre-tratamiento y análisis de datos multivariantes para la resolución de los problemas y objetivos (discriminaciones, correlaciones, etc.) implementados en esta Tesis, así como las cuestiones relacionadas con la validación de modelos serán detalladamente justificados y discutidos de forma específica en secciones posteriores de la presente Memoria.

\subsection{REFERENCIAS}

[1] O.I.V, Recueil des méthodes internationales d'analyse des vins et des moûts. , Office International de la Vigne et du Vin., Paris, 1990.

[2] Métodos oficiales de análisis en la Unión Europea, Diario oficial de las Comunidades Europeas. Ministerio de Agricultura, Pesca y Alimentación, Madrid, 1998. 
[3] M. A. Amerine \& C. S. Ough, Análisis de mostos y vinos, Acribia, 1976.

[4] J. García-Barceló, Técnicas analíticas para vinos, GAB, Moja-Olèrdola, 1990.

[5] J. Wirth, C. Morel-Salmi, J. M. Souquet, J. B. Dieval, O. Aagaard, H. Vidal, H. Fulcrand \& V. Cheynier, The impact of oxygen exposure before and after bottling on the polyphenolic composition of red wines, Food Chemistry, 2010, 123, 107-116.

[6] J. García-Barceló, Metodología de análisis de vinos y derivados, Sepsa, Villafranca del Penedés, 1976.

[7] M. Ruiz Hernández, Crianza y envejecimiento del vino tinto, Madrid Vicente, 1994.

[8] J. Ribéreau-Gayon, E. Peynaud, P. Sudraud \& P. Ribéreau-Gayon, Tratado de enología. Ciencias y técnicas del vino Hemisferio sur., 1980.

[9] T. C. Somers, Wine tannins-isolation of condensed flavonoid pigments by gel-filtration., Nature, 1966, 209, 368-370.

[10] R. B. Boulton, Methods for the assessment of copigmentation in red wines Reno, NV 1996.

[11] J. Cacho, Ciencia y Tecnología de los vinos del siglo XXI. Palencia, 2004.

[12] K. Holley, M. Pennington \& P. Phillips, Gas chromatography in food analysis: an introduction, Nutrition \& Food Science, 1995, 95, 10-12.

[13] M. Romero, A. Casp \& J. M. Carrasco, Determinación de componentes volátiles en vinos, Agroquímica y Tecnología de Alimentos, 1986, 26, 338-348.

[14] S. Villanueva, Integración de un sistema de medida de aromas y color para la caracterización de vinos tintos, Tesis doctoral, Universidad de Valladolid, 2006.

[15] S. Villanueva, A. Vegas, J. A. Fernandez-Escudero, M. Iniguez, M. L. RodriguezMendez \& J. A. de Saja, SPME coupled to an array of MOS sensors - Reduction of the interferences caused by water and ethanol during the analysis of red wines, Sensors and Actuators B-Chemical, 2006, 120, 278-287.

[16] K. Kolb \& L. Ettre, Static Headspace-Gas Chromatography: Theory and Practice, Wiley, 2006.

[17] M. Decker, Current practice of gas chromatography--mass spectrometry, Wilfried M.A. Niessen, 2001.

[18] R. Flamini \& P. Traldi, Mass Spectrometry in Grape and Wine Chemistry, Wiley, New Jersey, 2010.

[19] T. Cserháti, Chromatography of Aroma Compounds and Fragrances, Springer, Budapest, 2010.

[20] T. E. Acree, Gas-Chromatography Olfactometry, Eds.: C. T. Ho and C. H. Manley), 1993, pp. 77-94. 
[21] T. E. Acree \& E. H. Lavin, Future of gas chromatography/olfactometry, Abstracts of Papers of the American Chemical Society, 1999, 218, 37-AGFD.

[22] T. E. Acree \& J. Barnard, Gas-Chromatography Olfactometry And Charmanalysis, Eds.: H. Maarse and D. G. VanDerHeij), 1994, pp. 211-220.

[23] V. Ferreira, N. Ortin, A. Escudero, R. Lopez \& J. Cacho, Chemical characterization of the aroma of Grenache rose wines: Aroma extract dilution analysis, quantitative determination, and sensory reconstitution studies, Journal of Agricultural and Food Chemistry, 2002, 50, 40484054 .

[24] V. Ferreira, F. San Juan, A. Escudero, L. Cullere, P. Fernandez-Zurbano, M. P. SaenzNavajas \& J. Cacho, Modeling Quality of Premium Spanish Red Wines from Gas Chromatography-Olfactometry Data, Journal of Agricultural and Food Chemistry, 2009, 57, 7490-7498.

[25] M. P. Martí Borràs, Estudi de l'aplicació del nas electrònic i de la cromatografia de gasosolfactimetria a l'anàlisi de l'aroma de vins i aiguardents, Tesis doctoral, Universitat Rovira I Virgili, Tarragona, 2005.

[26] W. Grosch, Evaluation of the key odorants of foods by dilution experiments, aroma models and omission, Chemical Senses, 2001, 26, 533-545.

[27] W. Grosch, Detection of potent odorants in foods by aroma extract dilution analysis, Trends in Food Science \& Technology, 1993, 4, 68 - 73.

[28] E. Campo, V. Ferreira, A. Escudero \& J. Cacho, Prediction of the wine sensory properties related to grape variety from dynamic-headspace gas chromatography-olfactometry data, Journal of Agricultural and Food Chemistry, 2005, 53, 5682-5690.

[29] H. Guth, Quantitation and sensory studies of character impact odorants of different white wine varieties. , Journal of Agricultural and Food Chemistry 1997, 45, 3027-3032.

[30] M. Aznar, R. Lopez, J. Cacho \& V. Ferreira, Prediction of aged red wine aroma properties from aroma chemical composition. Partial least squares regression models, Journal of Agricultural and Food Chemistry, 2003, 51, 2700-2707.

[31] B. D'Acampora Zellner, P. Dugo, G. Dugo \& L. Mondello, Gas chromatographyolfactometry in food flavour analysis., Journal of Chromatography A, 2008, $1186,123-143$.

[32] A. Chaintreau, Quantitative use of gas chromatography-olfactometry: the GC-"SNIF" method, (Ed. R. Marsili), Marcel, New York, 2002, pp. 333-348.

[33] S. M. Van Ruth \& C. H. O'Connor, Evaluation of three gas chromatography-olfactometry methods: comparison of odour intensity-concentration relationships of eight volatile compounds with sensory headspace data., Food Chemistry, 2001, 74, 341-347.

[34] P. Pollien, L. B. Fay, M. Baumgartner \& A. Chaintreau, First attempt of odorant quantification using gas chromatography-olfactometry. , Analytical Chemistry, 1999, 71, 53915397.

[35] V. Ferreira, J. Pet'ka, M. Aznar \& J. Cacho, Quantitative possibilities of gas chromatography-olfactometry with simple measurements of intensity, 2003. 
[36] C. M. Delahunty, G. Eyres \& J. P. Dufour, Gas chromatography-olfactometry, Journal of Separation Science 2006, 29, 2107-2125.

[37] B. S. Mistry, T. Reineccius \& L. K. Olson, Gas Chromatography-olfactometry for the determination of key odorants in foods, (Ed. R. Marsili), Marcel Dekker, New York, 1997, pp. 265-292.

[38] M. J. Benito, M. C. Ortiz, M. S. Sanchez, L. A. Sarabia \& M. Iniguez, Typification of vinegars from Jerez and Rioja using classical chemometric techniques and neural network methods, Analyst, 1999, 124, 547-552.

[39] S. Villanueva, A. Guadarrama, M. L. Rodriguez-Mendez \& J. A. de Saja, Use of an array of metal oxide sensors coupled with solid phase microextraction for characterisation of wines Study of the role of the carrier gas, Sensors and Actuators B-Chemical, 2008, 132, 125-133.

[40] S. H. Hahn, N. Bârsan \& U. Weimar, Investigation of CO/CH4 mixture measured with differently doped $\mathrm{SnO} 2$ sensors, 2001, 78, 68.

[41] R. K. Sharma, P. C. H. Chan, Z. Tang, G. Yan, I. M. Hsing \& J. K. O. Sin, Sensitive, selective and stable tin dioxide thin-films for carbon monoxide and hydrogen sensing in integrated gas sensor array applications, 2001, 72, 160-166.

[42] M. Riu, M. Mestres, O. Busto \& G. J, Determination of 2,4,6-trichloroanisole in wines by headspace solid-phase microextraction and gas chromatography-electron-capture detection., Journal of Chromatography A, 2002, 977, 1-8.

[43] K. Chatterjee, S. Chatterjee, A. Banerjee, M. Raut, N. Pal \& H. S. Maiti, The effect of palladium incorporation on methane sensitivity of antimony doped Tin, Materials Chemistry and Physics, 2003, 81, 33-38.

[44] V. V. Malyshev \& A. V. Pislyakova, Investigation of gas-sensitivity of sensor structures to hydrogen in a wide range of temperature, concentration and humidity of gas medium Sensors and Actuators B-Chemical, 2008, 134, 913-921.

[45] K. Kalcher, J. M. Kauffmann, J. Wang, I. Svancara \& K. N. Vytras, C.; Yang, Z., Sensors based on carbon paste in electrochemical analysis: A review with particular emphasis on the period 1990-1993, Electroanalysis, 1995, 7, 5-22.

[46] R. N. Adams, Carbon paste electrodes, Analytical Chemistry 1958, 30, 1576.

[47] J. Lindquist, Carbon paste electrode with wide anodic potential range, Analytical chemistry, 1973, 45, 1006.

[48] M. E. Rice, Z. Galus \& R. N. Adams, Graphite paste electrodes. Effects of paste composition and surface states on electron-transfer rates, Journal of Electroanalytical Chemistry 1983, 143, 89-102.

[49] C. Urbaniczkya \& K. Lundströma, Voltammetric studies on carbon paste electrodes: The influence of paste composition on electrode capacity and kinetics Journal of Electroanalytical Chemistry and Interfacial Electrochemistry, 1984, 176. 
[50] V. Parra, T. Hernando, M. L. Rodriguez-Mendez \& J. A. de Saja, Electrochemical sensor array made from bisphthalocyanine modified carbon paste electrodes for discrimination of red wines, Electrochimica Acta, 2004, 49, 5177-5185.

[51] Guia técnica, 2010, http://www.princetonappliedresearch.com/.

[52] K. Toko, Electronic Sensing of Tastes, Electroanalysis, 1998, 10, 657-669.

[53] Y. Vlasov, A. Legin \& A. Rudnitskaya, Electronic tongues and their analytical application, Analytical and Bioanalytical Chemistry, 2002, 373, 136-146.

[54] H. Cabrera, J. J. Rodríguez, M. A. Rodríguez, J. E. Conde \& J. P. Pérez, Valencia, 2001, p. 43.

[55] L. Carmel, S. Levy, D. Lancet \& D. Harel, An eNose algorithm for identifying chemicals and determining their concentration Sensors and Actuators B-Chemical, 2003, 93, 67-76.

[56] C. Luyo, R. Ionescu, L. F. Reyes, Z. Topalian, W. Estrada, E. Llobet, C. G. Granqvist \& $\mathrm{P}$. Heszler, Gas sensing response of $\mathrm{NiO}$ nanoparticle films made by reactive gas deposition, Sensors and Actuators B-Chemical, 2009, 138, 14-20.

[57] C. M. Hsiung, A. Roy, B. Hermann, S. H. Zhang \& S. Malin, United Kingdom, 2000, pp. 71-72.

[58] M. L. Olvera, A. Maldonado \& R. Asomoza, Películas delgadas de SnO2:Ga utilizadas como sensores de oxígeno, Superficies y Vacío, 1999, 8, 33-36.

[59] C. Di Natale, A. Macagnano, F. Davide, A. D'Amico, R. Paolesse, T. Boschi, M. Faccio \& G. Ferri, An electronic nose for food analysis, Sensors and Actuators B-Chemical, 1997, 44, 521-526.

[60] C. Di Natale, A. Macagnano, E. Martinelli, E. Proietti, R. Paolesse, L. Castellari, S. Campani \& A. D'Amico, Electronic nose based investigation of the sensorial properties of peaches and nectarines, Sensors and Actuators B-Chemical, 2001, 77, 561-566.

[61] M. Wang, A. Perera \& R. Gutierrez-Osuna, Principal discriminants analysis for smallsample-size problems: application to chemical sensing, Eds.: D. Rocha, P. M. Sarro and M. J. Vellekoop), 2004, pp. 591-594.

[62] J. E. Haugen \& C. Ketecioglu, Electronic nose and artificial neural network, Rome, 202, pp. 120-121.

[63] T. Artursson, T. Eklöv, I. Lundström, P. Martensson, M. Sjöström \& M. Holmerg, Drift correction for gas sensors using multivariate methods, Journal of Chemometrics, 2000, 14, 711-723.

[64] M. Casale, N. Sinelli, P. Oliveri, V. Di Egidio \& S. Lanteri, Chemometrical strategies for feature selection and data compression applied to NIR and MIR spectra of extra virgin olive oils for cultivar identification, Talanta, 2010, 80, 1832-1837.

[65] R. Bro \& A. K. Smilde, Centering and scaling in component analysis, Journal of Chemometrics, 2003, 17, 16-33. 
[66] S. P. Gurden, J. A. Westerhuis, R. Bro \& A. K. Smilde, A comparison of multiway regression and scaling methods, Chemometrics and Intelligent Laboratory Systems, 2001, 59, $121-136$.

[67] J. Mendieta, M. S. DiazCruz, R. Tauler \& M. Esteban, Application of multivariate curve resolution to voltammetric data .2. Study of metal-binding properties of the peptides, Analytical Biochemistry, 1996, 240, 134-141.

[68] J. Ghasemi, A. Niazi \& R. Leardi, Genetic-algorithm-based wavelength selection in multicomponent spectrophotometric determination by PLS: application on copper and zinc mixture, Talanta, 2003, 59, 311-317.

[69] R. Leardi \& A. L. Gonzalez, Genetic algorithms applied to feature selection in PLS regression: how and when to use them, Chemometrics and Intelligent Laboratory Systems, $1998,41,195-207$.

[70] L. Xu \& W.-J. Zhang, Comparison of different methods for variable selection, Analytica Chimica Acta, 2001, 446, 477-483.

[71] K. Tang \& T. Li, Combining PLS with GA-PLS for QSAR, Chemometrics and intelligent laboratory systems, 2002, 64, 55-64.

[72] L. Davis, The Hanbook of genetic Algorithms, Van Nostrand Reinhold, New York, 1991.

[73] A. S. Barros \& D. N. Rutledge, Genetic algorithms applied to the selection of principal components, Chemometrics and Intelligent Laboratory Systems, 1998, 40, 65-81.

[74] R. Leardi, Genetic algorithms in chemistry, Journal of Chromatography A, 2007, 1158, 226-233.

[75] R. Leardi, Application of a genetic algorithm to feature-selection under full validation conditions and to outlier detection, Journal of Chemometrics, 1994, 8, 65-79.

[76] R. Leardi, R. Boggia \& M. Terrile, Genetic algorithms as a strategy for feature-selection, Journal of Chemometrics, 1992, 6, 267-281.

[77] J. F. Hair, R. E. Aderson, R. L. Tatham \& W. C. Black, Análisis Multivariante, Prentice Hall Iberia, 1999.

[78] J. Brezmes, Diseño de una nariz electrónica para la determinación no destructiva del grado de maduración de la fruta, Tesis Doctoral, Universitat Politécnica de Catalunya, 2002.

[79] K. H. Esbensen \& P. Geladi, Principal Component Analysis: Concept, Geometrical Interpretation, Mathematical Background, Algorithms, History, Practice, (Ed. Elsevier), 2009

[80] V. Parra, Diseño y aplicación de sensores electroquímicos basados en compuestos orgánicos electroactivos para una lengua electrónica: discriminación y clasificación de vinos., Tesis doctoral, Universidad de Valladolid, Valladolid, 2005.

[81] A. C. Olivieri \& N. M. Faber, Validation and Error, (Ed. Elsevier), 2009.

[82] C. A. Andersson \& R. Bro, Improving the speed of multi-way algorithms: Part I. Tucker3, Chemometrics and Intelligent Laboratory Systems, 1998, 42, 93-103. 
[83] A. Smilde, R. Bro \& P. Geladi, Multiway Analysis, Applications in the Chemical Science, Wiley, England, 2004.

[84] R. Bro, Review on multiway analysis in chemistry - 2000-2005, Critical Reviews in Analytical Chemistry, 2006, 36, 279-293.

[85] C. Cordella, R. Leardi \& N. R. Douglas, Three-way principal component analysis applied to noodles sensory data analysis, Chemometrics and Intelligent Laboratory Systems, 2010, $106,125-130$.

[86] C. Durante, M. Cocchi, M. Grandi, A. Marchetti \& R. Bro, Application of N-PLS to gas chromatographic and sensory data of traditional balsamic vinegars of modena, Chemometrics and Intelligent Laboratory Systems, 2006, 83, 54-65.

[87] R. Bro, PARAFAC. Tutorial and applications, Chemometrics and Intelligent Laboratory Systems, 1997, 38, 149-171.

[88] C. Burian, J. Bremes, M. Vinaixa, E. Llobet, X. Vilanova, N. Canellas \& X. Correig, MS-Electronic Nose Performance Improvement Using GC Retention Times And 2-Way And 3Way Data Processing Methods, Eds.: M. Pardo and G. Sberveglieri), 2009, pp. 105-108.

[89] D. C. Montgomery \& G. C. Runger, Applied Statistics and Probability for Engineers, John Wiley \& Sons, New York, 2002.

[90] G. Luciano \& T. Næs, Interpreting sensory data bycombining principal component analysis and analysis of variance, Food Quality and Preference, 2009, 20, 167 - 175.

[91] C. Acosta Muñoz, Análisis multivariado para la identificación de compuestos generadores de sabor y aroma en productos alimenticios., Tesis de Master, Universidad Nacional de Colombia, Manizales, 2010.

[92] R. J. Dos Santos Clímaco Pinto, Développement de nouvelles méthodes chimiométriques d'analyse. Application à la charatésisation spectroscopique de la qualité des aliments, Tesis doctoral, Institut des sciences et idustries du viviant et de l'enviromnement, Paris, 2009. 


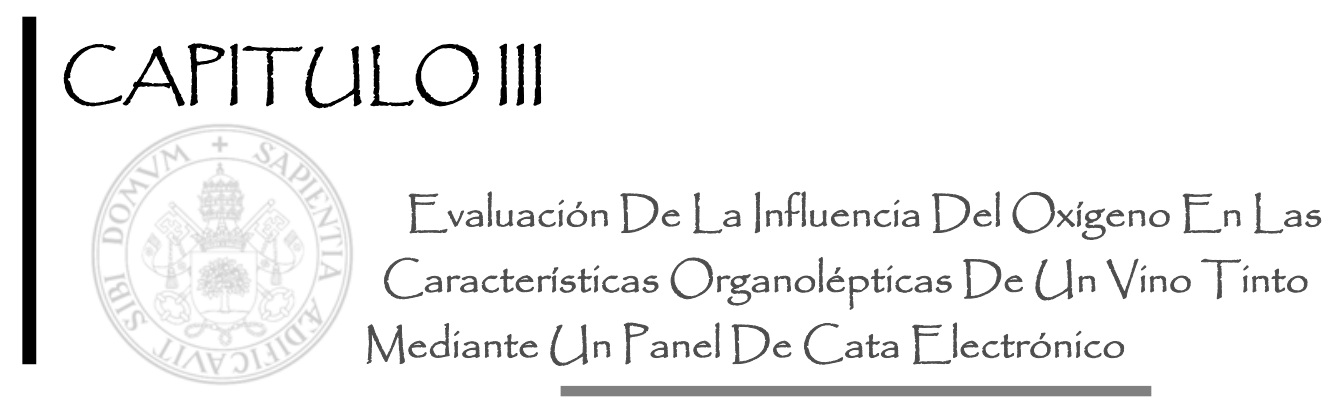

Página

3.1. GENERALIDADES $\quad 139$

3.1.1. Revisión teórica $\quad 141$

3.1.1.1. Efectos del oxígeno en el color del vino 141

3.1.1.2. Efectos del oxígeno en el gusto del vino 143

3.1.1.3. Efectos del oxígeno en el aroma del vino 143

3.1.1.4. Aplicación del tratamiento de microoxigenación 144

3.1.1.5. Efecto del oxígeno durante el envejecimiento en botella 145

3.1.1.6. Incorporación del oxigeno a través de los tapones sintéticos 146 porosos

3.2. DESARROLLO EXPERIMENTAL $\quad 149$

3.2.1. Características de las muestras de vino 149

3.2.2. Características de los tapones sintéticos porosos $\quad 150$

3.2.3. Medidas del oxígeno durante el envejecimiento en botella $\quad 154$

3.2.4. Medidas de los compuestos polifenólicos 155

3.3. RESULTADOS 156

3.3.1. Análisis Exploratorio $\quad 156$

3.3.1.1. Coordenadas CIELab. 157

3.3.1.2. Nariz electrónica. $\quad 159$

3.3.1.3. Lengua electrónica. $\quad 161$

3.3.1.4. Panel de Cata. 162

3.3.2. Análisis Supervisado $\quad 164$

3.3.2.1. Análisis Cualitativo: Clasificación (PLS-DA) 164

3.3.2.2. Análisis Cuantitativo: PLS2 168

$\begin{array}{lr}\text { 3.4. CONCLUSIONES } & 178\end{array}$

$\begin{array}{lr}\text { 3.5. REFERENCIAS } & 179\end{array}$ 



\subsection{GENERALIDADES}

Toda práctica enológica deber cumplir con una serie de normativas, algunas de carácter sanitario, otras de protección legal del consumidor y otras referidas a las características del producto elaborado. En este punto se asume que el uso técnicas alternativas no deben implicar un cambio inaceptable en la composición del producto tratado y deben asegurar la preservación de las características naturales del vino, permitiendo mejorar a la vez la calidad.

En este sentido las nuevas prácticas son siempre susceptibles de enfrentar opiniones entre los productores. En primer lugar, porque inciden en el perfil organoléptico y, en segunda instancia porque aceleran el tiempo de producción, variando significativamente la obtención de beneficios económicos del producto resultante. Entre las prácticas que están en uso cada vez más, se encuentran el envejecimiento con métodos alternativos, la microoxigenación, la aplicación de organismos orientados genéticamente, y otras técnicas algo más complejas como las resinas de intercambio iónico o el spinning cone column.

Al mismo tiempo que aparecen nuevas opciones para la elaboración del vino, se han ido desarrollando nuevos métodos analíticos, dentro de los cuales podemos nombrar específicamente las narices y lenguas electrónicas que como hemos indicado anteriormente ofrecen la posibilidad de evaluar las características organolépticas del vino de una manera rápida y objetiva. Dos de los principales intereses en nuestra investigación dentro este campo, han sido la validación de la capacidad que presentan estos sistemas para distinguir los matices diferenciadores de vinos elaborados con técnicas estándar y alternativas, y por otra parte, la evaluación de las correlaciones existentes entre los resultados que se obtienen por medio de estos sistemas electrónicos y los que entregan los análisis químicos y organolépticos tradicionales.

Las investigaciones que se describen en este capitulo se centran en el estudio de algunos de los efectos de la microoxigenación en la calidad del vino. En un capítulo posterior se abundará en el tema del uso de los métodos alternativos al envejecimiento en barrica. 
Durante el desarrollo de este capítulo se dará especial atención al estudio de la influencia que tiene el oxígeno en el vino, antes y durante el tiempo de su almacenamiento en botella. Debido a la relación existente entre oxígeno y polifenoles se han diseñado una serie de experimentos que abarcan un conjunto de muestras caracterizadas por que presentar dos niveles diferentes de contenido fenólico [1, 2]. Para obtener estos niveles se utilizará el mismo tipo de uvas para todas las muestras a embotellar, pero con dos técnicas de extracción del mosto: termovinificación o Flash release (FR) que consigue un producto con un alto contenido en polifenoles y la técnica de maceración tradicional (Trad) [3]. Estas dos series de muestras, a su vez fueron divididas en grupos de vinos con y sin microoxigenación. Respecto a la velocidad del paso del oxigeno cuando el vino está almacenado en botella, este parámetro se ha controlado mediante la combinación de tapones sintéticos con diferente porosidad y consecuentemente distinta velocidad de transferencia del oxigeno (OTR).

Concretamente, los objetivos que planteamos en esta parte de nuestra investigación básicamente fueron:

1. Diseñar un método de control objetivo y rápido que potencialmente permita determinar el oxigeno presente en el vino y sus efectos, en cualquiera de las etapas de su elaboración.

2. Evaluar el potencial de la nariz, lengua y ojo electrónico para distinguir, apoyándose en un análisis PCA, vinos con diferente capacidad antioxidante y niveles de oxígeno, consecuencia de la etapa de microoxigenación y del uso de distintos tipos de tapones sintéticos con diferente porosidad.

3. Emplear los métodos multivariantes PLS-DA para construir modelos de clasificación con los sistema de análisis sensorial electrónicos de acuerdo a los tres factores básicos determinados para este investigación: (a) contenido polifenólico (b) contribución de la microoxigenación e (c) influencia de la porosidad del tapón.

4. Establecer correlaciones mediante el método multivariante PLS2 entre los resultados obtenidos con los sistemas de análisis sensorial electrónico y resultados de las valoraciones respecto al contenido polifenólico y a los niveles de oxígeno durante el envejecimiento en botella.

5. Combinar los aportes que realiza cada uno de los tres sistemas empleados en este trabajo: nariz, lengua y ojo electrónicos para obtener una valoración conjunta de los efectos que tiene el vino el cambio en el contenido polifenólico y el nivel de oxígeno. 
A continuación citaremos algunas de las conclusiones alcanzadas hasta el momento por los diferentes grupos de investigación en torno a la influencia del oxigeno durante el proceso de elaboración del vino.

\subsubsection{Revisión teórica}

\subsubsection{Efectos del oxigeno en el color del vino}

Las modificaciones que tienen lugar en el color del vino están en función, entre otras, de las características de las uvas, del grado de extracción de antocianos y taninos, de las condiciones del medio y de las posteriores modificaciones de estas moléculas durante el envejecimiento. En otros términos los matices cromáticos del vino siempre tienen una significación relacionada con su origen, la edad del vino o con su estado de elaboración y conservación.

La maceración y la ubicación de los compuestos fenólicos dentro de la uva dan origen a una extracción de compuestos fraccionada, que además depende de la distinta solubilidad y capacidad de difusión de los compuestos y ello es función fundamental del grado alcohólico del medio.

En términos generales, la extracción de los compuestos fenólicos durante la maceración presenta un crecimiento continuo seguido por un proceso de saturación para el caso de los taninos y un decrecimiento en la concentración de los antocianos. El proceso total es mucho más complejo, pero se puede decir a grandes rasgos que la velocidad de extracción es proporcional al gradiente de concentración entre los sólidos y el mosto.

La disminución que presentan los antocianos puede ocurrir por degradación de las moléculas consecuencia del tipo de levaduras usadas [4], o debido a la existencia de partes sólidas de la uva [5] Existen otro tipo fenómenos relacionados con los antocianos como su polimerización con las procianidinas de la uva y/o su reacción con otros compuestos del vino como el ácido pirúvico, el acetaldehído, etc. Estos polímeros son más estables y además permiten la precipitación de materia colorante durante el envejecimiento en barrica aunque no durante el tiempo que el vino se conserve en botella [6-9].

Las reacciones de estabilización tienen su origen, la mayoría de las veces, en la formación de combinaciones entre antocianos y taninos. Así, el color de los vinos tintos es debido a la presencia, además de los antocianos libres, de combinaciones del tipo taninos-antocianos, de taninos condensados o de fenómenos de copigmentación. Es bien conocido que las modificaciones que se dan durante el 
envejecimiento se evidencian por la evolución del color hacia tonos más teja y la pérdida de astringencia.

En la unión entre antocianos y taninos normalmente interviene el acetaldehído, producto asociado al grado de etanol y oxigeno presente en el medio. Estas reacciones se traducen en una disminución del color rojo, pero al mismo tiempo generan compuestos de un color rojo-azulado. El etanal también puede dar origen a reacciones indirectas que implican la combinación entre los antocianos y los flavanoles, entre flavanoles, e incluso entre los propios antocianos, lo que genera reajustes en el peso molecular de los polímeros.

Por otra parte, también existe la posibilidad de originar compuestos vinilflavanoles que podrían reaccionar con los antocianos dando lugar a nuevos pigmentos, los denominados piranoantocianos. Estos piranoantocianos son compuestos que presentan una gran estabilidad química, son resistentes a la decoloración con el dióxido de azufre, apenas varían su coloración por las variaciones del $\mathrm{pH}$ y son abundantes en la composición de los vinos añejos [10, $11]$.

El oxígeno participa claramente en toda una serie de reacciones entre antocianos y flavanoles que se traducen en una estabilización de la materia colorante y una disminución de la astringencia. Aún así, es necesario señalar que no se conocen rigorosamente ni los mecanismos químicos de las reacciones que genera el oxígeno en el vino ni sus consecuencias organolépticas. Hasta el momento sólo se ha conseguido un importante conocimiento empírico de esta materia, especialmente en lo referente a la permanencia del vino en las barricas y la consiguiente asimilación del oxígeno, que permite la transformación de la estructura fenólica del vino y los beneficios en los aspectos sensoriales. Su influencia y explicación científica durante la etapa del envejecimiento en botella es un tema poco estudiado.

Aunque no es materia de este capítulo, es importante especificar que durante el envejecimiento en barrica, es conocido que los antocianos presentan dos tipos de reacciones: por una parte son oxidados, lo que implica una disminución de la componente roja del color, y por otra se produce una combinación entre antocianinas y procianidinas, lo que da lugar a una estabilización del color rojo. Son precisamente estas combinaciones de antocianinas-procianidinas las responsables de la coloración de los vinos tintos añejos así como de la capacidad del vino para equilibrar la capacidad oxidativa en la barrica. Por lo tanto, un vino tinto destinado a la crianza en barrica debe poseer una concentración elevada de antocianinas, de lo contrario la oxidación de las mismas provocará una pérdida importante del color rojo durante el envejecimiento. 
Los fenómenos relacionados con el envejecimiento en botella serán explicados con detenimiento en otros apartados de este capitulo.

\subsubsection{Efecto del oxigeno en el gusto del vino}

Al igual que las reacciones asociadas al cambio del color, consecuencia de la presencia de oxígeno en el vino son los antocianos, taninos y flavanoles, a nivel gustativo los principales compuestos que intervienen en sensaciones de amargor y astringencia. Los cambios que se generan en un vino joven con un carácter duro, áspero, astringente e incluso algo amargo, hacia un carácter más suave y menos astringente se deben en gran medida a la polimerización entre flavanoles. Estas nuevas moléculas, al parecer son menos reactivas con los receptores sensoriales de la lengua, lo que se puede traducir en sensaciones más suaves y equilibradas.

Durante el envejecimiento del vino es el acetaldehído el principal compuesto que contribuye a la polimerización de los flavanoles. Las moléculas que se forman por medio del acetaldehído no son tan reactivas como sus constituyentes y esto puede propiciar que los vinos sean menos astringentes. Las reacciones entre flavanoles dan origen a las procianidinas, las cuales a su vez, de acuerdo con su reactividad pueden interactuar con los antocianos. En caso de dar origen a nuevos compuestos, estos pueden influenciar el sabor del vino al ser compuestos poliméricos con un bajo número de unidades (entre 8 a 10 unidades), a las cuales los receptores sensoriales de la lengua son más sensibles; además estas moléculas consiguen impedir otro tipo de polimerizaciones.

\subsubsection{Efecto del oxígeno en el aroma del vino}

Estudiar el efecto del oxígeno a nivel aromático es mucho más complejo, porque la mayoría de reacciones que tienen lugar durante el envejecimiento del vino también dependen de otros factores como la temperatura del medio y de su $\mathrm{pH}$. Muchas cuestiones en este aspecto continúan si resolver, siendo motivo de constante discusión científica.

La base primaria para la oxidación del vino son los polifenoles, cuando estos compuestos reaccionan con el oxígeno pasan a formar las quinonas. Otros compuestos como el dióxido de sulfuro y al ácido ascórbico adicionados al vino son capaces de reducir las quinonas y de esta manera el oxígeno presente en el vino se va consumiendo, impidiendo la oxidación de otros compuestos. Las quinonas, además actúan como trampa de compuestos sulfurosos, compuestos que pueden modificar negativamente el aroma del producto. 
En este caso la presencia de acetaldehído también es importante como intermediario de la reacción entre compuestos volátiles como el furfural y el 5hidroximetilfurfural con los flavanoles. También se ha demostrado que pueden reaccionar directamente con antocianos como el malvidin-3-glucosa. Existen además muchas reacciones de los compuestos volátiles a nivel particular que se producen en un ambiente oxidativo durante el envejecimiento en barrica. Entre estas reacciones podemos nombrar la formación del sotolón, compuesto que se origina debido a la oxidación de la treonina. Un estudio llevado a cabo por Cerdàn et al. [12] demuestra que las concentraciones de etilbutirato, etiloctanoato decrecen durante los 18 primeros meses en barricas, mientras que la concentración del etilhexanoato y el etildecanoato se incrementan y ello es debido a la presencia de oxigeno en el medio.

\subsubsection{Aplicación del tratamiento de microoxigenación para vinos tintos}

La dosificación de oxígeno durante la fermentación y especialmente durante el envejecimiento en barrica busca la estabilización de la materia colorante, así como la suavización de la astringencia inicial a través de las combinaciones y polimerizaciones entre los antocianos y taninos [11]. Tradicionalmente, estas reacciones se desarrollan durante la crianza del vino en barricas de roble, en las que tiene lugar una microdifusión del oxígeno a través de la duelas o poros de la barrica [10].

La microoxigenación inducida es una opción alternativa que aporta al vino pequeñas cantidades de oxígeno de forma controlada, a través de un microdifusor poroso unido a una botella de oxígeno [13]. El oxígeno puede añadirse en función de las necesidades que se evalúen durante la vinificación. Al finalizar la fermentación maloláctica, por ejemplo, puede suministrarse cantidades entre 1-4 mg/L/día. También es una práctica común aplicar entre 1-6 $\mathrm{mg} / \mathrm{L} / \mathrm{mes}$ durante el periodo de envejecimiento.

Uno de los principales motivos para aplicación de esta técnica se debe a que este método contribuye a la aceleración del proceso de estabilización de la materia colorante. Ribéreau-Gayon [10] reportó un incremento en la intensidad del color de hasta el 50\% durante los cinco primeros meses de envejecimiento, en los cuales el vino fue microoxigenado. En sus experiencias compuestos como los antocianos y taninos presentaron una disminución de su concentración, y los compuestos sulfurosos no deseados como el dimetil sulfuro no mostraron ningún incremento en su concentración. 
El efecto de la microoxigenación a nivel sensorial no está totalmente definido. Por ejemplo a nivel empírico se sabe que cuando el vino recibe oxígeno aparentemente puede tener más estructura, especialmente durante los primeros seis meses del envejecimiento; si durante los siguientes meses se observara una disminución considerable de los niveles de astringencia, el proceso de microoxigenación debería entonces interrumpirse porque podría alcanzarse un promedio de polimerizaciones de procianidinas demasiado alto, y como consecuencia se podría llegar a tener vinos con amargor o demasiado secos.

\subsubsection{Efecto del oxígeno durante el envejecimiento en botella}

En vista de la controversia que ha causado el cambio de los corchos tradicionales por los tapones sintéticos porosos, tanto por los aspectos científicos-industriales vinculados, como por la implicación cultural, una parte de nuestra atención ha estado centrada en estudiar la influencia de distintos tapones en esta parte del proceso del envejecimiento de los vinos. En esta fase, el vino es almacenado en botella pasando de un medio oxidativo a un medio reductor, y a partir de este momento es cuando el tapón actúa como una barrera selectiva para el oxígeno.

Diferentes trabajos publicados han demostrado que la difusión del oxígeno durante el tiempo que el vino se encuentra almacenado depende en gran medida del tipo de tapón, ya que la permeabilidad al oxígeno varía mucho entre los diferentes tipos corchos, y esta heterogeneidad es uno de los principales factores que pueden contribuir a la variación entre los vinos en su fase de envejecimiento en botella $[10,14]$.

Las principales características de los tapones dependen de su composición, es decir, de si trata de un corcho tradicional o un tapón sintético; y estos últimos, a su vez de cada una de las configuraciones disponibles (diferente geometría, porosidad, etc.) $[15,16]$. En general, los tapones sintéticos porosos ceden el paso al oxigeno al interior de la botella a una velocidad relativamente alta [17]. En el caso de tapones de corcho natural, la mayor parte del oxígeno se difunde a través de su estructura celular a una velocidad relativamente baja [18]. En principio, es posible aprovechar las ventajas del diseño "a medida" en la fabricación de tapones sintéticos de manera que su estructura porosa aporte cantidades controladas y reproducibles de oxígeno al vino embotellado. Bajo esta idea los vinos, se pueden elaborar preestableciendo un valor específico de la velocidad de transferencia de oxígeno (OTR), ventaja que no puede considerarse en los corchos naturales tradicionales.

La cuestión del OTR ha generado polémica desde tiempo atrás. Pasteur, en sus estudios realizados en 1873, fue el primero en estudiar el efecto del oxígeno en el 
desarrollo del vino. En su opinión "el oxígeno es el enemigo más grande del vino", pero también aseguró que "el oxígeno hace al vino, el cual envejece bajo su influencia" [19]. Por otro lado, Emile Peynaud consideró que "es el proceso contrario a la oxidación, es decir la reducción, la etapa en la cual evolucionan los vinos en la botella" [20]. En esta misma línea se encuentran las investigaciones de Jean Ribereau-Gayon, quien en sus estudios encontró que las cantidades de oxígeno que se difunden por medio de los tapones de corcho natural en los vinos embotellados son insignificantes pero de gran importancia [9]. En la actualidad, se admite que un cierto grado de oxigenación continua y controlada parece beneficiar la maduración del vino respecto a las reacciones entre polifenoles que conducen a estabilizar el color, reducir la astringencia e incluso cambiar algunos de sus matices aromáticos [21].

Los resultados de las valoraciones sensoriales publicadas hasta el momento son controvertidas; explican por ejemplo que los vinos sellados con tapones sintéticos porosos presentan una tendencia a perder los atributos relacionados con aromas frutales y desarrollar un carácter oxidado incluso en períodos de almacenamiento cortos [22]. Por otro lado, los vinos sellados con tapones de rosca han mostrado una mayor puntuación para los aromas frutales, manteniendo también niveles más altos para los antioxidantes, pero al mismo tiempo sus aromas con carácter reducido prevalecieron y la evolución del color se presentó de manera más lenta. Estudios recientemente publicados por Kwiatkowski [23] sugieren que el desarrollo del carácter reducido se debe en mayor medida a la baja difusión de oxígeno a través de los tapones y no tanto al oxigeno presente en el espacio en cabeza entre el vino y el tapón.

\subsubsection{Incorporación de oxigeno a través de los tapones sintéticos porosos}

El trabajo realizado por Jean Ribéreau-Gayon en la década de los 30, es uno de los primeros estudios reportados en el área del OTR. Estos resultados determinaron que la entrada de oxigeno a través de los distintos corcho naturales era demasiado variable, con registros entre 0,10 a $0,38 \mathrm{~mL}$ de oxígeno en las primeras tres semanas y entre 0 y $0,07 \mathrm{~mL}$ en los cuatro meses subsiguientes. No obstante, desde la época en la que Ribéreau-Gayon realizó este estudio, tanto los procesos de embotellado de los vinos, como el desarrollo de nuevas encorchadoras y tapones, han mejorado significativamente permitiendo controlar esta variabilidad.

Citar también otra investigación importante realizada por la empresa Southcorp Vino. En este trabajo se demostró la variabilidad que presenta el paso del oxígeno al interior de la botella en función del tapón utilizado. En el estudio llevado a cabo sobre un conjunto de 35 muestras selladas con diferentes tipos de corchos 
naturales, se valoró que las permeabilidades se encontraban en un rango de 0,001 $\mathrm{mL} / \mathrm{O}_{2} /$ día hasta llegar a obtener valores poco usuales de $1 \mathrm{~mL} / \mathrm{O}_{2} /$ día. En contraste a estos resultados, Duncan et. al. encontraron valores de 0.01 $\mathrm{mL} / \mathrm{O}_{2} /$ día para los tapones sintéticos porosos, disponibles en aquel momento [24].

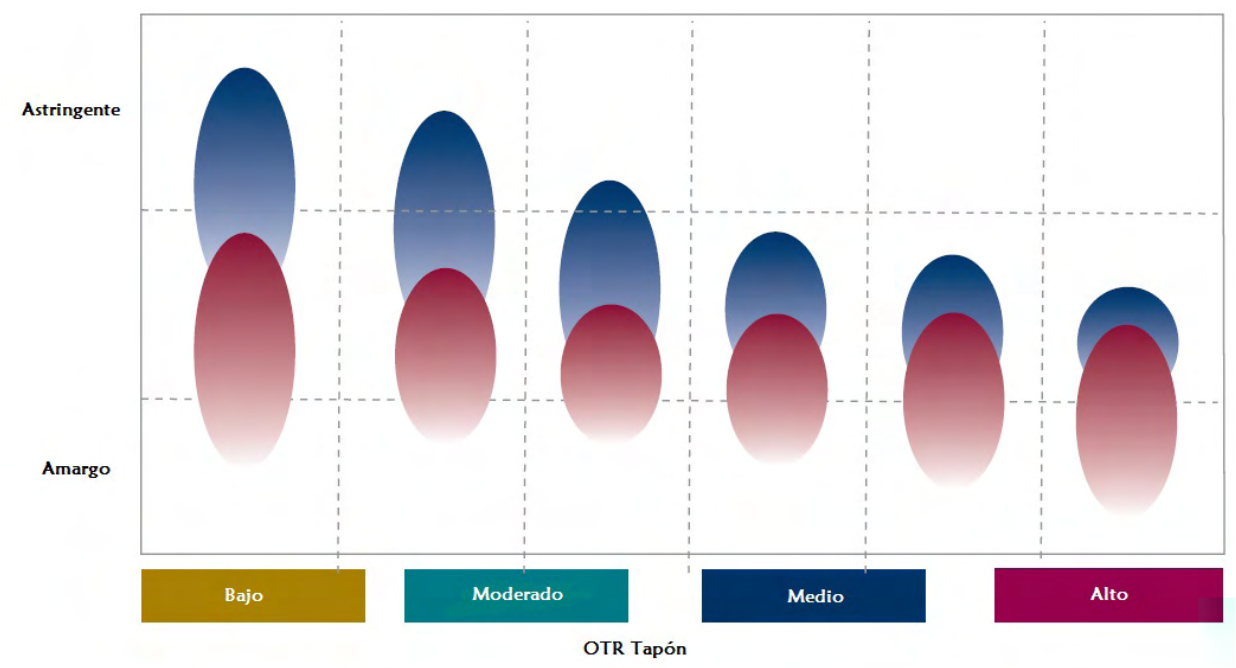

Figura 3.1. Cambios de la astringencia y el amargor en función del OTR.

Estos resultados y otros en esta línea han despertado una serie de preguntas en el campo de la enología, y entre ellas: ¿Cómo ha de ser la microoxigenación del vino de acuerdo con su naturaleza?, ¿Cada tipo de vino necesitará un OTR específico?, ¿Deberían las condiciones de almacenaje (temperatura, posición de la botella, tiempo de envejecimiento en botella) estar correlacionadas con la morfología del tapón?, etc. La contestación a estas preguntas da pie a plantearse la necesidad de disponer de métodos rápidos para el análisis de las características organolépticas de los vinos, resaltando la importancia de desarrollar un método de medida no invasivo y con mínimos errores experimentales. En esta línea, se basa la investigación que sintetizamos en este capítulo.

Buena parte de la atención a este tipo de cuestiones se ha invertido en la valoración sensorial de los efectos del OTR. Podemos decir que el efecto del OTR más conocido se asocia a los cambios en la acidez y el amargor. La Figura 3.1 explica como en función del OTR del tapón se puede presentar un cambio en las propiedades gustativas. Puede suceder por ejemplo, que un cambio en la acidez contribuya a que los polímeros condensados rompan sus cadenas y generen moléculas más pequeñas, lo cual se traduciría en un aumento del amargor. Por lo contrario un aumento en el grado de polimerización puede conducir a un incremento de la astringencia [25].

En parte, como consecuencia de los beneficios a nivel económico que genera el óptimo manejo de los vinos en botella, la industria enológica demuestra cada vez más interés en el uso de los tapones sintéticos porosos. Desde el año 2004 el 
consumo de estos tapones ha aumentado un $10 \%$ y ello ha sido debido a los avances en la tecnología de fabricación y a los resultados comparativos realizados en diferentes investigaciones. Un estudio publicado en año 2009 demuestra que los tapones sintéticos han llegado a ocupar un 30\% del mercado de las bodegas, especialmente de aquellas con la producción de grandes cantidades de vino. La Figura 3.2 indica como las grandes bodegas diversifican entre los cuatro tipos principales de tapones: corcho natural y técnico, tapón sintético y cierre de rosca.

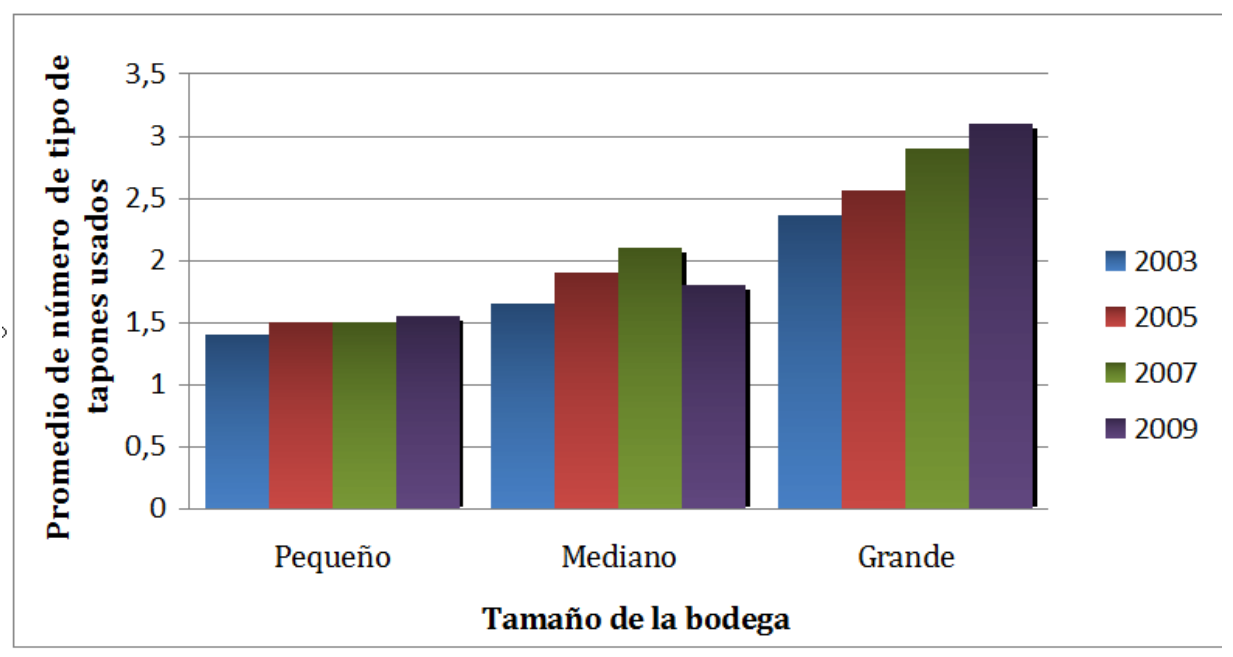

Figura 3.2. Incremento del uso de los diferentes tipos de tapones de acuerdo con el tamaño de las bodegas.

Hasta el momento se ha demostrado que existe una incidencia importante de la utilización del oxígeno o intervención por parte de quien elabora el vino, a nivel químico y por tanto a nivel organoléptico. Es importante señalar que en esta investigación no se pretende discutir las ventajas y desventajas del uso de un determinado tapón o cuales son las cantidades adecuadas de microoxigenación que se deben adicionar. Esta investigación solamente trata de identificar las posibilidades de nuestro sistema de medida para seguir la evolución del vino embotellado con diferentes tapones de origen sintético, y a través de herramientas quimiométricas, intentar predecir las consecuencias y características de los vinos, de acuerdo con las condiciones iniciales de elaboración. 


\subsection{DESARROLLO EXPERIMENTAL}

\subsubsection{Características de las muestras de vino}

Los vinos utilizados en este estudio se han elaborado utilizando la variedad de uva garnacha, añada del 2007. El proceso de vinificación se ha realizado en la estación experimental del Instituto Nacional de la Investigación Agraria (INRA) Pech Rouge (Gruissan, France). Un total de diez mil kilos de uva fueron cultivados, los cuales procedían de dos parcelas deferentes. Las uvas procedentes de la primera parcela $\left(22^{\circ}\right.$ Brix, $\left.\mathrm{pH}=3.6\right)$ se emplearon para la elaboración tradicional de vino (Trad) y los de la segunda parcela $\left(25^{\circ}\right.$ Brix, $\left.\mathrm{pH}=3.7\right)$ fueron usados para elaborar vinos con la técnica termovinificación (FR). La concentración de polifenoles resultantes puede relativizarse como baja en el primer caso y alta en el segundo. Cada uno de estos vinos (tradicional y termovinificación) se ha dividido en dos tanque para evaluar el efecto de la microoxigenación: en un tanque fueron adicionadas dosis controladas de oxigeno (Mox) y en el otro no (noMoX). Se tuvieron así cuatro tipos diferentes de vinos: FR, FR+Mox, Trad, Trad+Mox.

Tabla 3.1. Características de los vinos evaluados.

Microoxigenación Tipo de tapón

\begin{tabular}{|c|c|c|c|}
\hline \multirow{16}{*}{$\begin{array}{l}\text { Mosto } \\
\text { Garnacha }\end{array}$} & \multirow{8}{*}{$\begin{array}{l}\text { Método tradicional: } \\
\text { Nivel bajo de } \\
\text { polifenoles en } \\
\text { comparación el } \\
\text { método de } \\
\text { termovinificación }\end{array}$} & \multirow{4}{*}{$\begin{array}{c}\text { No } \\
\text { microoxigenados }\end{array}$} & Light 21 (T1) \\
\hline & & & Classic 21 (T2) \\
\hline & & & Classic 4 (T3) \\
\hline & & & Classic 1 (T4) \\
\hline & & \multirow{4}{*}{ Microoxigenados } & Light 21 (TM1) \\
\hline & & & Classic 21 (TM2) \\
\hline & & & Classic 4 (TM3) \\
\hline & & & Classic 1 (TM4) \\
\hline & \multirow{8}{*}{$\begin{array}{l}\text { Flash Release } \\
\text { (Termovinificación) } \\
\text { Nivel relativamente } \\
\text { alto de polifenoles. }\end{array}$} & \multirow{4}{*}{ Microoxigenados } & Light 21 (FM1) \\
\hline & & & Classic 21 (FM2) \\
\hline & & & Classic 4 (FM3) \\
\hline & & & Classic 1 (FM4) \\
\hline & & \multirow{4}{*}{$\begin{array}{c}\text { No } \\
\text { microoxigenados }\end{array}$} & Light 21 (F1) \\
\hline & & & Classic 21 (F2) \\
\hline & & & Classic 4 (F3) \\
\hline & & & Classic $1(\mathrm{~F} 4)$ \\
\hline
\end{tabular}

El tratamiento FR consiste en el despalillado y estrujado de las uvas a una temperatura de $95^{\circ} \mathrm{C}$ durante 6 minutos en condiciones de vacío (presión cercana 
a $60 \mathrm{hPa}$ ). Tanto para la maceración tradicional como para el ensayo de termovinificación, los mostos se distribuyeron en 2 tanques de fermentación de acero inoxidable de $50 \mathrm{hL}$ con control de temperatura, cantidades de sulfitos en torno a 5g.hL-1 y 500g de levadura (LBRouge, Lallemand). La tapa de los depósitos está diseñada de tal manera que permite mezclar el hollejo de las uvas con la fase líquida en el proceso de fermentación. Además, se ha introducido nitrógeno (20g.hL-1 DPA) después de una disminución de 30 puntos en el valor de la densidad. Una vez que la fermentación alcohólica ha finalizado (controlada por los azúcares reductores), los vinos fueron trasegados (adición de $\mathrm{O}_{2}$ : 0.3ppm). Se transfirieron a tanques de $20 \mathrm{hL}$ a los cuales se les añadió bacteria láctica (Lalvin VP41, Lallemand) para empezar la fermentación maloláctica. Al final de la fermentación malolática, los vinos se trasegaron a otros depósitos de acero (adición de $\mathrm{O}_{2}: 4 \mathrm{ppm}$ ) donde se incorporaron sulfitos en concentración de (3g.hL-1).

Cada uno de los vinos se transfirió desde los tanques de $20 \mathrm{hL}$ a cuatro tanques de un volumen de $2.7 \mathrm{hL}$ cada uno. Estos tanques fueron adaptados para llevar a cabo la microoxigenación con un sistema de 10-canales Oenode. Esta transferencia de oxigeno se incorporó en cantidades de $5 \mathrm{mg} \mathrm{O}_{2} \cdot \mathrm{L}^{-1} \cdot \mathrm{mes}^{-1}$ durante un período de 3 meses. Para el caso de las modalidades sin microoxigenación, los vinos fueron almacenados en tanques de características similares. Las cantidades totales de oxígeno introducido (adición debida al trasiego y a la microoxiganción) en estos 4 vinos (Tradmox, Trad, FRmox, FR), fueron estimadas como el resultado del promedio de cinco repeticiones diferentes, obteniendo los siguientes valores: $8.66,5.79,9,3.85 \mathrm{mg}^{-\mathrm{L}^{-1}}$, respectivamente.

Los vinos estudiados y su nomenclatura se resumen en la Tabla 3.1.

Posteriormente los vinos fueron almacenados en botellas de vidrio de $375 \mathrm{~mL}$ (Saint-Gobain bordelaise 39) en lugar de las botellas tradicionales (volumen de $750 \mathrm{ml}$ ) con el fin de amplificar el fenómeno de oxidación. Cada uno de los cuatro tipos de vinos se repartió en cuatro botellas con diferentes condiciones de OTR. Una primera serie de vinos se embotelló con tapones Nomacorc Light y otros tres vinos se almacenaron en botellas con corchos Nomacorc Classic, los cuales fueron designados con los códigos Light 21, Classic 21, Classic 4 y Classic 1. Todos fueron conservados en la misma habitación a temperatura constante $\left(23^{\circ} \mathrm{C}\right)$.

\subsubsection{Características de los tapones sintéticos porosos}

Los tapones suministrados por la empresa Nomacorc, Light 21, Classic 21, Classic 4 y Classic 1, se producen en un proceso de co-extrusión de polietileno de baja densidad (LDPE), material que es permeable al oxígeno y en su superficie estos 
tapones tienen un film de un compuesto diferente (silicona). Los materiales fueron elaborados bajo distintas condiciones y formulaciones de manera que se pudieran obtener tapones sintéticos que permitieran diferentes niveles de difusión del oxígeno. La Figura 3.3 muestra la capacidad de difusión del oxígeno de cada uno de los tapones sintéticos porosos; estos datos también fueron suministrados por la empresa Nomacorc.

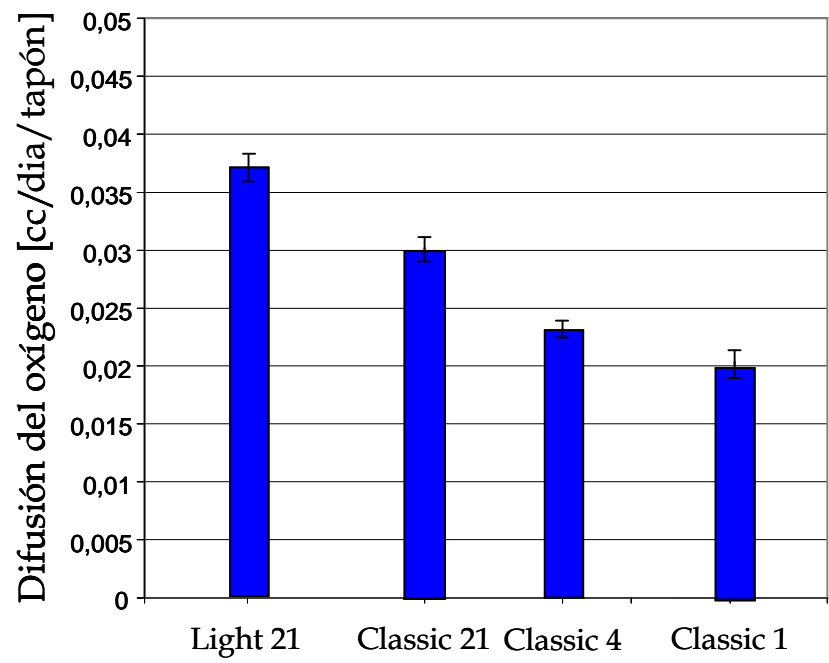

Figura 3.3. Difusión del oxígeno a través de los diferentes tapones sintéticos

Para controlar la capacidad de difusión del oxígeno del material es importante identificar cual es la propiedad macroscópica del material que propicia un mayor o menor nivel de oxígeno a través de su estructura. Hemos realizado una caracterización del material teniendo en cuenta tres propiedades importantes: el tamaño del poro, la densidad y el índice de la cristalinidad del polímero base. Comentar además que los tapones en los cuales se ha realizado este estudio tienen las mismas características de fabricación que la de aquellos que fueron utilizados en las botellas que contienen las muestras. Sin embargo los resultados que mostraremos a continuación son parte de un estudio independiente; es decir que estos tapones no estuvieron sometidos al proceso mecánico que supone su inserción en la botella, y además tampoco estuvieron en contacto con el vino.

El tamaño de los poros de cada tipo de tapón ha sido visualizado en nuestro departamento a partir de imágenes tomadas con un microscopio electrónico de barrido SEM (JSM-820 Jeol, Japan). La Figura 3.4 representa una de las imágenes obtenidas con estos tapones sintéticos. De la observación de esta mircrografía puede afirmarse que existe una distribución no homogénea del tamaño del poro, y que además este material no muestra un alto grado de conectividad en su estructura celular, es decir no hay canales abiertos que comuniquen directamente la parte superior e inferior del corcho para el paso del oxígeno. 


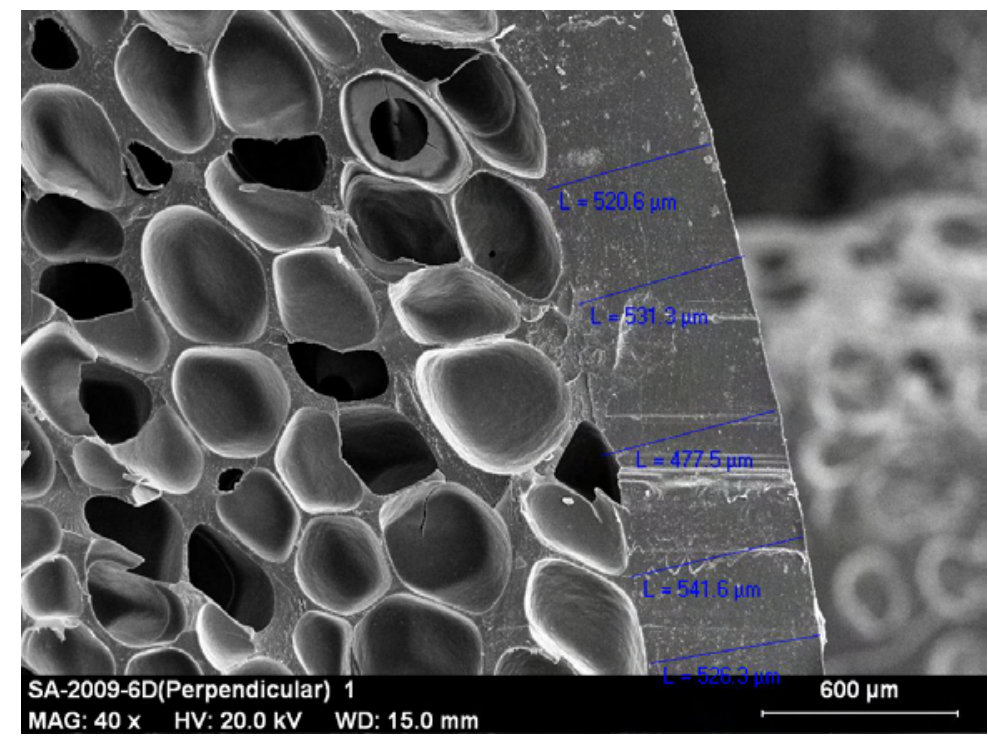

Figura 3.4 Micrografía SEM de los tapones sintéticos porosos utilizados en este estudio (dirección perpendicular al proceso de extrusión).

Otra posibilidad para el transporte del oxigeno viene determinada por la difusión de celda en celda a través de las paredes celulares. Esta propiedad de los materiales parecía podía ser evaluada haciendo una comparación de la densidad de cada tapón sintético porque en cuanto menor sea el número de paredes celulares a atravesar más sencillo sería el paso del oxígeno a través de su estructura. Así en principio se supuso que la densidad había de ser fundamental en el proceso de difusión de oxígeno a través del tapón sintético. Sin embargo una vez realizados el conjunto de medidas de la densidad en la balanza Metter AT261 (principio de Arquímedes), se pudo observar que para los valores de la capacidad de difusión del oxígeno en las muestras estudiadas se registra una densidad del material similar (Figura 3.5.).

Ahora bien, en materiales como estos tapones sintéticos porosos, el paso del oxigeno depende de dos factores principales. El primero de ellos es la estructura celular. El segundo es la permeabilidad intrínseca del polímero, de la cual podemos decir que es una característica controlada por su cristalinidad, característica que hemos determinado a partir de medidas de calorimetría diferencial de barrido (DSC). 


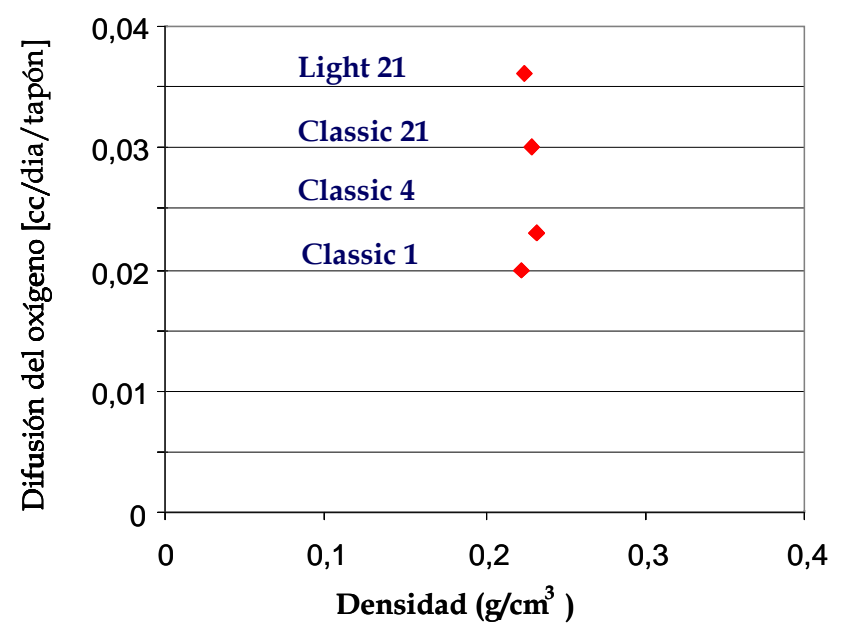

Figura 3.5. Medidas de la densidad en función de la capacidad de difusión del oxígeno de los diferentes tapones sintéticos.

En la Figura 3.6. hemos representado los termogramas correspondientes a las 4 muestras (tapones) utilizados en esta investigación. Los valores de la cristalinidad del polietilieno se calcularon a partir del pico endotérmico del segundo calentamiento, el cual es una repetición del primer barrido que se ha realizado para borrar la historia térmica de las muestras. De acuerdo con los resultados señalados en esta figura las diferencias entre los tipos de tapones son significativas respecto a su cristalinidad, la cual varía entre $34 \%$ para el tapón Light 21 y $45 \%$ correspondiente al tapón Classic 1.

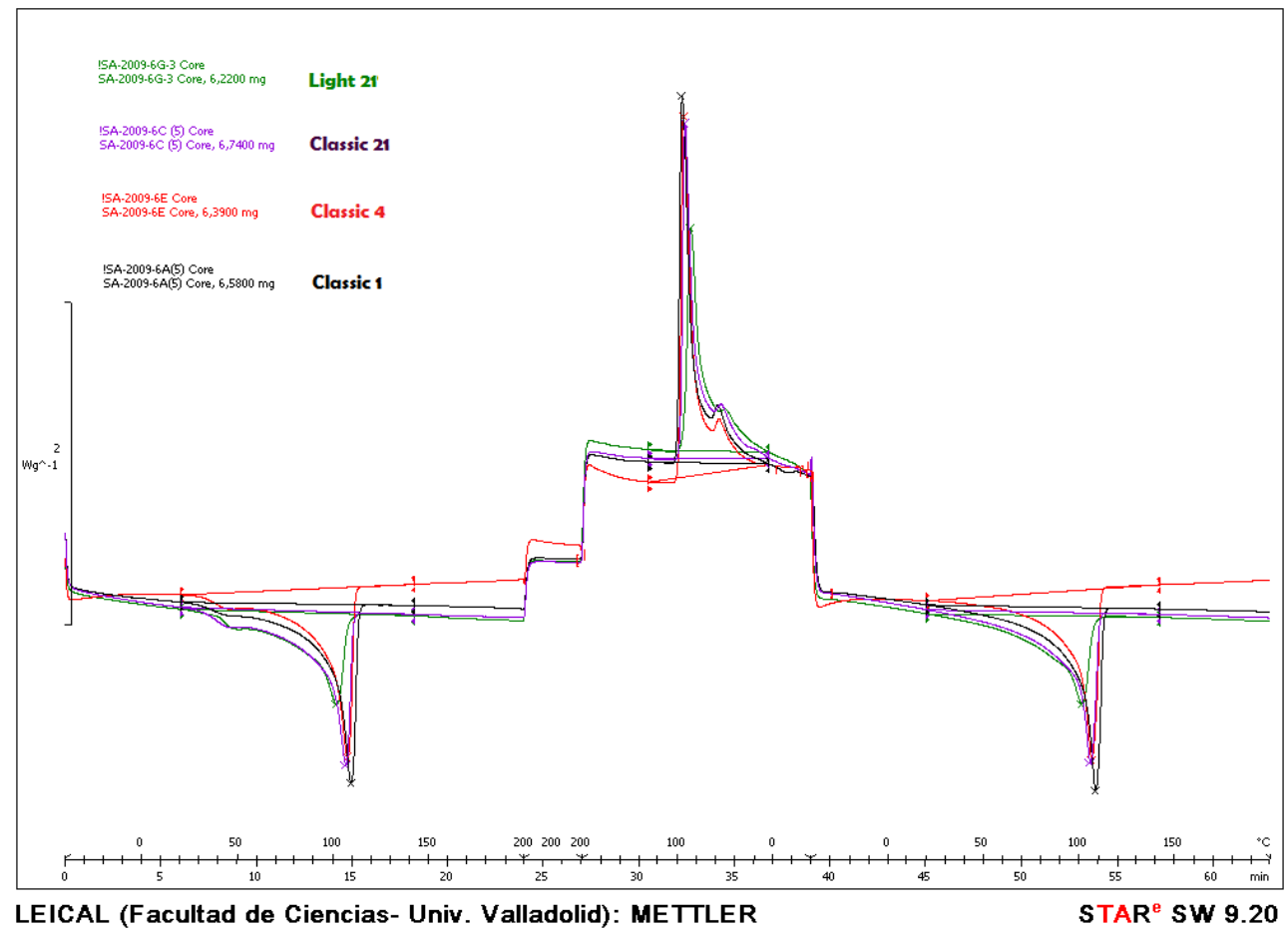

Figura 3.6. Resultados DSC para los tipos de tapones sintéticos porosos utilizados. 
A partir de estos valores se ha construido la Figura 3.7 la cual ilustra los valores de la capacidad de difusión del oxígeno en función de la cristalinidad del material. Como específica la figura, el grado de cristalinidad del polímero es la propiedad del material que más influencia tiene sobre la capacidad de difusión del oxígeno, confirmando así nuestra hipótesis de partida. Esto es debido a que el gas solo puede penetrar y difundirse en las zonas amorfas del polímero, por lo que cuanto menos zonas amorfas haya (mayor cristalinidad) menor ha de ser la difusividad del oxígeno.

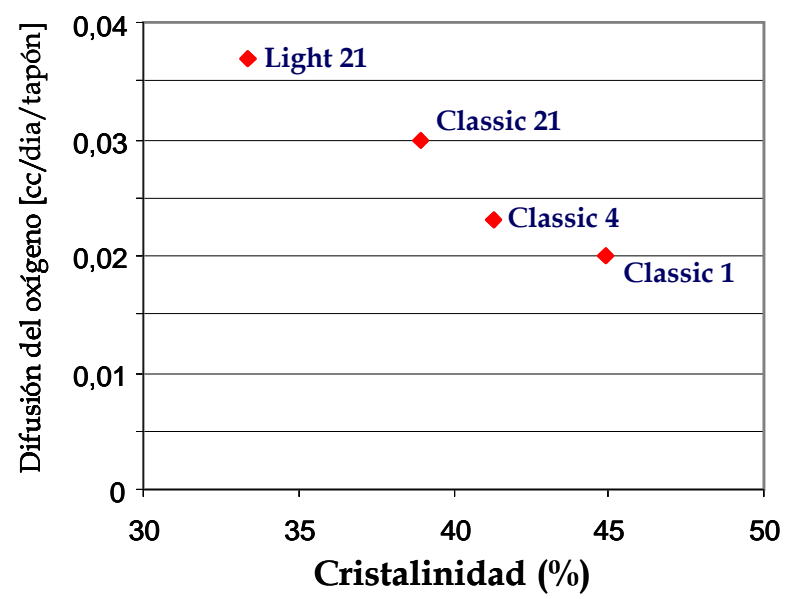

Figura 3.7. Medidas de la cristalinidad en función del OTR de los diferentes tapones sintéticos.

\subsubsection{Medidas del oxígeno en durante el envejecimiento en botella}

Los tres parámetros seguidos durante esta investigación, es decir el oxigeno disuelto (DO), el oxígeno en el espacio en cabeza (HO), y la contribución del tipo de tapón sintético a la exposición de oxígeno (CL) han sido valorados por el grupo Nomacorc utilizando un sistema denominado Fibox 3-Trace (Preseas Precision Sensing GMBH, Regensburg, Germany). El equipo se compone de un sensor de oxígeno (pastilla reactiva luminiscente) y esta equipado con una fibra óptica que lleva la luz hasta los sensores y recoge su respuesta luminosa, proporcional a la concentración de oxígeno que les rodea [26]. Un primer sensor se sitúa dentro de la botella y entra en contacto con el vino directamente; así se realiza la medida del oxígeno disuelto (DO) diez meses después de comenzar el envejecimiento en botella. Un segundo sensor es pegado en la parte superior de la botella en este caso para realizar las valoraciones respecto al espacio en cabeza (HO) [27]. El valor determinado para cada uno de los parámetros es el resultado obtenido del promedio de cinco repeticiones para cada tipo de medida. El 
parámetro CL está determinado por la capacidad de difusión del oxígeno y la cantidad de oxigeno inicialmente contenida dentro del tapón sintético poroso.

El resultado de los valores $\mathrm{DO}, \mathrm{HO}, \mathrm{CL}$ así como el tratamiento de datos de estas variables esta indicado en el apartado 3.3.2.2. (Tabla 3.11).

\subsubsection{Medidas de los compuestos polifenólicos}

A continuación citaremos los 22 parámetros valorados y su respectiva nomenclatura para estudiar el efecto del método de extracción del mosto y la dosificación del oxígeno con la microoxigenación y su control durante el envejecimiento en botella (Tabla 3.2).

Tabla 3.2. Parámetros químicos evaluados

Parámetro Químico

Nomenclatura

\begin{tabular}{|c|c|}
\hline Antocianos [mg. L-1] & $\mathrm{ACN}$ \\
\hline Taninos [mg. $\left.\mathrm{L}^{-1}\right]$ & TAN \\
\hline Relación taninos/antocianos & $\mathrm{T} / \mathrm{A}$ \\
\hline Catequinas [mg.L.-1] & CAT \\
\hline Ácido hidroxicinámico [mg.L-1] & HA \\
\hline Piranoantocianos (vitisinas) [mg. $\left.\mathrm{L}^{-1}\right]$ & CPA \\
\hline $\begin{array}{l}\text { Catequina, epicatequina y malvidina-3-O-glucósido unidos } \\
\left.\text { por puentes etilo [mg. } \mathrm{L}^{-1}\right]\end{array}$ & EB \\
\hline Flavonoles $\left[\mathrm{mg} . \mathrm{L}^{-1}\right]$ & FLV \\
\hline Piranoantocianos vinilfenólicos [mg.L-1 $]$ & $\mathrm{VP}$ \\
\hline Grado medio de polimerización & $\mathrm{mDP}$ \\
\hline Porcentaje de ácido gálico. & \%Gall \\
\hline Porcentaje de galato de epicatequina & \%EGC \\
\hline Intensidad del color & IC \\
\hline Intesidad de color corregida & ICcor \\
\hline Corrección de Hue & Tcor \\
\hline Color total de pigmentos & CTP \\
\hline Índice de polifenoles totales & IPT \\
\hline Pigmentos decolorables totales & PDT \\
\hline Edad química del vino & $\mathrm{ACV}$ \\
\hline Porcentaje de pigmentos decolorables por bisulfito & $\%$ PDSO2 \\
\hline Pigmentos totales & PV \\
\hline Color debido a la copigmentación & $\mathrm{CC}$ \\
\hline
\end{tabular}


Comentar además que este trabajo se realizó en colaboración con el "Centre de Recherche en Gestion de l'Oxygène" del "Institut Rhodanien d'Orange", en Francia y la empresa Nomacorc en Bélgica.

Como se indicó anteriormente, nuestro objetivo era establecer la correlación entre los parámetros del nivel de oxígeno y contenido polifenólico con los resultados obtenidos mediante los sistemas de evaluación sensorial electrónicos, utilizando un análisis de regresión lineal PLS2. Indicar que debido a la gran cantidad de variables asociadas al contenido fenólico, se llevaron a cabo análisis previos de varianza ANOVA, para identificar los parámetros más relevantes y así reducir el valor de la relación entre variables y muestras para el análisis PLS2.

Por el contrario, en relación con el oxígeno, existen solamente tres parámetros que fueron medidos directamente: DO, HO y CL. En vista de los objetivos de este estudio en particular, no consideramos necesario realizar esta selección preliminar con el ANOVA debido fundamentalmente a que el número de variables es mucho menor al número de muestras evaluadas. Es por ello que estas tres variables fueron directamente incluidas en el análisis PLS2 llevado a cabo en el apartado 3.3.2.2.

\subsection{RESULTADOS}

\subsubsection{Análisis Exploratorio}

Los espectros UV-Visible registrados para el conjunto de muestras evaluadas señalan principalmente un aumento en la intensidad de la absorción en función del aumento del OTR de los diferentes tapones (Figura 3.8). Los vinos que fueron sellados con los tapones Light 21 y Classic 21 presentaron los valores más altos en la intensidad de absorción, mientras que los vinos sellados con los tapones Classic 4 y Classic 1 registraron una menor intensidad en los espectros. Por otra parte, las diferencias entre Classic 4 y Classic 1 fueron poco significativas en comparación con las diferencias entre Light 21 y Classic 21. Esto fenómeno podría explicarse por el hecho de que vinos almacenados en botellas con tapones de mayor valor de OTR presentan una mayor difusión de oxígeno, y ello debido a que estas moléculas puede penetrar a través el tapón, recorriendo las zonas amorfas del polímero que le compone. Como se ha experimentado en la caracterización del material, un mayor porcentaje de cristalinidad del polímero (menor porcentaje de fase amorfa) implica un menor OTR del tapón. El aumento 
de oxígeno se traduce entonces en un incremento de la absorbancia de las muestras de vino, especialmente en la zona alrededor de los $520 \mathrm{~nm}$, propiciando que las reacciones entre antocianos sean más lentas. De esta manera se puede disminuir el porcentaje de antocianos altamente polimerizados y por tanto disminuir la probabilidad de precipitación de los polifenoles, con lo cual se tiene una mayor estabilidad en el color.

\subsubsection{Coordenadas CIELab}
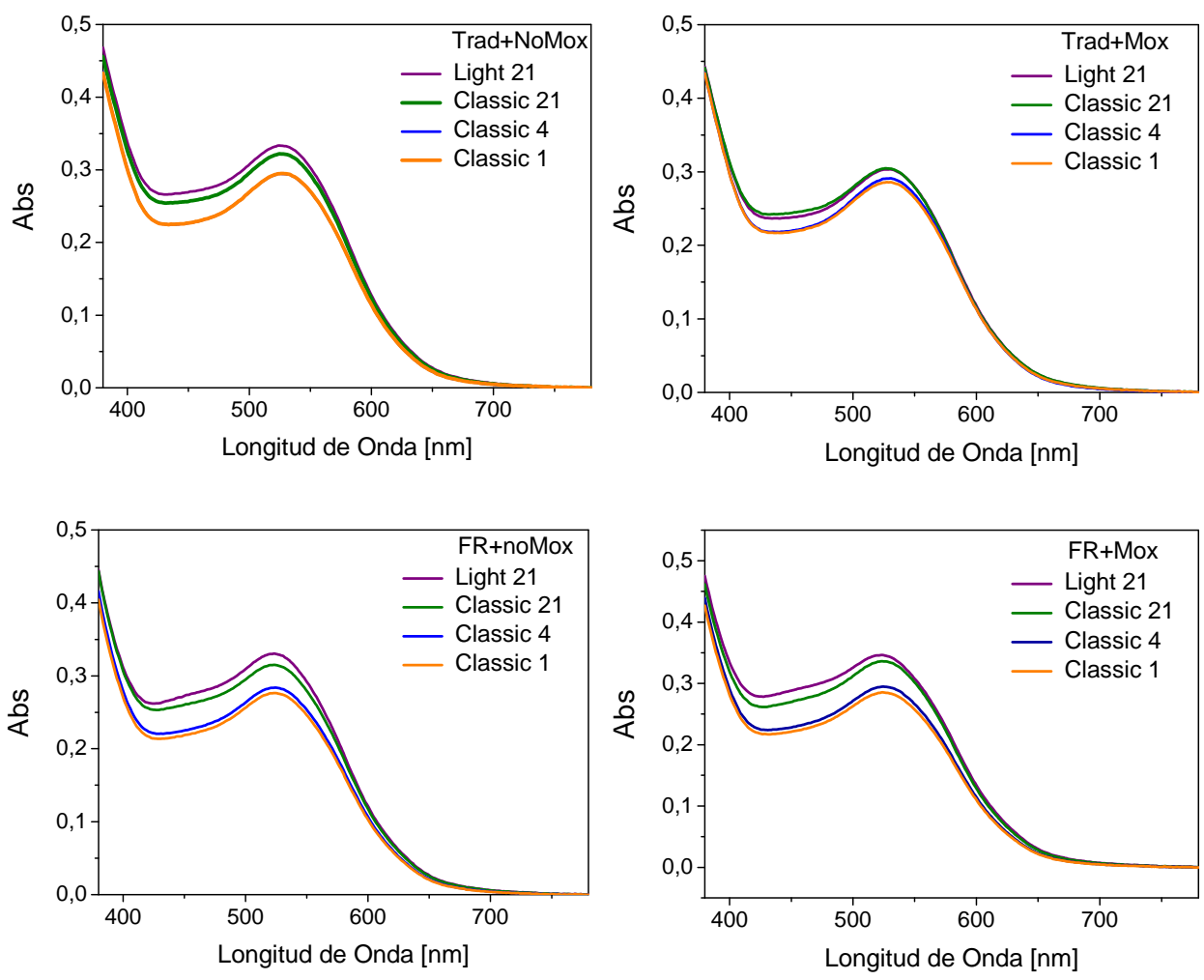

Figura 3.8. Espectros UV-Visible registrados para todo el conjunto de vinos.

Para continuar con el análisis de estos resultados, las señales fueron parametrizadas utilizando sus coordenadas CIELab. Los resultados obtenidos se especifican en la Tabla 3.3 y de ellos se deduce que el OTR afecta fuertemente a la luminosidad del color $L$ de los vinos. Los valores mas altos registrados para esta coordenada -refiriéndose 0 al negro y 100 al blanco- corresponden a vinos embotellados empleando los tapones con el OTR más bajo, Classic 4 y Classic 1 con valores entre 25.09 y 27.10. Los valores inferiores para el parámetro L correspondieron a los vinos embotellados con los tapones con un superior OTR: Light 21 y Classic 21, que registraron valores entre 21.10 y 24.59 . 
Tabla 3.3. Resultados de las coordenadas CIELab

\begin{tabular}{l||l|l|l|l|l|l|} 
Sample & $\mathrm{L}$ & $\mathrm{a}^{*}$ & $\mathrm{~b}^{*}$ & $\mathrm{C}$ & $\mathrm{H}$ & $\mathrm{S}$
\end{tabular}

\begin{tabular}{|c|c|c|c|c|c|c|c|}
\hline \multirow[t]{4}{*}{ Trad+noMicro } & Light 21 (T1) & 22,32 & 52,70 & 35,69 & 63,65 & 34,10 & 2,85 \\
\hline & Classic 21 (T2) & 24,59 & 54,20 & 37,15 & 65,70 & 34,42 & 2,67 \\
\hline & Classic 4 (T3) & 21,10 & 51,54 & 34,50 & 62,02 & 33,80 & 2,94 \\
\hline & Classic 1 (T4) & 23,32 & 53,38 & 37,39 & 65,18 & 35,00 & 2,80 \\
\hline \multirow[t]{4}{*}{ Trad+Micro } & Light 21 (TM1) & 23,22 & 53,38 & 36,30 & 64,56 & 34,22 & 2,78 \\
\hline & Classic 21 (TM2) & 24,29 & 54,04 & 36,04 & 64,96 & 33,70 & 2,68 \\
\hline & Classic 4 (TM3) & 22,22 & 52,65 & 35,43 & 63,46 & 33,94 & 2,86 \\
\hline & Classic 1 (TM4) & 23,98 & 53,64 & 37,72 & 65,57 & 35,12 & 2,73 \\
\hline \multirow[t]{4}{*}{ FR+noMicro } & Light 21 (FM1) & 25,40 & 54,99 & 36,10 & 65,78 & 33,29 & 2,59 \\
\hline & Classic 21 (FM2) & 25,55 & 55,06 & 35,17 & 65,34 & 32,57 & 2,56 \\
\hline & Classic 4 (FM3) & 25,09 & 54,64 & 35,96 & 65,41 & 33,35 & 2,61 \\
\hline & Classic 1 (FM4) & 26,45 & 55,15 & 37,59 & 66,74 & 34,28 & 2,52 \\
\hline \multirow[t]{4}{*}{ FR+Micro } & Light 21 (F1) & 25,13 & 54,78 & 35,98 & 65,54 & 33,30 & 2,61 \\
\hline & Classic $21(\mathrm{~F} 2)$ & 25,49 & 54,97 & 34,95 & 65,14 & 32,45 & 2,55 \\
\hline & Classic 4 (F3) & 25,98 & 55,05 & 36,11 & 65,83 & 33,26 & 2,53 \\
\hline & Classic $1(\mathrm{~F} 4)$ & 27,10 & 55,45 & 37,52 & 66,95 & 34,09 & 2,47 \\
\hline
\end{tabular}

La coordenada S, es una relación de la coordenada C y la coordenada L, y por tanto no presenta una tendencia clara que nos permita decir si aumenta o disminuye en función del OTR.

El efecto del tipo de estrujado de la uva es evidente en los valores registrados para la coordenada $a^{*}$. Esta coordenada es una medida del color rojo; en este caso y como era de esperarse, los vinos con mayor contenido polifenólico, -aquellos que fueron elaborados con la técnica de la termovinificación-, presentaron valores superiores a 54.64, mientras que para el caso de las muestras maceradas con el método tradicional, este valor osciló entre 51.54 y 54.20 unidades.

Finalmente, para el caso de las coordenadas $b^{*}$ (relacionada con el color amarillo), C (saturación o croma), y H (tonalidad) no se observó una tendencia clara que permita establecer si existe algún efecto del oxígeno o del contenido polifenólico en sus diferentes valores del envejecimiento en botella.

En general, es posible observar que los polifenoles del vino participan en diferentes reacciones debido a los cambios en la concentración de oxígeno presente en el espacio en cabeza de la botella, lo cual puede dar lugar a nuevos pigmentos y a un cambio en la intensidad cromática en los diferentes vinos. 


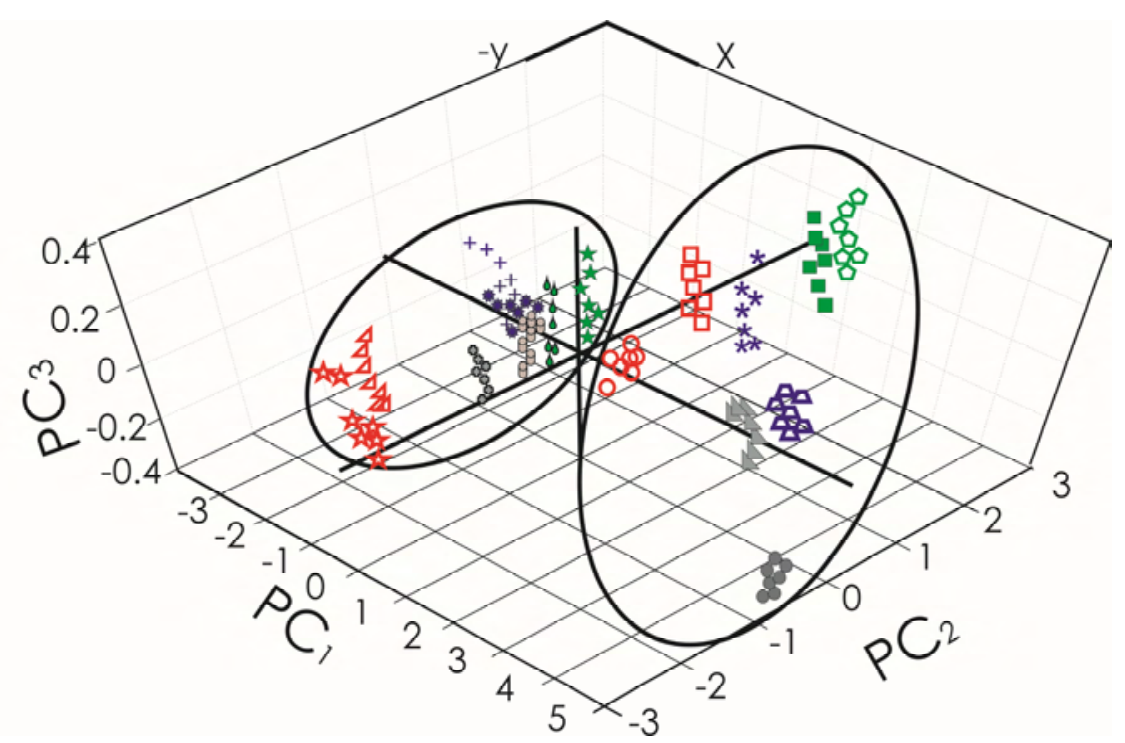

Figura 3.9. PCA de las coordenadas CIELab (69\%, 30\%, 1\%).

Vinos elaborados según el método de extracción tradicional y no microoxigenados $\mathrm{T}(\mathrm{T} 1 \boldsymbol{\Delta} \mathrm{T} 2 * \mathrm{~T} 2+\mathrm{T} 3 *)$. Vinos con método de extracción tradicional y microoxigenados TM (TM1 TM2。 TM3 $\Delta$ TM4 $\star$ ). Vinos con método Flash Realase y microoxigenados FM ( FM1・FM2 \& FM3・ FM4 ๘). Vinos con método Flash Realase y no microoxigenados F (F1 ๖ F2 $\mathrm{F} 3 \star \mathrm{F} 4 \bullet)$

La representación de los espectros en término de las 6 coordenadas CIELab nos ha permitido observar más claramente que es el OTR el factor que más afecta a las distintas características del color. Para comprobar este resultado se ha realizado un análisis PCA utilizando como variables de entrada las coordenadas CIELab. El gráfico de los "scores" del correspondientes PCA se muestra en la Figura 3.9. Como se puede observar, la primera componente principal (PC1), que contribuye al 69\% de la información, distingue los vinos dependiendo del tipo de corcho. Los vinos sellados con los tapones sintéticos con menor porcentaje de cristalinidad y mayor OTR (Light 21 y Classic 21) aparecen ubicados en la parte derecha de la gráfica, mientras que los vinos sellados con los tapones sintéticos Classic 4 y Classic 1 aparecen en la parte izquierda del gráfico. Estos últimos no se distinguen claramente, lo cual es consecuencia de la similitud de los espectro UV-Visibles registrados. Los resultados son coherentes con las condiciones iniciales de embotellado, ya que los vinos con tapones Classic 4 y Classic 1 dan paso a cantidades de oxígeno similares.

\subsubsection{Nariz electrónica}

El primer acercamiento a los datos resultantes de la nariz electrónica se realizó mediante un PCA. Los valores registrados para el punto máximo que se presenta en la señal del cambio de las resistencias de los sensores durante la inyección de los compuestos volátiles de los vinos, fueron los valores utilizados como 
variables de entrada para el análisis multivariante. Las técnicas empleadas son las decrritas en el canítulo II

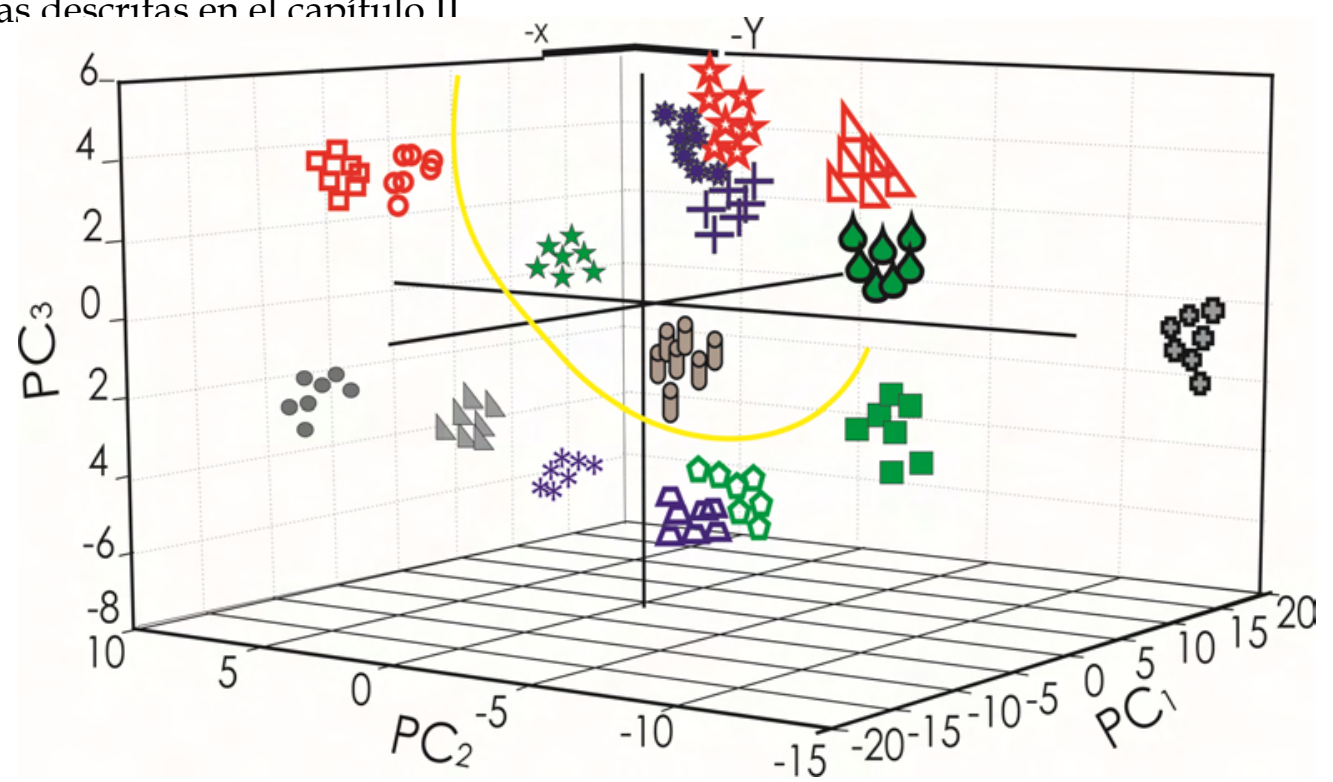

Figura 3.10. PCA de la nariz electrónica.

Vinos elaborados según el método de extracción tradicional y no microoxigenados T (T1

- $T 2 * T 2+T 3$ ( Vinos con método de extracción tradicional y microoxigenados TM

(TM1 TM2O TM3 $\triangle$ TM4 خ). Vinos con método Flash Realase y microoxigenados

FM (FM1•FM2 $\triangle F M 3$ FM4 ๘). Vinos con método Flash Realase y no microoxigenados $F(F 1 \square F 2 \square F 3 \star F 4$ ๑).

La Figura 3.10 muestra el gráfico de los "scores" para las tres primeras componentes principales ( $\mathrm{PC} 1=68 \%$, PCA2 $=18 \%$ y $\mathrm{PCA} 3=6 \%$ ). En general es posible diferenciar entre los tipos de vinos evaluados, a excepción de los vinos W3 y W4, para los cuales las señales registradas fueron bastante similares. Los vinos embotellados con un alto valor de OTR (tapones Light 21 y Classic 21) aparecen en la parte izquierda de la figura (eje $X$ negativo). En la región del eje $X$ positivo se encuentran los vinos embotellados con tapones que presentan un valor bajo de su OTR. Las diferencias en términos de microoxigenación o contenido polifenólico, a nivel visual de las PCs, parecen menos relevantes.

Los resultados muestran que el OTR ha tenido un impacto importante en las características aromáticas de los vinos. En efecto, de acuerdo con el OTR que se difunde a través de los tapones, estos vinos presentan distintas notas olfativas: los vinos almacenados con OTR de valores altos presentaron un carácter algo más frutal y, por el contrario, los vinos embotellados con OTR de valores más bajos presentaron notas con un carácter reductor. Estos resultados anteriormente explicados coinciden con la investigación de Soline Caillé et al. [28], efectuada valorando los efectos del OTR en un conjunto de vinos tintos, para los cuales se estableció que un mayor paso del oxigeno contribuye a mejorar el sabor del vino, y además se perciben menos defectos aromáticos que en los vinos almacenados con un OTR menor. 


\subsubsection{Lengua electrónica}

La Figura 3.11 muestra el gráfico de los "scores" de la PCA para la valoración realizada con la lengua electrónica según los protocolos definidos en el capítulo II. Se observa un solapamiento parcial entre los vinos estudiados; también se aprecian un cierto grado de discriminación entre vinos con alto contenido polifenólico (parte izquierda de la gráfica) y vinos con bajo contenido en polifenoles (parte derecha del gráfico). Además, los vinos no microoxigenados tienden a aparecer en la parte superior y los microoxigenados en la parte inferior del gráfico. El porcentaje de la varianza explicada obtenida para este análisis fue: $35 \%$ para $\mathrm{PC} 1,18 \%$ para PC2 y $11 \%$ para PC3.

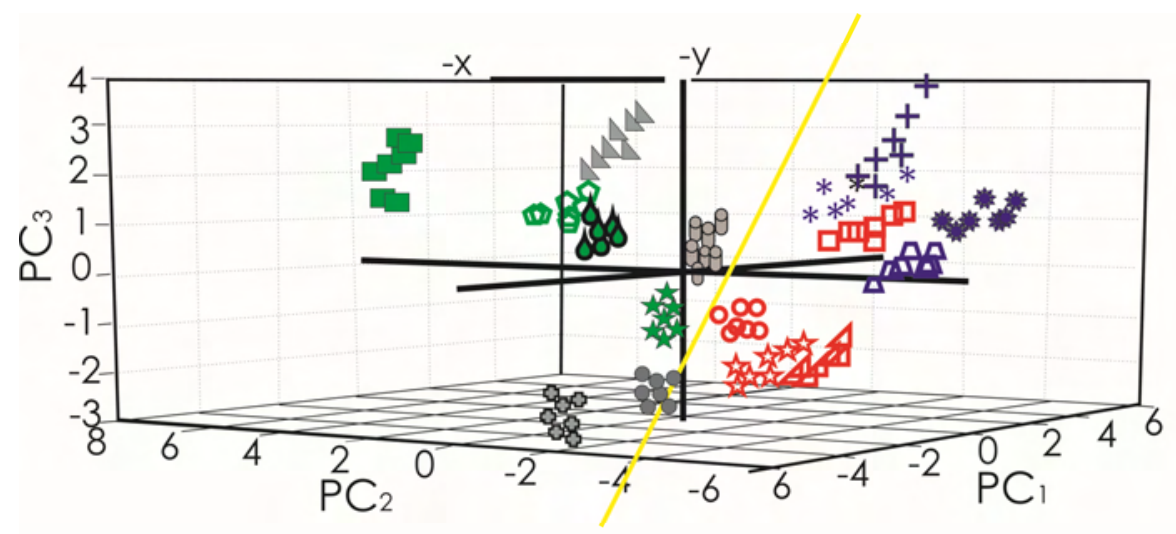

Figura 3.11. PCA de la lengua electrónica.

Vinos elaborados según el método de extracción tradicional y no microoxigenados T (T1 - T2* T2+ T3 ). Vinos con método de extracción tradicional y microoxigenados TM

(TM1 $\square$ TM2० TM3 $\triangle$ TM4 $\star$ ). Vinos con método Flash Realase y microoxigenados

FM (FM1•FM2 $\triangle F M 3 \ominus F M 4$ \#). Vinos con método Flash Realase y no microoxigenados $F(F 1 \square F 2 \square F 3 \star F 4 \bullet)$

Las variables utilizadas para el análisis con la lengua electrónica se seleccionaron a través de una descomposición del voltagrama de cada sensor en 10 kernels. Esto implica un número de variables seleccionadas $(8$ sensores $\times 10$ variables $=80$ variables) relativamente alto en comparación que las utilizadas en las experiencias con la nariz electrónica y las coordenadas CIELab.

El siguiente paso de nuestra investigación requirió la fusión de los datos de los tres sistemas. Como además se trata de una análisis exploratorio y no supervisado, era importante que el número de variables fuera lo más parecido posible entre cada uno de los sistemas a fusionar, de manera que ningún sistema condicione las tendencias generales para la visualización de los resultados. Por 
este motivo, se seleccionaron determinadas variables para cada sensor, teniendo en cuenta los resultados de los loadings del análisis PCA, escogiendo aquellas variables que aportaban una mayor cantidad de información (3 variables por sensor). Esta selección ha permitido tener un nuevo conjunto de 24 variables.

\subsubsection{Panel electrónico}

Un panel electrónico ha de combinar las señales provenientes de los 3 sistemas en una matriz de datos formada por 6 coordinadas CIELab (color), 15 variables extraídas de las señales generadas por los 16 sensores MOX (aroma) y 24 datos seleccionados a partir de las curvas voltamétricas (sabor) proporcionadas por los sensores electroquímicos (3 variables por cada sensor), para así emplearlas en el análisis de datos multivariante. Las primeras componentes principales, mostradas en la Figura 3.12., capturan el 29\%, 19\% y 12\% de la varianza total. Los clusters de la PCA son pequeños, mostrando que la varianza experimental es más pequeña que las diferencias existentes entre las muestras evaluadas. Esto significa que la fusión de las 3 redes de sensores mejora la discriminación de acuerdo con el tipo de tapón (distinto OTR), el contenido polifenólico y la microoxigenación. En la parte de eje $\mathrm{X}$ negativo del gráfico se pueden observa los vinos embotellados con tapones de alto OTR para los cuales el oxígeno puede penetrar y difundirse más fácilmente a través de las zonas amorfas del polímero (Light 21\% y Classic 21\%), mientras que los vinos embotellados con bajo nivel de OTR (Classic 4\% y Classic 1\%) aparecen en la región del eje X positivo. Al mismo tiempo, los clusters correspondientes a los vinos microoxigenados (vinos marcados con el color gris y rojo) tienden a aparecer en la parte derecha de la gráfica (eje Y negativo).

Respecto a los vinos con diferente contenido en polifenoles, estos aparecen bien separados en la PCA. Los vinos FM y F marcados con el color gris y verde, se encuentran ubicados en la parte derecha de la gráfica. Mientras que los vinos T y TM aparecen en la parte izquierda de la gráfica marcados con el color azul y rojo, respectivamente.

Es razonable que el sistema completo muestre una tendencia mayor a la dferenciación de los vinos de acuerdo con el tipo de extracción de compuestos volátiles que a otro factor como la microoxigenación. Las muestras de vinos procesadas mediante la técnica de termovinificación contienen una mayor cantidad de taninos. Esta concentración aumenta la astringencia, el grado de polimerización y el porcentaje de esteres del ácido gálico [27], compuestos que han demostrado tener buenas correlaciones con nuestros sistemas de valoración electrónica [29]. Respecto a la microoxigenación, debido a que esta técnica es utilizada para estabilizar el color, y además para producir la reacción entre 
taninos que a nivel sensorial significa una reducción de la astringencia. Esta formación compuestos a base de taninos se produce a través de puentes etilo debido a la reacción del oxígeno y el etanal [1]. Esto significa que hay una cierta dependencia de los dos factores; las características gustativas dependen de las técnicas de extracción, y el oxígeno presente en el medio facilita la expresión de estas características.

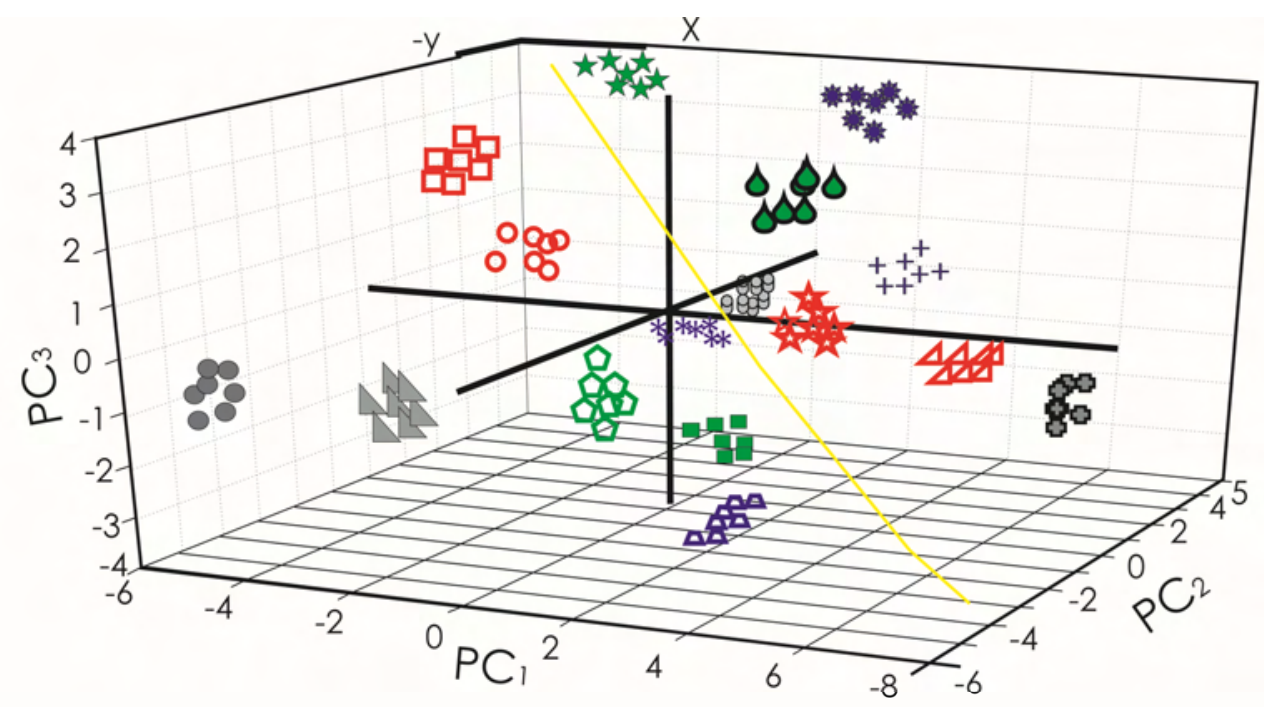

Figura 3.12. PCA del Panel de cata electrónico.

Vinos elaborados según el método de extracción tradicional y no microoxigenados $T$ (T1 - T2* T2+ T3 . Vinos con método de extracción tradicional y microoxigenados TM (TM1 TM20 TM3 $\triangle$ TM4 «). Vinos con método Flash Realase y microoxigenados FM (FM1•FM2 - FM3 FM4 \&). Vinos con método Flash Realase y no microoxigenados $F(F 1 \square F 2 \square F 3 \star F 40)$

Los resultados anteriores implican que la combinación de los sistemas electrónicos puede permitir la discriminación simultánea de acuerdo al tipo de corcho (gracias a la información provista por los sistemas de nariz y coordenadas CIELab) y de acuerdo a los niveles de polifenoles, asociando a la información fundamentalmente proporcionada por la lengua electrónica.

Una vez realizado el análisis exploratorio de los datos originados por los tres sistemas de caracterización sensorial electrónica y determinada la compatibilidad que existe entre los mismos cuando sus respectivos datos fueron fusionados, hemos realizado una evaluación de la capacidad de clasificación del sistema mediante un análisis PLS-DA. 


\subsubsection{Análisis supervisado}

\subsubsection{Análisis Discriminante por Mínimos Cuadrados PLS-DA}

Es importante resaltar que este análisis proporciona una valoración del potencial de los sistemas de análisis sensorial electrónico en la caracterización de vino, a pesar de que por razones experimentales, el número de muestras no es lo suficientemente amplio para obtener conclusiones definitivas, en términos estadísticos. Conseguir el número de muestras industriales (no de laboratorio) analizadas, ha sido una suerte, pero ello ha subordinado la población del que nos hubiera gustado disponer. En cualquier caso es válido para determinar las posibilidades que tienen nuestros sistemas en el seguimiento de diferentes características de los vinos tintos. Por este motivo todos los análisis llevados a cabo se basan únicamente en el "training set"; los modelos fueron validados y a continuación se seleccionó el número de variables latentes necesarias para realizar una correcta estimación de los diferentes parámetros.

Comenzaremos por el estudio de los módulos de análisis individuales, a continuación procederemos con la fusión de los datos y la construcción de un modelo de predicción PLS-DA con el sistema integrado. Es necesario precisar nuevamente de que el número de variables de la lengua electrónica es sensiblemente mayor que el de nariz y coordenadas CIELab, por lo que a la hora de realizar las estimaciones de las clases es factible prever un cierto desequilibrio en la contribución de cada dispositivo.

En este caso hemos empleado el PLS-DA para establecer modelos de predicción basados en el contenido polifenólico, el efecto de la microoxigenación y el tipo de corcho. Las siguientes tablas muestran los resultados para cada sistema; el último de los modelos se refiere al análisis realizado con la fusión de los datos de todos los sistemas.

Los resultados registrados en esta Tabla 3.4 demuestran que las coordenadas CIELab no se ven influenciadas por el contenido en polifenoles y tampoco por el uso de microoxigenación aplicado a los vinos analizados, razón por la cual los errores en la predicción son un poco más altos para estos dos factores. Por el contrario, el modelo establecido para la predicción de clases de acuerdo al tipo de tapón, ha mostrado mejores resultados para la discriminación y reconocimiento de vinos basados en su diferente OTR. 
Tabla 3.4. Modelo PLS-DA para las coordendas CIELab.

\begin{tabular}{|c|c|c|c|c|}
\hline & & Polifenoles & Mox & OTR \\
\hline \multirow{2}{*}{ Pendiente } & Calibración & 0.402 & 0.387 & 0.844 \\
\hline & Validación & 0.383 & 0.369 & 0.843 \\
\hline \multirow{2}{*}{ Offset } & Calibración & 0.300 & 0.307 & 0.078 \\
\hline & Validación & 0.310 & 0.313 & 0.077 \\
\hline \multirow{2}{*}{ Correlación } & Calibración & 0.634 & 0.622 & 0.919 \\
\hline & Validación & 0.605 & 0.593 & 0.914 \\
\hline \multirow{2}{*}{ Error residual } & Calibración & 0.387 & 0.391 & 0.197 \\
\hline & Validación & 0.398 & 0.403 & 0.204 \\
\hline \multirow{2}{*}{ Sensitividad } & Calibración & 0.875 & 0.771 & 0.833 \\
\hline & Validación & 0.854 & 0.771 & 0.812 \\
\hline \multirow{2}{*}{ Especificidad } & Calibración & 0.750 & 0.708 & 0.722 \\
\hline & Validación & 0.708 & 0.667 & 0.711 \\
\hline
\end{tabular}

Los parámetros reportados para la nariz electrónica muestran que ha sido posible obtener un modelo con 5 variables latentes, bajos errores de calibración y porcentajes de clasificaciones correctas aceptables. Los resultados indican que la nariz electrónica bajo este tipo de modelos supervisados es capaz de agrupar los vinos de acuerdo al OTR del tapón o el contenido polifenólico de los vinos (Tabla 3.5).

Tabla 3.5. Modelo PLS-DA para los resultados de la nariz electrónica.

\begin{tabular}{|c|c|c|c|c|}
\hline & & Polifenoles & Mox & OTR \\
\hline \multirow{2}{*}{ Pendiente } & Calibración & 0.732 & 0.566 & 0.740 \\
\hline & Validación & 0.702 & 0.526 & 0.720 \\
\hline \multirow{2}{*}{ Offset } & Calibración & 0.134 & 0.217 & 0.127 \\
\hline & Validación & 0.150 & 0.257 & 0.135 \\
\hline \multirow{2}{*}{ Correlación } & Calibración & 0.855 & 0.753 & 0.860 \\
\hline & Validación & 0.826 & 0.703 & 0.840 \\
\hline \multirow{2}{*}{ Error residual } & Calibración & 0.260 & 0.330 & 0.255 \\
\hline & Validación & 0.282 & 0.356 & 0.272 \\
\hline \multirow{2}{*}{ Sensitividad } & Calibración & 1.0 & 1.0 & 1.0 \\
\hline & Validación & 1.0 & 0.958 & 0.958 \\
\hline \multirow{2}{*}{ Especificidad } & Calibración & 1.0 & 0.958 & 1.0 \\
\hline & Validación & 1.0 & 0.938 & 1.0 \\
\hline
\end{tabular}

Durante el desarrollo de las investigaciones correspondientes a este capítulo hemos podido observar que las dos técnicas de extracción usadas para conseguir 
un contenido polifenólico diferente juegan un papel importante en los compuestos aromáticos que se extraen (libres y formas precursoras). Además, debido a las diferentes OTR que han sido empleadas, hemos observado que el "bouquet" de los diferentes vinos se modifica. Se sabe que la transmisión de la cantidad adecuada de oxígeno a través del tapón implica que los vinos envejezcan correctamente en la botella, sin problemas de oxidación o reducción remarcables.

Atendiendo a los resultados registrados en la Tabla 3.6 respecto a los parámetros del modelo PLS-DA registrados para los datos provenientes de la lengua electrónica, destacar que respecto al contenido polifenólico, se ha obtenido un coeficiente de predicción de 0.97 con 5 variables latentes. En el caso de hacer la agrupación en función de la microoxigenación, también se observó un coeficiente de predicción aceptable de 0.93 con 6 variables latentes. Por el contrario, la capacidad del sistema para clasificar vinos según el tipo de corcho OTR fue algo inferior, 0.88 con un error residual de 0.24 para un modelo con 6 variables latentes.

Estos resultados están en concordancia con aquellos publicados anteriormente, en los cuales se demostraba que existe una correlación entre la señal proporcionada por los sensores y el contenido polifenólico de los vinos y con los componentes con propiedades redox $[7,22]$.

Tabla 3.6. Modelo PLS-DA para los resultados de la lengua electrónica.

\begin{tabular}{|c|l|l|l|l|}
\hline & & Polifenoles & Mox & OTR \\
\hline \multirow{2}{*}{ Pendiente } & Calibración & 0.960 & 0.914 & \\
\cline { 2 - 5 } & Validación & 0.950 & 0.888 & 0.846 \\
\hline \multirow{2}{*}{ Offset } & Calibración & 0.020 & 0.043 & 0.877 \\
\cline { 2 - 5 } & Validación & 0.023 & 0.933 & 0.077 \\
\hline \multirow{2}{*}{ Correlación } & Calibración & 0.980 & 0.956 & 0.920 \\
\cline { 2 - 5 } & Validación & 0.971 & 0.933 & 0.877 \\
\hline \multirow{2}{*}{ Error residual } & Calibración & 0.100 & 0.147 & 0.196 \\
\cline { 2 - 5 } & Validación & 0.120 & 0.180 & 0.240 \\
\hline \multirow{2}{*}{ Sensitividad } & Calibración & 1.0 & 1.0 & 1.0 \\
\cline { 2 - 5 } & Validación & 1.0 & 1.0 & 0.986 \\
\hline \multirow{2}{*}{ Especificidad } & Calibración & 1.0 & 1.0 & 1.0 \\
\cline { 2 - 5 } & Validación & 1.0 & 1.0 & 0.958 \\
\hline
\end{tabular}

Con los resultados anteriores podemos asegurar que cada instrumento proporciona información complementaria que contribuye a la discriminación de los vinos estudiados. Hemos visto como la nariz y ojo electrónico son responsables de la sensibilidad al efecto causado por el efecto del corcho OTR en 
los diferentes vinos. Por otra parte, el sistema de sensores electroquímicos puede discriminar los atributos del sabor que están influenciados por la presencia de compuestos con actividad redox y por el carácter antioxidante del vino (contenido polifenólico y microoxigenación). Esta capacidad de discriminación puede ser mejorada cuando se fusionan los datos provenientes de estos 3 sistemas (Tabla 3.7).

Tabla 3.7. Modelo PLS-DA para los resultados del panel de cata electrónico.

\begin{tabular}{|c|l|l|l|l|}
\hline \multicolumn{2}{|c}{ Polifenoles } & \multicolumn{2}{c|}{ Mox } & OTR \\
\hline \multirow{2}{*}{ Pendiente } & Calibración & 0.973 & 0.923 & 0.964 \\
\cline { 2 - 5 } & Validación & 0.955 & 0.902 & 0.948 \\
\hline \multirow{2}{*}{ Offset } & Calibración & 0.013 & 0.039 & 0.018 \\
\cline { 2 - 5 } & Validación & 0.022 & 0.050 & 0.027 \\
\hline \multirow{2}{*}{ Correlación } & Calibración & 0.986 & 0.961 & 0.982 \\
\cline { 2 - 5 } & Validación & 0.980 & 0.943 & 0.973 \\
\hline \multirow{2}{*}{ Error residual } & Calibración & 0.082 & 0.139 & 0.094 \\
\cline { 2 - 5 } & Validación & 0.101 & 0.167 & 0.115 \\
\hline \multirow{2}{*}{ Sensitividad } & Calibración & 1.0 & 1.0 & 1.0 \\
\cline { 2 - 5 } & Validación & 1.0 & 1.0 & 1.0 \\
\hline \multirow{2}{*}{ Especificidad } & Calibración & 1.0 & 1.0 & 1.0 \\
\cline { 2 - 5 } & Validación & 1.0 & 1.0 & 1.0 \\
\hline
\end{tabular}

Se realizó además un análisis LDA para verificar los resultados obtenidos mediante PLS-DA (Tabla 3.8). Para el análisis LDA se utilizó la técnica leave-oneout para llevar a cabo el procedimiento de validación cruzada. Los porcentajes de clasificación registrados con este método son aceptables, sin embargo lo más importante es que estos valores permiten afirmar que tanto los resultados provenientes de la nariz electrónica como las coordenadas CIELab mostraron buenas correlaciones respecto a la valoración del OTR, mientras que los derivados de la lengua electrónica constituyeron el sistema de mejor seguimiento para todos los factores seguidos en esta investigación.

Tabla 3.8. Comparación para los porcentajes de clasificación entre el análisis PLS-DA y LDA

\section{$\begin{array}{lll}\text { CIELab [\%] } & \text { Nariz [\%] } & \text { Lengua [\%] }\end{array}$}

\begin{tabular}{|c|l|l|l|}
\hline Polifenoles $^{*}$ & 68,8 & 95,8 & 100 \\
\hline Mox* & 50 & 90,6 & 100 \\
\hline OTR $^{*}$ & 77,1 & 100 & 100 \\
\hline
\end{tabular}


Hasta el momento se han aplicado dos tipos de análisis multivariantes diferentes, el primero de ello para saber si los diversos sistemas son capaces de distingue entre los tipos de muestras seleccionados para esta investigación (PCA), y el segundo de ellos para evaluar la capacidad de clasificación de los mismos sistemas (PLS-DA). En vista de los resultados anteriores en los cuales se aprecia que la nariz y lengua electrónica detectan diferencias entre el conjunto de muestras debido a los distintos tipos de tapones, la microoxigenación aplicada y el tipo de extracción del mosto, se pensó en mejorar el tratamiento aplicando una prolongación del método PLS (PLS2) que permitiera conocer cuales son los parámetros con los cuales se presenta una mejor correlación. Los parámetros que tendremos en cuenta para los siguientes modelos son los derivados de la evaluación química (a nivel de contenido polifenólico) y las medidas del nivel de oxígeno durante el envejecimiento en botella.

\subsubsection{Análisis multivariante PLS2}

\section{Resultados de los parámetros químicos evaluados}

Los 22 parámetros químicos nombrados en el apartado 3.2.4. fueron registrados una vez habían pasado 10 meses del comienzo al proceso del envejecimiento en botella. Los resultados numéricos de estos experimentos pueden ser consultados en las Tablas 3.9 y 3.10 .

Tabla 3.9. Resultados de las medidas de los parámetros químicos para los vinos elaborados con el método tradicional de estrujado de la uva.

\begin{tabular}{|l|r|r|r|r|r|r|r|r|r|}
\multicolumn{1}{c}{ Vinos } & \multicolumn{1}{c}{ T1 } & \multicolumn{1}{c}{ T2 } & \multicolumn{1}{c|}{ T3 } & \multicolumn{1}{c|}{ T4 } & \multicolumn{1}{c|}{ TM1 } & \multicolumn{1}{c|}{ TM2 } & TM3 & TM4 \\
\hline ACN [mgL-1] & 122,58 & 111,60 & 91,82 & 85,59 & 120,27 & 107,19 & 88,06 & 82,88 \\
\hline TAN [mgL-1] & 462,46 & 462,02 & 441,20 & 478,84 & 439,31 & 489,35 & 422,30 & 458,29 \\
\hline T/A & 4,01 & 4,50 & 5,10 & 5,56 & 3,90 & 4,81 & 5,11 & 5,87 \\
\hline CAT [mgL-1] & 28,50 & 28,10 & 27,27 & 27,02 & 29,35 & 26,60 & 27,87 & 28,39 \\
\hline HA [mgL-1] & 148,82 & 150,06 & 146,93 & 147,15 & 151,56 & 148,49 & 144,48 & 145,28 \\
\hline CPA [mgL-1] & 1,963 & 2,028 & 2,147 & 2,097 & 1,878 & 1,847 & 2,116 & 1,948 \\
\hline EB [mgL-1] & 0,228 & 0,172 & 0,193 & 0,198 & 0,229 & 0,301 & 0,199 & 0,268 \\
\hline FLN [mgL-1] & 75,31 & 75,54 & 67,16 & 66,64 & 85,41 & 80,88 & 74,61 & 71,64 \\
\hline VP [mgL-1] & 0,551 & 0,542 & 0,706 & 0,671 & 0,826 & 1,484 & 0,587 & 1,386 \\
\hline mDP & 4,308 & 4,196 & 4,361 & 3,894 & 4,205 & 4,268 & 4,998 & 4,082 \\
\hline \% Gall & 5,973 & 6,074 & 6,638 & 5,576 & 5,462 & 5,401 & 5,506 & 6,077 \\
\hline \%EGC & 20,93 & 19,85 & 19,43 & 18,95 & 21,01 & 20,57 & 21,61 & 21,31 \\
\hline IC & 6,28 & 6,98 & 7,43 & 7,40 & 6,24 & 6,77 & 7,33 & 7,74 \\
\hline ICcor & 7,781 & 7,886 & 7,444 & 7,541 & 7,674 & 7,929 & 7,382 & 7,672 \\
\hline Tcor & 0,682 & 0,713 & 0,758 & 0,769 & 0,691 & 0,720 & 0,755 & 0,768 \\
\hline CTP & 11,74 & 12,17 & 9,66 & 11,37 & 10,84 & 12,55 & 9,50 & 12,46 \\
\hline
\end{tabular}


Tabla 3.9. Continuación. Resultados de las medidas de los parámetros químicos para los vinos elaborados con el método tradicional de estrujado de la uva.

\begin{tabular}{|l|r|r|r|r|r|r|r|r|r|}
\multicolumn{1}{c}{ Vinos } & \multicolumn{1}{c}{ T1 } & \multicolumn{1}{c|}{ T2 } & \multicolumn{1}{c|}{ T3 } & \multicolumn{1}{c|}{ T4 } & TM1 & TM2 & TM3 & TM4 \\
\begin{tabular}{|l|r|r|r|r|r|r|r|r|}
\hline IPT & 31,91 & 29,14 & 32,44 & 30,57 & 31,40 & 29,87 & 29,36 & 34,24 \\
\hline PDT & 10,14 & 10,52 & 7,99 & 9,78 & 9,30 & 11,02 & 7,85 & 10,80 \\
\hline ACV & 38,94 & 40,49 & 44,21 & 41,67 & 38,58 & 37,84 & 44,27 & 43,25 \\
\hline \%PDSO2 & 61,06 & 59,51 & 56,73 & 58,33 & 61,42 & 62,16 & 55,73 & 56,75 \\
\hline PV & 3,078 & 3,165 & 3,415 & 3,555 & 3,104 & 3,190 & 3,447 & 3,695 \\
\hline CC & 1,496 & 1,339 & 1,001 & 1,430 & 1,421 & 1,514 & 1,119 & 1,495 \\
\hline
\end{tabular}
\end{tabular}

Tabla 3.10. Resultados de las medidas de los parámetros químicos para los vinos elaborados con el método de termovinificación.

\begin{tabular}{|c|c|c|c|c|c|c|c|c|}
\hline Vinos & FM1 & FM2 & FM3 & FM4 & F1 & F2 & F3 & F4 \\
\hline $\mathbf{A C N}\left[\mathrm{mgL}^{-1}\right]$ & 111,82 & 92,79 & 75,85 & 74,07 & 119,57 & 99,15 & 83,73 & 90,54 \\
\hline TAN $\left[\mathrm{mgL}^{-1}\right]$ & 1171,58 & 1247,89 & 1109,00 & 1109,95 & 1192,49 & 1293,71 & 1178,77 & 1269,92 \\
\hline T/A & 10,99 & 14,02 & 15,32 & 16,64 & 10,44 & 13,59 & 14,71 & 14,54 \\
\hline CAT [mgL-1] & 57,53 & 52,27 & 52,69 & 51,21 & 55,36 & 53,60 & 52,23 & 46,53 \\
\hline $\mathbf{H A}\left[\mathrm{mgL}^{-1}\right]$ & 300,87 & 286,15 & 287,09 & 292,93 & 301,95 & 286,96 & 289,73 & 295,39 \\
\hline CPA [mgL-1] & 1,882 & 1,902 & 2,061 & 2,442 & 2,189 & 2,171 & 2,268 & 2,547 \\
\hline EB $\left[\mathrm{mgL}^{-1}\right]$ & 0,273 & 0,197 & 0,135 & 0,243 & 0,252 & 0,221 & 0,266 & 0,192 \\
\hline FLN [mgL-1] & 81,90 & 79,06 & 72,76 & 68,88 & 75,06 & 75,81 & 67,86 & 65,40 \\
\hline $\mathbf{V P}\left[\mathrm{mgL}^{-1}\right]$ & 0,744 & 1,045 & 0,693 & 0,785 & 0,543 & 0,706 & 0,748 & 0,729 \\
\hline $\mathrm{mDP}$ & 4,210 & 4,446 & 4,691 & 4,100 & 4,291 & 4,692 & 4,941 & 4,380 \\
\hline$\%$ Gall & 7,097 & 7,116 & 7,477 & 7,647 & 7,200 & 7,020 & 7,168 & 6,978 \\
\hline$\%$ EGC & 15,32 & 17,38 & 17,57 & 16,86 & 16,73 & 20,38 & 22,38 & 20,93 \\
\hline IC & 6,26 & 6,56 & 6,99 & 7,89 & 6,07 & 6,36 & 6,67 & 7,09 \\
\hline Iccor & 7,368 & 7,431 & 7,339 & 7,512 & 7,357 & 7,558 & 7,238 & 7,680 \\
\hline Tcor & 0,707 & 0,733 & 0,770 & 0,771 & 0,703 & 0,735 & 0,765 & 0,767 \\
\hline CTP & 11,13 & 12,28 & 9,38 & 12,92 & 10,81 & 12,74 & 10,19 & 13,39 \\
\hline PDT & 9,55 & 11,70 & 7,66 & 12,03 & 9,24 & 11,18 & 8,46 & 11,68 \\
\hline $\mathrm{ACV}$ & 41,52 & 42,31 & 46,96 & 43,03 & 40,72 & 40,59 & 47,30 & 44,16 \\
\hline IPT & 44,34 & 38,99 & 43,33 & 42,38 & 44,03 & 41,96 & 41,47 & 45,55 \\
\hline$\%$ PDSO2 & 58,48 & 57,69 & 53,04 & 56,97 & 59,28 & 59,41 & 52,70 & 55,84 \\
\hline PV & 3,091 & 3,182 & 3,535 & 3,794 & 3,001 & 3,088 & 3,428 & 3,839 \\
\hline $\mathrm{CC}$ & 1,170 & 1,287 & 1,029 & 1,326 & 1,276 & 1,362 & 1,031 & 1,407 \\
\hline
\end{tabular}

A modo de resumen, la Figura 3.13 ilustra algunas de las diferencias existentes entre los vinos, consecuencia de sus distintas condiciones de elaboración. Por ejemplo, los vinos elaborados con el método de termovinificación mostraron un 
mayor contenido de ácido hidroxicinámicos que las muestras elaboradas con el método tradicional. Un efecto similar se observó también para los niveles de las catequinas, taninos, polifenoles totales, entre otros. Por otro lado, los valores de los parámetros como los flavonoles, pigmentos totales, la intensidad del color aumentan en función del OTR del tipo de tapón utilizado. Esto puede interpretarse como resultado de la presencia de las diferentes cantidades de oxígeno y la posibilidad de producir nuevos derivados fenólicos.
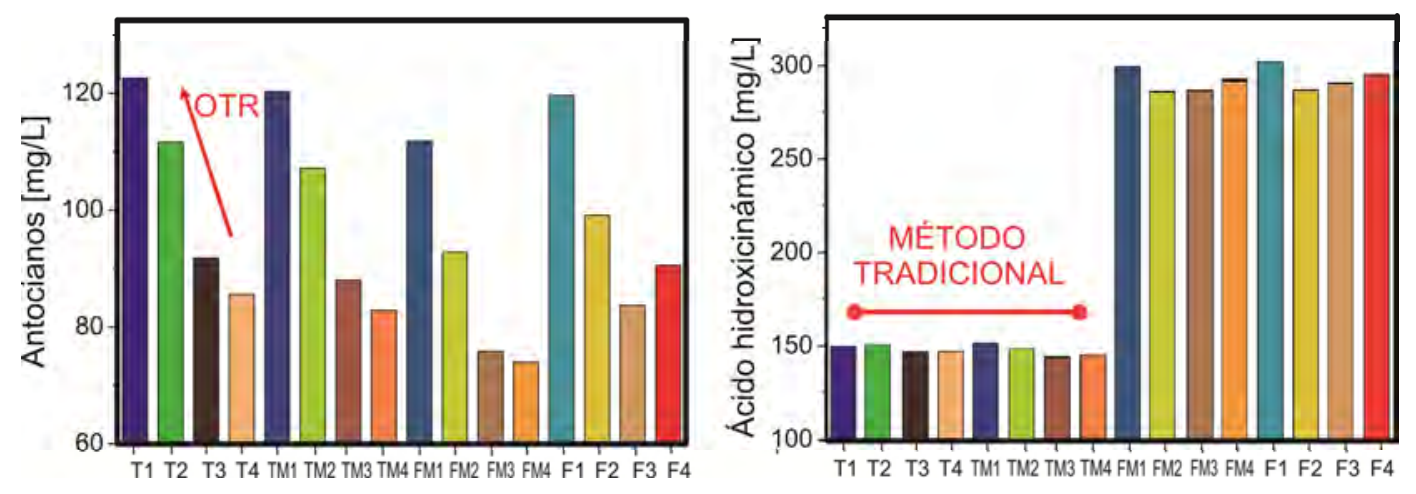

Figura 3.13 Resultados obtenidos para algunos de los parámetros evaluados en los vinos después de 10 meses de dar comienzo al envejecimiento en botella.

El incremento en el OTR también se asoció a una disminución en los niveles de los antocianos. Esto significa que los antocianos son los primeros compuestos en comenzar a oxidarse, mostrando una pérdida más acusada aquellos vinos estrujados por métodos tradicionales, para los cuales los taninos y el contenido de ácidos hidroxicinámicos eran inferiores. Estos resultados señalan, de momento, que tanto el OTR como el tipo de estrujado de la uva tienen un mayor efecto en la composición química del vino que la incorporación controlada de oxígeno durante la fermentación.

En cuanto a las medidas registradas en la Tabla 3.11 respecto al nivel de oxígeno: oxigeno disuelto $\mathrm{DO}$, oxigeno en el espacio en cabeza $\mathrm{HO}$ y la contribución del tipo de tapón utilizado $C L$, -este último parámetro también se encuentra descrito en la Figura 3.14-, podemos indicar con estos resultados que los tres parámetros respondieron principalmente al OTR asociado a cada tapón sintético. De acuerdo con otros estudios es importante puntualizar que la cantidad de oxígeno que se incorpora al vino durante un cierto período de tiempo no depende solamente del OTR, ya que inicialmente el oxígeno que entra en una botella de vino no está directamente relacionado con el oxígeno existente en su entorno atmosférico. En las primeras semanas, de hecho, hasta los tres primeros meses, el oxígeno que entra en la botella se libera fundamentalmente desde el interior del tapón.

Esto sucede básicamente porque los cierres están hechos de materiales porosos, que contienen aire, y por lo tanto oxígeno. Cuando el cierre se aprieta en el cuello 
de la botella, parte del oxígeno es expulsado fuera. Por lo tanto, la proporción total de oxígeno en el interior de la botella depende de esta cantidad inicial y por otra parte del OTR.

Tabla 3.11. Medidas del oxígeno en botella.

\begin{tabular}{|c|c|c|c|}
\hline & $\mathrm{DO} \mathrm{mg} / \mathrm{L}$ & $\mathrm{HS} \% \mathrm{O}_{2}$ & $\mathrm{CL}[\mathrm{mg} / \mathrm{L}]$ \\
\hline Light 21\% (T1) & 0.004 & 0.10 & 1.0989 \\
\hline Classic 21\% (T2) & 0.010 & 0.24 & 1.6367 \\
\hline Classic 4\% (T3) & 0.020 & 0.68 & 3.6477 \\
\hline Classic 1\% (T4) & 0.026 & 0.84 & 5.3294 \\
\hline Light 21\% (TM1) & 0.008 & 0.12 & 1.0998 \\
\hline Classic 21\% (TM2) & 0.004 & 0.20 & 1.6371 \\
\hline Classic 4\% (TM3) & 0.022 & 0.72 & 3.6657 \\
\hline Classic 1\% (TM4) & 0.034 & 0.84 & 5.4287 \\
\hline Light 21\% (FM1) & 0.008 & 0.14 & 1.0998 \\
\hline Classic 21\% (FM2) & 0.010 & 0.24 & 1.6731 \\
\hline Classic 4\% (FM3) & 0.032 & 0.70 & 3.6189 \\
\hline Classic 1\% (FM4) & 0.046 & 0.90 & 5.3887 \\
\hline Light 21\% (F1) & 0.008 & 0.12 & 1.0986 \\
\hline Classic 21\% (F2) & 0.012 & 0.24 & 1.6348 \\
\hline Classic 4\% F3) & 0.018 & 0.56 & 3.7230 \\
\hline Classic 1\% (F4) & 0.036 & 0.74 & 5.4207 \\
\hline
\end{tabular}

Podemos afirmar entonces que el proceso de difusión de oxígeno desde la atmósfera es muy lento, y que este fenómeno está fuertemente afectado por las características del tipo de tapón utilizado.

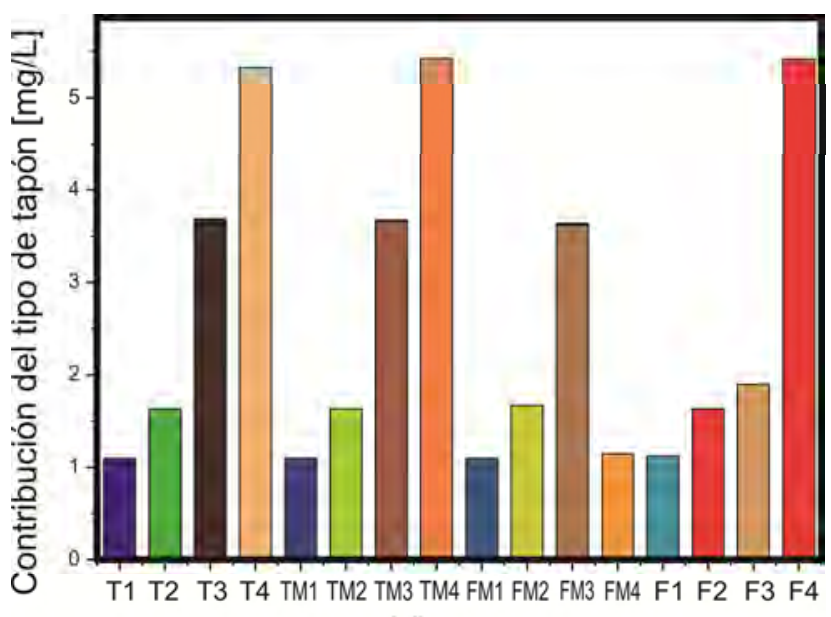

\section{Vinos}

Figura 3.14. Medidas del nivel de oxígeno para cada una de las muestras evaluadas. Contribución del tipo de corcho. 
Como se mencionó anteriormente, para el desarrollo de la regresión multivariante PLS2 es conveniente reducir el número de parámetros de manera que la relación entre variables del bloque $\mathrm{Y}$ con el número de muestras que forman el bloque $X$ sea óptima. Es por ello que en una primera fase, hemos realizado un estudio ANOVA para conocer cuales eran las variables más significativas para los tres factores dentro del proceso de elaboración del vino, que se han de tener en cuenta en esta parte de nuestra investigación.

Tabla 3.12. Resultados ANOVA para los parámetros químicos significativos

\begin{tabular}{|c|c|c|c|}
\hline OTR & Trad/FR & Mox/No Mox & OTR \\
\hline Antocianos [mgL-1] & * & n.s. & $* * *$ \\
\hline Taninos [mgL-1] & $* * *$ & n.s. & n.s. \\
\hline Relación taninos/antocianos & $* * *$ & n.s. & * \\
\hline Catequinas [mgL-1] & $* * *$ & n.s. & * \\
\hline Ácido hidroxicinámico [mgL-1] & *** & n.s. & * \\
\hline Piranoantocianos (vitisinas) [mgL-1] & * & n.s. & n.s. \\
\hline $\begin{array}{l}\text { Catequina, epicatequina y } \\
\text { malvidina-3-O-glucósido unidos } \\
\text { por puentes etilo }\left[\mathrm{mgL}^{-1}\right]\end{array}$ & n.s. & n.s. & n.s. \\
\hline Flavonoles [mgL-1] & n.s. & n.s. & $* *$ \\
\hline $\begin{array}{l}\text { Piranoantocianos vinilfenólicos } \\
{\left[\mathrm{mgL}^{-1}\right]}\end{array}$ & n.s. & n.s. & n.s. \\
\hline Grado medio de polimerización & * & * & $* *$ \\
\hline Porcentaje de ácido gálico. & ** & * & n.s. \\
\hline Porcentaje de galato de epicatequina & * & ** & n.s. \\
\hline Intensidad del color & * & n.s. & $* * *$ \\
\hline Intesidad de color corregida & ** & n.s. & * \\
\hline Correción de Hue & ** & n.s. & $* * *$ \\
\hline Color total de pigmentos & n.s. & n.s. & *** \\
\hline Índice de polifenoles totales & $* * *$ & n.s. & * \\
\hline Pigmentos decolorables totales & n.s. & n.s. & $* *$ \\
\hline Edad química del vino & $* *$ & n.s. & $* *$ \\
\hline $\begin{array}{l}\text { Porcentaje de pigmentos } \\
\text { decolorables por bisulfito }\end{array}$ & ** & n.s. & ** \\
\hline Pigmentos totales & n.s. & n.s. & *** \\
\hline Color debido a la copigmentación & * & n.s. & n.s. \\
\hline
\end{tabular}

*: $\mathrm{p}<0.05$ (significante), ${ }^{* *}: \mathrm{p}<0.01$ (muy significante); ${ }^{* * *}: \mathrm{p}<0.0001$ (considerablemente significante); n.s.: $\mathrm{p}>0.05$ (no significante).

El impacto que tienen las técnicas de extracción (tradicional o termovinificación), la microoxigenación, y el OTR en la composición fénolica y en el color del vino, 
fueron valorados mediante un análisis ANOVA. Los tres factores considerados tuvieron una influencia significativa en la mayoría de los parámetros de color y polifenoles analizados. Los resultados listados en la Tabla 3.12 destacan que el OTR ha desempeñado un papel significativo en 16 de los 22 parámetros que se enumeran, mientras que el método de estrujado de la uva afectó a 17 de ellos y la microoxigenación a tres.

La técnica de maceración de la uva (tradicional/termovinificación) fue el factor con el mayor número de variables estadísticamente significativas (16 parámetros). En cuanto al factor del OTR de cada tipo de corcho, los resultados mostraron que 15 de los parámetros medidos fueron significativas. Respecto al factor de la microoxigenación se observó que solamente tres parámetros fueron designados como estadísticamente significativos.

Todos los parámetros que mostraron un valor de $\mathrm{p}<0,0001$ fueron seleccionados para llevar a cabo la regresión PLS2. Basados en este criterio, los parámetros seleccionado fuero: antocianos, intensidad del color, pigmentos totales, ácido hidroxicinámico, catequinas, taninos, relación taninos/antocianos, índice de polifenoles totales y Hue corregido.

\section{Resultados de los modelos PLS2}

La técnica de análisis multivariante PLS2 fue utilizada para correlacionar los datos derivados de la nariz, lengua y ojo electrónico con los valores obtenidos por medio de métodos analíticos (contenido polifenólico y niveles de oxigeno).

Para cada uno de los tres sistemas de caracterización sensorial electrónicos se realizaron dos modelos diferentes, uno referente al contenido polifenólico y otro referente a las medidas del nivel de oxígeno durante el envejecimiento en botella. Es decir, se ha trabajado con los resultados de 6 modelos diferentes PLS2. Además se ha realizado un último modelo combinando los datos provenientes de la nariz y lengua electrónica con el objetivo de aprovechar la complementariedad que presentan estos sistemas para evaluar los fenómenos referentes al oxígeno y el contenido polifenólico. Como ya citamos anteriormente, los modelos PLS2 sólo se realizaron con el "training set" y fueron "cross-validados", de forma que se obtuviera una valoración fiable del error global de cada modelo.

Las Tablas 3.13 y 3.14 recogen los resultados de los parámetros estadísticos para los modelos de regresión PLS2 respecto a las coordenadas CIELab. En términos generales las correlaciones entre las medidas referentes al color y los parámetros químicos no son las ideales, aunque si existen ciertos compuestos como los antocianos, los pigmentos totales y parámetros como la intensidad del color que 
presentaron valores aceptables. Es difícil explicar porque no existe una buena correlación entre las coordenadas CIELab con compuestos como los taninos, y ello quizás sea debido a que pudo suceder que otros fenómenos destacaron más que los cambios que se registraron para estos parámetros. Entre los fenómenos que más incidieron podemos citar que, los vinos almacenados con tapones sintéticos con OTR más altos mostraron mayor intensidad de color, esto se debe a la conversión cada vez mayor de pigmentos derivados de los antocianos a compuestos como los piranoantocianos. Estas conclusiones además son coherentes con las observadas en el análisis sensorial realizado a este mismo conjunto de muestras publicado por S. Caillé et. al [30], en los que OTR alto se asoció con mayor intensidad de color.

Tabla 3.13. Resultados del análisis PLS2 de las coordenadas CIELab con los parámetros químicos.

\section{CIELab \\ RMSEC \\ RMSECV \\ $\mathrm{R}^{2}$ \\ $\mathrm{R}^{2} \mathrm{CV}$}

\begin{tabular}{|l|l|l|l|l|}
\hline Antocianos & 8.10277 & 8.63276 & 0.730 & 0.695 \\
\hline Intensidad del Color & 0.288239 & 0.306261 & 0.729 & 0.695 \\
\hline Pigmentos totales & 0.106971 & 0.113384 & 0.838 & 0.819 \\
\hline Ácido hidroxicinámico & 60.0112 & 62.2368 & 0.329 & 0.280 \\
\hline Catequinas & 10.5549 & 10.9287 & 0.311 & 0.264 \\
\hline Taninos & 307.346 & 319.42 & 0.335 & 0.284 \\
\hline $\begin{array}{l}\text { Relación entre taninos } \\
\text { y antocianos }\end{array}$ & 3.8959 & 4.03188 & 0.321 & 0.274 \\
\hline $\begin{array}{l}\text { Índice de polifenoles } \\
\text { totales }\end{array}$ & 4.93848 & 5.14875 & 0.354 & 0.300 \\
\hline Correción de Hue & 0.013742 & 0.014816 & 0.797 & 0.765 \\
\hline
\end{tabular}

Tabla 3.14. Resultados del análisis PLS2 de las coordenadas CIELab con las medidas del oxígeno.

\section{CIELab}

RMSEC

RMSECV

$\mathrm{R}^{2} \mathrm{CV}$

\begin{tabular}{|l|l|l|l|l|}
\hline Oxigeno Disuelto & 0.008247 & 0.008599 & 0.564 & 0.527 \\
\hline $\begin{array}{l}\text { Oxígeno en el espacio en } \\
\text { cabeza }\end{array}$ & 0.212895 & 0.219971 & 0.489 & 0.455 \\
\hline $\begin{array}{l}\text { Contribución del tipo de } \\
\text { tapón al nivel de oxígeno }\end{array}$ & 1.04099 & 1.07508 & 0.626 & 0.602 \\
\hline
\end{tabular}

Respecto a las correlaciones existentes entre la nariz electrónica y el contenido de polifenoles y el nivel de oxígeno, los valores se encuentran registrados en las Tablas 3.15 y 3.16, respectivamente. Es posible observar que se encontraron valores satisfactorios especialmente para uno de estos parámetros, la 
contribución del tipo de tapón al nivel de oxígeno (CL), que es el parámetro que permite calcular la cantidad de oxígeno que pasa al interior de la botella en función del OTR. En cuanto a modelo de regresión PLS2 basado en las nueve variables del contenido polifenólico, al parecer el sistema no es tan sensible como lo fue a las medidas del nivel de oxígeno.

Tabla 3.15. Resultados del análisis PLS2 de la nariz electrónica con los parámetros químicos.

\section{Nariz electrónica}

\section{RMSEC}

\section{RMSECV}

$\mathrm{R}^{2}$

$\mathrm{R}^{2} \mathrm{CV}$

\begin{tabular}{|l|l|l|l|l|}
\hline Antocianos & 9.47583 & 10.538 & 0.631 & 0.546 \\
\hline Intensidad del Color & 0.262298 & 0.28676 & 0.773 & 0.729 \\
\hline Pigmentos totales & 0.128925 & 0.144353 & 0.765 & 0.706 \\
\hline $\begin{array}{l}\text { Ácido } \\
\text { hidroxicinámico }\end{array}$ & 28.5066 & 31.6073 & 0.846 & 0.810 \\
\hline Catequinas & 5.11525 & 5.65095 & 0.835 & 0.799 \\
\hline Taninos entre & 125.105 & 167.686 & 0.834 & 0.798 \\
\hline $\begin{array}{l}\text { Relación } \\
\text { taninos y antocianos }\end{array}$ & 2.13875 & 2.37153 & 0.793 & 0.746 \\
\hline $\begin{array}{l}\text { Índice de polifenoles } \\
\text { totales }\end{array}$ & 3.02571 & 3.29219 & 0.753 & 0.708 \\
\hline Correción de Hue & 0.0190027 & 0.0212185 & 0.610 & 0.517 \\
\hline
\end{tabular}

Estos resultados sugieren que la nariz electrónica puede haber sido más sensible a los cambios que el oxígeno produce en la composición volátil a nivel global que a la concentración de un compuesto como tal. El anterior planteamiento puede justificarse en el hecho de que los sensores incluidos dentro de la red de nuestro sistema no son específicos, es decir que no están diseñados para responder de manera predominante a un compuesto en particular, lo que realmente sucede es que el conjunto de señales que arrojan todos los sensores forman un patrón particular para cada tipo de vino.

Tabla 3.16. Resultados del análisis PLS2 de la nariz electrónica con las medidas del oxígeno.

\section{Nariz electrónica}

\section{RMSEC RMSECV}

$\mathrm{R}^{2}$

$\mathrm{R}^{2} \mathrm{CV}$

\begin{tabular}{|l|l|l|l|l|}
\hline Oxigeno Disuelto & 0.0054922 & 0.00624741 & $\mathbf{0 . 8 0 6}$ & 0.752 \\
\hline $\begin{array}{l}\text { Oxígeno en el espacio en } \\
\text { cabeza }\end{array}$ & 0.0916162 & 0.103808 & $\mathbf{0 . 9 0 5}$ & 0.879 \\
\hline $\begin{array}{l}\text { Contribución del tipo de } \\
\text { tapón al nivel de oxígeno }\end{array}$ & 0.46004 & 0.520652 & $\mathbf{0 . 9 2 7}$ & 0.907 \\
\hline
\end{tabular}


Tabla 3.17. Resultados del análisis PLS2 de la lengua electrónica con los parámetros químicos.

\begin{tabular}{|c|c|c|c|c|}
\hline Lengua electrónica & RMSEC & RMSECV & $\mathrm{R}^{2}$ & $\mathrm{R}^{2} \mathrm{CV}$ \\
\hline Antocianos & 8.18863 & 9.76214 & 0.724 & 0.613 \\
\hline Intensidad del Color & 0.209776 & 0.250455 & 0.855 & 0.794 \\
\hline Pigmentos totales & 0.155966 & 0.173168 & 0.656 & 0.579 \\
\hline $\begin{array}{l}\text { Ácido } \\
\text { hidroxicinámico }\end{array}$ & 21.7954 & 27.0437 & 0.910 & 0.861 \\
\hline Catequinas & 4.2149 & 5.21074 & 0.88 & 0.829 \\
\hline Taninos & 105.104 & 129.524 & 0.921 & 0.880 \\
\hline $\begin{array}{l}\text { Relación entre } \\
\text { taninos y antocianos }\end{array}$ & 1.173139 & 2.08962 & 0.864 & 0.802 \\
\hline $\begin{array}{l}\text { Índice de polifenoles } \\
\text { totales }\end{array}$ & 2.56194 & 2.95237 & 0.823 & 0.765 \\
\hline Correción de Hue & 0.0161039 & 0.0188305 & 0.720 & 0.622 \\
\hline
\end{tabular}

Resultados similares fueron obtenidos al realizar un modelo PLS2 con los datos provenientes de lengua electrónica y los datos referentes al nivel de oxigeno y los parámetros químicos (Tablas 3.17 y 3.18). De acuerdo con los datos de la tabla 3.17 es aún más notable la capacidad de la lengua electrónica para predecir los valores químicos relacionados con el contenido polifenólico. Estos resultados están en buen acuerdo con otros trabajos publicados que indican que las respuestas observadas en los voltamogramas están relacionadas con o influenciadas por la composición química de los vinos [31-33]. Los electrodos modificados químicamente mostraron una alta selectividad cruzada ya que todos los proceso redox que se presentan están relacionados con los compuestos redox presentes en los vinos, como son los compuestos polifenólicos a los que hemos hecho seguimiento en este trabajo.

Tabla 3.18. Resultados del análisis PLS2 de la lengua electrónica con las medidas del oxígeno.

\section{\begin{tabular}{l|l|l|l|l} 
Lengua electrónica & RMSEC & RMSECV & $R^{2}$ & $R^{2} \mathrm{CV}$
\end{tabular}}

\begin{tabular}{|l|l|l|l|l|}
\hline Oxigeno Disuelto & 0.00448088 & 0.00524145 & 0.871 & 0.824 \\
\hline $\begin{array}{l}\text { Oxígeno en el espacio en } \\
\text { cabeza }\end{array}$ & 0.099238 & 0.11631 & 0.889 & 0.848 \\
\hline $\begin{array}{l}\text { Contribución del tipo de } \\
\text { tapón al nivel de oxígeno }\end{array}$ & 0.448832 & 0.524142 & 0.931 & 0.905 \\
\hline
\end{tabular}

En términos generales podemos hablar de complementariedad entre los sistemas, por ejemplo con la nariz electrónica se puede hacer un buen seguimiento a las 
medidas del oxigeno en botella, quizás por los efectos que produce este factor en la fase volátil del vino. En el caso de la lengua electrónica, reiterar que es el instrumento que mejor sigue las diferencias que se producen entre los compuestos químicos de acuerdo con el tipo de tapón sintético utilizado. Puede apreciarse también que la espectroscopía UV-Visible (color) es el sistema con el número más bajo de parámetros evaluados que es posible correlacionar.

En vista de los anteriores resultados, se ha realizado un modelo adicional que incluye la fusión de los datos provenientes de la nariz y lengua electrónica para evaluar la capacidad que tienen ambos sistemas de seguir simultáneamente los cambios en el contenido polifenólico y los niveles de oxígeno.

Tabla 19. Resultados del análisis PLS2 del panel de cata electrónico con los parámetros químicos y las medidas del oxígeno.

\begin{tabular}{|l|l|l|l|l|}
\hline \multicolumn{2}{|l|}{ RMSEC } & RMSECV & R $^{2}$ & R$^{2}$ CV \\
\hline Antocianos & 3.71009 & 4.6318 & 0.943 & 0.912 \\
\hline Intensidad del Color & 0.139295 & 0.159869 & 0.936 & 0.916 \\
\hline Pigmentos totales & 0.0826799 & 0.0949098 & 0.903 & 0.873 \\
\hline Ácido hidroxicinámico & 12.4443 & 15.4804 & 0.971 & 0.954 \\
\hline Catequinas & 2.80034 & 3.38893 & 0.951 & 0.928 \\
\hline Taninos & 53.903 & 68.9914 & 0.979 & 0.966 \\
\hline $\begin{array}{l}\text { Relación entre taninos y } \\
\text { antocianos }\end{array}$ & 0.928101 & 1.13424 & 0.961 & 0.942 \\
\hline $\begin{array}{l}\text { Índice de polifenoles } \\
\text { totales }\end{array}$ & 1.10591 & 1.41158 & 0.967 & 0.946 \\
\hline Correción de Hue & 0.00723751 & 0.00892215 & 0.943 & 0.915 \\
\hline Oxigeno Disuelto & 0.00336761 & 0.00399553 & 0.927 & 0.898 \\
\hline $\begin{array}{l}\text { HS \% } \mathrm{O}_{2} \\
\text { Contribución Cl }\end{array}$ & 0.0606004 & 0.0736087 & 0.959 & 0.939 \\
\hline
\end{tabular}

Los resultados anotados en la Tabla 3.19 demuestran que los parámetros estadísticos del modelo PLS2 mejoran al realizar la fusión de los datos. La combinación de sistemas permite evaluar directamente los efectos de las técnicas de estrujado de la uva, la micro-oxigenación y especialmente el efecto del OTR del tipo de tapón. Las mejores correlaciones se obtuvieron para los taninos y el ácido hidroxicinámico, que son compuestos activos que pueden modificar el gusto del vino, con atributos relacionados con el amargor. Característica sensorial que la lengua electrónica ya ha demostrado ser capaz de predecir [34, 35]. Así como los buenos resultados alcanzados para el parámetro que mide la contribución del tipo de tapón a los niveles de oxígeno presentes en el vino. Esta capacidad de nuestro sistema podría estar relacionada con la alta sensibilidad de 
los sensores de gases para medir los cambios en la composición volátil asociados a los cambios que se registran para los niveles de oxígeno en las distintas muestras de vino [36-39].

\subsection{CONCLUSIONES}

Se ha justificado la influencia de los distintos tapones sintéticos porosos en el proceso de oxigenación de los vinos en condiciones de envejecimiento en botella. Ello está asociado a la morfología de estos tapones de polietileno y en concreto a la relación fase amorfa/fase cristalina, entendiendo que existe una mayor permeabilidad del oxígeno a través de la parte amorfa del polímero.

La aplicación de un sistema de caracterización electrónica ha permitido evaluar las diferencias organolépticas que ocasiona dentro de un conjunto de vinos el cambio del método de extracción (tradicional o termovinificación), la aplicación de la microoxigenación y el uso de tapones de plástico espumado con diferentes niveles de permeabilidad al paso del oxigeno, durante el periodo del envejecimiento en botella.

De la misma manera la respuesta obtenida con este prototipo, especialmente la nariz y lengua electrónica, ha demostrado ser correlacionable con diversos parámetros físico-químicos, y parámetros referentes al nivel de oxígeno durante el envejecimiento en botella.

Una exploración estadística más particular de los resultados obtenidos para las muestras estudiadas nos permite extraer las siguientes conclusiones específicas:

El análisis de datos mediante PCA ha demostrado que es posible distinguir entre los diferente OTR, niveles de polifenoles y microoxigenación, tanto por parte de los sistemas individuales como por parte del panel electrónico, tras el pretratamiento de los correspondientes matrices de datos.

Respecto a los métodos supervisados como el PLS-DA, se ha evidenciado el potencial que pueden tener estos sistemas para valorar un conjunto de muestras en función del oxígeno presente y la cantidad de polifenoles. Específicamente podemos afirmar que:

Se ha demostrado la capacidad de la nariz electrónica y el ojo electrónico para seguir los cambios en los atributos sensoriales inducidos por el uso de tapones sintéticos porosos con diferente permeabilidad. 
En el caso de la lengua electrónica, podemos además decir que este sistema es más sensible a las propiedades organolépticas relacionadas con el estado de oxidación de los vinos. En este caso ha sido posible hacer un seguimiento a las características inducidas por la microoxigenación y el contenido polifenólico controlado durante la producción del vino.

El panel de cata electrónico es por lo tanto una herramienta adecuada para evaluar las diferencias que se producen en el vino debido a la aplicación de distintas técnicas de extracción (termovinificación/método tradicional), de métodos de microoxigenación y al uso de tapones sintéticos con diferentes OTR. El uso combinado de las coordenadas CIELab, la nariz y la lengua genera una evaluación más exacta de los fenómenos que tienen lugar en función de los cambios inducidos durante la elaboración del vino.

Los datos cuantitativos obtenidos a partir de un modelo de regresión PLS2 han demostrado que las respuestas de la nariz y lengua electrónicas presentan una buena correlación con el contenido de polifenoles y los parámetros relacionados con el nivel de oxígeno. Concretamente los parámetros con los que se obtuvo una mejor correlación fueron los taninos, el ácido hidroxicinámico y la contribución al paso del oxígeno del tipo de tapón utilizado.

En resumen, la capacidad de discriminación del panel electrónico mejora considerablemente cuando se fusionaron las señales de cada tipo de sistema electrónico.

Finalmente, desde un punto de vista enológico, es importante decir que investigaciones como esta demuestran que existe un efecto significativo del OTR de los tapones durante la evolución de los compuestos fenólicos, y por tanto es importante continuar en la búsqueda de alternativas como los tapones sintéticos que dan un aporte en el proceso de elaboración del vino. Además, herramientas como pueden ser el panel de cata electrónico contribuyen en la evaluación de las características organolépticas, en las diferentes fases de elaboración de los vinos.

\subsection{REFERENCIAS}

[1] V. L. Singleton, Oxygen with phenols and related reactions in musts, wines, and model systems-observations and practical implications, American Journal of Enology and Viticulture, 1987, 38, 69-77.

[2] A. L. Waterhouse \& V. F. Laurie, Oxidation of wine phenolics: A critical evaluation and hypotheses, American Journal of Enology and Viticulture, 2006, 57, 306-313. 
[3] C. Morel-Salmi, J. M. Souquet \& M. Y. C. Bes, Effect of flash release treatment on phenolic extraction and wine composition Analytica Chimica Acta, 2006, 54, 4270-4276.

[4] F. Palomero, A. Morata, S. Benito, M. C. González \& J. A. Suárez-Lepe, Conventional and enzyme-assisted autolysis during ageing over lees in red wines: Influence on the release of polysaccharides from yeast cell walls and on wine monomeric anthocyanin content Food Chemistry, 2007, 105, 838-846.

[5] M. Bourzteix, J. Mourges \& S. Aubert, Influence de la duree de maceration sur la constitution en polyphenols et sur la degustation des vins rouges, Connaisance Vigne Vin, 1970, 4, $447-460$

[6] C. A. Sims, R. P. Bates \& A. G. Arreola, Color, polyphenoloxidase, and sensory changes in banana juice as affected by heat and ultrafiltration, Journal of Food Quality, 1994, 17, 371-379.

[7] V. Cheynier, El color de los vinos tintos Wine Internet Technical Journal, 2003, 4, 1-9.

[8] H. Fulcrand, M. Dueñas, E. Salas \& V. Cheynier, Phenolic reactions in the course of winemaking and ageing, American Journal of Enology and Viticulture, 2006, 57, 298-305.

[9] J. Ribereau-Gayon, Dissolution d'oxygene dans les vins. In contribution a l'etude des oxidations et reductions dans les vins. Application a l'etude de vieillissement et des casses, Delmas, Bordeaux-France, 1933.

[10] P. Ribéreau-Gayon, Y. Glories \& D. Maujean A, The chemestry of wine, stabilization and treatments, Wiley, 1999.

[11] F. Zamora, Elaboración y crianza del vino tinto: aspectos científicos y prácticos, Mundi prensa, 2003.

[12] T. Garde-Cerdan, C. Lorenzo, G. L. Alonso \& M. R. Salinas, Employment of near infrared spectroscopy to determine oak volatile compounds and ethylphenols in aged red wines, Food Chemistry, 2010, 119, 823-828.

[13] M. Moutounet, P. Ducournau, M. Chassin \& T. Lemaire, Appareillage d'apport d'oxygène aux vins. Son Interêt technologique. , Paris, 1995, pp. 411-414.

[14] J. M. Cook, R. L. Karelitz \& D. E. Dalsis, Measurement of oxygen, nitrogen, and carbon dioxide in beverage headspace, Journal of Chromatographic Science, 1985, 23.

[15] P. Godden, K. Lattey, L. Francis, M. Gishen, G. Cowey, M. Holdstock, E. Robinson, E. Waters, G. Skouroumounis, M. Sefton, D. Capone, M. Kwiatkowski, J. Field, A. Coulter, N. D'Costa \& B. Bramley, Towards offering wine to the consumer in optimal condition;the wine, the closures and other packaging variables. A review of AWRI research examining the changes that occur in wine after bottling. , Wine Industry Journal, 2005, 20, 20-30.

[16] P. Lopes, C. Saucier \& Y. Glories, Nondestructive colorimetric method to determine the oxygen diffusion rate through closures used in winemaking. , Journal of Agricultural and Food Chemistry, 2005, 53, 6967-6973.

[17] P. Lopes, M. A. Silva, A. Pons, T. Tominaga, V. Lavigne, C. Saucier, P. Darriet, P. L. Teissedre \& D. Dubourdieu, Impact of Oxygen Dissolved at Bottling and Transmitted through Closures on the Composition and Sensory Properties of a Sauvignon Blanc Wine during Bottle Storage, Journal of Agricultural and Food Chemistry, 2009, 57, 10261-10270. 
[18] P. Lopes, C. Saucier, P. L. Teissedre \& Y. Glories, Main routes of oxygen ingress through different closures into wine bottles, Journal of Agricultural and Food Chemistry, 2007, 55, 51675170.

[19] L. Pasteur, Etudes sur le vin: ses maladies, causes qui les provoquent, procedes nouveaux pour les conserver et pour les vieillir, Royale, Paris, France, 1873.

[20] E. Peynaud, Connaissance et travail du vin, Dunod, Paris-Francia, 1981.

[21] V. Atanasova, H. Fulcrand, V. Cheynier \& M. Moutounet, Effect of oxygenation on polyphenol changes occurring in the course of winemaking., Analytica Chimica Acta, 2002, 458, $15-27$.

[22] G. Skouroumounis, M. Kwiatkowski, I. Francis, H. Oakey, D. Capone, B. Duncan, M. Sefton \& E. Waters, The impact of closure type and storage conditions on the composition, colour and flavour properties of a Riesling and a wooded Chardonnay wine during five years' storage. , Australian Journal of grape and wine Research, 2005, 11, 369-384.

[23] M. Kwiatkowski, G. Skouroumounis, K. A. Lattey \& E. Waters, The impact of closures, including screw cap with three different headspace volumes, on the composition, colour and sensory properties of a Cabernet Sauvignon wine during two years' storage, Australian Journal of Grape and Wine Research, 2007, 13, 81-94.

[24] B. Duncan \& A. Kleinig, Oxygen Transmission Analysis of Wine Bottle Closures., Southcorp Wines, 1999.

[25] M. A. Sefton \& R. F. Simpson, Compounds causing cork taint and the factors affecting their transfer from natural cork closures to wine - a review, Australian Journal of grape and wine Research, 2005, 11, 226-240.

[26] J. B. Dieval, M. Veyret, J. C. Vidal, O. Aagaard \& S. Vidal, Validation of noninvasive measurement of total package oxygen. , Bulletin de l’O.I.V, Zagreb, Croatia, 2009, p. 78.

[27] V. O’Brien, C. Colby \& M. Nygaard, Managing oxygen ingress at bottling, The Australian and New Zealand Wine Industry Journal, 2009, 24, 24-29.

[28] J. Wirth, C. Morel-Salmi, J. M. Souquet, J. B. Dieval, O. Aagaard, H. Vidal, H. Fulcrand \& $\mathrm{V}$. Cheynier, The impact of oxygen exposure before and after bottling on the polyphenolic composition of red wines, Food Chemistry, 2010, 123, 107-116.

[29] V. Parra, Diseño y aplicación de sensores electroquímicos basados en compuestos orgánicos electroactivos para una lengua electrónica: discriminación y clasificación de vinos., Tesis doctoral, Universidad de Valladolid, Valladolid, 2005.

[30] S. Caille, A. Samson, J. Wirth, J. B. Dieval, S. Vidal \& V. Cheynier, Sensory characteristics changes of red Grenache wines submitted to different oxygen exposures pre and post bottling, Analytica Chimica Acta, 2010, 660, 35-42.

[31] V. Parra, T. Hernando, M. L. Rodriguez-Mendez \& J. A. de Saja, Electrochemical sensor array made from bisphthalocyanine modified carbon paste electrodes for discrimination of red wines, Electrochimica Acta, 2004, 49, 5177-5185. 
[32] I. Apetrei, C. Apetrei, I. Nevares, M. Del Alamo, V. Parra, M. L. Rodríguez-Méndez \& J. A. De Saja, OIV Conference. ( Logroño) 2006.

[33] M. Gay, C. Apetrei, I. Nevares, M. del Alamo, J. Zurro, N. Prieto, J. A. De Saja \& M. L. Rodriguez-Mendez, Application of an electronic tongue to study the effect of the use of pieces of wood and micro-oxygenation in the aging of red wine, Electrochimica Acta, 2010, 55, 6782-6788.

[34] C. Apetrei, M. L. Rodriguez-Mendez, V. Parra, F. Gutierrez \& J. A. de Saja, Array of voltammetric sensors for the discrimination of bitter solutions, Sensors and Actuators B-Chemical, 2004, 103, 145-152.

[35] A. Legin, A. Rudnitskaya, L. Lvova, Y. Vlasov, C. Di Natale \& A. D'Amico, Evaluation of Italian wine by the electronic tongue: recognition, quantitative analysis and correlation with human sensory perception, Analytica Chimica Acta, 2003, 484, 33-44.

[36] C. Di Natale, A. Macagnano, F. Davide, A. D'Amico, R. Paolesse, T. Boschi, M. Faccio \& G. Ferri, An electronic nose for food analysis, Sensors and Actuators B-Chemical, 1997, 44, 521-526.

[37] K. C. Persaud \& J. P. Travers, Arrays of broad specificity films for sensing volatile chemicals, (Ed. Kress-Rogers), CRC Press, Frankfurt, 1997, pp. 563-592.

[38] M. G. O’Sullivan, D. V. Byrne, M. T. Jensen, H. J. Andersen \& J. Vestergaard, A comparison of warmed-over flavour in pork by sensory

analysis, GC/MS and the electronic nose, Meat science, 2003, 65, 1125-1138.

[39] T. C. Pearce, S. Schiffman, H. Troy Nagle \& J. W. Gardner, Handbook of Machine Olfaction: Electronic Nose Technology, John Wiley \& Sons 2003. 


\section{CAPITULOIV \\ Evaluación, Caracterización Y Análisis Comparativo de Vinos Tintos Tratados con Sistemas Alternativos a la Barrica de Roble Organolépticas de Un Vino Tinto}

Página

4.1. GENERALIDADES

4.1.1. Revisión teórica

4.1.1.1. Técnicas alternativas para el envejecimiento del vino

4.1.1.2. Efectos de la madera sobre el vino

4.1.1.3. Compuestos cedidos por la madera al vino

4.1.1.4. Tendencias actuales de consumo y el posicionamiento de métodos alternativos

\subsection{DESARROLLO EXPERIMENTAL}

4.2.1. Preparación de las muestras de vino

4.3. RESULTADOS

4.3.1. Parámetros Químicos 197

4.3.2. Coordenadas CIELab 200

4.3.3. Cromatografía de Gases Masas $\quad 204$

4.3.4. Cromatografía de Gases-Olfatometría 207

$\begin{array}{ll}\text { 4.3.5. Nariz electrónica } & 213\end{array}$

4.3.5.1. Análisis exploratorio. PCA 214

4.3.5.2. Análisis supervisado. PLS-DA 216

4.3.6. Correlaciones entre sistemas 221

4.3.6.1. Regresión Lineal por mínimos cuadrados PLS2 221

$\begin{array}{ll}\text { 4.3.6.2. Modelo Tucker3 } & 227\end{array}$

$\begin{array}{ll}\text { 4.3.6.3. ANOVA-PLS } & 236\end{array}$

4.3.7. Tratamientos de datos complementarios 240

4.4. CONCLUSIONES 249

4.5. REFERENCIAS $\quad 251$ 



\subsection{GENERALIDADES}

En este capítulo se aborda un viejo problema que en la actualidad ha cobrado un notable interés: el envejecimiento del vino en barrica y las alternativas que existen para sustituir o complementar esta práctica tradicional. Por tanto, hemos definido como objetivo básico de esta investigación, la implementación de un método de valoración organoléptica mediante sistemas de análisis sensorial electrónico y técnicas analíticas que permitan distinguir las principales características que cada tipo de envejecimiento le aporta a los vinos.

En relación con los vinos evaluados, nos hemos centrado en el envejecimiento de vinos tintos de Ribera del Duero mediante métodos tradicionales (barrica de roble) o métodos alternativos (depósito de acero con inserción de madera de roble). Especificar además que los resultados que recogemos a continuación están relacionados con muchos de los fenómenos físico-químicos referidos en el capítulo anterior.

La información concerniente al análisis de la fase volátil de los vinos con la nariz electrónica vendrá acompañada por los resultados de técnicas analíticas como la GC-MS y la olfatometría. Respecto al estudio realizado con lengua electrónica, precisar que estos resultados serán discutidos en profundidad en otra tesis doctoral realizada en nuestro grupo de investigación. Nuestra aportación a las experiencias que se realizan con la lengua electrónica ha sido la validación de herramientas quimiométricas, que permitirán mejorar la habilidad de interpretación del conjunto de señales provenientes del panel de cata electrónico (nariz + lengua + ojo electrónico).

En cuanto al análisis estadístico de los resultados se establecerán una serie de correlaciones entre los métodos de análisis realizados, y, fundamentalmente, una valoración comparativa de los productos elaborados en barrica estándar frente a los conseguidos con métodos alternativos, utilizando el mismo tipo de roble y grado de tostado. Esta correlación será referida fundamentalmente a las variables del aroma y color. El análisis estadístico de estas correlaciones, ha de suministrar información sobre la adecuada utilización de estas técnicas frente a la detección de elaboraciones alternativas objetivo de esta fase de nuestra investigación. 


\subsubsection{Revisión teórica}

En el transcurso del proceso de envejecimiento, la barrica somete al vino a tres efectos principalmente:

1. Una oxidación prolongada durante todo el añejamiento, en la que el oxígeno difunde desde el exterior a través de los poros de la madera hacia el vino. Algunos autores sostienen que esta difusión es mínima y que el mayor aporte de oxígeno se produce al abrir la barrica para el relleno. Este fenómeno dependerá principalmente de la clase de roble utilizado en la barrica y del tipo de cortado de la madera [1].

2. La evaporación de etanol y agua del vino hacia el exterior, en contracorriente a la difusión de oxígeno. La proporción etanol/agua evaporada depende de la temperatura y humedad relativa exterior, de manera que una mayor temperatura ambiental favorece la evaporación de agua, mientras que una humedad relativa alta favorece la del etanol. Esta difusión hacia el exterior dependerá, una vez más, del tipo de roble y además del modo de aserrado de las piezas de roble $[2,3]$.

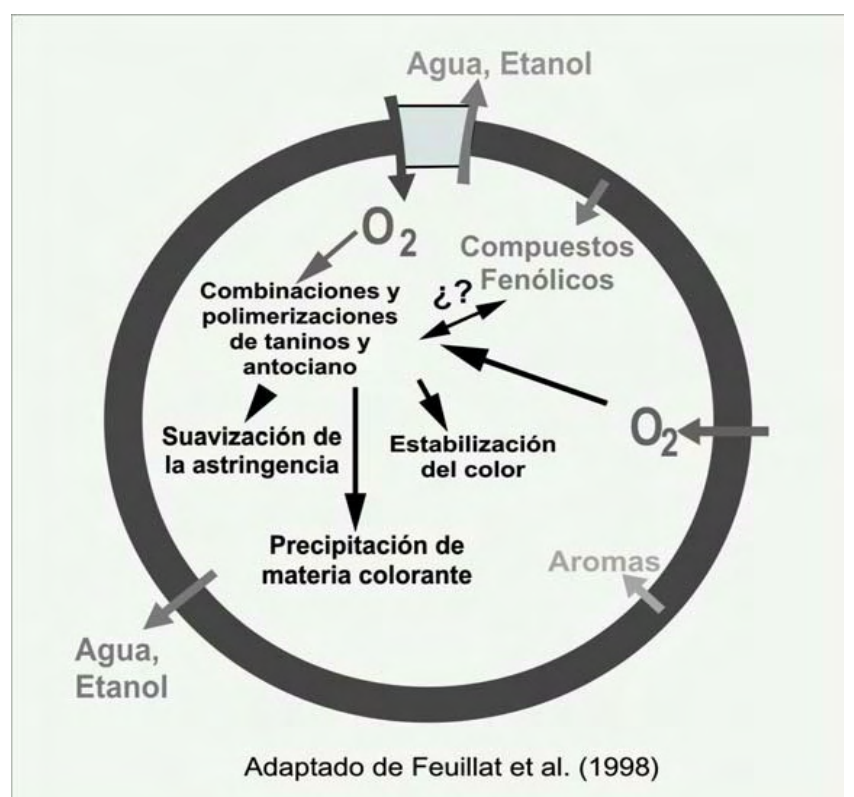

Figura 4.1. Influencia de la crianza en barrica sobre la evolución del vino. Tomado de F. Zamora [3]

3. La cesión al vino de sustancias contenidas en la madera de roble. La clase de sustancias que se extraigan dependerá del tipo de secado y quemado al que se haya sometido a la madera; mientras que la cantidad de sustancias extraídas dependerá del pH y la graduación alcohólica del vino contenido en la barrica. 
Muchas son las sustancias extraídas por el vino, provenientes del roble, pero gran cantidad de ellas no se extraen en cantidades suficientes para ser percibidas por el olfato humano. En todo caso, el análisis sensorial por correlaciones estadísticas indica que los atributos a vainilla, roble, madera, coco, medicinal, tostado, ahumado y especiado, son factores del sabor relacionados con la maduración de las vinos en barricas de roble [4-6].

La acción de estos tres fenómenos (oxidación, evaporación y cesión) causará una serie de transformaciones (Figura 4.1), que aportarán el bouquet definitivo durante el envejecimiento en barrica.

\subsubsection{Técnicas alternativas para envejecimiento del vino}

La utilización de virutas de roble para aromatizar vinos es una técnica sobradamente conocida y aplicada en bodega desde hace muchos años. Las posibilidades existentes en el mercado son muy amplias, la madera de roble con esta configuración se ofrece de distintas formas: las conocidas como virutas (oak chips), las porciones de madera cortadas formando cubos llamados cubes u oak beams, también encontramos polvo de roble (oak powder), trozos de madera granulada llamados pencil shavings o granulates, o también en bloques paralelepípedos (blocks o segments) [7]. Algunas de estas opciones se ilustran en la Figura 4.2.
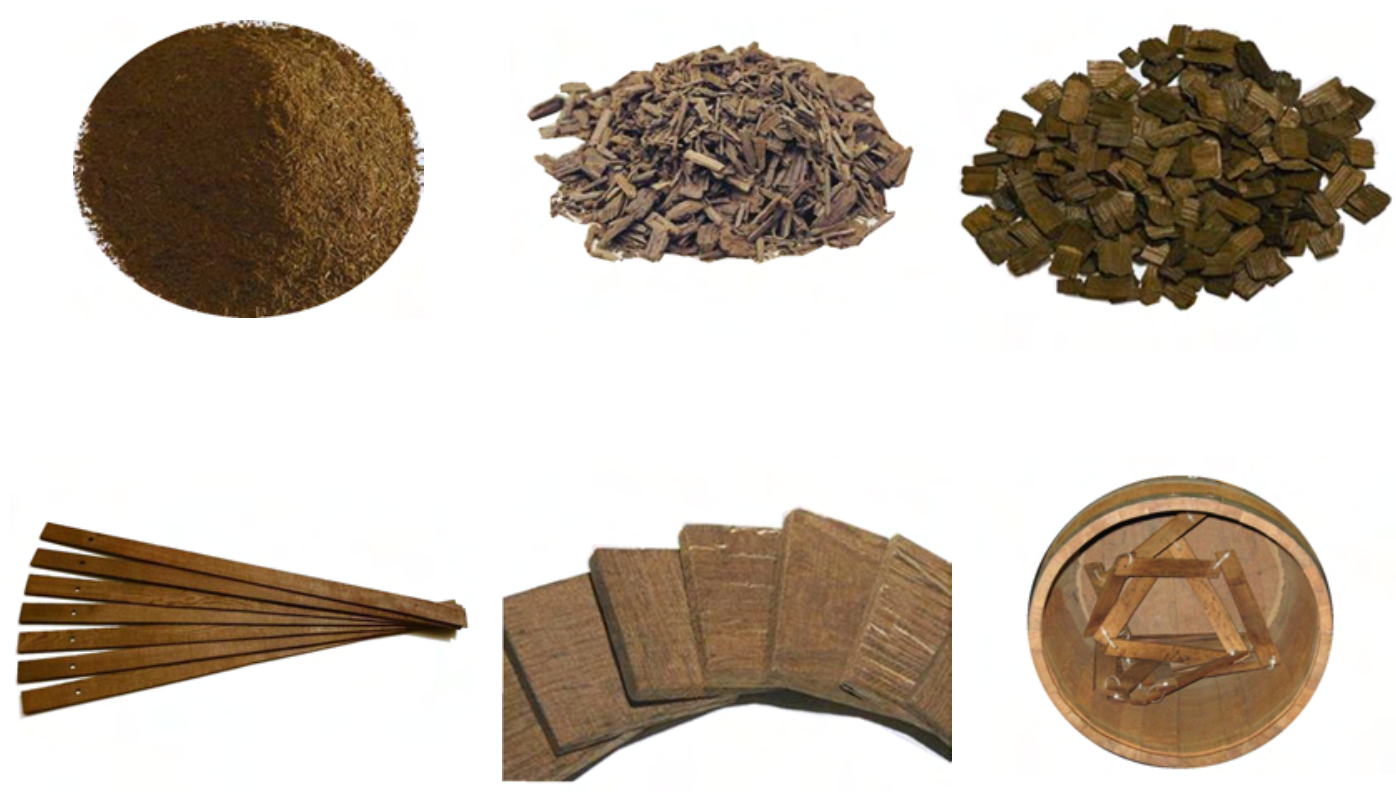

Figura 4.2. Tipos de madera utilizada para el envejecimiento con métodos alternativos. 
La parte más compleja que tiene la aplicación de esta técnica alternativa, es elegir la combinación adecuada que propicie la estructura aromática deseada, es por ello que en cada elaboración es necesario adaptar el tipo de madera y tostado, además de los momentos de adición de las porciones de madera.

Es bien sabido que durante la fermentación de vinos tintos el polvo, astillas o chips pueden adicionarse al encubado, teniendo notables efectos sobre la expresión aromática y el color. No obstante también es conocido que este método responde más a un sentido práctico que técnico, y que la eficacia del tratamiento puede ser inferior a una adición posterior al descube y prensado, según la duración y eficacia de la maceración. En cualquier caso, existe una notable volatilización de compuestos aromáticos arrastrados por el $\mathrm{CO}_{2}$ liberado durante la fermentación. Las distintas dosis se aplican en función del efecto que se busca utilizando cantidades en un rango de 1 a $10 \mathrm{~g} / \mathrm{L}$.

Durante la crianza, se pueden realizar aportes de madera en dosis inferiores con el objetivo de evitar un endurecimiento excesivo del sabor. El uso de duelas sumergidas aporta un enmaderado progresivo, pero con un cierto riesgo de endurecimiento por aportación tánica [8]. Cada vez es posible encontrar más opciones para este tipo de crianza; así recientemente se ha puesto en el mercado chips sin taninos, que limitan su eventual impacto sobre la estructura del vino.

En ocasiones también se aporta polvo o astillas de madera a las barricas tradicionales unas semanas antes del embotellado, con el objetivo de obtener un carácter más maderado de forma rápida. Este procedimiento tiene un riesgo de inestabilidad. En efecto, los elagitaninos disueltos se hidrolizan en unas semanas en el vino, liberando ácido elágico insoluble. En estos casos es imperativo asegurar que los vinos lleguen a un punto de estabilidad antes de proceder al embotellado

Así, el equivalente a una crianza de un año en barrica se estima corresponde a una formulación de 10 a $15 \mathrm{~g} / \mathrm{L}$ de chips de roble, aportados en diferentes momentos. En general, el coste de la madera en el envejecimiento alternativo puede oscilar entre 0,05 y $0,1 € / L$, frente a 0,5 y $1 € / L$ para la barrica [9]. Además, las posibilidades técnicas en principio son mucho mayores, puesto que en el método alternativo el enólogo puede jugar con las dosis, los tostados, la presencia o no de taninos y los momentos de aplicación.

\subsubsection{El efecto de la madera sobre el vino}

La madera utilizada durante el envejecimiento en barrica proporciona muy pocos compuestos fenólicos en comparación con los que tienen su origen en la uva. Entre los que aporta la madera se encuentran los ácidos y aldehídos fenólicos, 
estilbenos, y algunos taninos hidrolizables. Por otra parte estos compuestos varían de acuerdo al tipo de roble, el origen geográfico de la madera, el secado y grado de tostado. A estas variables se une la entrada de oxigeno por los poros de las duelas que da lugar a la oxidación del etanol para generar el acetaldehído que intervine en la condensación entre antocianos y taninos; así los niveles de etanol han de condicionar el nivel de estos compuestos en el vino [10]. Además la barrica permite la difusión de agua y etanol hacia el exterior, como se mencionó anteriormente, que puede escapar como vapor de acuerdo con las condiciones ambientales de la bodega, vino que se repone rellanado la barrica.

Cuando se utiliza un envejecimiento en depósitos de acero inoxidable con inserción de madera ha de utilizarse una dosificación controlada de oxigeno para conseguir la modificación de la fracción fenólica de los vinos, que repercuta finalmente en la mejora de las propiedades cualitativas y cuantitativas de los vinos. La estrategia en nuestra investigación ha sido regular el control de oxigeno en función del existente en un mismo vino envejecidos en barricas de idéntico roble y tostado al de la madera (chips) que ha sido sumergida en el depósito de acero; ello nos permitió eliminar la variable oxigeno en nuestras experiencias.

\section{Efectos sobre el color}

El aporte de la madera generalmente permite intensificar algunas características del color de los vinos. El efecto puede ser superior cuanto más temprano se aporte la madera. Se trata de un efecto ligado al aporte tánico y/o a compuestos colorantes (que incrementan la tonalidad parda). Los taninos reaccionan con los antocianos durante el inicio de la maceración, antes de la producción de alcohol por parte de las levaduras. En cualquier caso, si existe un incremento de color, este no es necesariamente estable, ya que la pigmentación desaparece con la aparición del alcohol por desorganización molecular. Para la estabilización de este color será necesaria la acción del oxígeno, generalmente mediante más control de la microoxigenación [3].

\section{Efectos sobre la estructura y el dulzor}

La madera aumenta el volumen global en boca por un incremento conjugado de la estructura y el dulzor. Con los chips, es posible incidir sobre este equilibrio de diversas formas:

El uso de madera sin taninos permite aportar dulzor.

El uso de madera sin tostar también aporta dulzor. 
La actividad microbiológica (alcohólica y maloláctica) permite limitar el impacto de la madera sobre la estructura.

Por contra, el uso de madera con taninos después de la fermentación permite aumentar la estructura de los vinos.

El dulzor lo aportan lactonas y polisacáridos en el caso de la madera sin tostar, compuestos de degradación de las ligninas en las maderas tostadas. El efecto en la estructura del vino lo aportan los taninos, pero también algunos compuestos volátiles que aparecen en los tostados intensos; como en el caso del 4-metil- 2,6dimetoxifenol que, a menudo, es responsable de sensaciones de dureza y sequedad [11].

\section{Efecto sobre la expresión aromática}

La expresión aromática es el primer elemento que nos viene a la mente cuando hablamos del enmaderado de vinos en cualquiera de sus versiones (polvo, chips, duelas, barricas, etc.). Notas afrutadas, especiadas, vainilladas o tostadas vienen a completar la paleta aromática de los vinos. Estos aromas provienen, bien de la degradación de compuestos de la madera durante el tostado, o bien de la madera en sí misma.

Los compuestos volátiles provenientes de la madera son numerosos pero en concentraciones bajas. Fundamentalmente el eugenol aporta caracteres especiados, la ß-ionona caracteres florales, las lactonas notas lácteas y afrutadas, etc.

Las maderas sin tostar son aromáticamente menos intensas que las tostadas, y permiten trabajar el volumen en boca limitando el impacto aromático. En este caso, es necesario ser cuidadoso con la calidad de la madera, ya que un secado deficiente proporciona «maderas verdes» con caracteres de serrín, secantes y vegetales.

La lignina se degrada durante el tostado dando lugar a fenoles volátiles y aldehídos aromáticos (guayacol, vainillina, siringaldehído), al mismo tiempo que las hemicelulosas dan compuestos furánicos (furfural, 5-metilfurfural: notas de frutos secos y almendra tostada).

Cada compuesto aromático aparece preferentemente a una temperatura concreta. Siempre que se consiga un tostado homogéneo, se obtienen maderas con características muy precisas, y en consecuencia, se permite el ajustado aromático deseado para los vinos. A la inversa, una mezcla de diferentes temperaturas de tostado aporta maderas complejas, que se aproximan bien a la complejidad 
debida al gradiente de tostado a lo largo del espesor de la madera en la barrica [6].

La fermentación alcohólica y maloláctica cambian el perfil aromático de la madera. Además de la absorción de compuestos volátiles por parte de los microorganismos, que disminuyen la intensidad aromática, existe también una transformación de ciertas moléculas: la vainillina se transforma en alcohol vainíllico, casi inodoro, a la vez que el furfural puede dar lugar a furfuriltiol, con olor acusado a café y con un umbral de percepción muy bajo [12, 13]. Este compuesto aparece a menudo cuando la fermentación maloláctica se efectúa en presencia de chips, duelas o barricas ricas en furfural.

Cuando se trabaja con chips, es necesario tener presente el antagonismo entre complejidad e intensidad. La puntualización de un característica aromática se podrá realizar con dosis bajas de chips, a la vez que un trabajo sobre la complejidad exigirá una dosificación superior de mezclas de diferentes tostados.

\section{Efectos sobre la expresión afrutada/vegetal}

La madera puede disminuir los caracteres vegetales de ciertos vinos. Mediante un incremento de la expresión afrutada: las whiskylactonas parecen aumentar la intensidad aromática afrutada, aportando notas cítricas y de coco cuando llegan a concentraciones elevadas. A concentraciones medias, la expresión afrutada del vino resulta reforzada durante la degustación. El efecto es más interesante después de la fermentación, puesto que la pérdida aromática por desplazamiento de $\mathrm{CO}_{2}$ es menor. Las whiskylactonas provienen sobre todo, de maderas sin tostar, que no aportan aromas característicos a madera [14]. En vinos blancos por el contrario, un aporte importante de whiskylactonas puede hacer pesado el perfil aromático; en estos casos puede ser mejor elegir maderas menos ricas en lactonas y más florales.

También puede existir un efecto de enmascarado ya que algunos aromas provenientes del tostado pueden ocultar notas vegetales. En este caso, se utilizarán maderas tostadas, sabiendo que también aportan caracteres enmaderados.

\subsubsection{Compuestos cedidos por la madera al vino}

Hay pocos estudios sobre la influencia que presenta la composición del vino en la extracción de los compuestos volátiles procedentes de la barrica, sin embargo empíricamente se conoce que no todos los vinos evolucionan de la misma 
manera en barricas de roble similares. Uno de los problemas para obtener conclusiones generales es que parte de las investigaciones que se han llevado a cabo hasta el momento y que hemos estudiado, cuentan con experimentos cuyos vinos sintéticos fueron preparados con diferentes porcentajes de alcohol. En otros casos, los resultados corresponden a tipo de uva y denominaciones de origen diferentes, dificultando en todo caso la posibilidad de establecer comparaciones.

Las Tablas 4.1-4.3 presentan un resumen de los resultados comunes entre diferentes investigaciones sobre los principales compuestos que intervienen durante el envejecimiento de los vinos tintos [15].

Tablas 4.1. Compuestos extraíbles de la madera.

\begin{tabular}{|c|c|}
\hline $\begin{array}{l}\text { Compuestos genuinos extraíbles de } \\
\text { cualquier parte de la madera }\end{array}$ & $\begin{array}{c}\text { Compuestos extraíbles } \\
\text { únicamente de la superficie de la } \\
\text { madera }\end{array}$ \\
\hline 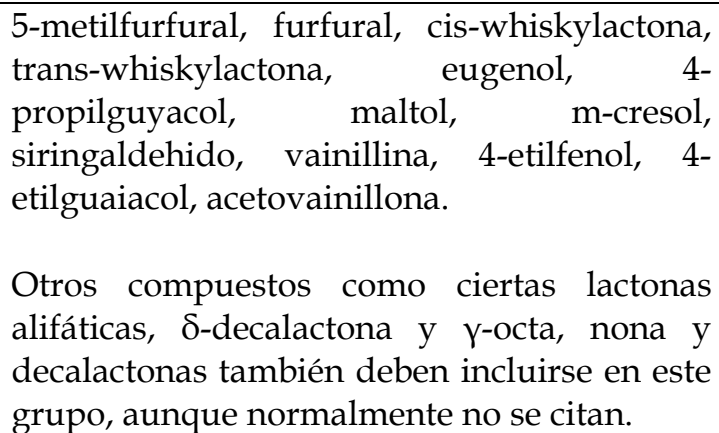 & $\begin{array}{l}\text { Son compuestos cuya concentración } \\
\text { en el vino después de unos tres } \\
\text { meses de contacto también es } \\
\text { siempre más alta que la del vino del } \\
\text { depósito de acero, pero que a partir } \\
\text { de ese tiempo la concentración no } \\
\text { sigue aumentando. A esta categoría } \\
\text { pertenecen productos del } \\
\text { metabolismo de los ácidos grasos. }\end{array}$ \\
\hline
\end{tabular}

En la siguiente tabla enumeramos compuestos que también se originan a partir de precursores existentes en el vino, es decir, sustancias que también aparecen en el vino mantenido en depósitos y madera de roble.

Tablas 4.2. Compuestos extraíbles de la madera y su relación con compuestos precursores existentes en el vino.

\begin{tabular}{|c|l|}
\hline $\begin{array}{c}\text { Por la acción de la } \\
\text { madera en los } \\
\text { precursores en el vino }\end{array}$ & $\begin{array}{l}\text { Acetovainillona, vainillado de metilo, furaneol, } \beta \text { - } \\
\text { damascenona, } \beta \text {-ionona, } \gamma \text {-ionona, linalol y } \\
\text { homofuraneol. }\end{array}$ \\
\hline Por acción microbiológica & $\begin{array}{l}\text { 4-etilfenol y 4-etilguayacol que se originan por acción } \\
\text { de las levaduras Brettanomyces/Dekkera sobre los } \\
\text { ácidos p-cumárico y ferulico }\end{array}$ \\
\hline Por oxidación & $\begin{array}{l}\text { Los dos ejemplos más representativos de este grupo } \\
\text { son sotolón y fenilacetaldehído. }\end{array}$ \\
\hline
\end{tabular}

El estudio de la evolución de los compuestos permite su clasificación en dos categorías de acuerdo con el tipo de envejecimiento aplicado: 
a) Compuestos que aumentan solo durante la crianza en barrica, y

b) Compuestos que se forman tanto en barrica como en depósito virutas.

\section{Compuestos que aumentan sólo en los vinos criados en barrica}

En esta categoría se agrupan los compuestos que provienen exclusivamente de la madera y aquellos otros ligados a procesos oxidativos [16]. La evolución que presentan los distintos compuestos aquí agrupados es muy diferente, de manera que están separados en función de su comportamiento en los diferentes periodos del envejecimiento.

Tabla 4.3. Compuestos presentes durante el envejecimiento en barrica (tiempos estimados).

\begin{tabular}{|c|l|}
$\begin{array}{c}\text { Compuestos que } \\
\text { presentan su máximo de } \\
\text { extracción a los seis } \\
\text { meses }\end{array}$ & $\begin{array}{l}\text { El furfural, 5-metilfurfural y la y-octalactona. } \\
\text { Compuestos con una cinética de extracción muy rápida. } \\
\text { No se observan variaciones significativas entre la del } \\
\text { roble francés del americano. }\end{array}$ \\
\hline $\begin{array}{c}\text { Componentes que } \\
\text { presentan el máximo de } \\
\text { extracción a los doce } \\
\text { meses }\end{array}$ & $\begin{array}{l}\text { El único componente del vino que responde a este } \\
\text { comportamiento es la trans-whiskylactona. La cesión de } \\
\text { este compuesto es mayor y más rápida cuando se utiliza } \\
\text { roble francés, su degradación comienza a partir de los } 12 \\
\text { meses. }\end{array}$ \\
\hline
\end{tabular}

\footnotetext{
Componentes que presentan el máximo de extracción entre doce y veinticuatro meses
}

Cis-whiskylactona, eugenol, 2,6 dimetoxifenol y 4propilguayacol.

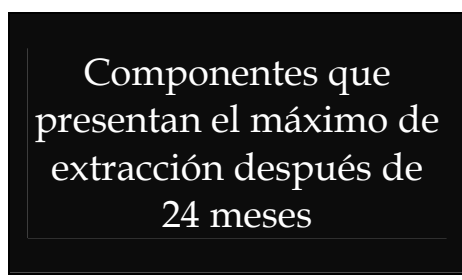

Incrementan continuamente su contenido en función del tiempo y no alcanzan un equilibrio. Maltol, 4-etil-2,6 dimetoxifenol y m-cresol pertenecen a este grupo. En caso del sotolon, esta sustancia no la cede la barrica por un proceso de extracción sino que se origina en ella por un proceso oxidativo.

Compuestos que presentan dos máximos de extracción
Dos productos responden a este comportamiento, el siringaldehido y la vainillina. El primer máximo se presenta en octubre, pasadas las temperaturas altas del verano y el segundo un año después.

Resumiendo, dentro de este conjunto de compuestos que contribuyen activamente en el aroma del vino durante su etapa de envejecimiento, existe un porcentaje de ellos que han sido continuamente reportados como compuestos marcadores, es decir que favorecen la identificación de diferencias entre un grupo de muestras evaluados durante el envejecimiento. Dentro de estos 
compuestos marcadores, en esta investigación se realizará un seguimiento especial al furfural, guaiacol, cis, trans-whiskylactona y eugenol, componentes fundamentales por su evolución durante el proceso.

\subsubsection{Tendencias actuales de consumo y el posicionamiento de métodos alternativos}

Hasta hace muy poco, los acuerdos comerciales bilaterales reconocían las prácticas enológicas mutuas aceptando las importaciones en territorio europeo de vinos sometidos a estos tipos de tratamientos, existentes desde 1983, pero prohibiendo curiosamente estas prácticas a los productores europeos. Desde 1993, se podían utilizar «a título experimental» virutas y otros granulados de roble respetando las obligaciones dictadas por el reglamento CEE 822/87, es decir, con un límite de $50.000 \mathrm{hl}$ por año, durante tres años como máximo y con la imposibilidad de exportar fuera del país de producción. Hipócritamente, la "experimentación" ha durado más de diez año. Desde 2006, la normativa ha evolucionado. Así el reglamento 1507/2006, de 11 de octubre de 2006, modifica el anterior $(1622 / 2000)$ permitiendo la utilización de trozos de madera de roble más o menos tostados, el $95 \%$ de los cuales debe superar $2 \mathrm{~mm}$. sin liberar substancias que puedan presentar un riesgo para la salud.

Algunas denominaciones de origen en España han rechazado este tipo de prácticas. En Estados Unidos, un mercado extremadamente maduro para la utilización de barricas y métodos alternativos, se han observado en los últimos años numerosos cambios. Algunas de las conclusiones que han sido publicadas anualmente por la revista WineBusiness [17-21]. Con el propósito de determinar tendencias en el uso de barricas de roble y las alternativas a este tipo de envejecimiento en este país. Estos resultados además ofrecen un panorama general en la industria enológica sobre su uso y una valoración definitiva extrapolable, quizás, a la industria vitivinícola en nuestro país. No se trata de un trabajo científico como tal, y ello implica que de cierta manera que los resultados deben ser utilizados sólo como un punto de referencia para posteriores reflexiones.

\section{Metodología del estudio llevado a cabo para conocer las posibilidades de los} métodos alternativos en USA.

Este estudio se basa en una encuesta anual que se realiza a diferentes bodegas en Estados Unidos. Hasta el momento se ha registrado un máximo de participación con 341 respuestas y un mínimo con 222 respuestas desde el año 2003 hasta el año 2010. Las bodegas encuestadas pertenecen a las regiones de California, 
Washington, Oregon, Nueva York, Ontario, Texas, Virginia y British Columbia. Se realizó una separación entre las bodegas de acuerdo con su producción. Alrededor del $50 \%$ de las bodegas fueron denominadas de tamaño pequeño, un $25 \%$ como medianas y el porcentaje restante fue asignado a bodegas grandes.

\section{Principales resultados del estudio}

La Figura 4.3 explica el cambio que se ha presentado en la compra de barricas para la crianza de vino desde el año 2005 hasta el año 2010. El cambio más amplio se registra entre el año 2008 y 2010, alrededor del 20\% de las bodegas disminuyeron la compra de barricas durante el año 2009. La primera suposición que se hizo para entender este caso, es el recorte en compras generado por la crisis general, además se muestra una recuperación para el año 2010. Sin embargo, es importante recordar que la producción de vino también está ligada a una cosecha anual. Una gran cosecha puede causar un aumento en las compras de barricas durante el año en cuestión, mientras que otra añada puede ser sorprendentemente menor y generar una disminución en la adquisición de barricas para el siguiente año. En general las diferentes bodegas justificaron esta disminución de la compra de barricas a circunstancias externas y no a un cambio drástico en las técnicas de envejecimiento de vinos tintos.

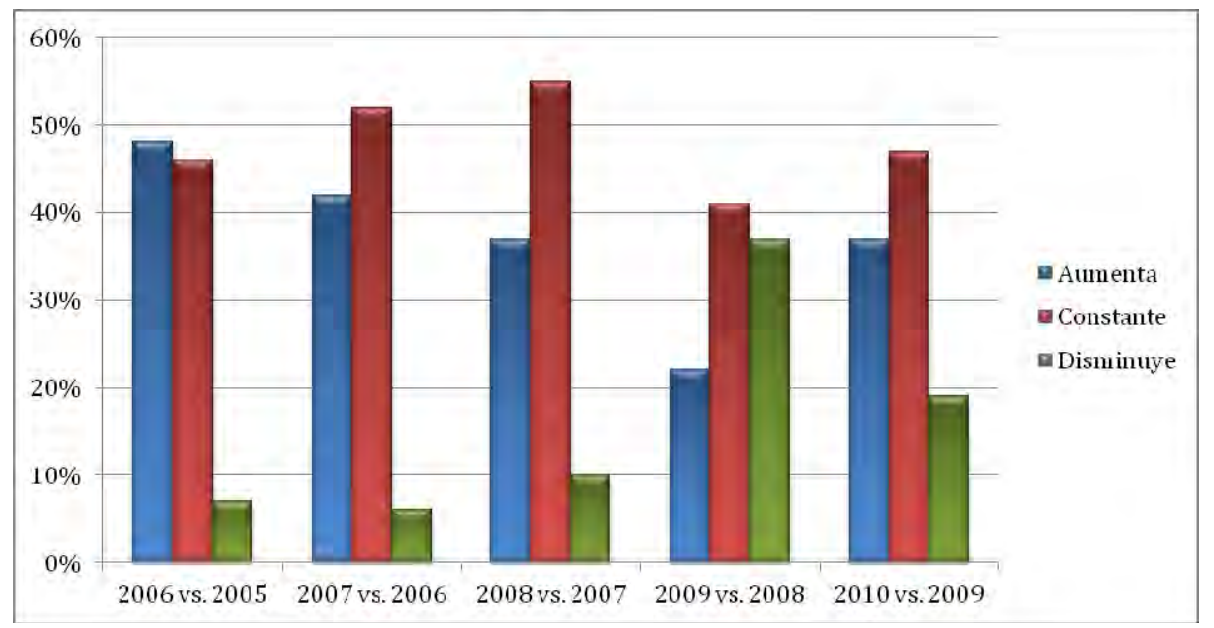

Figura 4.3. Cambios del porcentaje de compra de barricas en Estados Unidos

Algunas tendencias comienzan a consolidarse, por ejemplo, parece ser que la mayoría de las bodegas que van a abandonar por completo la crianza en barrica ya lo han hecho. Por otro lado, también se ha visto que las bodegas prefieren utilizar barricas para el vino que va a estar destinado a largos periodos de envejecimiento. 


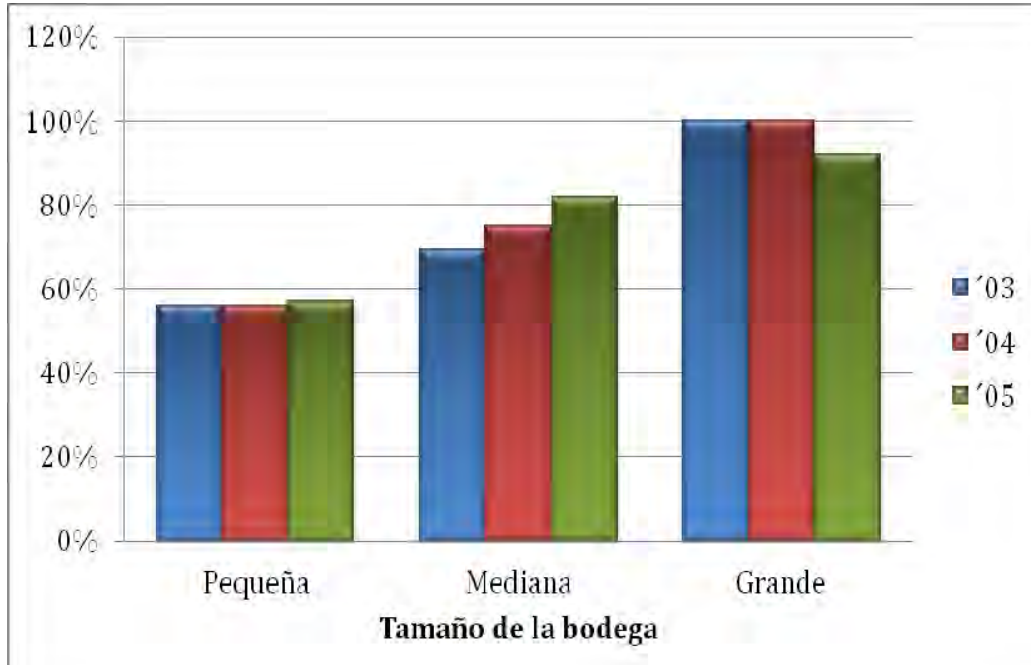

Figura 4.4. Porcentaje de uso de métodos alternativos en función del tamaño de la bodega en Estados Unidos.

La proporción de bodegas medianas (de 50.000 a 500.000 cajas/año) que utilizan soluciones alternativas aumenta de forma sistemática desde el año 2003 hasta el año 2005 (Figura 4.4). Desde el año 2005 hasta el año 2008 no se registra un aumento en el uso de alternativos, tal y como lo explica la Figura 4.5. En Estados Unidos desde el año 2004, el 95\% de los vinos de menos de 5 dólares utiliza sistemas de crianza alternativa. Desde 2003 se detecta una tendencia en su uso en la gama de precios 10-25 dólares (Figura 4.6).

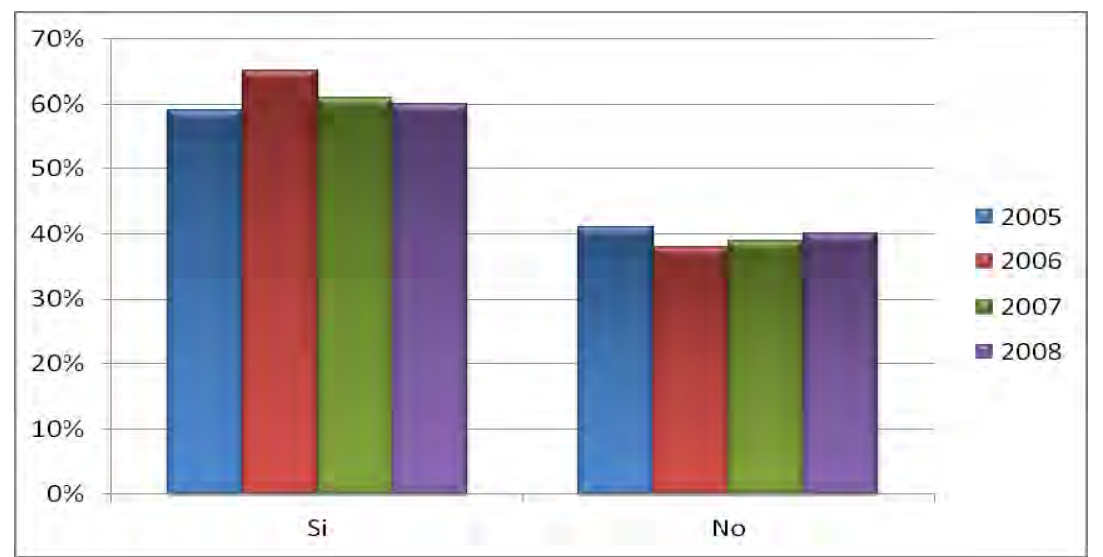

Figura 4.5. Porcentaje de bodegas que en algún momento han usado métodos alternativos.

Otro resultado interesante que se recoge en este estudio es el relacionado con la mircrooxigenación, ya que al parecer no es un método tan ampliamente aplicado como se esperaba. Sólo el 16\% de los encuestados indicaron que alguna vez han utilizado la microoxigenación. Parece ser que seguirá siendo una herramienta de las grandes bodegas. Las bodegas más pequeñas prefieren continuar con todo el proceso tradicional en barrica de roble para el envejecimiento de vinos tintos. 


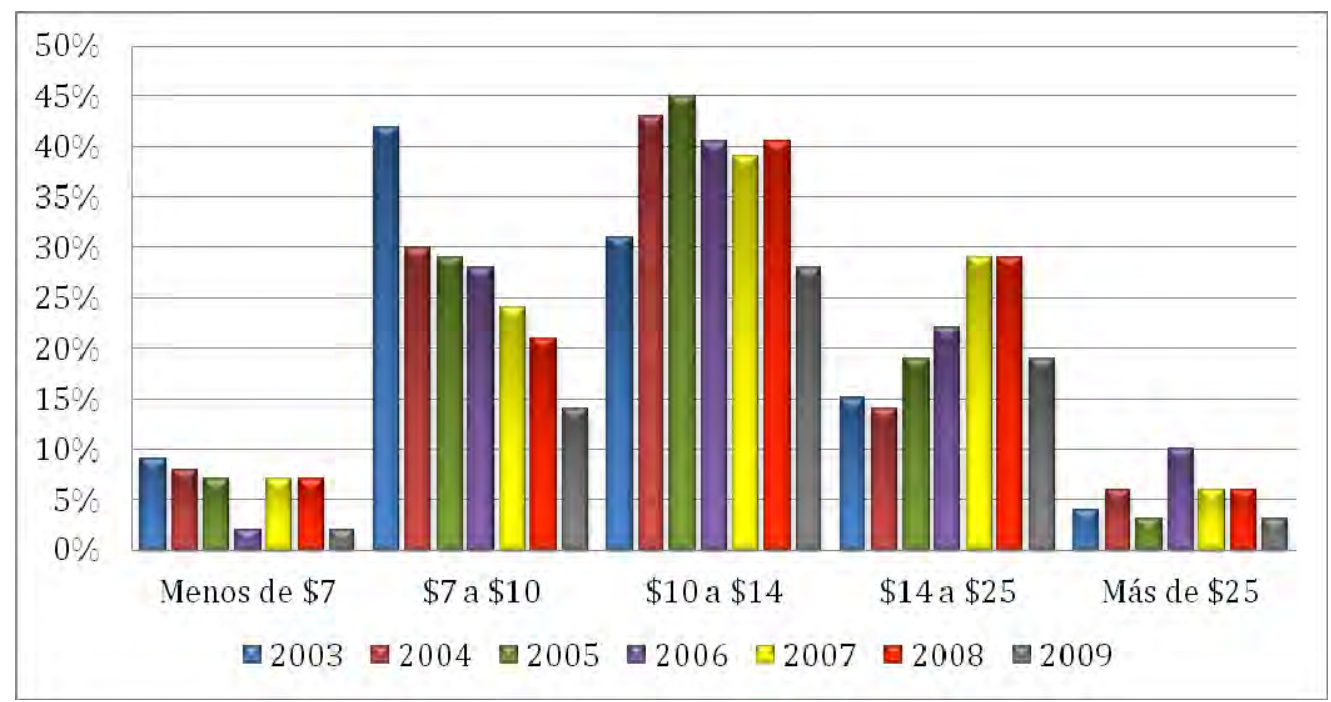

Figura 4.6. Uso de los métodos alternativos en función del precio de la botella del vino en el mercado.

\subsection{DESARROLLO EXPERIMENTAL}

\subsubsection{Preparación de la muestras de vino tinto}

La Tabla 4.4 registra las muestras de vinos elaboradas para el desarrollo de esta investigación. La nomenclatura asignada a cada deposito será utilizado durante este capítulo para hacer referencia a cada tipo de vino. En todos los casos se utilizó una uva variedad Tempranillo de la Denominación de Origen Ribera de Duero (cosecha 2006). Se emplearon un total de 225 L de vino por cada depósito de madera o acero inoxidable. Con el fin de hacer un seguimiento a los posibles errores experimentales durante la elaboración de los vinos, todas las experiencias fueron seguidas en dos depósitos equivalentes.

Para los vinos envejecidos con técnicas alternativas se utilizaron depósitos de acero inoxidable en los cuales se adicionaron pequeñas dosis de oxígeno controladas con un equipo que permitió regular en cada depósito las concentraciones de oxigeno. Para ello se aportó la cantidad oxigeno necesaria para igualar la lectura de la cantidad de oxigeno presente en las barricas testigo de roble. Se estudió el efecto del tamaño de la madera añadida utilizando en cuatro de los depósitos, chips y en los otros cuatro, staves. Al mismo tiempo 
dentro de cada grupo, la mitad de la muestras fueron envejecidas con madera de origen francés y la otra mitad con roble americano. Los periodos de muestreos que se realizaron durante esta investigación están descritos en la Tabla 4.5 y corresponden a etapas en las que los vinos están contenidos en barricas y posteriormente, después de 150 días, pasados a botella.

Tabla 4.4. Características de los vinos tintos.

\begin{tabular}{|c|c|c|c|c|c|}
\hline Depósito & Nombre & $\begin{array}{c}\text { Tipos de } \\
\text { depósitos }\end{array}$ & $\begin{array}{c}\text { Origen del } \\
\text { roble }\end{array}$ & $\begin{array}{c}\text { Tamaño de } \\
\text { la madera }\end{array}$ & $\mu \mathrm{O}_{2}$ \\
\hline 1 & $\mathrm{Ca}$ & \multirow{2}{*}{$\begin{array}{c}\text { Acero inoxidable } \\
\text { 220L }\end{array}$} & \multirow{2}{*}{ Americano } & \multirow{2}{*}{ Chips } & \multirow{2}{*}{ SI } \\
\hline 2 & $\mathrm{Ca}$ & & & & \\
\hline 3 & $\mathrm{Cf}$ & \multirow{2}{*}{$\begin{array}{c}\text { Acero inoxidable } \\
\text { 220L }\end{array}$} & \multirow{2}{*}{ Francés } & \multirow{2}{*}{ Chips } & \multirow{2}{*}{ SI } \\
\hline 4 & $\mathrm{Cf}$ & & & & \\
\hline 5 & Sa & \multirow{2}{*}{$\begin{array}{c}\text { Acero inoxidable } \\
220 \mathrm{~L}\end{array}$} & \multirow{2}{*}{ Americano } & \multirow{2}{*}{ Travesaños } & \multirow{2}{*}{ SI } \\
\hline 6 & Sa & & & & \\
\hline 7 & Sf & \multirow{2}{*}{$\begin{array}{c}\text { Acero inoxidable } \\
220 \mathrm{~L}\end{array}$} & \multirow{2}{*}{ Francés } & \multirow{2}{*}{ Travesaños } & \multirow{2}{*}{ SI } \\
\hline 8 & Sf & & & & \\
\hline 9 & $\mathrm{Ba}$ & \multirow{2}{*}{$\begin{array}{c}\text { Barrica roble } \\
225 \mathrm{~L} \\
\end{array}$} & \multirow{2}{*}{ Americano } & \multirow{2}{*}{ No aplica } & \multirow{2}{*}{$\mathrm{NO}$} \\
\hline 10 & $\mathrm{Ba}$ & & & & \\
\hline 11 & $\mathrm{Bf}$ & \multirow{2}{*}{$\begin{array}{c}\text { Barrica roble } \\
225 \mathrm{~L}\end{array}$} & \multirow{2}{*}{ Francés } & \multirow{2}{*}{ No aplica } & \multirow{2}{*}{$\mathrm{NO}$} \\
\hline 12 & $\mathrm{Bf}$ & & & & \\
\hline
\end{tabular}

Tabla 4.5. Periodos de muestreo.

\section{\begin{tabular}{l|l} 
Muestreo & Etapa de la vinificación
\end{tabular}}

\begin{tabular}{|c|l|}
\hline Toma 1: 30 días & Envejecimiento \\
\hline Toma 2: 90 días & Envejecimiento \\
\hline Toma 3: 150 días & Envejecimiento \\
\hline Toma 4: 330 días & Botella \\
\hline Toma 5: 480 días & Botella \\
\hline Toma 6: 600 días & Botella \\
\hline
\end{tabular}




\subsection{RESULTADOS}

\subsubsection{Parámetros Químicos}

Para ofrecer una visión global del proceso de envejecimiento del vino, algunos de los resultados de los parámetros analíticos del vino envejecido en barrica de roble americano se han representado en la Tabla 4.6. en función de todos diferentes periodos de muestreo, proporcionando de esta manera una mejor perspectiva de los cambios que a nivel general han presentado las muestras de vino.

Tabla 4.6. Representación de algunos de los parámetros químicos para un vino envejecido en barrica de roble americano.

\begin{tabular}{|c|c|c|c|c|c}
\hline $\begin{array}{c}\text { Graduación } \\
\text { alcohólica } \\
{[\%]}\end{array}$ & $\begin{array}{c}\text { Acidez } \\
\text { volátil } \\
{[\mathrm{g} / \mathrm{L}]}\end{array}$ & $\begin{array}{c}\text { Polifenoles } \\
\text { totales } \\
{[\mathrm{mg} / \mathrm{L}]}\end{array}$ & $\begin{array}{c}\text { Taninos } \\
{[\mathrm{mg} / \mathrm{L}]}\end{array}$ & $\begin{array}{c}\text { Catequinas } \\
{[\mathrm{mg} / \mathrm{L}]}\end{array}$ & $\begin{array}{c}\text { Antocianos } \\
{[\mathrm{mg} / \mathrm{L}]}\end{array}$ \\
\hline
\end{tabular}

\begin{tabular}{|c|c|c|c|c|c|c|}
\hline T1 & 13,42 & 0,55 & 2158,38 & 2,11 & 1270,2 & 608,56 \\
\hline T2 & 13,87 & 0,53 & 2222,13 & 2,08 & 553,2 & 585,81 \\
\hline T3 & 14,19 & 0,55 & 2100,25 & 2,13 & 799,0 & 512,75 \\
\hline T4 & 13,79 & 0,70 & 2276,72 & 2,40 & 783,8 & 427,88 \\
\hline T5 & 14,03 & 0,66 & 1658,00 & 1,14 & 861,0 & 381,00 \\
\hline T6 & 14,17 & 0,68 & 2316,25 & 2,40 & 928,8 & 323,75 \\
\hline
\end{tabular}

En los primeros meses de estancia en depósito o barrica de los vinos destinados a crianza, se obtiene un ligero aumento en la graduación alcohólica por evaporación diferencial del agua frente al etanol; según lo explicado por algunos autores este fenómeno corresponde a temperaturas altas y menor humedad relativa durante el verano [22].

En la acidez volátil se observa un aumento desde el primer registro que se realizó cuando se dio comienzo al envejecimiento en depósito hasta el momento que se realizó la última evaluación del envejecimiento en botella. Este cambio puede suceder por cualquiera de los siguientes fenómenos que tienen lugar durante el envejecimiento en barrica: la oxidación de etanol, la acción de las bacterias acéticas en presencia de oxígeno o la extracción de ácidos volátiles de la madera $[23,24]$.

El índice de polifenoles totales experimenta ligeras fluctuaciones durante su estancia en barrica, provocadas posiblemente por el balance resultante entre la 
extracción de fenoles de la madera y posterior precipitación de materia colorante, durante el periodo del envejecimiento en botella. El descenso de las formas libres es más rápido durante los primeros meses de crianza, puesto que la polimerización se ve favorecida en presencia de oxígeno [25] que es mucho más abundante durante la estancia del vino en barrica que durante el almacenamiento en botella.

Respecto a la evolución de los taninos en el vino a lo largo del añejamiento (Figura 4.7.), existe una diferencia -aumento- entre la cantidad inicial que se registra para estos compuestos en el periodo T1 y la cantidad final determinada para el periodo T6; sin embargo no podemos afirmar que este aumento cumpla con algún tipo de tendencia durante todo el proceso de evolución del vino. Estos resultados indican que existe una extracción de taninos hidrolizables del roble de acuerdo con lo apuntado por otros autores [26, 27].

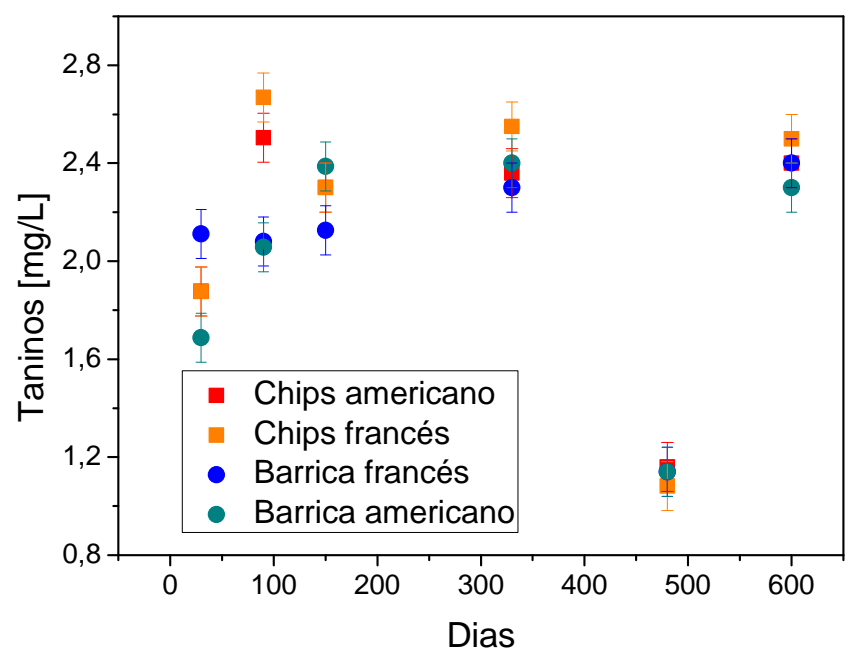

Figura 4.7. Medida de los taninos durante el envejecimiento de los vinos.

Se observa también que la cesión de taninos hidrolizables de la madera en la mayoría de casos es algo superior para los vinos envejecidos en roble francés, al tratarse de una madera más porosa y de grano ancho, que la de americano, que facilita la extracción de este tipo de compuestos. Esta diferencia en la extracción de fenoles entre robles de distinta procedencia ha sido registrada anteriormente en bibliografía de otros autores [28-30].

Un comportamiento similar se describe para las catequinas, lo cual indicaría un equilibrio de formación-destrucción entre formas poliméricas, los taninos, y moléculas más sencillas como son las catequinas.

Por lo contrario, en el caso de los antocianos libres existe una pérdida progresiva y acusada, como se demuestra en la Figura 4.8 [31]. En efecto, estos compuestos, 
responsables del color rojo intenso del vino joven, van polimerizándose entre ellos, especialmente durante la etapa del envejecimiento en botella, para dar sustancias de color rojo-teja que proporcionan estabilidad al color del vino [32, 33] y complejidad gustativa [34].

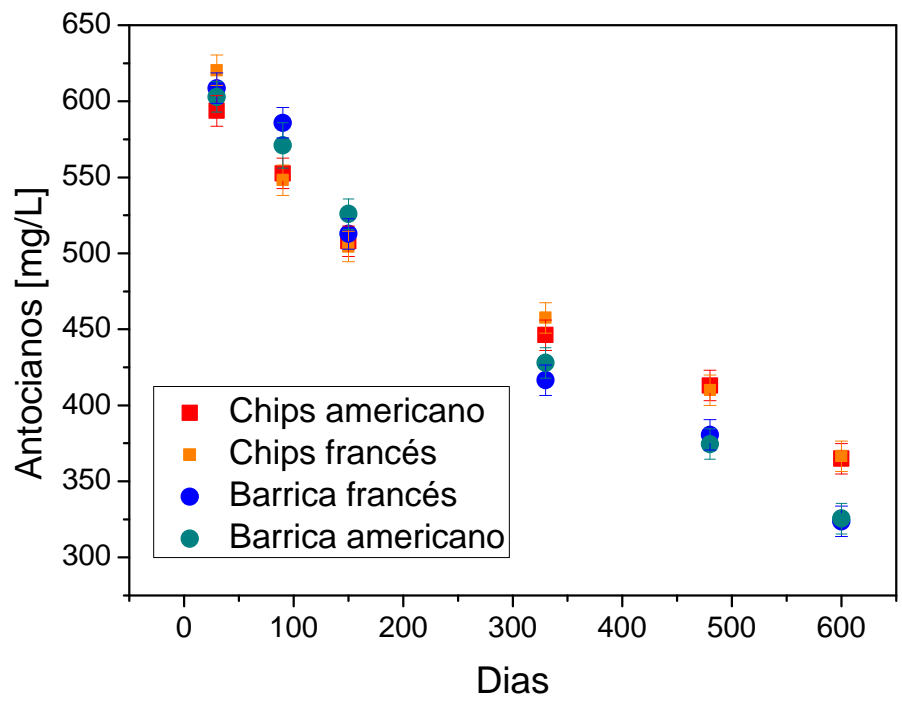

Figura 4.8. Medida de los Antocianos durante el envejecimiento de los vinos

Estos resultados también manifiestan que los antocianos tienen comportamiento similar para los dos tipos de envejecimiento (barricas, depósitos), sin embargo en el último periodo se muestran mayores inestabilidades para el caso de los vinos almacenados en los depósitos de acero.

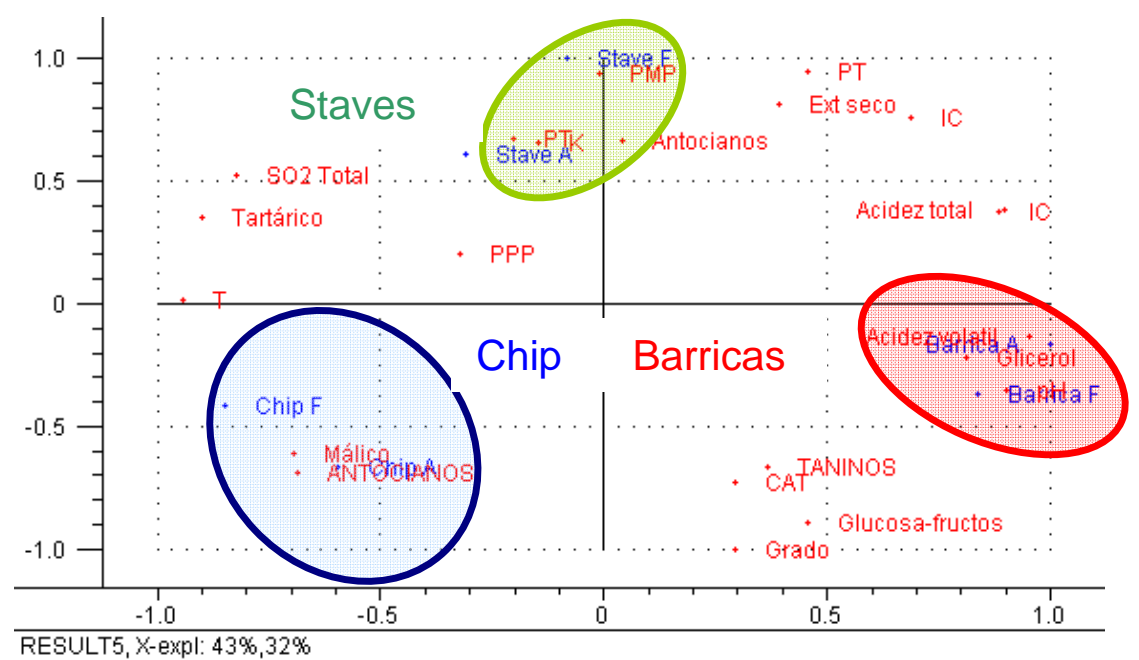

Figura 4.9. Análisis de componentes principales de parámetros enológicos en la toma 6.

A titulo de ejemplo, la Figura 4.9 muestra un análisis bi-plot de los parámetros químicos para el periodo de muestreo 6. Este tipo de representación permite superponer los resultados de las medidas químicas con los diferentes tipos de 
vinos. Tal y como se observa en la figura, la primera componente principal, que explica casi el $43 \%$ está muy relacionada con la acidez volátil, antocianos y los polifenoles muy polimerizados, entre otros, y es la componente que mejor describe las diferencias entre los tipos de envejecimiento para este caso en concreto.

Dentro de los objetivos marcados en este trabajo hemos utilizado esta evaluación como método de seguimiento al envejecimiento y como una herramienta para evaluar el potencial de nuestro equipo electrónico.

\subsubsection{Coordenadas CIELab}

Diferencias existentes entre el envejecimiento en depósito (espectros en color verde) y el envejecimiento en botella (espectros en color marrón).

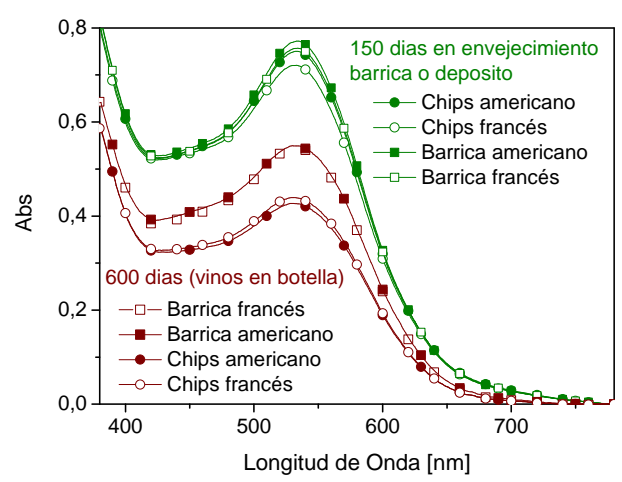

Evolución de un vino envejecido en barrica (espectros marcados con los círculos) y un vino tratado con métodos alternativos. Evolución del envejecimiento en botella.

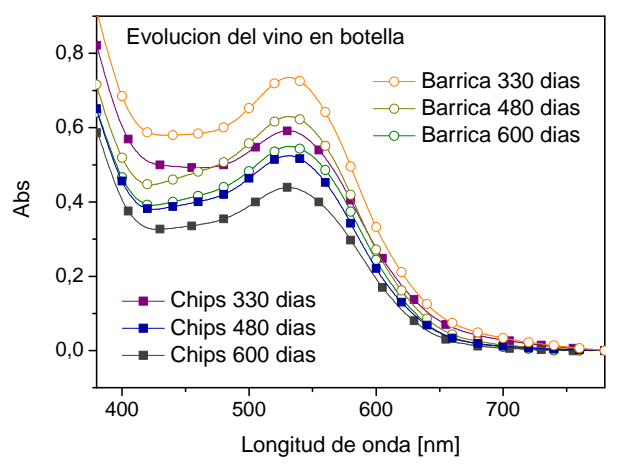

Figura 4.10. Espectros UV-Visible resgistrados para las muestras de vino durante el envejecimiento en barrica o depósito de acero inoxidable seguido del envejecimiento en botella.

Los resultados de las valoraciones que hemos realizado mediante técnicas de espectrometría UV-Visible durante el proceso de envejecimiento con madera y el tiempo de almacenamiento en botella están representados en la Figura 4.10. Estos resultados corresponden a los espectros registrados para las muestras que durante 150 días fueron envejecidas en barricas de madera con diferentes orígenes $\mathrm{o}$ en depósitos de acero inoxidable que contenían una cantidad equivalente de madera en términos de la superficie de la barrica tradicional. A continuación los vinos fueron almacenados en botellas durante 450 días. Los correspondientes espectros (Figura 4.10) muestran una disminución generalizada de la absorbancia para todas las longitudes de onda con el tiempo de 
almacenamiento en botella. Este resultado coincide con el hecho de que las muestras han pasado de estar en un medio oxidativo (barrica) a un medio reductor (botella), propicio para generar las reacciones de hidrólisis que ocasionan la transformación de los antocianos y por tanto un cambio en la coloración del vino [35].

Los anteriores resultados también indican que la evolución del color para esta serie de muestras concretamente, no depende tanto del tipo de madera como del tipo de envejecimiento utilizado. Además, se observa que las diferencias entre muestras comienzan hacerse más visibles con el tiempo del almacenamiento del vino en la botella.
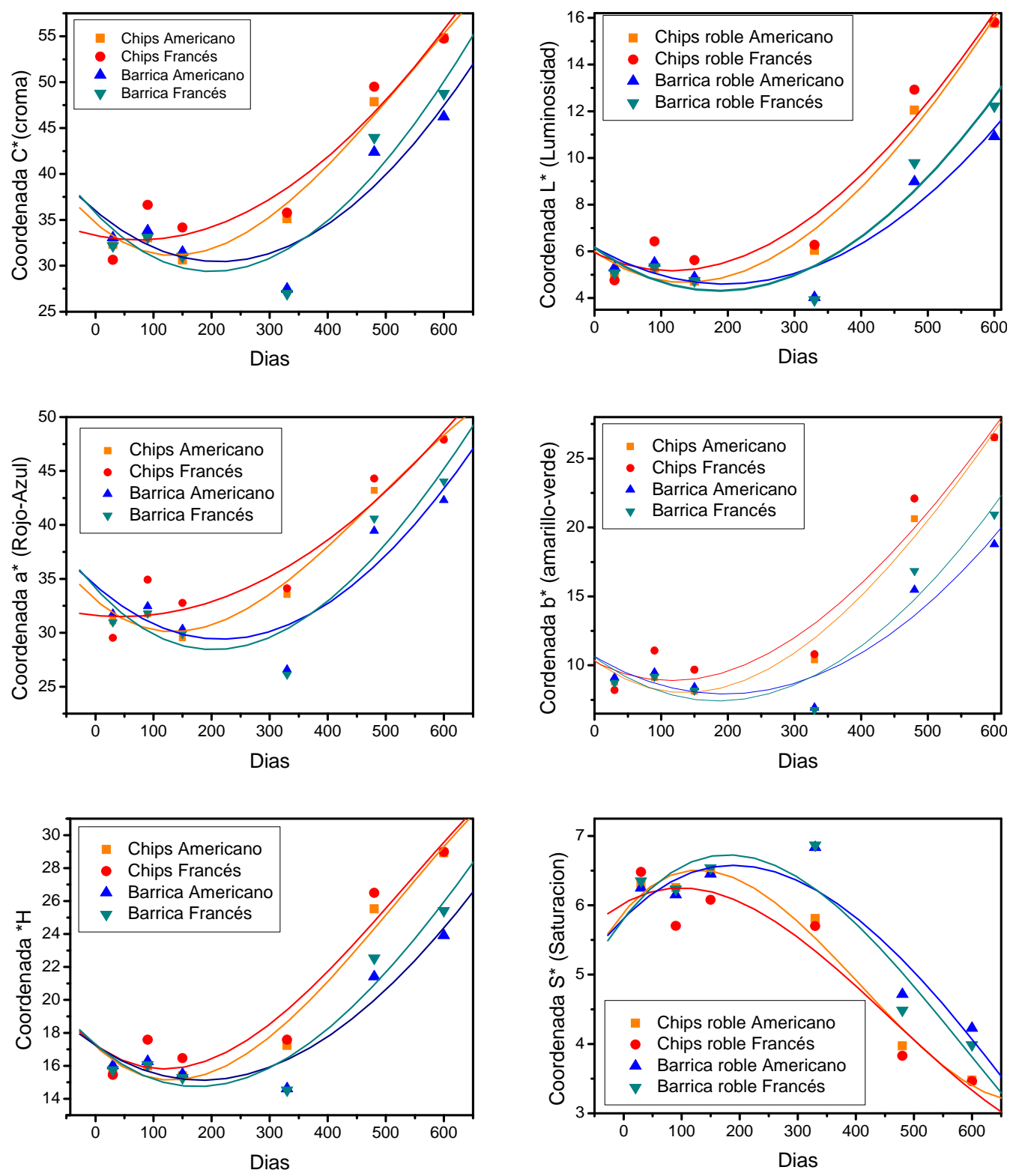

Figura 4.11. Evolución de cada una de las coordenadas CIELab durante el envejecimiento en barrica (o en depósito) y almacenamiento en botella. 
Con la información de los espectros y utilizando el método de análisis del color de las coordenadas CIELab estudiamos la evolución de cada coordenada de acuerdo al tipo de envejecimiento y madera utilizada (Figura 4.11.). Todas las coordenadas registran un aumento de su valor a excepción de la coordenada $\mathrm{S}$, que indica un paso del vino de un color rojo rubí a un rojo teja.

Esto podría estar relacionado con los antocianos no estabilizados (asociados al color rojo puro) y su capacidad de asimilación del oxígeno a través de las reacciones de condensación. Recordemos que en el caso de los vinos envejecidos en barrica el paso del oxígeno a través de las duelas y poros se presenta como un proceso natural, mientras que para el caso de los vinos envejecidos con métodos alternativos el incorporación del oxigeno se realizan por medio de dosificaciones periódicas. Al parecer, cada manera de aportar oxígeno al vino genera diferencias entre la cinética de las reacciones.

Por otra parte los resultados también muestran que los vinos envejecidos en depósitos de acero inoxidable con inserción de chips tienden a cambiar su color más rápidamente que los vinos envejecidos en barricas. Una explicación de este fenómeno puede derivarse de los compuestos químicos relacionados con el color rojo puro como son los antocianos no estabilizados [3], compuestos que pueden seguir evolucionando por su afinidad a las reacciones de condensación. En este caso los vinos en barricas parecen presentar mayor estabilidad posiblemente porque las reacciones de condensación ocurren en manera natural y lenta con el paso natural del oxígeno a través de las duelas y poros de la barricas mientras que en el envejecimiento alternativo las dosis de oxígeno fueron adicionadas de acuerdo a la cantidad de oxigeno que fuera medida en la barrica testigo a un determinado tiempo, generando así que las moléculas tuvieran menos tiempo para reaccionar con el oxígeno.

Las consecuencias de este proceso sólo son realmente evidentes en el momento en el que vino está en un medio reductor (botella), puesto que es en este medio cuando polifenoles muy polimerizados precipitan y los menos polimerizados continúan reaccionando. Si por algún motivo la cantidad de alguno de los dos tipos de moléculas es muy alta se genera un cambio más rápido y menos estable del color [36].

También se realizó un análisis de componentes principales (PCA) con los correspondientes valores de las coordenadas CIELab que se registraron de los vinos durante todo su proceso de envejecimiento; cada una de las valoraciones realizadas se repitió un total de siete veces. Los resultados de los análisis llevados a cabo durante todos los periodos de evaluación se muestran en la figura 8. Los datos utilizados como variable de entrada para el análisis PCA fueron preprocesados mediante los métodos autoscaling y $S N V$. 


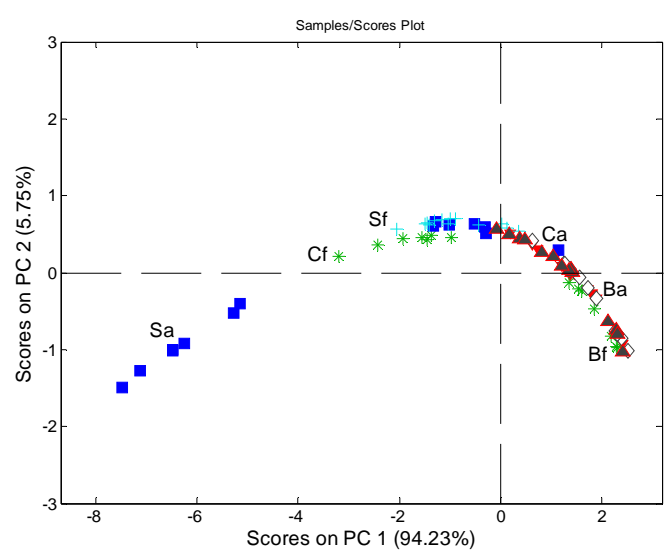

PCA para los datos registrados para T1

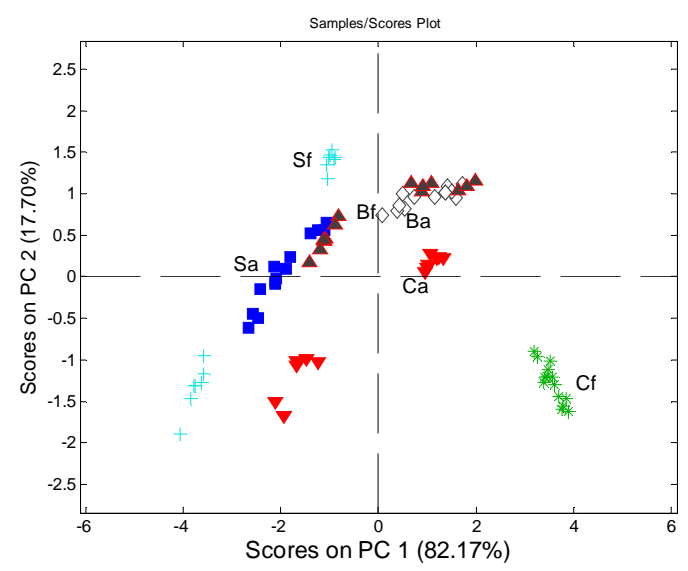

PCA para los datos registrados para T3

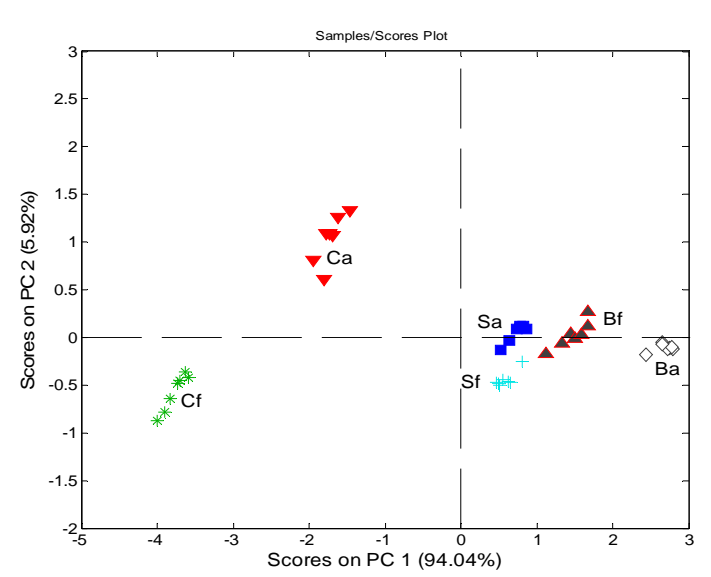

PCA para los datos registrados para T5

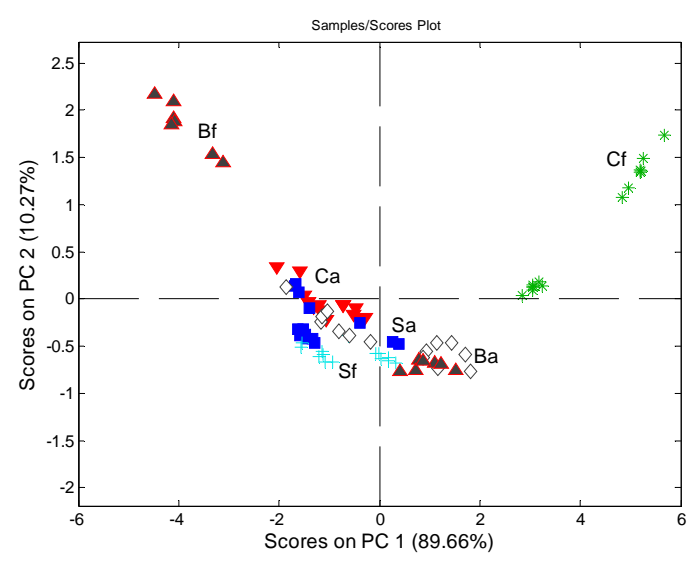

PCA para los datos registrados para $\mathrm{T} 2$

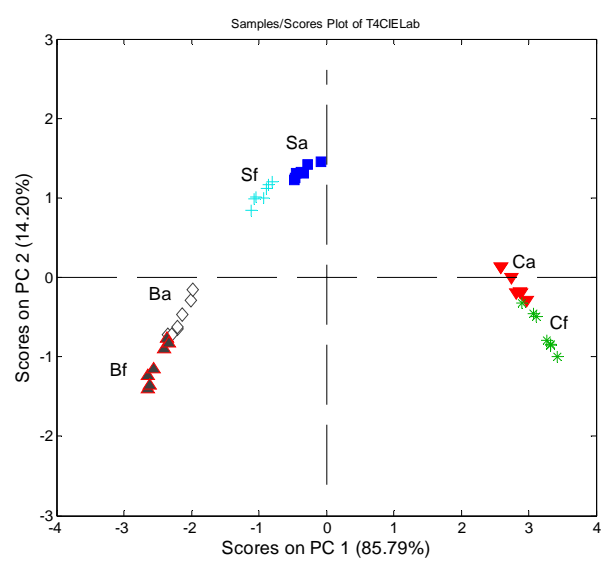

PCA para los datos registrados para T4

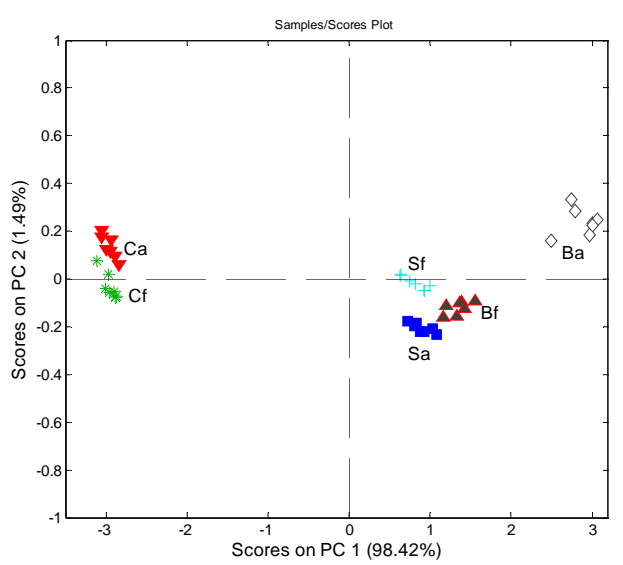

PCA para los datos registrados para T6

Figura 4.12. PCA para las Coordenadas CIELab de cada uno de los periodos de muestreo.

En todos los casos las dos primeras componentes registran en $99 \%$ de la información. Podemos decir que durante los primeros días, cuando dio comienzo el proceso del envejecimiento, es difícil distinguir entre las muestras en función de las propiedades colorimétricas de los distintos vinos, solamente es posible 
apreciar una tendencia a ubicar los vinos envejecidos con métodos tradicionales en la parte derecha del gráfico de los scores y los vinos envejecidos con métodos alternativos en la parte izquierda.

En el segundo periodo de muestreo si se pueden observar cambios en los vinos. En este caso la principal diferenciación está determinado por el origen de la madera. Los vinos elaborados con un mismo tipo de envejecimiento pero con madera de distinto origen están ubicados siempre a una distancia considerable entre si, por ejemplo las medidas correspondientes a las barricas de origen francés y americano presentan 6 unidades de diferencia en la gráfico de los scores.

Es importante resaltar que los vinos comienzan a parecerse un poco después de aproximadamente 150 días de haber dado comienzo al envejecimiento (T3). Sin embargo las diferencias entre tipo de envejecimiento se pueden identificar claramente en la PCA cuando los vinos pasaron a ser almacenados en botellas; los vinos envejecidos en barricas se ubican el parte izquierda del gráfico de los scores mientras que los vinos envejecidos con chips se ubican en la parte opuesta y los vinos envejecidos con staves en la parte superior de la PC2. Estas diferencias entre vinos envejecidos en barricas y en depósitos de acero con inserción de chips se mantienen para los dos periodos de evaluación siguientes (T5 y T6).

De igual manera se ha realizado un PCA con el conjunto de todos los datos, es decir se ha organizado una matriz con todos los periodos de muestreos para visualizar la evolución del color de los vinos durante el desarrollo de este proyecto. Las dos primeras PCs recogen aproximadamente el 100\% de la información y podemos afirmar que el modelo es capaz de seguir la evolución del vino, aunque no es posible identificar dentro de este modelo los diferentes tipos de vino porque prevalecen la información relativa a los cambios que se dan por el paso de un envejecimiento en depósitos a un almacenamiento en botella.

\subsubsection{Cromatografía de gases-masas GC-MS}

El estudio de la composición volátil se llevó a cabo haciendo un seguimiento a un grupo de compuestos volátiles comúnmente referenciados como marcadores del proceso de envejecimiento [16, 37-39]. Los compuestos volátiles evaluados utilizando GC-MS fueron los ya aludidos en el apartado 4.1.1.3.: furfural, guaiacol, eugenol, cis y trans-whiskylactona.

La cinética de asimilación de los compuestos por parte del vino durante todo el proceso de envejecimiento se puede visualizar en las Figuras 4.13 y 4.14. En el caso del furfural, la concentración se incrementa significativamente durante el 
envejecimiento y decae durante el periodo en botella. Además la concentración encontrada en barricas fue siempre más alta que la medida utilizando las técnicas alternativas [40].

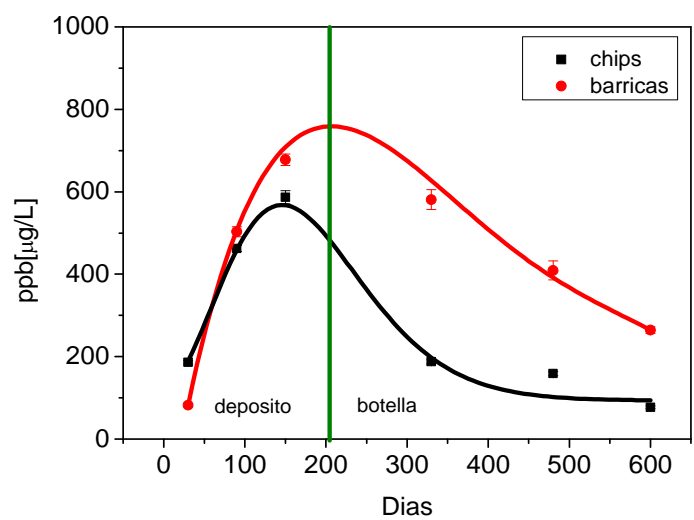

Evaluación del furfural en vinos envejecidos con madera de roble americano

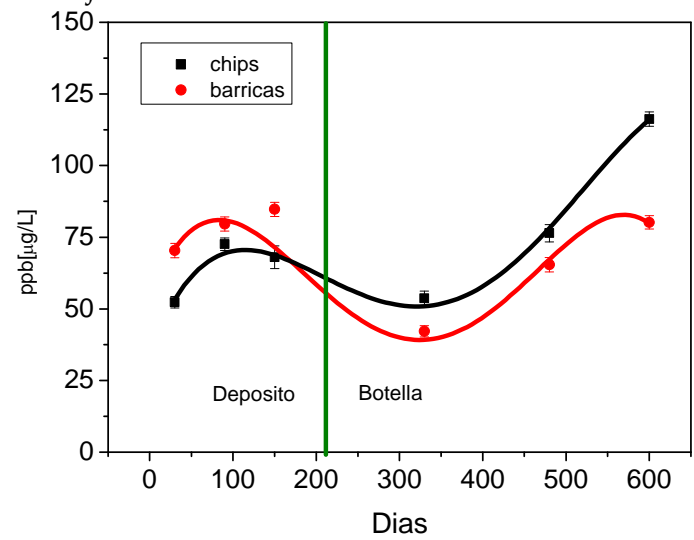

Evaluación del guaiacol en vinos envejecidos con madera de roble americano

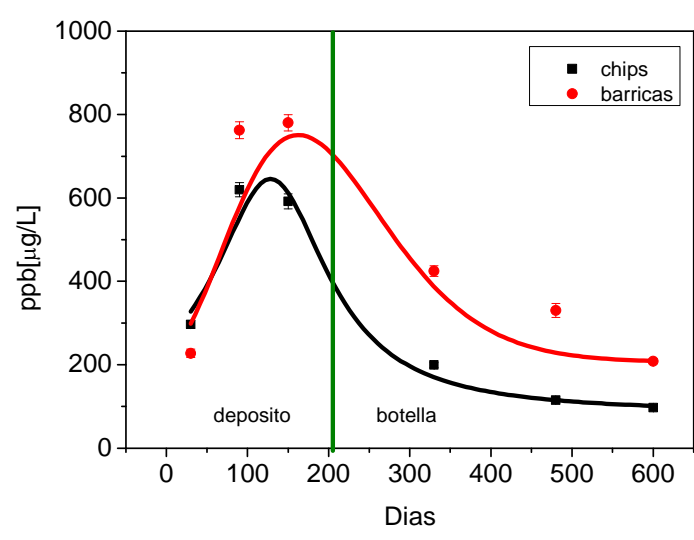

Evaluación del furfural en vinos envejecidos con madera de roble francés

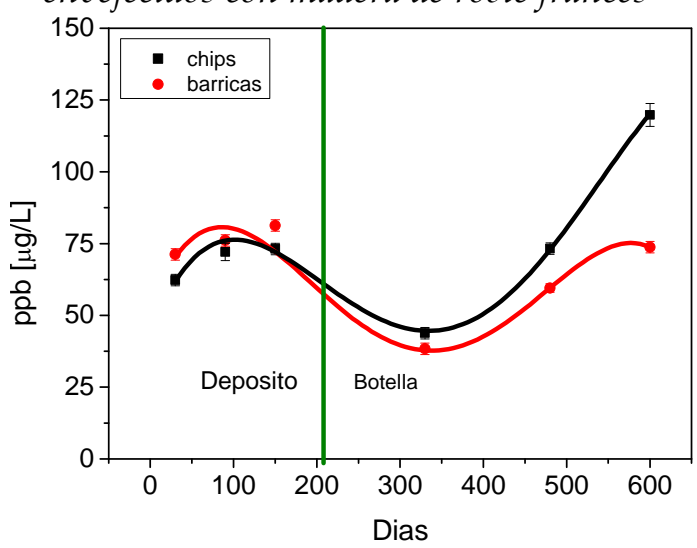

Evaluación del guaiacol en vinos envejecidos con madera de roble francés

Figura 4.13. Evolución del furufural y el guiacol durante la vinificación.

La reducción de la cantidad de furfural radica en la oxidación de este compuesto a un alcohol furfurílico [12]. También pudo haber ocurrir que este compuesto haya servido como precursor en la formación de otros productos de aldehídos furánicos. El comportamiento encontrado en nuestras experiencias coincide con las investigaciones de Rodríguez-Bencomo et al., según las cuales la disminución del furfural es más pronunciada para los vinos madurados en presencia de chips de roble $[40,41]$.

Respecto a los isómeros de la whislylactona y como es usual en vinos madurados con roble, se obtuvo que su concentración fue más alta cuando se había empleado roble americano. La relación de los isómeros cis/trans permite identificar claramente el origen de la madera, los vinos envejecidos con roble americano generalmente presenta un valor mayor [5, 42]. Para los vinos macerados con alternativos se obtuvo que la relación cis/trans fue 4,7 veces superior cuando se 
utiliza roble americano y menor (un valor de 3,3) cuando se aplica roble francés. En el caso de los vinos envejecidos en barricas esta relación es superior a 4,3 con roble americano e inferior a 0,9 con roble francés [43].

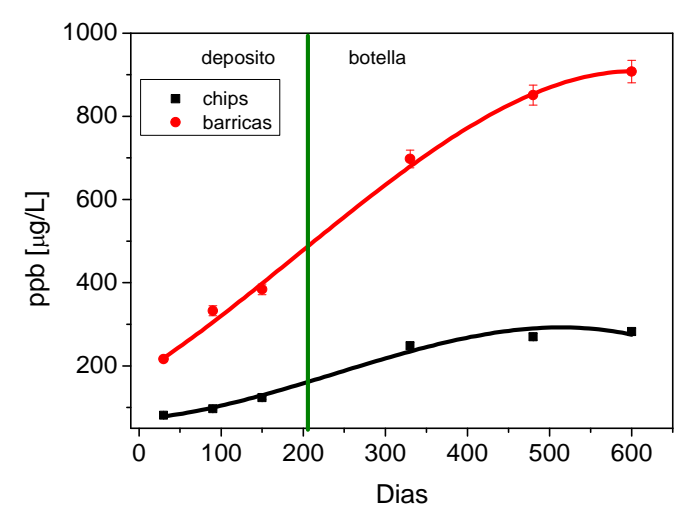

Evaluación de la cis-whiskyplactona en vinos envejecidos con madera de roble americano

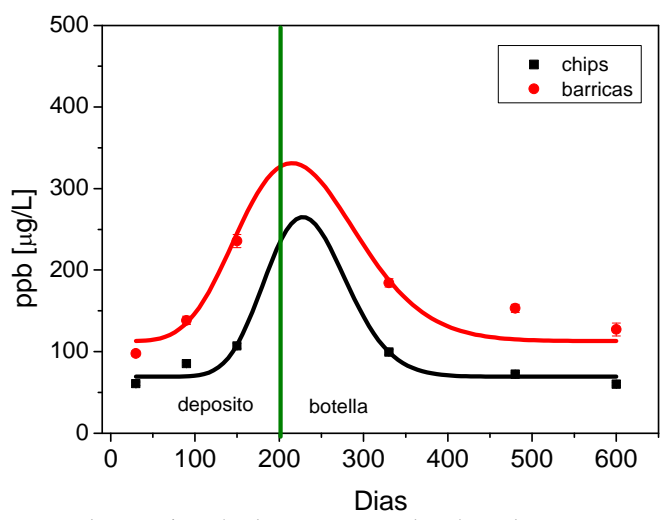

Evaluación de la trans-whiskyplactona en vinos envejecidos con madera de roble

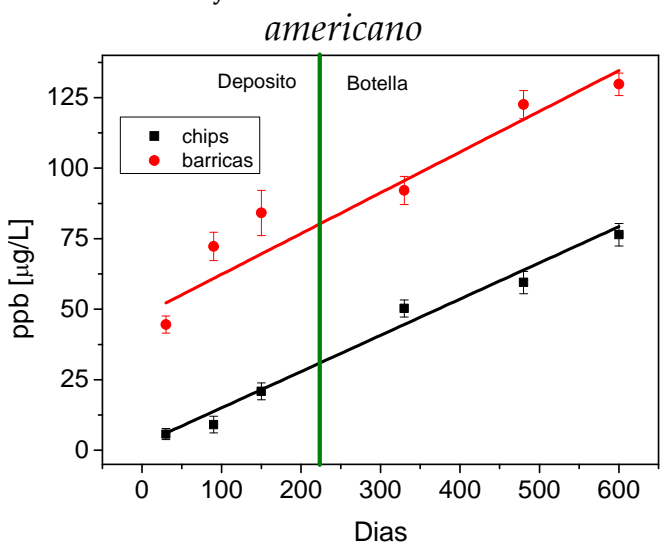

Evaluación del eugenol en vinos envejecidos con madera de roble americano

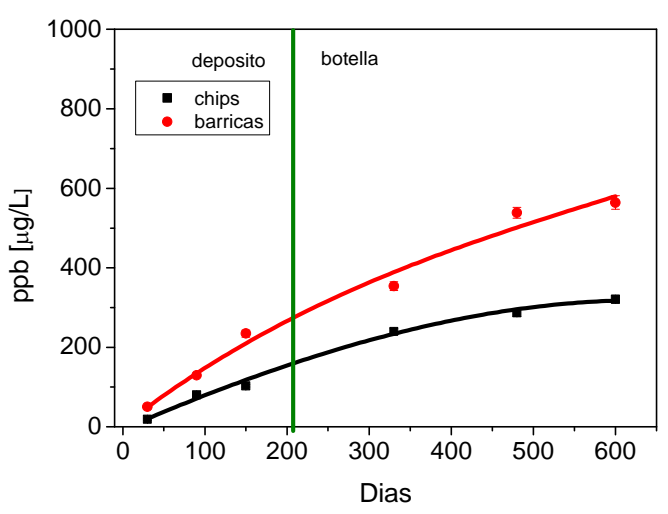

Evaluación de la cis-whiskyplactona en vinos envejecidos con madera de roble francés

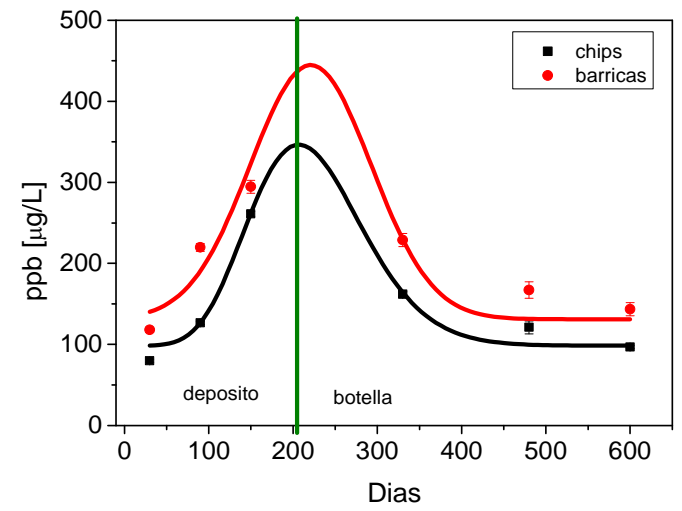

Evaluación de la trans-whiskyplactona en vinos envejecidos con madera de roble

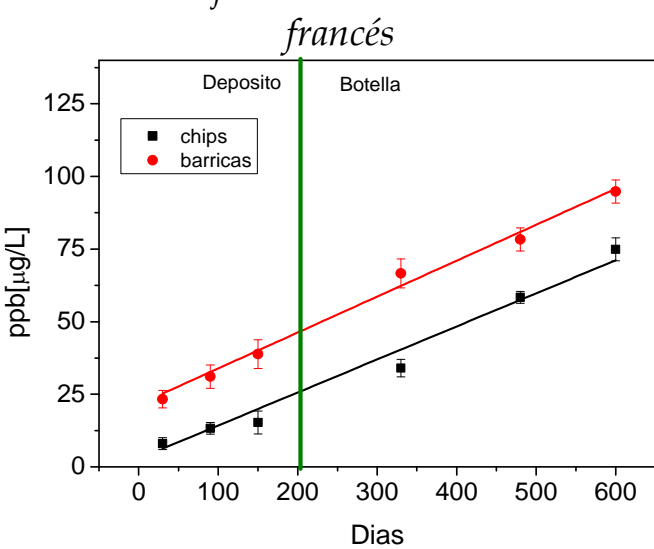

Evaluación del eugenol en vinos envejecidos con madera de roble francés

Figura 4.14. Evolución del furufural y la cis-whiskylactona durante la vinificación.

La concentración de whiskylactona, una vez que ha concluido el tiempo en contacto con la madera, continúa creciendo. Algunos autores justifican este 
comportamiento en la estabilidad de la molécula alifática de los ácidos lácticos y grasos no saturados que dan origen a la whiskylactona, porque al parecer esta molécula sólo comienza a degradarse después de dos años de maceración y ello es como consecuencia de la reducción de la actividad microbiológica del vino [16, 44].

El guaiacol, el eugenol y la cis-whiskylactona también muestran un incremento continuo durante los primeros días de la maceración (Figuras 4.13. y 4.14.) [45]. Las diferencias son más evidentes durante el periodo de almacenamiento en botella, mientras algunos compuestos como el furfural decaen, otros continúan aumentando su cantidad como es caso de la cis-whiskylactona y el eugenol.

El fenómeno descrito anteriormente sucede por dos causas principales, el primero de ellos se presenta porque algunos compuestos se descomponen formándose moléculas menos complejas y el segundo, porque hay cierto tipo de moléculas que son siempre susceptibles a reacciones de condensación [6, 46]. Al final del periodo en botella, se registra una tendencia a que los niveles para cada compuesto sean más altos en los vinos envejecidos en barricas que en los madurados con técnicas alternativas.

Los resultados analizados hasta ese punto nos permiten ver que existe una clara influencia en la composición volátil determinada por el tipo de madera o envejecimiento utilizado. Si bien hemos demostrado que existe este efecto a nivel de ciertos compuestos marcadores, no es una tarea fácil determinar cual es el efecto a nivel global que produce la variación de ciertos compuestos. En este punto radica parte de la importancia de nuestro trabajo: a partir de métodos de análisis multivariantes determinar las correlaciones existentes entre los efectos globales por medio de la nariz electrónica y los efectos en ciertos compuestos marcadores utilizando la GC-MS y la GC-Olfatometría.

\subsubsection{Cromatografía de gases-Olfatometría}

Es importante comentar en este aparatado que las condiciones experimentales (especialmente método de extracción) de esta técnica se han establecido en función de la metodología que ya sido optimizada para la nariz electrónica, puesto que es la técnica definida como objeto principal de estudio de esta tesis. Además los resultados que utilizaremos para la búsqueda de correlaciones entre ambos sistemas serán únicamente en aquellos aromas que hemos sido capaces de identificar en todos los periodos de muestreo. Por otra parte estos volátiles cuentan con valoraciones altas y bien definidas características de aroma.

Para la identificación de los compuestos responsables de los diferentes olores determinados por medio de la GC-O se ha utilizado también como referencia 
todos los resultados que se obtuvieron utilizando la técnica GC-MS. Esta comparación fue posible porque el mismo tipo de columna y programa de temperatura se ha empleado con ambos sistemas. La importancia de realizar este estudio de manera paralela radica en que en algunos casos era difícil definir el compuesto al que debíamos asociar determinado aroma porque pueden presentarse varios tipos de situaciones. Entre ellas, por ejemplo hay compuestos con alto impacto aromático que son difíciles cuantificar con un detector FID debido a que se encontraban en concentraciones inferiores al límite de detección de este sistema, o bien, los compuestos aparecen solapados dentro del cromatograma.

Ya indicamos en el Capítulo II en el apartado 2.1.2.2. (Condiciones Experimentales para la GC-O) que las medidas de olfatometria se realizaron en nuestro equipo de cromatografía Hewlett Packard HP 4890D, provisto de un detector FID, así como que idealmente habría sido óptimo poder acoplar el equipo olfatométrico al equipo Clarus 500 (PerkinElmer) de la Estación Enológica de la Rioja en Haro. En aquel apartado se discutió la complementariedad de nuestro trabajo usando ambos equipos.

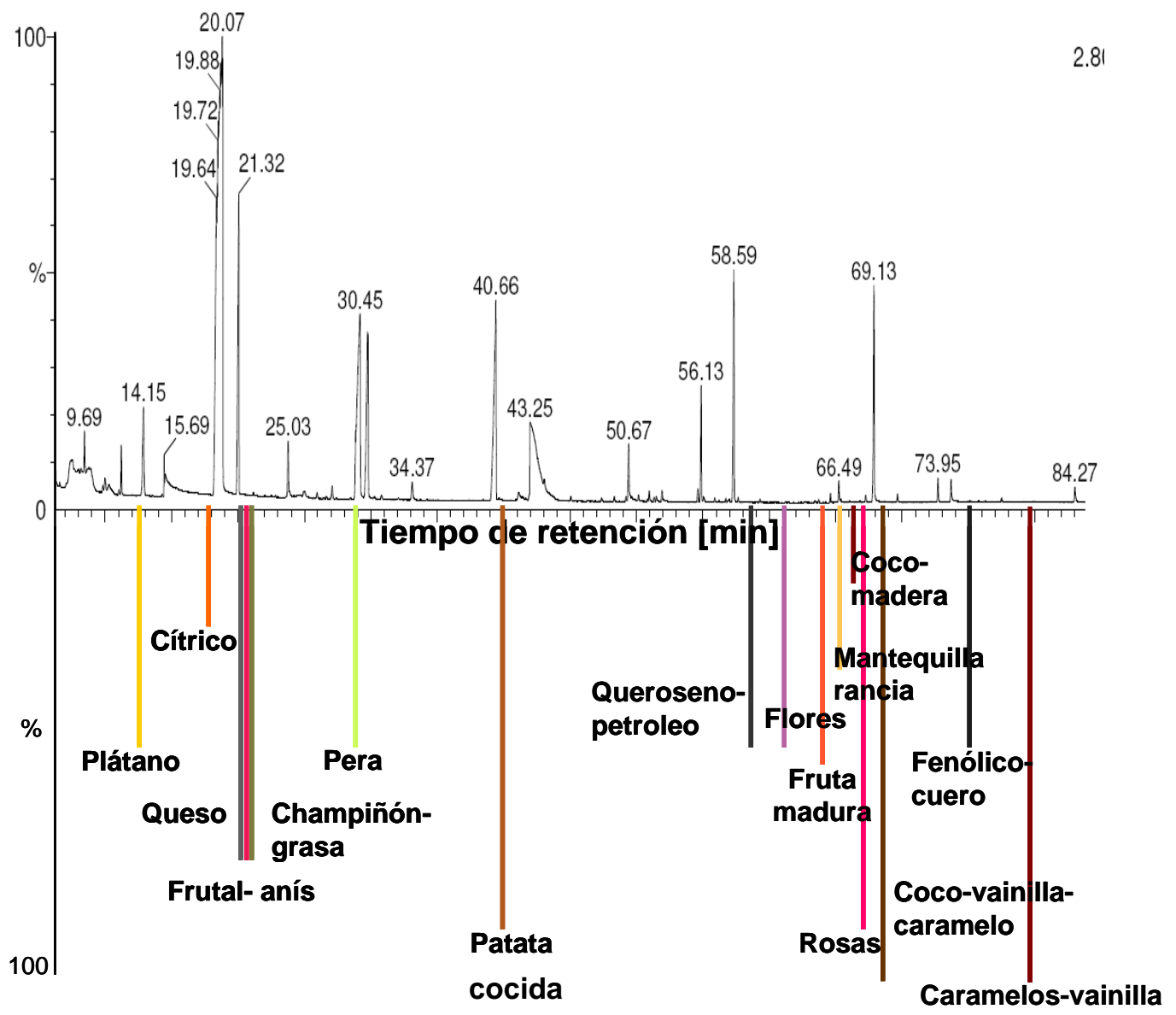

Figura 4.15. Ejemplo de un olfatograma registrado para la muestra de vino tinto envejecido en barrica. En la parte superior de la figura se ha dibujado el correspondiente 
cromatograma registrado en la Estación Enológica de la Rioja.

La Figura 4.15 ejemplariza el tipo de resultados que se han obtenido utilizando para una muestra de vino envejecida en barrica. Incidiendo en lo anteriormente dicho es posible observar que la intensidad percibida del olor de un compuesto no siempre depende de su concentración dentro de una matriz.

Así pues, con el objetivo de conocer cuáles son compuestos aromáticamente activos de los vinos y hacer una estimación de su importancia sensorial, se procedió a desarrollar una serie de experimentos mediante la técnica de dilución AEDA, en la que se utilizó la SPME para la obtención del extracto aromático.

La primera parte de este trabajo consistió en el entrenamiento para la olfatometría de dos miembros del grupo, partiendo con un caso sencillo, utilizando disoluciones hidroalcohólicas con cuatro o cinco compuestos de similares características aromáticas y tiempos de retención bastante separados. Los analistas también se entrenaron para realizar olfatometría de vinos en muestras de la Ribera del Duero a las que se añadieron en sus matrices pequeñas y controladas dosis de los compuestos furfural, guaicol, whiskylactona y eugenol.

Todos los resultados obtenidos por medio de la GC-O realizados durante el periodo del envejecimiento en botella, se explican por medio de gráficos tipo "tela de araña". Los diferentes descriptores sensoriales asociados con los compuestos reportados se indican en las Figuras 4.16-4.18.

Tal y como se puede observar en estas figuras, en general, se han seleccionado olores que aparecen en todos los tipos de vinos, cada uno con diferentes intensidades que contribuyen de manera diferente a su aroma global. Algunos de los compuestos identificados con mayores intensidades en los olores son el 3metil-1-butanol (olor a queso), 2-fenil etanol (olor a rosas), trans-whiskylactona (olor a coco-vainilla), los cuales forman parte del aroma terciario, es decir, se originan durante el proceso de envejecimiento del vino. Los vinos envejecidos con alternativos fueran vinos sometidos a microoxigenación, por tanto, existieron diferencias en las reacciones mediadas por el oxigeno, y esto explicaría porque no se detectaran olores marcados, especialmente los de las sensaciones relacionados con flores y rosas.

La Figura 4.17 muestra nuevamente que unos de los principales compuestos marcadores es la whiskylactona cuyo isomero trans presenta un olor a cocovainilla (e incluso caramelo cuando su concentración es muy alta). Este compuestos además permite apreciar diferencias entres los tipos de envejecimiento. El comportamiento es además coherente con otros resultados que aparecen publicados en la bibliografía $[6,47,48]$. 
a.

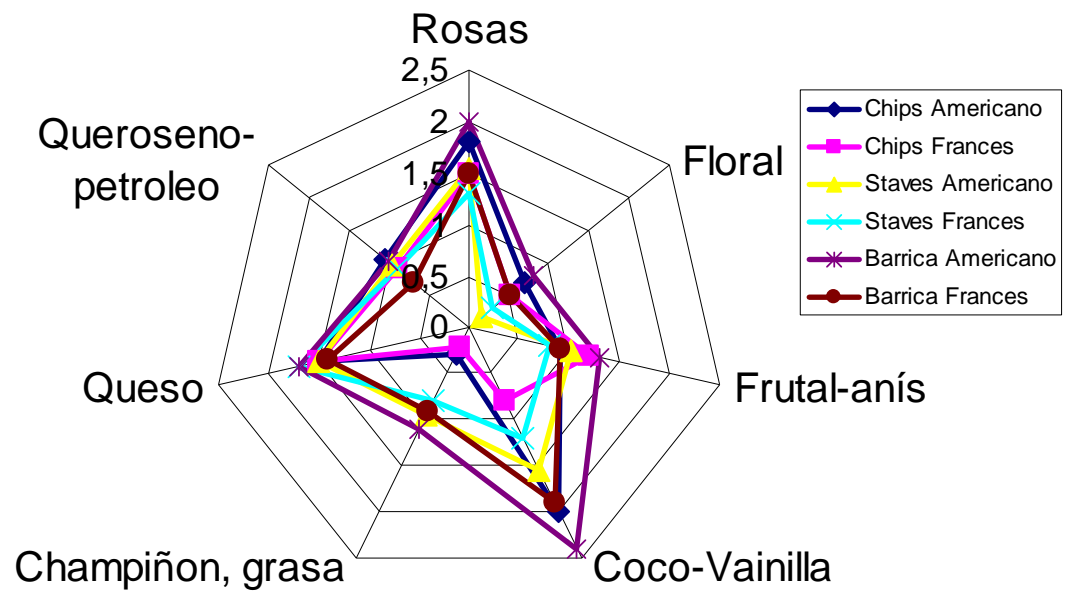

b.

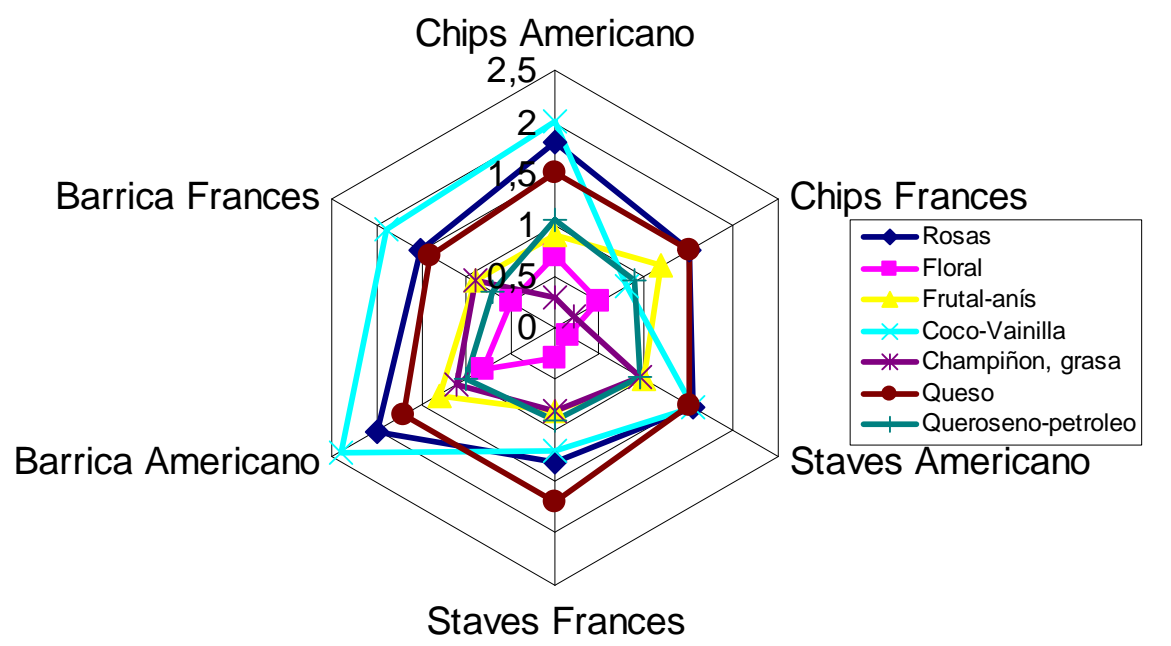

Figura 4.16. Representación tipo "tela de araña" de los resultados alcanzados con la técnica GC-O en función: $\boldsymbol{a}$. de los olores detectados $y$, $\boldsymbol{b}$. en función del tipo de muestra evaluada. Análisis para el periodo de muestro 4 (envejecimiento en botella).

Las representaciones muestran que las lactonas de roble y vainilla son las principales responsables de la presencia de notas de roble, coco, vainilla en los vinos. También es posible afirmar que existe otro grupo de compuestos que pueden ser cuantificados con la cromatografía de gases pero que es poco probable que alcancen los umbrales sensoriales detectables. Sin embargo, esto no quiere decir que no contribuyen al aroma del vino, algunos estudios han demostrado que las sustancias con similitudes químicas, como el eugenol y isoeugenol, puede tener efectos aditivos o acumulativos $[49,50]$.

El anterior comentario también se aplica a los fenoles volátiles que poseen con olores característicos ahumados, son compuestos que tienen mayor influencia cuando se mezclan con otros volátiles. Compuestos como el guaiacol y 4- 
metilguaiacol tienen concentraciones individuales que pueden estar por debajo de los umbrales sensoriales, sin embargo sus concentraciones combinadas pueden provocar un efecto de percepción sensorial.

a.

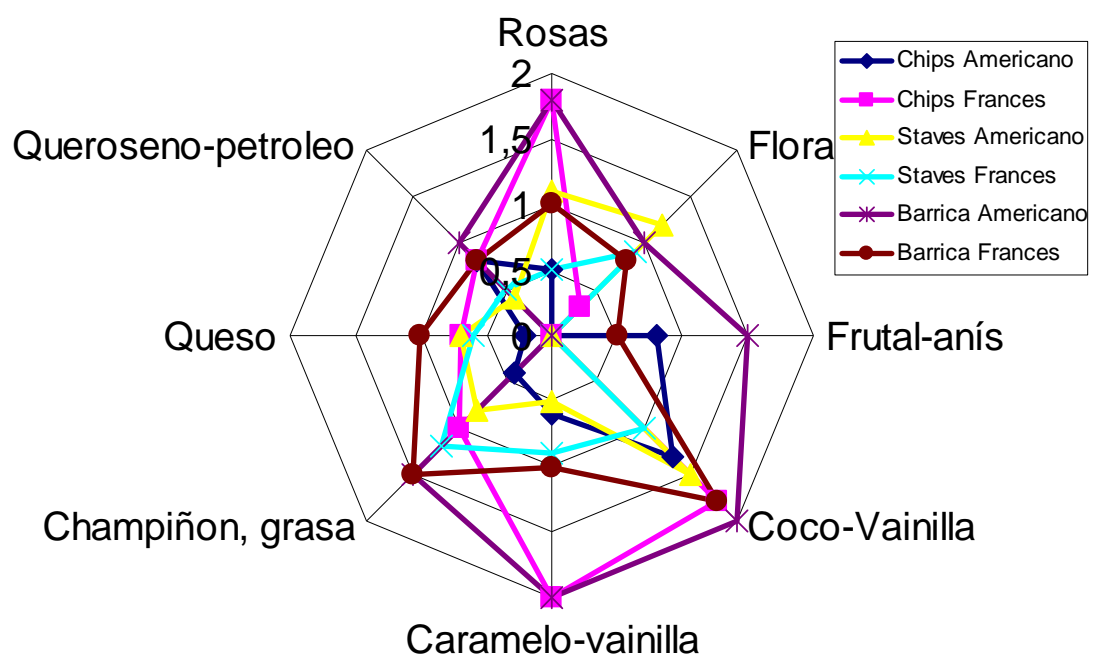

b.

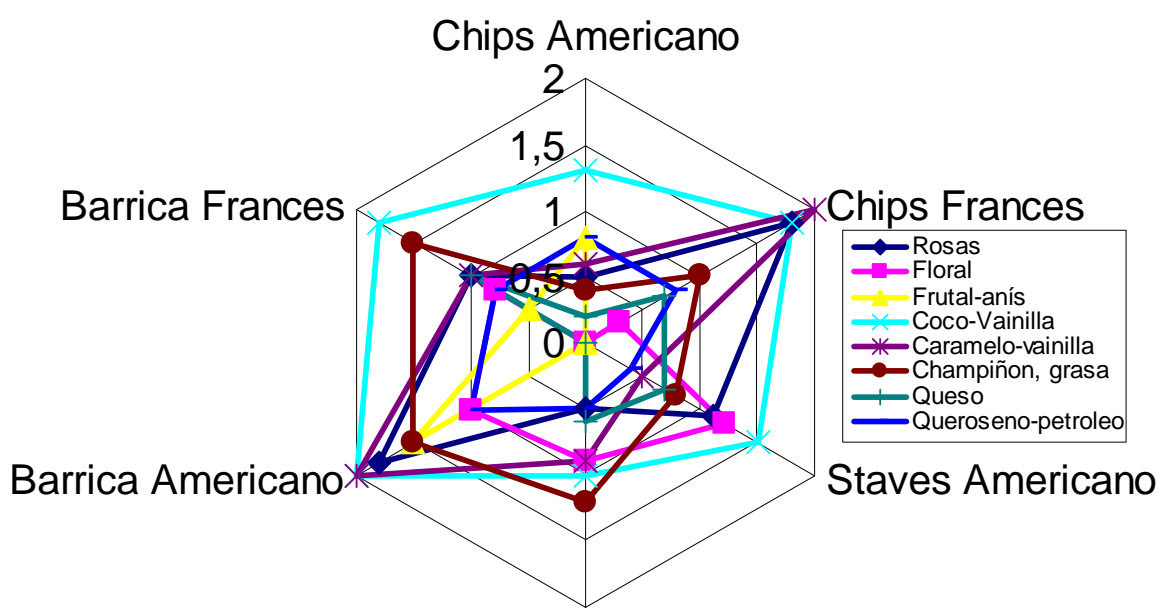

Staves Frances

Figura 4.17. Representación tipo "tela de araña" de los resultados alcanzados con la técnica GC-O en función: $\boldsymbol{a}$. de los olores detectados y, b. en función del tipo de muestra evaluada. Análisis para el periodo de muestro 5 (Envejecimiento en Botella).

La Figura 4.18 ilustra los resultados obtenidos para el periodo de evaluación número 6. Es posible observar que los olores con notas relacionadas con productos químicos, terrosos y madera tienden a dominar sobre los olores con notas a frutas y flores. Estos resultados sugieren que la interacción entre compuestos varia en función de su concentración dentro de la mezcla [51]. Además, a medida que la mezcla se hace más compleja parece ser que los fenómenos de supresión dominan sobre los fenómenos de adición o sinérgicos [52]. 
a.

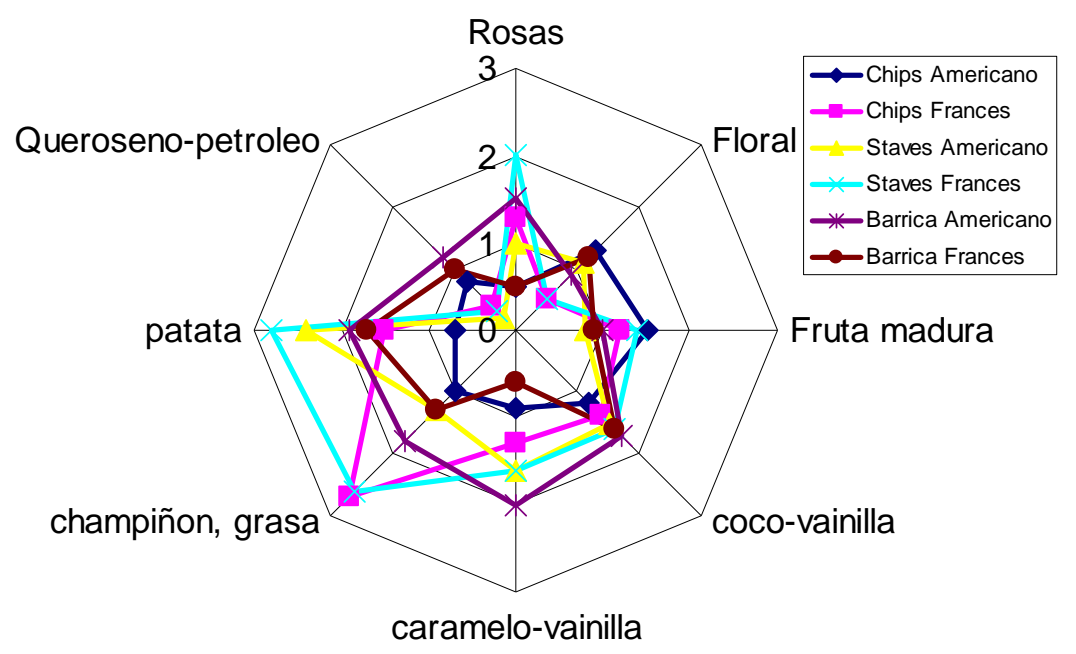

b.

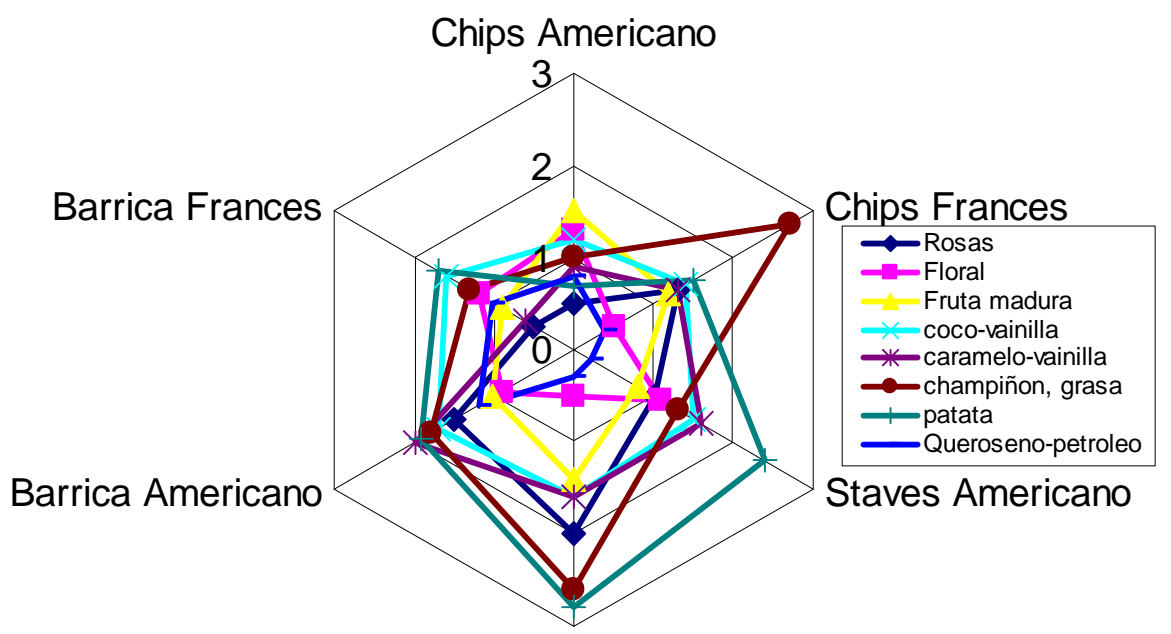

Staves Frances

Figura 4.18. Representación tipo "tela de araña" de los resultados alcanzados con la técnica GC-O en función: $\boldsymbol{a}$. de los olores detectados y, b. en función del tipo de muestra evaluada. Análisis para el periodo de muestro 6 (Envejecimiento en Botella).

El dominio de estas notas terrosas y a madera es más intenso en vinos envejecidos con métodos alternativos. En el caso de los vinos que fueron envejecidos en barricas de roble, estos presentaron un nivel algo mayor en el contenido de fenoles volátiles, los cuales ejercen un efecto importante en la expresión de las notas frutales [53]. En este contexto químico, la percepción de un rango amplio de notas, particularmente de las frutales, es muy compleja. Además, los grandes vinos tintos no muestran únicamente olores muy explícitos y específicos, sino que más bien muestran una amplia paleta de muchos olores de carácter más sutil. No es sorprendente, por tanto, que una modificación en las variables consideradas durante el envejecimiento tengo una repercusión importante en las características sensoriales del vino [54]. 
Los resultados vistos hasta aquí muestran que es posible determinar diferencias entre los tipos de envejecimiento (alternativos y barricas) utilizando la cromatrografía de gases masas y la olfatometría. El desarrollo de sensores, y los avances en el análisis multivariante y la fusión de los datos provenientes de técnicas analíticas pueden dar origen a un sistema de análisis sensorial que permita una valoración precisa de la calidad -en términos del proceso de elaboración- de un vino.

\subsubsection{Nariz electrónica}

Esta parte de la investigación esta referida a la utilización de la nariz electrónica para evaluar las diferencias entre la composición volátil a nivel global de las diferentes muestras de vinos elaborados para esta investigación. A titulo de ejemplo la Figura 4.19 esquematiza la respuesta del sensor TGS 885 durante el desarrollo de este estudio; el resto de los sensores tiene un comportamiento similar. Se puede observar que la intensidad máxima de la señal decrece con el paso del tiempo de envejecimiento (Figura 4.19a) y además que su valor es especifico para las dos formas de envejecimiento estudiadas (Figura 4.19b).

La disminución de la señal registrada puede estar asociada a una perdida de intensidad de aromas frutales que implica el paso de la crianza en barrica a botella lo cual se puede traducir en una mayor armonía con los compuestos que vienen de la madera, [55].

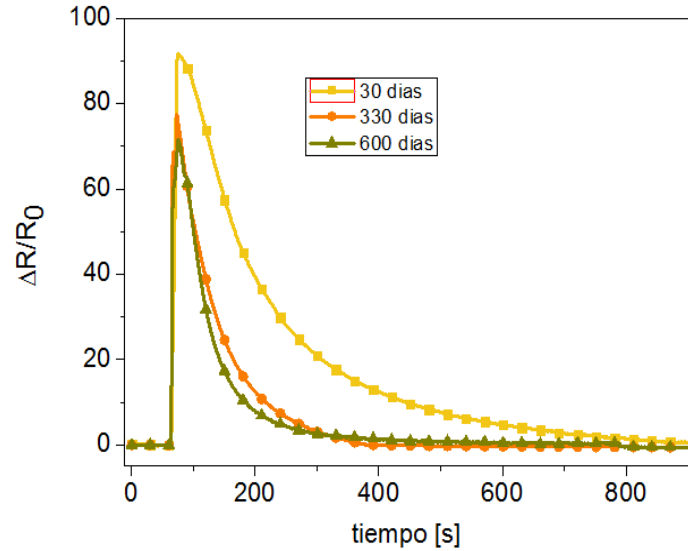

Figura 4.19a. Ejemplo de la respuesta del Sensor TGS 885 a la inyección de una muestra de vino durante su envejecimiento.

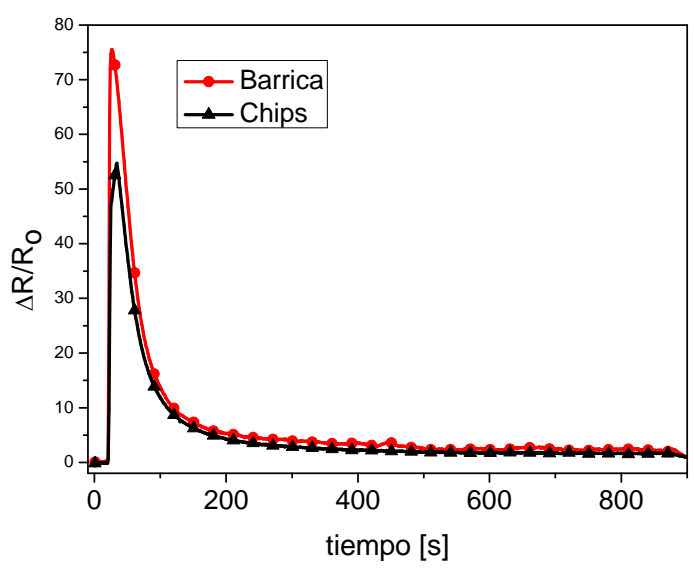

Figura 4.19b. Diferencias en la respuesta del sensor TGS 885 a la inyección de un vino envejecido en barrica y un vino envejecido en depósitos de acero inoxidable después de 600 días desde el comienzo del proceso de envejecimiento. 


\subsubsection{Análisis Exploratorio. PCA.}

Las respuestas de los sensores, la cantidad de muestras y el número de repeticiones por cada una tipo de vino, requieren la aplicación de herramientas del tipo PCA, que es además el punto de partida para el análisis de los resultados obtenidos con la nariz electrónica. Cada uno de los análisis PCA realizados se inicia con una matriz de datos que fue preprocesada usando los métodos $S N V$ y autoscaling. En la mayoría de los casos se recogió un 95\% de la varianza explicada con las primeras 4 PC. Los resultados de estos análisis se muestran en la Figura 4.20 .

Al igual que sucedió con los resultados de las coordenadas CIELab respecto al primer periodo de muestreo (Figura 4.12.), es decir 30 días después de dar comienzo al proceso de envejecimiento en depósito, los vinos no mostraron características que permitan diferenciarlos claramente utilizando este método estadístico (Figura 4.20). Pasados 60 días más de maduración del vino en barricas o en depósito, los vinos comenzaron a asociarse poco a poco de acuerdo al tipo de envejecimiento (periodo T2), es decir que los clusters referentes a los vinos envejecidos en barrica tienen posiciones adyacentes - de acuerdo con los scores de la PC1- mientras que los vinos envejecidos en depósito de acero inoxidable con inserción de "chips" y "staves" aparecen ubicados en el lado contrario, a excepción de los vinos envejecidos con "staves" de roble francés que parecen tener características próximas al con el vino envejecido en barricas de roble americano.

Durante los periodos tres y cuatro (T3 y T4) se comienzan a diferenciar claramente los vinos envejecidos en barrica de aquellos envejecidos en depósito de acero inoxidable con inserción de madera. El cambio en el número de muestras del PCA para la T4 (330 días) se deben a que una vez los vinos fueron embotellados, se mezclaron los vinos que pertenecían a un mismo tipo de madera y envejecimiento, pasando de tener un conjunto de 12 vinos a una nueva serie de 6 muestras.

Durante el tiempo que el vino permanece en botella comienzan a revalidarse las características aromáticas que diferencian un vino envejecido en barrica de roble de un vino envejecido en depósitos de acero inoxidable. Para el último periodo de evaluación además fue más evidente el efecto del tipo de origen de la madera. 


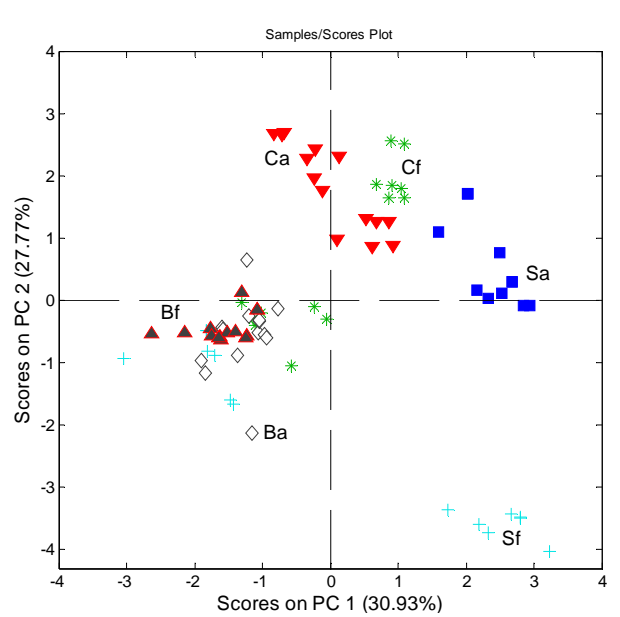

PCA período T1.

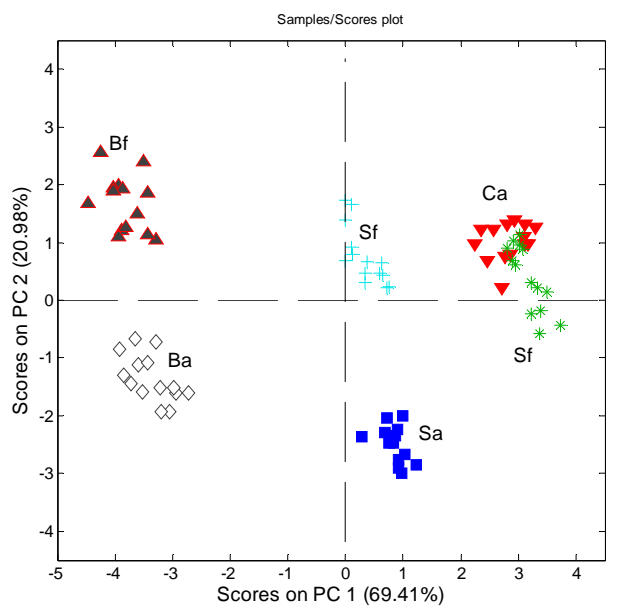

PCA periodo T3.

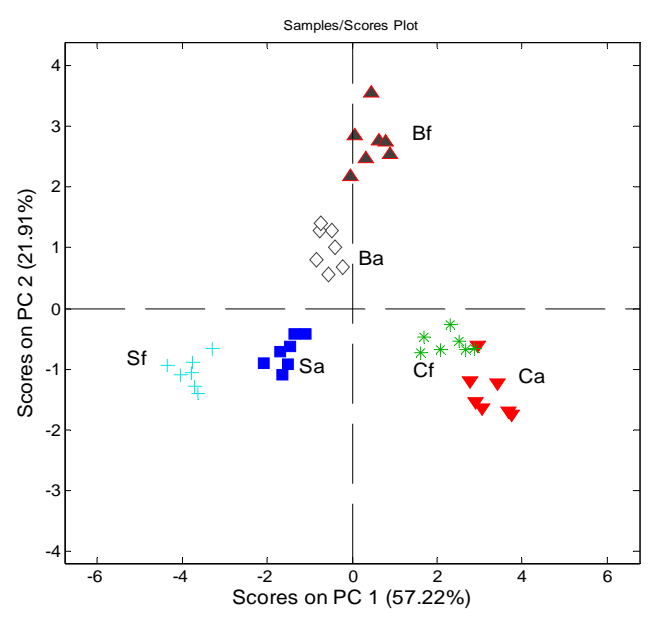

PCA periodo T5.

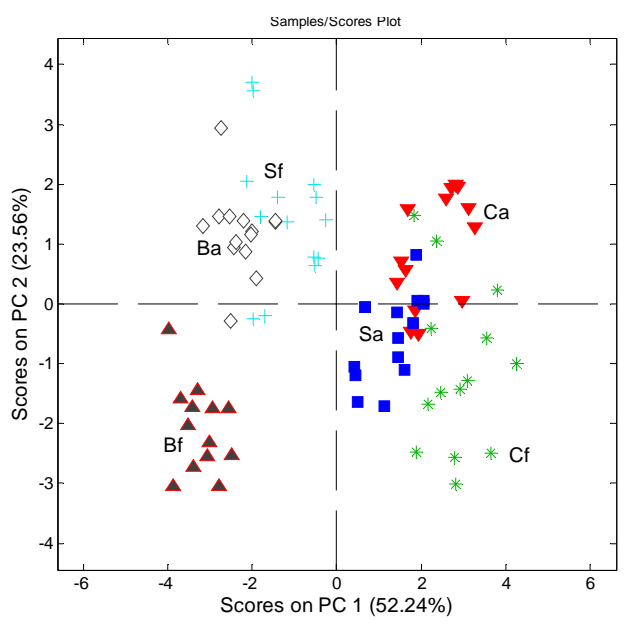

PCA periodo T2.

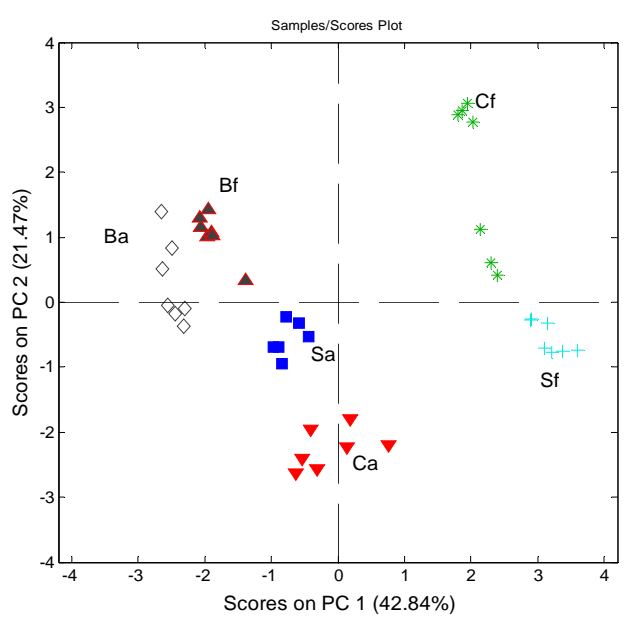

PCA periodo T4.

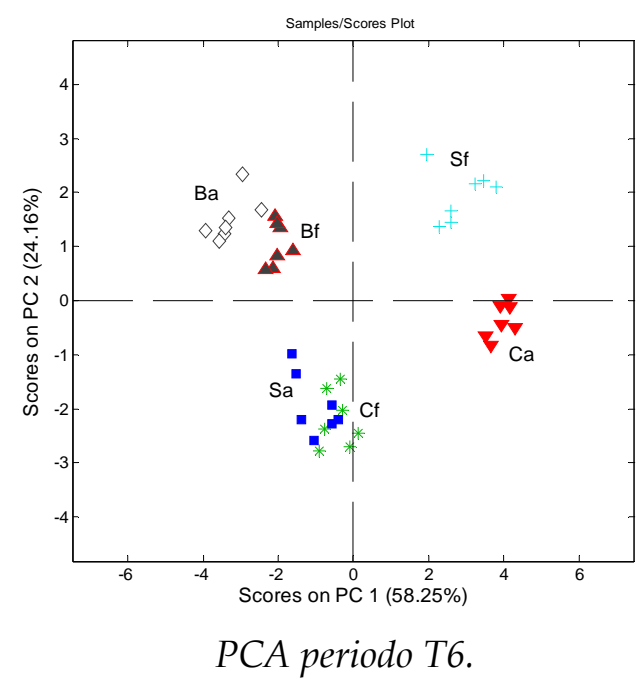

Figura 20. PCA para los resultados de la nariz electrónica de cada uno de los periodos de muestreo. Ca, Chips roble americano triangulo rojo. Cf, Chips roble francés asterisco verde. Sa, Staves roble americano cuadrado azul. Sf, Chips roble francés, cruz cian. Ba, Barrica roble americano rombos. Bf, Chips roble francés triangulo gris. 
En cada uno de los periodos de evaluación se observó una tendencia a discriminar entre tipos de envejecimiento; sin embargo un análisis conjunto de todos los periodos de muestreo sólo permitió una interpretación de la evolución de todas las muestras de vino y ninguna en función del tipo de envejecimiento o madera utilizada (Figura 4.21.). Fue por ello necesario desarrollar otro tipo de modelos quimiométricos complementarios a partir de los cuales se pudiera extraer más información, de manera que sus parámetros puedan explicar las posibles correlaciones con los demás sistemas analíticos empleados para la caracterización.

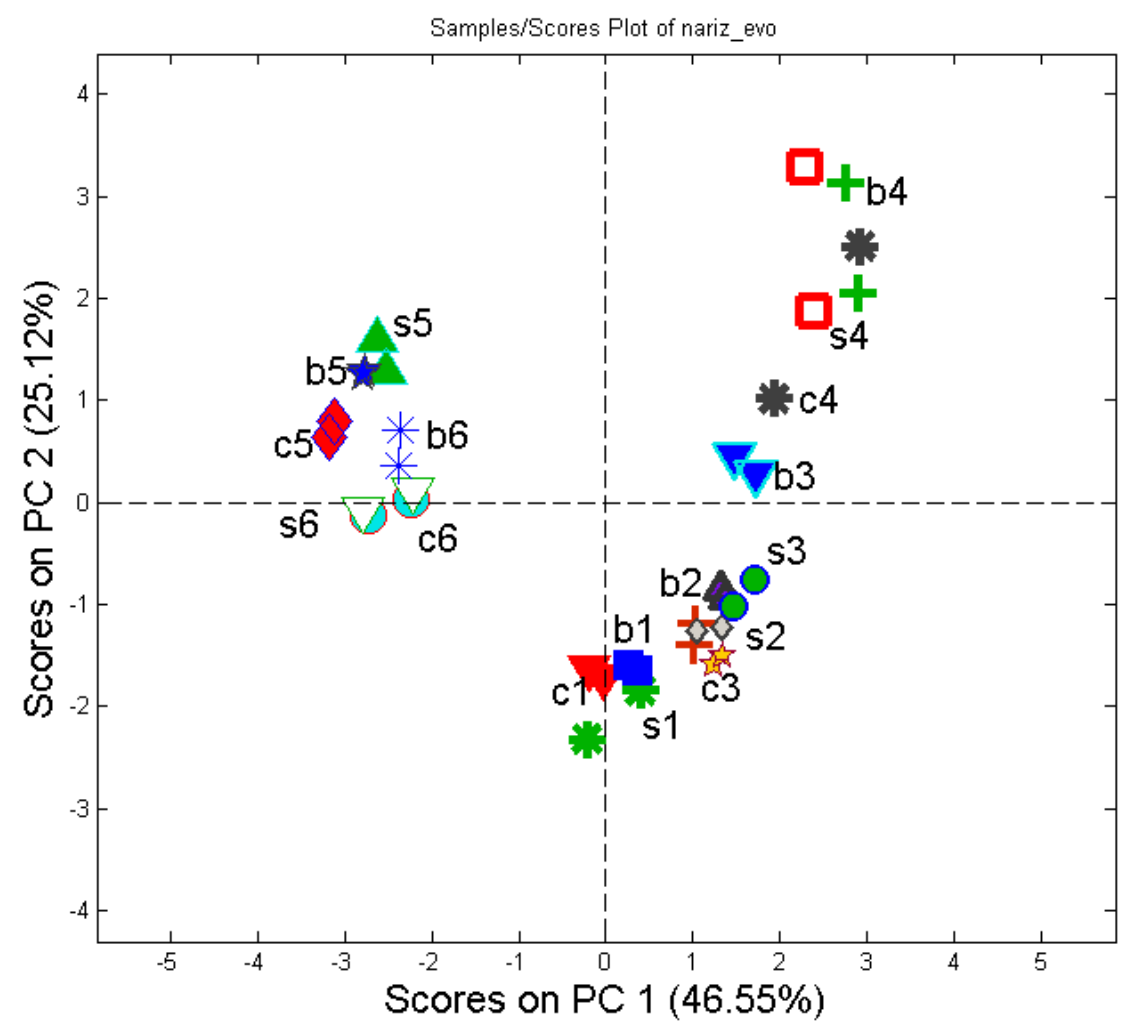

Figura 4.21. PCA resultados nariz electrónica. Estudio de la evolución de todas las tipos de vino. Las muestras marcadas con la letra c indican el envejecimiento con chips, la letras $s$ indican el envejecimiento con staves, la letra b envejecimiento en barrica, $b$. La numeración (1-6) indica el periodo de envejecimiento.

\subsubsection{Análisis supervisado. PLS-DA}

Las Tablas 4.7-4.12 explican los resultados obtenidos con los modelos PLS-DA de los diferentes análisis que se han llevado a cabo con la nariz electrónica. Recordemos que el PLS-DA corresponde con los métodos de reconocimiento supervisado de patrones y que es un método en el cual se emplean una serie de 
variables cualitativas o de categoría $Y$ para realizar una predicción con un conjunto de variables experimentales (sensores) $\mathrm{X}$. El número de variables $\mathrm{Y}$ es igual al número de clases a modelar, se les asigna un valor de 1 si las muestras pertenecen a ella y 0 en caso contrario [3]. Por lo tanto, al tratarse de múltiples variables a correlacionar, el algoritmo aplicado es PLS2, al que se le aplica un protocolo de validación cruzada total.

Una vez más, es necesario precisar que los valores numéricos han sido preprocesados usando los métodos SNV y autoescalado; además la categorización de los vinos se realizó de acuerdo con el tipo de envejecimiento aplicado (bloque Y).

El proceso de entrenamiento de los modelos fue evaluado utilizando el método de validación cruzada, leave one out. El número óptimo de variables latentes para el ajuste del modelo final se determinó mediante el parámetro RMSECV. De esta manera, fue posible predecir un valor para el bloque $\mathrm{Y}$ que puede ser interpretado como una medida de la diferencia entre clases. Cada valor de la predicción de una clase fue comparada con la curva de distribución bayesiana, y esto permitió determinar la probabilidad de que un objeto pertenezca a la clase original.

En ocasiones, el método PLS-DA es demasiado flexible y produce un ajuste forzado. Esto puede atribuirse en gran medida al modelado del ruido que traen los datos que afecta la capacidad de predicción del sistema. Este problema sólo se puede evitar con una validación completa, para que el porcentaje de predicción y la sensibilidad de los modelos no sean sobreestimados.

Como se mencionó anteriormente, la aplicación de modelos de clasificación en problemas reales requieren una estimación precisa de los diferentes parámetros. La validez real del modelo depende en gran medida de la calidad del conjunto de datos. Es importante por ello contar con una serie de datos representativos que cubran todas las fuentes de variabilidad dentro de las categorías. Por desgracia, un buen diseño de muestreo es muy raro, tanto por razones económicas, como por el hecho de que en el sector de la alimentación existen muchas fuentes de variabilidad que limitan un experimento.

A partir de los resultados expuestos anteriormente con el desarrollo de las diversas PCA, podemos afirmar que se distinguen seis clases, definidas por el tipo de envejecimiento. En esta ocasión, dado el tipo y cantidad de muestras disponibles de cada clase, decidimos comenzar con la aplicación PLS-DA como técnica de análisis multivariante de clasificación supervisada. Consecutivamente se realizará un análisis PLS2 para determinar si existe alguna correlación con los sistemas de caracterización analítica tradicional. 
Tabla 4.7. Resultados del análisis PLS-DA para los vinos evaluados el periodo de muestro T1.

\begin{tabular}{|c|c|c|c|c|c|c|}
\hline & $\begin{array}{c}\text { Chips } \\
\text { americano }\end{array}$ & $\begin{array}{l}\text { Chips } \\
\text { francés }\end{array}$ & $\begin{array}{c}\text { Staves } \\
\text { americano }\end{array}$ & $\begin{array}{l}\text { Staves } \\
\text { francés }\end{array}$ & $\begin{array}{c}\text { Barrica } \\
\text { americano }\end{array}$ & $\begin{array}{l}\text { Barrica } \\
\text { frances }\end{array}$ \\
\hline $\begin{array}{l}\text { Sensitividad } \\
\text { (Cal): }\end{array}$ & 1.00 & 0.86 & 0.93 & 1.00 & 1.00 & 0.93 \\
\hline $\begin{array}{l}\text { Especificidad } \\
\text { (Cal): }\end{array}$ & 1.00 & 0.96 & 1.000 & 0.94 & 0.814 & 0.886 \\
\hline $\begin{array}{l}\text { Sensitividad } \\
\text { (CV) }\end{array}$ & 1.00 & 0.86 & 0.93 & 1.00 & 1.00 & 0.93 \\
\hline $\begin{array}{l}\text { Especificidad } \\
(\mathrm{CV})\end{array}$ & 1.00 & 0.94 & 1.00 & 0.90 & 0.81 & 0.87 \\
\hline $\begin{array}{l}\text { Class. Err } \\
\text { (Cal): }\end{array}$ & 0 & 0.092857 & 0.035714 & 0.028571 & 0.0928571 & 0.0928571 \\
\hline $\begin{array}{l}\text { Class. Err } \\
(\mathrm{CV}):\end{array}$ & 0 & 0.1 & 0.0357143 & 0.05 & 0.0928571 & 0.1 \\
\hline $\mathrm{R}^{\wedge} 2:$ & 0.759 & 0.618 & 0.810 & 0.609 & 0.333 & 0.435 \\
\hline $\mathrm{CV} \mathrm{R}^{\wedge} 2:$ & 0.711 & 0.543 & 0.768 & 0.518 & 0.242 & 0.349 \\
\hline
\end{tabular}

Los resultados de los diferentes parámetros del modelo multivariante PLS-DA para el periodo de muestreo 1 se encuentran registrados en la Tabla 4.7. En este caso el parámetro $\mathrm{R}^{2}$ no muestra que exista una buena correlación entre los valores correspondientes al bloque $X$ (datos de la nariz electrónica) y el bloque $Y$ (parámetros de clasificación). Si bien este parámetro no es una medida de la calidad del modelo, si sería conveniente que mostrara valores más altos, ya que además este es un método supervisado, es decir, que está forzando el ajuste de los datos a una determinada clase.

Tabla 4.8. Resultados del análisis PLS-DA para los vinos evaluados el periodo de muestro T2.

\begin{tabular}{|c|c|c|c|c|c|c|}
\hline & $\begin{array}{c}\text { Chips } \\
\text { americano }\end{array}$ & $\begin{array}{c}\text { Chips } \\
\text { francés }\end{array}$ & $\begin{array}{c}\text { Staves } \\
\text { americano }\end{array}$ & $\begin{array}{c}\text { Staves } \\
\text { francés }\end{array}$ & Barrica & Barrica \\
Americano & Frances \\
\hline
\end{tabular}

\begin{tabular}{|l|l|l|l|l|l|l|}
\hline $\begin{array}{l}\text { Sensitividad } \\
(\mathrm{Cal}):\end{array}$ & 1.00 & 0.93 & 0.86 & 1.00 & 1.00 & 1.00 \\
\hline $\begin{array}{l}\text { Especificidad } \\
(\mathrm{Cal}):\end{array}$ & 0.97 & 0.99 & 0.91 & 1.00 & 1.00 & 1.00 \\
\hline $\begin{array}{l}\text { Sensitividad } \\
(\mathrm{CV})\end{array}$ & 1.00 & 0.86 & 0.86 & 1.00 & 1.00 & 1.00 \\
\hline $\begin{array}{l}\text { Especificidad } \\
(\mathrm{CV})\end{array}$ & 0.97 & 0.97 & 0.89 & 1.00 & 1.00 & 1.00 \\
\hline $\begin{array}{l}\text { Class. Err } \\
(\mathrm{Cal}):\end{array}$ & 0.014286 & 0.042857 & 0.114286 & 0 & 0 & 0 \\
\hline $\begin{array}{l}\text { Class. Err } \\
(\mathrm{CV}):\end{array}$ & 0.014286 & 0.085714 & 0.128571 & 0 & 0 & 0 \\
\hline $\mathrm{R}^{\wedge}$ : & 0.586 & 0.654 & 0.362 & 0.652 & 0.525 & 0.855 \\
\hline $\mathrm{CV} \mathrm{R}^{\wedge} 2:$ & 0.526 & 0.598 & 0.232 & 0.598 & 0.427 & 0.833 \\
\hline
\end{tabular}


Este método PLS-DA fue validado con 6 variables latentes que recogen el 95\% de la información del bloque $X$ que a su vezse relaciona con un $60 \%$ de la información del bloque $Y$, el cual especifica las clases existentes de acuerdo al tipo de envejecimiento.

Para el periodo de muestreo 2 (Tabla 4.8.), los resultados son similares a los señalados anteriormente. Se ha desarrollado un modelo con 5 variables latentes que acumulan el 99\% de la información del bloque X y nuevamente el 60\% de la información para el bloque $Y$.

Una vez han pasado 150 días a partir del comienzo del proceso de envejecimiento de los vinos, es posible observar que los distintos parámetros presentan una notable mejoría (Tabla 4.9), especialmente en el cálculo del error de clasificación después de realizar la validación cruzada. Para este modelo se obtuvieron valores de $R^{2}$ entre 0.885 y 0.908 . Estos resultados pueden ser explicados desde el punto de vista enológico, ya que es en esta etapa del proceso de envejecimiento cuando los vinos comienzan a asimilar de mejor manera todas las propiedades organolépticas que la madera le aporta al vino, permitiendo en este caso distinguir las características de cada tipo de envejecimiento.

Tabla 4.9. Resultados del análisis PLS-DA para los vinos evaluados el periodo de muestro T3.

\begin{tabular}{|c|c|c|c|c|c|c|}
\hline & $\begin{array}{c}\text { Chips } \\
\text { americano }\end{array}$ & $\begin{array}{l}\text { Chips } \\
\text { francés }\end{array}$ & $\begin{array}{c}\text { Staves } \\
\text { americano }\end{array}$ & $\begin{array}{l}\text { Staves } \\
\text { francés }\end{array}$ & $\begin{array}{c}\text { Barrica } \\
\text { Americano }\end{array}$ & $\begin{array}{l}\text { Barrica } \\
\text { Frances }\end{array}$ \\
\hline $\begin{array}{l}\text { Sensitividad } \\
\text { (Cal): }\end{array}$ & 1.000 & 1.000 & 1.000 & 1.000 & 1.000 & 1.000 \\
\hline $\begin{array}{l}\text { Especificidad } \\
\text { (Cal): }\end{array}$ & 1.000 & 1.000 & 1.000 & 1.000 & 1.000 & 1.000 \\
\hline $\begin{array}{l}\text { Sensitividad } \\
(\mathrm{CV})\end{array}$ & 1.000 & 1.000 & 1.000 & 1.000 & 1.000 & 1.000 \\
\hline $\begin{array}{l}\text { Especificidad } \\
(\mathrm{CV})\end{array}$ & 1.000 & 1.000 & 1.000 & 1.000 & 1.000 & 1.000 \\
\hline $\begin{array}{l}\text { Class. } \quad \text { Err } \\
\text { (Cal): }\end{array}$ & 0 & 0 & 0 & 0 & 0 & 0 \\
\hline $\begin{array}{ll}\text { Class. } & \text { Err } \\
(\mathrm{CV}): & \end{array}$ & 0 & 0 & 0 & 0 & 0 & 0 \\
\hline $\mathrm{R}^{\wedge} 2:$ & 0.855 & 0.908 & 0.895 & 0.877 & 0.871 & 0.885 \\
\hline $\mathrm{CV} \mathrm{R}^{\wedge} 2$ & 0.829 & 0.892 & 0.879 & 0.857 & 0.849 & 0.867 \\
\hline
\end{tabular}

En la Tabla 4.10, es posible observar como aumenta el error de clasificación y disminuye la correlación entre los bloques X y Y después de 140 días de haber dado comienzo al envejecimiento en botella. Esta disminución podría estar relacionada con el cambio de etapa en la elaboración del vino, ya que los vinos han pasado de estar en un medio oxidante (depósitos, barricas) a un medio 
reductor (botella), afectando sus propiedades sensoriales. Para este modelo se han determinado 4 variables latentes que capturan el $97 \%$ del bloque $X$ y el $68 \%$ del bloque $Y$.

Tabla 4.10. Resultados del análisis PLS-DA para los vinos evaluados el periodo de muestro T4.

\begin{tabular}{|c|c|c|c|c|c|c|}
\hline & $\begin{array}{c}\text { Chips } \\
\text { americano }\end{array}$ & $\begin{array}{l}\text { Chips } \\
\text { francés }\end{array}$ & $\begin{array}{c}\text { Staves } \\
\text { americano }\end{array}$ & $\begin{array}{l}\text { Staves } \\
\text { francés }\end{array}$ & $\begin{array}{c}\text { Barrica } \\
\text { Americano }\end{array}$ & $\begin{array}{l}\text { Barrica } \\
\text { Frances }\end{array}$ \\
\hline $\begin{array}{l}\text { Sensitividad } \\
\text { (Cal): }\end{array}$ & 1.000 & 1.000 & 1.000 & 1.000 & 1.000 & 1.000 \\
\hline $\begin{array}{l}\text { Especificidad } \\
\text { (Cal): }\end{array}$ & 1.000 & 0.971 & 1.000 & 1.000 & 0.686 & 0.943 \\
\hline $\begin{array}{l}\text { Sensitividad } \\
\text { (CV) }\end{array}$ & 1.000 & 1.000 & 1.000 & 1.000 & 1.000 & 0.857 \\
\hline $\begin{array}{l}\text { Especificidad } \\
\text { (CV) }\end{array}$ & 1.000 & 0.971 & 1.000 & 1.000 & 0.686 & 0.943 \\
\hline $\begin{array}{ll}\text { Class. } & \text { Err } \\
\text { (Cal): } & \\
\end{array}$ & 0 & 0.014286 & 0 & 0 & 0.157143 & 0.028571 \\
\hline $\begin{array}{ll}\text { Class. } & \text { Err } \\
(\mathrm{CV}): & \end{array}$ & 0 & 0 & 0 & 0 & 0.157143 & 0.1 \\
\hline $\mathrm{R}^{\wedge} 2:$ & 0.890 & 0.727 & 0.818 & 0.879 & 0.598 & 0.762 \\
\hline $\mathrm{CV} \mathrm{R}^{\wedge} 2$ & 0.854 & 0.616 & 0.711 & 0.843 & 0.360 & 0.634 \\
\hline
\end{tabular}

Tabla 4.11. Resultados del análisis PLS-DA para los vinos evaluados el periodo de muestro T5.

\begin{tabular}{|c|ccc|c|c|c|}
\hline & $\begin{array}{c}\text { Chips } \\
\text { americano }\end{array}$ & $\begin{array}{c}\text { Chips } \\
\text { francés }\end{array}$ & $\begin{array}{c}\text { Staves } \\
\text { americano }\end{array}$ & $\begin{array}{c}\text { Staves } \\
\text { francés }\end{array}$ & $\begin{array}{c}\text { Barrica } \\
\text { Americano }\end{array}$ & $\begin{array}{c}\text { Barrica } \\
\text { Frances }\end{array}$ \\
\hline
\end{tabular}

\begin{tabular}{|l|l|l|l|l|l|l|}
\hline $\begin{array}{l}\text { Sensitividad } \\
(\mathrm{Cal}):\end{array}$ & 1.000 & 1.000 & 1.000 & 1.000 & 1.000 & 1.000 \\
\hline $\begin{array}{l}\text { Especificidad } \\
(\mathrm{Cal}):\end{array}$ & 1.000 & 1.000 & 1.000 & 1.000 & 1.000 & 1.000 \\
\hline $\begin{array}{l}\text { Sensitividad } \\
(\mathrm{CV})\end{array}$ & 1.000 & 1.000 & 1.000 & 1.000 & 0.857 & 1.000 \\
\hline $\begin{array}{l}\text { Especificidad } \\
(\mathrm{CV})\end{array}$ & 1.000 & 1.000 & 1.000 & 1.000 & 1.000 & 1.000 \\
\hline $\begin{array}{l}\text { Class. Err } \\
(\mathrm{Cal}): \quad 0\end{array}$ & 0 & 0 & 0 & 0 & 0 \\
\hline $\begin{array}{l}\text { Class. Err } \\
(\mathrm{CV}):\end{array}$ & 0 & 0 & 0 & 0 & 0.071429 & 0 \\
\hline $\mathrm{R}^{\wedge} 2:$ & 0.857 & 0.886 & 0.558 & 0.903 & 0.900 & 0.917 \\
\hline $\mathrm{CV} \mathrm{R}^{\wedge} 2:$ & 0.805 & 0.824 & 0.423 & 0.856 & 0.850 & 0.875 \\
\hline
\end{tabular}

El modelo resultante con los datos registrados en el periodo de muestro 5 (Tabla 4.11), explican que la capacidad de discriminación de la nariz electrónica mejora con la tiempo de envejecimiento en botella. Esto podría indicar que los cambios que generan las reacciones entre los polifenoles pueden ser seguidas con nuestro sistema. En este periodo solamente los vinos envejecidos en barricas de roble 
americano presentaron un valor en el error de clasificación después de la validación cruzada. El modelo se determinó con 5 variables latentes que capturan el 96\% de la información para el bloque X y el $84 \%$ para el bloque $\mathrm{Y}$.

Tabla 4.12. Resultados del análisis PLS-DA para los vinos evaluados el periodo de muestro T6.

\begin{tabular}{|c|c|c|c|c|c|c|}
\hline & $\begin{array}{c}\text { Chips } \\
\text { americano }\end{array}$ & $\begin{array}{l}\text { Chips } \\
\text { francés }\end{array}$ & $\begin{array}{c}\text { Staves } \\
\text { americano }\end{array}$ & $\begin{array}{l}\text { Staves } \\
\text { francés }\end{array}$ & $\begin{array}{c}\text { Barrica } \\
\text { Americano }\end{array}$ & $\begin{array}{l}\text { Barrica } \\
\text { Frances }\end{array}$ \\
\hline $\begin{array}{l}\text { Sensitividad } \\
\text { (Cal): }\end{array}$ & 1.000 & 1.000 & 1.000 & 1.000 & 1.000 & 1.000 \\
\hline $\begin{array}{l}\text { Especificidad } \\
\text { (Cal): }\end{array}$ & 1.000 & 0.943 & 1.000 & 1.000 & 1.000 & 1.000 \\
\hline $\begin{array}{l}\text { Sensitividad } \\
(\mathrm{CV})\end{array}$ & 1.000 & 0.857 & 1.000 & 1.000 & 1.000 & 0.857 \\
\hline $\begin{array}{l}\text { Especificidad } \\
\text { (CV) }\end{array}$ & 1.000 & 0.714 & 1.000 & 1.000 & 1.000 & 1.000 \\
\hline $\begin{array}{ll}\begin{array}{l}\text { Class. } \\
\text { (Cal): }\end{array} & \text { Err } \\
\end{array}$ & 0 & 0.0428571 & 0 & 0 & 0 & 0 \\
\hline $\begin{array}{ll}\text { Class. } & \text { Err } \\
(\mathrm{CV}): & \\
\end{array}$ & 0 & 0.114286 & 0 & 0 & 0 & 0.0714286 \\
\hline $\mathrm{R}^{\wedge} 2:$ & 0.929 & 0.581 & 0.931 & 0.912 & 0.907 & 0.729 \\
\hline $\mathrm{CV} \mathrm{R} \mathrm{R}^{\wedge} 2$ & 0.883 & 0.407 & 0.877 & 0.849 & 0.816 & 0.470 \\
\hline
\end{tabular}

Finalmente, los resultados para el periodo de muestro 6 mostraron que la nariz electrónica sería capaz de distinguir entre los diferentes tipos de envejecimientos aplicados en este trabajo a los diferentes vinos, especialmente cuando se trata de envejecimientos para los cuales se ha utilizado una madera de origen americano. Este modelo ha mostrado una buena correlación entre los bloques X y Y; se han determinado 6 variables latentes que recogen el $99 \%$ de la varianza explicada del bloque $\mathrm{Y}$ que se relaciona con un $88 \%$ de la información que recoge el bloque $\mathrm{Y}$.

\subsubsection{Correlaciones entre sistemas}

\subsubsection{Regresión Lineal por mínimos cuadrados PLS2}

En términos de calidad, uno de los productos del sector de la alimentación en el que más se ha trabajado, es sin duda, el vino. El análisis fisicoquímico de las muestras puede aportar un tipo de información de gran interés para el enólogo. Así, este hecho nos proporciona una oportunidad única para comparar las diversas técnicas instrumentales analíticas con los sistemas de evaluación sensorial electrónicos artificiales. Esta comparación ha de permitir estimar la capacidad de nuestra nariz electrónica para facilitar información análoga o 
complementaria a la obtenida mediante aquellos procedimientos, de forma evidentemente más rápida, sencilla, objetiva y menos costosa. Con este fin, emplearemos las señales registradas por la red de sensores para construir modelos de predicción de diversos parámetros analíticos y sensoriales, mediante el uso del algoritmo PLS2, adecuadamente validado.

Como se ha demostrado con los distintos análisis fisicoquímicos y organolépticos, hasta el momento la práctica de nuevas técnicas de maduración se ha traducido en una modificación de los valores propios de los parámetros analíticos. En esta ocasión, el propósito no es distinguir o valorar la capacidad del sistema para clasificar los vinos de acuerdo con sus características de elaboración, en este punto el objetivo es determinar si la nariz electrónica es capaz de predecir ciertos parámetros obtenidos mediante análisis clásicos.

Para que un modelo PLS2 entregue buenos resultados es necesario que en la fase de entrenamiento se utilicen suficientes medidas para tener una buena representación estadística del conjunto de entrada. Al mismo tiempo, resulta interesante poder evaluar un número de medidas elevado para asegurarnos de el sistema realiza predicciones exactas. Teniendo en cuenta que la obtención de medidas de entrenamiento suele ser una tarea muy difícil, resulta sumamente interesante optimizar su uso en el proceso de reconocimiento de patrones. Por ese motivo, en todos los estudios de correlación y en esta tesis se utiliza una validación cruzada que ha de permitir determinar la capacidad de predicción de la nariz electrónica a partir de las señales de los sensores.

Tabla 4.13. Descripción de los diferentes modelos PLS2 obtenidos para cada periodo de evaluación llevado a cabo con los vinos tintos.

\begin{tabular}{|c|c|c|c|c|}
\hline $\begin{array}{c}\text { Periodo de } \\
\text { muestreo }\end{array}$ & Tiempo & $\begin{array}{c}\text { Nro. Variables } \\
\text { latentes }\end{array}$ & $\%$ bloque $X$ & $\%$ bloque Y \\
\hline $\mathrm{T} 1$ & 30 días & 6 & 95.72 & 73.56 \\
\hline $\mathrm{T} 2$ & 90 días & 4 & 95.61 & 57.91 \\
\hline T3 & 150 días & 4 & 98.91 & 93.52 \\
\hline $\mathrm{T} 4$ & 330 días & 6 & 96.89 & 91.91 \\
\hline T5 & 480 días & 6 & 95.02 & 79.12 \\
\hline T6 & 600 días & 4 & 97.04 & 77.12 \\
\hline
\end{tabular}

Reiteramos que por imposibilidad experimental no ha sido posible tener un alto número de muestras desde el punto de vista estadístico, por este motivo se han utilizado, validaciones cruzadas ("leave-one-out"). Es importante también mencionar nuevamente que para cada modelo PLS2, los datos del conjunto de entrenamiento son normalizados usando los métodos SNV y autoescalado.

La tabla 4.13 ilustra el número de variables latentes y el porcentaje de información que recogen para todos los modelos que se obtuvieron durante las diferentes medidas realizadas a los vinos de Ribera del Duero, tanto para el 
bloque $X$ como para el bloque Y. Para todos los resultados se tuvieron un máximo de 6 variables latentes que recogen el 95\% de la información del bloque $X$. Observando la relación entre bloque $X$ y $Y$, podemos afirmar que el modelo que recoge un menor porcentaje de la varianza explicada respecto al bloque $Y$, es el modelo resultante de las medidas realizadas en el periodo de muestreo 2.

Los resultados del periodo de muestreo 1 (Tabla 4.14) informan que los compuestos analizados con GC-MS generan un modelo con errores muy altos. Es posible observar que para compuestos como el furfural aunque existe un rango de valores amplio para las muestras analizadas, el error calculado puede llegar a superar el valor real de algunas de las muestras. Esta situación es admisible cuando se trata de los primeros días del envejecimiento, momento en el que los vinos aún no han comenzado a asimilar todas las propiedades que la madera puede aportarle.

Tabla 4.14. Resultados modelo PLS2 para el periodo de evaluación T1.

\begin{tabular}{|c|c|c|c|c|c|c|}
\hline \multirow[t]{2}{*}{ Parámetro } & \multicolumn{2}{|c|}{ Rango } & \multirow[t]{2}{*}{ RMSEC } & \multirow[t]{2}{*}{ RMSECV } & \multirow[t]{2}{*}{$\mathrm{R}^{\wedge} 2$} & \multirow[t]{2}{*}{$R^{\wedge} 2 \mathrm{CV}$} \\
\hline & Inferior & Superior & & & & \\
\hline Furfural & 82.27 & 1569.91 & 234.35 & 346.19 & 0.801 & 0.626 \\
\hline Guaiacol & 52.28 & 107.95 & 8.14 & 11.45 & 0.799 & 0.623 \\
\hline Cis-Whiskylactona & 0.38 & 216.30 & 45.57 & 59.33 & 0.671 & 0.394 \\
\hline Trans-Whiskyactona & 60.77 & 118.03 & 14.3 & 16.70 & 0.549 & 0.384 \\
\hline Eugenol & 5.77 & 49.57 & 8.5 & 9.28 & 0.695 & 0.600 \\
\hline $\mathrm{L}$ & 3.92 & 5.29 & 0.17 & 0.31 & 0.885 & 0.619 \\
\hline $\mathrm{a}$ & 25.90 & 31.77 & 0.70 & 1.32 & 0.888 & 0.614 \\
\hline $\mathrm{b}$ & 6.76 & 9.12 & 0.29 & 0.53 & 0.885 & 0.620 \\
\hline $\mathrm{C}$ & 26.77 & 33.1 & 0.75 & 1.41 & 0.888 & 0.615 \\
\hline $\mathrm{H}$ & 14.59 & 16.01 & 0.18 & 0.33 & 0.874 & 0.618 \\
\hline$S$ & 6.25 & 6.85 & 0.12 & 0.16 & 0.704 & 0.610 \\
\hline
\end{tabular}

Respecto a las medidas del color, todas las coordenadas CIELab presentan valores de correlación similares (entre 0.60 y 0.62), lo que significa que hasta este punto del proceso de evolución no existe una característica en los vinos capaz de relacionar el aroma y el color.

De la misma manera que ocurrió con los resultados PLS-DA para estas muestras de vino, los parámetros valorados en el periodo de muestreo 2 (Tabla 4.15) tienen un comportamiento similar a los registrados para el periodo de muestreo 1. Sin embargo en este caso, los valores de $\mathrm{R}^{2}$ han disminuido de manera general para las coordenadas CIELab.

La Tabla 4.16 (periodo de muestreo 3) presenta un aumento en la correlación para todos los parámetros; esto sucede cuando los vinos han asimilado todos los aportes que la madera entrega tanto a nivel del color como en el aroma. Es importante también destacar que en esta ocasión el error calculado ha sido el más 
bajo entre los tres periodos de muestreos evaluados durante el envejecimiento en depósito o barrica.

Tabla 4.15. Resultados modelo PLS2 para el periodo de evaluación T2.

\begin{tabular}{|l|r|r|r|r|r|r|r|}
\multicolumn{1}{c|}{ Parámetro } & \multicolumn{2}{c}{ Rango } & \multicolumn{1}{c}{ RMSEC } & \multicolumn{1}{c}{ RMSECV } & $\mathrm{R}^{\wedge} 2$ & $\mathrm{R}^{\wedge} 2$ CV \\
\hline Inferior & \multicolumn{3}{c|}{ Superior } & \multicolumn{3}{c|}{} \\
\hline Furfural & 462.40 & 1503.10 & 215.99 & 243.23 & 0.759 & 0.697 \\
\hline Guaiacol & 72.09 & 114.40 & 9.47 & 10.56 & 0.741 & 0.680 \\
\hline Cis-Whiskylactona & 0.79 & 332.42 & 12.62 & 118.80 & 0.100 & 0.025 \\
\hline Trans-Whiskyactona & 37.68 & 219.83 & 25.46 & 27.43 & 0.788 & 0.754 \\
\hline Eugenol & 9.12 & 72.24 & 14.40 & 15.33 & 0.523 & 0.462 \\
\hline L & 5.24 & 6.43 & 0.27 & 0.30 & 0.578 & 0.497 \\
\hline a & 31.54 & 34.93 & 0.77 & 0.84 & 0.573 & 0.492 \\
\hline b & 9.03 & 11.07 & 0.47 & 0.52 & 0.578 & 0.497 \\
\hline C & 32.82 & 36.64 & 0.87 & 0.95 & 0.574 & 0.492 \\
\hline H & 15.92 & 17.58 & 0.38 & 0.42 & 0.580 & 0.499 \\
\hline S & 5.70 & 6.28 & 0.13 & 0.14 & 0.577 & 0.496 \\
\hline
\end{tabular}

El cambio del envejecimiento en depósito al envejecimiento en botella de los vinos, ilustra que también existe un cambio en la correlación entre los datos de la nariz electrónica con los suministrados por el análisis cromatográfico y colorimétrico. Se ha presentado una disminución en la mayoría de parámetros que puede estar relacionada con el cambio de un medio oxidativo a un medio reductor y ello se traduce en un cambio en la composición global y en la cantidad de oxigeno disuelto en el vino. De manera que para el primer periodo de muestreo -de los vinos en botella-, los sensores de la nariz electrónica no fueron especialmente sensibles o tuvieron una respuesta más baja, que podría estar relacionado con la disminución del oxígeno disuelto en las muestras de vino [56].

Tabla 4.16. Resultados modelo PLS2 para el periodo de evaluación T3.

\begin{tabular}{|l|r|r|r|r|r|r|}
\hline \multicolumn{1}{c|}{ Parámetro } & \multicolumn{2}{c}{ Rango } & RMSEC & \multicolumn{1}{c}{ RMSECV } & $\mathrm{R}^{\wedge} 2$ & $\mathrm{R}^{\wedge} 2$ CV \\
\hline & Inferior & \multicolumn{1}{c}{ Superior } & \multicolumn{1}{c|}{} & \\
\hline Furfural & 586.60 & 1511.54 & 65.5987 & 70.0135 & 0.972 & 0.969 \\
\hline Guaiacol & 68.03 & 128.51 & 4.53855 & 4.81247 & 0.951 & 0.945 \\
\hline Cis-Whiskylactona & 2.84 & 383.87 & 29.3353 & 31.3064 & 0.943 & 0.935 \\
\hline Trans-Whiskyactona & 33.37 & 261.07 & 37.7703 & 40.0371 & 0.830 & 0.809 \\
\hline Eugenol & 15.30 & 84.12 & 9.25526 & 9.83566 & 0.839 & 0.819 \\
\hline L & 4.27 & 5.62 & 0.091396 & 0.0978295 & 0.957 & 0.951 \\
\hline A & 27.95 & 32.77 & 0.329673 & 0.352982 & 0.957 & 0.950 \\
\hline B & 7.36 & 9.69 & 0.157175 & 0.168232 & 0.957 & 0.951 \\
\hline C & 29.21 & 34.17 & 0.360123 & 0.385571 & 0.957 & 0.950 \\
\hline H & 14.75 & 16.47 & 0.10911 & 0.116695 & 0.961 & 0.956 \\
\hline S & 6.07 & 6.77 & 0.0424009 & 0.0453376 & 0.964 & 0.959 \\
\hline
\end{tabular}


Tabla 4.17. Resultados modelo PLS2 para el periodo de evaluación T4.

\begin{tabular}{|c|c|c|c|c|c|c|}
\hline \multirow[t]{2}{*}{ Parámetro } & \multicolumn{2}{|c|}{ Rango } & \multirow[t]{2}{*}{ RMSEC } & \multirow[t]{2}{*}{ RMSECV } & \multirow[t]{2}{*}{$R^{\wedge} 2$} & \multirow[t]{2}{*}{$\mathrm{R}^{\wedge} 2 \mathrm{CV}$} \\
\hline & Inferior & Superior & & & & \\
\hline Guaiacol & 38.37 & 61.66 & 3.59388 & 6.36084 & 0.852 & 0.609 \\
\hline Cis-Whiskylactona & 20.40 & 697.59 & 128.558 & 185.724 & 0.783 & 0.504 \\
\hline Trans-Whiskyactona & 31.00 & 228.7 & 15.6242 & 25.353 & 0.883 & 0.727 \\
\hline Eugenol & 33.98 & 93.20 & 14.5781 & 17.7491 & 0.623 & 0.399 \\
\hline $\mathrm{L}$ & 3.93 & 6.28 & 0.201499 & 0.463916 & 0.960 & 0.768 \\
\hline A & 26.12 & 34.10 & 0.633445 & 1.48367 & 0.966 & 0.796 \\
\hline B & 6.77 & 10.80 & 0.345472 & 0.795913 & 0.960 & 0.768 \\
\hline $\mathrm{C}$ & 26.99 & 35.78 & 0.70484 & 1.64736 & 0.965 & 0.792 \\
\hline $\mathrm{H}$ & 14.53 & 17.58 & 0.275988 & 0.628111 & 0.956 & 0.752 \\
\hline $\mathrm{S}$ & 5.70 & 6.87 & 0.1564 & 0.216566 & 0.912 & 0.812 \\
\hline
\end{tabular}

En el periodo de muestreo 5 (Tabla 4.18) es posible observar que un compuesto como el furfural no muestra una buena correlación a pesar de ser un marcador estándar del tipo de envejecimiento durante el almacenamiento en botella. Respecto a otros parámetros como las coordenadas CIELab, también se ha detectado una disminución de todos los parámetros relacionados con estas técnicas. Sin embargo, se han apreciado resultados muy importantes con otras variables como los referentes a la cis y trans-whiskylactona, que han mostrado un aumento significativo en los valores de correlación.

Tabla 4.18. Resultados modelo PLS2 para el periodo de evaluación T5.

\begin{tabular}{|l|r|r|r|r|r|r|r|}
\multicolumn{1}{c|}{ Parámetro } & \multicolumn{2}{c|}{ Rango } & \multicolumn{1}{c|}{ RMSEC } & RMSECV & $\mathrm{R}^{\wedge} 2$ & $\mathrm{R}^{\wedge} 2 \mathrm{CV}$ \\
\hline & Inferior & \multicolumn{1}{c}{ Superior } & & & & \\
\hline Furfural & 115.37 & 876.81 & 165.214 & 224.481 & 0.737 & 0.546 \\
\hline Guaiacol & 59.50 & 83.50 & 9.48733 & 12.4693 & 0.792 & 0.655 \\
\hline Cis-Whiskylactona & 38.90 & 851.15 & 58.2753 & 82.0592 & 0.950 & 0.902 \\
\hline Trans-Whiskyactona & 47.10 & 167.17 & 13.2937 & 18.7457 & 0.905 & 0.886 \\
\hline Eugenol & 58.38 & 122.53 & 6.27198 & 8.88358 & 0.941 & 0.883 \\
\hline L & 8.99 & 12.05 & 0.816746 & 1.1407 & 0.793 & 0.613 \\
\hline a & 39.46 & 44.30 & 0.938472 & 1.31102 & 0.786 & 0.601 \\
\hline b & 15.47 & 22.09 & 1.29941 & 1.81497 & 0.790 & 0.609 \\
\hline C & 42.38 & 49.50 & 1.42284 & 1.98762 & 0.788 & 0.605 \\
\hline H & 21.41 & 26.49 & 0.832183 & 1.16211 & 0.788 & 0.606 \\
\hline S & 3.83 & 4.72 & 0.124826 & 0.174224 & 0.785 & 0.600 \\
\hline
\end{tabular}

El último de los modelos (Tabla 4.19) ha ilustrado que los vinos después de más 400 días en botella presentaron mejores correlaciones entre la nariz electrónica y la cromatografía de gases que las correspondientes coordenadas CIELab. 
Tabla 4.19. Resultados modelo PLS2 para el periodo de evaluación T6.

\begin{tabular}{|c|c|c|c|c|c|c|}
\hline \multirow[t]{2}{*}{ Parámetro } & \multicolumn{2}{|c|}{ Rango } & RMSEC & RMSECV & $\mathrm{R}^{\wedge} 2$ & $\mathrm{R}^{\wedge} 2 \mathrm{CV}$ \\
\hline & Inferior & Superior & & & & \\
\hline Furfural & 73.20 & 885.12 & 90.9909 & 118.625 & 0.901 & 0.836 \\
\hline Guaiacol & 73.73 & 119.80 & 2.85114 & 3.98782 & 0.900 & 0.859 \\
\hline $\begin{array}{l}\text { Cis- } \\
\text { Whiskylactona }\end{array}$ & 76.60 & 907.50 & 30.4634 & 72.5503 & 0.979 & 0.975 \\
\hline $\begin{array}{l}\text { Trans- } \\
\text { Whiskyactona }\end{array}$ & 13.97 & 143.45 & 22.6579 & 36.4544 & 0.946 & 0.904 \\
\hline Eugenol & 43.13 & 129.73 & 5.00976 & 7.2631 & 0.951 & 0.912 \\
\hline $\mathrm{L}$ & 11.10 & 15.82 & 0.438086 & 1.01058 & 0.909 & 0.475 \\
\hline $\mathrm{A}$ & 42.48 & 47.99 & 0.547682 & 1.24719 & 0.905 & 0.424 \\
\hline B & 19.04 & 26.54 & 0.736425 & 1.69734 & 0.909 & 0.473 \\
\hline $\mathrm{C}$ & 46.55 & 54.82 & 0.796 & 1.82699 & 0.908 & 0.447 \\
\hline $\mathrm{H}$ & 24.14 & 28.99 & 0.572742 & 1.31 & 0.907 & 0.470 \\
\hline$S$ & 3.46 & 4.20 & 0.145321 & 0.260127 & 0.778 & 0.520 \\
\hline
\end{tabular}

Consideramos oportuno aclarar que esta capacidad de predicción podría ser aún más robusta y ajustada si dispusiéramos de matrices de calibración más significativas y parámetros fisicoquímicos a cuantificar que abarcaran un rango de concentraciones más amplio.

En definitiva, este experimento nos permite apreciar, no sólo selectividad cruzada de la red de sensores, sino también su sensibilidad cruzada frente a diferentes compuestos volátiles orgánicos en una muestra tan compleja como es el vino tinto.

Los resultados a partir de PCA y PLS-DA han posibilitado discriminar y clasificar las muestras siguiendo un patrón acorde con la evolución temporal del vino a lo largo de su elaboración, vislumbrándose además diferenciaciones según el tipo envejecimiento empleado en la vinificación. Además el análisis PLS2 ha permitido determinar las correlaciones existentes, entre el sistema de análisis sensorial electrónico y los sistemas de valoración analíticos tradicionales. En otras palabras, se ha podido evaluar la capacidad de la nariz electrónica para ser empleada como una herramienta para el control de calidad de vinos.

Cabe recordar en este punto que buena parte de nuestros objetivos se basan en la implementación de nuevos tratamientos de datos como una posibilidad para conseguir extraer más información de los experimentos realizados. Y por otra parte adicional se intenta cubrir la necesidad existente de evaluar en profundidad el potencial de los sistemas de análisis sensorial electrónicos para predecir características químicas y organolépticas de una muestra analizada. Para llegar a tal fin, a continuación daremos lugar a una discusión sobre las principales 
ventajas que tiene la aplicación de nuevos métodos quimiométricos en el entorno de las narices y lenguas electrónicas.

\subsubsection{Modelo Tucker 3}

A manera de resumen, hemos discutido hasta ahora los resultados de cuatro experimentos diferentes: las coordenadas CIELab, la cromatografía de gasesmasas, la cromatografía acoplada a un puerto de olfatometría y la nariz electrónica.

Salvo la GC-O todas las técnicas fueron utilizadas durante 6 periodos de evaluación a un conjunto de 12 vinos diferentes. Sólo aparecen reportados en esta tesis los resultados la técnica GC-O para el periodo del envejecimiento en botella ya que durante el periodo del envejecimiento en depósito de acero o barrica fue necesario realizar todo el proceso de entrenamiento y validación del método. Era la primera vez que este grupo de investigación se trabajaba en la búsqueda de correlaciones entre este sistema sensorial y la nariz electrónica.

La matriz de datos obtenida a partir de estas medidas está compuesta por tres dimensiones: muestras $\mathrm{x}$ parámetros $\mathrm{x}$ periodos de muestreo. Por este motivo fue necesario la aplicación de un método capaz de proporcionar un conocimiento complementario entre los resultados que traen cada una de las vías de este estudio, es decir que no se limite a el análisis en un único factor sino que además sea capaz de relacionar la información derivada por los otros factores.

Como se mencionó en el apartado 2.5.5, el modelo Tucker3 es uno de los métodos mas conocidos del análisis multi-way, y puede ser considerado una extensión del análisis bilineal del análisis de componentes principales PCA. En términos generales, uno de los objetivos de este trabajo fue aplicar una herramienta para el modelamiento de la información, proveniente de tres técnicas analíticas complementarias, con el fin de encontrar diferencias existentes entre vinos envejecidos en barricas y en sistemas alternativos. El método Tucker 3 en este caso ha permitido la interpretación conjunta de las relaciones entre variables durante toda la evolución de los vinos estudiados en esta investigación y ello se realizó a través del siguiente proceso:

\section{a. Preprocesado del conjunto de datos.}

Se evaluaron los efectos que pueden tener en el análisis final la aplicación de dos métodos de preprocesamiento diferentes: el j-escalado y el jk-escalado estableciendo una comparación entre los mismos. Para ambos casos el 
preprocesamiento tenía como finalidad eliminar las diferencias que tienen las variables debido a las escalas y unidades de medida de cada una de las técnicas utilizadas, corregir los posibles errores sistemáticos, reducir el ruido e incrementar el ajuste de los datos.

A través de cada tipo de preprocesamiento se han determinado desde dos enfoques distintos, las interacciones entre las muestras, variables y periodos de muestreo. El j-escalado removió variaciones artificiales de las variables sin eliminar diferencias entre los tiempos de muestreo, y de esta manera se pudo evaluar la evolución del vino; y el jk-escalado permitió identificar las características comunes de los vinos en todos los periodos de maduración debido tipo de envejecimiento y madera utilizada [57].

La dimensionalidad apropiada del modelo Tucker 3 para el j-scaling se determinó mediante un screen test y el criterio de la divergencia [58]. De acuerdo con los resultados que se representan de la Figura 4.22 se ha encontrando que el modelo óptimo corresponde a un factor para el modo A y dos para el modo B y C a los modos (1 2 ( 2 2). Los siguientes resultados reportan la matriz principal (proporcionales a la varianza explicada) y los conjuntos de arreglos básicos para la combinación de factores. Se ha encontrado que el modelo explica el 69,8\% de la varianza explicada.

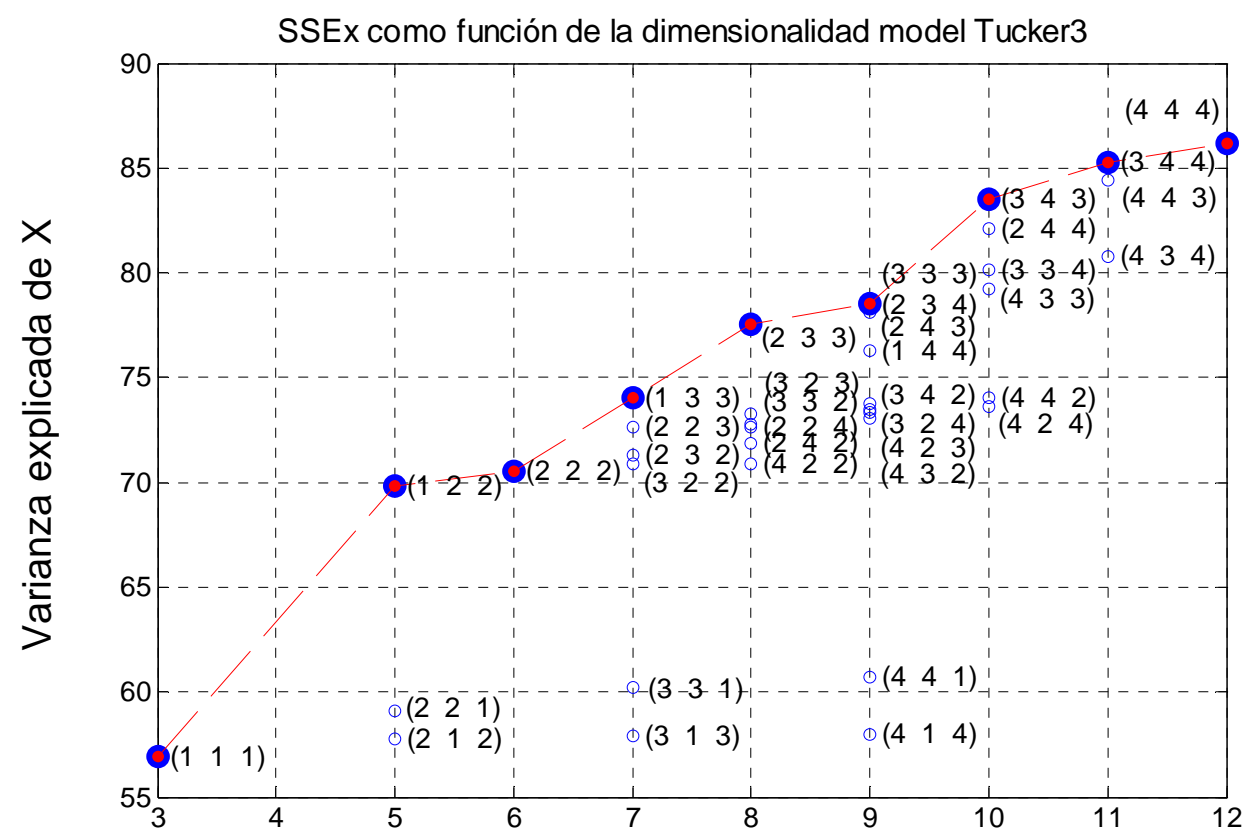

Dimensionalidad del modelo Tucker3 (numero total de componentes)

Figura 4.22. Tuckertest. Varianza explicada en función de la dimensionalidad del modelo para datos pretratados con el método j-scaling. 
En el caso de los resultados de la rutina Tucker cuando se aplica el jk-scaling y de acuerdo a los resultados que se muestran en la Figura 4.23, la selección de la dimensionalidad optima corresponde a (2 2 2). Para este modelo se obtiene un $39.7 \%$ de la varianza explicada; esta varianza explicada disminuye para el caso del jk-scaling porque el escalado entre periodos reducen la fuente de variabilidad propia de la evolución del vino.

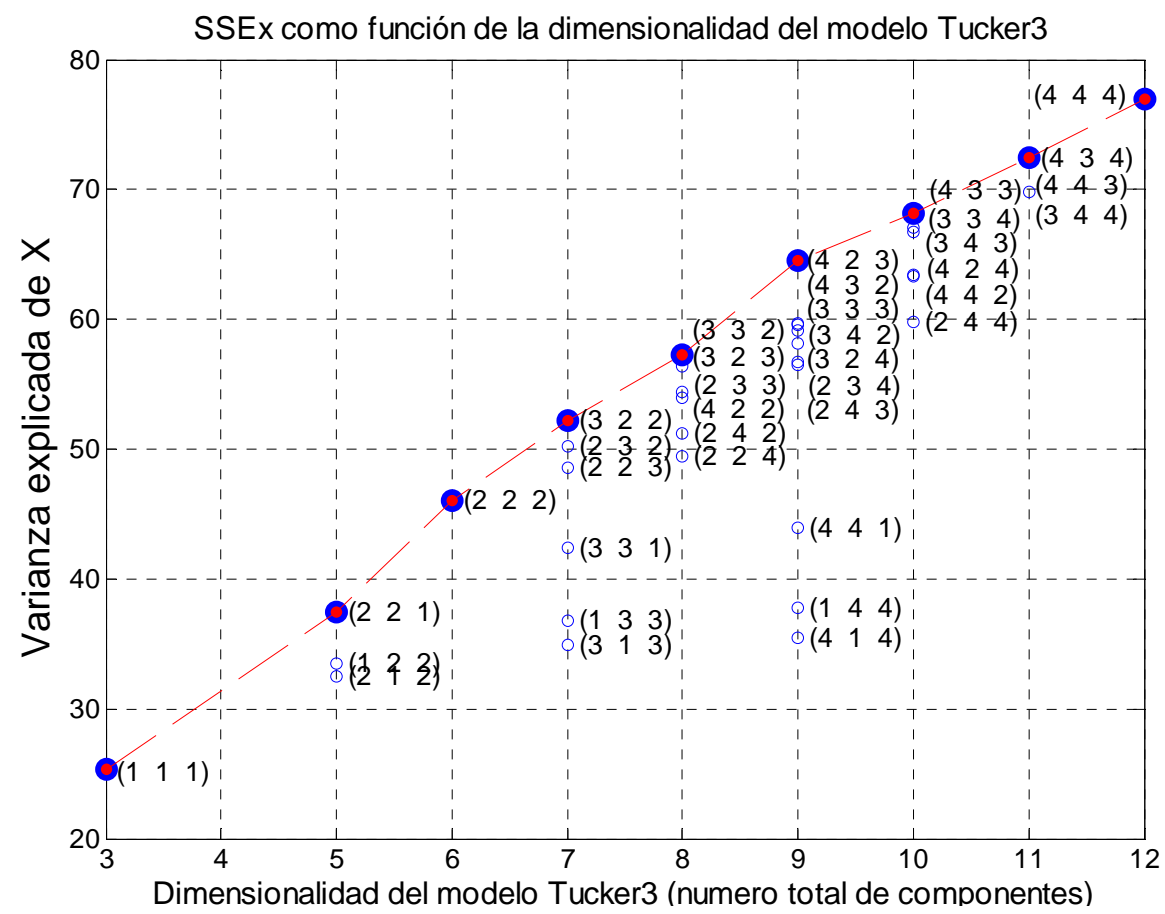

Figura 4.23. Tuckertest. Varianza explicada en función de la dimensionalidad del modelo para datos pretratados con el método jk-scaling.

$\begin{array}{lrrr}\mathrm{C}_{111} & \mathrm{C}_{121} & \mathrm{C}_{112} & \mathrm{C}_{122} \\ \mathrm{C}_{211} & \mathrm{C}_{221} & \mathrm{C}_{212} & \mathrm{C}_{222} \\ & & \text { j-escalado } & \\ 19.96 & 0.00 & 0.00 & -9.52\end{array}$

\begin{tabular}{lrrl}
\multicolumn{4}{c}{ jk-escalado } \\
11.8583 & 1.9633 & -2.9982 & 2.4275 \\
-1.9837 & 3.5446 & -2.4627 & -9.7645
\end{tabular}

\section{b. Análisis de los objetos (vinos)}

La interpretación de los resultados se fundamentó en las gráficas de los loadings combinada con la información de los elementos de la matriz principal. Los valores correspondientes a los loadings de los objetos para una sola componente 
de acuerdo con el modelo (l 12 2 $)$ seleccionado para el j-escaldo no se muestran diferencias significativas entre objetos (Figura 4.24a). Los vinos envejecidos con chips ( 1 y 2 ) tienen los loadings con valores más bajos, seguidos por los staves ( 3 y 4) y las barricas (5 y 6); las diferencias son pequeñas fundamentalmente porque la fuente de variación para este modelo depende de la evolución del vino, es decir que las diferencias principales cuando este tipo de preprocesado de datos es aplicado serán observadas en el análisis del gráfico referente a las condiciones (periodos de muestreo).

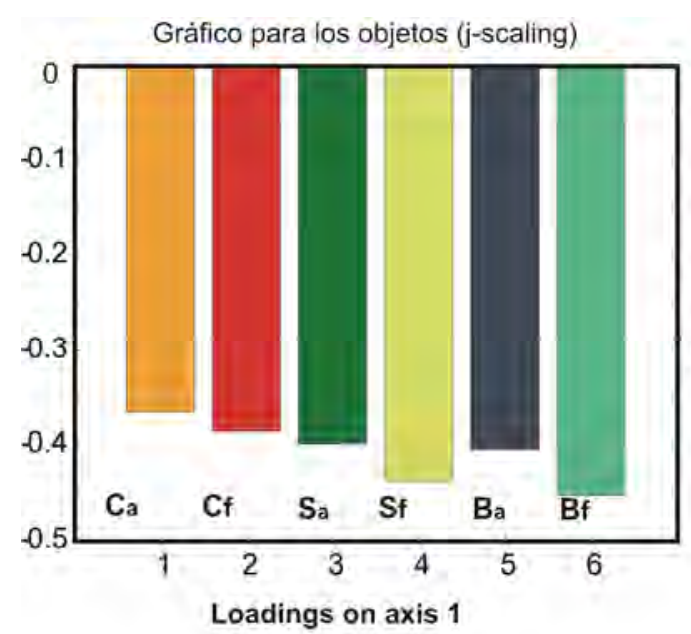

Figura 4.24a. Diagrama de barras para datos pretatados con el método j-scaling

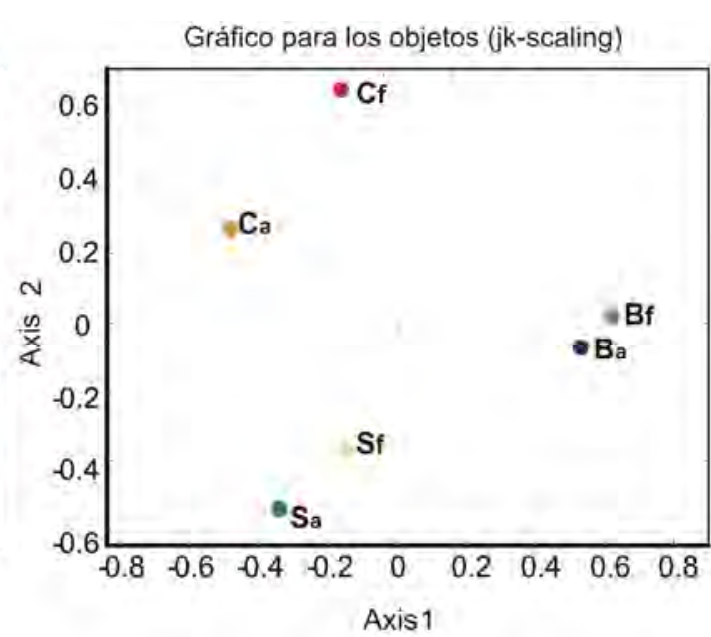

Figura 4.24b. Diagrama de los loadings para datos pretatados con el método jkscaling

En contraste con este resultado se analizaron los loadings utilizando el jk-escalado observando que para este caso su valores fueron más heterogéneos en ambos ejes, debido a las rotaciones ortogonales implican la búsqueda de información común y mayor descripción de las características de las muestras de vino para cada periodo de medida. De esta manera el jk-escalado ha generando un análisis en función de la diferencias entre tipos de envejecimiento y origen de la madera (Figura 4.24b). El eje 1 de la grafica que contiene los loadings que separan perfectamente entre tipos de envejecimiento, las muestras 1, 2, 3, y 4 tienen loadings con valores negativos y pertenecen al conjunto de muestras para las cuales se aplicó la adición de madera, mientras que las muestras 5 y 6 (barricas) tienen loadings con valor positivo. La relación entre el eje 1 y el eje 2 detecta el uso de madera de un determinado origen porque existe una pendiente en la dirección propia que hay entre dos muestras de un mismo tipo de envejecimiento. Las diferencias más claras entre roble americano y francés se observaron para los vinos envejecidos con chips y la más pequeñas para las barricas, especialmente cuando es aplicado el jk-escalado. 
Los objetos nunca llegan a superponerse, esto indica que las muestras tienen características que las hacen significativamente diferentes a nivel organoléptico. La diferencia entre loadings de los vinos envejecidos con chips y los envejecidos con barricas es más grande que la diferencia entre roble americano y roble francés para cada par de tipo de vinos. En general los vinos macerados con roble americano siempre tienen loadings más altos que el francés

\section{c. Análisis de las variables}

Tabla 4.20. Lista de variables evaluadas.

\begin{tabular}{|c|c|c|}
\hline & Variable & Código \\
\hline \multirow{6}{*}{$\begin{array}{c}\text { Espectroscopia UV- } \\
\text { Visible }\end{array}$} & Luminosidad & $\mathrm{L}^{*}$ \\
\hline & Intensidad de color rojo & $a^{*}$ \\
\hline & Intensidad de color amarillo & $\mathrm{b}^{*}$ \\
\hline & Saturación o croma & $\mathrm{C}^{*}$ \\
\hline & Tonalidad & $\mathrm{H}^{*}$ \\
\hline & Cantidad de negro u oscuridad & $S^{*}$ \\
\hline \multirow{5}{*}{$\begin{array}{c}\text { Cromatografía de } \\
\text { gases }\end{array}$} & Furfural & $\mathrm{F}$ \\
\hline & Guaiacol & G \\
\hline & Cis-whiskylactona & $\mathrm{CW}$ \\
\hline & Trans-whiskylactona & TW \\
\hline & Eugenol & $\mathrm{E}$ \\
\hline \multirow{9}{*}{ Nariz electrónica } & TGS822 & $\mathrm{S}_{1}$ \\
\hline & TGS825 & $\mathrm{S}_{2}$ \\
\hline & TGS880 & $\mathrm{S}_{3}$ \\
\hline & TGS2611 & $\mathrm{S}_{4}$ \\
\hline & TGS2620 & $\mathrm{S}_{5}$ \\
\hline & SP12a & $\mathrm{S}_{6}$ \\
\hline & SP19 & $\mathrm{S}_{7}$ \\
\hline & SP31 & $\mathrm{S}_{8}$ \\
\hline & SPMW1 & $\mathrm{S}_{9}$ \\
\hline
\end{tabular}

La nomenclatura que hemos usado para nombrar cada una de las variables se encuentra registrada en la Tabla 4.20. La Figura 4.25a muestra los loadings de las variables para el preprocesado j-escalado, la primera característica que se distingue es la amplia separación entre las variables del color y las variables relacionados con la fase volátil. Las coordenadas CIELab se encuentran agrupadas en un mismo cluster porque cada una de ella proyecta información similar dentro de todo el conjunto de variables utilizadas para la valoración de vino. Sólo la coordenada $S^{*}\left(S^{*}\right)$ tiene un valor de loading para el eje 1 opuesto a toda el conjunto y esto justifica en el hecho de que esta coordenada explica la 
saturación del color, es decir que su valor es inversamente proporcional al de la coordenada $L^{*}\left(L^{*}\right)$. El mismo comportamiento se puede observar para el preprocesado jk-escalado pero en este caso las diferencias quedan registradas en el eje 2 (Figura 4.25b).

Respecto a las loadings de las variables de la nariz electrónica (1-9) es importante destacar la correlación entre los resultados obtenidos a través de la respuesta de los diferentes sensores y la concentración de algunos compuestos químicos, ya seleccionados en el apartado 4.1.1.3, como son la whiskylactona (CW y TW) y el eugenol (E), volátiles con gran impacto en las características aromáticas del vino $[59,60]$.

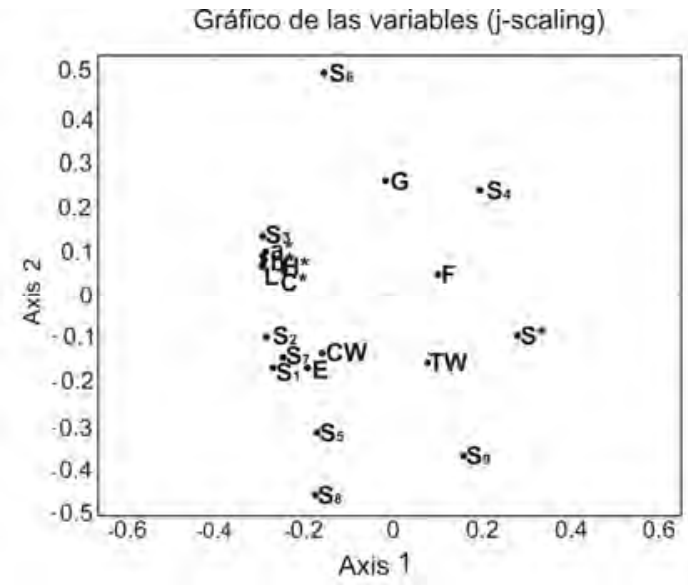

Figura 25a. Diagrama de los loadings para los datos pretratados con el método j-scaling.

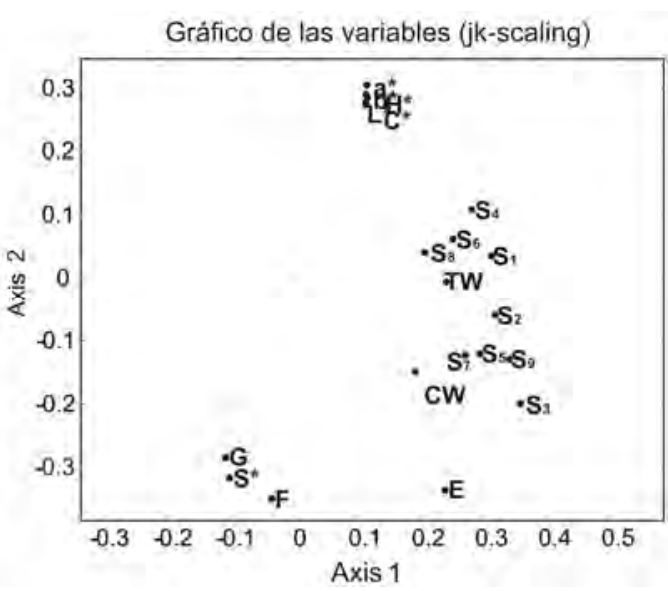

Figura 25b. Diagrama de los loadings para los datos pretatados con el método $j k$-scaling.

En contraste con estos compuestos el furfural (F) y el guaiacol (G) aparecen ubicados de manera opuesta presentado loadings positivos para ambos ejes del jescalado y mostrando que sólo dos sensores de la red podrían tener alguna correlación inversamente proporcional. Este fenómeno ya ha sido publicado en otros trabajos en los cuales se puede entender que una red de sensores MOS tiene una respuesta más baja a la inyección de compuestos como el guaiacol [61].

Además la grafica de los loadings reflejó la tendencia señalada por la cromatografía en la que habíamos visto que la whiskylactona y el eugenol presentaban patrones modelables que permitían distinguir entre tipos y orígenes de la madera, mientras que el patrón del guaiacol no permitió observar claramente esos efectos. Sin embargo el guaiacol es un compuesto que ha sido ampliamente reportado por su efecto sinérgico en la valoración aromática [48, 62]. 


\section{d. Análisis de las condiciones (periodos de muestreo).}

Los loadings registrados para las condiciones (periodos de muestreo) se encuentran registrados en la Figura 4.26a. Los índices más bajos ocurren a partir de los 330 días que corresponde a la transición entre el periodo en depósito a el periodo en botella (punto 4). Es un efecto que se debe principalmente a la variación en la concentración de oxigeno presente en cada tipo de proceso.

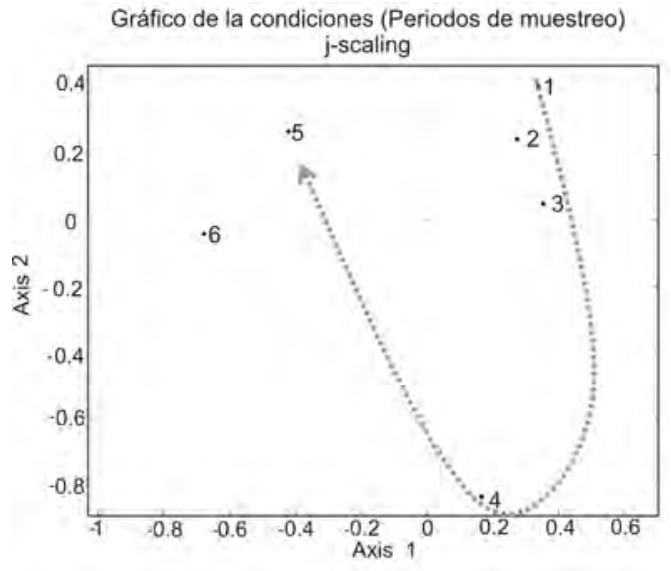

Figura 4.26a. Diagrama de los loadings para los datos pretatados con el método j-scaling.

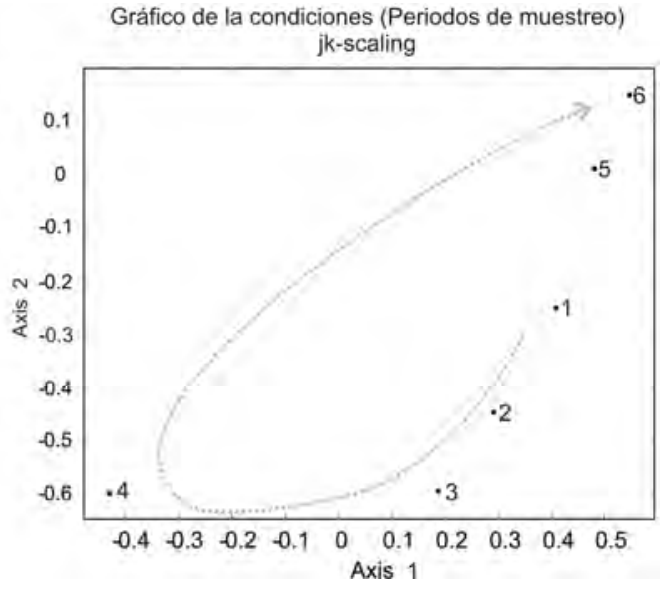

Figura 4.26b. Diagrama de los loadings para los datos pretatados con el método j-scaling.

La Figura 4.26b presenta los loadings resultantes del jk-escalado. Estos valores demuestran que las técnicas analíticas usadas detectan la transición entre las dos etapas de este estudio: depósito y botella. Las diferencias de los loadings encontradas entre periodos fueron pequeñas lo que demuestra la estabilidad del modelo. Aunque el punto 4 para los 330 días del envejecimiento presenta un valor negativo no indica que este periodo tenga mayor influencia en el análisis porque es la magnitud del loading la que se utiliza como medida de la consistencia de cada periodo dentro del modelo [63].

\section{e.. Interpretación conjunta objetos, variables y condiciones}

Hasta el momento ha sido demostrado que el modelo Tucker3 identifica a través de los loadings los distintos tipos de envejecimiento (análisis de objetivos), la relación entre las técnicas analíticas junto con la cohesión entre los resultados (análisis de variables) y la estabilidad del modelo (análisis de condiciones). 
Finalmente, para establecer un comportamiento global de las variables especialmente de aquellas que mejor definen las diferencias entre vinos se ha realizado un análisis triplot.

La Figura 4.28 muestra la superposición de los loadings de los tres factores. De acuerdo con los resultados previamente descritos es más oportuno realizar el análisis de la evolución utilizando el preprocesado de datos j-escalado y el análisis de las diferencias entre tipos de vinos utilizando el jk-escalado.

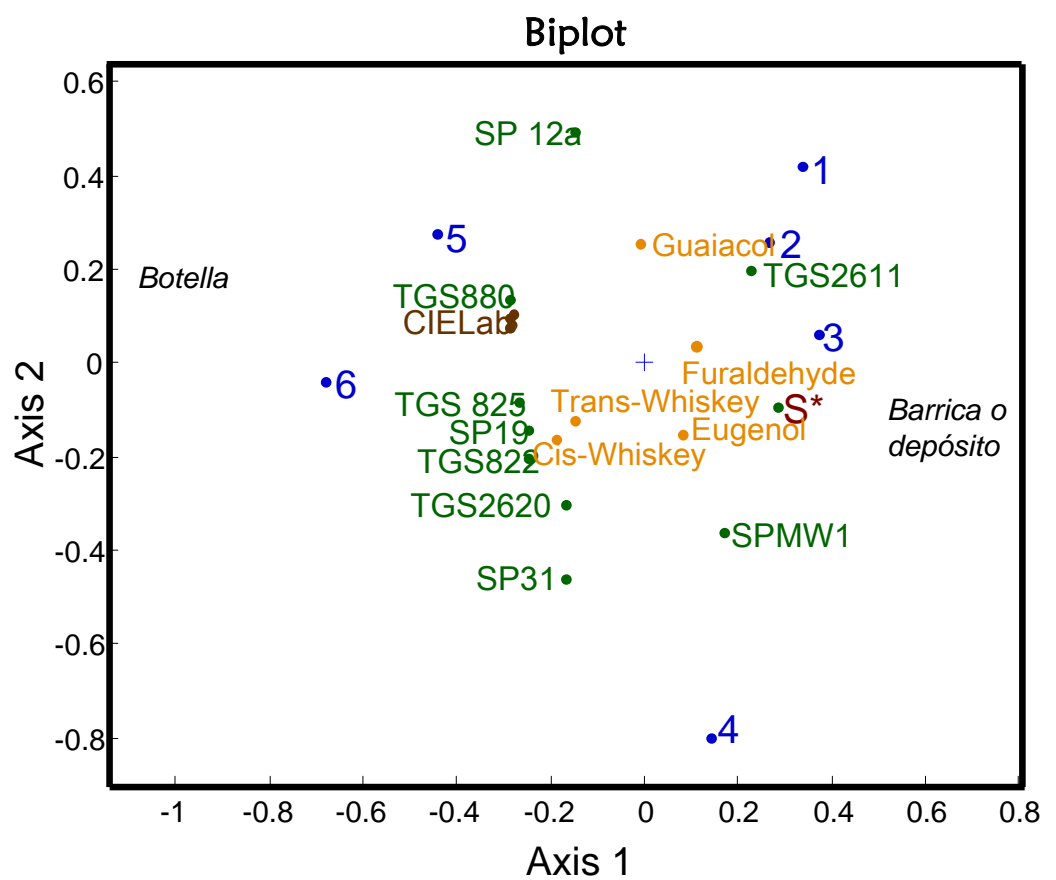

Figura 4.27. Biplot para el análisis conjunto de las variables y condiciones para el conjunto de datos pretratado con el método "j-scaling".

Los valores loadings del eje 1 del grafico 4.27 muestran una separación entre los dos procesos (deposito y botella). La primera etapa de la vinificación ubica los loadings en los ejes positivos y las etapas finales en los ejes negativos. También se pudo observar que los últimos periodos de la crianza en botella (5 y 6) están correlacionados con el cluster de las coordenadas CIELab debido a que fueron estos los parámetros analíticos, entre los seleccionados, que mejor representaron la evolución de los vinos.

En otro sentido, el eje 1 del grafico 4.28 que contiene los loadings del jk-escalado separa los vinos envejecidos en barrica ( 5,6 en el eje positivo) de los envejecidos con alternativos (1, 2, 3 y 4 en el eje negativo). Se pudo observar también que dentro de cada conjunto de vinos envejecidos con diferentes sistemas (chips, staves o barricas) es posible identificar el origen de la madera; en efecto aquellos que fueron macerados con roble americano $(1,3$ y 5$)$ tienen valores de loadings más altos que los correspondientes franceses $(2,4$ y 6$)$. Existe una relación de la anterior tendencia con las coordenadas CIELab que indica que a partir del 
periodo de evaluación número 4 se incrementan también las diferencias para los matices del color entre roble americano y francés

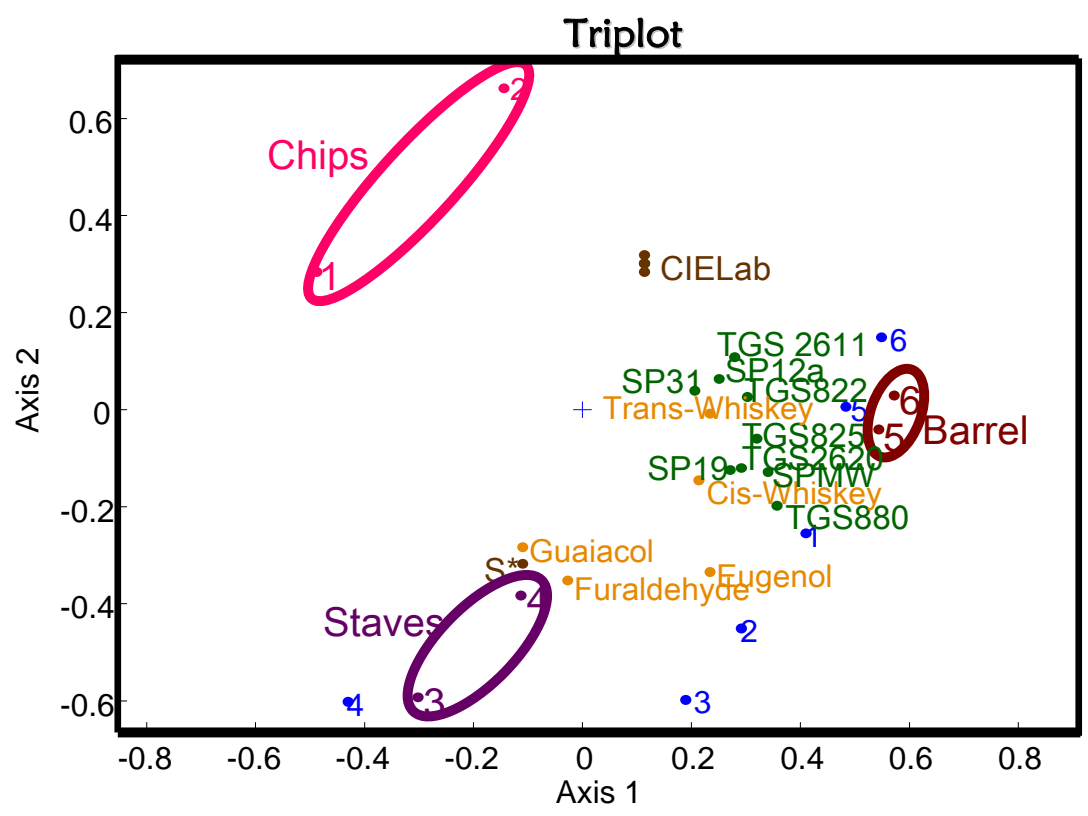

Figura 4.28. Triplot para el análisis conjunto de los objetos, variables y condiciones para el conjunto de datos pretratado con el método "jk-scaling".

Las propiedades aromáticas de los vinos envejecidos en barrica están directamente correlacionadas con las respuestas de la nariz electrónica y con compuestos como la whiskylactona y el eugenol como lo demuestra la agrupación de loadings en la parte derecha de la Figura 4.28. Los anteriores resultados indican que al final del envejecimiento los vinos en barricas pueden aparecer con mayores concentraciones de whiskylactona y eugenol. Esto puede explicarse porque al parecer la cinética de formación de compuestos es más lenta en la barrica en comparación con la que tiene lugar en vinos madurados con alternativos; lo cual también puede generar una aceleración de la degradación de compuestos para estos últimos vinos [12].

Por otra parte, Waterhouse et. al. han estimado que el cociente entre concentraciones de los isómeros de la whiskylactona permite conocer el origen de la madera [44]. Puede observarse que estos compuestos presentan la mismo patrón de separación (grado de inclinación) que aquel que tienen cada par de muestras envejecidas con un sistema igual -es decir muestras envejecidas en barrica o en depósitos con chips o staves- y tanto en madera de origen francés como en americano. La relación cis/trans es más alta para un vino madurado con madera de roble de origen americano que para uno envejecido en madera de origen francés; además en nuestro estudio se muestra que el efecto del origen del roble es más importante para los vinos madurados con inserción de chips que 
para los envejecidos en barricas, y estas diferencias se amplían con el tiempo en botella. Se puede entender este fenómeno recordando que el proceso de formación del isómero trans-whiskylacyona comienza a activarse antes y más rápido que el cis, pero también comienza a descomponerse antes, incluso cuando el compuesto cis continua con su proceso de formación. Como el proceso de absorción de compuestos fue más rápido con los chips se induce que las diferencias entre cis y trans-whiskylactona se amplíen en mayor medida al final del envejecimiento.

Finalmente los vinos macerados con técnicas alternativas utilizando staves presentaron menor composición volátil de whiskylactona y mayor de furfural, quizás porque el proceso tiene mayor variabilidad debido a la menor área específica de la madera en contacto con el vino.

\subsubsection{ANOVA-PLS}

ANOVA-PLSR se llevó a cabo para establecer la influencia de cada variable en la diferenciación de tipos de vinos, utilizando las ventajas del método ANOVA. Con la información procedente de este análisis (ANOVA) se realizó un modelo de regresión lineal PLS con el fin de evaluar la capacidad de predicción de los diferentes sistemas de evaluación. En consecuencia, demostramos que es posible valorar las correspondientes relaciones que existen entre la nariz electrónica, la GC-MS, GC-O y las coordenadas CIELab. Este tipo de tratamientos abren una posibilidad en la predicción de información con la nariz electrónica, especialmente con aquella que tiene un grado de complejidad mayor, como lo es la designación de características olfativas de las muestras evaluadas.

En primer lugar nos interesa conocer el nivel de significancia de las variables procedentes de los tres métodos (la nariz electrónica, GC-MS y GC-FIC-O) para distinguir entre un tipo de crianza con métodos tradicionales y una con alternativos, el origen de madera (francés o americano) y el seguimiento a la evolución del vino (determinar el periodo de envejecimiento). En la Tabla 4.21 se enumeran los efectos de cada variable en la discriminación de acuerdo con el sistema de crianza del vino mediante un ANOVA. Las tres técnicas parecen contribuir significativamente a la diferenciación entre las distintas características de los vinos ( $\mathrm{p}<0,005)$. El método ANOVA se aplicó a las variables derivadas de la evaluación de cinco compuestos cuantificados por cromatografía, seis atributos de aroma, seis coordenadas CIELab y la respuesta de los nueve sensores de gases que componen la nariz electrónica para los dos tipos de envejecimiento (factor 1), los dos diferentes orígenes de la madera (factor 2) y los dos periodos de envejecimiento (factor 3 ) que construyen el diseño factorial. 
Tabla 4.21. Resultados ANOVA

\begin{tabular}{|c|c|c|c|}
\hline & $\begin{array}{c}\text { Tipo de } \\
\text { envejecimiento }\end{array}$ & $\begin{array}{c}\text { Origen de la } \\
\text { madera }\end{array}$ & $\begin{array}{c}\text { Periodo del } \\
\text { envejecimiento }\end{array}$ \\
\hline TGS 822 & $* * *$ & $* *$ & NS \\
\hline TGS 825 & $* * *$ & * & NS \\
\hline TGS 880 & $* * *$ & NS & NS \\
\hline TGS 2611 & $* * *$ & NS & $* * *$ \\
\hline TGS 2620 & $* * *$ & $* *$ & $* * *$ \\
\hline $\mathrm{SP} 12^{\mathrm{a}}$ & $* * *$ & * & * \\
\hline SP 19 & $* * *$ & NS & NS \\
\hline SP31 & $* * *$ & $* * *$ & * \\
\hline SPMW1 & ** & * & ** \\
\hline Furfufal & NS & NS & $* * *$ \\
\hline Guaiacol & $* *$ & NS & $* *$ \\
\hline Cis-Whiskylactona & $* * *$ & $* * *$ & $* * *$ \\
\hline Trans-Whyskylactona & $* * *$ & $* * *$ & *** \\
\hline Eugenol & $* * *$ & * & $* * *$ \\
\hline $\mathrm{L}$ & $* * *$ & NS & NS \\
\hline A & $* * *$ & NS & NS \\
\hline $\mathrm{B}$ & $* * *$ & NS & NS \\
\hline $\mathrm{C}$ & $* * *$ & NS & NS \\
\hline $\mathrm{H}$ & $* * *$ & NS & NS \\
\hline$S$ & $* * *$ & NS & * \\
\hline Rosas & NS & $* *$ & * \\
\hline Floral & NS & NS & $*$ \\
\hline Frutal & $* * *$ & ** & NS \\
\hline Coco-Vainilla & $* * *$ & NS & $* *$ \\
\hline Caramelo-Vainilla & NS & $* *$ & NS \\
\hline Champiñon & NS & $* *$ & $* * *$ \\
\hline
\end{tabular}

De acuerdo con la tabla 4.21, la mayoría de las variables son capaces de identificar las principales diferencias entre los dos tipos de envejecimiento del vino (barricas o depósitos de acero inoxidables con inserción de chips) salvo compuestos analizados por medio de la GC-MS como el furfural y aquellos identificados por la olfatometría, como son el olor a rosas, floral, caramelovainilla y champiñón.

Las variables que tienen menor nivel de significancia son aquellas estudiadas por medio de la olfatometría. Esta situación pudo haberse presentado porque estas medidas tienen mayor incertidumbre analítica, lo cual se traduce en un incremento del error residual. 
El grafico bi-plot (Figura 4.29) establece las correlaciones existentes entre los sistemas para distinguir entre los tipos vinos analizados, todo esto en función de los factores que se han establecidos en este estudio. De este gráfico es posible observar que la mayoría de los sensores que constituyen la nariz electrónica tienen un papel importante en la diferenciación del tipo de envejecimiento que fue aplicado a los vinos (depósito o barrica). Otra característica interesante de este análisis es la relación existente en la identificación del origen de la madera con la cis-whiskylactona y el olor a caramelo-vainilla.

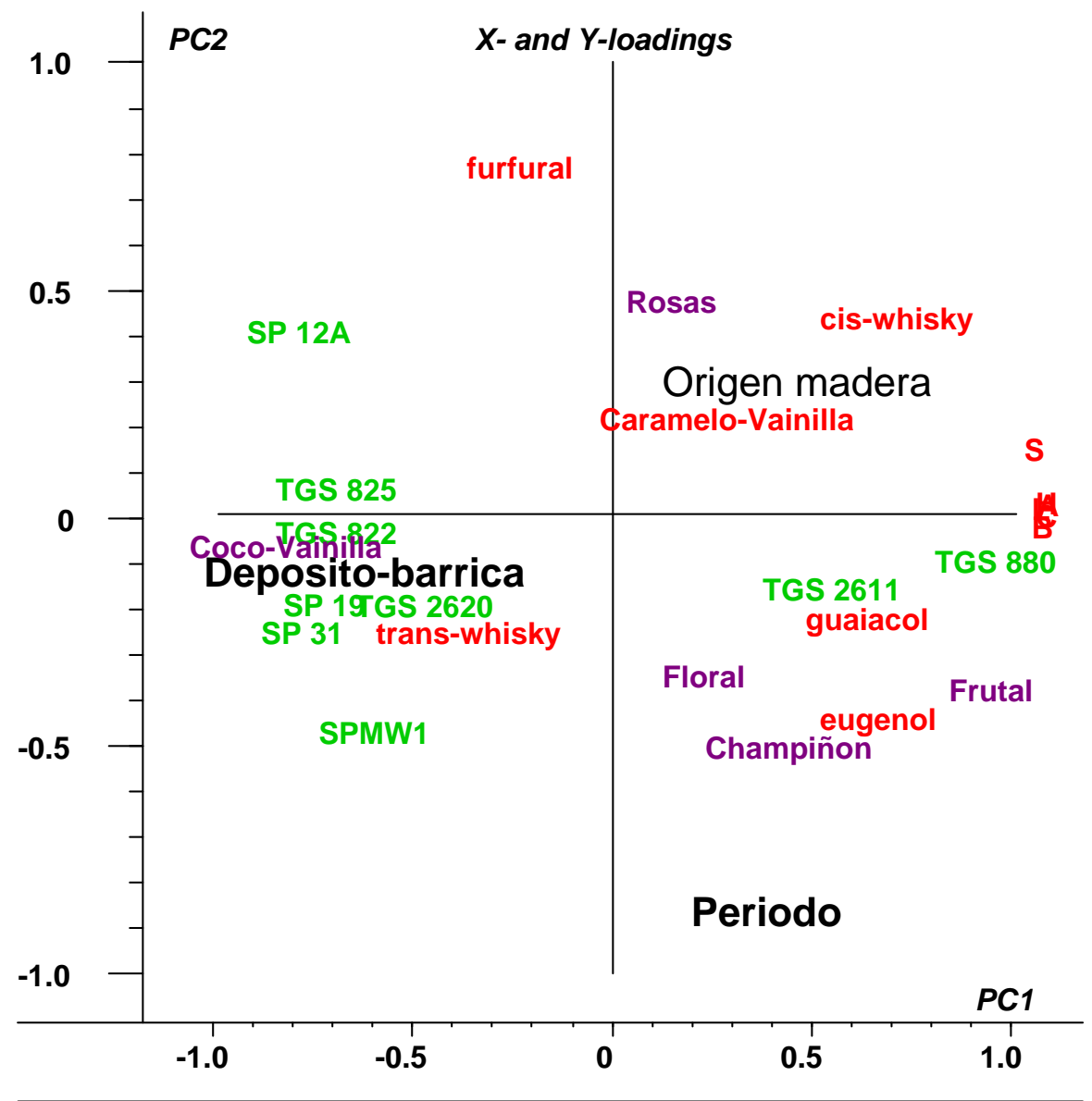

X-expl: 33\%,33\% Y-expl: 70\%,11\%

Figura 4.29. Gráfico Bi-plot derivado del ANOVA-PLS.

Ahora bien, existe un análisis aún más motivante, cuando se comparan los resultados de este grafico biplot con los derivados del modelo Tucker3 para el caso específico del jk-scaling. Se puede corroborar que la respuesta que entregan los sensores TGS 822, SP 19, TGS 2620 y SP 31 tienen un grado de correlación importante con la trans-whiskylactona. En el caso del grafico biplot del ANOVAPLS, la trans-whiskylactona se puede correlacionar también con el olor a cocovainilla que fue determinado por medio de la GC-O, que es justamente el aroma que caracteriza este compuesto [6]. 
Tabla 4.22. Resultados del ANOVA-PLS

\begin{tabular}{|c|c|c|c|c|c|c|}
\hline & Offset & Cal & RMSE & RMSEc & $R^{\wedge} 2$ & $\mathrm{R}^{\wedge} 2 \mathrm{cal}$ \\
\hline TGS 822 & $-6.9 \times 10^{-9}$ & $-8.2 \times 10^{-10}$ & 0.039 & 0.047 & 0.930 & 0.908 \\
\hline TGS 825 & $-2.5 \times 10^{-10}$ & $-1.1 \times 10^{-09}$ & 0.046 & 0.055 & 0.903 & 0.873 \\
\hline TGS 880 & $-6.9 \times 10^{-10}$ & $-6.7 \times 10^{-10}$ & 0.010 & 0.012 & 0.930 & 0.908 \\
\hline TGS 2611 & $-5.3 \times 10^{-9}$ & $-9.9 \times 10^{-9}$ & 0.035 & 0.042 & 0.897 & 0.864 \\
\hline TGS 2620 & $-8.7 \times 10^{-9}$ & $-6.7 \times 10^{-9}$ & 0.047 & 0.056 & 0.933 & 0.911 \\
\hline $\mathrm{SP} 12^{\mathrm{a}}$ & $-1.6 \times 10^{-9}$ & $-1.9 \times 10^{-9}$ & 0.012 & 0.014 & 0.868 & 0.826 \\
\hline SP 19 & $-6.2 \times 10^{-9}$ & $-7.3 \times 10^{-9}$ & 0.041 & 0.049 & 0.899 & 0.867 \\
\hline SP31 & $-1.9 \times 10^{-9}$ & $-2.1 \times 10^{-9}$ & 0.051 & 0.060 & 0.933 & 0.911 \\
\hline SPMW1 & $-2.0 \times 10^{-9}$ & $-5.9 \times 10^{-9}$ & 0.050 & 0.058 & 0.915 & 0.889 \\
\hline Furfufal & $-1.9 \times 10^{-8}$ & $-2.2 \times 10^{-8}$ & 0.200 & 0.237 & 0.744 & 0.673 \\
\hline Guaiacol & $-1.0 \times 10^{-8}$ & $-2.7 \times 10^{-9}$ & 0.100 & 0.120 & 0.944 & 0.927 \\
\hline $\begin{array}{l}\text { Cis- } \\
\text { Whiskylactona }\end{array}$ & $-3.5 \times 10^{-8}$ & $-5.1 \times 10^{-8}$ & 0.121 & 0.145 & 0.874 & 0.833 \\
\hline $\begin{array}{l}\text { Trans- } \\
\text { Whyskylactona }\end{array}$ & $-2.2 \times 10^{-9}$ & $-1.5 \times 10^{-9}$ & 0.053 & 0.064 & 0.956 & 0.942 \\
\hline Eugenol & $-1.75 \times 10^{-9}$ & $-1.8 \times 10^{-9}$ & 0.038 & 0.045 & 0.940 & 0.923 \\
\hline $\mathrm{L}$ & $-5.6 \times 10^{-10}$ & $-6.8 \times 10^{-11}$ & 0.028 & 0.033 & 0.901 & 0.878 \\
\hline A & $-4.5 \times 10^{-9}$ & $-3.7 \times 10^{-9}$ & 0.049 & 0.058 & 0.924 & 0.900 \\
\hline $\mathrm{B}$ & $-2.8 \times 10^{-9}$ & $-8.7 \times 10^{-10}$ & 0.036 & 0.043 & 0.900 & 0.870 \\
\hline C & $9.0 \times 10^{-10}$ & $-2.0 \times 10^{-9}$ & 0.054 & 0.065 & 0.917 & 0.890 \\
\hline $\mathrm{H}$ & $6.8 \times 10^{-10}$ & $-1.2 \times 10^{-9}$ & 0.036 & 0.044 & 0.918 & 0.891 \\
\hline $\mathrm{S}$ & $-4.4 \times 10^{-11}$ & $4.1 \times 10^{-10}$ & 0.020 & 0.022 & 0.936 & 0.915 \\
\hline Rosas & $-3.2 \times 10^{-8}$ & $-2.6 \times 10^{-8}$ & 0.583 & 0.700 & 0.400 & 0.207 \\
\hline Floral & $-7.9 \times 10^{-8}$ & $-6.5 \times 10^{-8}$ & 0.630 & 0.756 & 0.297 & 0.070 \\
\hline Frutal & $-2.8 \times 10^{-8}$ & $5.4 \times 10^{-8}$ & 0.511 & 0.613 & 0.546 & 0.399 \\
\hline Coco-vainilla & $-2.8 \times 10^{-8}$ & $-2.8 \times 10^{-8}$ & 0.390 & 0.469 & 0.540 & 0.389 \\
\hline $\begin{array}{l}\text { Caramelo- } \\
\text { Vainilla }\end{array}$ & $3.1 \times 10^{-11}$ & $-1.7 \times 10^{-10}$ & 0.650 & 0.779 & 0.255 & 0.015 \\
\hline Champiñon & 0.000 & $-1.2 \times 10^{-9}$ & 0.395 & 0.474 & 0.701 & 0.605 \\
\hline
\end{tabular}

En esta misma dirección podemos agregar que la comparación respecto al jscaling, señala que existe una correlación importante entre las coordenadas CIELab y el sensor TGS 880. Este hecho podría estar indicando que tanto las coordenadas CIELab como el sensor describen determinada característica de los vinos que no hemos incluido en esta investigación.

Respecto a los resultados del PLS registrados en la Tabla 4.22., decir que mediante estos valores es posible identificar las variables que más contribuyen a la diferenciación de las muestras en función de los tres factores determinados para el ANOVA. Estos resultados deberían ser considerados más bien como una información adicional, y no como un dato del cual sea propicio extraer conclusiones. Si se tiene en cuenta la naturaleza de nuestro problema sería hilar muy fino al afirmar que con este único análisis, es posible clasificar una muestra de vino en función de los tres factores aquí considerados. Es por ello, que la mayor parte de información de este estudio debería ser considerada desde el 
análisis del gráfico bi-plot y servirse de los valores de las correlaciones del PLS como un aporte para confirmar los resultados visuales.

\subsubsection{Tratamientos de datos complementarios.}

Implementación de un algoritmo genético acoplado al método de la regresión lineal por mínimos cuadrados (GA-PLS) para reducir el número de variables derivadas de la lengua electrónica.

Los resultados que presentaremos a continuación forman parte de un estudio complementario dentro de nuestro objetivo de aplicar nuevos métodos quimiométricos a los datos derivados de una red de sensores. En este caso ha sido aplicado un método para reducir el alto número de variables que hacen parte de las señales electroquímicas provenientes de la lengua electrónica, facilitar el modelamiento de los datos que arroja el sistema y mejorar su capacidad de clasificación.

El nuevo subconjunto de variables ha sido seleccionado mediante el método de algoritmos genéticos con regresiones lineales basadas en mínimos cuadrados parciales (GA-PLS). Este tipo de técnicas, como se ha mencionado anteriormente permite la búsqueda de variables óptimas a lo largo de toda la señal. Sin embargo, en comparación con los modelos clásicos, puede perder la eficiencia necesaria para evaluar en detalle un pico en concreto. El desarrollo de este trabajo supone un avance en la aplicación de los GA-PLS en señales electroquímicas, ya que ha sido diseñado un modelo que combina el método de búsqueda de soluciones aleatorias con el método secuencial, por medio de la función fitness de los GAPLS, para poder evitar que cualquier tipo de solución quede atrapada en una única región del voltamograma.

Para el desarrollo del GA-PLS ha sido necesario dividir en intervalos de variables la señal electroquímica; cada intervalo fue tomado como un único punto de entrada en el GA-PLS, el cual además venía acompañado del punto posicionado a su izquierda y a su derecha. Esta condición pretendía la evaluación de una región completa, al igual que la selección de un conjunto de variables que tuviera interés desde el punto de vista electroquímico.

La Figura 4.30 ilustra las respuestas que se obtuvieron cuando los sensores electroquímicos fueron sumergidos en un vino tinto. En la figura se puede observar que los electrodos basados en ftalocianinas muestran picos característicos correspondientes a la oxidación y la reducción de la ftalocianina a los potenciales $+0.6 \mathrm{~V}$ y $-0.3 \mathrm{~V}$ respectivamente. Un tercer pico puede ser observado para el potencial de $+1.0 \mathrm{~V}$, el cual de acuerdo con los resultados 
publicados se relaciona con la oxidación de compuestos polifenolicos presentes en los vinos [64]. Este tercer pico puede observarse claramente en el caso de los sensores basados en ftalocianinas de lutecio $\left(\mathrm{LuPc}_{2}\right)$ y cobalto $(\mathrm{CoPc})$. En el caso de la respuesta del sensor basado en Gadolinio $\left(\mathrm{GdP}_{2}\right)$, este pico está superpuesto con el pico correspondiente la oxidación del anillo de la ftalocianina. También se pudieron observar otras características de las señales como la influencia que tiene el átomo central coordinado del anillo de las ftalocianinas en los voltamogramas, recordando que la intensidad de los picos de reducción puede descender en el orden de la serie de los elementos de las tierras raras $(\mathrm{Lu}>\mathrm{Gd})$, lo que también permite confirmar el papel del material sensible en el comportamiento redox del sistema [65].

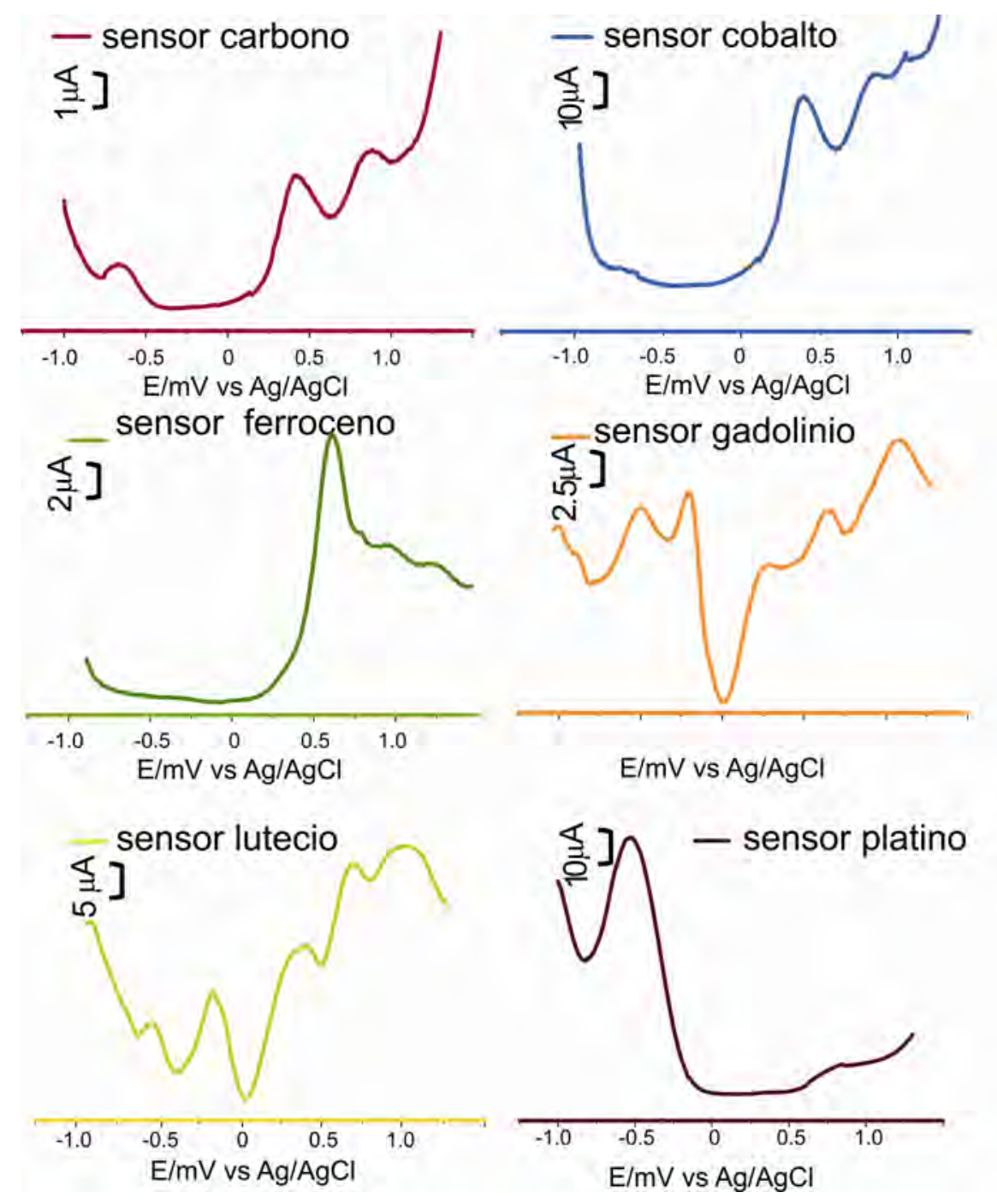

Figura 4.30. Señales características de la lengua electrónica. 
Para estudiar la estabilidad de los electrodos después de cada día trabajo, los sensores fueron expuestos a una solución electrolítica de $0.1 \mathrm{molL}^{-1} \mathrm{KCL}$. Luego, los coeficientes de variación de los picos fueron calculados obteniendo valores entre el $7 \%$ y $13 \%$ después de tres días de trabajo. Para evitar que estos porcentajes tengan un efecto en el posterior tratamiento de datos se ha aplicado el preprocesamiento de la varianza estándar SNV. Además se realizó un autoescalado por columnas para evitar las diferencias generadas a causa de la intensidad relativa entre las señales de los sensores.

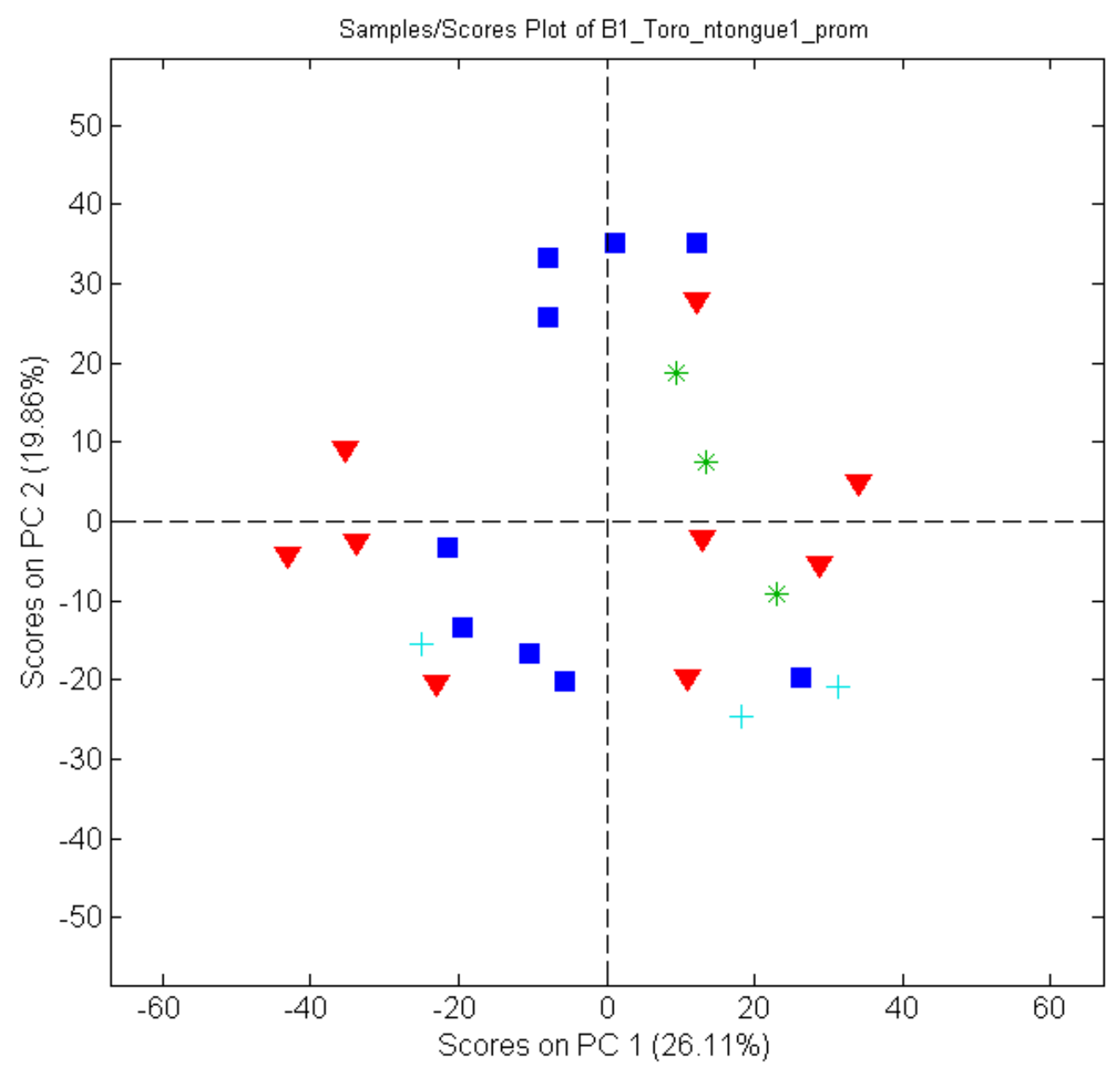

Figura 4.31. Gráfico de los scores para los vinos de la D.O. Toro. Los vinos marcados con el asterisco verde (T1) y el signo positivo azul (T2) pertenecen al conjunto de muestras envejecidas en barricas. Las muestras señaladas con el triangulo rojo (T1) y cuadrado azules (T2) corresponden a los vinos envejecidos con métodos alternativos.

Hasta el momento se ha demostrado que cada electrodo que constituye la lengua electrónica proporciona una repuesta particular para cada tipo de vino y estas respuestas sirven como punto de partida de una determinada caracterización analítica. Ahora bien, con el objeto de estudiar la capacidad de discriminación de 
esta red de sensores se han evaluado las muestras problemas bajo un total de siete réplicas por vino. A continuación, mediante los scores obtenidos con un PCA es posible visualizar parte de la información registrada por las 1980 variables (330 valores de intensidad por cada sensor registra) para los vinos analizados de las denominaciones de origen de Toro y Ribera de Duero (Figuras 4.31 y 4.32 respectivamente).

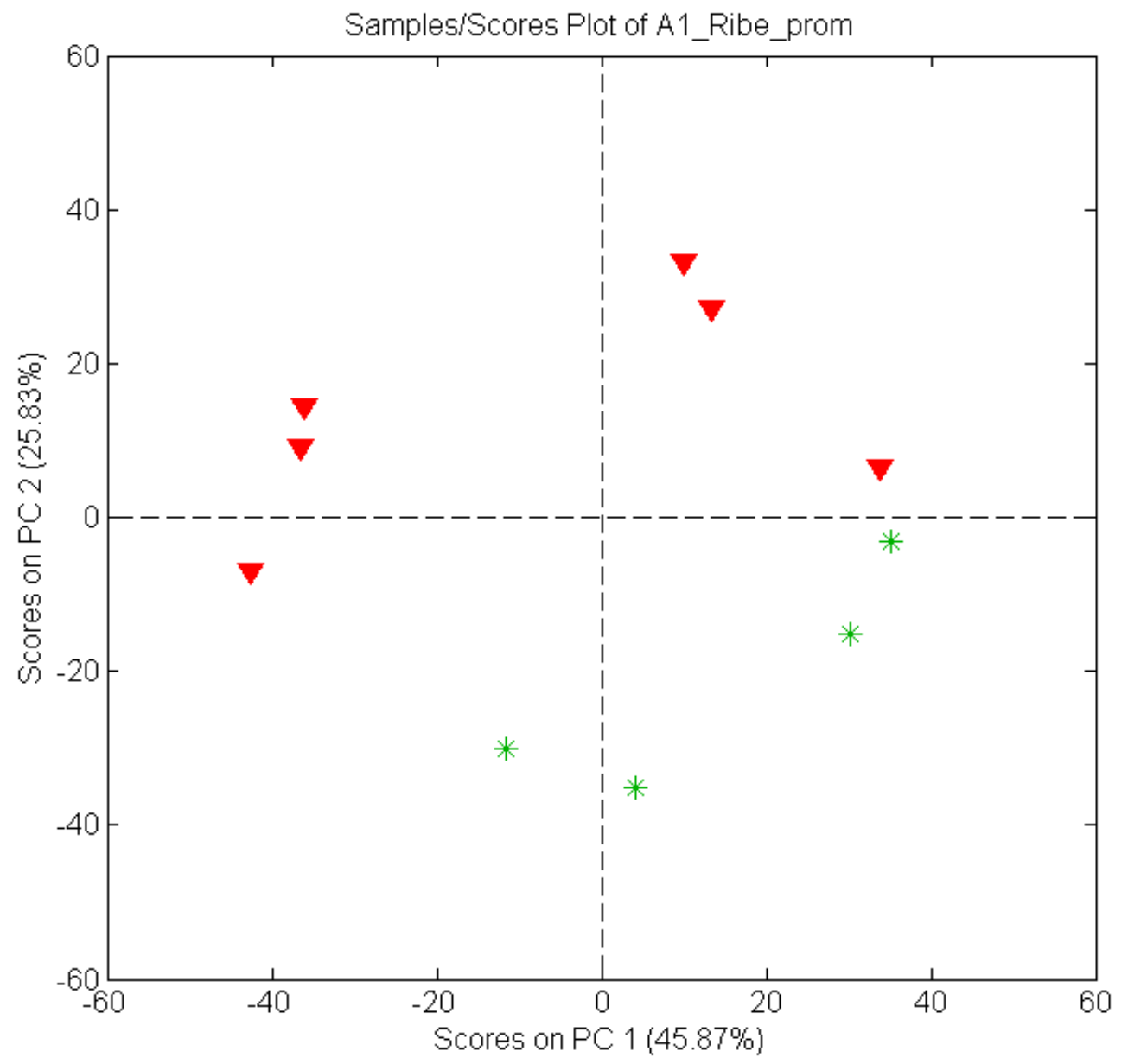

Figura 4.32. Gráfico de los scores para los vinos de la D.O. Ribera del Duero. Los vinos marcados con el asterisco verde pertenecen al conjunto de muestras envejecidas en barricas y las muestras señaladas con el triangulo rojo corresponden a los vinos envejecidos con métodos alternativos.

Respecto a los scores a los que hace referencia la Figura 4.31 con los resultados de las muestras evaluadas de la D.O. Toro durante el primer y tercer mes de envejecimiento, podemos decir que existe un solapamiento de los dos formatos de envejecimiento utilizados para este estudio. En la visualización de los datos para las dos primeras componentes principales que acumularon el $46 \%$ de la información, puede advertirse que los clusters que contiene los vinos envejecidos en barricas que están marcados con los asteriscos de color verde no se distinguen 
claramente de aquellos señalados con los triángulos rojos correspondientes a las muestras envejecidas en depósitos de acero inoxidable macerados con madera de roble, es decir que utilizando el conjunto de variables completo no es posible observar la discriminación en las dos primeras componentes principales, lo que no significa que dentro del restante porcentaje de información que arroja la matriz de datos no sea posible identificar las diferencias entre tipos de envejecimiento. El mismo fenómeno ocurre para las muestras evaluadas dos meses después, es decir no existe una buena diferenciación entre tipo de envejecimiento.

En contraste con los anteriores resultados puede observarse en la Figura 4.32, en la grafica de los scores para los vinos de la D.O. Ribera de Duero, donde a simple vista puede comprobarse la tendencia a discriminar entre los dos tipos de envejecimiento. Al igual que en el caso anterior el cluster que contiene las muestras envejecidas en barrica de roble corresponde a los puntos señalados con los asteriscos de color verde, los cuales se encuentran bien separados con respecto a los vinos madurados en depósitos de acero inoxidable (triángulos rojos) en función de la componente principal 2.

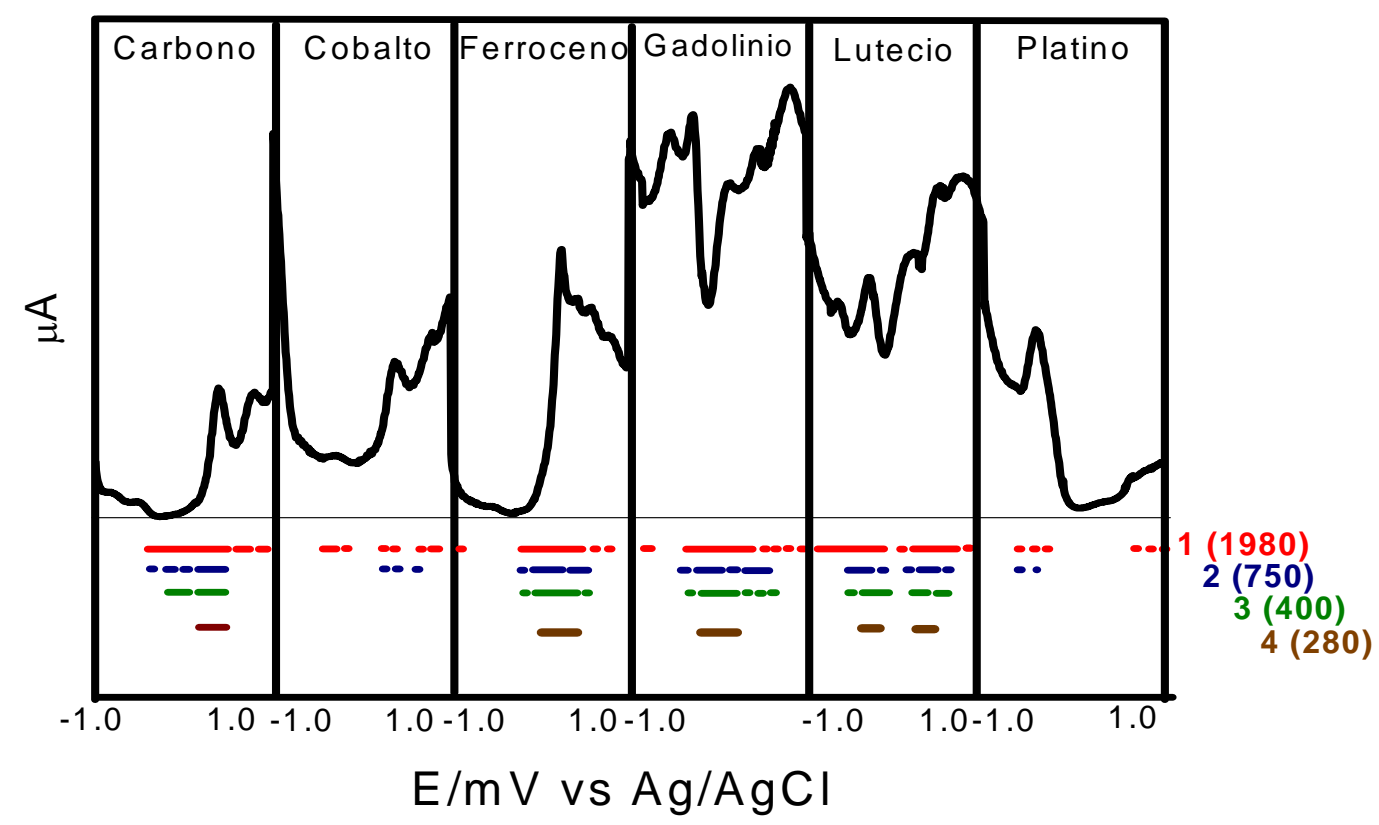

Figura 4.33. Esquema general de las diferentes etapas de la selección de variables realizadas con el método PLS-GA. Las líneas debajo de los voltamogramas indican las regiones que fueron seleccionadas cada vez que se aplicó el algoritmo.

Los resultados expuestos hasta ahora resaltaban que era posible extraer más información adicional que permitiera reflejar las características de las muestras analizadas. Por ello el siguiente paso de esta investigación fue el desarrollo de la reducción de variables a través de un GA-PLS. Como se mencionó anteriormente 
el número de variables es más grande que el número de muestras, motivo por el cual se ha desarrollado un método que utiliza el promedio de un intervalo de variables como punto de entrada, y en el cual la reducción de variables se ha efectuado en varias etapas. La figura 4.33 describe cada una de las etapas en las que el algoritmo genético buscó las zonas del voltamograma que aportaban la información que permitía una mejor clasificación de las muestras, de acuerdo al tipo de envejecimiento. Las líneas quebradas de las parte inferior de la figura indican las regiones con mayor impacto dentro de cada uno de los modelos realizados.

Etapa 1. Se utilizaron todos los datos obtenidos durante el desarrollo experimental, es decir un conjunto total de 1980 variables que registraron las diferencias existentes para 238 medidas (34 muestras con 7 replicas por cada una de las muestras). Este conjunto de muestras estaba dividido en las clases: barricas y alternativos; el algoritmo genético debía por tanto encontrar las zonas del voltamograma donde se podían identificar las diferencias ocasionadas por los distintos tipos de envejecimientos en los vinos tintos (Figura 4.34).

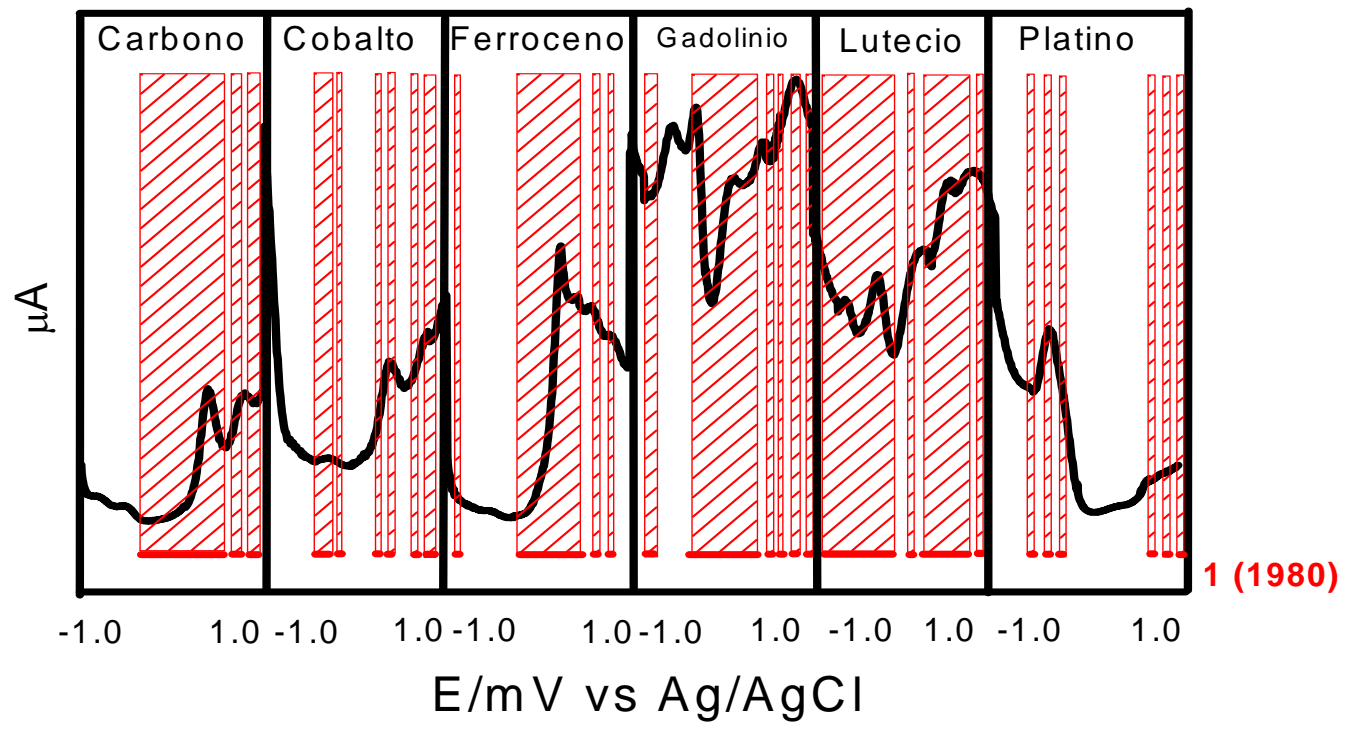

Figura 4.34. Etapa 1 del algoritmo genético.

Este primer análisis nos permitió concluir que los sensores basados en cobalto (CoPc) y platino aportan poca información para la clasificación de este conjunto de vinos de acuerdo al tipo de envejecimiento. También se pudo observar que la región alrededor del potencial de $+1.0 \mathrm{~V}$ que anteriormente había sido asociada al comportamiento electroquímico de los vinos, es justamente la región que los algoritmos genéticos han señalado como una zona importante para obtener la clasificación de acuerdo al tipo de envejecimiento de los vinos. 
Etapa 2. La región comprendida entre $+0.25 \mathrm{~V}$ y $+1.1 \mathrm{~V}$ fue utilizada para el desarrollo de un nuevo análisis de la red, con los electrodos basados en carbono, y aquellos modificados con $\mathrm{CoPc}_{2}, \mathrm{GdPc}_{2}$ y $\mathrm{LuPc}_{2}$, Respecto al electrodo de platino fue estudiado el rango comprendido entre $-0.8 \mathrm{~V}$ y $0 \mathrm{~V}$ y en cuanto al electrodo modificado con ferroceno, fue tomado un intervalo desde $+0.15 \mathrm{~V}$ hasta $+1.0 \mathrm{~V}$. Con el nuevo subconjunto de 750 datos se procedió a realizar el segundo modelo GA-PLS utilizando los mismos parámetros que fueron escogidos para el modelo anterior pero esta vez se redujo el tamaño de la ventana de datos; en esta serie de experiencias se estableció un intervalo de 5 variables para no suavizar demasiado la señal electroquímica. Los resultados de este segundo análisis confirman que los electrodos basados en $\mathrm{CoPc}_{2}$ y platino aportan poca información para la discriminación entre tipos de envejecimientos, y también se ha demostrado que el conjunto de variables se puede reducir aún más, especificando la importancia que tiene los picos centrales en esta clasificación (Figura 4.35.).

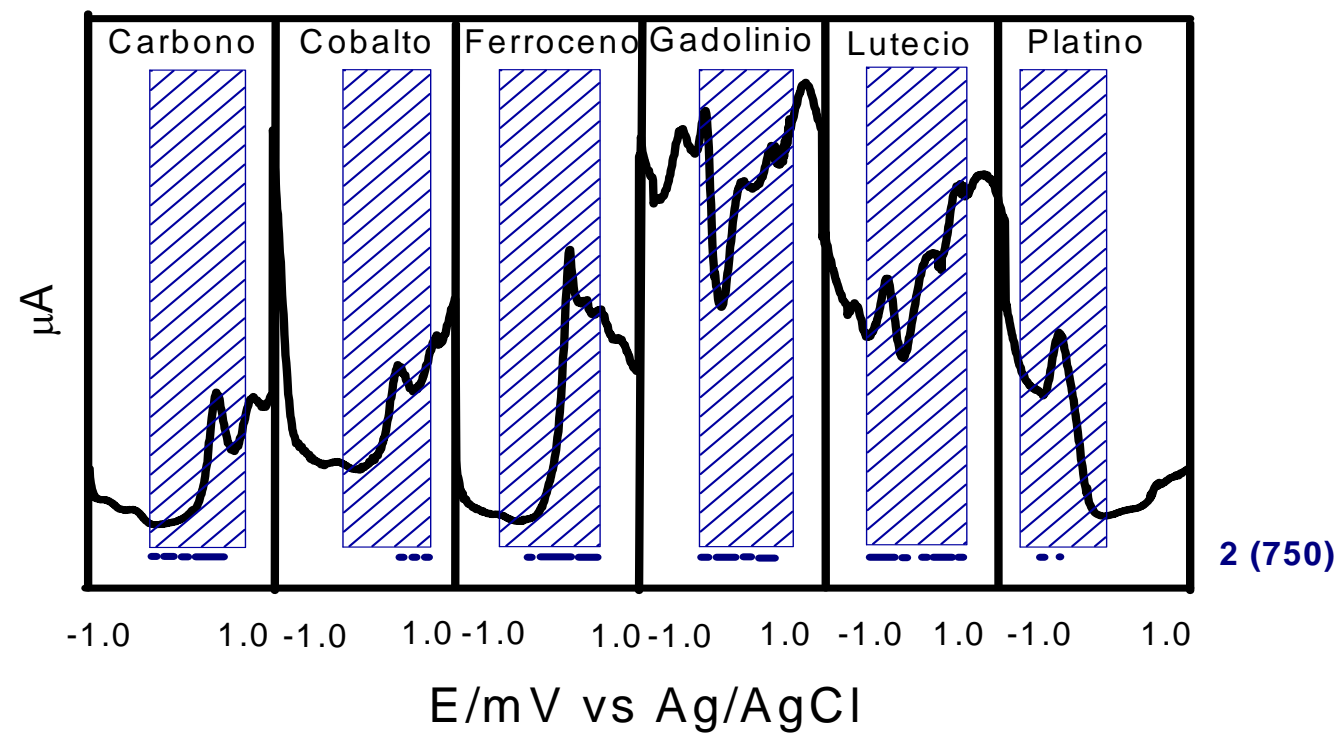

Figura 4.35. Etapa 2 del algoritmo genético.

Etapa 3. Para esta tercera etapa se utilizó un subconjunto de 400 datos. El nuevo subconjunto no contiene ninguna variable proveniente de los sensores de cobalto y platino. Para los demás sensores se tomó un total de 100 variables correspondientes a la región que contenía el valor máximo del pico asociado a la actividad electroquímica de los polifenoles del vino. El modelo GA-PLS se construyó utilizando sólo dos variables por ventana (Figura 4.36). 


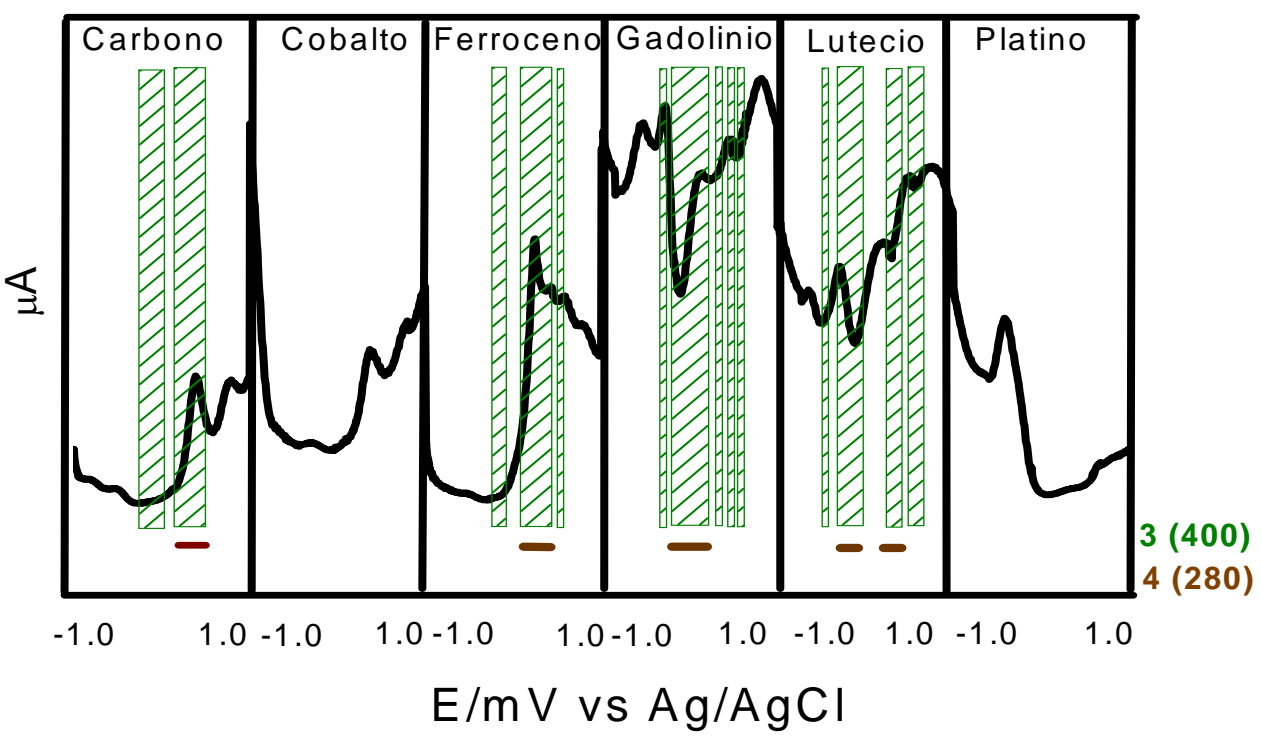

Figura 4.36. Etapa 3 del algoritmo genético.

Nuevamente con los resultados de este modelo se procedió a desarrollar una cuarta etapa. Como se puede observar en la Figura 4.36 en la parte que contiene las líneas quebradas marcadas con el número 4, se seleccionaron un total de 280 variables para toda la red de sensores.

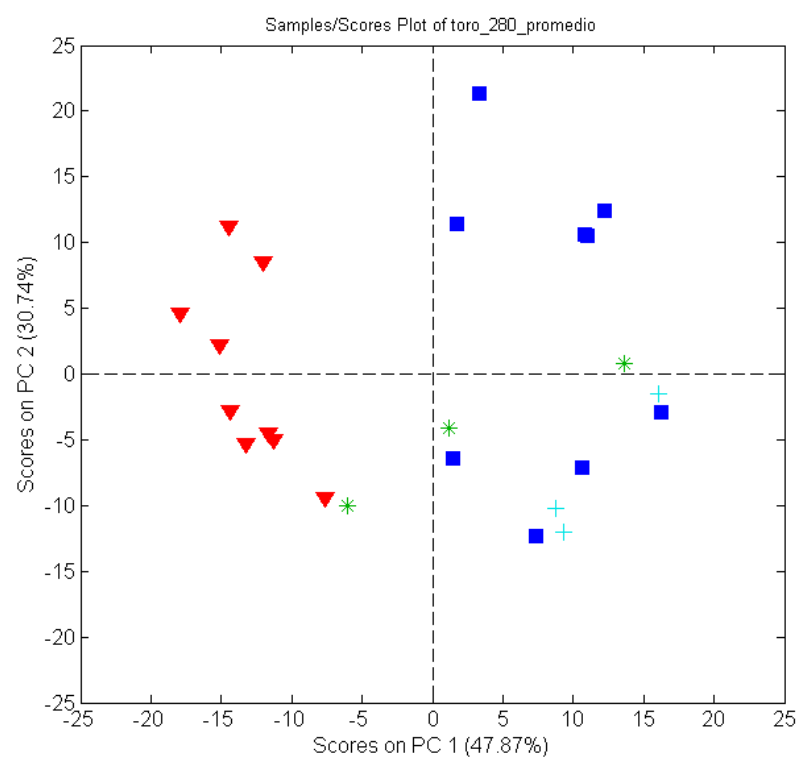

Figura 4.37. Gráfico de los scores para los vinos de la D.O. Toro con el conjunto de 280 variables seleccionadas por el método PLS-GA. Los vinos marcados con el asterisco verde

(T1) y el signo positivo azul (T2) pertenecen al conjunto de muestras envejecidas en barricas. Las muestras señaladas con el triangulo rojo (T1) y cuadrado azules (T2) corresponden a los vinos envejecidos con métodos alternativos. 
Para complementar este estudio se ha realizado un nuevo PCA con el nuevo subconjunto que contiene 280 variables. La Figura 4.37 ilustra el gráfico de los scores que retienen un $78.5 \%$ de información. En las dos primeras componentes principales, la distribución de los cluster para la discriminación en función del tipo de envejecimiento mejora notablemente en comparación con la distribución registrada cuando se realiza el PCA con el conjunto de datos completo. En este segundo PCA existe una visible diferenciación de los clusters, y en este caso las muestras que corresponden a los vinos envejecidos en barricas, representadas con un asterisco verde, están separadas claramente de las muestras maceradas con los trozos de madera, simbolizadas con el triangulo rojo.

Respecto a la nueva localización relativa de los clusters en el espacio establecido por las dos primeras PCs del subconjunto de datos de la D.O Ribera de Duero (Figura 4.38.), se puede decir que se conserva la tendencia o patrón de distinción que ubica los vinos envejecidos en barricas en la parte negativa de la PC2 y los vinos envejecidos con técnicas alternativas en la parte positiva de la PC2. Las dos primeras componentes principales recogen el $87 \%$ de la información.

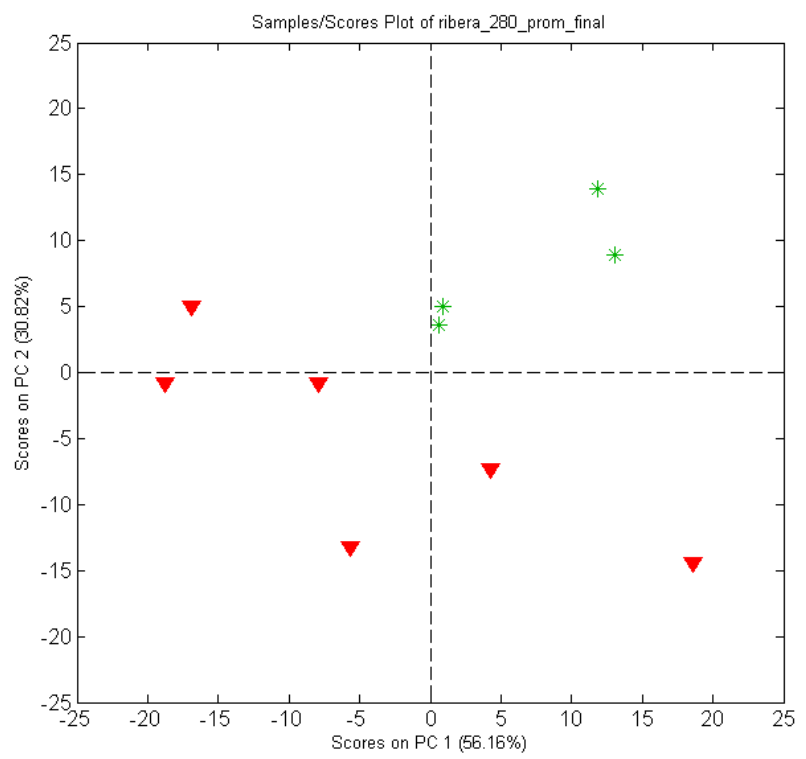

Figura 4.38. Gráfico de los scores para los vinos de la D.O. Ribera del Duero con el conjunto de 280 variables seleccionadas por el método PLS-GA. Los vinos marcados con el asterisco verde pertenecen al conjunto de muestras envejecidas en barricas y las muestras señaladas con el triangulo rojo corresponden a los vinos envejecidos con métodos alternativos.

En definitiva se puede observar que las diferencias que se han presentado indican por una parte que se puede mantener la capacidad de clasificación y por otra la fiabilidad del modelo GA-PLS para la reducción de variables. 
En resumen, con los interesantes resultados obtenidos en la discriminación y clasificación de los vinos envejecidos con diferentes métodos, se ha demostrado que nuestro sistema de sensores puede tener cabida como nueva herramienta de análisis de vinos, análisis no clásicos que pueden permitir al enólogo o bodeguero detectar, caracterizar o reconocer de forma global y rápida determinadas muestras de vino en función de sus necesidades, tras dotar al equipo del entrenamiento adecuado acorde con el problema particular que se plantee.

En este caso particular, nuestro sistema ha sido capaz de detectar las diferencias que se originan en el vino debido al cambio de un envejecimiento tradicional en una barrica de roble a un sistema alternativo como los depósitos de acero inoxidable con inserción de madera y una dosificación controlada de oxígeno. Todos los resultados reportados fueron además comparados con los obtenidos con técnicas analíticas tradicionales.

\subsection{CONCLUSIONES}

En este capítulo se han presentado resultados significativos de exploración, comparación y correlación respecto a la capacidad de caracterización de vinos por parte de nuestras redes de sensores y las técnicas analíticas clásicas. La exploración de los resultados obtenidos en los análisis de las muestras particularmente estudiadas nos lleva a validar las siguientes conclusiones:

- Los vinos evolucionan de manera diferente, dependiendo del tipo de envejecimiento y/o el origen de la madera de roble utilizada. A diferencia de otros trabajos publicados hasta el momento, en esta investigación se ha dado especial importancia al envejecimiento en botella, encontrando que es justamente en este periodo donde las diferencias se hacen más significativas.

- El estudio exploratorio mediante PCA ha demostrado una notable capacidad de diferenciación entre muestras de vinos; asimismo la aplicación de un PLS-DA como método supervisado de clasificación ha evidenciado la prometedora capacidad de predicción de muestras en función de su método de crianza.

- La aplicación de modelo más reciente como el Tucker3 al conjunto de datos derivado de tres técnicas analíticas diferentes ha permitido una interesante evaluación visual de las relaciones existentes entre la caracterización global 
del vino realizada con la red de sensores de gases y su composición química evaluada por medio de la GC-MS y UV-Vis.

El desarrollo de dos modelos basados en dos técnicas de preprocesamiento (j-scaling, jk-scaling) de datos diferentes nos ha permitido analizar el problema desde dos enfoques complementarios. El primero de ellos facilitó el estudio de la evolución de los vinos, mientras el segundo modelo nos permitió analizar las diferencias entorno al tipo de envejecimiento y origen de la madera para los vinos valorados.

- En esta misma dirección de la aplicación de nuevos métodos quimiométricos, se ha realizado un estudio específico del periodo del envejecimiento en botella a través del método ANOVA-PLS. Las características de este algoritmo nos han facilitado la combinación de los resultados de tres técnicas analíticas diferentes: GC-MS, GC-O y la nariz electrónica. Además, ha sido determinado el grado de significación de cada variable en función de los tres factores elegidos en esta investigación: el tipo de envejecimiento (depósito o barrica), el origen de la madera (americano o francés), y el periodo de envejecimiento (16 o 20 meses).

- Existe un resultado común a los diferentes métodos quimiométricos aplicados en las valoraciones obtenidas con las redes de sensores utilizadas. Los sensores TGS 822, SP 19, TGS 2620 y SP 31 han demostrado un grado de correlación importante con la trans-whiskylactona, determinados de manera particular por la valoración visual de los métodos Tucker3 y ANOVA-PLS y de una manera general durante todos los periodos de evaluación a través del análisis PLS2.

- En este capítulo también se ha mostrado que el método GA-PLS es una herramienta adecuada para la reducción de variables en las señales electroquímicas que proporciona nuestra lengua electrónica. Varias conclusiones se pueden deducir de este estudio, entre ellas que ha sido posible mejorar la capacidad de diferenciación de las distintas muestras de vinos evaluadas con este sistema, básicamente debido a la capacidad del método GA-PLS de identificar las zonas específicas de los voltamogramas asociadas a la actividad electroquímica de los sensores y de los polifenoles del vino. Para las futuras aplicaciones de la lengua electrónica, con este conjunto de datos reducidos, el modelamiento de los datos será más simple.

En síntesis, los resultados de los modelos GA-PLS consecutivos fueron coherentes; además el proceso de reducción de variables se realizó en varias etapas y bajo un cuidadoso proceso de validación para no sobreestimar la capacidad de clasificación del sistema 


\subsection{REFERENCIAS}

[1] M. Ortega-Heras, C. González-Huerta, P. Herrera \& M. L. González-Sanjosé, Changes in wine volatile compounds of varietal wines during ageing in wood barrels, Analytica Chimica Acta, 2004, 513, 341-350.

[2] P. Chatonnet, J. N. Boidron \& M. Pons, Maturation of red wines in oak barrels evolution of some volatile compounds and their aromatic impact, Sciences Des Aliments, $1990,10,565-587$.

[3] F. Zamora, Elaboración y crianza del vino tinto: aspectos científicos y prácticos, Mundi prensa, 2003.

[4] J. S. Camara, M. A. Alves \& J. C. Marques, Evolution of oak-related volatile compounds in a Spanish red wine during 2 years bottled, after aging in barrels made of Spanish, French and American oak wood, Analytica Chimica Acta, 2006, 563, 189-203.

[5] P. Hernandez-Orte, A. C. Lapena, A. Escudero, J. Astrain, C. Baron, I. Pardo, L. Polo, S. Ferrer, J. Cacho \& V. Ferreira, Effect of micro-oxygenation on the evolution of aromatic compounds in wines: Malolactic fermentation and ageing in wood, Lwt-Food Science and Technology, 2009, 42, 391-401.

[6] N. Loscos, P. Hernandez-Orte, J. Cacho \& V. Ferreira, Evolution of the aroma composition of wines supplemented with grape flavour precursors from different varietals during accelerated wine ageing, Food Chemistry, 2010, 120, 205-216.

[7] P. Arapitsas, A. Antonopoulos, E. Stefanou \& V. G. Dourtoglou, Artificial aging of wines using oak chips, Food Chemistry, 2004, 86, 563-570.

[8] M. J. Cejudo-Bastante, I. Hermosin-Gutierrez \& M. S. Perez-Coello, Microoxygenation and oak chip treatments of red wines: Effects on colour-related phenolics, volatile composition and sensory characteristics. Part II: Merlot wines, Food Chemistry, 2011, 124, 738-748.

[9] J. Béteau \& G. Roig Josa, Los chips de roble como herramienta de vinificación y crianza ACE Revista de Enología, 2006.

[10] M. Del Álamo, I. Nevares, L. Gallego, B. Fernández de Simón \& E. Cadahía, Micro-oxygenation strategy depends on origin and size of oak chips or staves during accelerated red wine aging, Analytica Chimica Acta, 2010, 660, 92-101.

[11] M. C. Llaudy, S. Roser Canals, M. Gonza Lez, J. M. Canals, C. Santos-Buelga \& F. Zamora, Influence of Micro-Oxygenation Treatment before Oak Aging on Phenolic 
Compounds Composition, Astringency, and Color of Red Wine., Journal of Agricultural and Food Chemistry, 2006, 54, 4246-4252.

[12] P. J. Spillman, A. P. Pollnitz, D. Liacopoulos \& K. H. Pardon, Formation and degradation of furfuryl alcohol, 5-methylfurfuryl alcohol, vanillyl alcohol, and their ethyl ethers in barrel-aged wines., Journal of Agricultural and Food Chemistry, 1998, 46, 657-663.

[13] V. L. Singleton \& A. C. Noble, Wine Flavor and Phenolic Substances, (Ed. G. Charalambous), ACS Symposium Series, 1976, pp. 47-70.

[14] M. Aznar, R. Lopez, J. Cacho \& V. Ferreira, Prediction of aged red wine aroma properties from aroma chemical composition. Partial least squares regression models, Journal of Agricultural and Food Chemistry, 2003, 51, 2700-2707.

[15] J. Cacho, El roble, la barrica, y la crianza del vino tinto, Institución "Fernando el Católico", Zaragoza, 2009.

[16] I. Jarauta, J. Cacho \& V. Ferreira, Concurrent phenomena contributing to the formation of the aroma of wine during aging in oak wood: An analytical study, Journal of Agricultural and Food Chemistry, 2005, 53, 4166-4177.

[17] C. Phillips, 2006 Barrel \& Oak Report. Use of alternatives continues to increase, not only as a means of cost-cutting but also as a new tool for producing quality wine, 2006, http://www.winebusiness.com/.

[18] C. Phillips, 2007 Barrels \& Oak Report 2007, http://www.winebusiness.com/.

[19] C. Phillips, 2008 Barrels \& Oak Survey Report. Barrels and alternative purchases remain stable, 2008, http:/ /www.winebusiness.com/.

[20] C. Phillips, 2009 Barrels \& Oak Survey Report: Recession Affects Barrel Purchases 2009, http://www.winebusiness.com/.

[21] C. Phillips, 2010 Barrel and oak Survey Report, 2010, http://www.winebusiness.com/.

[22] W. P. Venter \& G. F. Baumgarten, Influence of relative humidity and temperature on the chemical composition of brandy during maturation. Cape Town, 1-13., Cape

Town, 1987, pp. 1-13.

[23] J. W. Aiken \& A. C. Noble, Comparison of the aroma of oak- and glassaged wines, American Journal of Enology and Viticulture, 1984, 35, 196-205.

[24] N. Vivas, A. Lonvaud \& Y. Glories, Observations concerning the increase of volatile acidity in red wines whilst ageing in barrels, Journal of the Science of Technology Tonnellerie, 1995, 1, 81-122. 
[25] T. C. Somers, Wine tannins-isolation of condensed flavonoid pigments by gelfiltration., Nature, 1966, 209, 368-370.

[26] M. Moutounet, P. Rabier, J. L. Puech, E. Verette \& J. M. Barillene, Analysis by HPLC of extractable substances in oak wood, Sciences des Aliments, 1989, 9, 35-51.

[27] J. L. Puech, Extraction of phenolic compounds from oak wood in model solutions and evolution of aromatic aldehides in wines aged in oak barrels., American Journal of Enology and Viticulture, 1987, 38, 236-238.

[28] R. C. Schuetz, Humidity effects on evaporation and penetration in american and french white oak and its ethanol extract, Research Report. University of California, 1986, 54.

[29] V. L. Singleton, Some aspects of the wooden containers as a factor in wine maturation, Advances in Chemistry Series, 1974, 137, 254-277.

[30] J. M. Álvarez, Tanino: La revolución enológica, mito o realidad. , Enología, 2007, $1-15$.

[31] C. Dallas \& O. Laureano, Effects of $p H$, sulphur dioxide, alcohol content, temperature and storage time on colour composition of a young Portuguese red table wine, Journal of the Science of Food and Agriculture, 1994, 65, 477-485.

[32] G. Mazza, Anthocyanins in grapes and grape products, Critical Reviews in Food Sciences and Nutrition, 1995, 35, 341-371.

[33] P. Chatonnet, Productos alternativos a la crianza en barrica de los vinos. Influencia de los parámetros de fabricación y de uso, Enología, 2007, 3, 2.24.

[34] V. L. Singleton \& E. K. Trousdale, Anthocyanin-tannin interactions explaining differences in polymeric phenols between white and red wines, American Journal of Enology and Viticulture, 1992, 43, 63-70.

[35] M. E. Melendez, M. C. Ortiz, M. S. Sanchez, L. A. Sarabia \& M. Iniguez, Chemometric characterization of the claretes and rose wines of the certified denomination of origin Rioja using CieLab parameters, Quimica Analitica, 1999, 18, 119-126.

[36] V. Cheynier, El color de los vinos tintos Wine Internet Technical Journal, 2003, $4,1-9$.

[37] J. D. Carrillo, A. Garrido-López \& M. T. Tena, Determination of volatile oak compounds in wine by headspace solid-phase microextraction and gas chromatographymass spectrometry, Journal of Chromatography A, 2006, 1102, 25-36.

[38] V. Ferreira, I. Jarauta \& J. Cacho, Physicochemical model to interpret the kinetics of aroma extraction during wine aging in wood. Model limitations suggest the necessary existence of biochemical processes, Journal of Agricultural and Food Chemistry, 2006, $54,3047-3054$. 
[39] E. M. Díaz-Plaza, J. R. Reyero, F. Pardo \& M. R. Salinas, Comparison of wine aromas with different tannic content aged in French oak barrels, Analytica Chimica Acta, 2002, 458, 139-145.

[40] J. J. Rodríguez-Bencomo, M. Ortega-Heras, S. Pérez-Magariño \& C. González-Huerta, Volatile compounds of Red Wines Macerated with Spanish, American and French Oak Chips, Journal of Agricultural and Food Chemistry, 2009, $57,6283-6391$.

[41] J. J. Rodríguez-Bencomo, M. Ortega Heras, S. Pérez Magariño, C. González Huerta \& M. L. González San José, Importance of chip selection and elaboration process on the aromatic composition of finished wines. , Journal of Agricultural and Food Chemistry, 2008, 2008, 5102-5111.

[42] A. Zalacain, J. Marin, G. L. Alonso \& M. R. Salinas, Analysis of wine primary aroma compounds by stir bar sorptive extraction, Talanta, 2007, 71, 1610-1615.

[43] K. L. Wilkinson, G. M. Elsey, R. H. Prager, A. P. Pollnitz \& M. A. Sefton, Rates of Formation of cis- and trans-Oak Lactone from 3-Methyl-4-hydroxyoctanoic Acid, Journal of Agricultural and Food Chemistry, 2004, 52, 4213-4218.

[44] A. L. Waterhouse \& J. P. Towey, Oak Lactone Isomer Ratio Distinguishes between wines fermented in American and French Oak Barrels, Journal of Agricultural and Food Chemistry, 1994, 42, 1971-1974.

[45] A. B. Bautista-Ortín, A. G. Lencina, M. Cano-López, F. Pardo-Mínguez, J. M. López-Roca \& E. Gómez-Plaza, The use of oak chips during the ageing of a red wine in stainless steel tanks or used barrels: effect of the contact time and size of the oak chips on aroma compounds, Australian Journal of Grape and Wine Research, 2008, 14, 63-70.

[46] P. Chatonnet, I. Cutzach, M. Pons \& D. Dubourdieu, Monitoring toasting intensity of barrels by chromatographic analysis of volatile compounds from toasted oak wood, Journal of Agricultural and Food Chemistry, 1999, 47, 4310-4318.

[47] A. Escudero, J. Cacho \& V. Ferreira, Isolation and identification of odorants generated in wine during its oxidation: a gas chromatography-olfactometric study, European Food Research and Technology, 2000, 211, 105-110.

[48] V. Ferreira, F. San Juan, A. Escudero, L. Cullere, P. Fernandez-Zurbano, M. P. Saenz-Navajas \& J. Cacho, Modeling Quality of Premium Spanish Red Wines from Gas Chromatography-Olfactometry Data, Journal of Agricultural and Food Chemistry, 2009, 57, 7490-7498.

[49] S. M. Van Ruth \& C. H. O'Connor, Evaluation of three gas chromatographyolfactometry methods: comparison of odour intensity-concentration relationships of eight volatile compounds with sensory headspace data., Food Chemistry, 2001, 74, 341-347. 
[50] B. S. Mistry, T. Reineccius \& L. K. Olson, Gas Chromatography-olfactometry for the determination of key odorants in foods, (Ed. R. Marsili), Marcel Dekker, New York, 1997, pp. 265-292.

[51] B. Atanasova, T. Thomas-Danguin, D. Langlois, S. Nicklaus \& P. Etievant, Perceptual interactions between fruity and woody notes of wine, Flavour and Fragrance Journal, 2004, 19, 476-482.

[52] E. P. Köster, Intensity in mixtures of odorous substances, Rockefeller University Press 1969.

[53] P. J. Spillman, Oak wood contribution to wine aroma, University of Adelaide, Adelaide, 1997.

[54] M. T. Frangipane, D. De Santis \& A. Ceccarelli, Influence of oak woods of different geographical origins on quality of wines aged in barriques and using oak chips, Food Chemistry, 2007, 103, 46-54.

[55] J. Lozano, J. P. Santos, M. Aleixandre, I. Sayago, J. Gutierrez \& M. C. Horrillo, Identification of typical wine aromas by means of an electronic nose, Ieee Sensors Journal, 2006, 6, 173-178.

[56] C. Di Natale, R. Paolesse, A. Macagnano, A. Mantini, A. D'Amico, M. Ubigli, A. Legin, L. Lvova, A. Rudnitskaya \& Y. Vlasov, Application of a combined artificial olfaction and taste system to the quantification of relevant compounds in red wine, Sensors and Actuators B-Chemical, 2000, 69, 342-347.

[57] R. Bro, Multi-way Analysis in the Food Industry. Models, Algorithms, and Applications, Ph.D. thesis, University of Amsterdam, 1998.

[58] P. M. Kroonenberg, Three-Mode Principal Component Analysis: Theory and Applications, DSWO Press, Leiden, 1983.

[59] M. J. Cejudo-Bastante, M. S. Perez-Coello \& I. Hermosin-Gutierrez, Effect of wine micro-oxygenation treatment and storage period on colour-related phenolics, volatile composition and sensory characteristics, Lwt-Food Science and Technology, 2011, 44, 866-874.

[60] L. Castro-Vazquez, M. E. Alanon, E. Calvo, M. J. Cejudo, M. C. Diaz-Maroto \& M. S. Perez-Coello, Volatile compounds as markers of ageing in Tempranillo red wines from La Mancha DO stored in oak wood barrels, Journal of Chromatography A, 2011, 1218, 4910-4917.

[61] R. Jónsdóttir, G. Ólafsdóttir, E. Chanie \& H. J.E, Volatile compounds suitable for rapid detection as quality indicators of cold smoked salmon (Salmo salar). Food Chemistry, 2008, 109, 184-195.

[62] M. A. Sefton, P. J. Spillman, K. J. Pocok, Francis Il \& P. J. Williams, The influence of oak origin, seasoning, and other industry practices on the sensory 
characteristics and composition of oak extracts and barrel-aged white wines, Australian Grapegrower \& Winemaker, 1993, 335.

[63] R. Bro, Review on multiway analysis in chemistry - 2000-2005, Critical Reviews in Analytical Chemistry, 2006, 36, 279-293.

[64] H. Zou, A. Kilmartin, M. J. Inglis \& A. Frost, Extraction of phenolic compounds during vinification of Pinot Noir wine examined by HPLC and cyclic voltammetry, Australian Journal of grape and wine Research, 2002, 8, 163-174.

[65] Y. Vlasov, A. Legin, C. Rudnitskaya, C. Di Natale \& D’Amico, Non-specific sensor arrays («electronic tongue») for chemical analysis of liquids, Pure and Applied Chemistry, 2005, 77, 1965-1983 


\title{
CAPITULOV
}

\author{
Estudio de los Efectos de la Fermentación en \\ Barrica en la Composición Volátil de Vinos Blancos
}

Página

5.1. GENERALIDADES 257

5.1.1. Revisión teórica

5.2. DESARROLLO EXPERIMENTAL 261

5.2.1. Preparación de las muestras de vino 261

5.3. RESULTADOS 262

5.3.1. Parámetros Químicos $\quad 262$

5.3.2. Coordenadas CIELab 265

5.3.3. Cromatografía de Gases Masas 268

5.3.4. Olfatometría 270

5.3.5. Nariz electrónica $\quad 272$

5.3.5.1. Análisis supervisado. PLS-DA 274

5.3.5.2. Regresión Lineal por mínimos cuadrados PLS2 275

5.4. CONCLUSIONES 275

5.5. REFERENCIAS 276 



\subsection{GENERALIDADES}

En esta parte de la tesis se evalúan las características de vinos blancos fermentados en barricas nuevas y barricas "utilizadas", una práctica que seguida de una reducción en botella se está convirtiendo en una técnica habitual de elaboración de vinos de calidad. En el caso de las barricas usadas también se evaluará el efecto de la adición de chips de roble, las cuales han sido incorporadas con la idea de paliar el envejecimiento de la barrica por su uso precedente.

Al igual que los trabajos expuestos anteriormente, la caracterización de la composición volátil se ha realizado utilizando una nariz electrónica, la cromatografía de gases-masas y la olfactometría. Así mismo, se han utilizado medidas físico-químicas estándar y espectroscopia UV-Visible. Estas técnicas nos han permitido profundizar en los efectos que traen los diferentes mecanismos de fermentación en las características finales de los vinos blancos.

Los resultados de la nariz electrónica y de las coordenadas colorimétricas CIELab fueron analizados con técnicas quimiométricas para diferenciar los vinos blancos evaluados de acuerdo con el tipo de depósito usado para la fermentación. Respecto a la cromatografía de gases-masas, debido al buen desempeño de los compuestos seleccionados como marcadores en la investigación realizada con los vinos tintos, se ha realizado nuevamente un seguimiento a los mismos compuestos. Además, se adicionó el análisis de otros dos compuestos bastante conocidos por su influencia en las características organolépticas de los vinos blancos, el 5-metilfurfural y el 4-etilfenol.

Especificar que estos resultados hacen parte de un primer acercamiento a la aplicación de una nariz electrónica para la evaluación de vinos blancos. Esto significa principalmente que los siguientes resultados tienen un carácter exploratorio y pueden servir de punto de partida para futuras investigaciones en nuestro grupo investigación con un conjunto de muestras más amplio. 
El mercado actual de los vinos blancos tiene un interés creciente en nuestra Comunidad Autónoma y en concreto en la D.O. Rueda, cuna principal de estos vinos. De hecho, las ventas en litros de vinos blancos es creciente mientras los tintos y claretes sólo mantienen e incluso disminuyen en nuestra comunidad. De los 57 millones de botellas comercializadas en el año 2011, casi el 17\% han ido al exterior, siendo Alemania y Holanda los principales mercados de los vinos blancos de la D.O. Rueda.

Partiendo de los datos facilitados por las bodegas inscritas en la Denominación de Origen Rueda se confirma el incremento de las ventas en mercados exteriores, pasando de los 7.692 .158 botellas exportadas en 2010 a los 9.212 .902 del 2011. Y en relación con el mercado interno esta denominación ha experimentado en el 2011 un incremento del $12.3 \%$.

\subsubsection{Revisión teórica.}

La fermentación de los vinos blancos en barrica aunque parezca un método novedoso, es un sistema de vinificación tan antiguo como el empleo de este material en el contexto vinícola. Ya en la época romana se utilizaba este recipiente de madera tanto para el transporte o almacenamiento como en la fermentación del vino. El empleo de la barrica está mucho más vinculado al vino tinto que al blanco, pero en el proceso fermentativo es todo lo contrario, siendo los vinos blancos quienes lideran esta tipificación.

Generalmente, la fermentación alcohólica del mosto blanco se realiza en tanques de acero inoxidable, después de su respectiva clarificación. Este tipo de depósitos se utilizan principalmente para realizar un control más sencillo de la temperatura, evitando así posibles defectos finales en el vino. Una alternativa a este proceso, surge de combinar la fermentación alcohólica con el envejecimiento en barricas de roble, con el objetivo de mejorar el aroma del vino [1, 2].

De acuerdo con todo lo visto hasta el momento, el uso de la madera durante la maduración de los vinos contribuye favorablemente a su evolución organoléptica [3-5]. Además, durante el envejecimiento en madera se da lugar a una serie de cambios y de mejoras en sus caracteres sensoriales [6]. En el caso de los vinos blancos, se pretende también el desarrollo de otras propiedades interesantes, tales como el aumento de su capacidad antioxidante [7-9]. En otras bebidas como el coñac, whisky, aguardientes, vinagres, y mezcal ya se ha demostrado el aumento de la capacidad antioxidante, después de estar contacto con la madera [10-14]. Este hecho es debido una vez más a la importante cantidad de polifenoles 
extraídos por estas bebidas a partir de la madera durante el envejecimiento. La naturaleza y el contenido de los polifenoles de las maderas utilizadas durante la fermentación pueden determinar el aumento de la capacidad antioxidante durante el almacenamiento.

Por otra parte, entre las características sensoriales conocidas que aporta la fermentación en madera a los vinos blancos, en cuanto al color es posible afirmar que da lugar con a coloración más intensa, hacia los tonos amarillos dorados -oro nuevo, oro viejo-. Respecto a los aromas, los primarios de la fruta y los secundarios de la fermentación se fusionan con los aportados por la madera, conformando un sutil bouquet frutal más maduro, a veces exótico, resaltado por suaves notas de vainilla, especias y ligeros ahumados [5, 15-17]

Todo es cuestión de obtener una máxima armonía, equilibrio, elegancia y personalidad entre los elementos que componen el vino y los elementos que aporta la madera. En los vinos blancos, la función del roble es acunar con discreción y elegancia el carácter frutal del vino. No es de extrañar que muchos de estos vinos presenten ciertos problemas organolépticos, más por exceso de madera que por defecto. Los mejores vinos de este estilo deben mostrarse vivos al paladar, con presencia y cuerpo, con sabores más complejos, y no deben resultar cálidos ni empalagosos. Bien conservados, tienen una mayor longevidad que los jóvenes obtenidos por fermentación tradicional [18, 19].

En este sentido, una cuestión importante es controlar el grado de extracción de compuestos de madera, que depende del tipo de madera empleadas y su composición química [20]. Esta crianza en roble se hace a menudo junto con el uso de lías, que son residuos que forman en la parte inferior de los depósitos después de la fermentación alcohólica; de esta manera se busca disminuir el impacto sensorial de madera mediante la adsorción de algunos de sus componentes, según lo informado por Chatonnet et. al. [5]. Las lías son el resultado del proceso de autolisis que sufren las levaduras muertas. Técnicamente, este es un proceso natural y lento que se puede definir como la hidrólisis intracelular de biopolímeros bajo el efecto de las enzimas hidrolíticas asociadas a la muerte celular, que permiten la formación de productos de bajo peso molecular [21]. Los polisacáridos, proteínas y péptidos, aminoácidos y lípidos son liberados en el vino, favoreciendo su enriquecimiento desde el punto de vista organoléptico [22, 23]. A manera de ejemplo, citar las manoproteínas, compuestos que al parecer son capaces de mejorar la estabilización tartárica y proteica [24, 25]. La crianza con lías ha demostrado que es útil para proteger el color del vino de la oxidación por el consumo de oxígeno, y para aportar un equilibrio en el aroma final por su participación en la unión compuestos volátiles $[26,27]$. 
Asimismo comentar otros estudios que se han realizado sobre la influencia de lías en el aroma del vino. Chassagne et al. mostraron que los residuos de las lías son eficaces en la eliminación de 4-etilfenol y 4-etillguaiacol, compuestos conocidos por causar defectos organolépticos cuando se registran altas concentraciones [28]. Otros resultados publicados que relacionan el efecto de la madera y la levadura han demostrado que la retención de compuestos de la madera es proporcional al incremento de la cantidad de levadura adicionada al vino [29], también se ha comprobado que la combinación de estos dos factores favorece la unión y estabilización de compuestos aromáticos [4].

Otro trabajo realizado entorno a este tema, es un primer acercamiento llevado a cabo con una red sensores de gases para el seguimiento de la fermentación de vinos blancos. En esta investigación se ha demostrado que es posible obtener buenas correlaciones con las medidas registradas por la red de sensores y las medidas analíticas concernientes al grado alcohólico, la acidez volátil, y la concentración del acetato de etilo en el medio fermentativo [30].

Este capítulo de nuestra investigación tiene otro enfoque muy importante, la reutilización de barricas. Muchos experimentos confirman la regresión en extractos y efectos de maduración en barricas reutilizadas, para todo tipo de bebidas. Rejuvenecer una barrica eliminando las capas internas exhaustas ha dado efectos considerables, pero no produce el equivalente de una nueva barrica. Otro método de prolongar la vida de las barricas incluye la adición de piezas de roble o, de extractos comerciales del roble [31, 32].

Puech et. al. encuentran que brandys envejecidos 12 años dejan de extraer alrededor de un $94 \%$ de la lignina original, pero solo un $45 \%$ de los taninos originales de la madera de la barrica utilizada. Un vino sintético después de 18 meses deja un $98,2 \%$ de la lignina y un $74 \%$ de taninos no extraíbles [33, 34]. Skurikhin y Efimov reportan que las duelas de una barrica de roble europeo expuestas a un brandy por 30 años tienen como porcentajes en madera para las capas internas (expuestas) y las capas externas respectivamente, 0,8 y 6,2\% de taninos; 43,4 y $40,6 \%$ de celulosa; 29,0 y $29,4 \%$ de lignina y 25,9 y $24,7 \%$ de hemicelulosa [35]. Se puede deducir que, después de este período de tiempo, los extractivos solubles están bastante exhaustos en las capas expuestas de la duela, mientras que los polímeros estructurales grandes se disuelven en una proporción relativamente baja durante la maduración de la bebida.

Es conocido que las barricas nunca pierden sus efectos físicos y no parecen estar completamente exhaustas incluso sin el cepillado de la madera agotada, quizás debido al escamado y agrietado, que dan lugar a la aparición de nueva madera para un eventual contacto. Sin embargo, si pueden llegar a presentarse eventos poco deseables a partir del primer uso de una barrica como la solubilización de dióxido de azufre, acción microbiológica, hidrólisis, etc. El punto más difícil de 
controlar en la reutilización de una barrica es la actividad microbiológica, Chatonnet et al. han identificado 19 géneros y 27 especies de hongos y 2 géneros y al menos 4 especies de bacterias, la mayoría de ellos localizados en las capas superficiales de los tablones de roble expuestos entre 12 y 120 meses al aire libre [36]. Joseph y Marché aluden que la existencia de una actividad enzimática de naturaleza fúngica modifica notablemente el perfil polifenólico de la madera [37].

Es conveniente un conocimiento más profundo entorno a este tema, aunque se sabe que la edad de la barrica influye decisivamente en una menor cesión de compuestos al vino a medida que aumenta el número de veces que la barrica ha sido usada [38, 34]. Esta situación en principio debería corregirse con la adición de chips madera. Sin embargo, esta práctica conlleva otros fenómenos, poco explicados hasta ahora, como por ejemplo si la acción de oxígeno en la evaporación de agua y etanol y en la oxidación de compuestos es igual en las barricas viejas que en la nuevas, el efecto la obstrucción de los poros de la madera, no solo en el interior de la barrica, sino exteriormente por incrustación de polvo y suciedad, o los efectos microbiológicos, entre otros [39].

En resumen, el objetivo de la investigación han sido validar una vez más la bondad de nuestro sistema analítico y apoyo quimiométrico a través de:

i. Evaluar mediante una nariz electrónica y técnicas analíticas, los compuestos volátiles orgánicos de los diferentes vinos blancos estudiados, fermentados en barricas de roble nuevas y usadas, con y sin métodos alternativos (incorporación de chips).

ii. Realizar un seguimiento de su evolución durante la fermentación.

iii. Comparar la capacidad de discriminación del sistema electrónico con el obtenido con análisis químico tradicional, la espectroscopia UV-Visible, la GC-MS y la GC-O.

\subsection{DESARROLLO EXPERIMENTAL}

\subsubsection{Preparación de las muestras de vino}

La preparación de muestras se realizó en la Bodega de la Cooperativa Agrícola la Castellana (La Seca, Valladolid), partiendo de uvas variedad Verdejo, D.O. Rueda y utilizando distintos métodos para la fermentación alcohólica: depósitos de acero inoxidable y en barricas de roble. Los vinos fermentados en barrica fueron separados en dos clases: dos barricas viejas y dos barricas nuevas. Para envejecer 
el vino en las barricas de roble nuevas se utilizaron a su vez dos tipos diferentes de madera: americana (Quercus alba) y francesa (Quercus petrea); para el grupo de barricas de roble viejas se utilizó roble francés y en una de ellas se insertaron chips de roble francés (4 g. $\left.\mathrm{L}^{-1}\right)$. El seguimiento de la evolución de las muestras se efectuó mediante valoraciones cada 60 días, un número total de cuatro veces, es decir las muestras se evaluaron a los 30 días (T1), 90 días (T2), 150 días (T3) y 210 días (T4). En la Tabla 5.1 se resumen las características de los tratamientos realizados para la elaboración de los vino.

Tabla 5.1. Nombre y características de las muestras de vinos blancos evaluadas.

\begin{tabular}{|c|c|c|c|c|}
\hline $\mathrm{J}$ & VF & VFC & NA & NF \\
\hline \multicolumn{5}{|c|}{ Tipo de fermentación aplicada } \\
\hline \multirow{3}{*}{ Acero inoxidable } & \multicolumn{4}{|c|}{ Barrica } \\
\hline & \multicolumn{2}{|c|}{ Vieja } & \multicolumn{2}{|c|}{ Nueva } \\
\hline & $\begin{array}{c}\text { Roble } \\
\text { Frances }\end{array}$ & $\begin{array}{c}\text { Roble francés } \\
\text { con chips }\end{array}$ & $\begin{array}{c}\text { Roble } \\
\text { Americano }\end{array}$ & Roble Francés \\
\hline
\end{tabular}

\subsection{RESULTADOS}

\subsubsection{Parámetros Químicos}

Los vinos bajo estudio se analizaron por métodos químicos estándar, que incluyen el seguimiento de una serie de compuestos fenólicos provenientes tanto de la uva como de la madera, y que afectan directamente a las características organolépticas de los vinos. A manera de ejemplo, en la Tabla 5.2 se resumen algunas características valoradas en los vinos tras una permanencia de 30 días en contacto con madera. Los datos obtenidos para parámetros tales como el grado alcohólico, azucares reductores, acidez total y $\mathrm{pH}$ se encuentran entre los valores habituales para este tipo de vino y para esta denominación de origen. Además, la acidez total y el $\mathrm{pH}$, presentan valores correctos y adecuados para coadyuvar al estado de conservación de los vinos.

Varios de los valores obtenidos para los vinos fermentados en barrica contrastan con los valores que fueron medidos en el vino fermentado en depósitos de acero inoxidable, las diferencias apreciadas lógicamente tienen su origen en el uso de la madera durante la fermentación el vino [16]. Concretamente se ha encontrado durante esta parte del período de la fermentación en madera- que los vinos fermentados en barrica presentan diferencias en cuanto a la acidez total, volátil, el ácido málico, cítrico, el potasio, la glicerina, las catequinas y los taninos. 
Parámetros como el grado alcohólico y el azúcar muestran que no hay diferencias destacables de la graduación alcohólica entre los tratamientos. Se puedo observar que estos parámetros se mantuvieron prácticamente inalterables entre las mediciones efectuadas 30 días y 210 después de dar comienzo al ensayo, y que comparadas con las del vino fermentado en depósito de acero inoxidable no existen diferencias importantes.

Tabla 5.2. Características químicas representativas de los vinos 30 días después de comenzar la fermentación.

\section{PARÁMETRO}

$\mathrm{VF}$

VFC

NA $\quad$ NF

\begin{tabular}{|c|c|c|c|c|c|}
\hline Grado alcohólico [\% vol] & 12.87 & 12.75 & 12.72 & 12.86 & 12.67 \\
\hline Acidez total [g/L] & 5.77 & 6.34 & 6.93 & 6.67 & 6.40 \\
\hline Acidez volátil [g/L] & 0.28 & 0.30 & 0.32 & 0.33 & 0.31 \\
\hline Ácido Málico [g/L] & 1.43 & 1.24 & 1.30 & 1.23 & 1.25 \\
\hline Ácido cítrico [mg/L] & 248 & 229 & 232 & 223 & 223 \\
\hline Extracto Seco [g/L] & 19.8 & 19.7 & 18.8 & 19.7 & 19.1 \\
\hline Glicerina [g/L] & 6.5 & 4.9 & 4.8 & 4.9 & 5.1 \\
\hline Glucosa + fructosa [g/L] & 0.17 & 0.16 & 0.26 & 0.21 & 0.14 \\
\hline Azúcares [g/L] & 0.97 & 0.96 & 1.06 & 1.01 & 0.94 \\
\hline pH & 3.19 & 3.06 & 3.09 & 3.09 & 3.05 \\
\hline Potasio [mg/L] & 630 & 580 & 560 & 570 & 570 \\
\hline Catequinas [mg/L] & 13.3 & 8.9 & 12.8 & 12.6 & 11.2 \\
\hline Taninos totales [mg/L] & 0.162 & 0.148 & 0.143 & 0.158 & 0.153 \\
\hline
\end{tabular}

Respecto a la comparación de los efectos que existen en la composición química de los vinos entre barricas nuevas y viejas, los resultados indican que existen diferencias en los niveles de la acidez total y volátil. Generalmente, las concentraciones superiores de acidez volátil en un vino pueden ser atribuidas a la presencia de bacterias acéticas o levaduras del tipo Brettanomyces/Dekkera [40]. Sin embargo, dadas las condiciones de $\mathrm{SO}_{2}$ libre, $\mathrm{SO}_{2}$ total, $\mathrm{pH}$ y temperatura presentes durante el ensayo, parece existir una inhibición y buen control sobre la actividad bacteriana, por lo que se puede descartar una relación entre la concentración de acidez volátil y la presencia de bacterias acéticas en estos vinos. Este comportamiento de la acidez volátil también se podría atribuir a diferencias relacionadas con la proliferación de levaduras en estos tratamientos. Finalmente es importante señalar que las concentraciones de acidez volátil de los tratamientos obtenidas al final del ensayo no sobrepasan el umbral de percepción sensorial 0,7 g/L para este parámetro [40].

En cuanto a compuestos fenólicos como las catequinas y taninos, se presenta una diferencia cuando se utiliza una barrica vieja para la fermentación del vino, que además parece aumentar con el tiempo en el que vino está almacenado en las barricas. Este fenómeno puede estar relacionado con la dificultad que existe para 
el paso del oxigeno a través de los poros de la barrica cuando esta ya ha sido previamente utilizada [7-9, 17]. La Figura 5.1 representa algunas de las diferencias entre un vino blanco fermentado en una barrica de roble francés nuevo y en una barrica vieja, respecto a parámetros como el grado alcohólico, la glucosa + fructosa, las catequinas y los taninos.
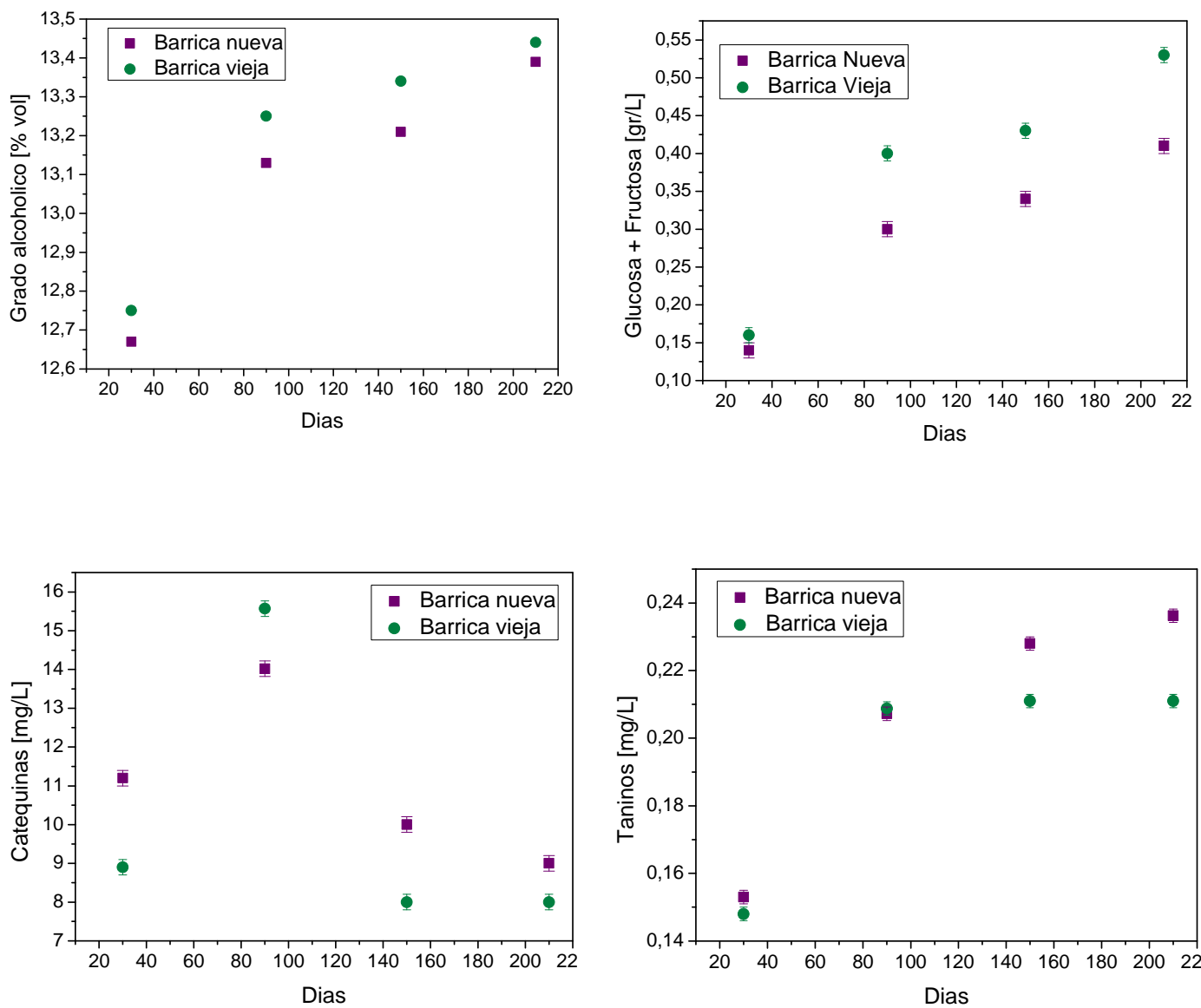

Figura 5.1. Representación de la evolución del grado alcohólico, la glucosa + fructosa, las catequinas y los taninos durante el tiempo de la fermentación en barricas.

Los resultados del conjunto de todos los parámetros analizados nos permite establecer que existen algunas características diferentes entre vinos fermentados en madera y el vino fermentado en tanques de acero inoxidable (manera tradicional). Sin embargo, esta información no es suficiente para explicar que produce las diferencias y cuál es el parámetro que permite distinguir siempre y bajo distintas circunstancias los diferentes tipos de fermentación. 


\subsubsection{Coordenadas CIELab}

Respecto a los aspectos visuales para realizar el seguimiento a la evolución del vino, se recurrió a medidas de la espectroscopia UV-Visible. Los resultados obtenidos se especifican en la Figura 5.2. La primera gran diferencia apreciable en estos resultados es la disminución en la absorbancia desde los primeros 30 días y los obtenidos 90 días después de haber dado comienzo al proceso de fermentación. A partir de este momento podemos afirmar que existe un cierto grado de estabilidad en el color de las muestras vinos, independientemente del tipo de fermentación aplicado.
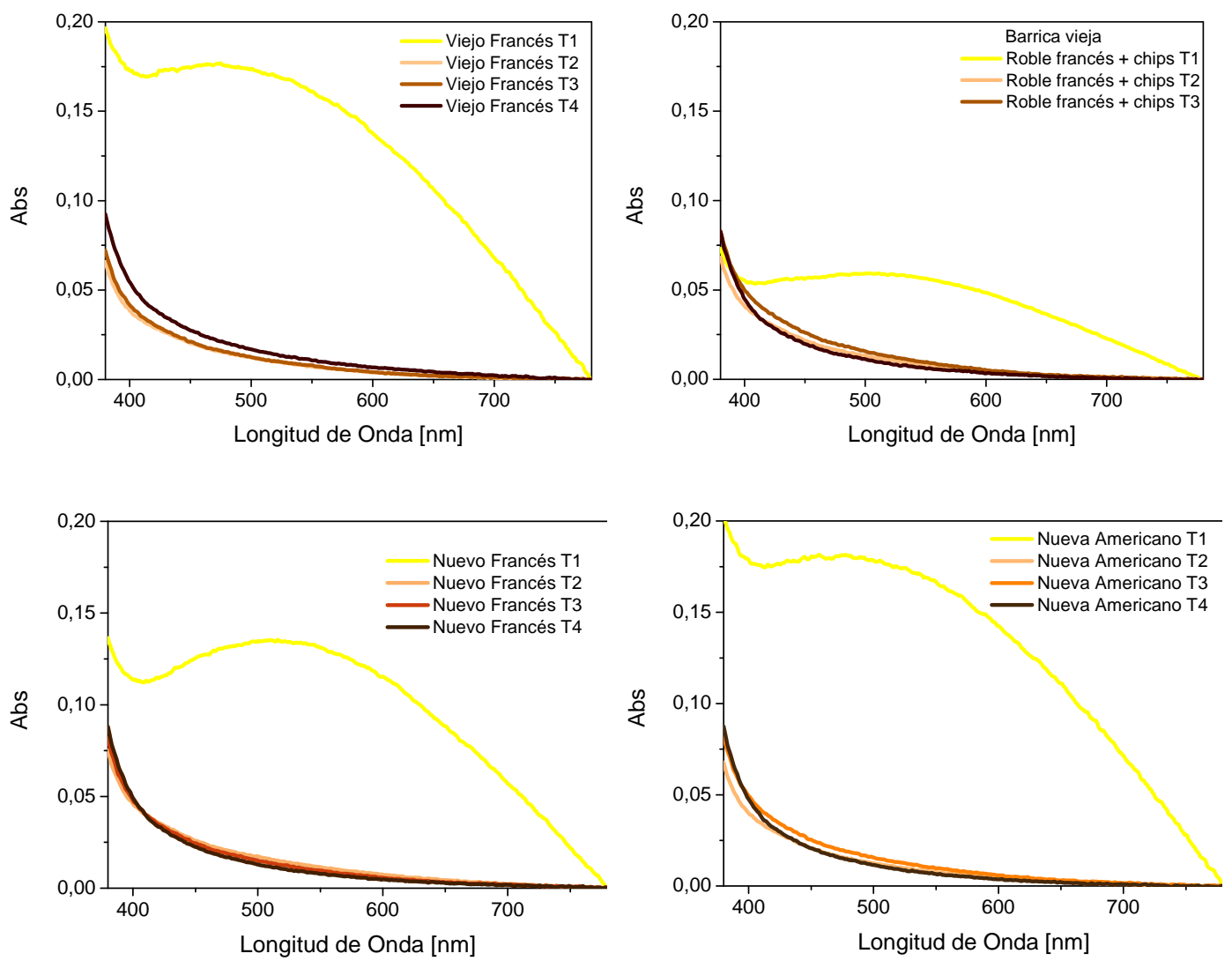

Figura 5.2. Espectros registrados durante el proceso de fermentación en barricas de vinos blancos.

Y en relación con la elaboración en barricas viejas a las que se les añadió chips de roble, los primeros espectros registrados para los vinos blancos (30 días después de comenzar la fermentación) indicaron algunas diferencias en las medidas de absorción alrededor de los $420 \mathrm{~nm}$. El vino envejecido en barrica de roble francés vieja con chips registraba mayor absorción a esta longitud de onda que los demás vinos analizados. Sin embargo durante el segundo ciclo de medidas (90 días 
después del comienzo de la fermentación) el valor de absorción decreció y las diferencias entre todos los vinos se acortaron.

Esto se debe principalmente a la presencia de oxigeno que propicia una reacción entre los taninos que son los compuestos que absorben en esta región del espectro. Los taninos consumen todo el oxigeno disuelto (fenómenos de oxidoreducción) para capturar los radicales libres y dar lugar a nuevos compuestos, con lo cual aunque todos los vinos tienen diferentes cinéticas de reacción dado las diferentes características de los depósitos para el paso del oxigeno, estos vinos alcanzan características cromáticas similares [41].

La Figura 5.3 demuestra que una vez han pasado 210 días de haber dado comienzo al proceso de la fermentación en barricas, las diferencias entre vinos son prácticamente inapreciables para los espectros UV-Visibles registrados. También es destacable el hecho de que el vino envejecido en una barrica vieja con adición de chips registra prácticamente el mismo espectro que los vinos envejecidos en barricas nuevas, ya sea roble francés o americano.

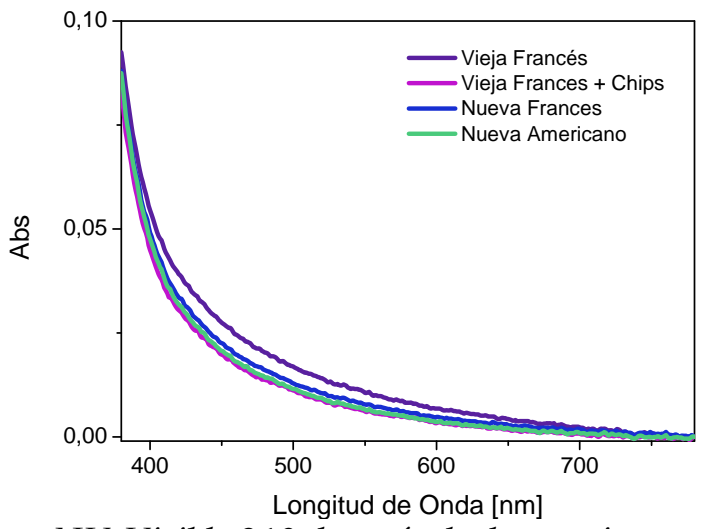

Figura 5.3. Espectros UV-Visible 210 después de dar comienzo a la fermentación en barricas.

En relación a las coordenadas CIELab, se observó un aumento en $L^{*}$ (luminosidad); los valores registrados los primeros días de la fermentación fueron de alrededor de 20 unidades hasta llegar a valores alrededor de las 90 unidades durante los últimos de la fermentación, por lo que los mostos eran más ligeros y menos marrón al final de la fase de contacto con la madera, independientemente de las condiciones de la barrica (nueva o usada).

Además, los valores de la coordenada $a^{*}$ (relacionada con el color rojo) disminuyeron, indicando una pérdida del color pardo en los mostos, de acuerdo con los valores de este parámetro. Este cambio observado en los mostos a lo largo de la fase de la fermentación puede ser debido a la precipitación, por polimerización, de pigmentos responsables del color. 
Con referencia a los parámetros $b^{*}$ (relacionada con el color amarillo) y C (croma, contribución de las $\mathrm{a}^{*} \mathrm{y} \mathrm{b}^{*}$ ) los valores, ambos parámetros han evolucionado de manera lenta, registrando valores alrededor de 21 unidades durante los primeros días de la fermentación y alcanzando valores de 17 unidades para los últimos días de este proceso. Esto significa que los vinos tienen un color más vivo y presentan un grado de estabilidad en la composición de sus taninos y las diferentes reacciones que tienen lugar durante la fermentación.

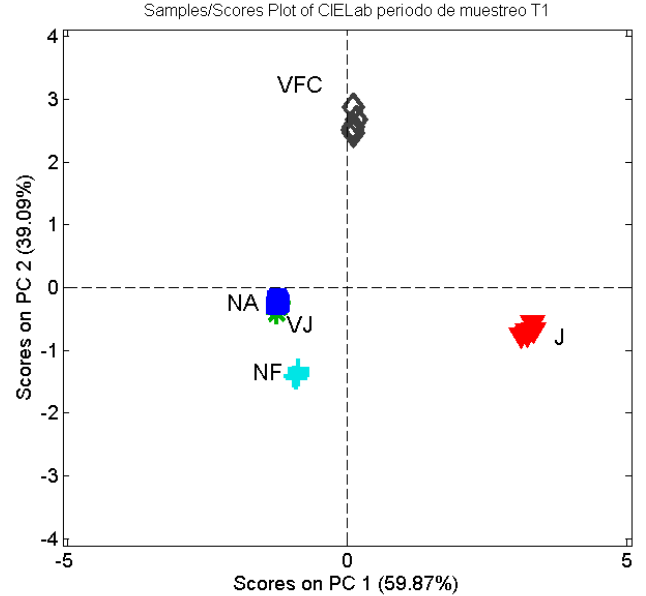

PCA periodo 1: 30 días.

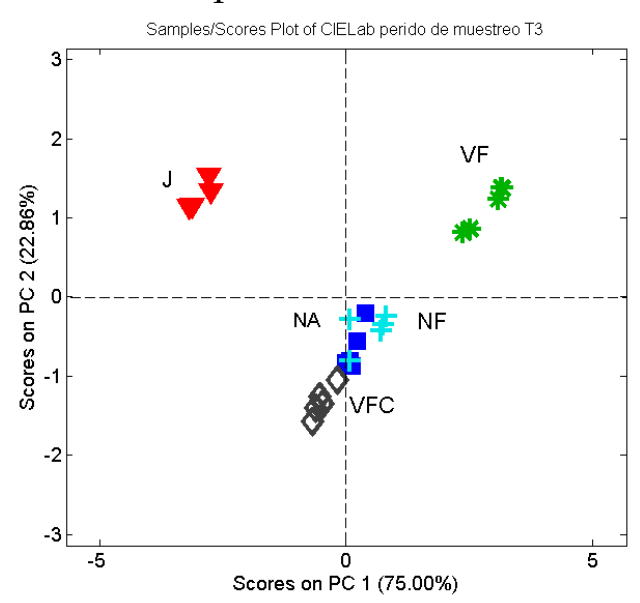

PCA periodo 3: 150 días.

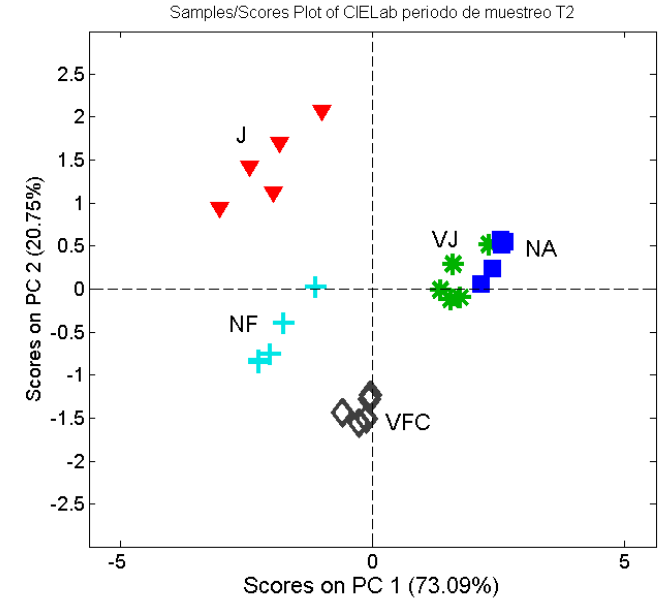

PCA periodo 2: 90 días.

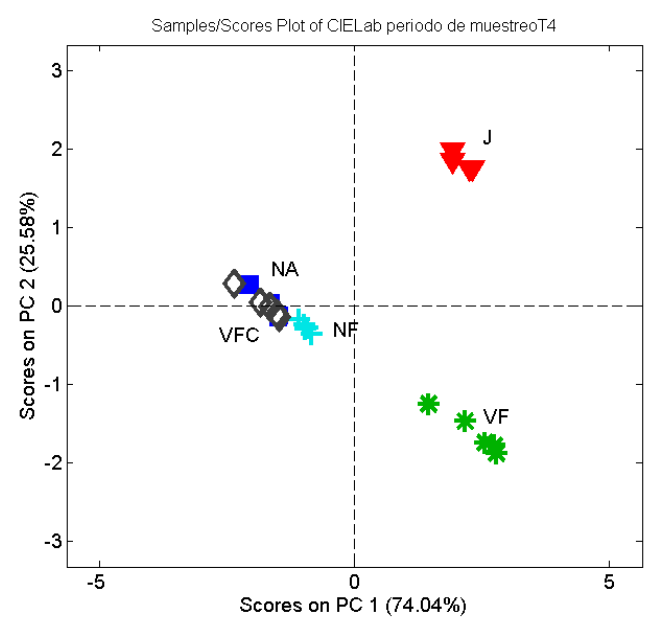

PCA periodo 4: 210 días.

Figura 5.4. PCA para las coordenadas CIELab durante la fermentación de los vinos blancos.

Los valores de las coordenadas CIELab fueron utilizados para realizar un PCA para los cuatros periodos de evaluación llevados a cabo durante esta investigación.

En la Figura 5.4 puede observarse una notoria separación de todos los vinos en función del tipo de fermentación aplicada, los vinos fermentados en depósitos de 
acero inoxidable (vinos señalados con triángulos rojos) aparecen claramente separados de los vinos fermentados en barricas viejas (asteriscos verdes, y con adición de chips rombos grises) y nuevas americano (símbolo cuadrados azules) y francés (símbolo cruz color cian) durante todo el tiempo en el que este experimento se ha llevado a cabo.

En cuanto a tendencias o patrones de distinción más sutiles, cabe reseñar la proximidad entre vinos fermentados en barricas de roble nuevas (americano y francés) con el vino fermentado en una barrica de roble viejo con inserción de chips durante los últimos periodos de evaluación. La posición del vino con chips VFC dentro del gráfico de los scores es siempre diferente al del vino que ha sido fermentado en la barrica de vieja sin la adicción de los chips (VF). Este resultado es interesante ya que muestra la posibilidad de aumentar el tiempo de las barricas.

\subsubsection{Cromatografía de gases masas}

Con el fin de encontrar diferencias entre el tipo de fermentación de los vinos, se midió mediante cromatografía de gases-masas la evolución de algunos de sus compuestos volátiles más significativos (Tabla 5.3). Puede observarse en esta tabla que las diferencias registradas entre el vino fermentado en acero inoxidable y los vinos envejecidos en barricas se amplían con el paso del tiempo durante el proceso de fermentación.

La concentración del furfural, 5-metilfurfural, cis y trans-whiskylactona y guaiacol, fue lógicamente más alta en los vinos fermentados en barricas. Comparando los diferentes métodos cabe comentar, que el nivel de asimilación de estos compuestos en los vinos fermentados en barricas nuevas es relativamente mayor que en las barricas viejas. Al mismo tiempo se puede observar que dentro del grupo de los vinos envejecidos en barricas de roble francés viejo, el depósito en el cual fueron insertados chips de roble tiene mayor contenido de todos los compuestos volátiles analizados; no obstante estos valores son inferiores a los valores registrados por el vino envejecido en la barrica de roble francés nueva. Este fenómeno puede estar relacionado con la cinética de cesión de compuestos de la madera [42] y la capacidad de paso de oxigeno a través de los poros y duelas de la barrica [4], que quizás podría paliarse en futuras experiencias con una adecuada microoxigenación.

La extracción de compuestos volátiles de la madera es finita, algunas investigaciones publicadas han demostrado que después de dos años la velocidad de extracción de estos compuestos disminuye drásticamente [43] y que además como puede ser comprobado en la Tabla 5.3 existen compuestos que 
presentan un mayor agotamiento como lo fueron el furfural y las whiskylactonas. Parece ser que las diferencias que existen en este conjunto de muestras evaluadas fueron más importantes debido a la capacidad de cesión de compuestos volátiles que al efecto que tiene el paso del oxigeno a través de la barrica y su capacidad de promover reacciones entre compuestos fenólicos.

Tabla 5.3. Resultados de la cuantificación de compuestos volátiles claves del envejecimiento en madera.

\begin{tabular}{|c|c|c|c|c|c|c|}
\hline Compuesto & Tiempo & $\mathrm{J}$ & VF & VFC & NA & $\mathrm{NF}$ \\
\hline \multirow{4}{*}{$\begin{array}{c}\text { Furfural } \\
{[\mu \mathrm{g} / \mathrm{L}]}\end{array}$} & 30 días & 74.9 & 159.6 & 191.5 & 4200.0 & 3411.7 \\
\hline & 90 días & 60.0 & 598.8 & 958.5 & 5453.8 & 1235.7 \\
\hline & 150 días & 63.4 & 782.5 & 1296.1 & 7510.6 & 4697.5 \\
\hline & 210 días & 61.0 & 952.7 & 1263.7 & 9149.4 & 5608.2 \\
\hline \multirow{4}{*}{$\begin{array}{l}\text { 5-Metilfurfural } \\
{[\mu \mathrm{g} / \mathrm{L}]}\end{array}$} & 30 días & 46.6 & 64.0 & 46.6 & 625.1 & 291.6 \\
\hline & 90 días & 47.8 & 68.1 & 82.4 & 750.1 & 90.5 \\
\hline & 150 días & 48.5 & 80.7 & 104.3 & 1148.2 & 424.2 \\
\hline & 210 días & 44.1 & 93.8 & 103.4 & 1132.4 & 422.5 \\
\hline \multirow{4}{*}{$\begin{array}{c}\text { Guaiacol } \\
{[\mu \mathrm{g} / \mathrm{L}]}\end{array}$} & 30 días & 18.1 & 25.2 & 20.7 & 19.9 & 20.1 \\
\hline & 90 días & 14.1 & 21.3 & 25.1 & 22.1 & 26.9 \\
\hline & 150 días & 9.8 & 23.9 & 26.9 & 16.0 & 10.8 \\
\hline & 210 días & 19.8 & 24.4 & 28.4 & 25.3 & 21.0 \\
\hline \multirow{3}{*}{$\begin{array}{c}\text { 4-etilfenol } \\
{[\mu \mathrm{g} / \mathrm{L}]}\end{array}$} & 90 días & 138.8 & 119.5 & 120.3 & 122.2 & 121.0 \\
\hline & 150 días & 149.1 & 151.1 & 149.9 & 149.2 & 149.5 \\
\hline & 210 días & 60.0 & 60.3 & 58.1 & 56.4 & 56.5 \\
\hline \multirow{4}{*}{$\begin{array}{c}\text { Cis-whiskylactona } \\
{[\mu \mathrm{g} / \mathrm{L}]}\end{array}$} & 60 días & 57.7 & 74.6 & 59.6 & 88.5 & 123.8 \\
\hline & 120 días & 30.9 & 44.7 & 53.7 & 386.2 & 58.7 \\
\hline & 150 días & 31.5 & 64.7 & 52.2 & 80.7 & 115.0 \\
\hline & 210 días & 28.1 & 74.3 & 51.6 & 78.0 & 114.2 \\
\hline \multirow{4}{*}{$\begin{array}{c}\text { Trans-whiskylactona } \\
{[\mu \mathrm{g} / \mathrm{L}]}\end{array}$} & 30 días & 64.7 & 156.7 & 193.2 & 467.7 & 130.3 \\
\hline & 90 días & 47.5 & 160.6 & 229.1 & 533.5 & 252.4 \\
\hline & 150 días & 43.8 & 178.1 & 243.3 & 475.1 & 127.1 \\
\hline & 210 días & 105.2 & 695.0 & 824.3 & 1590.9 & 390.2 \\
\hline \multirow{4}{*}{$\begin{array}{l}\text { Eugenol } \\
{[\mu \mathrm{g} / \mathrm{L}]}\end{array}$} & 30 días & 15.3 & 24.9 & 25.4 & 24.3 & 26.0 \\
\hline & 90 días & 14.6 & 19.2 & 23.7 & 18.9 & 24.1 \\
\hline & 150 días & 14.2 & 19.0 & 26.2 & 27.8 & 18.9 \\
\hline & 210 días & 15.7 & 24.0 & 31.0 & 32.9 & 21.6 \\
\hline
\end{tabular}

En el caso particular del furfural, y 5-metilfurfural, los principales componentes del grupo aldehídos furánico se forman principalmente durante el proceso de tostado de la madera a través de la degradación de los polisacáridos de la madera [44]. Es decir, que la superficie en contacto con el vino facilita su cinética de absorción y al mismo tiempo ocasiona que en los siguientes usos de la barrica, la presencia de este compuesto sea menor. 
Los distintos cambios registrados durante la fermentación de los vinos blancos se deben en parte, a la reducción en el consumo de nitrógeno asimilable por las levaduras durante su estancia en barricas de roble. De acuerdo con algunos autores, esta reducción genera un aumento y/o la aparición de alcoholes superiores. Asimismo, podría aumentar el contenido de ésteres, compuestos que intervienen en forma directa en las características organolépticas del vino [15, 26, $28,29,45,46]$.

\subsubsection{Olfatometria}

Se determinó el perfil aromático de las muestras de vinos blancos utilizando la técnica GC-O, puntuándose cada aroma de 1 a 3 según la intensidad establecida por el evaluador entrenado. Los resultados de estas valoraciones se muestran en una representación tipo tela de araña en las Figura 5.5 y 5.6.

En el caso de la olfatometría, y al igual que las demás técnicas revisadas hasta el momento, una vez han transcurrido 90 días de haber dado comienzo al proceso de fermentación, comenzaron hacerse evidentes algunas diferencias entre los vinos, de acuerdo con el tipo de depósito empleado. La Figura 5.5 representa el grupo de 5 compuestos identificados y cuantificados en función del valor de aroma apreciado.

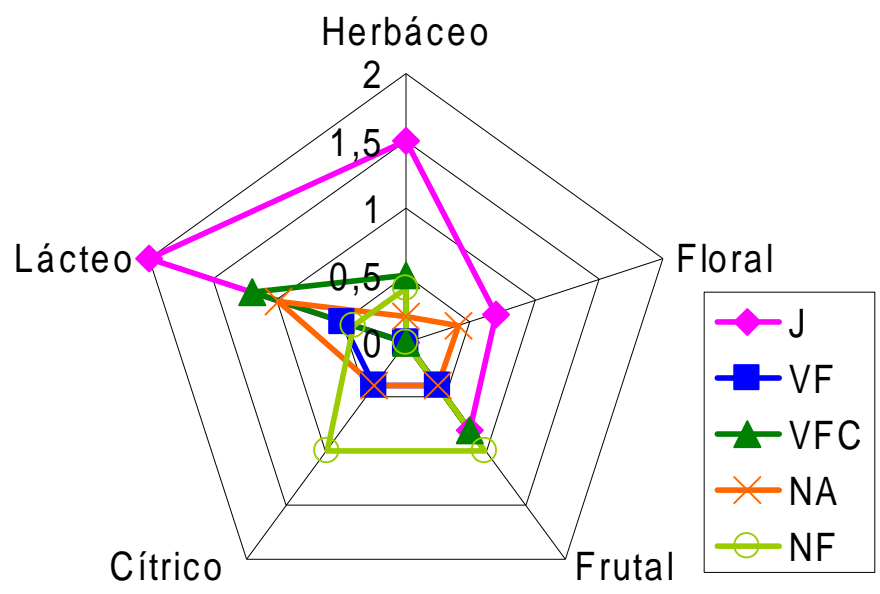

Figura 5.5. Tela de araña para los cinco compuestos con mayor intensidad de aroma evaluados por olfactometría 90 días después del inicio de la fermentación.

Dentro de la limitación cuantitativa de este método hay que decir que en contraste con los resultados obtenidos por cromatografía de gases masas, en donde se pudo ver un importante efecto de compuestos relacionados con la madera, la evaluación aromática mostró un bajo impacto aromático de los olores 
con características referentes a la madera y sus afines [47]. También se puede observar claramente la disminución de aromas lácteos y herbáceos en los vinos fermentados en barricas.

Por otra parte, un análisis conjunto de los resultados de la cromatografía de gases y la olfactometría permite inferir que la adición de chips de roble francés a una barrica no originó diferencias cuantitativas significativas con respecto a la barrica de roble francés en compuestos como el furfural y la cis-whiskylactona, sin embargo los perfiles aromáticos analizados por olfactometría en los vinos se muestran diferentes.

Respecto a los resultados de la evaluación olfatométrica 210 días (Figura 5.6) puede observarse como los vinos fermentados en barricas nuevas de roble americano y francés tienen un perfil algo similar si se comparan con el resto de muestras, con aromas fundamentalmente frutales. En el caso de los vinos envejecidos en barricas viejas sin adicción de chips, su perfil aromático es diferente en relación con los demás vinos que han sido fermentados en barica; se destaca en mayor medida su aroma lácteo y muy poco su aroma con características florales.

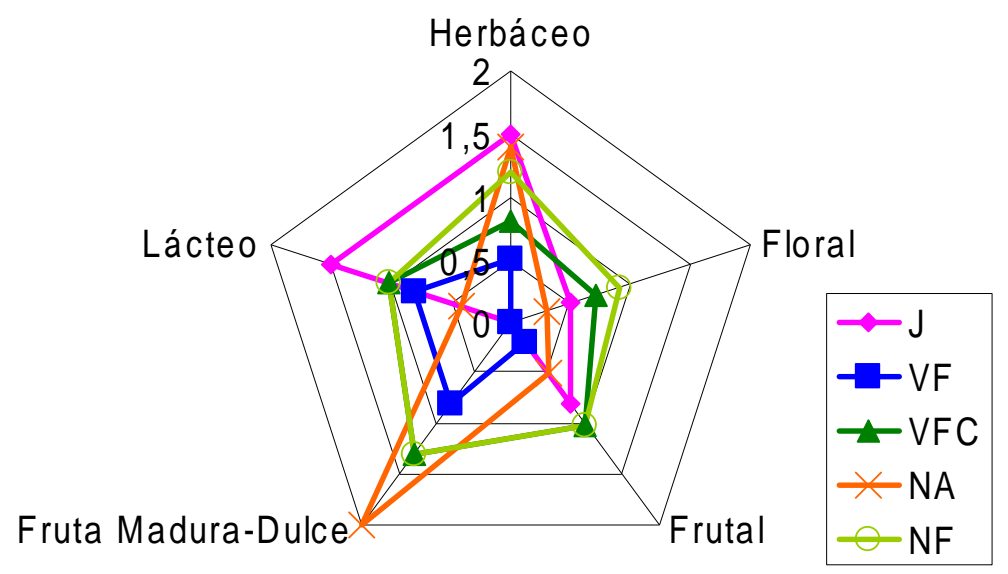

Figura 5.6. Tela de araña para los cinco compuestos con mayor intensidad de aroma, evaluados por olfactometría 210 días después del inicio de la fermentación.

A manera de conclusión sobre los diferentes perfiles aromáticos determinados para los vinos blancos, decir que las diferencias en el de aroma de los principales compuestos de los vinos no se pudieron establecer claramente. Además los aromas de la madera parecen estar atenuados debido a la presencia de las lías $[26,48]$. El efecto de la madera en este caso parece estar relacionado con el equilibrio en el aroma del vino, fenómeno que a su vez, podría estar conectado a un mayor contenido de terpenoides, lactonas, cetonas, aldehídos y ésteres, compuestos conocidos por afectar positivamente el aroma del vino [43, 47]. 


\subsubsection{La nariz electrónica}

La Figura 5.7 resume los resultados del PCA aplicado a la matriz de datos que recoge la variación de la resistencia eléctrica de los sensores al entrar en contacto con los volátiles de las muestras de vino inyectadas. Además, esta figura recoge los resultados obtenidos para los cuatros periodos de muestreo realizados en esta investigación.
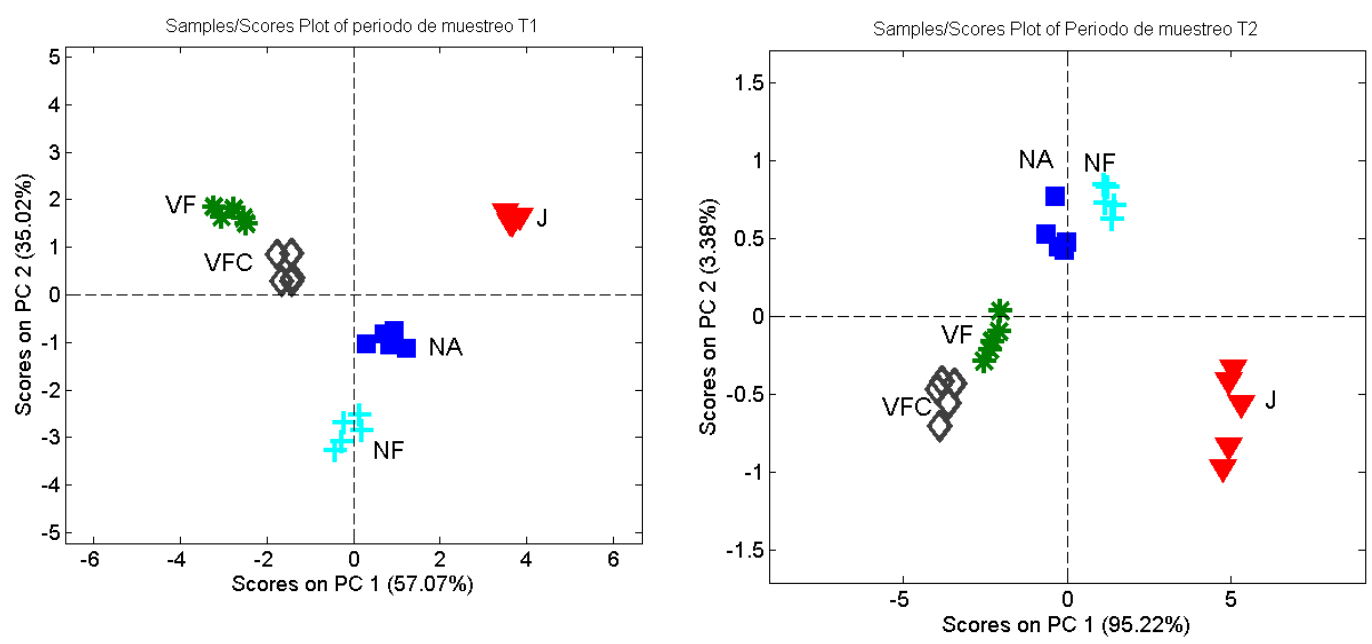

PCA periodo 1: 30 días.

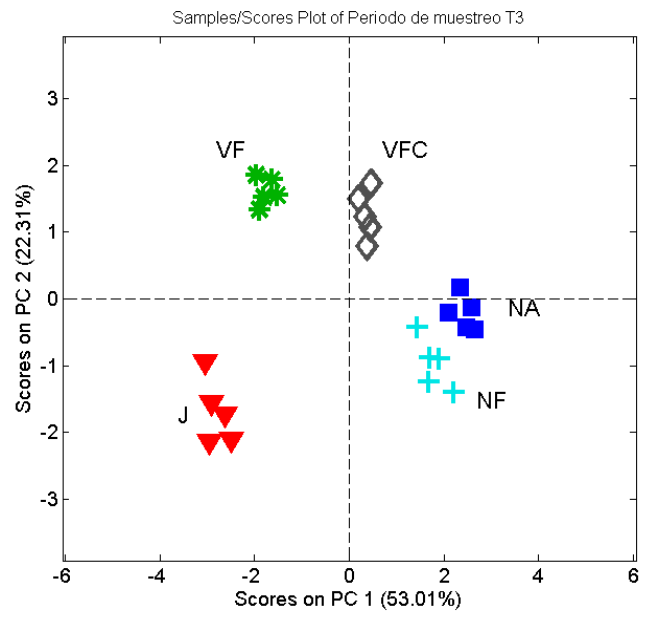

PCA periodo 2: 90 días.

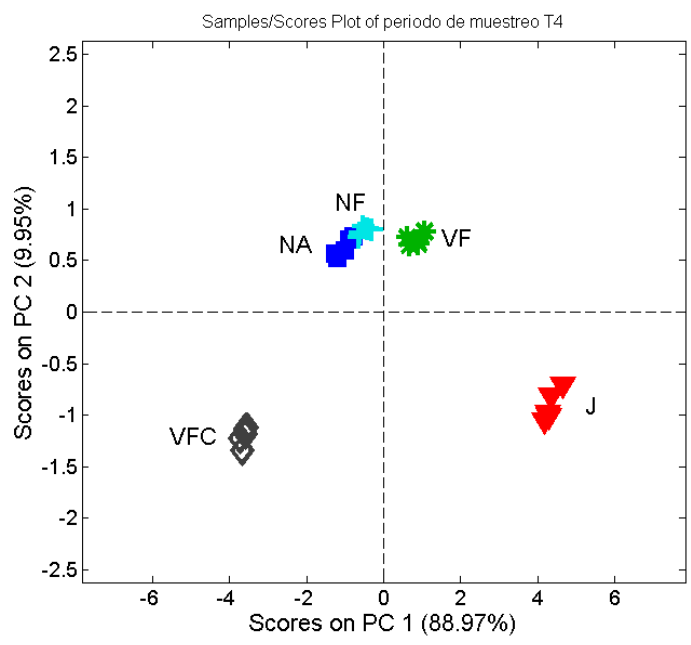

PCA periodo 3: 150 días.

PCA periodo 3: 210 días.

Figura 5.7. Análisis de componentes principales del conjunto de las medidas suministradas por los sensores resistivos (nariz electrónica).

Como se observa a nivel general en los diferentes PCAs, la presencia de madera y las diferentes configuraciones para la fermentación confiere a los vinos una composición química particular, que tiene un reflejo en la composición global de los volátiles. Esto permite discriminar los vinos fermentados en barricas de los de 
vinos fermentados en acero inoxidable. También se puede resaltar que los clústers son muy pequeños (lo que indica la elevada reproducibilidad de nuestra nariz electrónica.

En el caso de la primera de las evaluaciones realizadas (PCA periodo 1), las dos primeras componentes capturan el 57\% y 35\% de la varianza. Como se observa en esta primera figura, en el cuadrante superior derecho se encuentra localizada la muestra de vino fermentado en depósito de acero inoxidable (J, triangulo rojo); mientras que en el cuadrante izquierdo, se agrupan las muestras de vinos blancos fermentadas en las barricas que ya han sido utilizadas en otras ocasiones, con y sin adición de chips (VFC rombos grises y VF asteriscos verdes). Esto significa que con la información recogida por la PC1 podemos distinguir entre la fermentación de vinos blancos en depósitos de acero inoxidable y la fermentación en barrica. Respecto a la PC2, podemos distinguir los efectos en la composición global de los vinos de acuerdo con su fermentación en una barrica nueva o usada.

Para el segundo periodo de muestreo, es decir 90 días después del comienzo de la fermentación, la distribución de las muestras en el espacio descrito por las dos primeras PCs es similar al observado en la etapa anterior. En esta ocasión, las dos primeras componentes capturan aproximadamente el $98 \%$ de la información derivada de la red de sensores.

Continuando nuestro análisis para el tercer periodo de muestreo, que se refiere a los vinos una vez han transcurrido 150 días de haber dado comienzo al proceso de fermentación, cualitativamente se mantiene el perfil diferenciador entre vinos fermentados en barrica y el vino testigo fermentado en un depósito de acero inoxidable. Dentro de este gráfico, merece una especial mención la separación que comienza a establecerse respecto a la PC1 entre los vinos fermentados en barricas viejas; en este caso el vino fermentado en una barrica vieja con adicción de chips (VFC, rombos grises) parece presentar más características en común con los vinos fermentados en barricas nuevas (francés NF cruz color cian; americano NA cuadrado azul).

Finalmente, una vez han transcurrido 210 del proceso de fermentación los vinos presenta matices diferenciadores, las tendencias diferenciadoras entre clusters se mantienen. Destacar nuevamente el comportamiento de la muestra de vino blanco fermentado en una barrica de roble que ha sido utilizada en otras ocasiones, a la que además, se le adicionaron chips de roble para paliar el efecto del agotamiento de la madera. De esta manera, la red de sensores parece demostrar unas prometedoras cualidades en el ámbito de los vinos blancos, hecho indicativo de la versatilidad de este sistema de evaluación sensorial electrónico en la valoración de vinos de distinta naturaleza. Un próximo estudio 
con un grupo de muestras más amplio, nos permitiría abundar en la capacidad de este sistema para diferenciar cualidades globales en la fase volátil de los vinos.

\subsubsection{Análisis supervisado. PLS-DA}

Una investigación como la que hemos recogido hasta este punto también debe ser llevada a cabo para obtener una idea inicial acerca de la posibilidad de correlación entre procedimientos, considerando los análisis clásicos como métodos de referencia para nuestra red de sensores.

A partir de la experiencia arriba expuesta, podemos establecer a priori cuatro clases diferenciadas, definidas en función del periodo de evaluación (Tabla 5.4). En esta ocasión, dado el tipo y cantidad de muestras disponibles de cada clase, decidimos emplear PLS-DA como técnica de análisis multivariante de clasificación supervisada. Se observó una total clasificación de los vinos en función de los tiempos muestreos seguidos en esta investigación, capturando un $94 \%$ de la información contenida en el bloque $X$ y un $70 \%$ para el bloque $Y$ con las tres primeras VLs del modelo.

Con los resultados obtenidos hasta el momento se ha podido monitorizar el estado evolutivo del vino, caracterizar o reconocer de forma global las diferencias en función del tipo de fermentación empleado. Esto demuestra que tras dotar nuestro sistema de evaluación sensorial electrónico con un entrenamiento adecuado acorde con el problema particular que se plantee, potencialmente es una herramienta con múltiples posibilidades dentro de la industria enológica.

Tabla 5.4. PLS-DA para la clasificación de las muestras de vinos en función del tiempo que ha durado el proceso de fermentación de los vinos blancos.

\begin{tabular}{|l|l|l|l|l|}
\hline Parámetro & 30 dias & 90 días & 150 días & 210 días \\
\hline
\end{tabular}

\begin{tabular}{|l|l|l|l|l|l|}
\hline \multirow{2}{*}{ Sensitividad } & $\mathrm{c}$ & 0.920 & 1.000 & 1.000 & 1.000 \\
\cline { 2 - 6 } & $\mathrm{v}$ & 0.800 & 1.000 & 1.000 & 1.000 \\
\hline \multirow{2}{*}{ Especificidad } & $\mathrm{c}$ & 0.987 & 0.933 & 1.000 & 0.947 \\
\cline { 2 - 6 } & $\mathrm{v}$ & 1.000 & 0.933 & 1.000 & 0.947 \\
\hline \multirow{2}{*}{ Error clasificación } & $\mathrm{C}$ & 0.047 & 0.033 & 0 & 0.027 \\
\cline { 2 - 6 } & $\mathrm{v}$ & 0.1 & 0.033 & 0 & 0.027 \\
\hline \multirow{2}{*}{ RMSEC } & $\mathrm{C}$ & 0.207 & 0.309 & 0.11553 & 0.271 \\
\cline { 2 - 6 } & $\mathrm{v}$ & 0.506 & 0.531 & 0.503 & 0.520 \\
\hline \multirow{2}{*}{$\mathrm{R}^{\wedge} 2$} & $\mathrm{C}$ & 0.770 & 0.592 & 0.929 & 0.608 \\
\cline { 2 - 6 } & $\mathrm{v}$ & 0.754 & 0.544 & 0.921 & 0.573 \\
\hline
\end{tabular}

C: Calibración. V: Validación. 


\subsubsection{Regresión Lineal por mínimos cuadrados PLS2.}

Como punto final a esta investigación se ha realizado un modelo de calibración que nos permita saber si existe algún tipo de correlación entre las variables de un análisis cromatográfico (concentraciones de los compuestos aromáticos presentes en el vino) a partir de las medidas realizadas durante los cuatro periodos de muestreo seguidos en la fermentación con la nariz electrónica. Para ello, se ha realizado una correlación del tipo PLS2 entre las respuestas de los sensores de óxidos de metálicos y las concentraciones de los compuestos furfural, 5metilfurfural, guaicaol, cis-whikylactona, trans-whiskylactona y eugenol. En la Tabla 5.5 se enumeran estas variables y los coeficientes obtenidos para describir el modelo PLS2.

Tabla 5.5. Modelo PLS2 construido a partir de la respuesta de los sensores para estimar los compuestos orgánicos volátiles determinados por CG-MS para los cuatro periodos de muestreo valorados durante la fermentación.

Compuesto
\begin{tabular}{|l|l|l|l|l|}
\hline Furfural $[\mu \mathrm{g} / \mathrm{L}]$ & 11.7439 & 28.8751 & 0.842 & 0.363 \\
\hline 5-Metilfurfural $[\mu \mathrm{g} / \mathrm{L}]$ & 1.64327 & 4.91571 & 0.823 & 0.319 \\
\hline Guaiacol $[\mu \mathrm{g} / \mathrm{L}]$ & 0.0414689 & 0.0671166 & 0.399 & 0.193 \\
\hline Cis-whiskylactona $[\mu \mathrm{g} / \mathrm{L}]$ & 0.211119 & 0.499428 & 0.401 & 0.175 \\
\hline Trans-whiskylactona $[\mu \mathrm{g} / \mathrm{L}]$ & 1.69362 & 5.06426 & 0.786 & 0.261 \\
\hline Eugenol $[\mu \mathrm{g} / \mathrm{L}]$ & 0.0241212 & 0.112909 & 0.787 & 0.254 \\
\hline
\end{tabular}

Aunque la medida de las correlaciones a través del parámetro $\mathrm{R}^{\wedge} 2$ nos dice que existe una correlación aceptable de todos los compuestos volátiles seguidos en esta investigación, excepto el guaiacol, los resultados de la validación no confirman que el sistema sea capaz de predecir estos parámetros. Es posible que el reducido número de muestras empleado esté afectado la calidad del modelo PLS2, por tanto estos resultados pueden ser vistos como una primera aproximación para la aplicación de una nariz electrónica en el control de calidad de vinos blancos.

\subsection{CONCLUSIONES}

Esta investigación ha mostrado que la fermentación de vinos blancos usando barricas de roble permite mejorar características sensoriales de los vinos blancos, sin embargo estas nuevas características del vino no son fáciles de identificar 
usando técnicas de análisis tradicional y la tónica general de los enólogos es recurrir a métodos empíricos. Partiendo de estas ideas resaltamos como conclusiones más generales e importantes:

1. La nariz electrónica es un sistema objetivo, capaz de diferenciar los vinos blancos elaborados para esta investigación mediante los métodos de fermentación tradicional y métodos alternativos como la fermentación en barricas tanto nuevas como usadas.

2. La cromatografía de gases permitió describir la evolución de compuestos como el furfural, 5-metilfurfural y trans-whiskylactona y su importancia en la fermentación en barrica. Los vinos blancos envejecidos en barricas nuevas presentan siempre una mayor concentración de estos compuestos.

3. El incremento en la cantidad de cada uno de los volátiles de la madera no indicó un incremento en la intensidad de los aromas relacionados con la madera y sus afines; sin embargo este proceso favorece la integración de compuestos volátiles así como la disminución de características lácteas y herbáceas de los vinos.

4. Los anteriores resultados abren muchas y futuras posibilidades entorno a la aplicación de una nariz electrónica en el control de calidad de vinos blancos.

\subsection{REFERENCIAS}

[1] J. E. Bueno, R. A. Peinado \& M. Medina, Effect of a short contact time with lees on volatile composition of Airen and Macabro wines, Biotechnology Letters, 2006, 28, 1007-1011.

[2] M. Ortega-Heras, C. González-Huerta, P. Herrera \& M. L. González-Sanjosé, Changes in wine volatile compounds of varietal wines during ageing in wood barrels, Analytica Chimica Acta, 2004, 513, 341-350.

[3] P. Pontallier, Practiques Actuelles D’Elevage En Barriques Des Grands Vins Rouges, Journal International Des Sciences De La Vigne Et Du Vin, 1991, 16, 115-122.

[4] N. Vivas \& Y. Glories, Role of oak wood ellagitanins in the oxidation process of red wines during aging, American Journal of Enology and Viticulture, 1996, 47, 103.

[5] P. Chatonnet, D. Dubourdieu \& J. N. Boidron, Incidence of fermentation and aging conditions of dry white wines in barrels on their composition in substances yielded by oak wood, Sciences Des Aliments, 1992, 12, 665-685.

[6] J. S. Camara, M. A. Alves \& J. C. Marques, Evolution of oak-related volatile compounds in a Spanish red wine during 2 years bottled, after aging in barrels made of Spanish, French and American oak wood, Analytica Chimica Acta, 2006, 563, 189-203. 
[7] M. E. Alanon, L. Castro-Vazquez, M. C. Diaz-Maroto, I. Hermosin-Gutierrez, M. H. Gordon \& M. S. Perez-Coello, Antioxidant capacity and phenolic composition of different woods used in cooperage, Food Chemistry, 2011, 129, 1584-1590.

[8] I. Ruzic, M. Skerget, Z. Knez \& M. Runje, Phenolic content and antioxidant potential of macerated white wines, European Food Research and Technology, 2011, 233, 465-472.

[9] A. M. Alonso, D. A. Guillén, C. G. Barroso, B. Puertas \& A. García, Determination of of antioxidant activity of wine by products and its correlation with with polyphenolic content, Journal of Agricultural and Food Chemistry, 2002, 50, 5832-5838.

[10] H. Aoshima, H. Tsunoue, H. Koda \& Y. Kiso, Aging of whiskey increases 1,1-diphenyl-2picrylhydrazyl radical scavenging capacity, Journal of Agricultural and Food Chemistry, 2004, 52, 5240-5244.

[11] J. A. Avila-Reyes, N. Almaraz-Abarca, E. A. Delgado-Alvarado, L. S. Gonzalez-Valdez, G. V. del Toro \& E. D. Paramo, Phenol profile and antioxidant capacity of mescal aged in oak wood barrels, Food Research International, 2010, 43, 296-300.

[12] S. Cañas, V. Casanova \& A. P. Belchior, Antioxidant activity and phenolic content of Portuguese wine aged brandies, Journal of Food Composition and Analysis, 2008, 21, 626-633.

[13] C. Da Porto, S. Calligaris, E. Celotti \& M. C. Nicoli, Antiradical properties of commercial cognacs assessed by the DPPH test, Journal of Agricultural and Food Chemistry, 2000, 48, 42414245.

[14] J. A. Larrauri, C. Sánchez-Moreno, P. Rupérez \& F. Saura-Calixto, Free radical scavenging capacity in the ageing of selected red Spanish wines, Journal of Agricultural and Food Chemistry, 1999, 47 1603-1606.

[15] C. Munoz-Gonzalez, M. Victoria Moreno-Arribas, P. J. Martin-Alvarez, E. Bartra-Sebastian, A. Puig-Pujol, J. Garcia-Cazorla \& M. Angeles Pozo-Bayon, Volatile and sensory characterization of Xarel.lo white wines, Flavour and Fragrance Journal, 2011, 26, 153-161.

[16] M. S. Perez-Coello, M. A. Gonzalez-Vinas, E. Garcia-Romero, M. D. Cabezudo \& J. Sanz, Chemical and sensory changes in white wines fermented in the presence of oak chips, International Journal of Food Science and Technology, 2000, 35, 23-32.

[17] M. A. Sefton, P. J. Spillman, K. J. Pocok, Francis Il \& P. J. Williams, The influence of oak origin, seasoning, and other industry practices on the sensory characteristics and composition of oak extracts and barrel-aged white wines, Australian Grapegrower \& Winemaker, 1993, 335.

[18] H. Guth, Quantitation and sensory studies of character impact odorants of different white wine varieties. , Journal of Agricultural and Food Chemistry 1997, 45, 3027-3032.

[19] M. J. Cejudo-Bastante, I. Hermosin-Gutierrez, L. I. Castro-Vazquez \& M. S. Perez-Coello, Hyperoxygenation and Bottle Storage of Chardonnay White Wines: Effects on Color-Related Phenolics, Volatile Composition, and Sensory Characteristics, Journal of Agricultural and Food Chemistry, 2011, 59, 4171-4182.

[20] N. Vivas, Manuel de tonnellerie a l’usage des utilisateurs de futaille, Féret Éditions, France, 2002.

[21] T. L. Babayan \& M. G. Bezrukov, Autolysis in yeast, Acta Biotechnology, 1985, 5, 129-136. 
[22] M. A. Ducasse, R. M. Canal-Llauberes, M. D. Lumley, P. Williams, J. M. Souquet, H. Fulcrand, T. Doco \& V. Cheynier, Effect of macerating enzyme treatment on the polyphenol and polysaccharide composition of red wines, Food Chemistry, 2010, 118, 369-376.

[23] F. Palomero, A. Morata, S. Benito, M. C. González \& J. A. Suárez-Lepe, Conventional and enzyme-assisted autolysis during ageing over lees in red wines: Influence on the release of polysaccharides from yeast cell walls and on wine monomeric anthocyanin content Food Chemistry, 2007, 105, 838-846.

[24] S. Lubbers, B. Lèger, C. Charpentier \& M. Feuillat, Effect colloide proteteur d'extraits de parois de levures sur la stabilitè tartrique d'une solution hydroalcoololique modèle, Journal international des sciences de la vigne et du vin, 1993, 27, 13-22.

[25] E. J. Waters, W. Wallace, M. E. Tate \& P. J. Williams, Isolation and partial characterization of a natural haze protective factor from wine, Journal of Agricultural and Food Chemistry, 1993, $41,724-730$.

[26] R. Del Barrio-Galan, M. Ortega-Heras, M. Sanchez-Iglesias \& S. Perez-Magarino, Interactions of phenolic and volatile compounds with yeast lees, commercial yeast derivatives and non toasted chips in model solutions and young red wines, European Food Research and Technology, 2012, 234, 231-244.

[27] W. Pan, D. Jussier, N. Terrade, R. Y. Yada \& R. M. de Orduna, Kinetics of sugars, organic acids and acetaldehyde during simultaneous yeast-bacterial fermentations of white wine at different $p H$ values, Food Research International, 2011, 44, 660-666.

[28] D. Chassagne, M. Guilloux-Benatier, H. Alexandre \& A. Voilley, Sorption of wine volatile phenols by yeast lees, Food Chemistry, 2005, 91, 39-44.

[29] N. Jiménez Moreno \& C. Ancìn Azpilicueta, Binding of oak volatile compounds by wine lees during simulation of wine ageing, LWT - Food Science and Technology, 2007, 40, 619-624.

[30] M. Maciejewska, A. Szczurek \& Z. Kerényi, Utilisation of first principal component extracted from gas sensor measurements as a process control variable in wine fermentation, Sensors and Actuators B-Chemical, 2006, 115, 170-177.

[31] T. Rieger, Chips, staves, shaves, and other oak alternatives to traditional barrel use, Viney wine magnament, 1993, 19, 35-40.

[32] J. L. Puech, P. Rabier \& M. Moutounet, Principles of preparation and chemical composition of commercial oak wood extracts, (Ed. R. E. Kreibich), Proceedings of the 6th International Flavor Conference, Crete, Greece, 1990, pp. 159-168.

[33] J. L. Puech, C. Jouret, B. Goffinet \& (1985) Sci. Aliment., Evolution des composés phénoliques du bois de chêne au cours du vieilissement de l'Armagnac. , Sciences des Aliments 1985, 5, 379-391.

[34] J. L. Puech, Extraction of phenolic compounds from oak wood in model solutions and evolution of aromatic aldehides in wines aged in oak barrels., American Journal of Enology and Viticulture, 1987, 38, 236-238.

[35] I. M. Skurikhin \& B. N. Efimov, Aldéhydes aromatiques et alcools des alcools de Cognac, Bases biotechnologies 1972, 147-156.

[36] P. Chatonnet, J. N. Boidron \& D. Dubourdieu, Journal International des Sciences de la Vigne et du Vin, Nature et evolution de la microflore du bois de chêne au cours de son séchage et de son vieillissement a L`Air Libre, 1994, 28, 185-201. 
[37] E. Joseph \& M. Marché, Contribution à l étude du vieillissement du cognac, Connaisance Vigne Vin, 1972, 6 273-330.

[38] M. Moutounet, P. Rabier, J. L. Puech, E. Verette \& J. M. Barillene, Analysis by HPLC of extractable substances in oak wood, Sciences des Aliments, 1989, 9, 35-51.

[39] J. R. Mosedale \& J. L. Puech, Barrels. Wines, Spirits, and Other Beverages., UMR Sciences Pour L'oenologie. , Montpellier, France, 2003, pp. 393-403.

[40] B. W. Zoecklein, K. C. Fugelsang, B. H. Gump \& F. S. Nury, Análisis y produccion de vino, Acribia 2001.

[41] J. M. Álvarez, Tanino: La revolución enológica, mito o realidad. , Enología, 2007, 1-15.

[42] V. Ferreira, I. Jarauta \& J. Cacho, Physicochemical model to interpret the kinetics of aroma extraction during wine aging in wood. Model limitations suggest the necessary existence of biochemical processes, Journal of Agricultural and Food Chemistry, 2006, 54, 3047-3054.

[43] C. A. Azpilicueta, N. Jiménez \& A. González, Evolución de compuestos volátiles y de aminas biógenas en vinos envejecidos en barricas de roble, Enología, 2007, 4.

[44] P. Chatonnet, Discrimination and control of toasting intensity and quality of oak wood barrels, American Journal of Enology and Viticulture, 1999, 50, 479-494.

[45] S. M. Rocha, P. Coutinho, E. Coelho, A. S. Barros, I. Delgadillo \& M. A. Coimbra, Relationships between the varietal volatile composition of the musts and white wine aroma quality. A four year feasibility study, Lwt-Food Science and Technology, 2010, 43, 1508-1516.

[46] J. E. Welke, M. Zanus, M. Lazarotto, K. G. Schmitt \& C. A. Zini, Volatile Characterization by Multivariate Optimization of Headspace-Solid Phase Microextraction and Sensorial Evaluation of Chardonnay Base Wines, Journal of the Brazilian Chemical Society, 2012, 23, 678-687.

[47] M. T. Liberatore, S. Pati, M. A. Del Nobile \& E. La Notte, Aroma quality improvement of Chardonnay white wine by fermentation and ageing in barrique on lees. , Food research international., 2010, 43, 996-1002.

[48] E. Sartini, G. Arfelli, A. Fabiani \& A. Piva, Influence of chips, lees and micro-oxygenation during aging on the phenolic composition of a red Sangiovese wine Food Chemistry, 2007, 104, 1599-1604. 



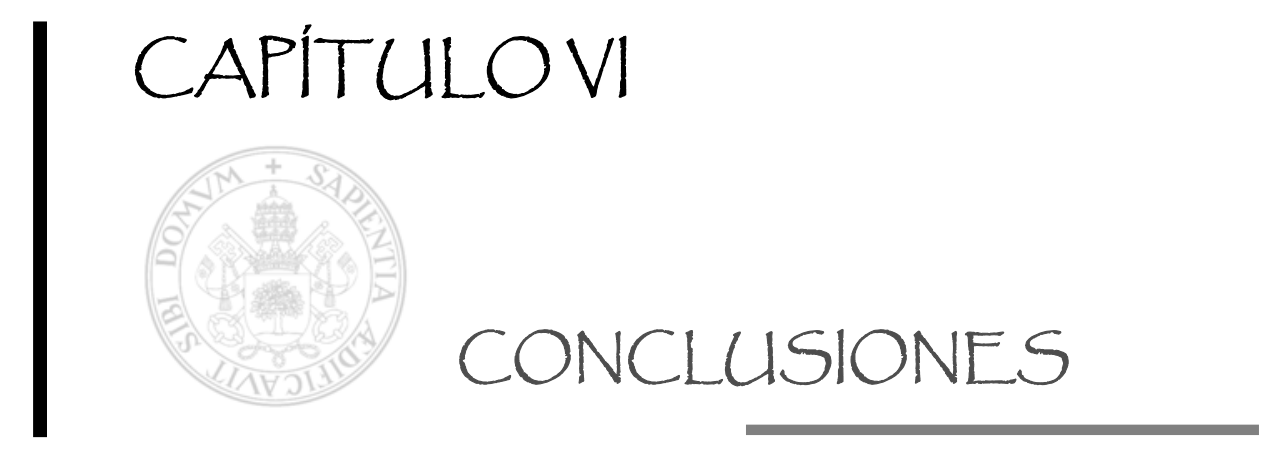

Página

6.1. CONCLUSIONES 



\subsection{CONCLUSIONES}

Esta tesis proporciona una descripción detallada sobre la implementación de nuevos métodos de análisis de datos en el campo de los sistemas de evaluación sensorial electrónico, los cuales entregaron soluciones fiables, robustas, y lo más importante adecuadas y adaptadas concretamente a la necesidad específica del problema de partida.

Además, en el desarrollo de esta tesis se ha dado igual importancia a la aplicación de los métodos quimiométricos tradicionales. De esta manera se ha evaluado y demostrado el potencial que tienen la nariz y lengua electrónica diseñadas en nuestro grupo de investigación para el control de calidad y caracterización organoléptica de vinos.

A continuación citaremos las principales conclusiones derivadas de la implementación y de la aplicación de estos sistemas de evaluación sensorial electrónicos a algunas problemáticas actuales en la industria vitivinícola.

Una descripción más detallada de estas conclusiones aparece al final de cada capítulo.

Conclusiones en el ámbito de las técnicas analiticas para la evaluación de compuestos volátiles orgánicos

$\checkmark$ Hemos demostrado que la metodología experimental diseñada en este grupo de investigación específicamente para el análisis de vinos, nos 
permite asegurar que se ha conseguido el desarrollo de una herramienta (hardware + software) de caracterización de aromas eficiente y objetiva.

$\checkmark$ Se ha realizado un análisis de la composición volátil de todos los vinos mediante la técnica analítica GC-MS, una tarea compleja debido a la gran cantidad de compuestos volátiles con diferentes propiedades químicas y organolépticas que conforman cada matriz. Se han seleccionando y evaluado un conjunto de compuestos característicos representativos de los aromas vinculados con el envejecimiento en madera. Esta técnica nos ha servido además como referente para evaluar la capacidad de detección de la nariz electrónica y nos ha aportado información valiosa para la posterior búsqueda de correlaciones entre sistemas de análisis.

$\checkmark$ Una combinación de la separación cromatográfica y posterior análisis olfatométrico nos ha permitido clasificar los compuestos químicos volátiles con mayor incidencia en la apreciación sensorial humana.

$\checkmark$ Una extrapolación de los métodos quimiométricas utilizados en nuestra investigación ha sido recoger experiencias desarrolladas en una investigación paralela de nuestro grupo (lengua electrónica) con algunas de las muestras analizadas con la nariz electrónica y ello ha complementado en manera importante los resultados de ambas investigaciones.

\section{Conclusiones en el ámbito de las experiencias analizadas}

La aplicación de este dispositivo electrónico ha permitido discriminar, clasificar y reconocer, mediante técnicas multivariantes no supervisadas (PCA) y supervisadas (PLS-DA) diferentes muestras de vino caracterizadas por poseer una amplia gama de atributos y matices organolépticos. Entre las series de muestras y parámetros diferenciadores, se han estudiado los siguientes problemas:

$\checkmark$ Vinos tintos elaborados con la misma variedad de uva pero vinificados con distintas técnicas de extracción (distinto contenido en polifenoles), diferentes grados de microoxigenación y envejecidos en botella con tapones sintéticos de diferente porosidad. 
Vinos elaborados con el mismo tipo de uva pero sometidos a dos proceso de envejecimiento diferente: la tradicional barrica de roble y los sistemas alternativos como el uso de depósito de acero inoxidable con inserción de madera con diferentes formas y tamaños. Para ambos caso se comparó también el efecto del origen de la madera, utilizando en este caso madera de roble americano y roble francés.

Vinos blancos fermentados en barricas nuevas y utilizadas. En el caso de las barricas usadas también se evaluó el efecto de la adición de chips de roble.

También indicar que las respuestas obtenidas con los prototipos de nariz y lengua electrónica dieron lugar a un alto índice de correlación con diversos parámetros físico-químicos, cromáticos y sensoriales, obtenidos mediante métodos estándar de análisis.

La combinación de información derivada del ojo, nariz y lengua electrónicos aporta mejores resultados respecto a los parámetros de cada uno de los modelos quimiométricos mencionados anteriormente. Además esta metodología ofrece ventajas en comparación con las técnicas analíticas tradicionales porque permite la caracterización organoléptica global de numerosos tipos de muestras industriales, las cuales pueden ser realizadas de manera más rápida, sencilla y objetiva.

\section{Conclusiones en el ámbito de nuevos tratamientos de datos aplicados a la nariz y lengua electrónica}

Se ha aplicado el método Tucker3 a los datos resultantes de la nariz electrónica, la GC-MS y la espectroscopía UV-visible. Este modelo difiere notablemente de los hasta ahora implementados en el área de las narices electrónicas, ofreciendo una interesante manera de visualizar las relaciones entre las respuestas de los sensores y la composición química de los diferentes tipos de vinos durante el proceso de maduración. La aplicación de este método, ofrece modelos más simples que utilizan menos parámetros, y que aportan más información sobre todo el conjunto de medidas que se realizaron.

Las conclusiones derivadas de este modelo no pueden generalizarse en vista del número de muestras relativamente bajo que se han utilizado. Sin embargo, en base a los valiosos resultados reportados en esta primera exploración, estudios futuros con un mayor número de muestras 
permitirán una comprensión más profunda y más amplia de las bondades de la aplicación de este método multi-way a los resultados procedentes de una red de sensores.

$\checkmark$ A través de la implementación método ANOVA-PLS, se ha presentado una posible solución al problema que implica tener un mínimo conjunto de muestras cuando se trabaja con datos multivariantes como los derivados de una red de sensores. De esta manera, se han combinando las bondades de dos métodos tradicionales en la quimiometría, un ANOVA que nos ha permitido evaluar el grado de significación de una variable para un factor de interés y las regresiones lineales por mínimos cuadrados que nos ha permitido evaluar la influencia de cada variable en la diferenciación de las muestras evaluadas.

Con este método ha sido posible analizar la tendencia entre los datos con una menor cantidad de experimentos. Al mismo tiempo se ha estimado, por una parte la capacidad de diferenciar entre el tipo de envejecimiento de las muestras evaluadas y su evolución durante este proceso, y de manera complementaria, las correlaciones existentes entre la nariz electrónica y los sistemas analíticos. Los resultados de este análisis fueron además coherentes con algunos de los derivados de la aplicación del método Tucker3.

$\checkmark$ Respecto a la lengua electrónica, dispositivo que genera matrices de datos con una gran cantidad de variables, decir que los resultados que se presentan en esta tesis demuestran que el número de variables puede ser reducidos sin perder información a través de la aplicación de un algoritmo genético acoplado a una regresión lineal.

En este sentido, se ha estudiado el efecto de diferentes métodos de preprocesamiento y se ha realizado una selección de características de los voltamogramas, consiguiendo mejorar así la discriminación visual entre muestras. A diferencia de los métodos tradicionales, el algoritmo PLS-GA que se ha aplicado en esta investigación permite analizar y seleccionar la información más relevante de la señal en un determinado punto y en la región alrededor del mismo. De esta manera ha sido posible seleccionar justamente la región de los voltamogramas relativa a la actividad electroquímica de los polifenoles del vino. En las futuras aplicaciones de la lengua electrónica, el método PLS-GA favorecerá un modelado de datos más simple, con menor ruido y con mayor capacidad para distinguir entre las muestras evaluadas. Es por ello que creemos que esta novedosa técnica tiene gran cabida dentro de la electroquímica. 


\section{Conclusiones en el ámbito de la enología}

Uno de los aspectos que destacan en esta Tesis Doctoral es la cuidadosa elección, elaboración a nivel industrial y caracterización de todas las muestras vino, tanto desde el punto de vista analítico, con la medida de los parámetros estándar y la cuantificación de sus compuestos volátiles, como el de la valoración sensorial derivada de la olfatometría. Todos estos resultados además fueron comparados y correlacionados con los derivados de los sistemas de evaluación sensorial electrónico. Entre las conclusiones más importantes relacionadas con las distintas problemáticas actuales del sector enológico tratadas en esta Tesis, podemos decir que:

Durante las últimas décadas, se han logrado importantes avances en la utilización de oxígeno durante la elaboración del vino, sin embargo el papel del oxígeno una vez los vinos han sido embotellados sigue siendo un desafío. El análisis estadístico de los datos obtenidos en el presente estudio demostró que existe un efecto significativo del OTR a través del tapón sintético en la evolución de los compuestos fenólicos y algunos cambios asociados de color de los diferentes vinos analizados.

En este sentido, podemos afirmar que es posible diseñar un tapón sintético que permita satisfacer las necesidades del enólogo en la elaboración del vino. En opinión de los fabricantes de estos corchos sintéticos cada vino debería tener una adecuada porosidad en el tapón. A través de la caracterización estructural de los tapones sintéticos que se producen en un proceso de co-extrusión del polietileno de baja densidad, hemos establecido una relación entre la cristalinidad de la matriz polimérica y el OTR, de manera que a medida que la cristalinidad del material incrementa, los valores del OTR decrecen.

$\checkmark$ Respecto a la investigación relacionada con el envejecimiento de vinos con métodos alternativos, los resultados hasta aquí alcanzados ponen claramente de manifiesto que es un proceso muy complejo, aún de difícil explicación. En nuestra opinión, el uso de métodos alternativos no debería estar orientando hacia la reproducción exacta del proceso que tiene lugar durante el envejecimiento en barrica, su enfoque debería estar más bien relacionado con el desarrollo de una herramienta complementaria, aprovechando además la diversidad de productos y posibilidades a las que se han llegado hoy día en este campo. Estamos ante una nueva tecnología para envejecer el vino, con ciertas ventajas (económicas) que permite obtener interesantes resultados tanto en el 
plano técnico como en el organoléptico. Es por otra parte un reto a nivel científico el avanzar en el conocimiento de esta alternativa.

Específicamente, los valores obtenidos a partir de la cromatografía de gases-masas para los compuestos aromáticos furfural, guaiacol, cis,transwhiskylactona y eugenol han demostrado que la trans-whiskylactona podría ser utilizada para distinguir entre vinos envejecidos en barricas o en depósitos de acero inoxidable con inserción de madera. Esta conclusión no pretende asegurar que realizando un seguimiento a un único compuesto en el vino podríamos detectar a que tipo de envejecimiento ha sido sometido. Se trata más bien de afirmar que la identificación de este compuesto como marcador del envejecimiento se convierte en una herramienta importante para la aplicación de dispositivos como la nariz electrónica en el control de calidad de vino debido a las excelentes correlaciones existentes y demostradas en el desarrollo de esta tesis.

$\checkmark \quad$ El estudio realizado sobre la fermentación de vinos blancos en barricas de roble confirman el importante efecto que tiene la interacción del vino blanco con la madera. Además, abre interesantes opciones acerca de la posibilidad de paliar el efecto del agotamiento de la barrica con la inserción de trozos de madera (chips). Estas experiencias constituyen un punto de partida de futuras investigaciones. 